\title{
ATTITUDES TO FOREIGN LANGUAGE LEARNING IN NEW ZEALAND SCHOOLS
}

\author{
Sandra Shearn
}

A thesis submitted for the degree of

Doctor of Philosophy in Linguistics

School of Linguistics \& Applied Language Studies

Victoria University of Wellington

Wellington, New Zealand 
VICTORIA UNIVERSITY OF WELLINGTON 
This thesis examines attitudes towards the learning of languages other than English and Maori among New Zealand school students in years 8 and 9, parents of year 9 students, and a wide range of teachers. The research examined the extent to which participants subscribed to certain commonly held views about second language learning, for example: that it is too hard for most students, that it serves no purpose for future employment, that languages are 'girls' subjects', and so on. The investigation adopted a theoretical framework derived chiefly from the social psychological literature concerning language learning attitudes and motivation.

Students were surveyed by means of questionnaires over two successive years in the same part of the country, so that it was possible to discover if the intentions of the year 8 students to study a foreign language when they started secondary school were carried out. Parents and teachers were interviewed to discover their experience of foreign language learning and their thoughts about its place in New Zealand schools and in their children's education. The findings are set against detailed information about each of the seven schools involved, the place of languages in the official curriculum framework and the Ministry of Education's efforts to promote language learning. For comparison, information is also presented on the recent history and current status of foreign language learning in the United Kingdom, the United States of America and Australia.

It was found that attitudes towards foreign language learning, of both adults and children, were mostly positive. Although many teachers were pessimistic about the views of their colleagues and students' parents, the majority of all the adults believed that language learning was desirable and possible for all or most students for a range of reasons. The majority also supported an earlier start to language learning, most favouring year 7 . The findings suggest that the main reason that the proportion of students starting a foreign language in year 9 remains around $50 \%$, and that retention rates in subsequent years continue to drop, is that languages are optional for most secondary students. This research found that choosing to study a language often meant sacrificing other subjects which students would like to have tried, and thus depended on strong intrinsic motivation. Although no participants claimed that language learning was more suitable for girls, it was found that the majority of students who opted for, and continued, language learning were girls, that boys tended to prefer practical subjects, and that, in the case of one secondary school, the minority of boys who were permitted to start a foreign language were discouraged from continuing by the general organisation and ethos of the school.

Ultimately, the research indicated that attitudes towards foreign language learning in schools involved a complex web of factors. External factors often outweighed even the most positive attitudes among students, parents and teachers when option subjects were chosen. The low level of language learning in New Zealand, contrasted with the importance it has in comparable countries, was shown to result not so much from negative attitudes but rather from barriers within the education system as a whole and individual school cultures. 


\section{Kiwis abroad...}

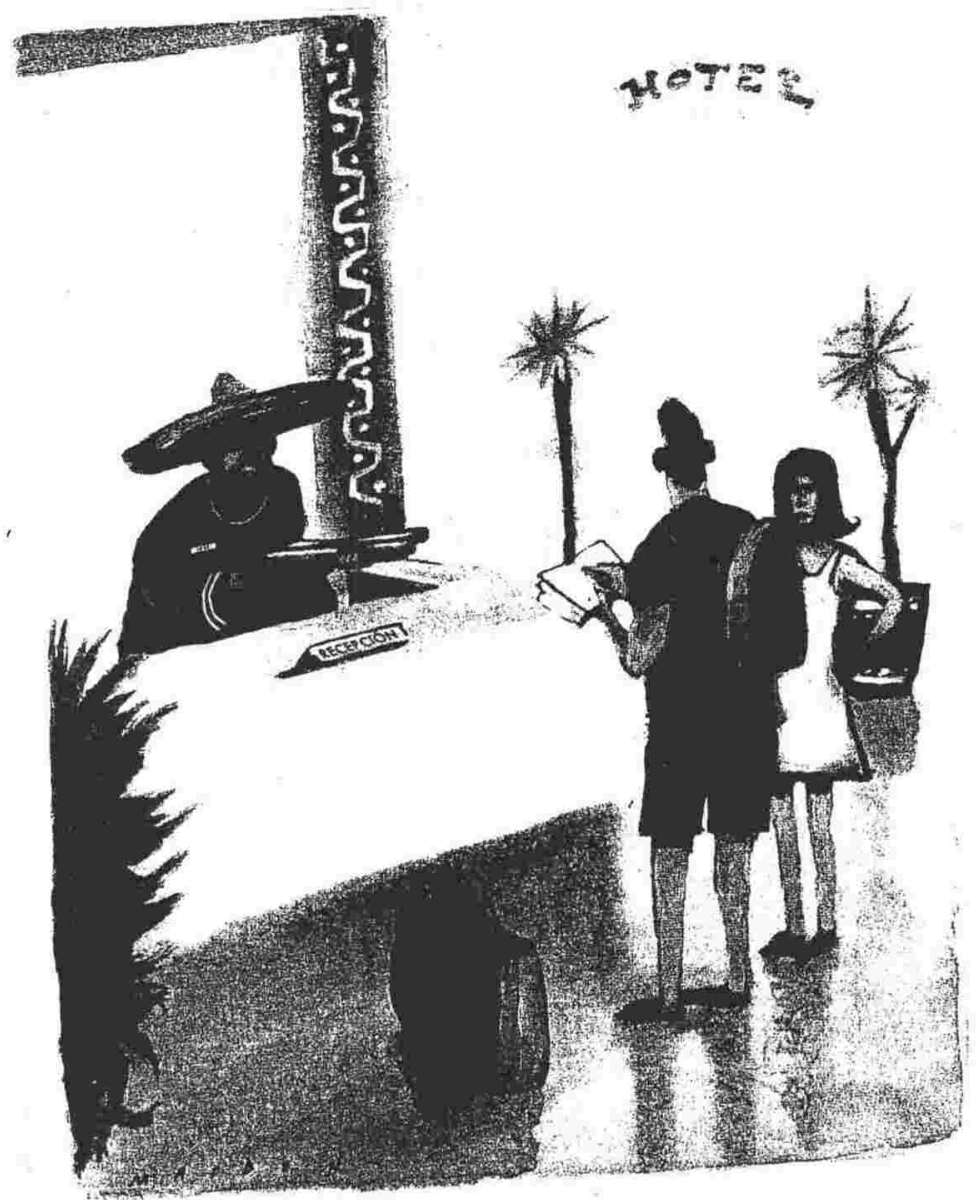

It was fast becoming clear to Sue that Jim's schoolboy Spanish wasn't going to cut the mustard. 


\section{ACKNOWLEDGEMENTS}

My grateful thanks go to all the schools, students and parents who participated in my pilot and final research projects, especially to the teachers, whose work pressures I well understand, who helped organise my visits or gave up precious free time to be interviewed.

I am also grateful for the help and encouragement I have received from past and present colleagues in the education field, especially those engaged in promoting language learning; my thanks to Sylvia Burch, Elizabeth Collins, Barbara Fitzsimons, Judith Geare at the Goethe Institute, Noeline Grant, Jo Guthrie and Catherine Hannigan from the language advisory service, Jenny O'Brien and Gail Spence at the Ministry of Education, Jean-Marc Philippeau at the French Embassy and Frances Salt at the Education Review Office.

At Victoria University I was supported by both academic and administrative staff in the School of Linguistics and Applied Language Studies, and by the Scholarships Officer, Maureen Penning. In the esoteric realm of statistics, I received invaluable advice from Megan Clark, Brian Dawkins and Edith Hodgen.

I thank the New Zealand Vice-Chancellors' Committee for the award of a Claude McCarthy Fellowship in 2001, which allowed me to spend five weeks carrying out valuable research, with hospitable support from Professor Heather Bowe, at Monash University. This enabled me to benefit from the time and expertise of Professor Michael Clyne at the University of Melbourne, Anne Eckstein at the Department of Education, Employment and Training, Dr. Margaret Gearing in the Education Department, Monash University, Madeleine Jenkins at the Victorian Curriculum and Assessment Authority, Elina Raso at the Catholic Education Office, and Judy Oakes at the Association of Independent Schools in Victoria.

I should also like to thank all my friends and fellow students, too numerous to name, who have been unfailing in their support and encouragement, but in particular Hilary Smith, Steve Haslett, Brenda Morrison, Murray Douglas, Meredith Marra and Alison McLeod. Loving thanks to my father, Norman Shearn, who has constantly been concerned for my financial wellbeing and ready to help, although never quite understanding why I gave up the security of a school teaching career.

Lastly, my greatest thanks are due to Professor Janet Holmes, who stimulated my initial interest in linguistics and has continued to mentor, encourage and challenge my studies. Without her I would not have pursued an MA, let alone contemplated embarking on a doctorate which, furthermore, I could not have undertaken without her support for my successful application for a Victoria University postgraduate scholarship. Merci beaucoup! 
CONTENTS

LIST OF APPENDICES X XV

LIST OF TABLES XVi

LIST OF GRAPHS AND FIGURES $\quad$ xxi

GLOSSARY Xxiii

\section{CHAPTER 1 Introduction}

1.1 Languages in New Zealand 1

1.2 Definitions of relevant terms 2

1.3 Languages in the New Zealand education system 2

1.4 Ministry of Education policy on languages $\quad 7$

1.5 Other views of foreign language learning (FLL) in schools 8

1.6 The aims of this research 9

\section{CHAPTER 2 Literature review}

2.1 Introduction 11

2.2 The nature and importance of attitudes 11

2.3 Attitudes towards languages $\quad 12$

2.4 Ethnocentrism, ethnolinguistic identity and ethnolinguistic vitality 13

2.5 Attitudes towards language learning $\quad 14$

2.6 Attitudes towards FLL 15

2.7 Attitudes and motivation 16

2.8 Motivation - beyond Gardner 19

2.9 Gender differences in attitudes towards FLL

2.9.1 Females dominate FLL 22

2.9.2 Girls enjoy FLL more than boys 23

2.9.3 Girls are more open to other languages and cultures 24

2.9.4 FLL is less relevant for boys $\quad 25$

2.9.5 Languages are more difficult for boys 25

2.9.6 Summary of gender differences 26

2.10 FLL in New Zealand

2.10.1 Official attitudes $\quad 26$

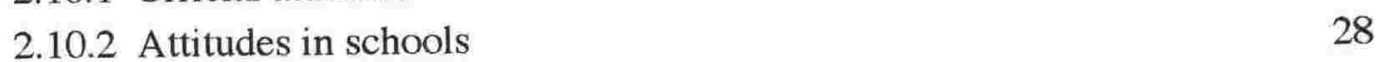

2.10.3 The attitudes of FL teachers $\quad 30$

2.10.4 The influence of gender $\quad 32$

2.11 The importance of languages for trade and tourism 32

2.12 Hypotheses to be tested 36

2.12.1 Reasons for choosing FLL 36

2.12.2 Reasons for not choosing FLL 36

2.12.3 Gender differences 36

2.12.4 Parental influence $\quad 37$

2.12.5 The influence of prior experience $\quad 37$

$\begin{array}{ll}\text { 2.12.6 The role of attitudes in students choices } & 37\end{array}$ 
CHAPTER 3 Foreign language education in England, the United States of America and Australia

3.1 Introduction

3.2 Foreign language education in England

3.2.1 Some history

3.2.2 Languages taught

3.2.3 Resistance to language learning

3.2.3.1 The choice of languages

3.2.3.2 The spread of English

3.2.3.3 Foreign languages are not 'useful' $\quad 41$

3.2.3.4 Lack of room for language learning $\quad 42$

3.2.3.5 The narrow nature of language learning 42

3.2.3.6 Language pedagogy $\quad 42$

3.2.3.7 Low achievement $\quad 42$

3.2.3.8 Low priority in schools $\quad 43$

3.2.4 Consequences $\quad 43$

3.2.5 Primary FLL 43

3.2.6 The National Curriculum $\quad 43$

3.2.7 The 1990s and beyond $\quad 45$

3.2.8 The Nuffield Inquiry $\quad 46$

3.2.8.1 "English is not enough." 46

3.2.8.2 "The UK needs competence in many FLs." 47

3.2.8.3 "Government has no coherent approach to languages." 47

3.2.8.4 "Secondary school pupils lack motivation or direction." 48

3.2.8.5 "People are looking for leadership to improve the nation's capability in languages." 49

3.2.8.6 "In spite of parental demand, there is still no UK-wide agenda for children to start FLs early." 49

3.2.9 The Nuffield Inquiry's recommendations $\quad 50$

3.2.10 The government response $\quad 50$

3.2.11 Specialist language colleges $\quad 50$

3.2.12 Other initiatives $\quad 51$

3.2.13 Summary 51

3.3 Foreign language education in the United States of America

3.3.1 Introduction 51

3.3.2 The state of FLL in the 1970s 52

3.3.3 The role of FL teachers $\quad 52$

3.3.4 The decline continued 53

3.3.5 The Presidential Commission $\quad 53$

3.3.6 The Commission's findings $\quad 54$

3.3.7 The Commission's recommendations $\quad 54$ 
vi

3.3.8 Reactions to the Presidential Commission's recommendations 55

3.3.9 Progress in the 1980s 55

3.3.10 Attitudes to FLL 56

3.3.11 Funding for FLL $\quad 57$

3.3.12 Languages at tertiary level 57

3.3.13 The 'English Only' movement 58

3.3.14 FLL in the 1990s 58

3.3.15 Development of content and achievement standards 60

3.3.16 Encouragement of bilingual education 61

3.3.17 Ongoing problems 61

$\begin{array}{ll}3.3 .18 \text { Summary } & 62\end{array}$

3.4 Foreign language education in Australia

3.4.1 The development of a national language policy 63

$\begin{array}{ll}3.4 .2 \text { National education policy } & 64\end{array}$

3.4.3 Current national goals for schooling 65

3.4.4 Current national funding for LOTEs $\quad 65$

3.4.5 Developments at state level 66

3.4.6 The reality behind the policies $\quad 66$

3.4.6.1 Changeability of policy 66

3.4.6.2 Lack of clear or realistic objectives $\quad 67$

3.4.6.3 Why LOTEs? $\quad 68$

3.4.6.4 Which LOTEs?

3.4.6.5 Lack of commitment to bilingualism $\quad 70$

$\begin{array}{ll}\text { 3.4.6.6 Teacher supply and training } & 71\end{array}$

$\begin{array}{ll}\text { 3.4.6.7 Other problems } & 73\end{array}$

3.4.7 Some indicators of what has been achieved in schools

$\begin{array}{ll}\text { 3.4.7.1 Victoria } & 73\end{array}$

3.4.7.2 Other states $\quad 75$

$\begin{array}{ll}3.4 .8 \text { The overall picture } & 76\end{array}$

$\begin{array}{ll}3.4 .9 \text { Summary } & 78\end{array}$

3.5 A final comparison $\quad 78$

\section{CHAPTER 4 Methodology}

4.1 Introduction $\quad 80$

$\begin{array}{ll}4.2 \text { Questionnaires versus interviews } & 80 \\ 4.2 .1 \text { For students } & \end{array}$

$\begin{array}{ll}\text { 4.2.1 For students } & 80 \\ \text { 4.2.2 For teachers and parents } & 80\end{array}$

4.3 Data gathering 81

4.4 Choice of location $\quad 81$

4.5 Student samples

4.5.1 Choosing the samples 82

4.5.2 The sample of year 8 students 82

4.5.3 The sample of year 9 students 83

4.6 Selection of teacher samples 83 
4.7 Selection of parent sample

4.8 Timing of the surveys

4.9 Development of student questionnaires

4.9.1 General considerations

4.9.2 Validity

4.9.2.1 Internal and external validity

88

4.9.2.2 Face validity

88

4.9.2.3 Content validity

88

4.9.2.4 Concurrent validity $\quad 89$

4.9.2.5 Response bias $\quad 89$

4.9.2.6 The importance of consistency 90

4.9.2.7 The placing of personal questions 90

4.9.3 Reliability

4.9.3.1 General considerations

4.9.3.2 Reliability of Likert scales 90

4.9.3.3 The importance of timing 91

4.9.3.4 The importance of consistency 91

4.9.3.5 Final thoughts 91

4.9.4 Ethical and practical considerations 91

4.9.5 Questionnaire form

4.9.5.1 The advantages of Likert scales 91

4.9.5.2 Building on previous research 92

4.9.5.3 Choice and wording of questionnaire items 92

4.9.5.4 Focus group discussions 92

4.10 The Year 8 questionnaire

4.10.1 Form and content $\quad 94$

4.10.2 Pilot survey 94

4.10.3 Pilot survey analysis

$\begin{array}{ll}\text { 4.10.3.1 Part 1 - Likert scale } & 95 \\ & 96\end{array}$

4.10.3.2 Part 2 - Yes/No/Comments 96

4.10.3.3 Part 3 - General questions $\quad 96$

4.10.4 Construction of the final version 97

4.10.5 Administration of the year 8 survey 99

4.11 The Year 9 questionnaire

4.11.1 Form and content 100

$\begin{array}{ll}4.11 .2 \text { Pilot survey } & 101\end{array}$

4.11.3 Pilot survey analysis and construction of the final version 101

4.11.4 Administration of the year 9 survey 101

4.12 Interviews

$\begin{array}{ll}\text { 4.12.1 General considerations } & 102\end{array}$

4.12.2 The problem of interviewer bias 102

4.12.3 Development of interview questions and procedures 103

$\begin{array}{ll}\text { 4.12.4 Pilot interviews } & 103\end{array}$

$\begin{array}{ll}\text { 4.12.5 Administration of teacher interviews } & 105\end{array}$ 
viii

4.12.6 Administration of parent interviews 105

4.12.7 Interviews - final thoughts 106

4.13 Data Analysis 106

\section{CHAPTER 5 Results and discussion - year 8 students}

5.1 Introduction

5.2 School profiles

5.2.1 Aster

109

5.2.2 Rose 110

$\begin{array}{ll}5.2 .3 \text { Salvia } & 110\end{array}$

5.3 The year 8 students

5.3.1 Numbers surveyed

5.3.2 Intermediate FLL experience 111

5.4 General attitudes towards FLL

5.4.1 Student comments on the opportunity to learn a FL 113

5.4.2 Beliefs about the difficulty of FLL 113

$\begin{array}{ll}\text { 5.4.3 The relative importance of FLL } & 114\end{array}$

5.4.4 Beliefs about the usefulness of FLL 115

$\begin{array}{ll}\text { 5.4.5 Ultimate interest in FLL } & 116\end{array}$

5.4.6 Summary of general attitudes to FLL 116

5.5 The influence of FLL experience on student attitudes 116

5.5.1 General reactions to FLL experience 116

5.5.2 Student opinions about skills development 117

$\begin{array}{ll}\text { 5.5.3 Attitudes towards FL teachers } & 117\end{array}$

$\begin{array}{ll}\text { 5.5.4 Attitudes to FL speakers and their cultures } & 118\end{array}$

5.5.5 Summary of attitudes to FLL experience 119

5.6 Year 8 student intentions regarding FLL in year 9

5.6.1 To study a FL or not

5.6.2 Reasons for intentions

$\begin{array}{ll}\text { 5.6.2.1 Reasons for responding 'yes' } & 119\end{array}$

5.6.2.2 Reasons for responding 'maybe' 120

5.6.2.3 More evidence of student interest in overseas travel 120

5.6.2.4 Reasons for not choosing FLL in year $9 \quad 122$

5.6.3 Intentions compared with beliefs about the usefulness, the relative importance and the difficulty of FLL 122

$\begin{array}{ll}\text { 5.6.4 Which languages students intended to study } & 124\end{array}$

5.6.4.1 Choices of those intending FLL in year $9 \quad 124$

5.6.4.2 Choices of those who might study a FL in year $9 \quad 124$

5.6.4.3 Reasons given for choices of language $\quad 124$

5.7 Student intentions compared with their ultimate interest in FLL

5.7.1 The availability of preferred languages $\quad 125$

5.7.2 Student intentions where preferred FL was available 125

$\begin{array}{ll}\text { 5.7.3 Student intentions where preferred FL was not available } & 127\end{array}$

5.7.4 Summary of comparison of intentions with preferred FLs 127 
5.8 Intentions for year 9 compared with previous experience of FLL

5.8.1 To study a FL or not

$\begin{array}{ll}\text { 5.8.2 The importance of enjoyment } & 129\end{array}$

$\begin{array}{ll}\text { 5.8.3 The influence of specific languages } & 129\end{array}$

5.8.4 Choice of future FL compared with language(s) studied 130

5.8.5 Other indications of the influence of previous FLL 131

5.8.6 Intentions compared with attitudes to FL speakers and their cultures 132

5.8.7 Summary of comparison of intentions with previous FLL 132

5.9 The influence of parents on intentions

5.9.1 General parental influence on subject choices 133

5.9.2 Student intentions compared with reported parental wishes 133

5.9.3 Student intentions compared with reported parental attitudes to the relative importance of English 134

5.9.4 Student intentions compared with parental FLL experience 134

5.10 The influence of intermediate schools and teachers on intentions

$\begin{array}{ll}\text { 5.10.1 School encouragement of FLL } & 135\end{array}$

$\begin{array}{ll}\text { 5.10.2 Teacher encouragement of FLL } & 135\end{array}$

5.10.3 Intentions compared with primary/intermediate schools 135

5.11 The influence of secondary schools and teachers on intentions

5.11.1 Schools which students expected to attend 136

$\begin{array}{ll}\text { 5.11.2 School influence on student intentions } & 136\end{array}$

$\begin{array}{ll}\text { 5.11.3 The influence of available languages } & 137\end{array}$

$\begin{array}{ll}\text { 5.11.4 The influence of different course lengths } & 139\end{array}$

$\begin{array}{ll}\text { 5.11.5 The importance of the FL teacher } & 140\end{array}$

5.11.6 Summary of comparison of intentions with future secondary school 140

5.12 The influence of peers and older siblings on intentions

$\begin{array}{ll}5.12 .1 \text { The influence of peers } & 140\end{array}$

$\begin{array}{ll}\text { 5.12.2 The influence of older siblings } & 141\end{array}$

5.13 Intentions for Year 9 compared with attitudes

5.13.1 Intentions compared with general attitudes to FLL 141

5.13.2 Intentions compared with attitudes to FLL experience 142

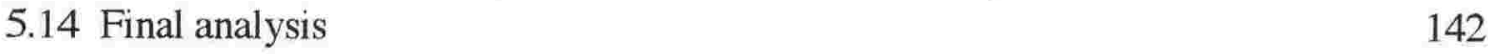

\section{CHAPTER 6 Results and discussion - year 9 students}

6.1 Introduction

6.2 School profiles

6.2.1 General information 146

$\begin{array}{ll}6.2 .2 \text { Arum } & 147\end{array}$

$\begin{array}{ll}6.2 .3 \text { Begonia } & 148\end{array}$

$\begin{array}{ll}6.2 .4 \text { Freesia } & 150\end{array}$

6.2.5 Gerbera 152

$\begin{array}{ll}\text { 6.2.6 Salvia } & 153\end{array}$

6.3 The year 9 sample

$\begin{array}{ll}\text { 6.3.1 Numbers surveyed } & 155\end{array}$ 
$\begin{array}{ll}\text { 6.3.2 Students' previous schools } & 155\end{array}$

6.3.3 Students' FLL decisions 156

6.4 General attitudes towards FLL

$\begin{array}{ll}\text { 6.4.1 Introduction } & 157\end{array}$

6.4.2 Beliefs about the difficulty of FLL 158

6.4.3 The relative importance of FLL 158

$\begin{array}{ll}\text { 6.4.4 The usefulness of FLL } & 160\end{array}$

6.5 The attitudes of students who were studying a FL

6.5.1 Reasons for choosing to study a FL 161

6.5.2 The languages students had chosen to study 164

6.5.3 Reasons for students' choice of language 165

$\begin{array}{ll}\text { 6.5.4 Beliefs about the difficulty of FLL } & 167\end{array}$

$\begin{array}{ll}\text { 6.5.5 The usefulness of FLL } & 167\end{array}$

6.5.6 Attitudes to speakers of the FLs studied 168

6.5.7 Attitudes to length of option courses 169

6.6 Intentions regarding FLL for year $10 \quad 169$

$\begin{array}{ll}\text { 6.6.1 Intentions compared with schools } & 170\end{array}$

6.6.2 Intentions according to FL studied in year $9 \quad 171$

6.6.3 Reasons for intentions to continue FLL 171

6.6.4 Reasons for intentions not to continue FLL or uncertainty 173

6.6.5 Intentions compared with attitudes to FLL 174

6.7 The attitudes of students who were not studying a FL

$\begin{array}{ll}\text { 6.7.1 General attitudes to FLL } & 174\end{array}$

6.7.2 Reasons for choosing not to study a FL 175

$\begin{array}{ll}\text { 6.7.3 Beliefs about the difficulty of FLL } & 175\end{array}$

$\begin{array}{ll}\text { 6.7.4 The relative importance of FLL } & 176\end{array}$

$\begin{array}{ll}\text { 6.7.5 The usefulness of FLL } & 176\end{array}$

$\begin{array}{ll}\text { 6.7.6 Attitudes to speakers of other languages } & 177\end{array}$

$\begin{array}{ll}\text { 6.7.7 Regrets about not studying a FL } & 177\end{array}$

$\begin{array}{ll}\text { 6.7.8 Possibility of future FLL } & 178\end{array}$

6.8 The influence of prior FLL on student option choices for year 9

$\begin{array}{ll}\text { 6.8.1 FLL experience at intermediate level } & 178\end{array}$

$\begin{array}{ll}\text { 6.8.2 Reasons for previous experience } & 179\end{array}$

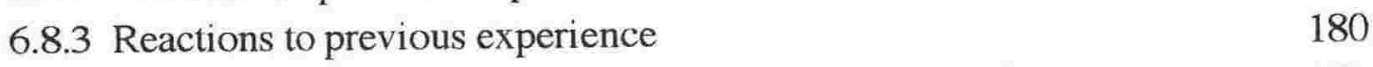

6.8.4 Reasons for reactions to previous experience 181

6.8.5 The effect on option choices for year 9 of enjoyment of, and confidence in, previous FLL experience 181

6.9 The influence of schools 182

6.10 The influence of siblings and peers 183

6.11 The influence of connections with or travel to non-English speaking places $\quad 184$

$\begin{array}{ll}6.12 \text { Final analysis } & 185\end{array}$

\section{CHAPTER 7 Comparison of year 8 intentions and year 9 choices}

7.1 Introduction 
7.2 The sample, their intentions and final choices

7.3 The influence of previous FLL experience

7.3.1 Numbers of students with previous FLL

189

7.3.2 The effect of previous experience on intentions and final choices 189

7.3.3 The importance of enjoyment of previous FLL experience 192

7.3.4 The effect of enjoyment on intentions and final choices 192

7.3.5 The importance of confidence in previous FLL experience 193

7.3.6 The effect of confidence on intentions and final choices 194

7.3.7 The influence of specific FLs on intentions 196

7.3.8 Did students carry out their intentions? 197

7.3.9 Summary of the influence of prior FLL experience on year 9 decisions 198

7.4 Student profiles 199

7.4.1 Students who intended to study a FL in year 9 but chose not to 199

7.4.2 Students who considered studying a FL in year 9 but decided against it 200

7.4.3 Students who did not intend to study a FL in year 9 but did choose a

FL option

202

7.4.4 Students who thought they might study a FL in year 9 and did so 203

7.4.5 Summary of profiles 203

7.5 Final analysis

204

\section{CHAPTER 8 The experiences, attitudes and influence of the parents of year 9 students}

8.1 Introduction

8.2 What students reported about their parents

8.2.1 Their parents' FLL experience

8.2.2 The influence of parental FLL experience on student choices 207

8.2.3 The general extent of parental influence on students' subject choices 207

8.2.4 Parental attitudes towards FLL in New Zealand schools 208

8.2.5 Parental attitudes towards FLL for their own children 208

8.3 The parent sample and their education

8.4 Parental FLL experience

8.4.1 FLs studied

8.4.2 Reasons for their FLL choices

8.4.3 Attitudes to their FLL experiences

8.5 Parental beliefs about the value of FLL in New Zealand schools 214

8.6 Parental attitudes towards the starting age for FLL 214

8.7 Parental attitudes towards the place of foreign language learning in their children's schools

8.8 Parental views on choosing year 9 options

8.8.1 Lack of information

8.8.2 Curriculum pressures

8.8.3 How option subjects were chosen

8.8.4 The extent of parental influence in the decision-making

8.8.5 Individual reasons for student choices as reported by their parents 
8.8.6.2 Family contacts with people from non-English speaking backgrounds

$\begin{array}{ll}\text { 8.8.6.3 Peer/sibling influence } & 221\end{array}$

8.8.7 Possible future FLL 222

8.8.8 Possible future career choices $\quad 222$

8.9 Teachers' views of parent attitudes towards FLL 222

8.10 A summary of general comments made by the parents interviewed

8.10.1 The need for languages other than English for the country's future $\quad 224$

8.10.2 The value of FLL for personal development 224

8.10.3 The value of FLL for cultural understanding 225

$\begin{array}{ll}\text { 8.10.4 What parents need to know } & 225\end{array}$

8.10.5 The need for an earlier start to FLL 225

$\begin{array}{ll}8.11 \text { Final analysis } & 225\end{array}$

CHAPTER 9 The attitudes of teachers towards foreign language learning and their influence over student choices

9.1 Introduction

9.2 Teachers' experience of and attitudes towards FLL

$\begin{array}{ll}\text { 9.2.1 School experiences } & 226\end{array}$

9.2.2 Teachers' attitudes to their school FLL experience 227

9.2.3 Further FLL by teachers not specialising in languages 228

$\begin{array}{ll}\text { 9.2.4 Teachers' reactions to FLL } & 228\end{array}$

9.3 Teachers' attitudes towards FLL for present-day students

9.3.1 How important is FLL? 229

9.3.2 Why is FLL important? 230

9.3.3 Which languages should New Zealand students learn? 231

9.3.4 Opinions about specific languages 233

9.3.5 The best starting age for FLL 234

9.3.6 Reasons for preferred starting ages 234

9.3.7 Should all students have some experience of SLL 235

9.4 Teachers' views of the current situation

9.4.1 Which students study FLs? 238

9.4.2 What governs student choices of FL? 239

9.4.3 Why students reject FLL in year 9 or beyond 240

9.4.4 The extent to which student choices were guided by schools 241

9.4.4.1 For students moving from Year 8 to $9 \quad 241$

9.4.4.2 For students moving from Year 9 to $10 \quad 241$

9.4.4.3 For senior students (years 11-13) 242

9.5 Teachers' views of their colleagues' attitudes towards FLL 243

9.6 Teachers' influence according to students $\quad 244$

9.7 Staffing issues

9.7.1 The general situation 
9.7.2 The principals' perspective

9.7.3 The language teachers' perspective $\quad 247$

9.7.4 The views of other teachers 248

9.8 General comments about education in New Zealand 249

9.8.1 General attitudes towards schooling 249

9.8.2 The limited nature of secondary education 249

9.8.3 More pressing demands on scarce resources 250

9.9 Final analysis 250

9.9.1 Children need to start SLL an an earlier age 250

9.9.2 All students should have some FLL at intermediate level 250

9.9.3 Other changes needed 251

9.9.4 The importance of gender 251

\section{CHAPTER 10 The role of gender in attitudes to FLL}

10.1 Introduction

10.2 Perceptions of gender differences in interest and ability in FLL

10.2.1 Student perceptions 253

10.2.2 Parents' perceptions 254

10.2.3 Teachers' perceptions 254

10.3 Year 8 intentions

10.3.1 To study a FL or not - the gender gap appears

10.3.2 Reasons for intending FLL 256

10.3.3 Reasons for being unsure about or not intending FLL in year $9 \quad 258$

10.3.4 FLs which year 8 students intended to study 258

10.3.5 Intended choice of FL compared with expected year 9 school 259

10.3.6 Intended choice of FL compared with preferred FL 259

10.3.7 Reasons given by year 8 students for their choice of FL 260

10.4 Year 9 decisions

10.4.1 To study a FL or not - the gender gap narrows 261

10.4.2 Reasons for the decisions of year 9 students

10.4.2.1 Reasons for choosing to study a FL 263

10.4.2.2 Gender perceptions of compulsion. 264

10.4.2.3 Reasons for choosing not to study a FL 265

10.4.3 FL choices made by year 9 students 267

10.4.4 Reasons given by year 9 students for their choice of FL 270

10.5 General attitudes to FLL experience

10.5.1 Girls enjoy FLL more than boys 270

10.5.2 Girls are less monocultural in outlook than boys 274

10.5.3 Boys find FLL more difficult than girls 276

10.5.4 Boys consider FLs less useful than girls
10.5.4.1 The relative importance of FLL

$\begin{array}{ll}\text { 10.5.4.1 The relative importance of FLL } & 279 \\ \text { 10.5.4.2 The relative importance of English } & 282\end{array}$

10.5.4.3 Awareness of transferable language skills 283

10.5.4.4 Awareness of the value of FLL for cultural understanding $\quad 284$ 
xiv

10.5.4.5 Usefulness of FLs for employment 285

10.5.5 Boys prefer other subjects $\quad 287$

10.6 External influences

10.6.1 The influence of parents

287

10.6.2 The influence of connections with or travel to non-English speaking places

291

10.6.3 The influence of peers and older siblings 291

10.6.4 The influence of prior FLL 291

10.6.5 The influence of schools 294

10.6.6 The influence of teachers 297

10.7 Beyond year 9

10.7.1 Student intentions for year $10 \quad 298$

10.7.2 Gender differences in general retention rates 301

10.8 Final analysis 301

CHAPTER 11 Conclusion

11.1 Discussion of hypotheses

11.1.1 Reasons for choosing FLL 303

11.1.2 Reasons for not choosing FLL $\quad 304$

11.1.3 Gender differences $\quad 305$

11.1.4 Parental influence $\quad 306$

11.1.5 The influence of prior experience $\quad 306$

11.1.6 The role of attitudes in students' choices 307

11.2 The outlook for the future - official encouragement for FLL in schools
11.2.1 The latest initiative

11.2.2 Support for Asian languages $\quad 309$

11.2.3 The Second Language Learning Project 309

$\begin{array}{ll}\text { 11.2.4 The new national qualifications } & 310\end{array}$

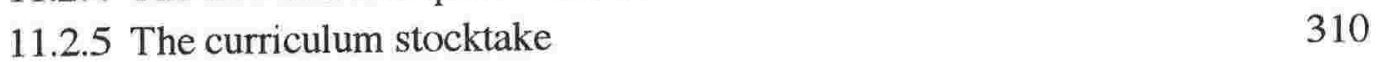

11.3 The remaining major barriers to FLL

11.3.1 The lack of continuity between primary and secondary education $\quad 313$

11.3.2 The lack of compulsory LOTE learning $\quad 313$

11.3.3 The lack of public support $\quad 314$

11.4 Suggestions for further research $\quad 315$

$\begin{array}{ll}11.5 \text { Final thoughts } & 315\end{array}$

REFERENCES 


\section{APPENDICES}

1. Interpreters - can they be trusted?

2. Surveyed schools by school type, decile rating, gender and ethnic balance

3. Sample information sheet and consent form (students and parents)

4. Sample information sheet and consent form (teachers)

5. Year 8 student survey questionnaire

6. Year 9 student survey questionnaire

7. Questions for primary and intermediate teacher interviews 357

8. Questions for secondary teacher interviews

9. A. Summary of parent interview schedule for parents to consider in advance

B. Parent interview schedule

10. Background information sheet

11. Chapter 5 graphs

12. The place of foreign languages in each secondary school

13. Chapter 10 graphs

14. Figure 2.

15. Year 8 questionnaire Parts 1 and 2 - response frequencies

16. Year 9 questionnaire Parts 2,3 and 4 - response frequencies 


\section{LIST OF TABLES}

1.1 How monolingual is New Zealand? 1

1.2 Proportions of secondary students in FLL 1994-1999 3

1.3 Year 9 FL enrolments 1994-1999 4

1.4 Proportions and dropout rates of one cohort of students of French and Japanese 4

1.5 Comparative dropout rates from French and Japanese in one cohort of students $\quad 5$

1.6 Gender imbalances in one cohort of FL students of French and Japanese 6

1.7 A comparison of gender imbalances in French in one cohort of FL students 7

1.8 A comparison of gender imbalances in Japanese in one cohort of FL students 7

2.1 Reasons cited by each gender for dropping out of FLL in England 24

2.2 Principal sources of visitors to New Zealand by language, 1991 and $2000 \quad 35$

3.1 1990 numbers of students entering GCSE and A level language examinations and percentage increases since 1988 in the UK 45

3.2 FL enrolments in public schools in the USA 1974-1994 59

3.3 Proportions (\%) of year 12 LOTE enrolments in Australian schools in the 10 most popular languages: 1991-1999 77

4.1 Numbers of teachers interviewed by type of school and gender 84

4.2 Numbers of teachers interviewed by responsibilities and gender 85

4.3 Numbers of parental interviews 86

5.1 Number of useful survey forms by school and gender 111

5.2 Students with FLL experience in years 7 or 8, 1998-99 112

5.3 Languages studied in years 7 or $8 \quad 112$

5.4 Numbers of students who studied more than one language 112

5.5 Year 8 comments on the chance to study a FL at intermediate school $\quad 113$

5.6 FLs students would most like to learn, if they had the opportunity 115

$\begin{array}{lll}5.7 & \text { Student intentions for year } 9 & 119\end{array}$

5.8 Reasons for choosing to study a FL in year 9 120

5.9 Reasons cited by students who said they might study a FL in year $9 \quad 120$

5.10 Languages chosen by students intending FLL in year 9 124

5.11 Students' intentions for year 9 if their preferred FL was possible at future $\begin{array}{ll}\text { secondary school } & 125\end{array}$

5.12 Year 9 choice of FL compared with reported FL preference 126

$\begin{array}{lll}5.13 & \text { Intentions to study French or Japanese compared with students' } \\ \text { ultimate interest } & 127\end{array}$

5.14 Students' intentions for year 9 if their preferred FL was not possible at
future secondary school

5.15 Year 8 students' intentions for FLL in year 9 compared FLs studied in $\begin{array}{ll}\text { year } 7 \text { or } 8 & 129\end{array}$

5.16 Year 8 students' choice of language for Year 9 compared with previous FL studied 
5.17 Student intentions for FLL in year 9 compared with responses to item

8.1.24: "What I've seen about languages I could learn next year makes them look really interesting"

5.18 Student intentions for FLL in year 9 compared with responses to item 8.1.23 "My parents want me to do a language next year."

5.19 Student intentions for FLL in year 9 compared with responses to item 8.1.25 "My parents think I should spend more time on my English than learning another language."

5.20 Student intentions for FLL in year 9 compared with parents' FLL at school in NZ

5.21 Student intentions for FLL in year 9 compared with year 8 school

5.22 Year 8 student choices of language according to intended school

5.23 Responses to item 8.1.15: “There isn't a good range of languages for me to choose from for next year"

5.24 Student intentions for FLL in year 9 compared with their general attitudes to FLL

5.25 Student intentions for FLL in year 9 compared with attitudes towards their FLL experience

6.1 The place of foreign languages in each surveyed secondary school - see Appendix 12

6.2 Arum FL student numbers 2000

6.3 Begonia FL student numbers 1997 to $2000 \quad 150$

$6.4 \quad$ Freesia FL student numbers in $2000 \quad 152$

6.5 Gerbera FL student numbers in $2000 \quad 153$

6.6 Salvia FL student numbers $2000 \quad 155$

6.7 Number of useful survey forms by school and gender 155

6.8 Which schools year 9 students came from 156

6.9 Numbers of surveyed students studying one or more FLs in $2000 \quad 157$

6.10 Student decisions about FLL compared with responses to item 9.2.12:

"Learning a foreign language is too hard for most students."

6.11 Comparison between responses to items concerned with the relative importance of FLL and student decisions to study a FL or not

6.12 Comparison between responses to items concerned with the usefulness of FLL and student decisions to study a FL or not

6.13 Reasons given by students for choosing FLL 162

6.14 Numbers of students studying each language 165

6.15 Numbers of year 9 students studying French and/or Japanese, by school 165

6.16 Languages mentioned as preferred alternatives or additional FLs 166

6.17 Reasons given for continuing FLL in year $10 \quad 172$

6.18 Reasons given for not continuing FLL in year 10 or being uncertain 172

6.19 Significant item responses in relation to student intentions for FLL in $\begin{array}{ll}\text { year } 10 & 173\end{array}$

6.20 Reasons for not studying a FL in Year 9 176

6.21 Students with FLL experience in Years 7 or 8, 1998-99, by year 9 school 179 
6.23 Reasons for enjoying or not enjoying FLL at intermediate level

6.24 Student decisions about FLL compared with responses to item 9.2.5, "My school's prospectus made learning a foreign language look really boring."

7.1 Student choices concerning FLL in year 9

7.4 Choice of FLL in year 9 compared with confidence in previous FLL

7.5 Intentions for FLL in year 9 compared with FLs studied at intermediate level

7.6 Intended FLs for study in year 9 compared with FLs studied at intermediate level

7.7 FLs chosen for study in year 9 compared with reported intentions in year 8

7.8 Proportions of students who intended FLL in year 9 compared with their final choices, according to their prior FLL experience

8.1 Student decisions regarding FLL in year 9 compared with their parents' experience of FLL at school

8.2 Student decisions regarding FLL compared with responses to item 9.2.3, "My parents think that studying a foreign language is a waste of time for New Zealand students"

8.3 Student decisions about FLL compared with responses to item 9.2.13, "My parents wanted me to do a foreign language this year"

8.4 Age range of parents

8.5 Ages at which parents left school

8.6 Educational qualifications of parents

8.7 Parents' FLL experience at school

8.8 Parents' feelings about their FLL or lack of it

8.9 Parents' thoughts about the value of FLL for present-day New Zealand school students

8.10 Parents' opinions about the best starting age for FLL

8.11 How Year 9 option subjects were chosen 217

8.12 How the eventual decisions were made regarding FLs 217

8.13 Students' travel experience overseas 220

8.14 Possible future overseas travel of students 220

8.15 Family contacts with people from non-English speaking backgrounds 221

8.16 Students' future language learning 222

8.17 Attitudes of secondary students' parents to compulsory and optional

9.1 FLs studied at school by teachers

$\begin{array}{lll}9.2 & \text { The value of their own FLL to teachers } & 228 \\ 9.3 & \text { Value placed on FLL for New Zealand students } & 230\end{array}$

9.4 Why FLL is important for New Zealand school students 231 
9.5 FLs considered the most appropriate for students

9.6 The best starting age

9.7 Reasons for starting FLL earlier than year $7 \quad 235$

9.8 Teacher opinions about the status of FLL in schools 236

9.9 Which students choose to study FLs 238

9.10 Reasons for not undertaking or continuing FLL 240

9.11 Advice about FLL given to senior students in relation to employment or tertiary courses

9.12 Teachers' attitudes towards FLL, according to their colleagues 244

10.1 Numbers and drop out rates of students, by gender, in one cohort studying French and Japanese 1994-1998

10.2 Responses by gender to survey item 8.2.16: "Boys are better at learning a language than girls."

10.3 Year 8 student intentions for FLL according to expected secondary school

10.4 Reasons for intending to study a FL in year 9

10.5 Student intentions for year 9 if preferred FL was possible at intended secondary school

10.6 Intentions to study French or Japanese compared with students' ultimate interest in FLL

10.7 Student decisions regarding FLL in year 9, by gender and school

10.8 Reasons for studying a FL in Year 9

10.9 Reasons for not studying a FL in Year 9

10.10 Responses by gender to survey item 9.4.9: "I'm glad I don't have to learn a foreign language this year."

10.11 Responses by gender to survey item 9.4.13: "I wanted to learn a foreign language but languages clashed with more important subjects", at Salvia and single sex schools.

10.12 Proportions of students in each language by gender

10.13 Numbers of students studying French and/or Japanese by school and gender

10.14 Proportions of girls and boys studying each FL 269

10.15 Positive reasons for choice of language(s) 270

10.16 Proportions of year 8 students reporting agreement or disagreement with survey items indicating reactions to FLL, by gender

10.17 Reasons for enjoying or not enjoying FLL at intermediate level, by gender

10.18 Responses by gender to survey item 9.3.2: "It's fun to learn another language."

10.19 Responses to the question: "Would you like to have studied a foreign language?"

10.20 Reasons why Year 9 students not in FLL would like to have learnt a FL

10.21 Proportions of year 9 students not in FLL reporting agreement or disagreement with survey items indicating attitudes to FLL, by gender

10.22 Responses by gender to survey item 8.2.7: "I'd like to know more about the life and customs of the people who speak the language." 
10.23 Proportions of year 9 students reporting agreement or disagreement with survey items indicating attitudes to people who speak FLs, by gender

10.24 Proportions of year 8 students reporting agreement or disagreement with survey items indicating perceptions of difficulty in FLL, by gender

10.25 Responses by gender to survey item 9.2.12: "Learning a foreign language is too hard for most students."

10.26 Proportions of year 9 students not in FLL reporting agreement or disagreement with survey items indicating perceptions of difficulty in FLL, by gender

10.27 Responses by gender to survey item 9.3.4: "I think I'll do well in my language learning this year."

10.28 Responses by gender to survey items 8.1.17 and 9.2.20: "There are lots more important things to learn than languages."

10.29 Responses by gender to survey item 9.2.11: "A foreign language should be part of the core curriculum at secondary school."

10.30 Responses by gender to survey item 8.1.12: "It's better for me to spend time doing more English than learning another language."

10.31 Responses by gender to survey item 9.4.2: "I need to concentrate on developing my English language skills."

10.32 Proportions of year 9 students in FLL reporting agreement or disagreement with survey items indicating perceptions of the transferability of language skills, by gender

10.33 Responses by gender to survey item 9.2.6: "Students who learn a foreign language are better at understanding another culture."

10.34 Responses by gender to survey item 9.2.8: "Lots of jobs involve working with people who don't speak English."

10.35 Year 8 student intentions and year 9 student choices regarding FLL compared with parents' FLL at school in NZ

10.36 Responses by gender to survey item 9.3.3: "My parents are pleased that I'm doing a foreign language this year."

10.37 Responses by gender to survey items 8.1.25, 9.4.8: "My parents think I should spend more time on my English than learning another language."

10.38 Year 8 student choices of FL for Year 9 compared with FL previously studied

10.39 Proportions of students in year 9 FLL by gender, according to responses to item 9.2.2, "I enjoyed learning a foreign language at intermediate school"

10.40 Responses by gender to survey item 9.2.5: "My school's prospectus made learning a foreign language look really boring."

10.41 Proportions of year 8 students reporting agreement or disagreement with survey items indicating reactions to FL teachers, by gender

10.42 Reasons given for continuing FLL in year 10

10.44 Students numbers in years 12 and 13 at single sex schools in 2000 


\section{FIGURES}

1. Model of hypothesised factors influencing student decisions about FLL

2. Interrelationship of main factors directly or indirectly influencing student attitudes towards FLL and decisions about FLL in year 9 - see Appendix 14

\section{GRAPHS}

5.1 Responses to item 8.1.12: “It's better for me to spend time doing more English than learning another language", compared with intentions for year 9

5.2 General student attitudes to FLL - see Appendix 11

5.3 Responses to item 8.2.13: "I'm not interested in meeting people who speak the language"

5.4 Attitudes to FLL experience - see Appendix 11

5.5 Responses to item 8.1.21, "I want to do a language next year because it'll give me the chance to go overseas on a school trip" compared with FLL intentions

5.6 Responses to item 8.1.17: “There are lots more important things to learn than languages", compared with intentions for year 9

5.7 Responses to item 8.1.6: "I think I'd find learning a language easy next year", compared with intentions for year 9

5.8 Intentions for FLL in year 9 compared with previous experience

5.9 Student intentions for year 9 compared with Y7/8 FLL experience see Appendix 11

5.10 Year 8 student intentions for FLL in year 9 compared with expected secondary school

5.11 Responses to item 8.1.7: "It's a pity that language options next year are only for half the year", compared with intentions for year 9

6.1 Student responses to item 9.3.13: "I'm only doing a foreign language because my school makes it compulsory."

6.2 Student responses to item 9.3.2: "It's fun to learn another language."

6.3 Student responses to item 9.3.12: "I'd like to know more about the life of the people who speak the language I'm learning."

6.4 Student responses to item 9.3.15: "I'm interested in meeting people who speak the language I'm learning."

6.5 Proportions of students, by school, intending to continue FLL in year 10 or uncertain

6.6 Student intentions for year 10 compared with FLs studied in year 9

6.7 Student responses to item 9.4.11: "I'm interested in meeting people who speak other languages." 
6.8 Responses to item 9.2.2: "I enjoyed learning a foreign language at intermediate school."

7.1 Student intentions for FLL in year 9

7.2 Proportions of students intending FLL in year 9 compared with previous FLL

7.3 Students' reported enjoyment of FLL at intermediate level

7.4 Proportions of students intending FLL in year 9 compared with enjoyment of previous FLL

7.5 Students' reported confidence in FLL at intermediate level

7.6 Proportions of students intending FLL in year 9 compared with confidence in their previous FLL

10.1 Student intentions for FLL in year 9, by gender

10.2 Choice of language for year 9 made by students intending to study a FL, by gender

10.3 Student decisions regarding FLL in year 9, by gender, for whole sample and sub-sample

10.4 Responses to item 9.3.13: "I'm only doing a foreign language because my school makes it compulsory", by school and gender

10.5 Responses by gender to survey item 9.4.7: "Most of my friends think that learning foreign languages is stupid."

10.6 Responses by gender to survey item 9.4.12: "I won't need to know any foreign languages in any jobs I might do."

10.7 Responses by gender to survey item 9.4.13: "I'm more interested in practical subjects than subjects with lots of reading and writing."

10.8 Responses by gender to survey item 9.2.3: "My parents think that studying a foreign language is a waste of time."

10.9 Student choices for year 9 FLL compared with experience of FLL during overseas travel

10.10 Year 8 student intentions for FLL in year 9 compared with previous experience, by gender - see Appendix 13

10.11 Student intentions for year 9 compared with Y7/8 FLL experience and gender - see Appendix 13

10.12 Year 8 student intentions for FLL in year 9 compared with expected secondary school and gender

10.13 Student intentions for year 10 by gender 


\section{GLOSSARY}

AATF American Association of Teachers of French

AATG American Association of Teachers of German

ACTFL American Council on the Teaching of Foreign Languages

ALLC Australian Language and Literacy Council

ALLP Australian Language and Literacy Policy

CAL Center for Applied Linguistics (USA)

DEET Department of Employment, Education and Training (Australia - now DETYA)

DETYA Department of Education, Training and Youth Affairs

DfEE Department for Education and Employment (UK)

DoE Department of Education (USA)

ERO Education Review Office (NZ)

EU European Union

FL Foreign language

FLL Foreign language learning

L1 First language, mother tongue

L2 Second (or further) language

LOTE(s) Language(s) other than English

LOTEM(s) Language(s) other than English or Mäori

MCEETYA Ministerial Council on Education, Employment, Training and Youth Affairs

MLA Modern Languages Association (USA)

MLJ Modern Language Journal

MoE Ministry of Education (NZ)

NALSAS National Asian Languages and Studies in Australian Schools NCLIS (USA)

NESB Non-English speaking background

NPL National Policy on Languages

NZALT New Zealand Association of Language Teachers

NZCTE New Zealand Council for Teacher Education

NZEI New Zealand Educational Institute

NZQA New Zealand Qualifications Authority

SLL Second language learning (Learning any language other than L1)

SLLP Second Language Learning Project

VCE Victorian Certificate of Education

VSL Victoria School of Languages 
The limits of my language mean the limits of my world. (Wittgenstein 1922, cited in Oxford Essential Quotations)

If a French car dealer wants to sell you a car, they will speak some English. If you want to sell them a car, they can speak only French. (Common saying, according to Jackson \& Peddie 1998: 44)

\subsection{Languages in New Zealand}

New Zealand is probably one of the most monolingual countries in the world. This is not necessarily a source of pride for a country dependent for its economic well-being on increasing trade with non-English speaking countries and increasing numbers of non-English speaking tourists. One academic who has carried out regular research into the use of and attitudes towards languages other than English in trade and tourism has called New Zealand a "country of foreign language illiterates" (Watts 1991: 12). Watts has proposed three possible reasons for this:-

- New Zealanders are not good at learning languages;

- New Zealanders are ethnocentric; or

- "New Zealanders have not recognised that mono-lingualism is a handicap in a world where economic strength and political influence is [sic] no longer monopolised by English-speaking nations" (Watts 1991: 11).

It is not credible that New Zealanders have some inherent deficiency when it comes to foreign language learning (henceforth FLL). Ethnocentricity seems an unlike explanation since New Zealanders have always travelled widely, seem reasonably knowledgeable about the rest of world and have ready media access to international affairs. Thus Watts (1991) blames the lack of will for the persistence of monolingualism, although it could also be attributed to laziness or the inherited British (and perhaps American) mentality that everyone ought to speak English and is probably able to do so these days.

Table 1.1 How monolingual is New Zealand?

\begin{tabular}{|l|c|c|}
\hline & $\mathbf{1 9 9 6}$ & $\mathbf{2 0 0 1}$ \\
\hline $\begin{array}{l}\text { Proportion of population who speak } \\
\text { English }\end{array}$ & $97.9 \%$ & $91.6 \%$ \\
\hline $\begin{array}{l}\text { Proportion of English speakers who } \\
\text { are monolingual }\end{array}$ & $86.0 \%$ & $83.7 \%$ \\
\hline $\begin{array}{l}\text { Proportion of whole population who } \\
\text { are monolingual in English }\end{array}$ & $83.9 \%$ & $76.8 \%$ \\
\hline Sources: Starks 1998, Statistics NZ 2002 \\
\hline
\end{tabular}

Neither publicly not privately has the country shown much interest in even knowing what languages New Zealanders can speak, let alone what languages might be valuable for 


\section{2}

improving the country's standard of education and hence the economy. Only in 1996 did the government decide to include in the census a question concerning language skills: "In which language(s) could you have a conversation about a lot of everyday things?" The results from the 1996 and 2001 censuses, see Table 1.1, show that the proportion of the population who can speak only English has decreased from over $83 \%$ to under $77 \%$, but that well over $80 \%$ of English speakers remain monolingual.

The Prime Minister herself has expressed concern that most New Zealanders speak only one language and, according to a press report (NZ Newspapers Association 2001), "believes the country needs to look at a national language strategy that would emphasise learning languages at school and in the curriculum". A large amount of work on just such a strategy was carried out for the Ministry of Education (MoE) a decade ago (Waite 1992), but the recommendations have largely been ignored in succeeding years.

\subsection{Definitions of relevant terms}

The terminology used to refer to the learning of languages other than a person's mother tongue (L1) or the dominant language of a country varies from country to country and even within New Zealand. In this research, 'foreign languages' (FLs) are taken to be languages not commonly spoken in New Zealand but studied for their cultural and/or economic importance in the world. In some of the literature they are referred to as 'international languages' (e.g. Waite 1992, R. Kaplan 1993, Shackleford 1996), 'modern languages' (especially in the UK and the USA) or LOTEMs, languages other than English or Mäori, after the official Australian term, LOTE (e.g. Peddie 1992, Benton 1996). Unlike the Australian usage, LOTEM is generally understood to exclude what are usually known as 'community languages' spoken by minority ethnic groups, such as Pacific Islanders or refugees, but in this research the term LOTE will be used to refer to any language other than English. Another term found in much of the literature, second language learning (SLL), is taken to refer, in the New Zealand context, to the learning of any language (an L2) which is not a person's L1, although the Education Review Office (ERO 1994) uses it to mean FLL. For many English speakers, Mäori is their L2. For most people from non-English speaking backgrounds (NESB) English is their L2 (or an additional language). The main FLs studied in New Zealand secondary schools are French and Japanese, with smaller numbers of schools offering German, Spanish, while a few students have the opportunity to learn Chinese (Mandarin) or Korean.

\subsection{Languages in the New Zealand education system}

The New Zealand Curriculum Framework states:

All students benefit from learning another language from the earliest practicable age.

Such learning broadens students' general language abilities and brings their own language into sharper focus. It enriches them intellectually, socially, and culturally, offers an understanding of the ways in which other people think and behave, and furthers international relations and trade. (MoE 1993b: 10) 
All NESB students will reap the above-mentioned benefits through learning English, which is compulsory for all students for most of their schooling. They will be particularly enriched if they also succeed in maintaining their L1 and any other languages they can already speak. English-speaking students will not necessarily enjoy the benefits of SLL, except perhaps by learning some Mäori, because there is no legal requirement to learn any LOTE in New Zealand schools. Schools are not even required to offer any LOTEs. In reality, most primary schools try to incorporate some Mäori into their programmes and the primary teachers' association, NZEI, has adopted a policy of Mäori learning for all New Zealand children (NZEI 2000), even though this is not yet a practical reality in all schools or for all primary teachers. Most secondary schools offer students at least one FL as well as Mäori.

Although one of seven Essential Learning Areas of the Curriculum Framework is called 'Language and Languages', the legal requirements for educating students in this area can be met entirely through the teaching of English. Traditionally, most students have not had the opportunity to begin FLL until year 9 (the first year of secondary school - about age 13). In most schools FLs are among optional subjects from which students have to choose a limited number. From 1987 to 1992 the proportion of secondary students studying a FL rose gradually from $22 \%$ to just over $26 \%$ (ERO 1994), but later statistics show that, since a peak of nearly $29 \%$ in 1994, the proportion steadily declined again until 1999, see Table 1.2 . Since statistics do not distinguish the students studying more than one FL, any percentages must be considered on the generous side.

\section{Table 1.2 Proportions of secondary students in FLL 1994-1999}

(MoE statistics)

\begin{tabular}{|l|c|c|c|c|c|c|}
\hline Year & 1994 & 1995 & 1996 & 1997 & 1998 & 1999 \\
\hline $\begin{array}{l}\text { Students in } \\
\text { FLL }\end{array}$ & $28.7 \%$ & $28.3 \%$ & $27.4 \%$ & $25.3 \%$ & $22.6 \%$ & $23.6 \%$ \\
\hline
\end{tabular}

As regards the choice of FLs available to students the Curriculum Framework promises that students "will be able to choose from a range of Pacific, Asian, and European languages, all of which are important to New Zealand's regional and international interests" (MoE 1993b: $10)$.

Despite the MoE's efforts to widen the range of FLs taught in schools, the majority of FL enrolments are in French and Japanese, which is not a reflection of the country's economic needs (Jackson \& Peddie 1998: 42). In 1994, for example, approximately $60 \%$ of year 9 students studied a FL, $85 \%$ of whom learnt French or Japanese. Most of the rest learnt German while very small numbers took Spanish or Chinese. There have been small numbers of students studying Indonesian and Russian in a handful of schools over the past decade but the proportions have never exceeded $0.1 \%$ (including students in all years 9-13) and Russian has now disappeared from New Zealand schools. At present, the teaching of Korean is limited to a few pilot schemes in Auckland. 
As Table 1.3 shows, there has been very little change in the pattern of year $9 \mathrm{FL}$ enrolments since 1994 and this pattern will be reflected in the results of this research.

Table 1.3 Year 9 FL enrolments 1994-1999 (MoE statistics)

\begin{tabular}{|l|c|c|c|c|c|c|}
\hline & $\mathbf{1 9 9 4}$ & $\mathbf{1 9 9 5}$ & $\mathbf{1 9 9 6}$ & $\mathbf{1 9 9 7}$ & $\mathbf{1 9 9 8}$ & $\mathbf{1 9 9 9}$ \\
\hline French & $26.2 \%$ & $24.6 \%$ & $22.7 \%$ & $21.1 \%$ & $21.4 \%$ & $23.9 \%$ \\
\hline Japanese & $24.9 \%$ & $24.0 \%$ & $23.0 \%$ & $21.5 \%$ & $18.0 \%$ & $19.2 \%$ \\
\hline German & $7.4 \%$ & $8.0 \%$ & $8.2 \%$ & $6.7 \%$ & $6.4 \%$ & $6.5 \%$ \\
\hline Spanish & $0.4 \%$ & $0.3 \%$ & $1.4 \%$ & $1.4 \%$ & $1.6 \%$ & $2.1 \%$ \\
\hline Chinese & $1.0 \%$ & $0.5 \%$ & $0.8 \%$ & $0.9 \%$ & $0.7 \%$ & $0.7 \%$ \\
\hline
\end{tabular}

Retention rates in FL courses after year 9 are poor. Typically, in recent years about half of year 9 students have started FLL but approximately half of them have not continued it the following year. In most succeeding years large proportions of students continue to drop out of FL classes. Table 1.4 shows the proportions of the 1994 cohort who gave up FLL over years 9 to 12 .

Table 1.4 Proportions and dropout rates of one cohort of students of French and Japanese (MoE statistics)

\begin{tabular}{|c|c|c|c|c|}
\hline & \multicolumn{2}{|c|}{ Student numbers } & Proportion of & \\
\cline { 2 - 5 } Year/Level & $\begin{array}{c}\text { Studying } \\
\text { F + J }\end{array}$ & Total & $\begin{array}{c}\text { group in } \\
\mathbf{F}+\mathbf{J}\end{array}$ & $\begin{array}{c}\text { Dropout } \\
\text { rate }\end{array}$ \\
\hline $\mathbf{1 9 9 4 / Y 9}$ & 26342 & 51472 & $51 \%$ & \\
\hline $\mathbf{1 9 9 5 / Y 1 0}$ & 12934 & 51580 & $25 \%$ & $51 \%$ \\
\hline $\mathbf{1 9 9 6 / Y 1 1}$ & 7164 & 54319 & $13 \%$ & $45 \%$ \\
\hline $\mathbf{1 9 9 7 / Y 1 2}$ & 3876 & 46674 & $8 \%$ & $46 \%$ \\
\hline $\mathbf{1 9 9 8 / Y 1 3}$ & 2512 & 35383 & $7 \%$ & $35 \%$ \\
\hline
\end{tabular}


Furthermore, as Jackson and Peddie point out:

It is well-known that many schools have (compulsory) 'taster' courses in Year Nine, which quite normally include one or more languages. This in turn means that a significant number of Year Nine students are likely to be studying an international language for only one semester or less. (1998: 41)

While French and Japanese were fairly equally popular at year 9, Table 1.5 shows that Japanese was much more popular than French in years 12 and 13 for this same cohort of students. Dropout rates were much higher for French at all levels except from year 12 to 13 , a pattern which has continued in many schools, as this research will show.

Table 1.5 Comparative dropout rates from French and Japanese in one cohort of students (MoE statistics)

\begin{tabular}{|c|c|c|c|c|}
\hline & $\begin{array}{c}\text { Student } \\
\text { numbers }\end{array}$ & $\begin{array}{c}\text { Dropout } \\
\text { rates }\end{array}$ & $\begin{array}{c}\text { Student } \\
\text { numbers }\end{array}$ & $\begin{array}{c}\text { Dropout } \\
\text { rates }\end{array}$ \\
\cline { 2 - 5 } Year/Level & \multicolumn{2}{|c|}{ Japanese } & \multicolumn{2}{c|}{ French } \\
\hline $\mathbf{1 9 9 4 / Y 9}$ & 12832 & & 13510 & \\
\hline $\mathbf{1 9 9 5 / Y 1 0}$ & 6799 & $47 \%$ & 6135 & $55 \%$ \\
\hline $\mathbf{1 9 9 6 / Y 1 1}$ & 4046 & $40 \%$ & 3118 & $49 \%$ \\
\hline $\mathbf{1 9 9 7 / Y 1 2}$ & 2435 & $40 \%$ & 1441 & $54 \%$ \\
\hline $\mathbf{1 9 9 8 / Y 1 3}$ & 1552 & $36 \%$ & 960 & $33 \%$ \\
\hline
\end{tabular}

In sum, very few students are pursuing FLL in senior classes and most of those study Japanese. This has important consequences for students and teachers of other languages. Classes may disappear, especially after year 11. Students may therefore give up FLL or be obliged to study by correspondence, which does not suit all students. No matter how good the available Correspondence School courses, students miss out on the natural interaction and immediate feedback which build skills and confidence. In other cases two or three levels of students may be combined in one class, which creates particular problems for teacher and students, a situation which rarely occurs in any other subject areas. In addition, specialist FL teachers are frequently obliged to teach other subjects even if they lack the relevant training or qualifications. 
Table 1.6 Gender imbalances in one cohort of FL students of French and Japanese (MoE statistics)

\begin{tabular}{|c|c|c|c|c|}
\hline \multirow{2}{*}{ Year/Level } & \multicolumn{2}{|c|}{ Numbers in F+ J } & \multicolumn{2}{c|}{ Gender balance } \\
\cline { 2 - 5 } & Girls & Boys & Girls & Boys \\
\hline $\mathbf{1 9 9 4 / Y 9}$ & 15164 & 11178 & $58 \%$ & $42 \%$ \\
\hline $\mathbf{1 9 9 5 / Y 1 0}$ & 8066 & 4868 & $62 \%$ & $38 \%$ \\
\hline $\mathbf{1 9 9 6 / Y 1 1}$ & 4833 & 2331 & $67 \%$ & $33 \%$ \\
\hline $\mathbf{1 9 9 7 / Y 1 2}$ & 2735 & 1141 & $71 \%$ & $29 \%$ \\
\hline $\mathbf{1 9 9 8 / Y 1 3}$ & 1752 & 760 & $70 \%$ & $30 \%$ \\
\hline
\end{tabular}

Another relevant factor is that, if students do not start it in Year 9, very few will have the opportunity for FLL in later years, although a few schools do offer beginner courses at Year 12. In particular this applies to Chinese, but it appears that such classes are frequently started for, and even limited to, overseas fee-paying students or new Asian immigrants. In some schools FLL is compulsory for one or two years, although this may apply only to more able students where classes are streamed, a system which tends to perpetuate the idea, noted by the ERO (1994: 11), that languages are too difficult for most students.

One other notable factor apparent in any examination of the FL enrolment statistics is the gender imbalance. FL classes are dominated by girls, especially beyond year 9 . Table 1.6 shows, for example, the differences in the numbers of boys and girls who studied French or Japanese among those who entered secondary school in 1994.

The generally better retention rates in Japanese seem to be largely due to the fact that boys are more inclined to continue studying this language than French, as shown in Tables 1.7 and 1.8. In years 12 and 13 in the cohort represented in the tables girls outnumbered boys in French by more than 3:1. Girls still outnumbered boys in senior classes of Japanese, but by less than 2:1. The role which gender plays in attitudes towards FLL and student choices will be discussed in detail in Chapter 10. 
Table 1.7 A comparison of gender imbalances in French in one cohort of FL students (MoE statistics)

\begin{tabular}{|c|c|c|c|c|c|}
\hline \multirow{2}{*}{ French } & \multicolumn{3}{|c|}{ Student numbers } & \multicolumn{2}{c|}{ Gender balance } \\
\hline Year/Level & Girls & Boys & Total & Girls & Boys \\
\hline $\mathbf{1 9 9 4 / Y 9}$ & 8038 & 5472 & 13510 & $59 \%$ & $41 \%$ \\
\hline $\mathbf{1 9 9 5 / Y 1 0}$ & 3957 & 2178 & 6135 & $64 \%$ & $36 \%$ \\
\hline $\mathbf{1 9 9 6 / Y 1 1}$ & 2172 & 946 & 3118 & $70 \%$ & $30 \%$ \\
\hline $\mathbf{1 9 9 7 / Y 1 2}$ & 1105 & 336 & 1441 & $77 \%$ & $23 \%$ \\
\hline $\mathbf{1 9 9 8 / Y 1 3}$ & 740 & 220 & 960 & $77 \%$ & $23 \%$ \\
\hline
\end{tabular}

Table 1.8 A comparison of gender imbalances in Japanese in one cohort of FL students (MoE statistics)

\begin{tabular}{|c|c|c|c|c|c|}
\hline \multirow{2}{*}{ Japanese } & \multicolumn{3}{|c|}{ Student numbers } & \multicolumn{2}{c|}{ Gender balance } \\
\hline Year/Level & Girls & Boys & Total & Girls & Boys \\
\hline $\mathbf{1 9 9 4 / Y 9}$ & 7126 & 5706 & 12832 & $56 \%$ & $44 \%$ \\
\hline $\mathbf{1 9 9 5 / Y 1 0}$ & 4109 & 2690 & 6799 & $60 \%$ & $40 \%$ \\
\hline $\mathbf{1 9 9 6 / Y 1 1}$ & 2661 & 1385 & 4046 & $66 \%$ & $34 \%$ \\
\hline $\mathbf{1 9 9 7 / Y 1 2}$ & 1630 & 805 & 2435 & $67 \%$ & $33 \%$ \\
\hline $\mathbf{1 9 9 8 / Y 1 3}$ & 1012 & 540 & 1552 & $65 \%$ & $35 \%$ \\
\hline
\end{tabular}

\subsection{Ministry of Education policy on languages}

Although detailed recommendations for a National Language Policy were made by Waite (1992) in a report commissioned by the MoE, such an overall policy has never been formulated. Therefore any language planning that is occurring in the country is proceeding without any national guidelines or setting of priorities (R. Kaplan 1993, Jackson \& Peddie 
1998). What remains of the project to develop a national languages policy is a 'languages-in education policy' with a number of separate developments within the MoE (Shackleford 1996). ERO (formerly the school inspection branch of the Education Department) carried out a small scale survey of the state of FLL in secondary schools and was clearly disappointed by what they found in 1994 :

If the future economy of New Zealand is dependent on people with skills in languages other than English and Mäori then the issue of second language provision, enrolment and retention in New Zealand schools needs to be addressed now.

(ERO 1994: 17)

Despite the lack of official policy towards FLL in schools, it must be acknowledged that much has been done to support and encourage it. The MoE has produced curriculum statements for the teaching of Chinese, Japanese, Korean Spanish and Samoan, and the French and German statements are under development. Another ongoing MoE initiative is the Second Language Learning Project (SLLP), launched in 1995 and designed to both increase the number of students learning a LOTE in Years 7 to 10, and to improve retention rates in senior secondary language programmes. Funds have been made available to improve the language teaching skills of existing teachers in the schools and to provide technical and educational support, but specifically not for the employment of specialist language teachers, nor for native speakers to be brought into the schools.

The SLLP has successfully encouraged the expansion of FLL in Years 7 and 8, with the help of specially produced teaching materials, the International Language Series, in French, German, Spanish and Japanese, designed for use by teachers who do not necessarily have FL skills themselves. The development of similar materials for Chinese is underway. In addition, the MoE extended the advisory support available to teachers by appointing a team of regional language advisers in 1998, in addition to the national advisers in Chinese, French, German, Japanese and Spanish, a factor particularly welcomed by the New Zealand Council for Teacher Education:

The impact of the advisers has been a significant and memorable step in the history of the teaching of international languages in New Zealand. (NZCTE 1998: 59)

Much of the work of the regional advisers focusses on developing FLL at intermediate level and on encouraging cooperation between secondary schools and their local primary and intermediate schools and coordination of FL teaching programmes. While the SLLP has increased the amount of FLL at intermediate level it has not always been welcomed by secondary FL specialists, some of whom remain sceptical about the value of the programmes and the skills of the teachers involved. Furthermore, large gaps remain between secondary schools and their feeder schools in terms of coordination or continuity of FLL programmes.

\subsection{Other views of foreign language learning in schools}

FL teachers have long been concerned about the lack of overall planning for the provision of 
FLL by the Government, more specifically the MoE, and about the fact that for approximately half of each year 9 intake FLL will never be part of their school education. FL teachers and advisers have gathered a good deal of information and opinion on what are perceived to be the barriers to FLL, most of which could be termed structural barriers in schools (for example, curriculum demands, the qualifications system, staffing problems and timetabling) rather than hostile attitudes. Nevertheless, it could be assumed that negative attitudes create the barriers or, at best, unconsciously allow them to be created or perpetuated. It has been interesting to observe occasional articles in the press which seem to indicate that New Zealanders are concerned about their lack of language skills (e.g. Stirling 1999). Some articles have specifically focussed on efforts by parents to seek FLL for their children, if necessary outside school (O'Flaherty 2001, Schumacher 2001, Walsh 2001).

\subsection{The aims of this research}

As a FL teacher with about 20 years experience in New Zealand schools I have a strong personal interest in this research. I have experienced the frustrations of seeing the length of FL courses reduced, while some of my best and most enthusiastic students have abandoned FLL because of timetable constraints or pressure to continue more 'useful' subjects. I have struggled with mixed-level classes and, with no time allowance, endeavoured to help isolated students obliged to study by correspondence. Finally, I have had countless conversations with colleagues and parents of students over the years about the fact that New Zealand values FLL so poorly, compared with other countries, and what might be done about it. Any conversation on the topic, even with people not directly involved in education, will elicit a variety of opinions as to why students do not choose to study a FL or drop out at the earliest possible moment. Most of these are reflected in the literature, to be reviewed in Chapters 2 and 3 , and the hypotheses which I formulated, see 2.12 .

The principal aim of my research was to discover what 11-13 year old students thought about FLL and the extent to which their attitudes influenced their choice of optional subjects, in relation to their educational goals and to the opportunities available to them at their local secondary schools. I hoped to identify the nature and the principal sources of attitudes to FLL among samples of year 8 and 9 students, parents and teachers. My underlying aim was to assist those who believe in the essential value of FLL in schools to promote it more successfully, by identifying the main disincentive(s) for such learning.

In the light of the above aims, I decided to investigate the following specific research questions:

- What do Year 8 students know/believe/think about FLL?

- Do they intend to study or continue studying a language in Year 9?

- What are the main reasons for their intentions?

- When they reach Year 9 have the same students chosen to study a language or not?

- What are the reasons for their choices?

- Who or what are the main influences on their choices? 
The theoretical framework for the investigation of attitudes was developed through my reading, mostly in the field of social psychology, and is presented in Chapter 2. In order to provide a basis for comparison with what happens in other English-speaking countries, Chapter 3 examines the place of FLL in Australia, the UK and the USA. How attitudes can be measured is discussed in Chapter 4 which details my methodology. Chapters 5-10 present the results from my student surveys and my interviews with parents and teachers and discussion of the findings. My final chapter summarises the results of this research, drawing conclusions about my hypotheses and discusses the current place of FLL in New Zealand schools. Lastly, recommendations are made for further action and research. 


\section{CHAPTER 2 LITERATURE REVIEW}

The sum of human wisdom is not contained in any one language, and no single language is CAPABLE of expressing all forms and degrees of human comprehension. (Pound 1934: 18, author's capitals)

\subsection{Introduction}

Since this research focusses on attitudes to FLL I began by exploring the notion of 'attitudes' and how they might be investigated. Then I examined literature pertaining to attitudes to languages and the concepts of ethnocentrism, ethnolinguistic identity and ethnolinguistic vitality which might explain different attitudes towards languages. Next I focussed on attitudes towards learning languages and specifically FLs. Much of the literature about attitudes to language learning is incorporated into research into motivation, especially Gardner (1985) and others who have extended his work. Lastly I sought literature concerning the place of FLL in New Zealand.

\subsection{The nature and importance of attitudes}

The investigation of attitude has largely been undertaken by social psychologists, in particular Fishbein and Ajzen (1975), Tajfel (1981, 1982), Eiser (1984, 1987, Eiser \& van der Pligt 1984) and Ajzen (1988). Fishbein and Ajzen (1975) provide a thorough overview of literature concerned with the nature of attitudes, how they can be measured and the value of such measurement. They assert that attitudes are closely linked to 'intentions to behave' and are based on what one believes to be the consequences of an action, how one evaluates those consequences, one's knowledge about what others expect and the degree to which one wishes to comply with those expectations.

This approach to attitudes has been called the "means-end analysis" (Cooper \& Fishman 1974), according to which a person develops an attitude towards a particular 'referent', an idea or course of action, for example, in relation to what that person wants to achieve. An attitude is explained as "a composite of the perceived usefulness of the referent with respect to his goals weighted by the relative value he places on each goal" (Cooper \& Fishman 1974: 7). While there are close links between beliefs, attitudes, intentions and actual behaviour, it cannot be assumed that attitudes towards an action are specifically related to actual behaviour (Fishbein \& Ajzen 1975: 520, Ajzen 1988: 133-134). According to Cooper and Fishman, "only modest relationships are found between attitude measures and the overt behaviors which such scores are designed to predict" (1974: 10). Nor can it be assumed that changing beliefs will lead to changed attitudes although attitudes are learned and can be changed (Fishbein \& Ajzen 1975, Lett 1977). These theories may be particularly useful in relation to this research since there may be no direct association between what students think about FLL and what they actually do about it when entering secondary school.

The other principal view of attitude, "the classical explication" (Baker 1992: 12) attributed to Plato, is that it is a hypothetical construct consisting of three factors:cognitive, affective and conative (Cooper \& Fishman 1974: 7, Ajzen 1988: 4). The cognitive aspect refers to what 
one thinks or believes about something. The affective component concerns one's feelings, which as Baker points out, may not be in harmony with one's beliefs. For example, an overtly stated rational belief about the value of learning a particular language may conceal an underlying prejudice or anxiety about such a course of action. The conative component refers to both readiness for action, that is intentions to behave, and actual actions or behaviour.

Baker is not sure that it is possible to accurately gauge attitudes:

Doubt has to be expressed whether deep-seated, private feelings, especially when incongruent with preferred public statements, are truly elicited in attitude measurement. (Baker 1992: 12)

Furthermore, it is often stressed that it is futile to investigate attitudes in isolation from any social context, since 'attitude-toward-object' cannot be separated from 'attitude-towardsituation (Cooper \& Fishman 1974: 11). Investigation of attitudes can explain both the choices made by individuals between alternative objects or actions, and the reasons for different evaluations of the same object or action by other individuals (Eiser \& van der Pligt 1984). Even if an individual has a favourable attitude towards a particular behaviour, and believes that it would be approved of by significant others, the individual may lack the necessary resources or opportunities to act as they would wish (Ajzen 1988: 133-134). It may well be the case that some students who are passionately interested in learning a FL do not find themselves in a school situation where such study is feasible.

Discussion of 'attitude' by different theorists of ten overlaps that of 'motivation'. In addition, the measurement of attitudes is considered problematical. However, Baker (1992), referring specifically to the study of bilingualism, asserts that the social psychological concept of attitude is important for three reasons. Firstly, attitude is a commonly used term. Secondly, "a survey of attitudes provides an indicator of current community thoughts and beliefs, preferences and desires", and can "provide social indicators of changing beliefs and the chances of success in policy implementation" (Baker 1992: 9). Thirdly, "attitude has repeatedly proven a valuable construct in theory and research, policy and practice" (1992: 10). Baker bases his investigations into attitudes towards Welsh on Gardner (1985), whose work will be examined in detail below. To a large extent, this investigation of attitudes is based on premises similar to Baker's.

\subsection{Attitudes towards languages}

Most research into attitudes towards languages has fallen into two main areas. One deals with attitudes towards variation in one particular language used within a society, usually the dominant one: variations in style, register, regional accent, and dialect, for example. The other deals with attitudes towards different languages used in a society, comprising different ethnolinguistic groups, where questions arise concerning language policy and planning, assimilation and bilingualism, language maintenance and shift, and where SLL is required of or desired by substantial numbers of people. Cooper and Fishman (1974: 6) sum up 
language attitudes as "those which influence language behavior and behavior towards language". Attitudes, they add, may be towards a given language as a whole, towards particular features of a language, towards the use of the language or to the language as a marker of a particular group. In all cases, it could be said that attitudes towards a language are inseparable from attitudes towards the people who speak it (Holmes 1976). Attitudes towards languages can therefore be linked to the notion of ethnocentrism, which may have some bearing on attitudes to FLL in New Zealand.

\subsection{Ethnocentrism, ethnolinguistic identity and ethnolinguistic vitality}

According to Giles, Bourhis and Taylor (1977) and Lett (1977) language is an essential feature of social identity and a different language is a marker of another group. Ethnocentrism necessarily involves discrimination of some sort against those perceived as belonging to an 'outgroup' (Tajfel, Flament, Billig \& Bundy 1971, cited by Bourhis \& Giles 1977). The more distant an 'outgroup' is, in cultural terms, the more likely attitudes towards it will be based on stereotypes, and the more negative the stereotypes, the more negative the attitudes (Coleman 1996: 101). Tajfel (1981) shows how children develop evaluations of national and ethnic groups other than their own, without direct contact or any other any real knowledge on which to base such evaluations, concluding that children are highly sensitive to the socially prevailing attitudes towards 'outgroups'. These findings are supported by Barrett and Short (1992) who also found that affective responses tend to remain relatively unchanged by the increase in knowledge. What is particularly interesting, in the light of opinions which emerged in this research about the best starting age for FLL, is that the older children, 8-10 years, were less negative and less inclined to stereotype foreigners than the younger children.

Although the concept of ethnocentrism may help to explain negative attitudes towards any sort of SLL, it is used largely to explain violent conflict between nations or ethnic groups. The concept of ethnolinguistic identity, based on Tajfel's (1974) social identity theory and introduced by Giles and Johnson (1987) seems more appropriate for this research. According to Gardner and Clément (1990), language is an important aspect of ethnic group identity within a multiethnic society, especially for minority groups who may be struggling to maintain their identity. In fact, the notion had already been explored by Taylor, Meynard and Rheault (1977) who identified social psychological factors among French Canadians which "could be construed as effective barriers to the process of learning [English]" (1977: 102). While this notion has relevance for New Zealand society, as far as Maori and other community languages are concerned, it may have little bearing on the question of FLL.

Another concept, developed by Giles, Bourhis and Taylor (1977), to explain attitudes towards language variation and SLL, and how they affect behaviour, is ethnolinguistic vitality (EV). The concept is defined as "that which makes a group likely to behave as a distinctive and active collective entity in intergroup situations" (1977: 308), and is once again based, in part, on Tajfel's (1974) theory of social identity. The categorisation of people by ethnic group is a "fundamental process which serves as a basis for people's attitudes and behaviour towards others" (Giles et al. 1977: 325). Once again, though, investigations are 


\section{4}

based on the behaviour of minority groups in the face of a dominant other language, and the extent to which group members, for example Welsh speakers (Bourhis \& 1977), emphasise their identity in their language use. The concept of EV is clearly valuable for sociolinguistic studies of language maintenance and shift, accommodation and code-switching, as well as for the social psychological study of attitudes (Harwood, Giles \& Bourhis 1994), but possibly not so useful in a study of attitudes to FLL.

\subsection{Attitudes towards language learning}

However attitudes towards languages develop, they clearly influence SLL and FLL (Giles \& St. Clair 1979, Gardner \& Clément 1990). Much of the research into attitudes towards language learning has concerned SLL in societies which are bi- or multilingual, for example in Canada (e.g. Gardner \& Lambert 1972, Gardner 1985, Labrie \& Clément 1986), and in Wales (Jones 1949, 1950, 1966, Baker 1988, 1992). Ball, Giles and Hewstone (1984), studying L2 acquisition in relation to the theory of intergroup behaviour as well as Gardner's theories of motivation, note that:

The implications for second language acquisition by members of a dominant group (i.e. the circumstances typical of formal 'modern language' teaching) have yet to be considered. (1984: 675)

Attitudes towards acquiring an L2 have been considered in relation to the concepts of additive and subtractive bilingualism (Lambert 1974). The extent to which a minority community is prepared to learn a dominant language may depend on the extent to which they feel that they may lose their L1, rather than gain an additional skill. Cook (1991) cites the example of Chilean refugees she taught in England who were disappointed at their lack of progress in learning English:

However much they consciously wanted to learn English, subconsciously they saw it as committing themselves to permanent exile and thus to subtracting from themselves as Chileans. (Cook 1991: 74)

On the other hand, if an L2 is perceived to have a high status or if speakers of the language are seen to be attractive, a member of another speech community may have positive attitudes towards learning the L2. Thus language attitudes may facilitate or hinder learning (Bradac \& Giles 1991: 2) and social context is crucial:

learning another language is a sociopolitical process and one which ... involves learners testing another's culture in their mouths and hearing themselves sound like a member of a respected or disrespected group. (Bradac \& Giles 1991: 9)

The extent to which an L2 is perceived to be desirable will depend to a great extent on the public treatment of the language by government, business and schools for example (Ryan, Giles \& Sebastian 1982: 7). A change in societal attitudes can lead to changes in individual attitudes towards learning a language. In Hong Kong, for example, as Chinese has 
gradually displaced English as the main language of instruction, school students have become less interested in learning English, except for purely instrumental reasons, although many parents still want English medium instruction for their children (Lai 1999: 282). Attitudes to language learning in schools may reflect wider political attitudes towards what constitutes a proper education and the appropriate allocation of resources and:

participants in formal language study programs may view efforts to change their language behavior as assertions of undue power on the part of institutions or employers (McGroarty 1996: 28-9)

Once again, most of the research is concerned with situations quite different from that of FLL in New Zealand schools, but it is undoubtedly important to consider the role of the attitudes of officialdom and the wider society towards the study of LOTEs in influencing what students themselves decide.

\subsection{Attitudes towards FLL}

The two main groups of attitudes towards FLL which have been identified and investigated are:

- attitudes towards the language learning process itself and the effect of achievement on further language acquisition (largely research into motivation);

- attitudes towards a specific target language community and culture and the effect of learning on such attitudes, (that is, non-linguistic outcomes of FLL, including "deliberate attempts to modify ethnocentric attitudes or to foster more positive attitudes toward cultural diversity in general" (Lett 1977: 294).

These are relevant to this research only if it is found that some surveyed students have already have some experience of FLL before reaching secondary school. Such experience will undoubtedly influence their attitudes towards undertaking further FLL in year 9, be it of the same or a different FL.

Lett applauds the longitudinal research being carried out in Canada by Gardner and his associates, but points out that "attitudes formed in a bilingual situation may have very little in common with those formed in a monolingual environment" (Lett 1977: 294). Lett also emphasises the need for before-and-after studies when attempting to measure the effect of FLL on attitudes since:

The fact that a collection of foreign language students may exhibit less ethnocentrism than a collection of nonforeign language students may be attributable to relevant differences between the groups of students that existed before any of them had an opportunity to study foreign languages, and that helped to determine which students would study foreign language and which would not. (Lett 1977: 294, my italics)

It was particularly difficult to find research into attitudes towards FLL among students who had not had any such experience, largely because in most Western countries FLL is a compulsory part of the school curriculum. The main exception is the USA where for several 
decades FLL was optional, or even nonexistent, in secondary institutions. Even so, most of the research has been into reasons for poor retention rates in FL classes. Hofman (1974), in a review of Gardner and Lambert's (1972) theories of integrative and instrumental motivation (see 2.7 below), claims that studies thus far had shown that students studying a FL without direct contact with the target language group "must perhaps fall back on the primacy of their own needs, one of which is simply that of doing what parents and school want them to do" (Hofman 1974: 118). According to Turner (1974), the factors which caused American students to not take up FLL or to drop out at the first possible opportunity were:

- lack of motivation because FLL is seen as irrelevant;

- lack of interest among parents; and

- lack of support from administration staff who were not convinced of the value of FLL.

These findings suggest that in New Zealand the most important influences on student behaviour regarding FLL, that is, their decision to study a FL or not, will be external, not students' own attitudes. To many FL teachers in New Zealand today the factors listed by Turner (1974) will sound familiar.

More recently FLL has been investigated in Hungary among secondary school learners of English (Dörnyei 1990a, 1990b, Clément, Dörnyei \& Noels 1994) and learners of five European languages (Dörnyei, Nyilasi \& Clément 1996). The latter research showed that English was the most popular FL because of "sociocultural aspects associated with the US" (Dörnyei 1998: 129). Three distinct dimensions to successful language learning are identified by Dörnyei: integrative motivation, linguistic self-confidence, and appraisal of classroom environment which, he claims, confirms "language teachers' intuitive knowledge that what goes on in the classroom will considerably affect the learners' affective predisposition" (1996: 75). Similarly, Chambers (2000) found that, for learners of English in Germany and learners of German in England, the quality of the teacher, as evaluated by the students, was a key factor in their attitude towards their FL classes. Once again, it is the case that the attitudes under investigation were those of students already involved in FLL, not students faced with the choice of learning a FL or not, as is the case in New Zealand. More evidence of attitudes towards language learning in the UK, the USA and Australia will be examined in detail in Chapter 3.

\subsection{Attitudes and motivation}

In general, research into attitudes towards L2 learning has been subsumed into investigations into motivation and differing levels of achievement among students, i.e. the attitudes investigated have been those of students already in the learning situation. It cannot be assumed that attitudes which have been shown to promote language acquisition are necessarily the same as those which encourage students to opt into language learning, but it seems reasonable to assume that they may depend on some common factors. This research will, therefore, be investigated in some depth.

The foundation work in the field of SLL motivation was carried out by Gardner and various 
colleagues, culminating in Gardner (1985) which presents his socio-educational model of SLL. This model is designed to explain the role of attitudes and motivation, along with other factors, in the different levels of achievement obtained by individual students in the same, formal learning situations. Gardner explains the nature of attitudes in social psychological terms, discusses ways of measuring them and identifies those attitudes which he finds most relevant in SLL. According to Gardner's model, not only do attitudes affect the progress which students make, but their experience of SLL affects their attitudes towards the language studied, the culture it represents and the learning process itself. Attitudes are entirely independent of intelligence and aptitude, although these are obviously important factors in a student's progress. Gardner reviews an extensive amount of research in the field, mostly in North America, and presents the results of a series of studies carried out by himself and his colleagues. Despite differences in methods and situations, he finds considerable consistency in all the results.

According to Gardner, SLL, unlike all other school subjects, is a social psychological phenomenon in that it involves changes in behaviour. An L2 "does not represent an aspect of the individual's own cultural heritage" (1985: 7). All other subjects can be learnt in one's mother tongue but not languages, at least not since the general abandonment of the grammartranslation method of teaching. Not only does Gardner find that attitudes towards SLL are closely linked to achievement, but also that it is "highly likely" (1985: 42) that attitudes towards other school subjects are not necessarily related to achievement in those subjects. Students' attitudes towards hearing and producing strange sounds and linguistic structures will affect the extent of their proficiency in the L2 and their adaptation to new cultural ideas.

To understand the nature and importance of attitudes towards SLL, Gardner asserts that it is necessary to look at the context of the learning, not just the classroom and the school, but the wider social milieu. As he states, "motivation has a social dimension reflecting the individual's reactions to outgroups in general and the other language community in particular" (1985: 168). Gardner's description of the general social backgrounds revealed in the research undertaken in the U.S. A. and Canada, suggests similarities with New Zealand, where SLL is not the norm for all students. Gardner reports that even in Canada, where official importance is given to bilingualism in French and English, only about half of primary and secondary students studied an L2 (1985: 3). Students in formal classes had little or no contact with communities or individuals who spoke the languages studied. Gardner also cites the general belief that North Americans are apathetic towards SLL or not good at it. Even in Europe where SLL has always been the norm, there are considerable differences in individual achievement and significant drop-out rates once such study is no longer compulsory.

Gardner did not aim to study what happens in the classroom, although he admits that teachers, their methods and the courses they use must have an effect on student motivation and progress. He wanted to identify what students bring with them, by way of attitudes and motivation and the role of these, alongside individual intelligence, language aptitude and 'situational anxiety' (depending on a student's personality), in L2 acquisition. He also 
investigated where such attitudes might come from, claiming that most are fostered in the home and immediate social environment, and reinforced by peers. "Attitudes are clearly influenced by many factors in the student's upbringing" (1985: 43). Gardner asserts that many attitudes may be dormant until students are actually involved in the SLL process.

It could be argued that, if SLL is optional, attitudes towards FLs in general or specific target languages will influence the choices made. An interest in learning more about, say, French speakers and how they live, as much as an interest in their language, could influence a student's choice to study French. This supposition is supported by one of Gardner's earlier findings (Gardner \& Smythe 1975) that:

students who drop out of second language study have a priori less favourable attitudes toward the other language community than those who continue language study, and it seems possible that such differences could also characterise those who elect initially to study or not study a second language (cited in Gardner 1985: 46).

Later evidence also supports the importance of interest in both learning a specific language and language learning in general. Gardner (1985), examined data from over thirty studies carried out in Canada among English-speaking students learning French and found that the two attitude measures most closely allied to achievement and continued learning of the language were the attitude towards learning French and a general interest in FLs. It needs to be noted that Gardner never makes it clear whether the research he reviewed or carried out himself involved students who had chosen to study a language or who were obliged to be in language classes.

Like Cooper and Fishman (1974) and Baker (1992), Gardner states that attitudes have three components - beliefs about an object, emotional reactions to it and behaviour towards it. He puts it thus:

an individual's attitude is an evaluative reaction to some referent or attitude object, inferred on the basis of the individual's beliefs or opinions about the referent (1985: 9, author's italics)

Thus a student's attitude towards SLL can be measured by examining stated beliefs and opinions about such learning, or the prospect of undertaking such learning. Gardner offers the helpful comment that it is easier to measure attitudes towards more specific objects, such as 'learning French' than more general ones such as 'learning another language'.

Although Gardner discusses attitudes and motivation separately, his definition of motivation (1985: 10) makes it clear that attitudes form an essential part of this concept. Motivation involves effort in class, a desire to learn and favourable attitudes towards what one is trying to learn and towards the learning process. Gardner identifies two general orientations of motivation (although he admits the possibility of others). With integrative orientation one strives to learn a language for mainly social reasons, such as interest in another culture or 
desire for contact with that culture, or for personal interest in the language itself. Instrumental orientation is more concerned with the usefulness of a particular language for employment, for example. One of Gardner's principal findings is that integrative orientation is closely linked with successful language acquisition.

Gardner (1985: 109) cites the main influences on students attitudes identified by Oskamp (1977) as parents, the peer group, "direct indoctrination in school", the mass media and "general expectations or beliefs in the cultural community". Gardner and Clément, referring back to the findings of Gardner, Lalonde and Pierson (1983), note:

a positive association between the extent to which students perceived school officials to be favourably predisposed toward second language course objectives and integrativeness (1990: 509)

General beliefs about SLL may relate to its perceived difficulty. If a community believes that languages are particularly difficult subjects, SLL may be reserved for more academic students, as used to occur regularly in New Zealand, but Gardner argues that this is a selffulfilling prophecy:

if the cultural belief is that it is very difficult to learn a second language, the general level of achievement will be low...If, on the other hand, it were expected that most individuals would learn a second language, the general level of achievement would be high" (1985: 146-7)

Gardner clearly agrees that parents have the greatest influence on children's attitudes, calling them "the major intermediary between the cultural milieu and the student" (1985: 109). Parents can actively encourage or discourage a student's efforts to learn an L2 by what they say, but they can be equally influential in passive ways by, for example, revealing negative attitudes towards a target language or culture. Such passive discouragement may outweigh specific exhortations to work hard in class, complete homework, and so on. On the other hand, positive passive influence tends to encourage an integrative orientation towards SLL which, as already discussed, Gardner found most favourable to success.

Lastly, one part of Gardner's (1985) socio-educational model of SLL not yet mentioned, but relevant in the New Zealand context, is the assertion that achievement in SLL leads to improved attitudes towards the language itself, the language learning process and the target community. Students who might not otherwise choose to study an optional FL, because of the perceived difficulty of such study, may change their attitude once they have had the chance to try it and succeed.

\subsection{Motivation - beyond Gardner}

As Crookall and Oxford (1988) predicted, Gardner's ideas have had a profound influence and much subsequent work has attempted to build on his socio-educational model to improve understanding of what motivates students to persist in their efforts to acquire an L2. 


\section{0}

Theoreticians and researchers (e.g. Skehan 1989, Crookes \& Schmidt 1991, LarsenFreeman \& Long 1991, Baker 1992, Dörnyei 1996, Oxford \& Shearin 1996, Williams \& Burden 1997, Agnihotri, Khanna \& Sachdev 1998), have sought to define a wider range of orientations than Gardner's (1985) integrative and instrumental ones to which attitudes and motivation might be attributed.

Oxford (1996) presents a series of papers which "chronicles a revolution in our thinking about what makes students want to learn languages" (1996: 1) but which aims to extend Gardner's ideas, not reject them. Gardner himself (Gardner \& Clément 1990, Gardner \& MacIntyre 1993, Tremblay \& Gardner 1995) has expanded his theories to include many more variables, paralleling work by other researchers. Gardner and Clément stress the role of the teacher who "may be perceived as representing the second language-speaking group for students living in unilingual communities and, consequently, be an important agent of attitude formation and change" (1990: 495). Gardner and MacIntyre (1991: 4) admit that "the old characterisation of motivation in terms of integrative versus instrumental is too static and restricted".

Once again discussion is almost entirely about in-class motivation and it is still the case that "most research on motivation has been conducted in second rather than foreign language learning contexts and in North American or European cultural settings" (Schmidt, Boraie \& Dassabgy 1996). Nevertheless I considered it valuable to try and pinpoint aspects of findings about attitudes which could be relevant for the FLL learning situation in New Zealand.

For Crookes and Schmidt (1991) motivation consists of four internal, attitudinal factors and several external or behavioural factors, the most relevant of which, for my research, is that the student decides to undertake language learning. The attitudinal factors are:

- interest in the L2 based on existing attitudes, experience, and background knowledge on the learners' part;

- relevance, which involves the perception that personal needs such as achievement, affiliation, and power are being met by learning the L2;

- expectancy of success or failure; and

- outcomes, i.e., the extrinsic or intrinsic rewards felt by the learner (cited in Oxford 1996: 5).

Oxford and Shearin (1996), drawing on various areas of psychology, go even further in widening the variables relevant to motivation in L2 learning, summarising them under seven headings: attitudes, beliefs about self (for example, aptitude for SLL), goals (including how they are established - by learner, teacher, institution), involvement, assistance/environment (including the institutional and cultural), performance links, and demographics/personal (including previous language and travel experiences) (1996: 143-4). Given the definitions of attitude previously discussed, consisting of three factors (beliefs, feelings and readiness to act), it is hard to understand what precisely Oxford and Shearin (1996) mean by 'attitudes' in isolation from the other listed variables. 
Research into the influence on motivation of the broad concepts of integrative and instrumental orientation has produced conflicting results. Some studies have shown achievement in SLL to be most closely associated with integrative orientation (Gardner 1985). Others have concluded that FL students usually have insufficient contact with a target language or community to develop clear attitudes towards that community and, hence, a desire to integrate with them (Dörnyei 1990a, 1990b). It has been suggested that:

the less contact there is between the learners and the target language community - as is often the case in instructed language acquisition - the more likely the learners are to display an instrumental orientation. (Hotho \& Reimann 1998: 132)

This may particularly be the case in a geographically isolated country like New Zealand, especially when our nearest neighbours mostly speak English, or languages not offered in schools, although this situation is beginning to change.

In later research, Dörnyei (1996) identified a lack of instrumental motivation in his results. $\mathrm{He}$ concludes that such motivation is only relevant where there are short-term practical benefits for students. "If by such benefits we mean job- or salary-related motives, instrumental motivation is actually very often not too relevant to school kids" (Dörnyei 1996: 76). Dörnyei goes on to offer a new framework for language learning motivation involving three levels to represent social, personal and educational dimensions:

- the social, or language, level "concerns ethnolinguistic, cultural, affective, intellectual, and pragmatic values and attitudes attached to the target language", which incorporates the traditional concepts of instrumental and integrative motivation;

- the personal, or learner, level concerns personality traits related to language or learning in general;

- the educational, or learning situation, level concerns all aspects of the classroom, including the teacher, teaching style and materials and the group dynamic of the class. (Dörnyei 1996: 76-77)

Many of the increased number of motivational variables identified by various theorists remain to be tested by empirical research, according to Hotho and Reimann (1998: 133), but Dörnyei (1998) offers an extensive overview of theoretical developments and empirical studies in the field of motivation, concerning both SLL and FLL in many parts of the world during the previous decade. He presents a table comprising thirteen motivation constructs which he has examined, synthesising them under seven headings or dimensions, with references to all the relevant studies:

- affective/integrative dimension

- instrumental/pragmatic dimension

- macro-context-related dimension

- self-concept-related dimension

- goal-related dimension

- educational context-related dimension 
- significant others-related dimension Dörnyei (1998: 128)

With regard to the last of these dimensions Dörnyei cites the importance of parental influence on student motivation, identified by Gardner, Masgoret and Tremblay (1997), commenting that "this issue has been somewhat neglected during the past decade relative to its paramount importance in shaping learner motivation" (Dörnyei 1998: 130). It is hard to imagine in the New Zealand context that parental influence, direct or indirect, would not be significant in forming student attitudes towards LOTE learning.

At this point in my review of the literature I felt that there was an enormous and bewildering range of factors involved in the development of attitudes and motivation towards SLL, although I suspected that in many cases it was a matter of relabelling, or of breaking down the broad concepts of integrative and instrumental orientation into subgroups. Noels (2001), for example, examines the idea of identifying intrinsic and extrinsic orientations and the extent to which these might parallel the integrative and instrumental, concluding that this is not the case. In particular, integrative orientation could be seen as extrinsic in that SLL is seen as useful for achieving the goal of interacting with another community (Noels 2001: 51). More usefully, Noels points out that some intrinsic motivation has little to do with either integrative or instrumental orientation. Some people want to learn an L2 out of interest in the language per se or for the pleasure of the language learning process, an aspect which, as noted above, Gardner (1985) includes in integrative orientation.

It needs to be reiterated that most of the literature reviewed concerning attitudes towards SLL has focussed on understanding what motivates students to achieve and continue their studies, not what motivates them to begin SLL in the first place. Nevertheless, there are valuable insights in all such work as to the nature, importance and origin of attitudes to L2 learning and how they can be measured. Commenting on differences in levels of achievement among L2 learners, Agnihotri et al. (1998: 17) state that "individual differences in second language learning are an index of a learner's social background, experiences and aspirations". It is obvious that a similarly complex combination of factors must influence students choices to study an L2 or not.

\subsection{Gender differences in attitudes towards FLL}

\subsubsection{Females dominate FLL}

Many studies over the last thirty years, in various parts of the world, have found that female school students have more positive attitudes towards FLL (Gardner 1985, Wright 1993). In Britain, for example, languages tend to be perceived as 'female' subjects. As a consequence more girls continue studying languages than boys, with proportionately larger numbers of female graduates and language teachers, which tends to reinforce the perception and offer few male role models in FLL (Wright 1993: 44, Clark \& Trafford 1995: 318). A large survey of over 19,000 university students of languages in several European countries found that the majority of students were female, for example, $73 \%$ of those studying French and $65 \%$ studying Russian (Coleman 1996). Gender discrepancies in the study of such subjects as 
science and technology were addressed in the UK in the 1980s but "very little note has been made of the considerable gulf between boys and girls in the uptake and performance in modern languages" (Clark \& Trafford 1995: 315). Carr (1997) noted the "lop-sided gender profile of language classrooms in Australia" and Waite (1992) pointed out that "there has as yet been no effort made to encourage male students into the languages area" in New Zealand, compared with the compulsory status of mathematics and science for all students.

French is the most commonly taught FL in Britain and it tends to be associated with things feminine, such as fashion, perfume and cooking (Pritchard \& Loulidi 1994), which may be a reason for boys' rejection of FLL, but other studies (Phillips 1982, Powell \& Batters 1985, Pritchard 1987) have shown that offering German instead of French makes little difference to boys' attitudes. Boys, as much as girls, consider German a 'harsh' language, find it more difficult than French and are less likely than girls to be pleased to have the opportunity to learn it (Pritchard 1987).

When asked directly, both girls and boys firmly reject the idea that FLL is more important to one group or that one group is more capable of FLL (Powell \& Batters 1985). Higher socio-economic status tends to produce more favourable attitudes, regardless of gender, and systematic gender differences probably result from parents passing on the accepted values of the wider society (Burstall 1979). In the USA (Muchnick \& Wolfe 1982, Bacon \& Finnemann 1992) and in Japan (Morizumi 1999) there are more favourable attitudes and motivation towards FLL among girls .

In Australia (Zammit 1992, Carr 1997) LOTEs are regarded as girls' subjects. In Victoria, FL programmes are stronger in independent, especially girls', schools (Peddie 1993, Ashenden \& Milligan 2001). A study into the attitudes of 13-15 yearolds towards seven specific languages (European and Asian) revealed significantly more positive attitudes among girls (Zammit 1993). Only in the case of Modern Greek were boys' attitudes equally positive and their attitudes were particularly negative towards French and Italian. The 2000 LOTE enrolment figures in Victoria showed that in Years 11 and 12 girls involved in FLL outnumbered boys by two to one and it appears that "boys just don't want to learn languages" (Ashenden \& Milligan 2001). Similar imbalances have been shown in FLL in New Zealand schools (Waite 1992, Vine 1993, ERO 1994). More evidence of gender differences in New Zealand is discussed in 2.10 .4 below.

\subsubsection{Girls enjoy FLL more than boys}

In the 1970 s when attempts were being made to introduce language learning (i.e. French) into English primary schools, significantly more positive attitudes were found among girls than boys (Burstall 1979). After one year of study, $83 \%$ of girls enjoyed French, compared with only $67 \%$ of boys, and girls received more encouragement than boys from their parents to learn French. A study of English secondary students after their first year of compulsory FLL (Powell \& Batters 1985) 
found that $30.4 \%$ of girls and $20.2 \%$ of boys rated a FL as a popular subject, and twice as many girls as boys rated a FL as their favourite or 'next favourite' subject.

Table 2.1 Reasons cited by each gender for dropping out of FLL in England (Adapted from Aplin 1991: 9)

\begin{tabular}{|l|c|c|}
\hline Reason for giving up FLL & $\begin{array}{c}\text { Proportion of } \\
\text { girls }\end{array}$ & $\begin{array}{c}\text { Proportion of } \\
\text { bovs }\end{array}$ \\
\hline Other subjects preferred & $71.3 \%$ & $71.7 \%$ \\
\hline Dislike of languages & $33.8 \%$ & $46.7 \%$ \\
\hline Low test marks & $36.3 \%$ & $25.0 \%$ \\
\hline Dislike of teacher & $13.8 \%$ & $20.8 \%$ \\
\hline Career guidance & $26.3 \%$ & $15.8 \%$ \\
\hline
\end{tabular}

Another survey in Britain of 200 secondary students, aged 16-19, investigating why they had given up FLL after 2-5 years of study, revealed that the main reason for equal proportions of girls and boys was their preference for other subjects (Aplin 1991). Among other reasons, however, there were some marked differences between the two groups, see Table 2.1. Girls clearly enjoyed languages and liked their language teachers more than boys did. Other research has also shown that boys seem to be more influenced than girls by their dislike of a teacher (Clark and Trafford 1995, Carr 1997). According to Aplin (1991), girls were discouraged from further FLL more by lack of success or by careers guidance which persuaded them that other subjects would be more useful to them. Nearly $29 \%$ of girls, compared with under $13 \%$ of boys would have liked the opportunity to start a new language, and more girls, 26\%, than boys, 18\%, regretted having given up FLL (Aplin 1991: 10) .

\subsubsection{Girls are more open to other languages and cultures}

Girls may enjoy FLL more than boys because they are, for some reason, more interested in languages and cultures other than their own. A British study (White \& Gray 1997) into how one secondary school was coping with an enormous increase in cultural and linguistic diversity found that the younger students were most open to the diversity as an opportunity for learning, but that it was always valued more by the girls and by students who spoke more than one language. As they grew older, all the students became less open to cultural and linguistic diversity, but this was noticeably more apparent among the monolingual boys. The school was committed to "empowering students through de-mystifying language" by encouraging and valuing bilingualism (White \& Gray 1997: 6) and the researchers found that the more languages the students spoke, the less divergence in attitudes there was between the 
girls and boys (1997: 13), which could be a powerful argument for encouraging or insisting that all boys study a second language.

\subsubsection{FLL is less relevant for boys}

Aplin expressed surprise at finding that girls were more likely than boys to have been influenced by career guidance into giving up languages "in view of the stereotyped views of male and female occupations" (1991: 8). This traditional view is reflected in the claim that different attitudes "undoubtedly stem from the different employment expectations of boys and girls and the extent to which foreign language learning can be perceived as relevant to their occupational requirements" (Burstall 1979: 140). Although the bias against FLL among boys may be sustained by the view that FLs are less relevant for male occupations, or that FLs in general are not useful for jobs, it is also possible that society considers it more important for boys to prepare for a career than girls (Waite 1992: 74). Hence boys may be more likely to be advised or encouraged to choose 'useful' subjects whereas girls can afford to indulge in subjects they enjoy.

Girls and boys seem to see FLs as relevant for different purposes. When Aplin (1991) asked what would have persuaded students to continue FLL, twice as many girls as boys selected travel to a country speaking the target language. $25 \%$ of all students would have been swayed by "closer connections with career opportunities" (Aplin 1991: 10). Powell and Batters (1985) found that girls considered FLL more important, showing a greater desire to meet speakers of the target language. However, Clark and Trafford found that "typically, the more able pupils, both boys and girls, had higher career aspirations and could appreciate the value of the study of a foreign language" (1995: 320), and that lower ability students saw no use in FLL as far as future employment was concerned, as opposed to possible use in travel.

In Australia boys and their parents consider FLL, especially of European languages, largely irrelevant for their lives and more specifically for their future careers (Carr 1997). In New Zealand there is evidence that boys are actively discouraged from studying languages as languages are given a low profile in many boys' schools (NZCTE 1998).

\subsubsection{Languages are more difficult for boys}

Students in the UK consider FLs more demanding than other subjects because of the amount of concentration and vocabulary learning required (Clark \& Trafford 1995: 321). This seems to be particularly a problem for boys who prefer to make the minimum efforts, or are actually disruptive, in class, but Clark and Trafford found that the lack of effort could be attributed to some extent to the quality of teachers, their methodology and the course contents. Similar problems were identified in Australia by Carr (1997) in addition to problems created by the lack of continuity between primary and secondary FLL. Girls may also be frustrated by the repetitiveness of learning, the irrelevance of some topics and the lack of contact with native 
speakers (Clark \& Trafford 1995), but they tend to have more positive attitudes to written and oral work in the target language than boys (Powell \& Batters 1985).

Whatever the reasons for their lack efforts in FLL, boys in the UK achieve less well in exams. The 1992 GCSE results showed "a considerable disparity" between boys and girls, with $26 \%$ of boys gaining grades A-C in modern languages compared with $43 \%$ of girls (DFE 1993, cited by Clark \& Trafford 1995: 315). In Australia, boys seem to find FLL more difficult than girls, which may cause their dislike of the subject and be the most common reason for abandoning it (Zammit 1992, Carr 1997).

Ultimately, it is not clear from the literature whether boys' dislike of FLL leads to poorer efforts and hence to lower levels of achievement, or some natural lack of aptitude leads to poor results and hence relatively poor attitudes to FLL. More research is obviously needed in this area.

\subsubsection{Summary of gender differences}

Overseas research shows that perceptions are still widespread that languages are somehow 'feminine' subjects. It has been pointed out that research into the existence of predestined biological sex differences in languages are "scant and inconclusive" (Loulidi 1990: 40). Different attitudes to FLL of girls and boys derive from sociocultural pressure. That is, "social norms do not seem to conflict with girls learning a language, whereas they do for boys" (Loulidi 1990: 42). Loulidi goes so far as to claim that this type of sex-stereotyping is perpetuated by schools. This is seen as particularly crucial in the area of careers guidance where advisers may be "biased by preconceived notions, which would no doubt influence the kind of advice or guidance they may offer" (1990: 42) and hence limit the opportunities for both girls and boys instead of helping them to widen their horizons and their opportunities in life. Anecdotal evidence indicates that gender is also an influential factor in subject choices in New Zealand schools, which will be discussed in 2.10 .4 below.

\subsection{FLL in New Zealand}

\subsubsection{Official attitudes}

Despite the work of Waite (1992), commissioned by the MoE, New Zealand has no stated national languages policy, and not even in education has a coherent, explicit policy regarding the teaching of LOTEMs in schools been developed. Positive statements about the value of FLL were made in various political statements and policy documents during the 1990s. The Minister of Education, addressing an Asia 2000 seminar, commented as follows on the recently published Curriculum Framework:

...while encouraging the study of a second language from the earliest practicable age, the curriculum stops short of requiring that all students learn a second language in the school years. Despite this, it is my firm intention to 
do all that I can to encourage the study of foreign languages - particularly Asian languages - in the primary school. (Smith, cited in Hollis 1993: 26)

The Minister also launched Education for the 21st Century which specified that the number of FL students was one indicator of "the school system's ability to provide the skills needed by New Zealand's economy" (MoE 1993a: 2). The discussion document also proposed ambitious targets for FLL:

The proportion of school students able, by the end of form four, to converse with and write to a native speaker of a language other than English and Maori about simple, everyday matters will by

1995 be 36 percent;

1998 by 40 percent;

2001 be 50 percent. (MoE 1993a: 27)

The same Minister later stated that:

Successful participation in trade, tourism, education, diplomacy and cultural exchanges demands language fluency. We need to reflect the changes in New Zealand's international trading situation by including the languages and cultures of our Asian trading partners. (Smith, cited in ERO 1994: 4)

An investigation into the state of FLL in secondary schools found that provision was very uneven around the country, that FLL is often viewed as "an adjunct to the core curriculum, perhaps little more than a frill" and that the education system is perpetuating its "perceived elite status" (ERO 1994: 15). ERO clearly believes that if the Government is serious about improving FL skills to overcome the mono-lingualism which "isolates New Zealanders from effective communication with the international community of which they are a part" (ERO 1994: 4), it is essential to provide incentives for students, parents and school boards and improve the supply of FL teachers, especially in Asian languages. ERO sums up their opinions of the gulf between government rhetoric and action thus:

If the future economy of New Zealand is dependent on people with skills in languages other than English and Maori then the issue of second language provision, enrolment and retention in New Zealand schools needs to be addressed now. The gaining of increased proficiency in a second language by more students in more schools will not happen by accident. (1994: 17)

It was, therefore, encouraging when the Minister of Foreign Affairs and Trade announced details of the SLLP to the NZALT Annual Conference (McKinnon 1995), emphasising the great changes in communications and trade relationships in the previous 30 years and the consequent vital need for FLL. Despite this he specifically 
stated that the new scheme would not become compulsory. In 1998, the MoE stated that it had no intention of making any SLL compulsory, declaring that it is not possible to ensure that anyone will value it, or that "students will be enriched socially, intellectually and culturally [a reference to the Curriculum Framework] by simply mandating these things in policies or curriculum statements, because these things exist in the hearts and minds of people" (Chamberlain 1998: 14).

The SLLP, beginning with Spanish and Japanese materials, was welcomed by Benton (1996: 95) as "a clear thrust to expand the opportunities for students to study languages thought to have strategic importance for commerce and diplomacy", but he remained sceptical of overall attitudes to LOTEs, stating that:

“there have always been tacit assumptions underlying the de facto (author's italics) policies.. One of these... is the widely held belief that English is the only essentially important language: other languages may have tactical significance, but English is the one that really matters." (Benton 1996: 95)

Benton did consider realistic the official attitude that schools should be encouraged to offer FLL without any specifications about which languages should be taught because he supported that idea that it was more important to create an environment where FLL is normal than to teach specific FLs (1996: 96).

\subsubsection{Attitudes in schools}

By searching NZALT publications and talking to colleagues I was able to locate some research into attitudes to FLL at various levels in New Zealand schools. At intermediate schools Mahon (1989) found generally positive attitudes to FLL. Most principals and teachers favoured giving all students the opportunity for FLL to develop understanding and tolerance of individual difference. Nearly two thirds of the surveyed parents were in favour of FLL but mainly for instrumental reasons. Those against believed that students whose English was inadequate should not study a FL. The students were keen to learn a FL for future travel or work. In a survey of year 8 students Fitzsimons (1999) discovered a high level of interest in studying one or two LOTEs the following year. An evaluation of the initial phase of the SLLP (Peddie, Gunn \& Lewis 1999) noted that "schools were virtually unanimous in terms of their strong support for the project, their claims of its benefits, and their desire to continue" (Peddie et al. 1999: 122). Schools reported that the majority of students and parents also responded positively to the introduction of SLL (Peddie et al. 1999: 105). Other findings in the project evaluation were that:

- over $90 \%$ of schools believed that all students should have the opportunity of LOTE learning while $72 \%$ thought it should be compulsory (Peddie et al. 1999: 106);

- the only reasons for dropping SLL programmes were concerns about resources, the 'crowded curriculum', and teacher availability (Peddie et al. 1999: 107-109); and 
- there were likely to be ongoing problems of 'articulation', i.e. coordination between primary and secondary schools in regard to SLL programmes (Peddie et al. 1999: 87).

In secondary schools, ERO (1994) found that students' choice of FLL depended mostly on interest in a particular language and culture, often with future travel in mind. Parents and friends also influenced their choices. Most students who had given up FLL had lost interest, but one quarter had done so because of a frustrating lack of space in their timetables. Only a minority cited difficulty or unsatisfactory teaching. The main reasons for not undertaking FLL were the perceived difficulty of languages and lack of usefulness for obtaining jobs. ERO concluded that "parental involvement, encouragement and support are important in the decision-making process of the student beginning or continuing with second language study" (ERO 1994: 12).

Research by Holt, Maeda, Sasai, Shaw, Waller and Young (2001) identified instrumental orientation as the most significant factor in year 10 students' decisions about FLL for year 11. Other important factors which encouraged continued FLL were enjoyment of and confidence in prior learning, personal interest in a language and culture, and parental encouragement. Positive parental attitudes were clearly associated with socio-economic status. The main reasons given by students for not studying a FL in year 10 , or not continuing in year 11 , were a lack of interest combined with the belief that FLL was of not vocational value and the relative difficulty of FLL, particularly of Japanese.

Whether the SLLP is improving FLL student numbers at secondary schools requires long term research (Peddie et al. 1999: 110), but some small-scale surveys have assessed the impact of prior FLL on student choices for year 9. Vine (1993) found mostly positive attitudes among students who had attended a bilingual EnglishJapanese primary school. One third had not continued Japanese in year 9, mostly because they had found it difficult, but they had all started French instead. Another school found that students with previous experience did not seem to have been put off by the change from 'fun short taster' courses to the 'more serious academic requirements' of secondary FLL (NZCTE 1998). Particularly positive attitudes were found among year 9 students at a high decile, girls' school (Guthrie 1998), where the majority had chosen a language option, and those with prior experience were much more likely to have opted for two languages than those without. However, whether students have their 'taster' course in years 7, 8 or 9, the general trend identified by the language advisers (NZCTE 1998) is for students to feel at the end of their course that they have 'done' languages and choose 'more attractive options'.

Knufermann (1993) agrees with Benton (1996) that it is not important which languages are initially studied because of the uncertain future of trade and tourism. FLL has long-term benefits rather than immediate usefulness, enabling students: 
"to ask questions, to develop a sense of inquisitiveness, to understand, identify and solve problems not solely from a national point of view, but from a multiple perspective (Knüfermann 1993: 71).

According to Knüfermann, it is only by clarifying these objectives that the attitudes of school students, parents, principals and Boards of Trustees towards FLL will improve.

\subsubsection{The attitudes of FL teachers}

FL teachers have expressed their attitudes in numerous articles in their newsletters and New Zealand Language Teacher over many years, and teachers' anxiety about the perceived threats to their subjects are a regular topic of discussions among colleagues. They express frustration with the perceived gulf between official statements which appear supportive of FLL and a lack of concrete support for any improvement in FL teaching. The NZALT President (Williams 1994) welcomed the words of the Minister of Education (Smith 1994), cited above, hoping that FL teachers' skills and expertise would finally be properly appreciated. She noted, however, that professional development for FL teachers had only ever been voluntary self help by individuals or the language teacher associations, and listed the many impediments to the improvement of FLL for which the Government was responsible: the lack of research and policy development, insufficient teacher training and a dearth of resources. Williams also claimed that some school principals were introducing 'new' (usually Asian) languages without adequate teachers or resources which could only do more harm than good. She welcomed the proposed SLLP but anticipated the problems which have subsequently emerged. Two years later, Williams (1996) noted a lack of incentives to language graduates to enter teaching, a lack of good training programmes and a generally poor public perception of teaching as a career. She was scathing about some of the new short courses being offered, taught by untrained native speakers, sometimes voluntarily, in schools trying to establish a competitive edge in the local community (Williams 1996:6).

Williams (1996: 9) also observed a disturbing trend in secondary schools where, according to advertised vacancies in the Education Gazette, some Heads of English were being made responsible for other languages, thereby removing career opportunities for FL teachers and increasing the demands on English departments. Even more disturbingly, Spence (1997: 2), claimed that FL teachers were being held accountable for low class numbers and poor examination results which often resulted from factors beyond their control, such as timetabling or module structures. More impediments to improved FLL identified by Spence (1998), include the slow, piecemeal development of curriculum and assessment, the disappearance of some tertiary language courses, a neglect of research showing that SLL improves overall learning, and a concern with economic rather than educational value.

Boekman (1998) blamed the neglect of FLL partly on the devolution of responsibility 
for school programmes onto local communities who may lack the necessary longterm vision or understanding of educational needs of the country. Any subject areas not perceived by parents or Boards of Trustees as of immediate, economic relevance (i.e. job qualifications) are relegated to "non-productive, airy-fairy, cissy qualifications" (Boekman 1998: 18 - author's italics). Another problem is the inequitable provision of FLL opportunities which vary greatly according to the size and location of schools (ERO 1994, Diane Johnson 1998). Suitable teachers may not be available or student numbers are too small to warrant a class, a particularly common situation at senior levels. FLL in New Zealand is, in fact, a "fragile activity" (Diane Johnson 1998: 73). The cutting of senior language classes is also considered inequitable for the minority of students who wish to pursue FLL and who need:

the guaranteed right for each individual to learn a second language as one of the workplace skills considered essential for the citizen of the future. (Spence 1998: 15)

After years of fruitlessly lobbying the MoE for clear national policy, NZALT produced its own Policy on Language Teaching and Learning in Secondary Schools (NZALT 1995) to encourage and assist schools to teach FLs. NZALT also commissioned a report to investigate the economic benefits of learning international languages (Jackson \& Peddie 1998). Teachers naturally welcomed the announcement (Curnow 1998) that the Ministry had contracted the NZALT to develop official guidelines for schools to develop FLL policies. This has proved to be a very slow process and the document (MoE 2002b) has taken four years to reach schools.

Johnson (1994) probably reflected the scepticism of most FL teachers by claiming that an increase in FLL would would only occur if there were a large public relations exercise, but that this would depend on the efforts of FL teachers. Campaigns, such as the International Languages Week in July 2002, have been generated by FL teachers in conjunction with interested bodies like the Alliance Française or the Goethe Institute, for example. Owing to the basic indifference of the government, Johnson suggested (1994: 33) that, as the world became a global village, New Zealand risked becoming the 'village idiot'. Her 1998 survey of 230 FL teachers asked them to report their perceptions of the attitudes of their school managers and their non-language teaching colleagues towards FLL. While $75 \%$ of respondents reported that their school managers considered languages "indispensable, very important or important", $46 \%$ reported that their colleagues did not attribute much importance to languages (Johnson 2001: 76). Johnson also found that $98 \%$ of the FL teachers believed that there were specific issues creating barriers to FLL in schools, the most important of which were:

- community perceptions of language;

- a general lack of interest in languages;

- a lack of a national languages policy; and

- timetabling issues. (Johnson 2001: 77) 
Little has been done to widen the range of languages available to schools, especially Asian languages which the Government has declared important for New Zealand. One tertiary teacher of Chinese has been highly critical of the government over the "dismal state of the NZ curriculum where Chinese studies is concerned and how the curriculum ill-prepares students for the realities of globalisation" (Haddon 1998: 26). She accused the government of "cultural naiveté", claiming that they had little understanding about China or about language learning in general and bemoaned the lack of progress by NZQA to recognise Chinese as a University Bursary subject. According to Haddon (1998: 26), the government does not promote FLL because their "policies regarding internationalism and language-learning belong in the Dark Ages".

\subsection{The influence of gender}

Guthrie (1998) noted that the particularly secure place of FLs at her school probably reflected "the national tendency for SLL to be strongest in single-sex schools, and girls' schools in particular". The gender imbalance is particularly noticeable in coeducational schools where senior languages classes are often dominated by girls (ERO 1994: 10). According to Holt et al. (2001) students are more likely to continue FLL in year 11 if they are female and if they attend single sex schools. Some of the recent findings of the National Education Monitoring Project (NEMP) suggest that differences in attitudes to FLL between boys and girls may relate to differences in their general development in language work. When tested for different language skills, year 4 and 8 girls scored significantly higher than boys on some tasks (NEMP 1998: 41, 2000: 66) and girls reported more positive attitudes to certain aspects of literacy. In no cases did the boys outperform the girls or show more positive attitudes.

\subsection{The importance of languages for trade and tourism}

It is obvious that the attitudes of the wider community, especially of employers, to FL skills will have a bearing on which FLs, if any, are taught in schools (Waite 1992). These attitudes will, in turn, affect the advice given by school careers advisers to students. Various investigations have been carried out into the FL needs of commercial organisations, or the extent to which they value FL skills in their employees. According to Watts (1991: 11), a lack of language skills and cultural understanding can lead to a lack of suitable negotiators and hinder access to essential market information, and, in his opinion, New Zealanders involved in international trade were still "by and large, stubbornly monolingual."

Massey University has undertaken regular surveys on the use of languages by exporting firms since 1966, and there have been signs of employers recognising the importance of FL skills through the 1980s and 1990s. By 1986 exporters had increased their use FLs from $11 \%$ to $78 \%$, especially in publicity materials, but three quarters of firms were still using English for most written or spoken communications. Among companies which used FLs in their publicity material, only a minority were interested in courses to improve the FL proficiency of their staff (Watts 1987). 
An extensive survey of organisations involved in tourism and trade in 1985 found that demand for Japanese language skills outstripped supply (Levett \& Adams 1987), and that companies tended to hire part-time native speakers as they were more effective than New Zealand staff in selling to the Japanese, and because Japanese tourists prefer to be looked after by Japanese. Companies felt that the presence of Japanese nationals helped New Zealanders to learn appropriate skills in language and culture, but were frustrated that such people were not always permitted to enter the country. Ironically, while all the organisations dealing with Japan valued language and cultural skills, two thirds of them did not consider them important in the selection or promotion of staff. Later research found evidence that employers were seeking graduates who had studied languages and business-related subjects, although they were in short supply (Watts 1991: 14). However, Enderwick and Gray claimed that, although "the largest New Zealand companies appear to place a higher value on foreign language skills than their US counterparts" (1992: 64-66), these skills were considered relatively unimportant when it came to recruiting staff. Although graduates believe that it is valuable to develop language alongside business skills, "employment opportunities for business and foreign language specialists remain limited" (Enderwick \& Gray 1992: 67). According to Knüfermann (1993), tertiary students are not always given sufficient guidance and encouragement to combine FLL with other 'marketable' skills, such as management, politics or science.

Exporters and tourist organisations are sometimes reluctant to communicate directly with customers in FLs and tend to use the services of interpreters and translators (Watts 1992), the sometimes hazardous limitations of which are illustrated by Ingram (2001) (see Appendix 1). Some organisations, while not rating knowledge of a FL as very important, do admit the need to understand their customers' way of life, especially notions of politeness. Positive attitudes are required at every staff level, not just among senior management, a point supported firmly by Capper (cited in Callister 1993) who discovered that employees who most need to use Japanese, those with the most contact with Japanese visitors, are the least likely to have studied it. Students learning Japanese tend to be those aiming for management positions in export industries, whereas "the students most likely to use the language, but least likely to learn it, are those who will become housemaids, bartenders or tour bus drivers" (Callister 1993: 34). Other companies recognise that one cannot assume that customers, even if they can speak English, will want to do business in English, or that they will view favourably people who can or want to use only English (Watts 1992).

According to Watts (1992), tourist operators, airlines and hotels especially value the use of FLs in attracting and assisting overseas visitors and tend to take more notice of language proficiency when recruiting staff than exporters, but he found a "considerable mismatch" between the perceived language proficiency needs of various sectors of the tourism industry and actual proficiency (1992: 18). Except in airlines and duty free shops, less than 19\% of tourism personnel had adequate language skills and Watts inferred that NESB visitors might experience considerable difficulty obtaining help and advice. 
(1991) is a lack of communication between educators and employers about the FL skills needed in business and the continued promotion of languages which are not the most relevant for successful competition in international markets. Crocombe, Enright and Porter blamed the limited government commitment to FLL, adding:

A central priority of our education system should be providing young New Zealanders with the skills necessary to become productive members of society in an increasingly competitive world. This means an increased focus on mathematics, technological subjects and languages. (Crocombe, Enright \& Porter 1991: 168)

In 1990, a series of articles in Export News, the official publication of TRADENZ, showed that exporters were being encouraged to understand that successful trade depends on a knowledge of the language and culture of the target country (Stark 1990, Waugh 1990). Barber (1990a) urged exporters to develop friendship and loyalty through personal contacts and $(1990 \mathrm{~b}, \mathrm{c})$ stressed the importance of language skills . The Ministry of Foreign Affairs and Trade (1995: 41) reiterated this advice when it reported on the importance of Latin America and the Caribbean for trade and investment. To deal with most of the countries in the area, "access to Spanish language skills is necessary or essential".

The importance of effective communication for successful overseas business was also emphasised by Enderwick and Akoorie (1996). Miscommunication between the management of an organisation and its overseas staff, affiliates or customers can lead to expensive failures, and training is needed, including facts, language, cultural differences and an examination of people's assumptions and their capacity to adapt. An organisation operating internationally will encounter sharp cultural contrasts, the most obvious manifestation of which is the languages spoken in different markets. (Enderwick \& Akoorie 1996: 154)

In 1991, the New Zealand Tourism Board (NZTB) reported that it would be focussing its attention on key non-English speaking markets and would be "actively involved in ensuring that there is sufficient capacity to train New Zealanders for all of the skilled positions required" (1991: 19). A later report (NZTB 1996) noted that tourism was by far the largest earner of foreign exchange for $\mathrm{NZ}$ and that, while the major sources of visitors remained Japan, the USA and Australia, countries of growing importance included non-English speaking ones such as Taiwan and Korea. The NZTB stated that one of their principal challenges was improving cultural understanding of our visitors, particularly those from China, Korea and Taiwan, in the 50+ age group, who need reassurance over possible language difficulties, and for whom "a high level of guidance and interpretation are key factors" (1996: 22). Table 2.2 shows how accurate the board's predictions were.

In his analysis of progress towards a national language policy, Shackleford states that more investigation is needed into the attitudes of exporters and tourism companies in New Zealand towards FL skills (1996: 163). My review of the above literature suggests that there has been a considerable amount of such investigation in the last 15 years. An examination of the economics of FLL reports "a number of recent studies, especially in Australia, which have 
strongly argued for firms developing a greater awareness of the economic benefits of language skills in the workplace" (Jackson and Peddie 1998: 5). It is possible that only when employers start to demand more language skills among school leavers and graduates that the MoE will put more pressure on schools to provide FLL for more students. What is needed, perhaps, is what Callister recommended nearly a decade ago: that employers be educated as to the benefits of employing people with such language skills (1993: 34).

In the end, as Jackson and Peddie (1998) admitted, it is difficult to identify clear economic benefits from FLL since "the full benefits of the study of language both from an individual and social perspective ... fall into the category of "things that cannot be measured by money", the point made by Knüfermann (1993). This fact "should not be seen to lead inevitably to an under-estimation of the economic worth of any particular form of language study" (Jackson \& Peddie 1998: 12). In the light of all the above, my research involved seeking the views of teachers responsible for careers guidance to ascertain what messages students were receiving about the value of FLL for future study and employment.

Table 2.2 Principal sources of visitors to New Zealand by language, 1991 and 2000 (Sources: Waite (1992), Statistics NZ web site)

\begin{tabular}{|l|l|c|c|}
\hline Language & $\begin{array}{c}\text { Source of } \\
\text { visitors }\end{array}$ & $\begin{array}{c}\text { \% for year } \\
\text { ended June 1991 }\end{array}$ & $\begin{array}{c}\text { \% for year ended } \\
\text { December 2000 }\end{array}$ \\
\hline English & Australia & 34.0 & 32.1 \\
\hline & USA & 15.8 & 10.9 \\
\hline & UK & 8.5 & 11.2 \\
\hline & Canada & 3.7 & 1.8 \\
\hline & Total & $\mathbf{6 2 . 0}$ & $\mathbf{5 6 . 0}$ \\
\hline Japanese & Japan & 10.9 & 8.5 \\
\hline Chinese & Singapore & 1.6 & 2.0 \\
\hline & Taiwan & 1.3 & 2.3 \\
\hline & China & 0.3 & 1.9 \\
\hline & Total & $\mathbf{3 . 2}$ & $\mathbf{6 . 2}$ \\
\hline Korean & Korea & Not given & 3.7 \\
\hline German & Germany & 2.8 & 2.9 \\
\hline Others & & 21.1 & 22.7 \\
\hline
\end{tabular}




\subsection{Hypotheses to be tested}

Personal experiences combined with ideas derived from the above literature led me to formulate the following hypotheses:

\subsubsection{Reasons for choosing FLL}

Hypothesis 1. Students have a particular interest in language and languages. (Gardner 1985, ERO 1994, Kern 1995)

Hypothesis 2. Students who choose to study a particular FL are more interested in learning about the target community or communities and their culture(s) than in how useful the language will be. (ERO 1994, Kern 1995, Dörnyei 1996)

These attitudes may have been developed by prior language learning, travel or parental encouragement, for example. Such attitudes may explain why secondary students do not continue FLL for more than one or two years as they come under pressure to focus on subjects which are seen to be more relevant for employment or further study.

\subsubsection{Reasons for not choosing FLL}

Hypothesis 3. Most students do not see the need to learn a FL for the purposes of future employment. (Turner 1974, ERO 1994, Burch 1999, Drop-out rates shown by MoE statistics)

Students tend to regard secondary education more as preparation for future employment than as broad individual development. The emphasis is on core subjects, which do not usually includes FLs, and optional subjects may be seen as less important. There is limited understanding of the skills and knowledge to be gained from FLL. Even if employers and careers advisers understand them, this may not be not reflected in the curriculum framework nor in the information provided to prospective Year 9 students and their parents.

Hypothesis 4. Students think that languages are 'difficult' subjects and therefore only suitable for the most intelligent students. (Turner 1974, ERO 1994, Calderon et al. 2000)

This is probably reinforced by schools which stream students and make FLL a compulsory subject for the top academic streams.

\subsubsection{Gender differences}

Hypothesis 5. Girls have more favourable attitudes towards FLL than boys. (Burstall 1979, Powell and Batters 1985, Aplin 1991, Waite 1992, Baker 1992, Ager et al. 1993, Fullerton \& Ainley 2000) 


\subsubsection{Parental influence}

Hypothesis 6. Parents' attitudes towards FLL in general, based on previous experience or lack of it, are an important influence on student attitudes. (Turner 1974, Hofman 1974, Waite 1992, ERO 1994, Dörnyei 1998)

Hypothesis 7. Parents' attitudes towards specific languages they have studied have an influence on student attitudes. (ERO 1994)

Parental attitudes are generally significant in student choices (Oskamp 1977, Gardner 1985, Williams \& Burden 1997), and probably depend largely on the socioeconomic status of families. They are likely also to reflect a society's indifference towards the value of FL skills (Turner 1974, Burstall 1979, Enderwick \& Gray 1992, Diane Johnson 1998). General community attitudes tend to reach students mostly through their parents (Gardner 1985).

\subsubsection{The influence of prior experience}

Hypothesis 8. Prior experience of FLL will improve a student's attitudes towards FLL but not necessarily encourage continued study. (Burch 1999).

\subsubsection{The role of attitudes in students choices}

Hypothesis 9. Ultimately, attitudes towards FLL play only a minor role in final subject choices for Year 9. (Waite 1992, ERO 1994, Williams \& Burden 1997, Diane Johnson 1998)

\subsection{Theoretical framework}

The above literature on the development of attitudes towards SLL or FLL, in New Zealand and elsewhere, has examined a wide range of factors, at times confusing and too numerous to investigate in this project. In addition, not all the factors discussed could be considered relevant to New Zealand society or our education system. Therefore, in the interests of simplicity and feasibility, I chose, as a basis for developing survey and interview questionnaires, a theoretical framework involving a complex of three broad groupings of variables which seemed likely to have the greatest influence on students' decisions to undertake FLL or not in year 9 in New Zealand schools. These variables are derived from the framework of motivational factors proposed by Williams and Burden (1997: 138-40) which, according to Dörnyei (1998: 126) represents, "the most detailed treatment of the particular issue in the L2 literature". The factors to be investigated feature frequently in the social psychological literature reviewed above, as it relates to the learning of any language other than a student's L1. This research was designed to investigate the relative importance of each factor in the New Zealand context and the extent of any possible relationship between the factors. 
38

The principal variables, summarised in diagrammatic form in Figure 1, are the following:

- student attitudes to: - language learning in general

- target languages available to students

- target language communities and cultures

- the influence of significant others: - parents

- teachers

- peers and siblings

- the broader context: - national curriculum and effects on local school organisation

- local school attitudes to FLL

- societal expectations and attitudes (especially of employers)

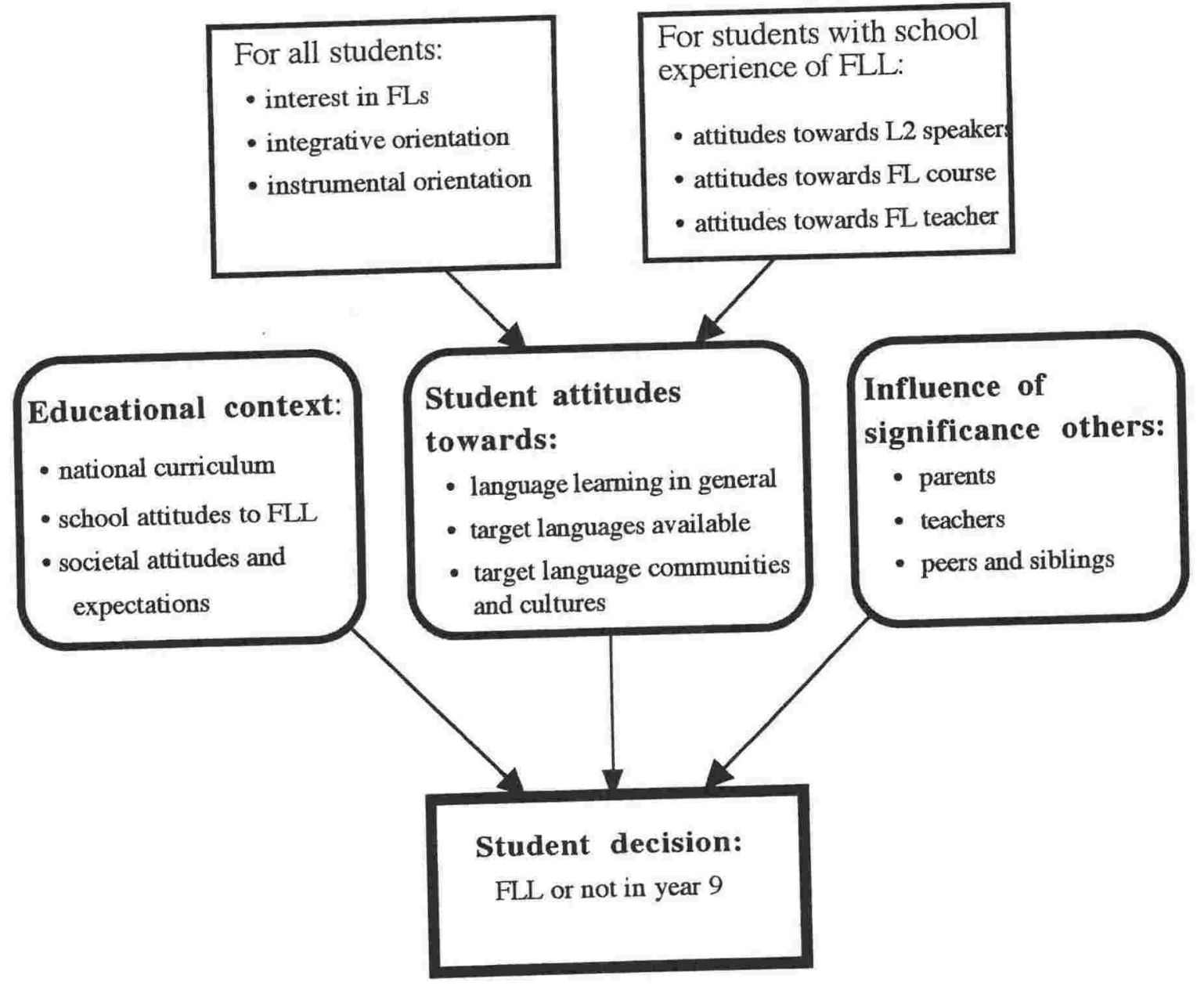

Figure 1. Model of hypothesised factors influencing student decisions about FLL (based on Tremblay and Gardner 1995 and Williams and Burden 1997) 
Student attitudes will be investigated in more detail according to the six aspects in Tremblay and Gardner's (1995) model of motivation. It is interesting that this model still includes the terms 'integrative orientation' and 'instrumental orientation', although, as noted above, Gardner and MacIntyre (1991) expressed doubt about their continued relevance. Whatever other theorists have suggested by way of labels for different types of motivation it seems that, in the end, motivation with regard to FLL can still be broadly classified under these terms and they remain a useful starting point. It is proposed, therefore, to explore student attitudes to FLL in relation to the following aspects of motivation which are also incorporated into Figure 1:

- those which apply to all students: - interest in foreign languages

- integrative orientation

- instrumental orientation

- those which apply to students with previous experience of FLL in school:

- attitudes towards L2 speakers

- attitudes towards L2 course

- attitudes toward L2 teacher

Although the last three may prove to be irrelevant to many of the student participants in this research it is essential to include them because of the MoE's encouragement of FLL at intermediate level through the SLLP, discussed in section 2.10.1 above.

\subsection{Conclusion}

As a result of reviewing the material in this chapter I concluded that New Zealand students' attitudes to FLL must result from a complex interaction among many factors: their social backgrounds, experiences, personal interests and aspirations, and the influence of significant others. I also surmise that there may be no direct association between student attitudes and the decisions they make about optional subjects on entering secondary school, given the many external pressures on their subject choices. 

THE UNITED STATES OF AMERICA AND AUSTRALIA

In societies where the majority of the population is monolingual, as in many Anglophone countries including the United States, there is often an underlying assumption that monolingualism - especially in English - represents an ideal natural state whereas multilingualism represents a temporarily abnormal condition. (Wiley 1996)

\subsection{Introduction}

This chapter examines the place of FLL in the education systems of the three predominantly English-speaking countries with which New Zealand has the closest cultural links, at least for the majority of the population. A comparison with what is happening in New Zealand schools shows that, whatever the inadequacies perceived by champions of FLL in these countries, they are far ahead of New Zealand in encouraging, if not mandating, FLL.

\subsection{Foreign language education in England ${ }^{1}$}

\subsubsection{Some history}

Although FLL has always been part of secondary education in England, the English "have a reputation, perhaps not ill-deserved, for being poor linguists" (Wright 1993: 39). Traditionally and up to the 1960s, FLL was generally the prerogative of social and academic elites (Wright 1993). Students in the public school system (private schools) and grammar schools (state secondary schools which selected the most academic children) would usually study a FL. State education is organised by Local Education Authorities (LEAs) in each county and in the 1970s many LEAs began to merge grammar and secondary modern schools into comprehensives, in which all or most students had some language learning for up to three years. Not all grammar schools disappeared, and in comprehensive schools students were less likely to pursue FLL as far as examination level (i.e. for 5 years). Hence FLs remained associated with more academic or privileged students. Since the 1970s various attempts have been made to increase the range of FLs taught in schools, to encourage more students to continue FLL beyond compulsory years, or to introduce FLL into primary schools.

\subsubsection{Languages taught}

Few FLs have traditionally been available to students, mostly French and German. To some extent this system is self-perpetuating since most language graduates and, therefore, potential teachers have studied French. The problem of diversifying the languages taught is also one of suitable resources and the availability of relevant qualifications. Examination boards in Britain are independent, profit-making companies and, not unreasonably, reluctant to set examinations for minority FLs. According to Wright (1993: 46) entries for some traditionally taught "small" languages, for example Russian, dropped drastically in the early 1980s and others were abandoned.

${ }^{1}$ Actually England and Wales. Scotland and Northern Ireland have independent education systems within the UK. 
On the other hand, minority languages, such as Arabic, Bengali, Chinese and Hindi, have increasingly been available in schools and enthusiastically received by local communities . Unfortunately, unlike many Australian states, but similar to the New Zealand situation, the government has been reluctant to fund such classes, declaring it the responsibility of the communities themselves to maintain their own languages. Others, however, did see the value of expertise in African and Asian languages and cultures for diplomacy and commerce (Wright 1993: 48-49). Examination boards towards the end of the 1980s began to develop relevant examinations and in many institutions community languages have equal status with the traditional European ones, even if they have not replaced them.

\subsubsection{Resistance to language learning}

\subsubsection{The choice of languages}

One of the causes of resistance to FLL may be the dilemma about which FLs it is desirable to learn (Wright 1993). Although France is Britain's nearest neighbour, it is not the main travel destination for most British children. It is a holiday destination only for "affluent middle class professionals who rent houses, camp and tour or ski in winter" (Wright 1993: 43). This seems to reinforce French as a subject for the socially privileged, not relevant for the masses. According to Wright, working class families are more likely to visit Spain, Greece, North Africa or the USA. The large ethnic minority groups are naturally more likely to visit their countries of origin.

\subsubsection{The spread of English}

Another issue is the growth of English as an international language, dominating many technological, commercial and industrial fields. Quirk (1990) claims that, for the first time in British history, there is a generation under the delusion that "because one particular language, English, seems to be very widely understood, no other language need be learned". Those students who do want to learn a FL tend to do so for access to a certain culture, not to acquire a lingua franca (Wright 1993: 46).

\subsubsection{Foreign languages are not 'useful'}

It is only recently that FL skills have started to be valued. In the early 1980s a study found "entrenched prejudice against professional qualifications which had been 'watered down' by the inclusion of the study of a foreign language" (Wright 1993: 45, citing Wright 1986). Liston and Reeves (1985) posed the question:

Do we show any signs at all as a nation of becoming more "languageliterate' in our business dealings and do our business leaders, particularly at the very top, think it worth the effort to try to do so? (1985: 4) 


\subsubsection{Lack of room for language learning}

The traditional British education system has been criticised for being very narrow in its last two years compared with the rest of Europe. Students usually take A level examinations in only 2 or 3 subjects, rarely including a language, whereas in Europe it is normal to take 8 or 9 subjects, including at least one language. This has led to "a lack of flexibility and a cultural and linguistic insularity which have repeatedly been shown to have an adverse effect on managerial efficacy and export performance" (Liston \& Reeves 1985: 26).

\subsubsection{The narrow nature of language learning}

Traditional A level language courses have been very academic and literary, designed largely as a selection tool for higher education. However, from 1986 FLs were included in the Technical and Vocational Initiatives in some areas which showed the value of FLL for industry and commerce. Funding was also provided for computers in Modern Languages departments. These initiatives may have helped to redress the gender balance in FLL (Wright 1993). There have always been more females than males studying and teaching FLs despite the efforts of inspectors and LEAs to change the situation while encouraging more girls to study science and technology.

\subsubsection{Language pedagogy}

After the extensive changes to the secondary school system (from grammar + secondary modern to comprehensives) and the introduction of FLs for all (or most) students, the Inspectorate found that most FL teachers were unable to cope with low ability students and that serious methodological problems needed to be addressed (Wright 1993: 47). There was a failure to adopt FL teaching methodology to suit a wider range of students (Trim 1999: 10). This was obviously a major cause of students viewing FLs as uninteresting, irrelevant or 'difficult' subjects. There was, however, in the 1970s an extensive move away from the traditional 'grammar-translation' method to more communicative approaches.

\subsubsection{Low achievement}

Although students do not seem to value learning unless they obtain a qualification at the end of a course, numbers continuing FLL beyond compulsory years remained small. $60 \%$ of students were dropping FLL after three compulsory years with nothing to show for their studies (Trim 1999: 10). Those who sat examinations were not very successful. so that the numbers remaining in FLL beyond the first examination level were even smaller. For example, in the school year 1986-7, about $34 \%$ of students took a $16+$ examination in French and $9 \%$ in German. Of those, less than half achieved a grade considered sufficient to progress to a higher level. In the same year only about $2 \%$ of those taking an $18+$ examination attempted A-level French 
and under 1\% German (Wright 1993: 41).

\subsubsection{Low priority in schools}

Because of the tendency to judge schools by their examination performances, many principals see FLL as a problem area because it is more difficult to get good marks. Consequently, overtly or covertly, languages may be given low priority which inevitably lowers their status in the eyes of students and parents. High dropout rates at $16+$ may lead to such small numbers that classes are cancelled, a situation familiar to New Zealand FL teachers, which in turn further lowers the status of languages (Downes 2001).

\subsubsection{Consequences}

As a result of low numbers of senior FL students, considerable competition grew among tertiary institutions to recruit the most able linguists. Poor retention rates threatening teachers' jobs were a major incentive for them to reconsider their methodology and materials, to foster students' interest and enjoyment. In their extensive survey of attitudes towards and use of languages in government, professional, commercial and educational establishments, Liston and Reeves (1985: 134) were pleased to note the increase in research into languages and linguistics, the development of new teaching methods and especially plans to introduce radically new higher education programmes. Their general impression of the state of affairs was, however, gloomy. They considered the lack of FLL harmful to commercial interests and that:

for our political, diplomatic and intelligence services the cultural impoverishment and the poor linguistic infrastructure at school level are matters of equally grave concern (Liston \& Reeves 1985: 28)

\subsubsection{Primary FLL}

Private preparatory schools (for students of about 8 - 13 years of age destined for public schools) have traditionally taught languages, but in state primary schools attempts were not made to introduce FLs until the 1970s. Schemes were never very successful for lack of coordination with local secondary schools. As in Australia and New Zealand, teachers were faced with classes of varying abilities and experience in FLL and treated them all as beginners, which did nothing to enhance the interest or enjoyment of more advanced students. Budget restrictions in the mid 1980s gave LEAs an excuse to cease primary language teaching (Wright 1993: 43-44) if they chose.

\subsubsection{The National Curriculum}

In 1988 the government introduced extensive reforms to state education, designating a broad core curriculum and assessment targets in order to stop early specialisation and raise standards, with the further, political motive of increasing central government control (Wright 1993: 49). FLs became part of the core curriculum for the first 
5 years of secondary education, Years 7-11, although it is not obligatory to sit the GCSE examination in a language. Students are allowed to change language after three years and may take a course such as European Studies with only a smattering of language, often the choice of the less able or less motivated students (Smith 1995). This still means that all students have at least three years of FLL and, for the first time, all students are entitled to learn FLs (Brumfit 2000: 94). FLL is no longer the preserve of the academic or social elite.

The new emphasis on FLs was largely due to economic developments in Europe where, since 1992, there has been free movement of goods, services, capital and people and an increased demand for linguistically competent professionals in many fields. Consequently tertiary education increasingly incorporated FLs into other courses (Wright 1993: 51). According to the National Curriculum:

Schools must offer ... one or more of the official working languages of the European Union (Danish, Dutch, Finnish, French, German, Modern Greek, Italian, Portuguese, Spanish, Swedish). Schools may, in addition, offer any other modern foreign language (DfEE/QCA 1999).

Unfortunately for the minority ethnic communities their languages do not have equal status since schools cannot offer non-EU languages unless one of the above FLs is also offered. As Ager et al.(1993: 205) point out, Britain has seemed happy to use immigrant labour to support economic progress but less happy to adapt to the country's subsequent cultural and linguistic diversity. Which language(s) schools choose to offer depends on local demand, the LEA and school principals. Schools with limited resources and well-established French or German departments are likely to simply continue offering only these languages.

As a result of the new National Curriculum the numbers of students entering language examinations increased greatly between 1988 and 1990. Table 3.1 shows, however, that French still dominated, although other languages were gaining rapidly in popularity.

Under the National Curriculum there is no statutory requirement to teach a FL at primary level although the curriculum sets out guidelines for students in Years 5 and 6 , noting that:

The learning of a foreign language in primary school provides a valuable educational, social and cultural experience for all pupils. (DfEE/QCA 1999)

The Curriculum also suggests that there should be links with other subjects in order to reinforce knowledge, skills and understanding developed in other areas. Many consider that Year 7 is a late start for FLL (Gregory 2000, Saunders 1998), but it is still, of course, two years earlier than most FLL in New Zealand. 
Table 3.1 1990 numbers of students entering GCSE and A level language examinations and percentage increases since 1988 in the UK

\begin{tabular}{|l|c|c|}
\hline A level entries & $\mathbf{1 9 9 0}$ & $\begin{array}{c}\text { Increase over } \\
\text { two years }\end{array}$ \\
\hline French & 27359 & $34 \%$ \\
\hline German & 9619 & $36 \%$ \\
\hline Spanish & 3896 & $57 \%$ \\
\hline Modern Greek & 257 & $11 \%$ \\
\hline Urdu & 503 & $54 \%$ \\
\hline GCSE entries & & \\
\hline French & 279081 & $5 \%$ \\
\hline German & 84827 & $12 \%$ \\
\hline Spanish & 25870 & $36 \%$ \\
\hline Modern Greek & 812 & $108 \%$ \\
\hline Bengali & 1330 & $121 \%$ \\
\hline $\begin{array}{l}\text { Source: Schools Examination and Assessment } \\
\text { Council statistics division, cited by Wright (1993: 53) }\end{array}$ \\
\hline
\end{tabular}

\subsubsection{The 1990s and beyond}

Despite the profound social and economic changes taking place in Europe and globally, the British remain "disastrously monoglot" (Quirk 1990), stuck in a "monolingual loop" (King 1999: 25), their attitudes to FLL still characterised by profound complacency and "resolute monolingualism" (Wright 1993: 53). There still exists "a trend of indifference to modern languages" (Saunders 1998: 65) and the "implicit assumption that monolingualism is the norm" (Brumfit 2000: 97-98). The current climate was neatly summed up by Lo Bianco: 
What is impressive, or astonishing, ... is how hard the struggle for languages education is in the UK. ... Public esteem for languages is the critical matter. Too many people in the UK see efforts to improve and extend bilingualism as a kind of 'special pleading' or, worse, a form of creeping 'Europeanisation'.

(Lo Bianco 2000: 1)

The EU is considered a multilingual society and the national languages of all member states are deemed equally valuable. One of the key features of the EU is the freedom to live and work anywhere, but this is feasible only "if Europeans can directly interact economically, socially and politically across language and cultural barriers" (Mackiewicz [President of the European Language Council] 2001). "Those who will be able to exploit the free movement of labour are those with marketable skills and language competence. The monolingual is not likely to be on the move either geographically or hierarchically." (Wright 1993: 52). Continental Europeans are taking advantage of their language skills in the "increasingly integrated European labour market" (Lo Bianco 2000: 7), but UK individuals cannot move so easily and UK companies must recruit from abroad to obtain the necessary language skills. According to a debate, "Languages at Work", held at the University of Westminster, May 2001, there is a substantial "mismatch between the public perception of the employability of Modern Languages graduates and the reality: they are in fact the second most successful group in finding graduate jobs after professional degrees such as medicine and law, and twice as successful as computing graduates" (Nuffield 2001a).

\subsubsection{The Nuffield Inquiry}

General concern about the state of FLL in the UK led to an inquiry, begun in 1998 by the Nuffield Foundation, to investigate the FL capability and needs of the country and the adequacy of existing policies. Two of the main findings were that "English is not enough" and that "Young people from the UK are at a disadvantage in the recruitment market" (Nuffield 2000). The following paragraphs 2.8.1 - 2.8.6 take headings from the Nuffield report (shown in italics).

\subsubsection{English is not enough. Young people from the UK are at a disadvantage in the recruitment market.}

The Nuffield report recognises the advantages for a country of speaking a global language but "exclusive reliance on English leaves the UK vulnerable and dependent on the linguistic competence and the goodwill of others" (Nuffield 2000). It has been pointed out that modern business management theory increasingly stresses the importance of human communication and that, therefore, FL is perceived as an essential part of such communication (King 1999: 22). All tertiary students, regardless of their area of specialisation, need the opportunity to learn FLs. "The graduates of today cannot know what language-related demands they will be confronted with in future 
employment" (Mackiewicz 2001). Hence there is a need to encourage more independent language learning and the development of language learning skills for future learning.

\subsubsection{The UK needs competence in many FLs - not just French - but the education system is not geared up to achieve this.}

Nine out of ten children stop learning FLs at 16.

With a more secure place in the National Curriculum, languages have "an enhanced position in the national consciousness as an important subject for the future economic health of the nation" (Saunders 1998: 63), but it has, ironically, made it difficult to study more than one language for GCSE and French remains the most widely taught language. "Curricular, financial and staffing pressures mean that we teach a narrowing range of languages, at a time when we should be doing the opposite" (Nuffield 2000). According to Brumfit (2000), 172 languages were active in inner London schools in 1987 and any policy on language in education needs to take into account such information. "Recognition of all languages active within Britain is a necessary precondition for starting the process of language learning" (Brumfit 2000: 98).

Furthermore students are generally not successful in FLL. An analysis of examination results shows that students entering for the three most popular FLs, French, German and Spanish, tend to achieve less than in other subjects. This tends to perpetuate the idea that FLL is difficult and to be avoided as soon as possible. In any case, the nature of post- 16 education is so narrow that "pupils must either specialise in languages or abandon them" (Lo Bianco 2000: 7). Overall, levels of competence achieved by school students are considered inadequate (Nuffield 2000).

The situation is not helped by the fact that, for a variety of reasons, FL teachers find it increasingly difficult to take students abroad (Downes 2001). Other teachers are reluctant to release pupils because of course work deadlines, parents are becoming more anxious about safety and less willing to receive foreign guests, and principals are loath to release teachers because of the shortage or lack of suitable replacement staff.

\subsubsection{Government has no coherent approach to languages. University language departments are closing, leaving the sector in deep crisis. \\ The UK desperately needs more language teachers.}

The Department for Trade and Industry has expressed concerns about the shortage of linguists and many degree programmes which include FLs have been short of students (Gregory 2000: 77). More seriously for the future of 
language teaching, there have been shortages of secondary trainee language teachers and reduced numbers of primary teacher training courses offering a language specialism. The Nuffield report speaks of "a vicious circle of inadequate supply" (Nuffield 2000). Long term planning and coordination of aims and resources remain inadequate. According the Nuffield report, "the scene remains a patchwork of often unrelated initiatives" with "no rational path of learning from primary school to university and beyond" (Nuffield 2000).

\subsubsection{Secondary school pupils lack motivation or direction.}

"Current provision does not motivate and too many pupils...see language learning as irrelevant" (Nuffield 2000). People may pay lip service to the value of language learning but employers and universities "have long since scrapped any requirements for modern language study beyond the age of 16 " (Saunders 1998: 65). In universities "foreign language competence is still not universally accepted as a prerequisite of professional life" (King 1999: 23-24). More positive publicity is needed about the successes of language graduates in employment (Gregory 2000: 78).

That FLs are still perceived as 'difficult' subjects, compared "with 'new' subjects such as psychology and media studies" (Gregory 2000: 78), can be attributed partly to teaching methods. For example, "languages are still a subject area where the memorising and recall of a great deal of abstract knowledge are still required" (Saunders 1998: 65) There is still a demand for "error-free performance" (Trim 1999: 14), the sort of perfection which would not be expected in, say English, nor perhaps of native speakers of similar ages and experience. This discourages students' from taking risks so that even after five years of learning they remain unwilling to use the language for fear of making mistakes. Saunders puts the responsibility squarely on language teachers to raise the performance of their students by all possible means to show that all students can succeed and encourage more students to continue FLL.

Another barrier to adequate FLL is seen in the traditional way in which schools still divide learning into separate subject areas (Brumfit 2000: 104), and the way they are timetabled, organisational constraints which are all too familiar to New Zealand language teachers. In the compulsory years of schooling "the pressure on the timetable of an increasingly centralised curriculum makes it difficult to give enough time to a subject where exposure to language is a critical factor" and timetabling does not allow sufficient frequency of language classes to maintain learning momentum (Downes 2001). "A radical rethink of the learning day, the nature of learning activities, the role of the teacher and of technology will be inevitable" (King 1999: 26). 
Some teachers have succeeded in making FLL more attractive to students by making it more relevant and accessible, but not enough (King 1999, Brown 2000). Much has been done by such organisations as the Centre for Information on Language Teaching and Research (CILT), the Nuffield Foundation and the Council of Europe to develop new teaching methodologies and resources, and more suitable language learning objectives, especially practical communication, in order to improve attitudes and achievement (Trim 1999: 10-11). The Council of Europe has been developing a Common European Framework of Reference for the teaching, learning and assessment of FLs (Trim 1999: 16).

\subsubsection{People are looking for leadership to improve the nation's capability in languages.}

Adults are keen to learn FLs but are badly served by an impoverished system.

"Attitudes have become markedly more positive in recent years" (Nuffield 2000). A National Opinion Poll in 1998, cited by the Nuffield Foundation's web site, found that $81 \%$ of adults over 15 believed that more should be done to promote FLL and $86 \%$ agreed that it was useful to have at least some knowledge of a FL. The government has recognised the importance of lifelong learning but there is little funding nor coherent planning for this (Nuffield 2000).

\subsubsection{In spite of parental demand, there is still no UK-side agenda for children to start FLs early.}

"There is a widespread public perception, backed by research, that learning another language needs to start earlier if the next generation is to achieve higher standards (Nuffield 2000). In fact, FLL at primary level (known as Early Teaching of Modern Languages - ETML) has increased rapidly but without any national policy and "little evidence of clear planning" (Gregory 2000: 77). Although FLL is normal in the primary curriculum in most other European countries, the government is reluctant to support ETML nationally because even in secondary schools there is a shortage of teachers. Consequently, "there are as many different approaches to teaching primary languages as there are schools and teachers" (Gregory 2000: 88).

Despite the problems, interest in early FLL (mostly French but also Welsh and German) has increased because of the introduction of European Awareness in the National Curriculum, increased foreign travel, schools competing for students in response to parental demand (shown, for example, by the "spectacular growth of private clubs" teaching FLs - Gregory 2000: 85) and the development of technology allowing more direct links with schools abroad. The BBC has introduced children's programmes to support FLL and publishers have followed their example with suitable resources. 
Some LEAs have developed their own schemes. Scotland has set an example with their successful pilot primary FLs scheme (Gregory 2000: 78-79). CILT held the first Primary Languages Show in 1997 which provoked "unprecedented interest" (Gregory 2000: 80).

The National Primary Languages Network, which has existed for over ten years, has drafted a proposal for the national introduction of FLL into primary schools, taking into account language awareness, progression, FLs other than French and teacher training. Most radically they have proposed that all initial teacher training programmes should require students to have GCSE in a FL(Gregory 2000: 80). The Nuffield report strongly supports an early start to enhance "literacy, citizenship and intercultural tolerance" (Nuffield 2000).

\subsubsection{The Nuffield Inquiry's recommendations}

- Designate language a key skill

- Drive forward a national strategy

- Appoint a languages supremo

- Raise the profile of languages

- Give young children a flying start

- Improve arrangements in secondary schools

- Make languages a specified component of the 16-19 curriculum

- Reform the organisation and funding of languages in higher education

- Develop the huge potential of language learning in adult life

- Break out of the vicious circle of inadequate teacher supply

- Establish a national standards framework for describing and accrediting language competence

- Co-ordinate initiatives linking technology and languages

\subsubsection{The government response}

The government finally responded to the Nuffield Inquiry findings in January 2001, welcoming the report and stating that the issues addressed are "crucial to our competitiveness as a nation and the ability of our citizens to prosper in an increasingly global community" (Department for Education and Employment (DfEE) 2001). The government claimed to be committed to "improving the status of and access to Modern Foreign Languages, particularly in education and training" (DfEE 2001). Objectives were specified, covering curriculum and qualifications, resources, teacher supply, links with business and adult education and cooperation with international bodies.

\subsubsection{Specialist language colleges}

Specialist language colleges received frequent mention in the government's response to the Nuffield report as the main source of improvement in FLL. The first such 
college opened in 1995 and there were 99 at the beginning of 2001. Nine more were to be established (DfEE 2001). These colleges receive special funding to improve the teaching and learning of modern FLs and diversify the range of languages taught. They work with neighbouring schools to disseminate good practice and share resources, and assist programmes in local primary schools. It is proposed to extend links with other schools and the wider community by developing such activities as masterclasses, language clubs and video-conferencing. Colleges are encouraged to develop innovative forms of learning and alternative methods of recognising student achievement.

\subsubsection{Other initiatives}

As 2001 was the European year of Languages, designed to promote linguistic and cultural diversity and language learning (Mackiewicz 2001), a variety of special projects were instigated in the UK. The first major initiative was a pilot scheme to increase primary language learning, supported by the embassies of France, Germany and Spain (Nuffield 2001b). The Nuffield Foundation, as a result of what they considered to be "the enthusiastic and positive response" to their inquiry, announced the investment of $£ 500,000$ to further the development of language learning (Nuffield 2001c).

\subsubsection{Summary}

New Zealand supporters of FLL will recognise most of the above problems and agree with many of the recommendations made by the Nuffield report. They may, however, despair when comparing the situation in New Zealand because, whatever the inadequacies in British FL education, all secondary students are, in theory, benefitting from at least five years of language learning, and an increasing number of primary children are also involved.

\subsection{Foreign language education in the United States of America}

\subsubsection{Introduction}

The USA has always had a multicultural and multilingual population, including Native Americans (155 of whose languages still survive). The founders of the country did not believe that language was a symbol of nationhood and many believed that laws about languages could run counter to the basic right of freedom of speech and therefore neither the Declaration of Independence (1776) nor the Constitution (1789) make any reference to language. English is, however, the de facto official language. "All other languages, variously called 'minority', 'heritage', 'ethnic', or 'native'...play roles limited to home, church, community or tribe", except Spanish which is widely used in Florida and the southwest and whose speakers have considerable political power (Dutcher 1995 internet document, section II Language Policy - Status and Role of First Languages... para. 1). According to researcher, James Crawford (1997), the United States has never had a language policy, consciously planned and national in scope, relying on ad hoc responses to immediate needs or political pressures - often contradictory and inadequate to cope with 
changing times.

According to Schulz (1998 internet document para. 1) the United States "may be the only nation in the world where it is possible to complete secondary and (author's italics) postsecondary education without any foreign language study whatsoever". (Schulz obviously had no knowledge of the New Zealand education system.) Despite enormous sums spent on various forms of FL education each year, "no federal agency is charged with coordination decisions, resources, or research in this area" (Crawford 1997). Crawford highlights the enormous growth of bilingual education, "mainly the transitional variety", designed to turn all children into English speakers alongside federal programmes to encourage FLL among the native English speakers. In many cases, Spanish being the most obvious, the same language is being eliminated in one group and encouraged in another. Crawford believes that "neither approach has been very successful in cultivating fluent bilingualism" (1997 internet document para. 2) despite official recognition that language skills are important for American national security and international trade.

\subsubsection{The state of FLL in the 1970s}

The Constitution places the responsibility for education on individual states but federal legislation has, from time to time, "influenced state action by providing funds for meeting specific objectives" (Dutcher 1995). Articles about the state of FLL during the 1970s paint a picture which would be familiar to New Zealand language teachers today. Language requirements to enter or graduate from college or university had been relaxed or abolished (Lippmann 1974). Language teachers were struggling to attract and keep students in their classes (Lawson 1971). They often had to cope with combined, or 'multi-level' classes (Brannon and Cox 1976, Strasheim 1979). Some argued for compulsory FLL (Birch 1971, Paquette 1971, Ziegler 1975), while others urged greater efforts to promote the value of FLL (Rolland 1974, Preller 1974, Meyer 1975, Knop 1975). There was indifference among students, nonlanguage teachers and the general public (Edgerton 1971, Altman 1974), and negative attitudes among administrative staff in educational institutions (Turner 1974, De Felippis 1979). Klayman (1975) attributes indifference to the association of FLs with ethnic minorities and immigrants who are expected to assimilate to the majority, English-speaking culture. This entails adopting such values as equality, meaning uniformity, and success, measured in material terms and acquired through studying only 'useful' subjects.

\subsubsection{The role of FL teachers}

Secondary and tertiary FL teachers tended to blame themselves for falling student numbers and recommended radical revisions of curriculum, materials and teaching methods, and activities to promote FLL (Lawson 1971, Edgerton 1971, Rolland 1974, Preller 1974, Reeves 1974, Allen 1975, Grittner 1975). A lack of clear goals was also criticised (Bouffard 1980 ). Others blamed FL teachers' negative attitudes towards students, who were of ten considered either too lazy or insufficiently intelli- 
gent to succeed in language classes (Lippmann 1974). FL teachers often demanded perfection from their language students, which obstructed real communication, destroyed confidence and was a great disincentive to continued study (Walker 1976). On the other hand, some investigations found positive attitudes to FLL, for example, among students and parents in New York state (Papalia 1978), and the general public in Fayetteville, Arkansas (Clark and Rudolph 1981). Encouraging signs were identified in the changing nature of language courses and the growth of 'vocational community colleges', but the need remained for language teachers, not just to 'sell' their 'product' as strenuously as possible, but also to find out what students wanted to learn (Walker 1976: 141). Walker noted "devastating apathy" (1976: 141) among high school teachers with regard to surveying student attitudes.

\subsubsection{The decline continued}

Despite attempts to address the problem of declining student numbers during the 1970s, the overall situation did not improve. A 1978 Gallup poll into public attitudes towards public schools, reported in Foreign Language Annals 11 (6), revealed the low esteem in which FLs were held. For example, knowledge of a FL was in last place in a list of skills and knowledge deemed necessary for graduation from high school for students not planning to go on to further education. Bryant (1978) reported a continued decline in student numbers and an increasing number of tertiary institutions dropping language requirements. He went so far as to call the language teaching profession "imperiled" and to call for "positive and extraordinary" action (1978: 11), including the payment of people to lobby the government and businesses and to instigate a nationwide publicity campaign.

\subsubsection{The Presidential Commission}

In April 1978, a Presidential Commission was appointed to investigate the state of FLL because of widespread concern that the overseas responsibilities of the USA were increasing but the knowledge of FLs and the understanding of international affairs were declining (Perkins 1978: 621). The Commission had, as its four objectives, to:

- Recommend means for directing public attention to the importance of FL and international studies for improvement of communications and understanding with other nations in an increasingly interdependent world;

- Assess the need in the United States for FL and area specialists, ways in which FL and international studies contribute to meeting these needs, and the job market for individuals with these skills;

- Recommend what FL area studies programs are appropriate at all academic levels and recommend desirable levels and kinds of support for each that should be provided by the public and private sectors;

- Review existing legislative authorities and make recommendations for changes needed to carry out most effectively the Commission's recommendations. (Carter 1978) 
Many studies and projects were undertaken to provide background information for the Commission, including a national survey of FL teaching in secondary schools in 1978 and 1979, a survey of State laws and regulations concerning language study, and a survey of attitudes towards FLL (Eddy 1979). The American Council on the Teaching of Foreign Languages (ACTFL) called for "the establishment of a national policy to preserve, develop, and strengthen second language education in this country, so as to enhance the status of the United States in the family of nations". This resolution was sent to President Carter and all members of congress. Paul Simon, one of the instigators of the Commission and one of the three Members of Congress on the Commission, assured the ACTFL that "this will be one of the central issues addressed by the President's commission".

\subsubsection{The Commission's findings}

The President's Commission reported to President Carter (Commission on Foreign and International Studies 1979) with an optimistic note from the Chairman to the effect that the commission's activities had "stimulated an increasing interest in improving our prospects for real competence in FL and improved understanding of the world outside our borders" among a wide range of people and institutions. However, the Commission reported "scandalous incompetence in foreign languages", "dangerously inadequate understanding of world affairs" (Perkins 1979: 13) and "the epidemic elimination of language requirements" (Perkins 1979: 19). It also offered some dismal statistics (Perkins 1979: 13):

- only $15 \%$ of high school students were studying a FL (24\% in 1965) and the number was steadily declining;

- $25 \%$ of high schools offered no FLL

- only one in 20 public high school students of French, Russian or German ever continued into a third year;

- only $8 \%$ of colleges and universities still had a FL requirement for admission (34\% in 1966);

- only a handful of approximately 900 American business representatives in Japan had a working knowledge of Japanese.

\subsubsection{The Commission's recommendations}

The report contained extensive recommendations for teacher training and support, increasing and strengthening FL courses from Kindergarten to Twelfth Grade (K-12) and in tertiary institutions, research and development of curriculum and resources, encouraging international educational exchanges, researching business and other employment needs, improving official and private organisation of relevant studies, and increasing public interest and support. The Commission was clearly in favour of some sort of compulsory FLL in schools:

All students, including minority-group members who often have less opportunity for language study, should be participating in the study of foreign languages and international subjects. (Perkins 1979: 25) 
The Commission recommended the establishment of a National Council on Foreign Language and International Studies (NCLIS), a body that still exists to promote "international education" (NCLIS 1999). The Commission also called for more collaboration at secondary school level between teachers of FLs and social studies (and many other relevant subjects). Strasheim agreed that interdisciplinary cooperation was "an educational idea...whose time has come" (1980: 59), but saw three major obstacles:

- subject compartmentalisation

- confusion about responsibility for curriculum development (school, state, federal?)

- lack of knowledge and/or communication among teachers

\subsubsection{Reactions to the Presidential Commission's recommendations}

There was strong theoretical support for the Commission's recommendations. The Council on Basic Education firmly endorsed them, stating that it had always favoured compulsory FLL. (Reported in Foreign Language Annals 13 (4), 330). The May 1980 issue of the Curriculum Report of the National Association of Secondary School Principals was devoted entirely to FLs (reported in Foreign Language Annals 13(4), 332). Support was also expressed by the Commission on Humanities in their 1980 report (reported in Foreign Language Annals 13(5), 427). However, the Secretary of Education, Terrel Bell, clearly saw that his country had a long way to go. He described the lack of FLL as a "major national disgrace", adding "We are a bunch of monolinguistic bumpkins and American education is to blame" (Bell 1981, reported in Foreign Language Annals 15 (3), 219). One piece of evidence he probably had in mind was a 1980 survey which had found that only 8 states required high schools to offer FLs and none required students to study any (Ryan 1984).

\subsubsection{Progress in the $1980 \mathrm{~s}$}

Progress was very slow but many steps were taken by official and professional bodies to promote FLL in American schools. For example, the Department of Education (DoE) supported an extensive survey into the supply and demand for FL teachers and teacher training (Brickell and Paul 1982). In 1983 the National Commission on Excellence in Education reported to the Secretary of Education that language learning for all children should begin at elementary level and continue for 4 to 6 years. Also in 1983, the DoE's National Advisory Board on International Education Programs called for every student to have the opportunity to begin FLL in the earliest years of formal education and to continue that same language to reach a useful level of proficiency. This would require among other things, the integration of FLL across all levels, the development of clear standards of proficiency in language skills, and research into methodology and the language needs and use of adults, especially in their employment.

Similarly, in 1985, the Council of Chief State School Officers declared that all ${ }^{2}$ This encompasses a wide range of language fields, including all types of language education, the classics, exchanges, research, technology, business education and translation (NCLIS 1999) 
students should have the opportunity to learn a language other than English, including non-Western ones, starting at elementary school and continuing through secondary to reach an acceptable level of proficiency, especially in speaking and listening. Furthermore, the Council believed that teachers should have first hand experience of culture of languages they teach. Recommendations made by the Council to Federal Agencies (1985: 245) included "comprehensive assistance to international education and second language study at the elementary and secondary level" for planning programmes, developing curriculum and assessment, teacher training, and exchanges. The Council particularly supported the teaching of Russian, Japanese, Portuguese, Arabic and Chinese.

The National Institute for Education set up CLEAR (Center for Language Education and Research) in 1985, based at UCLA, to carry out research into second and FLL, improve teaching methods, develop materials, and encourage community involvement (Reported in Foreign Language Annals 18 (6), 526).

\subsubsection{Attitudes to FLL}

Teachers were were beginning to see changes in attitudes away from the old idea that FLs were only for an academic elite (Koppel 1982), but the Director of International Education Programs at the DoE in 1983, although convinced that FL study was part of basics education, stated:

it is not clear that we will ever obtain the FL education our Nation needs until a fair number of Americans believe that foreign languages are worth studying. (Whitehead 1985: 7).

Articles related to FLL during the 1980s, and even into the 1990s, are characterised by the sense that secondary teachers were still having to fight for support for their subjects, from students, parents and school administrators (for example, Koppel 1982, Campana 1983, Cooper 1985). Even articles ostensibly about practical ideas for the classroom seem to have the ulterior motive of attracting and keeping more students by, for example, emphasising the development of communicative competence (Nussenbaum 1983, Ramage 1990) or stressing the need to include more cultural studies to motivate students (Wilkes 1983, Pfister 1989, Dodick 1996). Other commentators perceived growing good will towards FLL among high school administrators but still put the onus on teachers to promote FL study (Weatherford 1982, Beard 1984, Crawford-Lange 1985). FL teachers and their programmes were still being blamed by some secondary school principals for high drop-out rates and FLs were still perceived as too difficult, irrelevant for most students and competing poorly with other subjects (Crawford-Lange 1985: 14).

On the other hand, others reported increasing interest in, and even state requirements for, elementary school FL programmes, although developing these was a "challenging task" and of ten a "bewildering process" (Met 1985: 473). Baranick and 
Markham (1986), in a survey of attitudes towards FLL, found 54\% of elementary school principals in support, especially for Spanish, although it did not have high priority. Principals needed encouragement from parents, students and colleagues. Of those against FLL, most cited lack of time, but about a third would be prepared to accept it as extra out of class programme. (cf De Felippis 1979)

\subsubsection{Funding for FLL}

Federal funding for major initiatives remained a problem, unless FLL could be linked to political and economic policy (Groennings 1980). In support of increased FLL, Congressman Paul Simon introduced the Foreign Language Assistance for National Security Act 1983, which was passed by Congress but rejected by the Senate. Nonetheless, an optimistic note was struck in an address to the Joint Annual Meeting of ACTFL, AATF, AND AATG late 1984:

There has never been a time in my memory when there has been a more open moment, a more encouraging climate for the development of a fresh American national policy with respect to the teaching of foreign languages.(Lambert 1985: 379)

Lambert saw growing interest from government agencies, and "major private foundations (1985: 383), and called on teachers to organise their priorities and strategies to make the best of whatever funds came their way. The federal government remained reluctant to provide more assistance, with the view that "private sources should compensate for decreases in federal support for education" (Dandonoli 1986: 54), but the 1988 Foreign Language Assistance Program granted money to states to develop model teaching programmes to improve FLL by (Dutcher 1995).

\subsubsection{Languages at tertiary level}

At tertiary level there was evidence that colleges and universities through the 1980s discussed reinstating or strengthening FL requirements for entering or graduating, mostly from arts courses, or at least facilitating FLL within other programmes (Bugos 1980). By 1981, an MLA survey found that "nearly seventy institutions had restored a foreign language requirement, either for entrance or for the B.A." (Whitehead 1985: 6). Huber (1992, cited in Dutcher 1995) reported that, by the academic year 1988-9, over $25 \%$ of tertiary institutions required entrants to have done some language study. Nearly $35 \%$ of institutions required language study for all student $\mathrm{s}$ and another $40 \%$ for some. Spanish was most popular, followed by French, German and Japanese.

While FLs continued to be considered most relevant for arts degrees, there were growing calls for them to be associated with business studies. Hoegl (1986: 284) spoke of "a large and increasing number of jobs in the American labor market requiring proficiency in other languages and international understanding", but noted 
the dearth of suitable applicants for employment in the State Department, the Department of Defense, the CIA and business in general. He claimed that jobs in US companies abroad were mostly filled by foreigners, and asserted that lack of cultural understanding was a big barrier to successful overseas business. In contrast, Voght and Schaub (1992) considered that studies combining languages and business had been firmly established in U.S. higher education.

\subsubsection{The 'English Only' movement}

Since the 1970 s there have been numerous proposals to make English the official language at federal level, all of them defeated. In some state constitutions English is an official language (Dutcher 1995: 9), but an attempt to amend the constitution of Arizona to make English the official language was overruled by a federal judge as an infringement of the right to free speech. However, other states have introduced resolution or statutes declaring English the official language (17 by 1995 according to Dutcher (1995:9)).

The 'English Only' movement is attributed in part to the greatly increased diversity of the US population in the $1980 \mathrm{~s}$, including a particularly large increase in native Spanish speakers (Crawford (1997). By 1990, almost $8 \%$ of the population aged 5 or over spoke Spanish and another 5\% another language other than English ${ }^{3}$ (U.S. Bureau of the Census, cited by Dutcher 1995). Spanish is the only language other than English supported by television, 60 channels in 1993, (Broadcasting and Cable yearbook, cited by Dutcher 1995). Supporters of English Only believe that one official language is needed to maintain the unity of the country, regarding minority languages at best as a source of problems, at worst as a threat to the American, English-speaking way of life. Some have demanded that only English should be used "at the ballot box, in the classroom, and in the workplace" (Dutcher 1995: 11).

\subsubsection{FLL in the 1990s}

In 1990s, the general trends include:

- increased FLL in schools;

- development of content and achievement standards;

- encouragement of bilingual education;

- ongoing problems of inadequate funding, teacher training, and teacher supply;

- continued reintroduction of FL requirements at tertiary level with changes in the nature of courses and assessment;

- recognition of globalisation of business and need for language skills alongside others;

- ongoing controversy over 'English Plus' versus 'English Only'.

By the mid 1990s it was reported (Dutcher 1995) that FLs were included in all state curriculums. Forty states required schools (usually secondary) to offer at least two 
years FLL but none made it compulsory. The other ten states had some sort of FLL requirement but only for the most able students, for example, those wishing to enter tertiary education. By 1998, at least six states required all elementary schools to offer some FLL and some states had made FLL compulsory, for one or two years (District of Columbia, New York), for 'academically able' students (Louisiana), or for high school graduation (Maryland, Oregon) (Lewelling and Rennie 1998b).

What happens at school level is still largely dependent on individual school boards but much has been done at national level to encourage more and better FLL. It was estimated (Dutcher 1995) that, by the mid 1990s, about 39\% of upper secondary (grades 9-12), 24 of lower secondary (grades 7-8) and 6\% of primary students were studying a FL. Table 3.2 shows FL enrolments in grades 9-12 in public schools as a percentages of all students over a twenty year period.

A national survey of FL teaching in approximately $6 \%$ of public primary and secondary schools, (Center for Applied Linguistics 1997), showed that there had been an increase in the number of primary schools teaching a FL over ten years, from $22 \%$ to $31 \%$, with about half the students benefitting from FLL. The proportion of secondary schools involved had remained fairly stable, 87\% in 1987 and $86 \%$ in 1997, with $51 \%$ of public school students involved. By $1997,79 \%$ of primary and $93 \%$ of secondary schools were offering Spanish. The teaching of French remained fairly popular in secondary schools, still in $64 \%$ of schools, but had declined considerably in primary schools, from $41 \%$ to $27 \%$. Other areas of increase were Spanish for Spanish Speakers, Japanese, Italian and Sign Language (both at primary level), Russian (secondary).

Table 3.2 FL enrolments in public schools in the USA 1974-1994

\begin{tabular}{|l|c|c|c|c|c|c|}
\hline Language & $\begin{array}{l}\text { Fall } \\
\mathbf{1 9 7 4}\end{array}$ & $\begin{array}{l}\text { Fall } \\
\mathbf{1 9 7 8}\end{array}$ & $\begin{array}{l}\text { Fall } \\
\mathbf{1 9 8 2}\end{array}$ & $\begin{array}{l}\text { Fall } \\
\mathbf{1 9 8 5}\end{array}$ & $\begin{array}{l}\text { Fall } \\
\mathbf{1 9 9 0}\end{array}$ & $\begin{array}{l}\text { Fall } \\
\mathbf{1 9 9 4}\end{array}$ \\
\hline Spanish & 11.9 & 11.6 & 12.5 & 18.8 & 23 & 26.4 \\
\hline French & 6.9 & 6.1 & 6.9 & 9.2 & 9.6 & 9.1 \\
\hline German & 2.8 & 2.3 & 2.1 & 2.5 & 2.6 & 2.7 \\
\hline Italian & 0.3 & 0.3 & 0.4 & 0.4 & 0.4 & 0.4 \\
\hline Russian & 0.1 & 0.1 & Less than $0.05 \%$ & 0.1 & 0.1 \\
\hline Japanese & 0 & 0 & 0 & 0 & 0.2 & 0.3 \\
\hline All modern FLs & 22.1 & 21.6 & 21.9 & 31.1 & 36.1 & 39.4 \\
\hline Source: U.S. Department ( Education & & & \\
\hline
\end{tabular}


Programmes varied but the Center for Applied Linguistics (CAL 1997) noted that only $7 \%$ of all primary schools "offered instruction in which the students were likely to attain a high level of fluency, as recommended in the goals of the national standards" (see below). On the other hand, CAL found an increase at secondary level of advanced courses, recognising previous learning or native speaker ability, and the use of target FLs in class, but hours of instruction, conversation classes and any use of target FLs for teaching other subjects remained very limited. CAL also reported that "more than half of the schools not currently teaching languages were interested in doing so in the future".

\subsubsection{Development of content and achievement standards}

There has been a large amount of activity to encourage FLL through the improvement of curriculum and assessment, resources, teacher training and public promotion by teachers, administrators, academics, and through various official and teacher organisations. Legislation was passed in 1994, Goals 2000: Educate America Act, with the general aim of improving student achievement. This funded the development of goals and standards in all core subject areas, including FLs. In 1996, the National Standards in Foreign Language Education Project produced recommendations for all levels from Kindergarten to Grade 12 (K-12). This work, and its support for extended FLL through primary and secondary school, "galvanized the field of foreign language education", resulted in a greater focus on FLL for meaningful uses in real life situations and "generated a dynamic discussion on a compelling rationale for language education for all students" (Lewelling and Rennie 1998a).

Traditionally, language assessment was mostly a fairly informal process carried out by class teachers, there being no national programme of assessment required of states or schools, but Dutcher (1995) reported that 17 states had developed or planned to develop FL performance standards, and about 40 states had developed or were working on content standards. There are standard tests available nationally for students who wish to show what they have achieved in order to enhance their applications for college or university entrance, but the tests are limited in the skills they assess and available in a limited number of FLs. In addition, ACTFL has produced Proficiency Guidelines for secondary and post-secondary levels. According to Lewelling and Rennie (1998b), even where statewide assessments exist, most are voluntary.

What would seem extraordinary to those familiar with education systems in Britain, Australia or New Zealand is the complete lack of national or state qualifications at which school education is aimed. CAL (1997) reported that "curricula at all school levels tended to be developed by the teachers at the school". Only 37\% of primary and $62 \%$ of secondary schools reported that their FL teachers were aware of the national Standards for Foreign Language Learning, and only about half of those indicated that their FL curricula had changed accordingly. 


\subsubsection{Encouragement of bilingual education}

The Improving America's Schools Act, 1994, provided grants to states and schools under the 1988 Foreign Language Assistance Program, reauthorised in 1994, to improve language education, including Native American education. The act also provided assistance for bilingual education based on three principles:

(a) all children can learn to high standards;

(b) linguistically and culturally diverse children and youth must be provided with the equal opportunities to learn that are provided for all students; and

(c) proficiency in two or more languages should be promoted for all students. (Dutcher 1995)

In the 1994-5 school year, two-way (i.e. a combination of ESL and FL learning) bilingual programmes were being supported by federal funds in 61 local education agencies, mostly Spanish L2, but also Cantonese, French, Korean, Japanese, Nàvajo, Portuguese and Russian (Dutcher 1995). Dutcher also reported that in 1995 187 schools were offering English-speaking students partial or full immersion programmes in 26 states, mostly in Spanish, but also in Arabic, Cantonese, French, German, Hawaiian, Japanese, Mandarin and Russian. Such programmes have continued to grow in popularity, owing to parental demand. The proportion of primary schools nationwide offering such programmes increased from $2 \%$ to $8 \%$ between 1987 and 1997, supported by educators who say "the approach is one of the only ways for US-born students to achieve a high level of proficiency in a foreign language" (Mathews 2000).

In addition, as in Australia, there are many private ethnic schools teaching languages and culture, usually once a week, mostly at weekends, organised by community or church groups. According to Dutcher (1995) no recent data on these schools exists but Fishman and Markman (1979, cited by Dutcher 1995) reported that there were over 5000, of which over one third used Greek, German, Hebrew or Yiddish. Some community languages are now moving into public schools as part of bilingual programmes. Dora Johnson (1998) mentions Cantonese and Korean for L1 speakers, and Swahili "being taught occasionally because it provides historical enrichment, particularly in districts with a large population of African Americans".

\subsubsection{Ongoing problems}

The increase in FLL has not been matched by an adequate teacher supply. As in New Zealand, the majority of the teaching profession is approaching retirement. It was predicted that by about 2000 the number of language teachers leaving the profession would be greater than those entering, and that the shortage would be particularly acute for Spanish. The rapid growth in FLL in primary schools has exacerbated the situation (Dutcher 1995), a situation very similar to that which obtains in Australia. Various measures have been taken to address the problem, including: 
- financial incentives,

- recruitment abroad,

- increasing use of distance learning, especially for less commonly taught languages and particularly for Japanese,

- increasing class sizes,

- reducing language choice to make better use of available teachers,

- facilitating the recognition of qualifications of overseas-trained, native speakers, especially of Japanese

- increased in-service training for language and teaching skills.

According to CAL (1997), only $19 \%$ of primary schools reported that all their FL teachers were certified to teach a language at that level, but many states did not require any such qualifications and little relevant training was available. Other problems identified by CAL (1997) at all levels of schooling were lack of funding and suitable resources, class sizes and the inadequate 'sequencing' from primary to secondary schools, what the Australians call 'articulation', which is also a growing problem in New Zealand.

\subsubsection{Summary}

While FLL in U.S. schools has increased in the last 30 years, many problems and much dissatisfaction remain, mostly familiar to New Zealand language teachers. The CAL (1997) survey concluded that:

there is still reason for concern about the limited number of $\mathrm{K}-12$ longsequence language programs that are designed to educate students linguistically and culturally to communicate successfully in the U.S. and abroad.

Similarly, Rogers and Arn (1998: 347) claim that:

the United States is in some ways ill equipped to take an effective role in the international community. Most college students do not develop an understanding of even one FL or culture and little has been done to expand business students' FL knowledge.

However, the federal government DoE funds various means of improving FLL, some of which would be the envy of New Zealand FL teachers including:

- seven national FL resource centres

- model teaching programmes

- advanced teacher training

- the National Endowment for the Humanities, which provides scholarships and funds intensive summer courses, research projects and materials development (Boston 1998) 
The 'English Only' movement continues and has made progress in some states, but it is countered by a vigorous 'English Plus' movement (Crawford 1997, Farr 1997), supporters of which regard diversity as an asset and encourage the retention of minority languages as well as FLL, with the development of English proficiency for all. In other words, bilingualism is valuable for all individuals and for the country. This is an ideal which many would like to see as an educational goal in New Zealand.

\subsection{Foreign language education in Australia}

\subsubsection{The development of a national language policy}

Australia started working towards a national policy language in the 1980s, with support from both ethnic and educational groups (Liddicoat 1996: 4). Developments culminated in the National Policy on Languages (NLP) (Lo Bianco 1987), substantially a language-in-education policy, "in which the equity and economic rationales were evenly balanced" (Liddicoat 1996: 4).

The NLP advocated the promotion of nine languages of regional and global importance, which might also be community languages (Lo Bianco 1987: 147). Development also began of the Asian Studies Program, to encourage the particular study of Asian languages and cultures in schools. Other moves to encourage and assist the learning of LOTEs included the establishment in 1989 of the National Languages Institute of Australia (NLIA) with four research centres, a major review of languages in tertiary education (Leal 1991) and a national conference in 1990, "Language is Good Business" (Shannon 1991) which "raised the profile of languages in Australia" (Liddicoat 1996: 7).

Owing to a change in Commonwealth Government policy (Ozolins 1993; Moore 1996) the national policy was amended in 1991 and renamed Australia's Language: The Australian Language and Literacy Policy (ALLP). This policy remains substantially in place and, as the name suggests, more emphasis is placed on English language and literacy for all Australians than on the learning of LOTEs. However, Goal 2 (of four) states:

The learning of languages other than English must be substantially expanded and improved to enhance educational outcomes and communication within both the Australian and the international community. (Department of Employment, Education and Training 1991: 14)

The ALLP recognised the need to "strike a balance between the diversity of languages which could be taught and the limits of resources that are available" (DEET 1991: 15) and specified 14 priority languages "of broader national interest to Australia" on which Commonwealth assistance will be focused, divided into:

a) significant community languages: Aboriginal languages ${ }^{4}$, Italian, Greek,

German, Spanish and Vietnamese

${ }^{4}$ Underlined languages are in addition to the 9 listed as priorities by the NLP 1987 
b) languages of regional and economic importance: Chinese, Indonesian/ Malay, Japanese, Korean, Russian and Thai

c) languages of wider economic and cultural importance: French and Arabic

Each state ${ }^{5}$ was to nominate 8 of the above languages for priority development for which Commonwealth Government assistance would be provided. In fact, five of the languages (French, German, Japanese, Indonesian and Chinese) were already priorities in all states (DEET 1991: 16). The policy's aim was that, by the year 2000, $25 \%$ of Year 12 students should be studying a LOTE, (compared with fewer than 12\% in 1991) (DEET 1991: 17). Increases in funding were also promised for Ethnic School Programmes, for higher education to promote Asian languages and teacher training in LOTEs and a number of other LOTE education areas (DEET 1991: 18).

\subsubsection{National education policy}

Major national efforts have been made to improve schooling by the Australian Education Council, comprising the Ministers of Education from all states and the Commonwealth Government. They agreed on ten common goals, released as the Hobart Declaration in 1989, which included the establishment of eight key learning areas for the compulsory years of schooling (up to Year 10), one of which was LOTEs. Subsequently a Statement and Profile were produced for each key area to assist states in the work already underway to develop their own curriculum frameworks and assessment procedures.

The LOTE Statement made clear that there should be no "artificial distinctions" between different LOTEs, nor should any kind of hierarchy be seen, but:

It is essential that the curriculum of each State and Territory includes a balanced range of languages. Individual systems and schools need to decide which languages to offer, taking into account the needs of individual learners, the local community and Australia as a nation. (Curriculum Corporation 1994: 3)

The Statement presented a detailed, idealistic picture of a LOTE learning environment which catered for all students with a great diversity of backgrounds and linguistic ability, with adequate resources, time allocation, continuity of programmes between all levels of education and qualified teachers (Curriculum Corporation 1994: 3-4). The statement also provided a justification of LOTE learning, probably aimed mostly at parents (Curriculum Corporation 1994: 4-5) and stressed the impor-tance of parental involvement in their children's education. Above all, the LOTE statement affirmed that LOTE learning is "an essential part of a broad and balanced education for all learners" for the benefit of "the individual, the Australian community and Australia as part of the international community" (Curriculum Corporation 1994: 5).

Commonwealth funding was focussed on the study of Asian languages and cultures 
in 1994 when the Council of Australian Governments (COAG) developed the National Asian Languages and Studies in Australian Schools (NALSAS) strategy. The Council's report (COAG 1994) designated Japanese, Mandarin, Indonesian and Korean as priority FLs, with the major aim that $60 \%$ of all school students in Years 3-10 would be studying one of these four FLs by 2006. A similar proportion of Year 12 students in LOTE classes should eventually be studying one of the priority Asian FLs.

\subsubsection{Current national goals for schooling}

The national goals for schooling were revised in 1999 as the National Goals for Schooling in the 21st Century, known as the Adelaide Declaration which remains the basis of national education policy (ABS 2001: 410-412) and confirms the eight key learning areas for the compulsory years of schooling. It includes the goal that:

all students understand and acknowledge the value of cultural and linguistic diversity, and possess the knowledge, skills and understanding to contribute to, and benefit from, such diversity in the Australian community and internationally. (MCEETYA 1999)

Within this framework individual states remain free to develop their own education systems, including curriculum and assessment procedures. Development has been ongoing in all states during the last decade. Within each state considerable independence is given to government schools to develop their own priorities and programmes (ABS 2001: 412), while non-governmental systems, notably the Catholic system (which accounts for $20 \%$ of students nationally), and independent schools, are not subject to Commonwealth or state guidelines regarding curriculum. Within each system individual schools are free to choose the languages they offer, according to local and individual needs and the resources available to them.

\subsubsection{Current national funding for LOTEs}

The Commonwealth Government is currently providing funding to states for specific education programmes. One priority for 2001-2004 is "Improving outcomes in specific targeted teaching and learning areas" (Department of Education, Training and Youth Affairs 2000: 69), one of which is languages. There are two elements in this area: the NALSAS strategy and the general LOTE element. Grants made for both elements depend on a commitment from education systems and individual schools to the Adelaide Declaration.

NALSAS funds are paid to government education authorities, Catholic Education Commissions and Associations of Independent Schools twice a year on a per capita basis of school enrolments in the four targeted FLs, "chosen on the basis of regional economic forecasts made by the Department of Foreign Affairs and Trade" with the aim of improving "Australia's capacity and preparedness to interact internationally, in particular with key Asian economies" (DETYA 2000: 89). 5\% of the $\$ 30 \mathrm{~m}$ per 
annum promised until the end of 2002 is set aside to fund specific collaborative projects. Of the eight projects currently under development only two are concerned with language learning as opposed to the broader area of Asian Studies (NALSAS 2001). LOTE funds are paid once a year to education authorities which are responsible for facilitating resource development or teacher training, or for distributing money to schools according to individual applications.

\subsubsection{Developments at state level}

The Commonwealth Government had hoped that all states would endorse the implementation of the Statements and Profiles in 1993 but, owing to political disagreements, states agreed only to publish them, "leaving it up to the discretion of each education authority as to how they would be modified or used" (McCollow and Graham 1997: 65). By mid-1996, all states had to some extent implemented the recommendations (McCollow and Graham 1997: 72) and developments have continued since then. Some examples of state policies follow in section 3.4.7. In general, states aim to have all students experiencing some LOTE learning, at least up to Year 10. Starting ages vary from Preparatory to Year 4. Deadlines for programme development also vary from 2000 to 2007.

\subsubsection{The reality behind the policies}

While enormous efforts have been made to promote LOTE learning in schools, and while the status of LOTEs looks high in Australian education compared with New Zealand, progress has been accompanied by considerable disquiet amongst academics and the teaching profession. Specific criticisms of LOTE policies and progress in implementation have centred on:

- the changeability of policy

- the lack of clear or realistic objectives

- why LOTEs?

- which LOTEs?

- a lack of commitment to bilingualism

- problems of teacher supply, training, attitudes

\subsubsection{Changeability of policy}

Changeability has been "one of the more unfortunate aspects of Australia's language policies" (Clyne 1997: 68). LOTE policy is characterised by its "volatility" and a "propensity for ad hoc decision making" (Liddicoat 1996: 7). Progress has been hindered by economic and political changes at national and/or state level, leading to general uncertainty about the place of LOTE learning in schools (Djité 1994, Supit 1996, Scarino 1996). A major concerns is that, unlike the NLP which was based on a broad consensus of opinion from many quarters with long-term goals, the ALLP is subject to the short-term political aims of whichever party is in power at national level (Herriman 1996). 
Implementation failures are attributed to the inconsistent adherence to policy by politicians and officials who have tended to focus on languages seen as economically useful, particularly Japanese and Indonesian, and to the attitudes of business people who remain sceptical about the value of language skills because of the spread of English (Phillipson and Skutnabb-Kangas 1994). The Editor of Babel (the Australian language teachers' journal) reflects the feelings of language professionals in his comments on national languages policy:

It would be interesting to map the political rhetoric (at both state/Territorial and national levels) that has occurred since 1987, as well as the action and inaction taken by our various Governments in the area of languages since the heady days of that first national policy. It seems that when it comes to planning and implementing anything as important as long-term (author's italics) educational policy, it's all a bit too hard for our pollies, who usually can't see much beyond the next election (Vale 2000-01: 3).

\subsubsection{Lack of clear or realistic objectives}

Over a decade ago, Stefanik (1990: 38) identified "an obvious gap" between what the public wanted and what educational administrators could or intended to provide. As Djité (1994: 53) recognised:

it is one thing to make policy statements and quite another to be able to adequately implement those policies to achieve the desired outcome(s).

The ALLP maintains the rhetoric of language learning linked to multiculturalism but downplays issues more forcefully represented in the NPL, such as language maintenance, bilingual education and language services (Liddicoat 1996: 5). Policy makers show little understanding about the importance of language learning for social reasons in a multicultural society. National policy is seen, at best, as "a loose set of guidelines that can guide priority areas of spending by the Commonwealth government" (Herriman 1996: 36). There is disagreement over what proficiency levels students can reasonably be expected to reach and the aims of the NALSAS strategy are considered particularly unrealistic, given "the inadequate number of contact hours and the lack of an integrated approach for languages in the curriculum" (Romano 1995: 23). Quinn (1998: 13) talks of "minuscule" time allocation and a lack of qualified teachers noting the:

curious expectation, not only that anyone is going to actually learn the language this way, but that there will be an emerging generation of competent bilinguals whose skill will somehow make a difference to 
our trading relationships with Asia".

The national language policy goals are considered ambitious and unrealistic, and future planning needs to focus on quality, not just of language education but of the policy process itself Scarino (1998). Scarino claims that the growing emphasis on proficiency goals is at the expense of cultural goals, rather than integrating the two.

\subsubsection{Why LOTEs?}

Policy implementation may have been uneven owing to disagreement over, or lack of understanding of, the benefits of LOTE learning, although research has shown widespread support for the principal of SLL for all. A public opinion poll commissioned by the Office of Multicultural Affairs in 1989 produced strong support for the statement that "all Australians should have the chance to learn English and another language" (Stefanik 1990: 35). Strong support was also found for language services in, for example, broadcasting, courts, hospitals and the supply of government information. Respondents were fairly evenly divided about the relative value of European and Asian languages, but, not surprisingly, there was firm support for the teaching of community languages, in their own schools, among all the NESB groups. The teaching of the languages of the major cultural groups in the country through regular schools received substantial support from all respondents.

Australia has moved away from the traditional national policy of assimilation of immigrants from non-English speaking background to the acceptance of multiculturalism, but according to Foster and Harman (1992) "British Australia" has been replaced by "Australian Australia". That is, the country has forged a new identity for itself but is still basically English-speaking. The numerous languages in the country are confined largely to the domains of family and home and, Australia tends to ignore " the linguistic and cultural diversity of more than twenty per cent of its population and of it nearest neighbours" (Foster and Harman 1992: 40). Even among language professionals, attitudes to language learning range from "enthusiastic optimism to disillusioned scepticism" about the progress away from a "monolingual assimilationist world view" (Phillipson and Skutnabb-Kangas 1994). There is even "entrenched" resistance to language learning among some educationalists" (Supit 1996: 12).

The Australian Language and Literacy Council (1994, cited in ALLC 1996) criticised the "dreadful myth of Australian schooling that only bright children can learn another language" and blamed failures on poor teaching. Elsewhere it was claimed that noted that "some sectors of the community remain generally dismissive of the importance of LOTE" (Djité 1994: 53). Djité 
stressed the need for ongoing research into language attitudes and usage for the development of "a practical approach to a cost-effective and long term language policy" (1994: 54). The situation of the late 1990s was summed up by Scarino (1996):

We are still a long, long way away from the situation where languages are accepted as an integral part of the education of all young people because of their social and cognitive value.

Another problem identified by Scarino's (1998: 12) is that so many reasons are given for promoting LOTEs in schools that they appear "exaggerated and therefore open to questioning" . In addition, she claims that the changes, resulting from the ALLP, "from a philosophy of linguistic and cultural pluralism...to the strong emphasis on English as the dominant language" has led to confusion about the rationale for language learning. The growing emphasis on English literacy, which barely acknowledges the possibility or value of literacy in another language, tends to reinforce the attitudes that students whose English skills are weak, for whatever reason, cannot study another language.

\subsubsection{Which LOTEs?}

According to the 1991 Census (Kipp et al. 1995: 1), there were 150 LOTEs spoken in Australia, not counting about 150 Aboriginal languages. Of these, only about 30 LOTEs were spoken regularly in homes, businesses and professional practices, by nearly 15\% of the population (Romano 1995: 24). This large number made it particularly difficult for Australia to decide which languages should be fostered. The ALLP recognises the huge diversity of languages in the country but claims that it is not possible, "given limited teaching, curriculum and financial resources, to spread them thinly in an attempt to accommodate all interests", hence the choice of 14 priority languages (DEET 1991: 16). Since the implementation of the ALLP in 1992 there has been a shift of emphasis to languages of economic significance.

The development of the NALSAS strategy (COAG 1994) has created controversy. Moore (1996: 481) claims that "pluralist aspirations no longer have a place in Commonwealth-sponsored endeavours". The promotion of four Asian languages has been at the expense of other LOTEs (Clyne 1997, Scarino 1998) rather than increasing the overall amount of LOTE learning. NALSAS signals that language education for reasons other than economic ones is less important and recent Australian governments have generally been reluctant "to view languages funding as an investment rather than a cost" (Lo Bianco et al.1999).

It has been argued that Indonesian/Malay should be the only NALSAS 
priority language taught in primary or lower secondary level (Kirkpatrick 1995) because Japanese, Korean and Chinese are too difficult for "nonbackground" speakers of those languages. Kirkpatrick believes that students who spend years studying a 'difficult' language will not achieve even a basic proficiency and will therefore become disillusioned. This may reinforce the idea that language learning is only for the brightest and may even lead to prejudice against all things associated with the cultures involved. Kirkpatrick's arguments were countered by Williss (1995) and Gao (1996) who blamed low levels of achievement on inadequate resources and poor attitudes to language learning. They also argue that Kirkpatrick underestimates the ability of young children and the enjoyment they get out of meeting the challenge of language learning

Schools may be torn between the wishes of local communities and national or state priorities. Schools may not have the resources, especially teachers, to provide for the most important local community LOTEs and may therefore, not unnaturally, opt for programmes which attract state or Commonwealth financial support. Ultimately, therefore, the argument is not about which languages are the most suitable or desirable for schools to teach, but about the resources needed in schools to make teaching programmes effective. The major concern has to be the adequate supply of trained and qualified teachers (see 3.4.6.6 below).

\subsubsection{Lack of commitment to bilingualism}

Phillipson and Skutnabb-Kangas, vigorous advocates of bilingualism, cautiously welcome the progress made towards achieving language policy goals as "a significant advance for those who value bilingualism not only for minorities but also for the linguistically dominant group (1994: 125). However, while large number of languages are being learnt, there is very little genuine bilingual education. Overall, figures for FLL and SLL remain "lamentably low compared with continental Europe" and Phillipson and Skutnabb-Kangas remain particularly concerned about the future of Aboriginal languages (1994: 125-6).

It has been claimed that bilingual education is rare and that "the language rights of minorities continue to be only vaguely recognised by Australian education systems" (Romano 1995: 23). An investigation of language maintenance among NESB communities found that:

despite the mostly positive attitude towards multiculturalism and a national policy of LOTEs for all, the responsibility for L1 maintenance falls mainly on the communities themselves; young people are not motivated to maintain or learn their community language because LOTEs have low status and do not enhance job 
prospects (Kipp et al. 1995: xv).

Support for community languages is considered important for social and family cohesion and community identification by Kipp et al. (1995: xv-xvi), but language policy is seen as "subject to a set of economic concerns that might override the claims of language rights in education" (Herriman 1996: 36). The ALLP has "minimal regard for bilingual education except as a possible aid to literacy in transitional programmes" (Herriman 1996: 57). The strong emphasis on literacy for all Australians means literacy in English, as shown by the spending programmes set in place (Herriman 1996: 59).

Fostering bilingualism through language maintenance programmes is also judged to be a more efficient means of increasing proficiency in 'useful' languages than SLL for English-speaking students (Crawford 1995, Kipp et al. 1995, Liddicoat 1996). Encouraging bilingualism would be of benefit both to individual communities and the whole country. In this context it should be noted that the state of Victoria has introduced separate examination syllabuses for Chinese and Japanese as L1 and L2.

\subsubsection{Teacher supply and training}

Two major reports have examined the issue of teacher supply and training, especially for the development of primary LOTE learning. Languages at the Crossroads (Nicholas et al. 1993), made many recommendations to the Commonwealth Government for the promotion of LOTE learning, but the National Board of Employment, Education and Training (NBEET) commissioned another report from the ALLC (1996) because of the "systemwide, long-term historical failure" to produce satisfactory teaching which affected LOTE learning more than any other curriculum area" (Cavalier 1996: xi). The Preface to the report is at pains to point out that language teachers were not to blame for their inadequate training, nor the lack of time given to LOTE learning in schools.

The ALLC came to some scathing conclusions about the "chasmal proportions" of the gaps between policy objectives and what is happening in schools and teacher training institutions, speaking of "grievous weaknesses in teacher quality and supply" (ALLC 1996: 180). The report was particularly critical of the NALSAS strategy for its "ambitious and massively expensive policy goals", for which there were insufficient teachers fluent in the priority languages, with the necessary cultural knowledge and the competence to teach them. It also suggested that the narrow focus on only four Asian languages may turn students away from LOTE learning altogether. Where the greatest diversity of languages is encouraged, as in Victoria and the ACT, there has been the greatest increase in student numbers (ALLC 1996: 16). 
Furthermore, the ALLC report suggested that "frustrated and underachieving teachers and students can only further undermine the already precarious situation" (1996: 181). Frustration among teachers may be due their lack of involvement in the decision-making processes concerning LOTE policy and planning and lack of appropriate curricula (Rizvi 1997, Scarino 1998). Teachers need to see that the development of curriculum and assessment procedures will improve their own skills and methods as well as student achievement. Otherwise "the national curriculum initiatives may be just another example of reforms that withered at the classroom door" (McCollow and Graham 1997: 73). Many teachers saw the NALSAS strategy "as having been developed by people with no 'corporate memory' of the success and failures of similar reform initiatives" (Rizvi 1997: 118).

Large problems of LOTE teacher supply, poor quality of language competence and methodology, and lack of funding for teacher training are reported by Djité (1994), Herriman (1996) and Kirkpatrick (1997). It has been noted that one of the key elements of language policies in other countries is an integrated approach to language teaching from pre-school to teacher training, which does not exist in Australia (Romano 1995: 23) .

Teacher recruitment remains a problem (Supit 1996), partly because the career of teaching was perceived to have low status, poor rewards and to be too stressful (Kipp et al. 1995: 17). Personal communications from education personnel in Victoria indicated that:

- many LOTE teachers are leaving the profession early because of general frustration with the job.

- keen young teacher trainees are being deterred from pursuing a career in LOTE teaching, of ten turning to ESOL instead, because they meet inadequate teachers and programmes in schools.

- some teacher trainees are instructed not to use the target language in class, ostensibly because the students cannot cope but in reality because the class teachers lack proficiency and do not wish to be shown up by the trainees.

This reluctance to use the FL as the language of instruction has been found particularly among teachers from minority ethnic groups in primary schools either because it is not seen as the 'correct' way to teach a language in their culture, or because they have too low expectations of students' ability to understand and learn (Clyne et al. 1995). In secondary schools it may be a hangover from the traditional grammar-based method of teaching LOTEs through English (Crawford 1999).

For successful implementation of LOTE programmes primary schools need teachers with sufficient knowledge and training in the languages, culture, 
sociolinguistics and language teaching methodology (Clyne et al. 1995). Students need a "communication-rich environment', where they have sufficient, comprehensible input (spoken or read), opportunities to use the FL and the sense of needing to use it. In Victoria all three education authorities provide extensive opportunities for teacher training and in-service development and assistance with resources, but it is always the case that requests for assistance must be initiated by schools or individuals. They are not initiated by officials.

\subsubsection{Other problems}

Other barriers to progress in developing successful LOTE programmes include:

- a great lack of suitable resources for most LOTEs (Djité 1994, Williss 1995);

- a lack of 'articulation', that is, continuity between primary and secondary school programmes, (Djité 1994, Romano 1995, Clyne et al. 1995 Crawford 1999);

- classes combining beginners and students with previous LOTE knowledge, either from home (especially at primary level) or previous study (especially at secondary level) (Crawford 1999);

- devolution of decision-making to individual schools, which may perceive LOTEs as difficult to fit into the already 'crowded curriculum' or as a barrier to achieving the principal aim of English literacy, and may make only token gestures towards state policy, offering plausible excuses, such as the unavailability of suitable teachers ${ }^{6}$;

- in primary schools, a lack of understanding of or support for LOTE programmes, including disagreement over what is most desirable: 'content-based' or 'language-object' classes (Clyne et al. 1995, Gearon 2001);

- lack of public consultation about the NALSAS strategy (Clyne 1997) and disregard for "the deep ambivalence that exists in the Australian society about its engagement in Asia (Rizvi 1997: 117);

- the view that linguistic diversity is not a necessary part of a multicultural society, or that multiculturalism is really only for ethnic minorities, not for the dominant white, English-speaking society (Lo Bianco et al. 1999).

\subsubsection{Some indicators of what has been achieved in schools 3.4.7.1 Victoria}

Victoria expects all government schools to provide LOTE programmes for all students up to Year 10, although this may not yet have been fully achieved.

\footnotetext{
${ }^{6}$ In Victoria there is no inspection system of state schools and it is possible for schools to claim LOTE funding for minimal or even non-existent programmes. (Clyne 2001 - personal communication)
} 
According to Eckstein (2000), in 1999 at least 95\% of all students in Years 38 were learning a LOTE. In about half the secondary schools LOTE learning became optional in Year 9, and the proportion of LOTE students was 61\% in 1999, with 35\% in Year 10 (Victoria DEET 2000: 41).

Victoria has the additional resource of the Victorian School of languages (VSL), founded in 1935, which provides instruction after school (in 33 centres in 2001, in 40 languages) or by distance education (in French, German, Greek, Indonesian, Italian and Japanese). For many languages the VSL is the only school providing tuition in LOTES for the Victorian Certificate of Education (VCE). Approximately one third of Year 11 and 12 students study through VSL, in after hours classes. Instruction is also available in more than 50 languages through about 200 after-hours ethnic schools supported by government funding, on condition that schools develop curriculum and assessment policies in line with the latest Curriculum and Standards Framework (Board of Studies: 2000).

Victoria has introduced a range of initiatives to encourage LOTE learning, including:

- bonus points for LOTE studies for Year 12 students wanting to enter university;

- annual awards for teachers, schools and students (Eckstein 2000: 9).

The proportion of Year 12 students in Victoria sitting VCE in a LOTE in 2000 was 19\%, much higher than in any other state (Jenkins 2001), but this is not a reflection of higher numbers of students continuing LOTE study beyond the compulsory years as there is a considerable influx of both overseas students and students from ethnic minorities wishing to obtain a qualification in their home language.

There is more senior LOTE learning in non-government schools than government schools, and more in independent schools than in Catholic schools (Fullerton and Ainley 2000). According to Oakes (2001), independent schools in Victoria do not pay much attention to state priorities for LOTE learning. French and German have been the most commonly taught languages but more and more schools are introducing Chinese, Japanese and Indonesian. In 2001 nearly $87 \%$ of independent schools in Victoria were offering LOTEs, $30 \%$ offered French but the popularity of German had dropped. LOTE learning had increased in primary schools and schools which had not taught a LOTE in the past were tending to introduce an Asian language. Indonesian is particularly popular as it seems the easiest of the NALSAS languages. While no more than about $35 \%$ of schools made LOTE learning compulsory at any level (mostly at Year 8), in 2000 over $31 \%$ of 
Year 12 students were studying a LOTE, a very high proportion compared with government schools.

Catholic schools follow much more closely national and state recommendations concerning curriculum. In Victoria LOTE learning has long been particularly strong, as more than one third of all their students are from an NESB background (Raso 1997), including a substantial Italian community. The Catholic Education Commission of Victoria (CECV) firmly supports LOTE learning as "an important component of a quality education" and aims to increase participation in LOTE learning in years 11 and 12. (Catholic Education Office 2001), although individual schools remain independent in their choice of language and the degree of compulsion. About $90 \%$ of primary and all secondary schools have LOTE programmes (Raso 2001).

The most popular language taught in Catholic primary schools is Italian, although for most students it is no longer their L1 (Raso 2001). The most popular Asian language is Japanese, with Indonesian a close second at secondary level and increasingly popular at primary level (CECV 2000). In 2000 over $97 \%$ of students in years 3-10 were studying a LOTE (CECV 2000). Over $31 \%$ were studying a NALSAS language, i.e. the CECV was just over halfway to achieving the Commonwealth target of $60 \%$ studying a NALSAS language by 2006 (CECV 2000: 6). As in government schools, there is a large drop-out rate in Year 11 but at year 12, LOTE enrolments increased from $9.6 \%$ in 1995 to $13.7 \%$ in 2000 (Raso 2001), about one third of whom were were in a NALSAS language.

\subsubsection{Other states}

\section{Australian Capital Territory}

All schools, primary and secondary are expected to offer LOTEs. In most primary schools LOTEs are taught in years 1-6 and most secondary schools make LOTE learning compulsory in year 7 (Victorian Department of Education and Training: 2002).

\section{New South Wales}

LOTE learning is compulsory in years 7-10, but optional in primary schools (Victorian Department of Education and Training: 2002).

\section{Northern Territory}

All schools are required to offer LOTE learning (Victorian Department of Education and Training: 2002).

\section{Queensland}

LOTE has been compulsory in Years 6-8 since 1989 (Crawford 1999). 


\section{South Australia}

In $199768 \%$ of school students were studying a LOTE, compared with $31 \%$ in 1990. The current goal is LOTE learning for all primary students by 2004 (in $84 \%$ of schools in 1999) and for secondary students up to Year 10 by 2007 (in 92\% of schools in 1999). (MCEETYA 1999).

\section{Tasmania}

The state began introducing LOTEs in Year 3 in primary schools in 1996 which had extended to Year 6 by 1999, with over $60 \%$ offering an Asian language (MCEETYA 1999). About $75 \%$ of primary students were learning a LOTE in 2002 (Victorian Department of Education and Training: 2002).

\section{Western Australia}

All students in Years 3-7 were engaged in LOTE learning in 2001 (Education Department of Western Australia 2001). From 2003 it is compulsory for all schools to offer a LOTE from years 3-10 (Victorian Department of Education and Training: 2002).

\subsubsection{The overall picture}

There has been little success in persuading more students to study a LOTE beyond Year 10, nor in changing the languages being studied. The proportion of Year 12 LOTE enrolments remained fairly stable from just under $12 \%$ in 1991 to $13.2 \%$ in 1999 (DETYA 2000) The last figure represents $9.2 \%$ of boys and $16.7 \%$ of girls, showing a typical gender bias, but it should be noted that the girls outnumbered the boys in year 12 in 1999 by over two to one. French, German and Japanese remained in the top four languages studied (see Table 3.3) although French declined as Japanese increased in popularity, and Chinese overtook Italian for fourth place in 1992. Progress varied considerably among states. Senior LOTE learning increased in all states except South Australia and New South Wales from 1993 to 2000. Increases were particularly large in Western Australia and Tasmania and student numbers for Japanese in Queensland and Northern Territory doubled over the same period. Students were more likely to study a LOTE in one of the capital cities than in a rural area or in other cities (Fullerton \& Ainley 2000: 26-28). Even in Victoria, where LOTEs at senior levels are the strongest, in 1999 they were studied by only $14 \%$ of students in Year 11 and $12 \%$ in Year 12 in government schools (Victoria DEET 2000: 50). This is, however, approximately twice the usual number of senior FL students in New Zealand.

Among university entrants in Victoria in 1995 and 1999, only one key learning area had been studied by fewer students - Health \& Physical Education (Calderon et al.2000: 114). Knowledge of a LOTE is not a prerequisite for entry to or completion of any university course in Australia. According to Jenkins (2001), there is no talk of establishing any such requirement. Work is, however, in progress to develop assessment criteria for LOTEs in the context of vocational education qualifications in 
tourism, health and social services, for example, where translators and interpreters are in demand. (Jenkins 2001).

The report on the first four years of the NALSAS strategy reported a $50 \%$ increase in the study of Asian languages from 1994 to 1997 and that 2,500 teachers had been trained in Asian languages (MCEETYA 1999, cited on NALSAS web site). It is apparent, however, that the development of programmes in Chinese and Korean lag far behind those in Indonesian and Japanese (MCEETYA 1999). Japanese is the most widely taught language in Queensland and Indonesian in the Northern Territory.

Table 3.3 Proportions (\%) of year 12 LOTE enrolments in Australian schools in the 10 most popular languages: 1991-1999

\begin{tabular}{|l|c|c|c|c|c|c|c|c|c|}
\hline Language & 91 & $\mathbf{9 2}$ & $\mathbf{9 3}$ & $\mathbf{9 4}$ & $\mathbf{9 5}$ & $\mathbf{9 6}$ & $\mathbf{9 7}$ & $\mathbf{9 8}$ & 99 \\
\hline French & 24 & 20 & 19 & 17 & 18 & 17 & 16 & 17 & 17 \\
\hline Japanese & 15 & 18 & 19 & 20 & 21 & 22 & 21 & 22 & 22 \\
\hline German & 14 & 12 & 11 & 10 & 11 & 11 & 11 & 11 & 11 \\
\hline Italian & 12 & 10 & 9 & 9 & 9 & 9 & 9 & 8 & 8 \\
\hline Chinese & 9 & 11 & 10 & 11 & 10 & 10 & 10 & 10 & 11 \\
\hline Indonesian & 6 & 5 & 5 & 5 & 6 & 7 & 8 & 8 & 8 \\
\hline Greek & 4 & 8 & 7 & 7 & 6 & 5 & 5 & 4 & 4 \\
\hline Vietnamese & 4 & 4 & 5 & 5 & 5 & 4 & 4 & 3 & 3 \\
\hline Spanish & 3 & 3 & 3 & 4 & 3 & 3 & 3 & 3 & 3 \\
\hline Arabic & 1 & 2 & 2 & 3 & 2 & 2 & 2 & 2 & 2 \\
\hline Other & 7 & 9 & 10 & 9 & 9 & 9 & 11 & 11 & 12 \\
\hline
\end{tabular}

Data from DETYA Annual Report 1997 and MCEETYA National Report on Schooling 1999 


\subsubsection{Summary}

Implementation of Australian policy since 1987 has been hindered by many factors, although there the amount of LOTE learning in schools has increased considerably since the mid-1990s when it still held a marginal place in the school curriculum (Djité 1994: 52, Romano 1995: 23). Increases have been very uneven both geographically and in terms of the languages taught. Herriman blames slow progress on "years of neglect by governments and educational authorities" (1996: 59). Ten years after his original NLP (1987) Lo Bianco was more optimistic than other commentators but admitted that "the reality of language study, however, as against the policy positions, which are generally positive, is far from satisfactory" (1997: 112). He also admitted that the majority of adult Australians remained steadfastly monolingual English speakers but hoped that the "more assertive policies of more recent years may turn this around" (1997: 113).

On the positive side, language policies may be irreversible because mainstream Australians as well as ethnic groups benefit from multiculturalism and probably identify with this, and even with multilingualism (Clyne 1997: 70). LOTE learning for all is now generally accepted (Parkinson 1999), although arguments continue over the choice of languages, teaching methods and starting ages. Considerable dissatisfaction remains about the slow progress of language policy implementation in schools and the ongoing problems involved, especially the drop-out rates after Year 10 , but it must be noted that, compared with what is happening in New Zealand schools, Australia is far advanced in its language learning programmes.

\subsection{A final comparison}

The current place of FLL in New Zealand schools is probably more similar to the USA than the UK or Australian situations. New Zealand inherited much of its traditional education system from the UK, where French was, and remains, the most commonly taught FL. Despite New Zealand's geographical distance from Europe its only substantial concession to this and to its current socio-economic position in the world in terms of FLL has been the development of Japanese teaching, and French retains a dominant position in the languages curriculum. The UK may lament the high drop-out rates from FLL and the country's apparent lack of FL skills, but its population, in terms of LOTE learning, is far in advance of New Zealand.

Although it has a much more multicultural population, the majority view in the USA seems to be that being American means speaking English, and that, as English is becoming more and more the language of globalisation, there is little need for people to speaks LOTEs. This is probably similar to the views of many Pakeha New Zealanders. There is also, however, in the USA growing support for L1 maintenance and over the last 20 years a growing recognition of the need for FL capabilities, if only in the interests of defence policy.

On the other hand, Australia, which shares many geographical and historical similarities with New Zealand, also has a much more multicultural heritage and has moved strongly in the 
direction of the UK model (common in Europe) of requiring several years of compulsory FLL. To some extent, this policy is derived from a strong tradition, especially in the main cities, of L1 maintenance among immigrants, something which New Zealand immigrants have struggled to achieve owing to small numbers of individual cultural groups and lack of official support. There are signs that New Zealand (Evans 2002, MoE 2002b) is taking notice of developments in Australia, particularly in the context of the recent curriculum stocktake which will be discussed in chapter 11 . 


\section{CHAPTER 4 METHODOLOGY}

One language is none at all, two are not too many, and only the third brings a real feel for the world. (Hanimann 2001)

\subsection{Introduction}

Attitudes are abstract concepts and cannot be directly observed (Lett 1997, Oppenheim 1992) They have to be inferred from the way people behave or what they say and I needed to explore the relevant literature to select the best means of measuring attitudes among school students. Schuman and Presser (1981) investigated many attitude surveys to discover the effects on responses of variables such as question form, question order, the presence of 'Don't know' options, and the problem of acquiescence - the tendency for people to agree with whatever opinions are presented to them, regardless of content. Although they examined interview surveys about broad social issues, Schuman and Presser (1981) offer many useful ideas concerning written questionnaire construction. Many of their findings are now incorporated into advice given in more recent manuals.

This research largely took the form of a 'causal-comparative study' (Gall et al. (1996), by investigating and comparing the reasons why students did or did not intend or decide on FLL when they started secondary school in 2000 . The groups were thus self-selected by the nature of their intentions and the choices of option subjects they made. I supplemented data from student questionnaires with information collected from various sources, including interviews with teachers and a small sample of parents of year 9 students.

\subsection{Questionnaires versus interviews}

\subsubsection{For students}

Given the questions to be answered and the hypotheses I wished to test, and the desirability of surveying as many students as possible within necessary time and cost constraints, I decided that written questionnaires administered to whole classes were the most effective way to investigate student attitudes. Once distributed, questionnaires cannot be modified and have, therefore, built-in consistency, which is essential in considerations of reliability and validity, discussed in more detail below. Consistent wording also permits easier analysis of responses. The fact that I administered all the student surveys ensured consistency in the procedure and I could offer consistent on-the-spot assistance to students, in the event of misunderstandings. Neither could have been guaranteed if a postal survey had been used. All these points will be discussed in more detail below, including the choice of Likert scales for the main parts of the student surveys.

\subsubsection{For teachers and parents}

I decided that the attitudes of adults would be elicited more efficiently through interviews, which allow for deeper probing of the issues and more flexibility of questioning to suit individual respondents, who can, in turn, give more spontaneous responses (Bailey 1987, Wray, Trott \& Bloomer 1998). Interviews are more 
personal and allow for the creation of a sympathetic environment, particularly because they usually take place at a time and place most convenient to the respondents.

I aimed to interview a sample of class teachers and other key personnel in all participating schools, such as principals, teachers in charge of languages, year 9 Deans and careers advisers, with three aims in view:

- to supplement the factual knowledge, already gleaned from available documents, about the place of FLL in each school;

- to identify teachers' attitudes towards FLL;

- to ascertain, particularly in secondary schools, whether there was any difference between what students and their parents were told about FLL and what actually happened in each school.

I thought it valuable to investigate whether, if there was FLL at any of the primary schools in the area, there was any communication or cooperation between primary and secondary schools over course content or methodology. I was also interested in discovering if secondary FL teachers viewed classes containing students with varying amounts of previous FLL as a problem or a challenge.

I decided to interview a small sample of parents of year 9 students to ascertain if the children accurately reflected their parents' experience of and attitudes towards FLL, and the extent of their parents' influence on their option choices. I hoped to obtain a general picture of what parents thought about FLL in schools in general, or specifically for their children.

\subsection{Data gathering}

There were seven stages in the data gathering process:

1. year 8 student survey in the second half of 1999 ;

2. staff interviews in the same schools;

3. collection of information about local secondary schools, from prospectuses and option subject booklets sent to prospective students' homes or schools, or given out at open days, meetings and so on;

4. year 9 student survey in the same area early in 2000 ;

5. staff interviews in the same secondary schools;

6. collection of general information about all the schools from ERO reports (student numbers, gender balance, ethnic backgrounds), and the $\mathrm{MoE}$ (decile ratings which indicate the predominant socio-economic status of the schools' catchment areas);

7. parent interviews.

\subsection{Choice of location}

Student attitudes may depend partly on what FLs are offered by their local schools, the length of courses and the status of FLL (compulsory or optional). It is likely that no two 


\section{2}

areas have the same range of languages available in the same types of school, under the same conditions, another reason for not claiming that my results reflect what is happening throughout New Zealand. In the main cities it would be difficult to select a workable number of year 8 classes representative of the large number of schools, of ten serving limited catchment areas in socio-economic terms. It would also be difficult to follow up students in year 9 where there are many secondary schools to choose from. By contrast, in a small area there might be too few secondary schools to allow a comparison between single sex and mixed schools, for example.

\subsection{Student samples}

\subsubsection{Choosing the samples}

By the nature of their organisation, schools lend themselves to the convenient technique of 'cluster' sampling (Gall et al. 1996), provided that students are willing to participate and have their parents' permission, and that classes are of mixed ability and from a mix of social backgrounds. If the sample can be considered representative of the wider population 'convenience' sampling is commonly used and legitimate (Gall et al. 1996: 228). My research does not, however, claim that the attitudes to FLL which I identified are necessarily representative of the attitudes throughout New Zealand. It is, rather, an observational study of two groups of students, chosen largely for convenience, in a limited and accessible area. I selected a medium-sized city where, I estimated, students came from a wide range of socioeconomic backgrounds, and where it was feasible to aim to survey all or most of the year 8 and 9 students.

\subsubsection{The sample of year 8 students}

The major factor governing the selection of schools was their willingness to participate in the research. There are many primary schools in the city, but over $88 \%$ of year 8 students in 1998 (MoE figures) attended three intermediate schools and an integrated, Catholic, year 7 to 13 school. Therefore I sought permission from these four schools to carry out the year 8 survey. Unfortunately, two of the intermediate schools declined to participate and so I approached alternative schools. Only two primary schools in the area had appreciable numbers of year 8 students. Of these, one private girls' school declined to participate unless the survey took no more than 15 minutes of class time, which was not possible. The other, a large, semi-rural year 1 to 8 school was very pleased to be invited to participate.

Thus the sample of year 8 students came from three different types of school: a full primary, an intermediate and a year 7 to 13 school. Although the decile ratings were relatively high, ranging from 5 to 8 , all the schools had students from a wide range of backgrounds. None offered the same opportunities for FLL. The primary had virtually none; the intermediate was trying to give all classes some experience of Spanish or Japanese but two of the eight year 8 classes were not involved in 1999 , although some of the students had had some FLL in year 7; in the Catholic school all intermediate students had a term of French one year and a term of Japanese in the 
other, except one class in each year where Maori was studied instead of one of the FLs.

I delivered information letters and consent forms for distribution to all year 8 students and their parents to the participating schools at the end of August 1999, and arranged to conduct the intermediate school survey in the last week of term 3 and the other two in the first week of term 4. All the schools were extremely co-operative. The great majority of invited students took part , a sample total of 330, with a good gender balance. This sample yielded 308 useful surveys, almost exactly half girls and half boys.

\subsubsection{The sample of year 9 students}

There are six secondary schools in the city two large, single sex schools and four mixed, including the Catholic school. I sent letters to all the schools in June 1999 inviting their participation in the research. All but the smallest of the co-educational schools agreed. The smallest had only 5\% of the year 9 students in the city in 1999 and only four of the 330 year 8 students surveyed indicated that they would be attending that school in 2000. Only 14 year 8 students indicated that they would be attending secondary schools outside the city, such as private boarding schools. It seemed reasonable to assume that a fairly broad range of socioeconomic backgrounds was represented by the five participating schools, although the decile ratings were even higher than those of the year 8 schools, ranging from 6 to 9 . It also seemed reasonable to assume that the majority of students who participated in the year 8 survey would also be 'captured' in the year 9 survey.

The eventual proportions of participants varied from school to school, depending on time and timetable constraints, and on the level of cooperation in each school. All the schools except the boys' school allowed me to survey year 9 classes one at a time, in a manner similar to that used for the year 8 survey. The boys' school allowed only students who had returned the parental consent forms to participate, most of whom were from the top few streams (of 13, divided according to ability) and involved in FLL. As a result, the year 9 sample was not as representative as the year 8 sample in terms of gender balance or ability level. For example, in the single sex schools less than one quarter of the boys could be surveyed, compared with two thirds of the girls. The final sample comprised 821 students. As with the year 8 survey, I discarded survey forms if they were incomplete or extremely inconsistent, or if English was obviously not the L1 of the students and their parents. This left 664 useful surveys, 59\% girls and $41 \%$ boys. Details of the ethnic and gender proportions of students in all the surveyed schools, and their decile ratings, are given in the table in Appendix 2.

\subsection{Selection of teacher samples}

In each school, I tried to interview a range of teachers, according to both their positions in the administrative hierarchy and their subject responsibilities, in order to build up an accurate 
picture of the place of FLL within the school and teacher attitudes towards it. While I cannot claim that these schools and the attitudes of their teachers represent those of the whole country, all the information and opinions obtained can be set against the larger picture built up by language teachers and advisers throughout New Zealand, by ERO (1994), and by Peddie, Gunn and Lewis (1999), discusșed in chapter 2.

At the time of the preliminary visit to each school I requested a list of teachers with their responsibilities. The whole staff was usually informed by the principal of the timing and purpose of my visit. Between class visits I contacted as many teachers as possible. I supplied each one with written information about the project, a consent form and the preliminary questionnaire to fill in prior to an interview, and, if they agreed, we arranged a convenient time and place to meet.

At the primary school I was able to interview all the relevant staff members. Given the larger numbers in the other schools and limited time I had to target a sample and interview as many as possible, according to their availability and willingness to participate. Although I tried to achieve some degree of age and gender balance, this was inevitably limited, partly by the fact that, at pre-secondary level in particular, the teaching profession is predominantly female. To some degree, therefore, this was a random sample. As Table 4.1 shows, I managed to interview 56 teachers including all the school principals except one who was unfortunately away at the time of my visit.

Table 4.1 Numbers of teachers interviewed by type of school and gender

\begin{tabular}{|l|c|c|c|c|}
\hline Type of school & Years & Female & Male & Totals \\
\hline Primary & $1-8$ & 3 & 1 & 4 \\
\hline Intermediate & $7-8$ & 4 & 3 & 7 \\
\hline Integrated mixed secondary & $7-13$ & 8 & 4 & 12 \\
\hline Mixed high school (2) & $9-13$ & 9 & 6 & 15 \\
\hline Single sex high school (2) & $9-13$ & 12 & 6 & 18 \\
\hline Totals & & 36 & 20 & 56 \\
\hline
\end{tabular}

Table 4.2 shows the numbers of teachers according to their responsibilities and gender. 'Other senior management' includes two intermediate deputy principals, both involved in FL teaching. 'Other FL teachers' includes two intermediate classroom teachers and 'Others' 
include a wide range of teachers from heads of department (HODs) to assistant teachers, representing most subject areas.

In general, depending on the size of a school and the amount of FLL offered, there may or may not be a separate languages department. If such a department does not exist there is usually a 'teacher in charge' of each language without the status of HOD and responsible to the HOD of another department, often English, which was the case at one of the surveyed schools. One crucial consequence of this is that FL teachers may not be involved in middle management meetings and may therefore have a limited voice in discussions about curriculum development. It could also be interpreted as a sign that FLs occupy a subordinate place in the curriculum.

Table 4.2 Numbers of teachers interviewed by responsibilities and gender

\begin{tabular}{|l|c|c|c|}
\hline Teacher positions & Female & Male & Totals \\
\hline Principal & 2 & 4 & 6 \\
\hline Other senior management & 3 & 1 & 4 \\
\hline Dean/Guidance/ Careers adviser & 11 & 4 & 15 \\
\hline FL HODs & 3 & 1 & 4 \\
\hline Other FL teachers & 7 & 4 & 11 \\
\hline Others & 10 & 6 & 16 \\
\hline Totals & 36 & 20 & 56 \\
\hline
\end{tabular}

\subsection{Selection of parent sample}

Consent forms for the year 9 students included a request that parents indicate if they were willing to be interviewed in connection with the survey. Originally I aimed to interview only parents of students who took part in both the year 8 and 9 surveys, with the further aim of obtaining a balance for each of the following factors:

- school

- gender

- experience or lack of FLL prior to year 9, compulsory or optional

- whether student was studying a FL in year 9 or not

- parents' experience of FLL. 
The choice of parent interviewees proved problematic. It was first restricted by the number of consent forms returned. The parents of 75 children agreed to be interviewed. Most of the surveyed intermediate students had had some FLL experience, and mostly as part of their school curriculum, not chosen as an optional extra. Finally, in order to cover all the schools with a reasonable gender balance, and in order to include some parents whose children had no prior FLL, I decided to include in the pool parents of students whose children had taken part only in the year 9 survey. I had assumed that for year 9 students FLL would be optional and available for all, but since this did not prove to be the case I tried to take this factor into account also in the selection of interviewees. Among the students whose parents were willing to be interviewed only 14 were not studying a FL in year 9. Thus I decided to approach all these and select 16 of the rest to give a total of 30 interviews. The chief remaining problem was the large number of boys with no previous experience of FLL. In order to narrow down the number I decided to do a rough estimate of their attitudes to select a keen FL student, a reluctant FL learner and one with a 'middling' attitude. Table 4.3 shows the number of interviews carried out according to students' gender and whether or not they were studying a FL in year 9.

Once I had selected the families I telephoned them to confirm that they were still willing to be interviewed. Only one or two dropped out at this stage but I was able to replace them with other families which gave a reasonably well balanced sample. I made arrangements to meet one or both parents in each family at a time and place most convenient to them, within a three or four day period. In some cases I had to make further telephone calls closer to the time to confirm the meetings and in the final event, two mothers proved unavailable.

I offered to send each family a summary of the interview topics so that they could think about their responses in advance (see Appendix 9A), which most of them welcomed. All the parents interviewed proved to be from monolingual, English-speaking backgrounds. All were born in New Zealand, except one mother from Australia and one father from the USA, and English was the L1 of all of their parents, although interviews revealed that a few had NESB grandparents or more distant forebears.

\section{Table 4.3 Numbers of parental interviews}

\begin{tabular}{|l|c|c|c|}
\hline Students & $\begin{array}{c}\text { In FLL in } \\
\mathbf{9 9}\end{array}$ & $\begin{array}{c}\text { Not in FLL } \\
\text { in } \mathbf{9 9}\end{array}$ & Total \\
\hline Girls & 8 & 3 & 11 \\
\hline Boys & 8 & 9 & 17 \\
\hline & 16 & 12 & 28 \\
\hline
\end{tabular}




\subsection{Timing of the surveys}

Timing of the students surveys was crucial. Students in year 8 with no experience of FLL may not have thought about it before considering which secondary school they are going to attend and what choice of optional subjects they will have in year 9. Most secondary schools start their 'recruitment' campaigns in July or August, sending out prospectuses and holding open days or evenings for students and parents to visit, meet teachers and discuss courses. Option subject teachers, in particular, usually feel the need to 'advertise' their subjects in the most favourable light, putting on colourful and lively displays of teaching materials and students' work.

I planned, therefore, to survey the year 8 students towards the end of term 3 or early in term 4 so that the majority would have decided which school they would attend and what optional subjects they might take the following year. I anticipated that they would be aware of the possibility of studying one or more languages and have formed some sort of opinion about this. Any attempt to identify their attitudes to FLL would therefore have more validity. For the year 9 survey I proposed to administer the questionnaire as early in the year as possible so that students would still have a clear memory of how they had chosen their option subjects, what they took into consideration and who they discussed their ideas with.

Timing of teacher interviews was not so crucial but, for practical purposes, I conducted them when I was in each school to administer the student surveys. My major concern was to minimise the inconvenience to teachers in order to ensure their co-operation. Interviews with parents needed to follow a preliminary analysis of the year 9 questionnaires, for reasons discussed above.

\subsection{Development of student questionnaires}

\subsubsection{General considerations}

The major concern, stressed by much of the literature (e.g. Hatch \& Lazaraton 1991: 15 ) is that all terms used in questionnaires are clearly defined in the context of the specific research to provide consistency, clear understanding by respondents and the possibility of replicating the research at a later date. Consistency of communication by the researcher and understanding by respondents will elicit consistent communication of adequate responses, but respondents must be both able and willing to respond (Fowler 1995: 4). Investigators should not assume that attitudes to the topic under investigation already exist and acknowledge that a survey may, if not create an attitude, crystallise one that the respondent was not previously conscious of (Schuman \& Presser 1981:252). Respondents will be most co-operative and helpful if they appreciate the need for accurate responses, are assured of the confidentiality of their responses, and believe that these will be valued and used appropriately Fowler (1995: 30, 33). 


\subsubsection{Validity}

\subsubsection{Internal and external validity}

For a survey and its results to be useful the question of validity must be examined. To be internally valid the results of the research must derive from the method by which they are obtained. To be externally valid, the design of the survey must be such that the results can be generalised to a wider population (Nunan 1992: 17). Since the latter was not my intention, this notion of validity is not relevant. Another type of validity irrelevant for this research is 'predictive validity', because I did not intend to predict the future behaviour of students, based on their attitudes (Henerson et al. 1987: 144, Gall et al. 1996: 251).

\subsubsection{Face validity}

Burns (1997: 278) believes that 'face validity' is particularly important in the measurement of attitudes and usually obvious, but that, nevertheless, great care needs to be taken to ensure that all items are relevant to what is being measured. De Vaus (1995: 56) suggests asking a panel of 'experts' to judge the definitions of concepts involved, the relevance of items used and the adequacy of the coverage of the concepts. It was to meet such concerns that I organised focus group discussions with small groups of year 9 students early in the process of developing the questionnaires. I also needed to be confident that my survey was worthwhile, that I was using the most appropriate instrument to measure attitudes and that I was likely to obtain accurate information (Henerson et al.1987: 133). Feedback from all individual adults with whom I discussed the project, whatever their interest in or experience of FLL, indicated that the research was extremely worthwhile.

\subsubsection{Content validity}

I took care to select statements for the Likert scales which were relevant to the factors representing positive and negative attitudes to FLL to ensure that all items were based on my hypotheses and the variables to be investigated (Gall et al. 1996: 250-53). Any inference concerning student attitudes needed to derive logically from students' agreement or disagreement with the statements (Henerson et al. 1987: 136). It was also important to have a balanced representation of the factors identified (Oppenheim 1992: 181, De Vaus 1995: 56), although Henerson et al. (1987: 141) recommend that the more relevant factors be represented by more statements. This would have resulted in unwieldy questionnaires, given the number of relevant factors identified in the course of this research. For the year 8 survey I followed the advice of Oppenheim (1992) by including roughly equal proportions of positive and negative statements and some items representing extremes of attitude. Unfortunately, negative statements proved more difficult for students to cope with, especially if they wanted to disagree with them. Consequently I tried to avoid negatives in the year 9 survey and create statements with negative 
connotations by semantic means.

\subsubsection{Concurrent validity}

The overall validity of an instrument can be reinforced if the results from one measure correlate with those from another involving the same participants. The concept of concurrent validity has limited relevance for my research in that I wanted to compare parents' and their children's responses to items concerning parental attitudes towards FLL and their influence in their children's subject choices. Possible discrepancies between what parents and students reported would not necessarily invalidate responses because students might have misrepresented their parents through ignorance rather than intention.

\subsubsection{Response bias}

In any survey there may be response bias towards what participants think will please the researcher or reflect positively on themselves. Responses may vary in honesty, depending on the co-operation of respondents, their sense of personal adequacy, their sense of freedom from threat and the care they take in filling in a questionnaire (Burns 1997). In the case of school students there may be a particular risk of bias if the person administering the questionnaire resembles a figure of authority. I always encouraged students to be as honest as possible and assured them that their responses would be completely confidential, in particular that no teacher, fellow student nor parent would see their questionnaires. I also assured students that their responses would have no personal repercussions and that there were no 'right' answers. In the case of adult interviewees, it is true that all were aware of my credentials and interest in the topic, but at no stage did I sense that they felt constrained in what they could tell me.

Another type of bias may arise from a pattern of responses known as 'acquiescence'. People may consistently agree with any statement, regardless of content. According to Fowler (1995) this is mostly a problem with less educated people, which might apply to school students. One recommended method for overcoming the problem is to word about half of the items in such a way that agreeing with them will represent a negative attitude, while agreeing with the rest will represent a positive attitude, that is, varying the 'polarity' (Lett 1977, Oppenheim 1992, Scholfield 1995). Furthermore, items need to be in random order to oblige participants to give each one careful thought (Hatch \& Lazaraton 1991: 38). Participants therefore need adequate time to consider their responses (Henerson et al. 1987: 136). I took all these points into consideration in the composition and administration of the surveys. 


\subsubsection{The importance of consistency}

The validity of responses is reduced if participants do not understand statements or instructions, or if surveys are administered in an inconsistent manner (Hatch \& Lazaraton 1991: 38) . As discussed above, I carried out all the surveys and interviews and, for the students, I used a consistent set of clear, simple instructions.

\subsubsection{The placing of personal questions}

Hatch and Lazaraton (1991: 38) recommend leaving questions on personal information until last. Although there is a risk that participants may tire of filling in a questionnaire and tend to make mistakes or omit items towards the end, this is not so likely with personal questions which are generally straightforward to answer and which most people are happy to answer. I followed this advice for the year 8 survey but the last part tended to be the most carelessly tackled. As the year 9 survey was divided into sections in a slightly different manner I found it easier to give students the personal questions first. As it was the most straightforward section it possibly gave students confidence that they could cope with the rest of the questionnaire.

\subsubsection{Reliability}

\subsubsection{General considerations}

Ideally an instrument is sufficiently reliable if an independent researcher who replicated the same study or analysed the same data were to reach the same conclusions (Nunan 1992: 17, Burns: 259). As this is not feasible it is important to ensure that:

- all items are clear and unambiguous,

- there are enough items to minimise the role of chance in the responses, and

- all items measure the same thing (Lett 1977: 283).

\subsubsection{Reliability of Likert scales}

Survey statements need to lead to clear interpretation of positive and negative attitudes according to whether participants agree or disagree with them. Oppenheim (1992: 183) suggests verifying that all items can be translated into 'I like' or 'I dislike' statements, which should eliminate items that are too vague or neutral. Schuman and Presser (1981: 305) consider that the stronger the attitudes expressed, the less likelihood of random responses, hence the more reliable the survey. Gall et al. (1996: 253) also stress the need for the researcher to be confident that the results are a true reflection of attitudes, that is that the participants have been honest. Oppenheim (1992: 200) and De Vaus (1995: 55) support the notion that Likert scales designed to measure a single concept are inherently reliable. In any case, all measuring instruments need to be pretested for reliability (Henerson et al. 1987: 152). 


\subsubsection{The importance of timing}

Participants need to have sufficient awareness of the concepts being investigated in order to have an opinion to express (De Vaus 1995: 54). For this the timing of the surveys was crucial, as discussed in 4.8 above.

\subsubsection{The importance of consistency}

It is clear that any survey needs to be administered consistently for reliability as well as validity (Schuman \& Presser 1981: 305, Henerson et al. 1987: 147, De Vaus 1995: 55). De Vaus also warns of the risk of unpredictable errors resulting from distractions. I received considerable assistance from class teachers in all schools in minimising distractions.

\subsubsection{Final thoughts}

Ultimately, it is encouraging that Wray et al. (1998) claim that validity and reliability standards can be more relaxed for survey questionnaires and interviews because the collection of information is highly structured and the aim is to collect responses to build up a collective picture of the situation, not to investigate any individual cases.

\subsubsection{Ethical and practical considerations}

I approached school principals for permission to carry out investigations in their schools and I sought written consent from parents for their children's and their own participation. All information letters and consent forms for students, their parents and teachers (for samples (see Appendices 3-4) were developed in line with Victoria University's Human Ethics Guidelines in order to assure all participants of anonymity. All individuals were given the right to refuse to participate and to withdraw any information provided at any stage until the project was completed.

Since this research is only concerned with the attitudes of students whose L1 is English, I considered requesting that NESB students be withdrawn from participating classes, but this could have caused disruption to classes and supervision problems for teachers. I decided that it was practical and tactful to allow all students in a class, provided they or their parents did not object, to participate in the survey so that none felt unfairly excluded.

\subsubsection{Questionnaire form}

\subsubsection{The advantages of Likert scales}

I developed questionnaires in the form of Likert scales, "one of the most popular and widely used approaches to attitude measurement" (Lett 1977: 278) Students were_asked to read_a_series of statements and to indiconto the 
questions (Scholfield 1995: 63). Evidence suggests that the two types of questionnaire ultimately produce similar information (Gall et al. 1996: 295).

\subsubsection{Building on previous research}

The use of pre-existing attitude scales where possible is recommended by Fishbein and Ajzen (1975: 108, cited by Lett 1977: 285) and Gall et al. (1996). Although I found a number of scales with relevant items (Gardner \& Lambert 1972, Gardner 1985, Baker 1992, Coleman 1996, Schmidt, Boraie \& Kassabgy 1996) I did not identify any suitable, pre-existing scales nor any directly comparable research which used attitude scales. Burstall (1979) carried out extensive research using statements requiring simple Yes/No responses but asking respondents to express their opinions on some sort of continuum can produce more useful information (Busch 1993, Fowler 1995).

\subsubsection{Choice and wording of questionnaire items}

For constructing a scale labelled 'strongly agree', 'agree', 'disagree' and 'strongly disagree', Henerson et al. (1987), like most authorities, suggest including a mid-point, such as 'don't know' or 'uncertain'. Others advocate leaving out the mid-point (Lett 1977, Fowler 1995) so as to force respondents to make a judgement. While survey items must reflect the hypotheses to be tested and the theoretical framework for the research (Nunan 1992, Fowler 1995), they must also relate to ideas which respondents understand and are likely to have an opinion about (Busch 1993, Scholfield 1995). If they genuinely have no opinion, for lack of knowledge, there can be a separate category to mark apart from the scale items (Fowler 1995: 163). As discussed above, the timing of the student surveys and thorough piloting were designed to obviate this problem, although some year 9 students with no prior FLL and whose FL courses were only for one or two terms had not started FLL at the time of the survey. In the end I followed the advice of Henerson et al. (1987), with a mid-point labelled 'not sure' and a separate category of 'no opinion' which students were advised to select if survey items were not relevant to them.

As far as the individual questionnaire items are concerned, I initially drew up a long list of possible items, based on my own ideas or gleaned from other sources in the literature, modifying them as seemed necessary for the New Zealand context. The items were designed to test the concepts presented in my hypotheses, discussed in 2.12 , and those presented in the theoretical framework, see 2.13 .

\subsubsection{Focus group discussions}

According to Oppenheim (1992: 184) it is possible:

to develop complex attitude scales for use with school children, 
provided extensive pilot work has ensured that the children are aware of the topics of concern, and that they do have opinions about them.

I therefore attempted to identify the most appropriate concepts and wording for both the questionnaires and the instructions, to ensure maximum comprehension and reliable responses. In order to ensure that items were relevant and would be interpreted in the same way by all the students, I followed Fowler's (1995) advice and arranged discussions with small groups of students, similar to the targeted groups, so as to put them at ease, stimulate communication, and allow everyone to make a contribution, while it remained easy to keep the discussions focussed. The focus groups were organised with one unstreamed class of year 9 students in a mixed, state school, where FLL is entirely optional, during core class periods so that there was a mixture of language and non-language students, with a range of abilities. I provided the students with letters seeking written permission from their parents to participate and about half the class, eight boys and four girls, took part and I recorded the discussions for later analysis.

With a view to addressing my research questions (see 1.6) concerning what students know, believe or think about FLL, what reasons they may have for choosing to study a FL or not, and what might influence their choices, I aimed to investigate how the focus groups talked about:

- FLL, for themselves and for others;

- particular FLs they were studying or had previously studied;

- what FLL involves;

- what sort of people learn FLs, which ones and why; and

- how they chose their option subjects

Most of the students appeared willing to give honest opinions, although one or two were rather reticent. Few had much awareness of what FLL involved or why it might be valuable, although most had had some language learning experience, chiefly Maori or Japanese, at primary or intermediate school. Students seemed to have very little knowledge of, or interest in, what their peers were doing at their own school or at other schools in respect of FLL. Some believed that there is a need for Asian languages in New Zealand's business dealings.

After a general discussion about various aspects of FLL and how they had chosen their option subjects, I presented the students with a sample section of a questionnaire and some general questions, and they listened to the proposed instructions. They seemed to have no problem understanding what they needed to do. All understood the notion of confidentiality but several needed an explanation of the reasons for this, which they happily accepted. All groups favoured the inclusion of a 'not sure' alternative as well as a 'no 
opinion' column. The students made some useful suggestions for clarifying some of the general questions, and the whole process enabled me to identify questions which might be difficult for students to respond to, about their parents' FLL experience for example, and useful questions which I had omitted. In particular, I realised that I needed to ask year 8 students which secondary school they would be attending so as to follow up the same students as far as possible.

\subsection{The Year 8 questionnaire}

\subsubsection{Form and content}

After an analysis of the focus group discussions I modified the content and wording of many items and decided to have three parts for the pilot survey:

1. a Likert scale consisting of approximately 100 items;

2. a set of statements about previous FLL experience to which students could simply respond 'Yes' or 'No' and add any comment they wished; and

3. about a dozen general questions to gather personal information about each student.

I worded the Likert scale statements as clearly and simply as possible. I designed instructions to encourage students to respond as honestly as possible and assure them of the confidentiality of their responses. Although Wray et al. (1998: 294) recommend that questionnaires should begin with the simplest, non-threatening items and, if possible, 'grab the attention' of the readers so that they want to respond, it seemed better to make the final order of items entirely random to encourage careful thought about each one and avoid any pattern to the responses. As a final check for the comprehension level of the pilot survey, I asked an early childhood educator to proofread it and she made some helpful suggestions.

\subsubsection{Pilot survey}

I tested the preliminary questionnaire on year 8 students. Henerson et al.(1987: 86) suggest at least 50 respondents for this stage of piloting, although Oppenheim (1992: 195) suggests double that number. For practical purposes, I hoped that two or three classes would be sufficient, but, in the event, the intermediate school which I approached allowed all eight classes to participate. I supplied information letters and consent forms for the students and their parents which were distributed and collected by the class teachers. The eventual sample numbered $220,88 \%$ of the year 8 students in the school. Owing to the length of the pilot survey, half the classes did part 1 , while the rest did parts 2 and 3 .

I experimented by having the pilot administered by two different people, myself and a much younger university student, to half the classes each, to discover if that had any influence on the way the students responded to the questionnaire. Analysis of the results showed no noticeable differences in the way students responded which 
could be attributed to the class they were in or to the person administering the survey.

As well as pretesting the items themselves, it was important to ensure that all the instructions were clear so that no misunderstanding interfered with the student responses (Scholfield 1995, Wray et al. 1998). I thought it best at first to have the instructions written for the students but also read out, but I decided that this would add unnecessarily to the large amount of paper that the students had to cope with and that it was better to deliver the instructions orally, in as simple and informal manner as possible. I had also thought that reading aloud each item would help any students who had reading problems or whose L1 was not English (NESB), although the latter's responses were not relevant to the purposes of this research and it would not matter if their questionnaires were incomplete. In fact there were very few nonPakeha students in the classes and only one or two NESB students. Students did not seem shy about asking for help when they needed it and reading aloud all the items in part 1 of the survey with the first class proved to be much too slow and tedious for the majority of students who simply went ahead at their own speed. I began the same process with the second class but stopped and asked what the students preferred. It was clear that they wished to work at their own pace without the distraction of hearing items being read out. Each class teacher could easily identify and assist individual students where necessary. In some classes a teacher aide was present to help a special needs child.

All the students and teachers were co-operative. There were very few problems and very few survey forms were incomplete. Well over half the 118 students (64\%) who completed part 1 of the survey took the trouble to offer comments in the space provided at the end. Of the 46 negative comments, over half complained that it was repetitive, which was to be expected given the nature of the items being trialled. Five considered it too long which was also inevitable. Both problems would be largely overcome in the final version of the survey. Of the 30 positive comments, 12 thought the Likert scale was fine, especially as they just had to tick boxes. Others found it 'interesting', 'easy', 'relevant' and 'thought provoking'.

\subsubsection{Pilot survey analysis}

\subsubsection{Part 1 - Likert scale}

Of the 118 questionnaires, 6 were incomplete and a further 21 were eliminated for being inconsistent. The responses of the remaining 91 students were analysed according to the process recommended by Henerson et al. (1987). The responses were scored, 1 to 5 with 6 for 'No opinion' and the scores totalled for each student to identify the top $25 \%$ and bottom $25 \%$, that is those with the clearest favourable or unfavourable attitudes. The responses of these students (approximately 50) were then charted to identify which items most clearly discriminated between favourable and unfavourable attitudes. The majority of students tended to agree about the majority of 
items, probably owing to the apparently homogeneous background of the students. This, however made the choice of items for the final version of the survey somewhat problematic.

When deciding how many items to include in the final scale I took into account Busch's claim (1993: 735) that there is no ideal number, this being dependent on the respondents' familiarity with the ideas and their educational and socioeconomic background. Lett (1977: 280) and Henerson et al. (1987: 87) suggest that 20 items is sufficient, provided that all the variables which it is intended to measure are represented. My final selection of items was guided by the clear set of instructions provided by Henerson et al. (1987: 8788) and the advice of De Vaus (1995: 255) to check against the general pattern of pilot responses. If a particular item seems to be contradictory to this pattern, he claims, it is probably measuring something different and should be discarded. Through the process of analysis I the reduced the original 82 items to 25 .

\subsubsection{Part 2 - Yes/No/Comments}

This part of the survey, about previous or current FLL experience, contained 33 YES/NO items with space alongside each one for comments. At the beginning was a space for students to say which language they were focussing on and what they had liked most or least about FLL. At the end was space for general comments about the questionnaire.

102 students were presented with this part of the survey. Four declined to participate as they had never learnt another language at school, not even Maori. 47 students responded with respect to Maori, 46 to learning a FL. Six questionnaires were excluded from the analysis because they were ambiguous or did not make sense. The 93 usable questionnaires were first analysed by a simple manual count of YES and NO items to ascertain which items elicited the most divergent opinions. Then the 46 relating to FLL were analysed separately, by computer count. The most significant items had also shown up in the initial analysis although there were fewer of them. These 46 questionnaires contained only 6 comments on the questionnaire itself, four positive, including "Cool!" and "This was a good thorough questionnaire. Good luck with the rest of your research!!!". One expressed interest in the items about gender differences. One objected to having to write so much although they had been told that they did not have to fill in the 'Comments' column. Another suggested that some items were not relevant and others had no clear 'yes' or 'no' response.

\subsubsection{Part 3 - General questions}

The same 102 students presented with Part 2 were also given Part 3. Three indicated that they had done no L2 learning, one of which was unusable. Of 
the remainder, 53 had focussed on Maori in Part 2, although 13 had also done some FLL, and 47 had focussed on a FL in Part 2, 36 of whom had also learnt some Maori. (The questionnaire asked them to include it.) The students' answers were analysed according to their L2 focus in Part 2 and to their gender. Three students omitted to state their gender because they, like several others, forgot to turn over the page and the name of their future school gave no clue. I had to consider carefully how to avoid the problem of missed pages in the final version.

Part 3 gave a great deal of information about students' attitudes to their language learning experience and their future intentions, as well as relevant details about their family backgrounds. It also produced an enormous variety of information about the amount of time spent on L2 learning in primary schools which was not always clear but did give an indication of the lack of any consistent approach among schools. One of the most interesting findings was that the somewhat negative perceptions revealed by the teachers regarding the value of the FLL being undertaken in the school and their fear that it might put students off future learning seemed groundless. Only eight students stated that they would not be studying a language the following year although others were unsure.

Some questions caused confusion, especially those regarding choices, and had to be reworded. Students did not always understand whether questions referred to the past or future, for example. A question about penfriends was dropped as it produced very little useful information because they were almost entirely English-speaking. About one third of the students added extra comments at the end about language learning, mostly very positive. Only about a dozen commented on the questionnaire itself, mostly positively, for example:

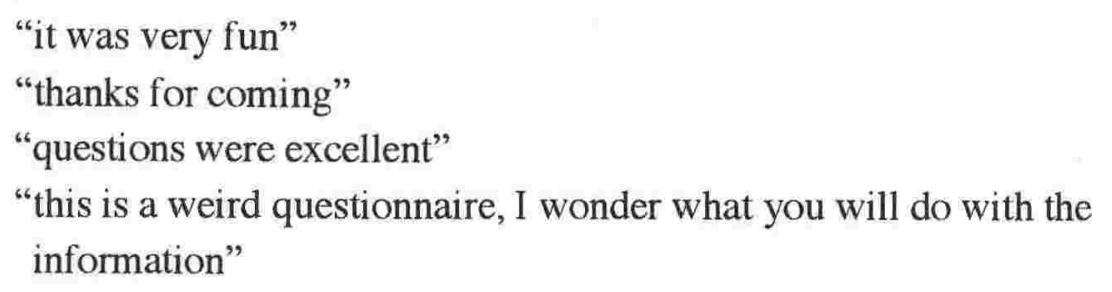

\subsubsection{Construction of the final version}

In developing the final version of the year 8 survey I took into account all the criticisms made by students about the pilot survey, and all the confusion shown in their responses. In particular, I decided to convert Part 2 into another Likert scale. When I compared the items with the variables to be investigated I found that not all were well represented by significant items, especially 'attitudes toward L2 teacher'. I therefore made some adjustments to produce a total of 15 items to form Part 2 to be presented only to those students with experience of FLL. 
In both parts of the revised Likert scale I modified the wording of some items to create the recommended balance between those where agreement represented positive or negative attitudes, and finally I put the statements into random order within each part. Although Fowler (1995) strongly recommends that the final version of any questionnaire be piloted, I had insufficient time to do this. However I presented it at a seminar in the School of Linguistics and Applied Language Studies where some useful suggestions were made which led to further minor revisions.

The final version, presented in Appendix 5, was designed to discover if year 8 students thought that:

- FLL is interesting (including interest in general FLL, specific FLs, travel, foreign people and cultures) (items ${ }^{7} 8.1 .7,8.1 .21,8.1 .24,8.2 .3,8.2 .7$, 8.2.8, 8.2.13);

- FLL is useful (for employment and transferability of language skills) (items $8.1 .2,8.1 .13,8.1 .19,8.1 .22,8.2 .4,8.2 .5,8.2 .10,8.2 .14)$;

- FLL is difficult (items 8.1.4, 8.1.6, 8.1.8, 8.2.1, 8.2.2, 8.2.11);

- FLL is more or less important than other subjects at secondary school (especially English and Maori) (items 8.1.12, 8.1.14, 8.1.17); or

- FLL is mostly for girls (because they are more interested or find FLL easier) (items 8.1.11, 8.2.16).

Questionnaire items were also designed to test the relative strength of influence on student attitudes and choices of:

- prior FLL at intermediate school (Part 2 of year 8 questionnaire, Part 1 of year 9 questionnaire, section A, questions 11-13, item 9.2.2);

- students' parents $(8.1 .10,8.1 .23,8.1 .25)$;

- school information, organisation, timetabling, subject choice, etc. (items 8.1.1, 8.1.15, 8.1.18);

- teachers (items 8.1.9, 8.1.16); and

- peers, including older siblings $(8.1 .3,8.1 .5,8.1 .20)$.

As can be seen from the above details, in all cases, an attempt was made to test each concept by means of several questions and/or statements. In addition to the above, in part 3 of the year 8 survey students were asked open-ended questions about:

- their family background;

- their parents' experience of FLL;

- their contacts with speakers of LOTEs in New Zealand or overseas; and

- their experience of overseas travel.

All students were asked why they were, or were not, studying a FL, what they thought about the idea, what they thought about particular FLs and what their intentions were in regard to FLL for the following year. In order to refine the questionnaires, to ensure relevance and ease of understanding and responding for all students, the concepts were discussed by student focus groups and pilot surveys were carried out, as discussed below.

\footnotetext{
${ }^{7}$ Item numbers refers to questionnaire documents in Appendices 5 and 6. For example, 8.1.1 refers to the Year 8 questionnaire, part 1, statement 1.
} 


\subsubsection{Administration of the year 8 survey}

I made preliminary visits to the schools at the end of August 1999 to meet the contact person arranged by correspondence and to deliver the information letters and consent forms for students and parents which the contact person undertook to distribute. We made arrangements for the surveys to take place at the end of term 3 or early in term 4, whichever was more convenient for the school. Survey visits took one or two days according to the size of the school.

All three schools agreed to allow all year 8 students who were at school and who agreed to participate, and whose parents had not declined permission, to be surveyed. In each case I arrived to find a timetable organised for class surveys and class teachers expecting me. The visits generally proceeded very smoothly and all the teachers seemed happy for time to be devoted to the survey, usually 20 to 30 minutes. The primary school was particularly pleased to be involved because they were not used to being included in such projects. My senior teacher contact reported that the students were pleased that someone was interested in their opinions. In each class students were reminded that they did not have to take part in the survey if they did not wish to, and some did decline. I did my best to encourage all participants to complete the questionnaires in a satisfactory manner and most were extremely cooperative, appearing to take the survey seriously and provide honest opinions. I told students to ask for assistance if they were not sure how to complete the forms but there were very few questions, and in most cases class teachers kept an eye on students they knew to have difficulty reading and understanding, and discreetly helped them where necessary.

Several problems became apparent as the first surveys were carried out. One of the questions in part 3 needed a 'Don't know' option and I advised students to write that in if 'Yes' or 'No' were inappropriate. The failure to provide a space for students' names on the first two sheets proved problematic as not all students remembered to name all their sheets, despite frequent requests to do so. Many students were obviously used to putting only their first names on work and so I had to request their full names. As I collected the questionnaires I tried to glance through them to check that they were complete because students tended to miss one particular side (only 4 items). I had to provide extra guidance for Part 3, where alternative routes through the questions depending on YES or NO responses, which was not always clear to the students.

As each class finished I asked their teacher if I could offer sweets as a small 'thank you'. This was welcomed by all the students and most of the teachers too. On finishing each visit I thanked the contact teacher for their assistance and presented them a book token for the school library. At the year 7-13 school the contact teacher offered to organise the following visit for the year 9 survey, an offer which I gratefully accepted. 


\subsection{The year 9 questionnaire}

\subsubsection{Form and content}

In the interests of consistency, and mindful that this survey was intended as a followup to the year 8 survey, I kept the format and content of the year 9 questionnaire as close as possible to the previous one described in 4.10.4, allowing for amendments needed for the change of context. I made other changes in the light of the experience of administering the year 8 survey and difficulties which showed up as the year 8 responses were analysed.

The questionnaire was divided into four parts. The first part of the Likert scale, similar to the Part 1 of the year 8 questionnaire, was for all students. Two versions of the second part were constructed for students who were or were not studying a FL at any time in year 9. The general questions were similar to those in the year 8 questionnaire but I decided to administer this first so that both parts designed for all students would be tackled together before I distributed the appropriate Part 3 or 4 to individuals.

The questionnaire, presented in Appendix 6, was designed to discover if year 9 students thought that:

- FLL is interesting (including interest in general FLL, specific FLs, travel, foreign people and cultures) (items 9.2.2, 9.3.2, 9.3.6, 9.3.9, 9.3.10, 9.3.12, 9.3.14, 9.3.15);

- FLL is useful (for employment and transferability of language skills) (items $9.2 .4,9.2 .6,9.2 .7,9.2 .8,9.2 .11,9.2 .15,9.2 .19,9.3 .1,9.3 .5,9.3 .16$, 9.3.18);

- FLL is difficult (items 9.2.1, 9.2.12, 9.3.4, 9.3.8);

- FLL is more or less important than other subjects at secondary school (especially English and Maori) (items 9.2.14, 9.2.20); or

- FLL is mostly for girls (because they are more interested or find FLL easier) (items 9.2.9, 9.2.17).

Questionnaire items were also designed to test the relative strength of influence on student attitudes and choices of:

- prior FLL at intermediate school (Part 2 of year 8 questionnaire, Part 1 of year 9 questionnaire, section $\mathrm{A}$, questions 11-13, item 9.2.2);

- students' parents (items 9.2.3, 9.2.13, 9.2.18, 9.3.3, 9.3.11);

- school information, organisation, timetabling, subject choice, etc. (items 9.2.5, 9.2.16, 9.3.13); and

- peers, including older siblings (9.2.10, 9.3.7, 9.3.17).

As in the case of the year 8 questionnaire, an attempt was made to test each concept by means of several questions and/or statements. In addition to the above, in part 1 of the year 9 survey, students were asked open-ended questions about:

- their family background;

- their parents' experience of FLL;

- their contacts with speakers of LOTEs in New Zealand or overseas; and 
- their experience of overseas travel.

As in the year 8 survey, all students were asked general questions about why they were, or were not, studying a FL, their general beliefs and feelings about FLL and particular FLs and their intentions in regard to FLL for the following year. The year 9 survey was also piloted as described below.

\subsubsection{Pilot survey}

I arranged a small scale pilot at a large co-educational high school in the Wellington area at the end of term 4 1999. Fourteen students in one unstreamed year 9 class, including some FL students, agreed to complete the survey with their parents' permission. My main aim was to ensure that students understood the procedure and all the items, and that they felt free to seek clarification if necessary. The survey took about half an hour to administer, as estimated. After completion I held a brief, informal discussion with two of the girls, selected by the form teacher as representing different ability levels, to probe further whether there were ambiguities or other possible areas of misunderstanding.

\subsubsection{Pilot survey analysis and construction of the final version}

There were very few incomplete questionnaires and only one student had trouble following instructions in part 4 (because of the timing of the survey). Questions from students during the process and the following discussions proved very helpful in identifying problems. It was evident that I needed to make it clearer that the survey referred only to FLs, not English or Maori. I decided to specify which languages were relevant for each school being surveyed. I also needed to clarify that Part 3 was for all students studying a language in year 9, either at the time of the survey or later in the same year if the school being surveyed had short option or module subjects. Students needed reminding to check that items had not been omitted, that responses were on the right line and that any changes they made were clear. I subsequently made minor adjustments to the questionnaires and my administration checklist. The final version is given in Appendix 6.

\subsubsection{Administration of the year 9 survey}

I contacted secondary schools by fax early in Term 1, 2000, to ascertain when initial visits would be convenient for the delivery of information letters and consent forms. I asked whether it would be possible to survey all the year 9 classes, and how many students would be involved. For the purposes of printing, I made a rough calculation of student numbers based on MoE figures for previous years.

As schools responded I made detailed arrangements for each visit according to what was convenient for each school. As mentioned above, the visit to the 7-13 school was organised by the same contact as in 1999. At the other schools the either the principal or the HOD languages made the arrangements. The surveys took place between the last week of February and the first week of April. In four schools I was able to survey each year 9 class in turn, but the boys' school took a different 
approach. Only boys who had returned the consent forms were allowed to participate, all together in a large lecture theatre. This meant that the supervising teacher and I were unable to ensure that students were following instructions or assist individuals in the same way as possible during class surveys. Apart from that instance, students and teachers were extremely cooperative and the visits went very smoothly. I was able to foresee and avoid most potential problems.

\subsection{Interviews}

\subsubsection{General considerations}

There are advantages and disadvantages to interviewing (Bailey 1987: 174-175), compared with other methods of obtaining information and opinions. Interviews can be more complex and flexible, allowing for probing and the appropriate selection and ordering of initial and follow-up questions. They also allow the interviewer to observe non-verbal behaviour. Interviews usually obtain good response rates, with more spontaneous and complete answers, but they may take more time and be less convenient. Interviewees may feel obliged to co-operate, or less convinced of the confidentiality of the process. Flexibility can lead to a less standard wording and ordering of questions, and make analysis and comparison of respondents more difficult, or even less valid.

While preparing the interview questions and process I took into account the same factors as those discussed above with regard to the student surveys. I hoped that interviews arranged at a convenient time and place for each interviewee, with minimum risk of disruption or distraction, would elicit maximum co-operation from both parents and teachers. I intended to achieve consistency in the administration of the interviews by carrying them all out myself, although I predicted that I might have to reframe questions, or supply varying amounts of background information, according to the knowledge and experience of the participants. I realised that some participants might not feel able to answer all the questions. I anticipated that parents with limited education and little or no contact with FLs, might have given little thought to the matters under investigation prior to participating, but I wanted to ensure all participants that their responses were relevant and valuable for my research. I assured them all, in writing and orally before the interviews, that their response would remain confidential.

\subsubsection{The problem of interviewer bias}

Depending on circumstances, interviewees may be unduly influenced by an interviewer because of, for example, age, gender, dress or accent (De Vaus 1995). I presumed that where teachers were interviewed by another teacher, myself, such factors would be minimised, although they might influence parents, depending on their background. It is preferable that respondents perceive an interviewer to be as neutral as possible about the topics under discussion in order to elicit the fullest and frankest responses, but Nunan (1992) and Burns (1997) recommend giving a brief explanation of the purpose of any research and answering questions that may arise 
about it.

I anticipated that I could not appear entirely neutral, given the background information I supplied to all potential participants, but I intended to be as informal and impartial as possible in reacting to interviewees' responses in order to encourage them to speak freely. I asked all participants for permission to tape record their responses in order to avoid any suggestion that, being biased, I might misrecord their responses.

\subsubsection{Development of interview questions and procedures}

Structured interviews, with a set list of questions to be followed in a set order, ensure that all respondents are asked the same questions, and permit easier analysis, consistency and comparability (Nunan 1992: 149-51). However, I needed interviews to be sufficiently open-ended for a wide range of teachers and parents, whose personal experience of FLL, and connections with students involved in such learning would vary considerably. Therefore the interview schedules took a semistructured form. Questions were developed for parents of year 9 students and teachers, designed to elicit the adults' beliefs and feelings about all aspects of FLL:

- for New Zealand school students in general;

- in the schools with which they were involved; and

- (in the case of the parents) for their own children.

In preparation for the interviews I also followed the suggestion (Nunan 1992, Burns 1997) to prepare a checklist of questions so that I could keep track of what had been covered without adhering to a rigid question order. This also allowed me to note additional non-verbal information or intrusive environmental factors to supplement the tape recordings. As an inexperienced interviewer, I found particularly helpful advice in Burns (1997) for putting interviewees at their ease, keeping an interview going smoothly, encouraging elaboration of responses and showing appreciation for the participants' time and effort.

\subsubsection{Pilot interviews}

It is essential that interviewees understand questions in the same way as an interviewer does, and that potential confusion or ambiguity is eliminated through pretesting (Nunan 1992, Fowler 1995). Initially, I developed a range of possible interview questions, principally aimed at teachers, and tried them out informally with two teachers. I interviewed a FL teacher and then a non-language teacher interviewed me. In this way a considerable amount of useful discussion arose about the nature of the questions and how satisfactory it was both to ask and answer them. Subsequently, I substantially revised the question wording and ordering. In addition, following Burns' (1997) recommendations to start with specific, simple questions which all respondents could answer, I constructed a short, preliminary written questionnaire which teachers could complete in advance and which could then serve as a starting point for each interview. This sought information about each 
teacher's position in the school, personal language learning experiences and general opinions about the place of LOTE learning in New Zealand schools.

For all the adult interviewees I also produced a background questionnaire to obtain factual details of age, gender, country of birth, L1 and L2 learning experiences, school leaving age, educational qualifications and current employment, see Appendix 10 .

I piloted the resulting interview schedule with a selection of secondary school teachers, and the use of the preliminary questions and answers proved helpful. I invited interviewees to comment on all aspects of the interview, and considered all their comments, which were entirely favourable, when developing the final interview questions and procedures. I did not record the pilot interviews and this highlighted the difficulties of trying to make notes while keeping the interviews flowing and on track.

As recommended by De Vaus (1995), I piloted the interview questionnaire a third time with four intermediate school teachers in order to ensure that the questions were appropriate for teachers at this level. The process provided me an insight into the general attitudes among staff and aspects of the school organisation relating to FLL which might affect student attitudes and choices. It appeared that students, parents and teachers were not necessarily receiving the same messages about why primary or intermediate schools were offering FLL or how much such learning was really valued. Lastly, the pilot interviews provided an efficient guide to the time required, about half an hour for each teacher.

Ideally, questionnaires should be retested if they are to be used with different types of people (De Vaus 1995), but this was not done for the parent interviews. Since some of the teachers involved in piloting were also parents they were able to comment from a parent's point of view. All the questions were carefully examined for their relevance to parents and were either eliminated or adapted as necessary. In particular, questions relating to curriculum, timetabling and option systems, which were clear to teachers, needed careful introduction to avoid problems for parents. In the end, two versions of the parent questionnaire were designed, depending on whether FLL was optional or compulsory at their child's secondary school (see Appendix 9B). The areas focussed on were:

- how their child's option subjects had been chosen;

- how the decision to study a FL or not was arrived at, and what they and their child felt about this;

- parents' experience of learning or using LOTEs in New Zealand or overseas; and

- parents' opinions about the place of FLL at their child's school and in New Zealand schools in general. 
Similarly, there are two versions of the teachers' questionnaire, one for teachers of year 7 and 8 students (see Appendix 7) and the other for secondary teachers (see Appendix 8). The latter includes a separate section designed solely for teachers in charge of FLL in their schools. Teachers were asked about :

- their own experiences of FLL;

- their knowledge of and opinions about FLL in their own school;

- their thoughts about FLL in New Zealand schools in general; and

- their beliefs about the attitudes towards FLL of colleagues, students and their parents.

\subsubsection{Administration of teacher interviews}

At the intermediate school most of the interviews went smoothly, with few interruptions from students or telephones, and all the teachers were generous with their time and their comments. At the Catholic school all the staff approached were extremely co-operative and most expressed an interest in knowing the results of the survey. At the primary school the senior staff members were very happy to cooperate and eager to know the results of the investigations. The other two teachers, while not reluctant to talk to me, showed little knowledge of or interest in the topic of FLL. Only the Principal seemed aware of the SLLP although the latest Gazette in the staffroom contained a new notice inviting schools to apply for support.

At the secondary schools all the teachers approached were more than willing to be interviewed, although some could spare only a little time. One or two were surprised to be approached because they had nothing to do with the FL programme or year 9 students, but nonetheless had plenty to say about the matter. I was able to conduct most interviews in a quiet, private situation but some took place in the staffroom, which did not seem to inhibit the participants.

Teachers often had not had the time to fill in the consent forms and preliminary questionnaires, and in some cases had mislaid them. Fortunately I had foreseen the possibility of this and supplied new forms at the start of the interviews. All the teachers except one agreed to be recorded, which made the interview process much easier than if I had had to make extensive notes. It proved very useful to have a checklist of the questions to keep track of what had been covered and to note additional questions which arose during the conversations.

\subsubsection{Administration of parent interviews}

The interviews took place over two separate periods of 3-4 days. At the last moment, three parents were unavailable. One mother, a teacher, was ill but gave some opinions over the telephone and sent me written responses to the interview topics. Of the remaining two, one forgot and was not at home at the arranged time. She was most embarrassed but was out of town for the rest of the available time. The other did not respond to several phone messages, possibly because a relative was in hospital. I sent written questions to both but received no responses. 
I interviewed in person the parents of 27 students: three couples, one solo father and 24 mothers. In a few cases male partners were hovering in the background or arrived during the interview and added some comments. Interviewees were happy to be recorded and fill in the background information sheets. Most of the interviews took place in homes in quiet circumstances. The reading room of the public library first thing in the morning proved to be a particularly noisy place for one interview!

\subsubsection{Interviews - final thoughts}

Although all the interviewees were aware of my background, or enquired about it during the course of the interviews, this did not seem to inhibit the expression of their opinions about the place of FLL, either generally in New Zealand schools or for their own children or students. Most interviewees appeared relaxed and were very generous with their time.

\subsection{Data Analysis}

As far as possible I number coded the data collected from students and used spreadsheets to calculate simple descriptive statistics. With regard to the Likert scale items, in most cases only small numbers of students selected the 'strongly agree' or strongly disagree' options. For the purposes of the analysis such responses have been counted with the 'agree' and 'disagree' responses respectively because this research was designed to obtain a broad picture of student attitudes rather than test the relative strength of these attitudes. Where particularly large numbers of students revealed strong views either way these have been discussed separately.

Some of the student survey questions produced a wide range of opinions held by small numbers of participants. It seemed more reasonable to summarise this qualitative data on spreadsheets and carry out manual calculations to establish relevant statistics. In some cases I summarised student opinions under broad relevant headings. Later, following the advice of statistical experts, I transferred all the quantitative data to SPSS spreadsheets in order to cross tabulate various responses, largely to investigate which variables appeared to have the greatest influence on year 8 student intentions and year 9 student choices regarding FLL. Wherever possible, student responses were also cross tabulated with gender. All percentages relating to student responses are based on the number of students who expressed an opinion, unless otherwise specified, and the percentages have been rounded to the nearest whole number. Where students omitted items, probably through carelessness, they were counted as having no opinion. All cross tabulations were tested for statistical significance using a chi-square test and the results presented as recommended by the experts I consulted.

All the interviews were recorded on cassettes. Initially, the intention was to transcribe these but in the end it was agreed that this would not be necessary. Spreadsheets were therefore created for the two groups of interviewees and their responses and comments abbreviated to some extent. However, many of their own words and phrases were recorded, especially where they provided particularly useful or colourful descriptions of the adults' beliefs or feelings. The parent data was summarised under the following headings: 
- name

- relationship to student

- gender of student

- student's school

- whether language learning was compulsory at the school

- what, if any FL the student was studying or going to study during year 9

- when and where the interview took place

- where the parent (s) were born and, if not in New Zealand, the age at which they came to the country

- the $\mathrm{Ll}$ of both parents

- the gender and age group of the parent being interviewed

- school leaving age of the parent(s) and their highest educational qualifications

- their current employment

- how the student's option choices had been made

- how the decision about FLL was arrived at in the family

- the student's previous FLL experience

- the student's travel experience

- FLL progress being made by the student

- whether the student would continue FLL in year 10

- the student's future aims for work or travel

- the possible influence of siblings or peers

- the family's contacts with NESB people

- FLL experience of the parents

- parents' travel or business contacts overseas

- main reason for student's FLL

- whether student would have preferred another FL

- whether the parent(s) would have preferred another FL or another subject altogether

- the place of FLL in the student's school

- the place of FLL in New Zealand schools in general

- the value of FLL

- whether New Zealand produces enough speakers of FLs

- other comments

The responses of teachers were recorded under the following headings:

- school

- gender

- how long they had worked in the school

- what classes they taught

- their L1

- their FLL experience

- reasons for having learnt a FL or not

- whether they had enjoyed their FLL

- what they thought they had gained from FLL

- their subsequent feelings about usefulness of FLs 
- their subsequent feelings about their FLL efforts

- any FL teaching experience

- how important they thought FLL is now for school students

- reasons for this rating

- what FLs New Zealand students should learn

- reasons for these preferences

- the best starting age for FLL

- whether FLL should be optional or compulsory

- how much FLL students should have

- what sort of students tend to choose FLL at secondary school

- the place/history of FLL at their own school

- the good and bad points of FLL at their own school

- the views of teachers, student and parents about such FLL

- any problems of teacher supply for FL classes

- the ideal place of FLL in New Zealand schools

- other comments

In addition, secondary school teachers were asked about what advice was given to students about FLL, either prior to entering Year 9 or at any later stage.

The results of the year 8 student survey are presented in the following chapter. Chapter 6 presents the findings of the year 9 student survey. Chapter 7 examines the sub-sample of students who participated in both surveys and presents a comparison between their intentions for FLL for their first year of secondary school and the decisions they actually made in this regard. Chapters 8 and 9 respectively present the findings of the parent and teacher interviews, while chapter 10 examines the role of gender in attitudes towards FLL. The final chapter contains a detailed discussion of the findings in relation to the hypotheses presented in 2.12 and sums up the state of play of FLL in New Zealand schools current at the time of writing. The major barriers to FLL are identified and suggestions made for further research 


\section{CHAPTER 5 RESULTS AND DISCUSSION - YEAR 8 STUDENT ATTITUDES TO FOREIGN LANGUAGE LEARNING AND INTENTIONS FOR YEAR 9}

Whatever the method, only desire can make a student learn a language, desire and necessity. (Kaplan 1993: 131)

\subsection{Introduction}

This part of my research aimed to answer the following questions:

- What did year 8 students know/believe/think about FLL?

- Did they intend to study or continue studying a FL in year 9?

- What were the main reasons for their intentions?

The results of the survey of year 8 students produced two major surprises and substantial evidence to support some of my hypotheses. The first surprise was the amount of FLL built into the curriculum of two of the three surveyed schools. This was probably not typical of the area since, as far as I could ascertain, the other two intermediate schools in the city had FLL only as an optional extra. The second surprise was how positive were the attitudes of students and teachers towards FLL. I analysed all responses to individual survey items in relation to students' stated intentions for year 9 in order to identify those attitudinal factors which were most closely associated with their intentions. I also examined the results for gender differences, which will be discussed in detail in Chapter 10. Unless otherwise stated, all responses to the Likert scale items have been grouped so that 'agreed' includes 'strongly agree' and 'disagree' includes 'strongly disagree'.

In order to establish a clear background to the student responses, I first present profiles of the schools, (for full details of ethnic and gender balance of students and school decile ratings see Appendix 2), followed by some basic facts about the students and their FLL experience. Then I examine the general attitudes of all students to FLL and the attitudes of those with FLL experience to that experience. Finally I investigate students' intentions regarding FLL in year 9, the reasons given for their intentions and the possible influence of a range of factors on these intentions.

\subsection{School Profiles}

\subsubsection{Aster}

This was a semi-rural, full primary school, decile 8, with 333 students in 1999, of whom 45 were in year 8 in three composite classes (i.e. mixed year 7 and 8 students). Children were from a variety of backgrounds, including university employees, commuters to the nearby city and farming families. In 1999 there was no formal language teaching, but a system of optional club activities was instigated for an hour a week in term two for year 7 and 8 students which included Spanish. Between 20 and 30 students had opted for Spanish but only a dozen could be accommodated, including 6 involved in my survey. The school hoped to repeat the clubs in term 4, and give more students the opportunity of learning some Spanish. 


\subsubsection{Rose}

This was a large intermediate school with approximately 225 year 8 students in eight whole classes and two composite classes in 1999. All classes were of mixed ability. The school considers itself multicultural and approximately $8 \%$ of the students surveyed were from homes where a LOTEM was spoken. The school considered its programmes student-centred and aimed to stimulate success through "challenges, adventures and diversity" (year 2000 prospectus), including offering FLL. While it was not possible to interview the principal, several of his staff reported that he was keen for the school to offer something different from its rivals to attract students (some referred to FLL as a "marketing tool"). In 1997, the school had started "outof-school classes' in German, French, Japanese and Spanish, one hour a week, open to all but for a fee. Over 60 students were involved in that year. In mid 1998 the school became part of the MoE's SLLP, teaching Spanish to two classes for one hour a week.

In 1999 Spanish was being taught by two enthusiastic senior teachers going into two classes each. Japanese was also being taught by class teachers with support from a Japanese teacher from the neighbouring secondary school. The school continued to offer the possibility of learning French and German outside normal class time, for a fee. Half a dozen students were studying French but there were not enough takers to warrant the employment of a German tutor. The school hoped to offer French and German for whole classes the following year if the courses promised by the MoE were ready, and at least one teacher with had expressed interest in teaching it to her class.

Students did not have much choice about FLL, nor which language they could study, except for the after class French lessons, as arrangements were made on the practical basis of which teachers were available and willing to teach which language. Student comments on their survey forms indicated that some teachers had asked their classes what they thought about learning a FL and may even have offered them the choice of Spanish or Japanese. In 1999, two of the year 8 classes were not learning a FL although some of the students had had FLL experience in the previous year.

\subsubsection{Salvia}

This was an integrated Catholic school, year 7 - 13 with a total of 885 students in 1999. There were 143 year 8 students in 1999, in four whole classes and one composite intermediate class - the bilingual Maori class. According to the year 8 survey responses, about $70 \%$ were expecting to remain at the school for their secondary education.

The school had a module programme for year 7 and 8 students, including languages. In 1999, year 7 students had a term of French and year 8 students a term of Japanese, both of which were optional subjects from year 9 to 13. The module programme was in its third year in 1999 and had been started for a variety of 
reasons. It took advantage of the presence of specialist teachers and facilities in the senior school and of fered the intermediate students a wider range of experiences than that available in some other intermediate or primary schools. To some extent this was a PR strategy to help attract families to the school. It also allowed students staying at the school to make more informed choices about FLL when they reached year 9 as they would know the FL teachers and understand what was involved in FLL. This type of situation may be a disadvantage for a department which teaches optional subjects if students have taken a dislike to, for example, the only French teacher. On the other hand it gives language teachers the chance to 'win' students for future classes. The latest available ERO report was particularly complimentary about the intermediate department: "Students have a clear sense of direction and purpose in their learning." (ERO 1997: 8)

All the modules were part of the intermediate programme although the word 'compulsory' was not used. One teacher indicated that, if there were objections from parents, a student could be withdrawn but this had not happened. However, it emerged from the student survey that the year 8 bilingual students, doing Japanese in 1999, had learnt Maori instead of French in 1998.

\subsection{The year 8 sample}

\subsubsection{Numbers surveyed}

330 students took part in the survey which produced 308 useful sets of responses, representing about $93 \%$ of those surveyed and nearly $76 \%$ of the year group in the three schools in 1999, see Table 5.1. 22 survey forms were discarded, four because they were incomplete or extremely inconsistent, the rest because English was not the $\mathrm{L} 1$ of the students or their parents.

Table 5.1 Number of useful survey forms by school and gender

\begin{tabular}{|l|c|c|c|c|c|}
\hline School & Girls & Boys & Totals & $\begin{array}{c}\text { Total 1999 } \\
\text { cohort* }\end{array}$ & $\begin{array}{c}\text { \% of 1999 } \\
\text { cohort }\end{array}$ \\
\hline Aster & 19 & 18 & 37 & 47 & $79 \%$ \\
\hline Rose & 69 & 83 & 152 & 217 & $70 \%$ \\
\hline Salvia & 63 & 56 & 119 & 143 & $83 \%$ \\
\hline Totals & 151 & 157 & 308 & 407 & $76 \%$ \\
\hline * 1 March 1999 figures from MoE
\end{tabular}

\subsubsection{Intermediate FLL experience}

The numbers of students involved in FLL during either year at intermediate level are shown in Table 5.2. That the figures are not typical for the district became clear when the year 9 students surveyed in the following year responded to a question 
about prior FLL. That much larger sample of 664 , including students from all three intermediate schools in the city and many different primary schools, rural and urban, showed that only $51 \%$ of students had had such experience.

Table 5.2 Students with FLL experience in years 7 or 8, 1998-99

\begin{tabular}{|l|c|c|c|}
\hline Y8 school & $\begin{array}{c}\text { Students } \\
\text { surveyed N }\end{array}$ & $\begin{array}{c}\text { No. with } \\
\text { FLL }\end{array}$ & $\begin{array}{c}\text { Proportion } \\
\text { with FLL }\end{array}$ \\
\hline Aster & 37 & 6 & $16 \%$ \\
\hline Rose & 152 & 126 & $83 \%$ \\
\hline Salvia & 119 & 118 & $99 \%$ \\
\hline Totals & 308 & 250 & $81 \%$ \\
\hline
\end{tabular}

Table 5.3 shows the languages which students had studied. Most students did not have a choice of language.

Table 5.3 Languages studied in years 7 or 8

\begin{tabular}{|l|c|c|c|c|}
\hline Y8 school & French & German & Spanish & Japanese \\
\hline Aster & $1^{*}$ & 0 & $5^{*}$ & 0 \\
\hline Rose & $6 *$ & $2 *$ & 87 & 57 \\
\hline Salvia & 96 & 0 & 0 & 91 \\
\hline Totals & 103 & 2 & 92 & 148 \\
\hline $\begin{array}{l}\text { Proportion of all } \\
\text { students }\end{array}$ & $33 \%$ & $1 \%$ & $30 \%$ & $48 \%$ \\
\hline * Denotes optional language learning \\
\hline
\end{tabular}

Table 5.4 Numbers of students who studied more than one FL

\begin{tabular}{|l|c|}
\hline Y8 School & Total \\
\hline Rose & 23 \\
\hline Salvia & 70 \\
\hline Totals & 93 \\
\hline $\begin{array}{l}\text { Proportion of all } \\
\text { students }\end{array}$ & $30 \%$ \\
\hline
\end{tabular}


What is perhaps even more surprising than the number of students who had already learnt a FL was the number who had had the opportunity of learning more than one, shown in Table 5.4. As explained above, the study of French and Japanese was built into the intermediate programme for most students at Salvia.

\subsection{General attitudes towards FLL}

\subsubsection{Student comments on the opportunity to learn a FL}

Since the majority of students surveyed already had some experience of FLL during year 7 or 8 their opinions about the value of FLL for New Zealand students must be coloured by their experience. Also, given that most students did not have a choice about studying a FL or which FL they might study, it is not relevant at this point to discuss the hypotheses which relate to the reasons students may have for choosing or not choosing FLL, or for choosing a specific language. The few students at Aster who had opted to learn Spanish as their 'club' activity did so equally out of general interest in SLL and an interest in Spanish. A few students at the other schools offered comments about the opportunity for SLL, which throw some light on their attitudes, see Table 5.5.

Table 5.5 Year 8 comments on the chance to study a FL at intermediate school

\begin{tabular}{|l|c|}
\hline Comments & $\begin{array}{c}\text { Number of } \\
\text { mentions }\end{array}$ \\
\hline General interest, something different & 15 \\
\hline Sounded cool, fun & 11 \\
\hline Interest in specific FL & 7 \\
\hline Useful for travel, jobs & 7 \\
\hline
\end{tabular}

Nine items in part one of the survey, which all students completed, were designed to ascertain their general attitudes towards FLL. Three items related to the perceived difficulty of FLL and whether it was associated with more intelligent students. Three items dealt with the relative importance of FLs compared with English, Maori and other subjects, and three required students to consider the importance of LOTEMs for jobs.

\subsubsection{Beliefs about the difficulty of FLL}

In relation to item 8.1.4, "People think you're clever if you can speak another language", students were fairly evenly divided in their opinions. $37 \%$ agreed while $40 \%$ disagreed $(\mathrm{N}=300)$. Item 8.1.8, "Knowing more than one language is confusing", drew much more disagreement, $48 \%$, as against $35 \%$ in agreement $(\mathrm{N}=$ 301). Lastly, students were asked to respond to a more personal item concerning confidence in their own ability to cope with more FLL. The majority were uncertain, 
although some may have been reluctant to appear boastful about their ability. Evidence of how easy or difficult students found FLL at intermediate school will be discussed in 5.5.1.

\subsubsection{The relative importance of FLL}

Nearly one third of students (93) disagreed with item 8.1.17, "There are lots more important things to learn than languages", but 128 (43\%) agreed, suggesting that FLL is low on their list of priorities, which may be a key reason why more students in general do not opt for FLL at secondary school. When asked to consider if it were better for them to spend more time on their English than on FLL, 125 (42\%) of respondents disagreed, and again there was a significant association $(p<.001)$ between the responses to this item and student intentions for year 9, see Graph 5.1. Only $26 \%$ of students agreed with item 8.1.14, "Learning Maori is more important than a foreign language in New Zealand", while $46 \%$ disagreed $(\mathrm{N}=300$ ). Responses to this item were significantly associated $(\mathrm{p}<.002)$ with intentions regarding FLL for year 9.

Graph 5.1 Responses to item 8.1.12: "It's better for me to spend time doing more English than learning another language", compared with intentions for year 9

(For association between item responses and intentions $\mathrm{p}<.001$ )

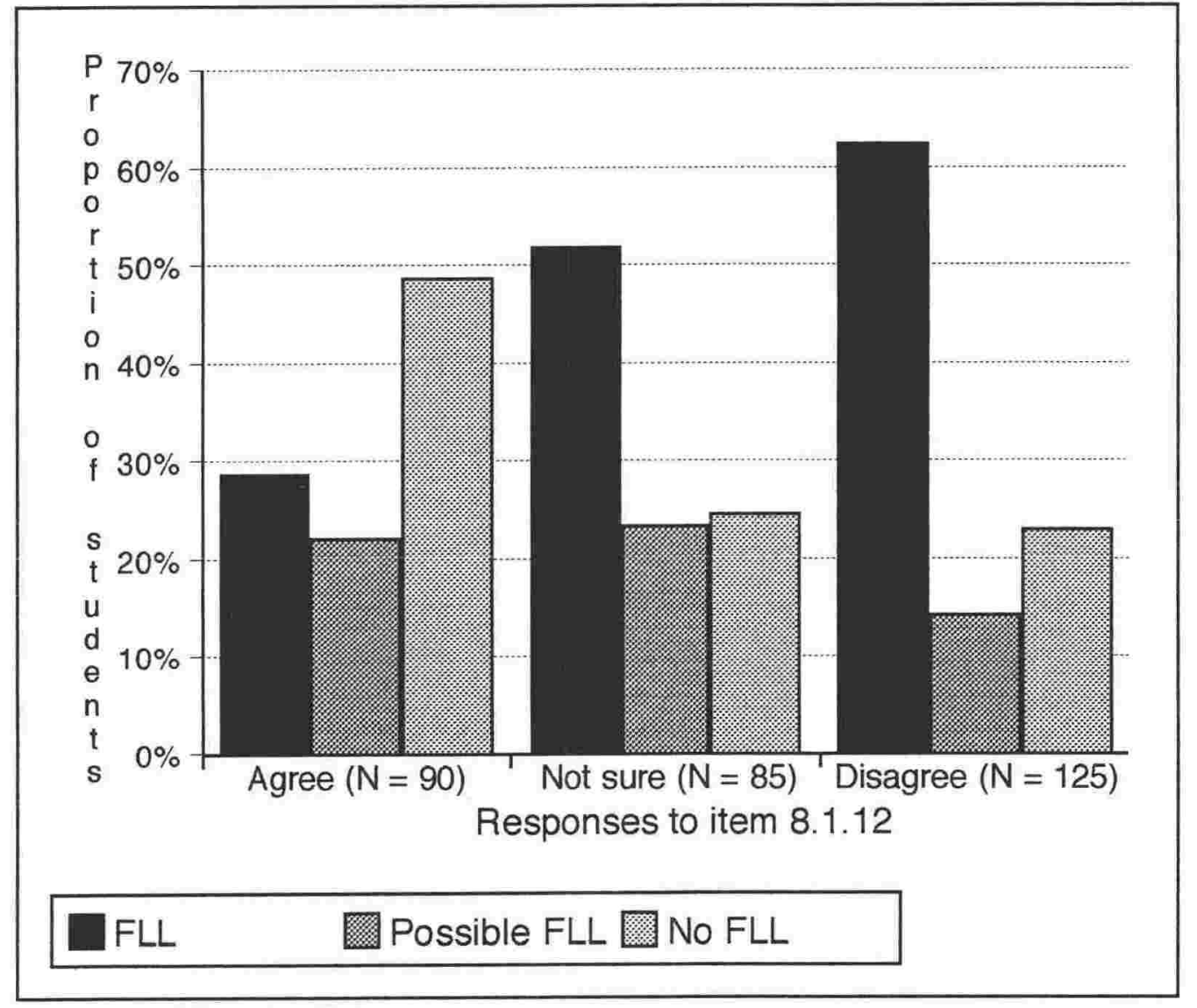


The majority of the 138 students who believed in the greater value of FLL, 59\%, were intending to study a FL, but so were one third of the 26 students who gave Maori higher priority. This suggests that they had a general interest in language learning, but it would be difficult for such students to study both Maori and a FL, given timetable constraints in most secondary schools, just as it is of ten difficult or impossible to study more than one FL.

\subsubsection{Beliefs about the usefulness of FLL}

The majority of students, $60 \%(\mathrm{~N}=302)$, disagreed with item 8.1.13, "Everybody speaks English these days", including one of the largest proportions of "strongly disagree', over $23 \%$, of all the survey items. A similar majority of students, $56 \%$, thought that knowing another language would help them get a good job (item 8.1.19, $\mathrm{N}=300$ ). This similarly included a particularly large proportion, $19 \%$, who strongly agreed with the statement. A small majority of students, $51 \%$, agreed that many jobs involve working with NESB people (item 8.1.22, N = 297). Student comments on the usefulness of FLL included:

"It's fun to talk in a different language because you might get the chance to visit that country..."

"It's a good thing to take if you want to get into tourism."

"[Languages] can get you somewhere in life."

"I think languages are helpful if you go overseas..."

"It is fun and is good for you because you can go and visit other places or you can teach it."

Table 5.6 FLs students would most like to learn, if they had the opportunity

\begin{tabular}{|l|c|}
\hline Languages cited & No. of students \\
\hline French & 55 \\
\hline German & 17 \\
\hline Spanish & 45 \\
\hline Chinese & 9 \\
\hline Japanese & 75 \\
\hline Other European* & 26 \\
\hline Other Asian $\dagger$ & 4 \\
\hline Pacific Island\# & 6 \\
\hline African $\diamond$ & 4 \\
\hline * Most often: Dutch, Italian, Irish, Russian \\
$\dagger \begin{array}{l}\dagger \text { Filipino, Indian, Korean \& Taiwanese } \\
\text { \# Tongan (3), Fijian, Niuean \& Samoan } \\
\diamond \text { Egyptian, Afrikaans }\end{array}$ \\
\hline
\end{tabular}




\subsubsection{Ultimate interest in FLL}

All students were asked what language they would most like to learn if they could learn any language at all. Table 5.6 shows the wide range of languages which were chosen. Most students, however, nominated languages which are available in schools, possibly because those were languages they were aware of or knew something about. Others clearly chose languages because of their family backgrounds. Allowing for the fact that 14 students mentioned more than one language, these responses from 227 students, (73\% of the sample) seem to show a great interest in LOTEMs.

\subsubsection{Summary of general attitudes to FLL}

General attitude scores were calculated for the 270 students who responded to all nine of the items included in this measure and the results are shown in Graph 5.2 (Appendix 11). Almost $85 \%$ of students have a score of $24 / 45$ and almost $54 \%$ score the median of 29 or above, which indicates that the year 8 students have generally positive attitudes towards FLL. Hardly any students complained about having to study a FL but one girl made the following comment:

"I think that languages are good to learn but if you don't want to learn them you shouldn't have to."

On the other hand, another girl commented very positively:

"It is important to learn another language and it should be compulsory."

\subsection{Attitudes towards FLL experience}

I hypothesised that prior experience of FLL might improve students' attitudes towards FLL and so all students with such experience at intermediate school were asked to complete the second part of the survey. Five items were designed to ascertain their general attitude to this experience. Four items related to the development of language skills and another four investigated students' reactions to their language teachers and their organisation of classes. Lastly, two items attempted to measure student attitudes towards speakers of foreign languages and their cultures. As well as responding to the Likert scale items, 29 students offered comments on their FLL experience, largely positive.

\subsubsection{General reactions to FLL experience}

Nearly half of all the students, $46 \%$, agreed that learning another language was easier than they expected (survey item 8.2.1, $\mathrm{N}=235$ ), but among those intending to continue FLL $(\mathrm{N}=117), 60 \%$ agreed with the same item, a significant association $(p<.03)$, suggesting that the unexpected ease of FLL may have encouraged them to continue. Students were also asked to respond to items indicating how difficult they found reading and writing a different language (items 8.2.11 and 8.2.2 respectively). $33 \%$ of students $(\mathrm{N}=235)$ agreed that reading the FL was too hard and $49 \%(\mathrm{~N}=$ 236) agreed that "trying to write it was really difficult". When asked to consider 
whether they would like to continue studying the same language (item 8.2.3), $44 \%$ $(\mathrm{N}=234)$ indicated that they would. Thus the degree of difficulty may have discouraged a proportion of students from continuing FLL.

Students were also asked to respond to the prospect of trying a different language in the following year (item 8.2.8). $36 \%$ of the 240 who responded agreed that they had enjoyed language learning so much that they would like to try another one, and the rest were evenly divided between uncertainty and disagreement. It is not possible to know whether those who disagreed with the statement were claiming that they had not enjoyed their FL or that they were simply not interested in learning another FL.

\subsubsection{Student opinions about skills development}

Students were asked to consider items which suggested that studying FL might help them listen and read more carefully, understand English better and also make future FLL easier. Reactions to the item 8.2.5, "Learning another language helps me listen more carefully", were evenly divided across the scale of 'agree', 'not sure', 'disagree'. There was more doubt about the value of FLL for improving reading. Less than one quarter of students agreed that "Learning another language helps me read more carefully" (item 8.2.14, $\mathrm{N}=234$ ), and $41 \%$ were unsure. Over half the students, $53 \%$, disagreed with the idea that learning another language would help them understand English better (item 8.2.4, N = 235), while only a small proportion, under $18 \%$, agreed. Only $29 \%$ of the students believed that their FLL experience would help them with future FLL (item 8.2.10, $\mathrm{N}=236$ ).

\subsubsection{Attitudes towards FL teachers}

In order to ascertain student attitudes towards their FL teachers the survey asked them to respond to items about class organisation, the effectiveness of the materials used, how much emphasis the teacher put on accuracy and how much fun the lessons were. The students were fairly evenly divided in their responses to item 8.2.12, "Our language lessons could have been better organised", although the largest proportion, $38 \%$, disagreed $(\mathrm{N}=237)$. However, $64 \%$ of the 117 students intending to continue FLL disagreed, a significant association ( $p<.02)$. The majority of students, 55\%, appreciated the value of the materials used in FL classes in that they disagreed with the suggestion that such materials did not help them learn (item 8.2.15, N = 229). Some students offered comments on their FL classes which throw more light on their attitudes. For example:

"Our language lessons are boring. We didn't use videos or tapes."

Nearly half the students disagreed that their teacher was "too fussy about us saying things correctly" (item 8.2.6, $\mathrm{N}=236$ ), while $30 \%$ were critical of this aspect of FLL. It is possible that teachers were not emphasising correct pronunciation or that they were doing it so subtly that students did not notice. In fact, one boy commented that what he liked most about the language he was learning was "the funny words 
and mixups that can happen".

When asked if their language teacher had made their lessons fun, (item 8.2.9) 49\% of students agreed $(\mathrm{N}=234)$ and only about one quarter disagreed. According to their individual comments, fun is certainly an important element in FLL and the responses to this item had a significant association with student intentions $(p<.02)$.

\subsubsection{Attitudes to FL speakers and their cultures}

Half the students responded positively to an item (8.2.7) which indicated their interest in knowing more about speakers of the FL they were studying and their culture, while only $22 \%$ were against the idea $(\mathrm{N}=236)$. Responses to the suggestion that students were not interested in meeting speakers of the language they had been studying (item 8.2.13) indicated even more positive attitudes, producing some of the firmest opinions of the whole survey, as shown in Graph 5.3. Only $11 \%$ of students agreed with the statement, which shows considerable openness towards LOTEM speakers. One girl stressed her enthusiasm by commenting that she would "really like to meet different people that speak different languages."

\section{Graph 5.3 Responses to item 8.2.13: "I'm not interested in} meeting people who speak the language'(No opinion =9)

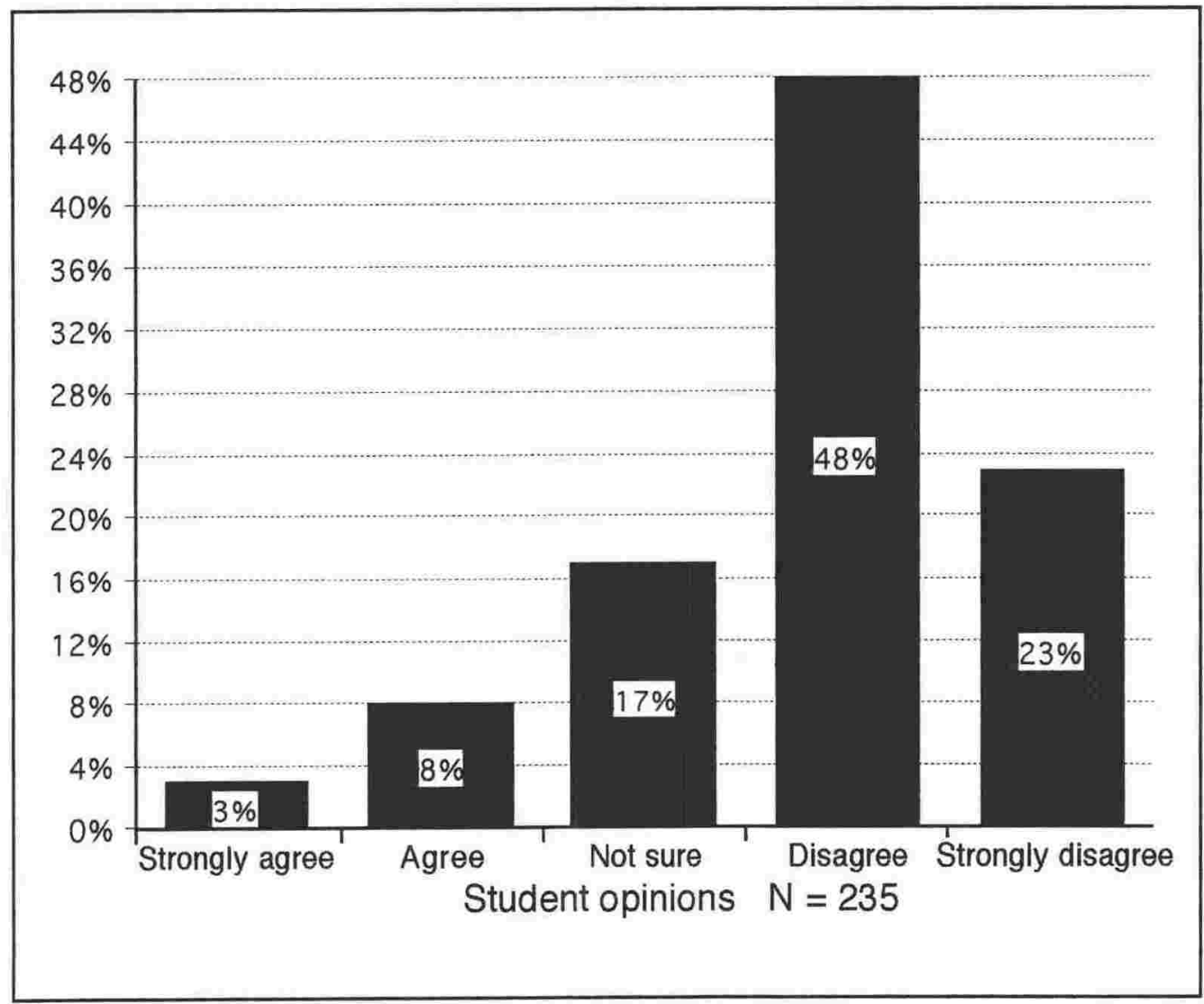




\subsubsection{Summary of attitudes to FLL experience}

Attitude scores were calculated for the students who responded to all ten of the items discussed so far in this section and the results are shown in Graph 5.4 (Appendix 11). Over $85 \%$ of the 208 students who responded to all the relevant items had a score of $25 / 50$ or above and about $56 \%$ scored the median of 30 or above, which indicates that the year 8 students had equally positive attitudes towards their experience of FLL at intermediate school FLL as they did to FLL in general. The most positive comment came from one of the girls:

"It rocks. I think everyone should have a chance to do it."

\subsection{Year 8 students intentions regarding FLL in year 9}

\subsubsection{To study a FL or not}

All the students were asked to respond to the question, "Will you study a language next year?". As shown in Table 5.7, nearly half the students reported that they would study a FL in year 9, consistent with the typical national average, about one third said 'no', and $19 \%$ were unsure.

Table 5.7 Student intentions for year 9

\begin{tabular}{|l|c|c|c|}
\hline \multirow{2}{*}{} & \multicolumn{3}{|c|}{ Students will study a FL in year 9 } \\
\cline { 2 - 4 } & Yes & Maybe & N o \\
\hline N & 150 & 59 & 96 \\
\hline Proportion & $49 \%$ & $32 \%$ & $19 \%$ \\
\hline \multicolumn{4}{|l}{ Total N = 308, missing data 3 } \\
\hline
\end{tabular}

\subsubsection{Reasons for intentions}

\subsubsection{Reasons for responding 'yes'}

The reasons that students gave for intending to study a FL in year 9 are set out in Table 5.8. Not all students gave reasons and some cited more than one. The reason mentioned most was a wish to continue something they had enjoyed at intermediate school, or to build on what they had already had achieved. As all the reasons listed are based on the students' own words, they are open to interpretation. Other reasons cited by a few students were the influence of parents, siblings, friends or overseas connections, or the desire for something new and challenging. 
Table 5.8 Reasons for choosing to study a FL in year 9

\begin{tabular}{|l|c|}
\hline Comments & $\begin{array}{c}\text { Number of } \\
\text { mentions }\end{array}$ \\
\hline To build on prior experience, enjoyment, success & 55 \\
\hline Interest in specific FL, culture & 31 \\
\hline Useful for jobs & 19 \\
\hline For travel & 17 \\
\hline Sounds cool, fun & 14 \\
\hline General interest in FLs, cultures & 9 \\
\hline
\end{tabular}

\subsubsection{Reasons for responding 'maybe'}

Reasons cited by students for indicating that they might study a FL are shown in Table 5.9. Again, students were particularly keen to build on their previous experience. The influence of previous FLL on intentions for year 9 will be explored below in section 5.8. Other comments were fairly equally divided between interest in a specific language, interest in travel and the idea of having fun. Other reasons mentioned by only 1 or 2 boys, included overseas connections, influence of older sibling, usefulness for jobs.

Table 5.9 Reasons cited by students who said they might study a FL in year 9

\begin{tabular}{|l|c|}
\hline Comments & $\begin{array}{c}\text { Number of } \\
\text { mentions }\end{array}$ \\
\hline To build on prior experience, enjoyment, success & 16 \\
\hline Interest in specific FL, culture & 9 \\
\hline For travel & 9 \\
\hline Sounds cool, fun & 7 \\
\hline General interest in FLs, cultures & 5 \\
\hline
\end{tabular}

\subsubsection{More evidence of student interest in overseas travel}

A particularly high proportion of students responded positively to item 8.1.21: "I want to do a language next year because it'll give me the chance to go overseas on a school trip." $38 \%$ agreed and $21 \%$ strongly agreed $(\mathrm{N}=$ 
295), although only one secondary school prospectus mentioned trips to Japan and no others referred to any overseas trips. Students might know about overseas travel opportunities through friends or family, but the survey item may have put the idea into their heads, or they naturally associated FLL with travel. There was, in any case, a significant association $(p<.001)$ between the responses to this item and student intentions, shown in Graph 5.5. $63 \%$ of the 174 students who agreed with the statement intended to study a FL. This does not necessarily mean that travel was a major factor in their option choice, but probably another indication of an interest in travel among those interested in FLL, indications of which appeared in Tables 4.8 and 4.9 discussed above.

Graph 5.5 Responses to item 8.1.21, "I want to do a language next year because it'll give me the chance to go overseas on a school trip" compared with FLL intentions

(For association between item responses and intentions $\mathrm{p}<.001$ )

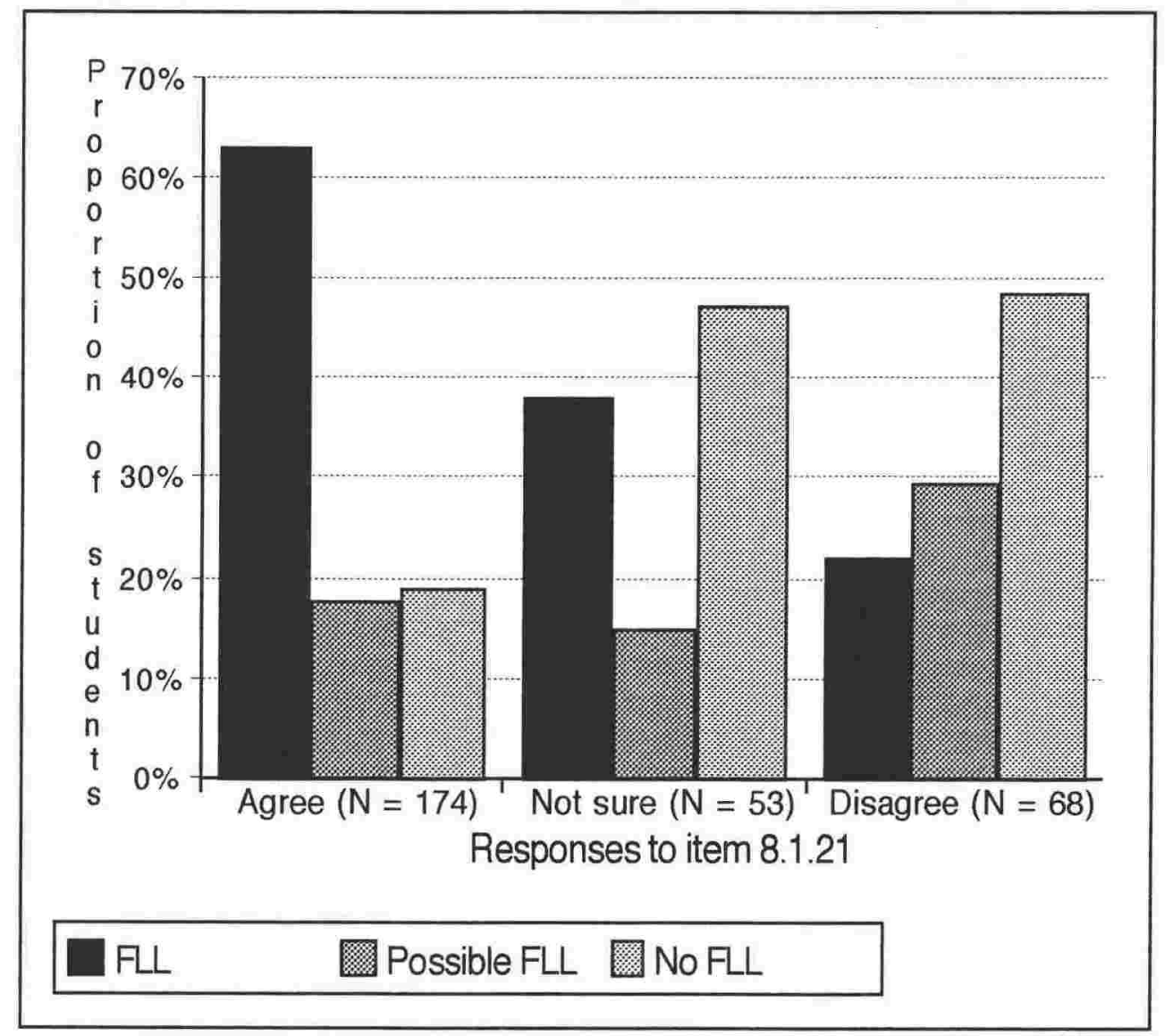




\subsubsection{Reasons for not choosing FLL in year 9}

The most common reason cited for not intending to study a FL was a preference for Maori, mentioned by 16 students. Only 3 students said that FLL would be a waste of time or would reduce the time available for other options. One boy thought that FLL would be too difficult and one girl was content with what she had already learnt.

\subsubsection{Intentions compared with beliefs about the usefulness, the relative importance and the difficulty of FLL}

There was little association between the students' responses to the two survey items concerning the usefulness of FLL, discussed above, and their intentions for year 9. While the majority of students believed that knowing another language would help them get a good job and that many jobs involve working with people who do not speak English, these beliefs did not necessarily encourage them to study a FL. Students may realise that most immigrants to the country do not speak the FLs commonly offered in schools, or they may simply consider other subjects as more useful for job qualifications.

\section{Graph 5.6 Responses to item 8.1.17: "There are lots more important} things to learn than languages", compared with intentions for year 9

(For association between item responses and intentions $\mathrm{p}<.001$ )

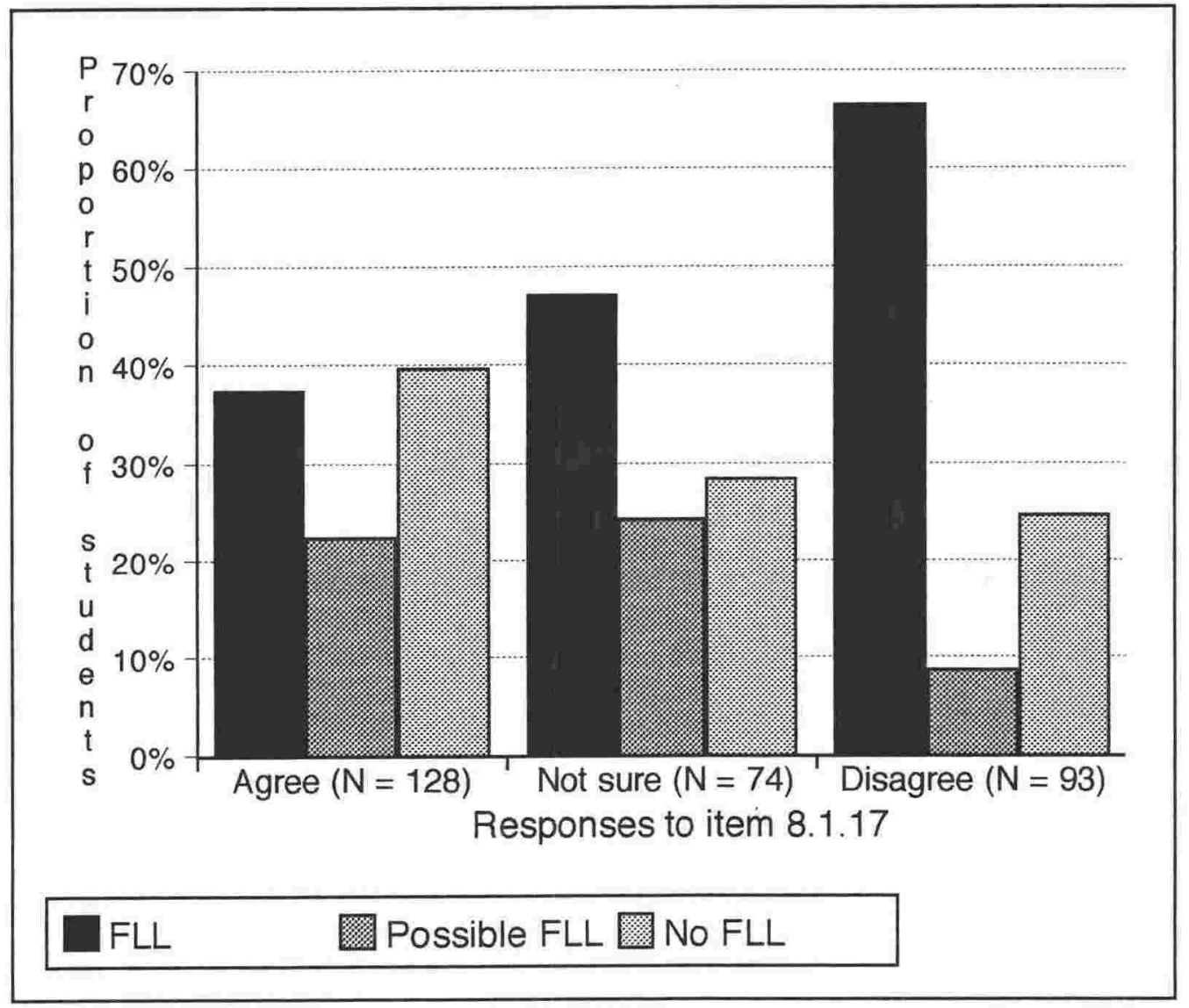


However, the perceived greater value of other subjects did not necessarily dissuade students from FLL. For example, 38\% of the 128 students who agreed that there are many more important things to learn than languages still intended to study a FL and another $22 \%$ were considering it. Even $29 \%$ of the 90 students who agreed that it was better to spend time learning more English than a FL were intending to study a FL in year 9. There was, however, a significant association, between student responses to item 8.1.17 and student intentions for year 9, see Graph 5.6. In general, the findings support the hypothesis that students who choose FLL do so out of personal interest, not for some future usefulness.

Similarly, and not surprisingly, there was a significant association $(\mathrm{p}<.001)$ between students' confidence in their ability to cope with future FLL and their intention to study a FL in year 9, see Graph 5.7.

Graph 5.7 Responses to item 8.1.6: "I think I'd find learning a language easy next year", compared with intentions for year 9 (For association between item responses and intentions $p<.001$ )

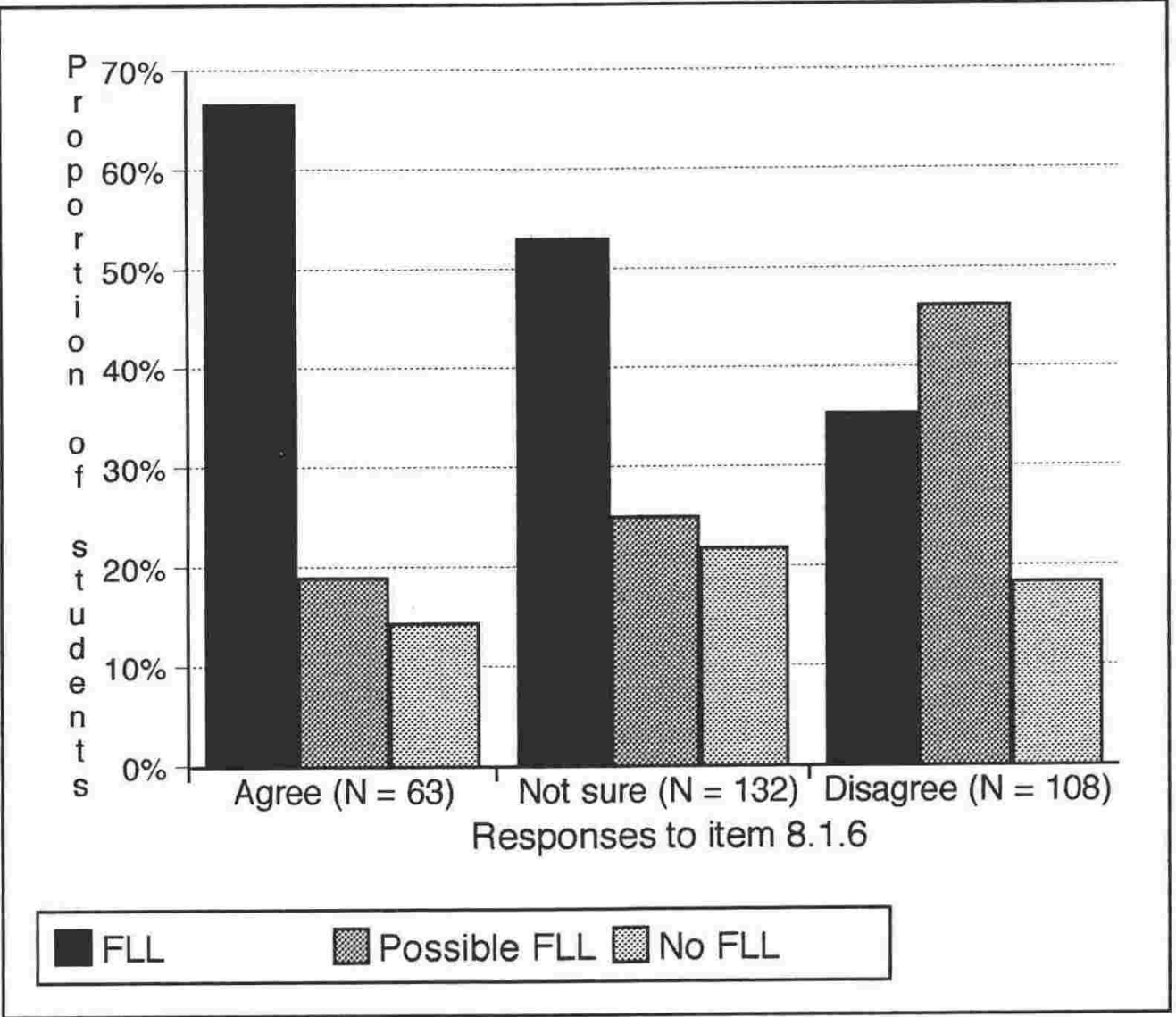




\subsubsection{Which languages students intended to study}

When students were asked which language they would or might study, most indicated only one language. If they mentioned two, sometimes including Maori, it was not always clear whether they intended to study more than one or they had not decided which one to study.

\subsubsection{Choices of those intending FLL in year 9}

Table 5.10 shows the proportions of students who intended to study one of the languages available at the local secondary schools. This shows a surprising imbalance in favour of Japanese which is not typical nationally. There are usually fairly similar proportions of students opting for French and Japanese, and German is often much more popular. Spanish is not widely available and was only offered by one secondary school in the survey area. Most undecided students were hesitating between a European language or Japanese. Three students mentioned Chinese but, as far as could be ascertained, it was not available in year 9 at any local secondary schools. Eight students were considering Maori instead of a FL.

Table 5.10 Languages chosen by students intending FLL in year 9

\begin{tabular}{|l|c|c|c|c|c|}
\hline & \multicolumn{5}{|c|}{ Languages chosen } \\
\hline \multirow{2}{*}{$\begin{array}{c}\text { Proportion } \\
\text { of students } \\
(\mathbf{N}=150)\end{array}$} & French & German & Spanish & Japanese & $?$ \\
\cline { 2 - 6 } & $17 \%$ & $9 \%$ & $6 \%$ & $52 \%$ & $15 \%$ \\
\hline
\end{tabular}

\subsubsection{Choices of those who might study a FL in year 9}

These students were much more undecided. Only half, 30 students, named one language and half chose Japanese. Of the rest, two thirds specified no language while the remainder mentioned various combinations of European languages, Japanese and Maori.

\subsubsection{Reasons given for choices of language}

Very few students gave reasons for their choice of language and most comments were about Spanish or Japanese. All but one made about Japanese focussed on its usefulness, for jobs, particularly tourism, although there were obviously some misconceptions. One boy stated "it is the most spoken language in the world". One girl said Japanese was the most useful language available. Most comments about Spanish referred to previous experience of learning the language. Two boys recognised that Spanish is widely spoken. Each of the four boys who commented on their choice of German had a different reason for his choice: 
"I've always wanted to go there"

"the main car manufacturers are there"

"it's a pretty rich country"

"easy to learn".

Only a few vague reasons were given for choosing French, such as: "a good language", "it would be cool", but one boy thought that it was widely spoken and one girl wanted to go to France.

\subsection{Student intentions compared with their ultimate interest in FLL}

\subsubsection{The availability of preferred languages}

As reported above in 5.4.5, students showed great interest in FLL when given a hypothetical free choice of language, but most of the languages they nominated are not available in schools. However, two thirds of students chose a language which would be available at the secondary school they expected to attend.

\subsubsection{Student intentions where preferred FL was available}

One might expect that the majority of those whose first choice was available would choose to study a FL in year 9, and that perhaps the third of students for whom their first choice was not available might decide against FLL. This did not prove to be the case. Table 5.11 shows that about one third of the 151 students who could have studied their preferred FL had decided against any FLL and only 37\% intended to study their preferred FL. A few had decided on a different language, showing a tendency to opt for Japanese in preference to an available European language which they most wished to learn.

Table 5.11 Students' intentions for year 9 if their preferred FL was possible at future secondary school

\begin{tabular}{|c|c|c|}
\hline Intentions & $\mathbf{N}$ & $\%$ of $\mathrm{N}$ \\
\hline Intending to study preferred FL & 56 & $37 \%$ \\
\hline Not sure which FL to study $\dagger$ & 9 & $6 \%$ \\
\hline Will study another FL* & 10 & $7 \%$ \\
\hline Not sure about FLL & 28 & $19 \%$ \\
\hline Will not study a FL & 48 & $32 \%$ \\
\hline Total & 151 & \\
\hline $\begin{array}{l}\text { † Most mentioned Japanese } \\
\text { * All opted for Japanese }\end{array}$ & & \\
\hline
\end{tabular}


Students intentions for year 9 were compared with their stated preferred language and the results are shown in Table 5.12. Although French and Japanese were available at all the local secondary schools, only $36 \%$ of students who wanted to learn French and 55\% of those interested in Japanese were intending to study a FL in year 9, and students interested in French were proportionately twice as undecided about future FLL as those wanting to learn Japanese. Students interested in other European or Asian languages, however, were more likely to opt into FLL, although they would probably have to study a language other than their first choice, which suggests that they were motivated by a strong interest in FLL per se.

Table 5.12 Year 9 choice of FL compared with reported FL preference

\begin{tabular}{|l|c|c|c|c|}
\hline \multicolumn{2}{|c|}{} & \multicolumn{3}{|c|}{ Future FL learning } \\
\hline $\begin{array}{l}\text { Students' FL } \\
\text { preference }\end{array}$ & N & $\%$ Yes & $\%$ Maybe & $\%$ No \\
\hline French & 55 & $36 \%$ & $31 \%$ & $33 \%$ \\
\hline Spanish & 45 & $60 \%$ & $18 \%$ & $22 \%$ \\
\hline German & 17 & $76 \%$ & $12 \%$ & $12 \%$ \\
\hline Other European & 26 & $62 \%$ & $4 \%$ & $35 \%$ \\
\hline Japanese & 75 & $55 \%$ & $16 \%$ & $29 \%$ \\
\hline \begin{tabular}{l} 
Other Asian† \\
\hline Other*
\end{tabular} & 12 & $58 \%$ & $25 \%$ & $25 \%$ \\
\hline $\begin{array}{l}\dagger \\
\text { * Mostly Chinese } \\
\text { NB Some students mentioned more than one language }\end{array}$ \\
\hline
\end{tabular}

A more detailed analysis of student intentions with regard to French and Japanese shows substantial differences, not just in whether students would opt for FLL but also in their choice of language. Table 5.13 shows that nearly half the students whose preference was for Japanese but less than one quarter of those interested in French were intending to study the same language. These analyses seem to suggest that some subtle influence was persuading students that it was more acceptable to study Japanese than French. 
Table 5.13 Intentions to study French or Japanese compared with students' ultimate interest

\begin{tabular}{|c|c|c|c|c|}
\hline Student reports & French & $\%$ & Japanese & $\%$ \\
\hline Would like to study & 55 & & 75 & \\
\hline Intend to study & 12 & $22 \%$ & 35 & $47 \%$ \\
\hline May study* & 18 & $33 \%$ & 17 & $23 \%$ \\
\hline Will/may study another FL $\dagger$ & 7 & $13 \%$ & 1 & $1 \%$ \\
\hline Will not study a FL & 18 & $33 \%$ & 22 & $29 \%$ \\
\hline \multicolumn{5}{|c|}{$\begin{array}{l}\text { * Students who reported that they would or might study a FL which } \\
\text { might be their preferred language. } \\
\dagger \text { Students who reported that they would or might study a FL but it would } \\
\text { not be their preferred FL, or no choice was specified. }\end{array}$} \\
\hline
\end{tabular}

\subsubsection{Student intentions where preferred FL was not available}

Of the 63 students who were interested in learning a FL which would not be available at their intended secondary school, the majority (59\%) were prepared to study another FL instead, see Table 5.14. Only a few students remained undecided about future FLL and a few more did not intend it at all. On the whole, therefore, students keen on FLL were not dissuaded by the fact that their first choice of language was not available to them.

Table 5.14 Students' intentions for year 9 if their preferred FL was not possible at future secondary school

\begin{tabular}{|l|c|c|}
\hline Intentions & N & \% of total \\
\hline Will study another FL & 37 & $59 \%$ \\
\hline Not sure about FLL & 10 & $16 \%$ \\
\hline Will not study a FL & 16 & $25 \%$ \\
\hline Total & 63 & \\
\hline
\end{tabular}

\subsubsection{Summary of comparison of intentions with preferred FLs}

There were clearly factors influencing student intentions other than their desire to learn a specific FL. Where students were undecided about FLL or their choice of FL this might have been the result of considerations about the relative interest, value or 
difficulty of learning any or a specific FL, compared with other options available to them. They could have been waiting to see what combinations of options were possible in year 9. It is clear that Japanese was seen as a more acceptable FL to study than French, even if students ultimately wished to learn French. Various other possible influences will be discussed in the following sections.

\subsection{Intentions for year 9 compared with previous experience of FLL}

\subsubsection{To study a FL or not}

Although the majority of year 8 students had had some experience of FLL, this factor had little effect overall on students' intentions, despite the fact that it was one of the reasons cited most often for continuing FLL. Graph 5.8 shows that only a slightly larger proportion of the 248 students with previous experience, $50 \%$, intended to study a FL in year 9 , compared with $46 \%$ of students without such experience. Even more marked is the difference between the 'no' and 'maybe' responses. Students without previous experience were more likely to have decided against FLL in year 9, $37 \%$, compared with $30 \%$ of those with such experience. It must be recalled that students without previous FLL experience were in a minority in this sample, only $19 \%$ (57 students).

\section{Graph 5.8 Intentions for FLL in year 9 compared with previous} experience (Missing data $=3$ )

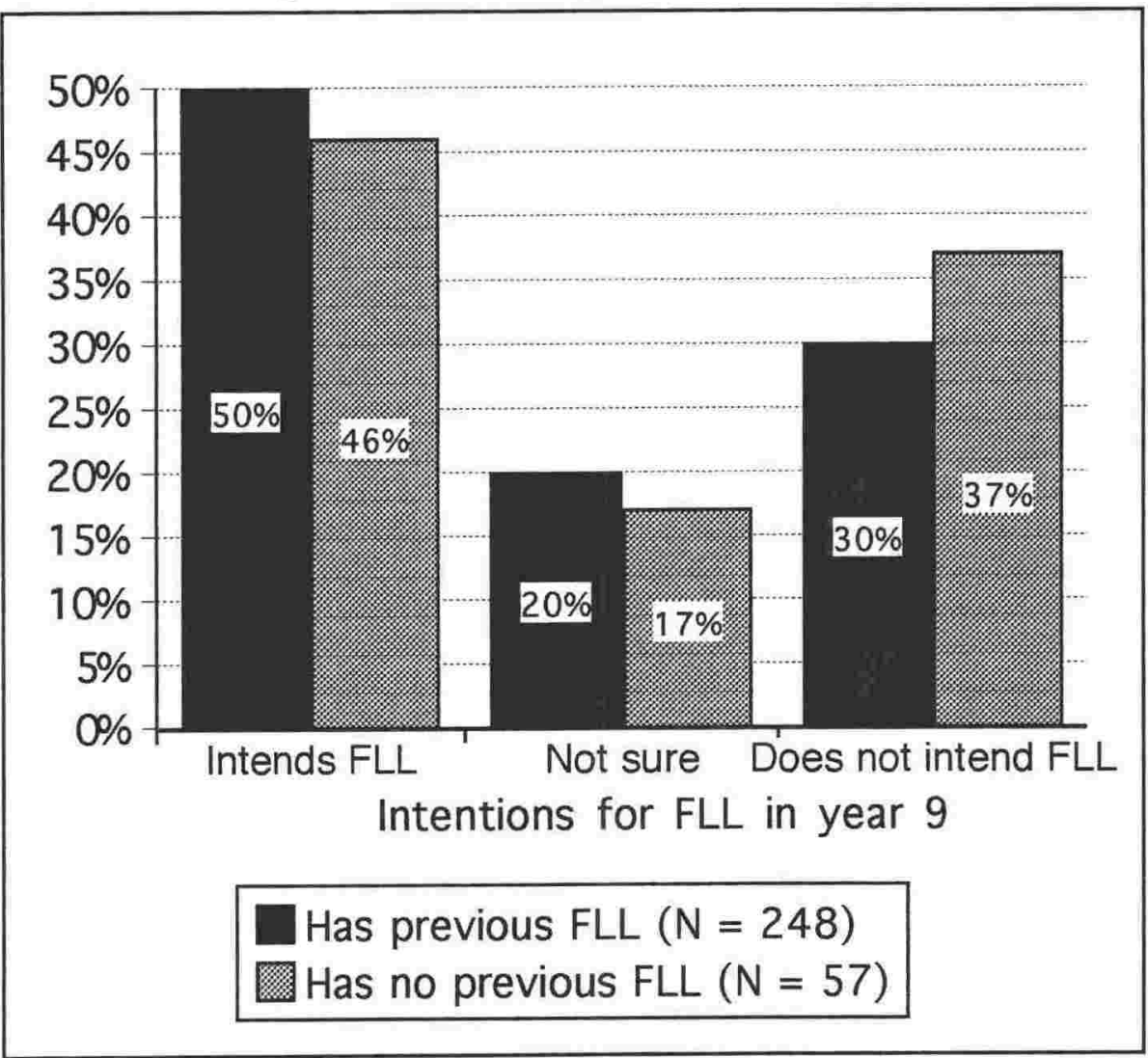




\subsubsection{The importance of enjoyment}

Item 8.2.8, "I enjoyed language learning so much I'd like to try another language", proved to have a significant association $(\mathrm{p}<.001)$ with student intentions. 63 of the 85 (74\%) students who agreed with this statement intended further FLL, but even 18 of the $75(24 \%)$ who disagreed and 36 of the $79(46 \%)$ who were unsure were intending further FLL. It is clear, however, that having enjoyed their prior experience of FLL had very positive effect on students' attitudes to further FLL, although lack of enjoyment did not necessarily put students off, presumably because they envisaged trying another language or because a change of school and teacher might make a difference

\subsubsection{The influence of specific languages}

Of the students who had studied one or two of the three main languages offered in the intermediate schools, French, Spanish and Japanese, those most certain that they would continue FLL were those who had learnt both Spanish and Japanese, see Table 5.15, for reasons which are discussed below. Of that group, nearly two thirds intended to study a FL in year 9 and most of the remainder were undecided. On the other hand, learning French only or French and Japanese tended to polarise students' views of FLL and over one third of students with such experience had decided against further FLL. The most undecided students were those who had studied only Spanish, or Spanish and Japanese, which could be explained by the limited availability of Spanish .

Table 5.15 Year 8 students' intentions for FLL in year 9 compared with FLs studied in year 7 or 8

\begin{tabular}{|l|c|c|c|c|}
\hline \multirow{2}{*}{$\begin{array}{l}\text { Prior FLL } \\
\text { by FL }\end{array}$} & \multirow{2}{*}{$\begin{array}{l}\text { Student } \\
\text { numbers }\end{array}$} & \multicolumn{3}{|c|}{ Intending FLL in year 9 } \\
\cline { 3 - 5 } French & 29 & 17 & 1 & 11 \\
\hline Spanish & 69 & 28 & 21 & 20 \\
\hline Japanese & 58 & 30 & 12 & 16 \\
\hline $\begin{array}{l}\text { French + } \\
\text { Japanese }\end{array}$ & 69 & 34 & 10 & 25 \\
\hline $\begin{array}{l}\text { Spanish + } \\
\text { Japanese }\end{array}$ & 18 & 11 & 5 & 2 \\
\hline
\end{tabular}


Graph 5.9 (Appendix 11) shows the differing intentions of students according to whether they had experience of:

- only one language,

- only a European language,

- only Japanese,

- Japanese plus a European language, or

- no experience of FLL.

The prior learning of Japanese, alone or with another language, seems to have a slightly greater positive influence on student intentions. This could be for several reasons:

- they found the Japanese language and/or culture inherently interesting,

- they enjoyed the challenge of something very different,

- they enjoyed the lessons owing to particularly good teaching, or

- they had decided that Japanese was the most useful language to learn.

The proportion of students having learnt only one European language and intending FLL in year 9 was the same as that of students with no FLL experience, but it is clear that students without that experience were the most likely not to opt for FLL in year 9.

Student responses to item 8.2.3, "I don't want to learn more of the same language", proved to have a significant association $(\mathrm{p}<.001)$ with student intentions. Not surprisingly, the majority of the 104 students who disagreed with this statement, $69 \%$, intended to study a FL in year 9 . What is more interesting is that $32 \%$ of both the 56 who agreed and the 74 who were unsure still intended some further FLL, presumably of a different FL. Only $41 \%$ who agreed with the statement stated a definite intention not to undertake further FLL. Thus, a negative experience of learning a particular FL does not seem to have put the majority of students off intending, or at least considering, FLL at secondary school.

\subsubsection{Choice of future FL compared with language(s) studied}

Table 5.16 shows the numbers of year 8 students intending to study the available FLs, compared with their prior experience. The majority opting to study French, Spanish or Japanese had already had some experience of these languages. Most students intending to change language or starting FLL for the first time were opting for Japanese.

The choice of Spanish seemed most influenced by students' previous experience, judging by the comments they made to explain their year 9 choice:

"It was fun and interesting"

"Our teacher made Spanish fun and easy to understand and helped us when we were unsure. It was easier than I thought it would be."

"Learning Spanish was cool"

"Spanish is primo!" 
Table 5.16 Year 8 students' choice of language for Year 9 compared with previous FL studied

\begin{tabular}{|l|c|c|c|c|}
\hline $\begin{array}{l}\text { Year 9 } \\
\text { choice }\end{array}$ & $\begin{array}{c}\text { Intending } \\
\text { FLL }\end{array}$ & $\begin{array}{c}\text { Continuing } \\
\text { same FL }\end{array}$ & $\begin{array}{c}\text { Starting new } \\
\text { FL }\end{array}$ & $\begin{array}{c}\text { No previous } \\
\text { FLL }\end{array}$ \\
\hline French & 26 & 15 & 6 & 5 \\
\hline Spanish & 14 & 11 & 1 & 2 \\
\hline Japanese & 78 & 47 & 20 & 11 \\
\hline German & 9 & 1 & 7 & 1 \\
\hline
\end{tabular}

\subsubsection{Other indications of the influence of previous FLL}

All the students were asked to respond to the statement, "What I've seen about languages I could learn next year makes them look really interesting", item 8.1.24. This was originally designed to ascertain what they had observed on visits to local secondary schools or what they had learnt from school prospectuses. When it became clear that most students had already done some FLL it could be inferred that their responses to this item might, at least in part, reflect their reactions to that experience. In fact, agreement with this item was much stronger among the 55 students without prior FLL experience than among the 239 who had learnt a FL, and who expressed an opinion. $31(56 \%)$ and $106(44 \%)$ respectively agreed that FLL at their intended secondary school looked interesting.

Table 5.17 Student intentions for FLL in year 9 compared with responses to item 8.1.24: "What I've seen about languages I could learn next year makes them look really interesting" (No opinion $=14)$

\begin{tabular}{|l|c|c|c|c|}
\hline \multirow{2}{*}{$\begin{array}{l}\text { Item } \\
\text { responses }\end{array}$} & N & \multicolumn{3}{|c|}{ Intending FLL in year 9 } \\
\cline { 3 - 5 } Agree & 137 & $69 \%$ & $17 \%$ & $14 \%$ \\
\hline Not sure & 77 & $42 \%$ & $19 \%$ & $39 \%$ \\
\hline Disagree & 80 & $25 \%$ & $22 \%$ & $53 \%$ \\
\hline Total & 294 & & & \\
\hline
\end{tabular}

For association between item responses and intentions $\mathrm{p}<.001$ 
There proved to be a highly significant association between this item and student intentions $(p<.001)$, see Table 5.17. 69\% of the 137 students who agreed with the statement intended to study a FL in the following year, compared with only $25 \%$ of those who disagreed. This, of course, begs the question of why the latter intended to study a FL. It might be the result of school or parental pressure, for example, both of which will be discussed below. $52 \%$ of those who disagreed with the statement did not intend further FLL, compared with only $14 \%$ of those who agreed. These responses suggest that very few students who were interested in FLL, and had some FLL experience, were put off engaging in such learning by other factors.

\subsubsection{Intentions compared with attitudes to FL speakers and their cultures}

As reported in 5.4, the majority of students showed positive attitudes towards speakers of the FLs they had been studying and to their cultures. These attitudes proved to have, not surprisingly, significant association $(\mathrm{p}(<.01)$ with student intentions. For example, 69 of the 117 students (59\%) who agreed with item 8.2.7, "I'd like to know more about the life and customs of the people who speak the language", were intending to study a FL in the following year, while just under half of the 53 who disagreed did not intend such study. What is perhaps more interesting is that $34 \%$ of students who disagreed and $45 \%$ of the 66 who were unsure still intended some FLL in year 9. This could mean that they intended to change FL or that it was the FLL itself that most interested them, rather than the cultural aspects. On the other hand they might have felt under some external pressure to choose a FL option.

As discussed in 5.5.4, student responses to item 8.2.13, "I'm not interested in meeting people who speak the language" revealed even more positive attitudes than item 8.2.7. A large proportion of the 168 students who disagreed with the item, 98 $(58 \%)$, intended FLL, a significant association ( $p<.001)$ between student interest in meeting speakers of the FL they have studied and their intention to continue FLL. This may relate to the interest shown in overseas travel, discussed in 5.6. It is, of course, impossible to tell from the data which came first - the desire to travel the interest in FLL.

\subsubsection{Summary of comparison of intentions with previous FLL}

The above findings show that prior FLL experience did not seem to have a considerable influence on students' intentions to study a FL or not in year 9. Typically, about half the students intended to study a FL in $\mathrm{y} 9$, with or without previous experience. This does not support the FL advisers' surmise that many students give up FLL after intermediate level because they feel they have learnt enough and want to try other subjects (NZCTE 1998). However, the lack of FLL experience produced a larger proportion of students definitely not intending to study a FL in y9. Thus prior experience may have had a slightly positive effect on student attitudes. 
Students who did intend FLL were interested in other languages and cultures and meeting people who speak other languages. They had enjoyed their prior learning, although enjoyment per se did not guarantee that students would continue studying the same FL or take up any other language. Students who had learnt Japanese were those most likely to continue FLL and most students, with or without previous FLL experience, intended to study Japanese, even if their ultimate preference was to learn another language and even if that was possible. Thus there were clearly external factors influencing students' intentions and of ten outweighing their preferences.

\subsection{The influence of parents on intentions}

\subsubsection{General parental influence on subject choices}

Out of 303 students, 65 strongly agreed (a particularly large proportion) and another 114 agreed (a total of 59\%), that their parents did not mind what subjects they chose the following year (item 8.1.10). Only 80 (26\%) disagreed. Thus the majority felt able to make their subject choices independently of their parents. Whether this represents parents' indifference or their encouragement of individual responsibility on the part of their children is open to debate. Parental influence may, in fact, be considerable but unperceived by students. What parents thought about this will be discussed in the analysis of the parent interviews in Chapter 8.

\subsubsection{Student intentions compared with reported parental wishes}

When asked if their parents wanted them to study a FL in the following year, item 8.1.23, one third of students were unsure but about $41 \%$ agreed $(\mathrm{N}=293)$. There was a significant association $(p<.001)$ between these responses and student intentions for year 9, see Table 5.18. Over three quarters of students who thought their parents wanted them to study a FL intended to do so, while $60 \%$ of those who disagreed that their parents wanted them to study a FL did not intend such study. It appears that, not surprisingly, parental encouragement had a strong positive influence on the choice to study a FL, whereas parental indifference or opposition had a slightly weaker negative influence.

Table 5.18 Student intentions for FLL in year 9 compared with responses to item 8.1.23 "My parents want me to do a language next year."

\begin{tabular}{|l|c|c|c|c|}
\hline \multirow{2}{*}{$\begin{array}{l}\text { Item } \\
\text { responses }\end{array}$} & N & \multicolumn{3}{|c|}{ Intending FLL in year 9 } \\
\cline { 3 - 5 } Agree & 119 & $76 \%$ & $12 \%$ & $12 \%$ \\
\hline Not sure & 99 & $42 \%$ & $26 \%$ & $31 \%$ \\
\hline Disagree & 75 & $20 \%$ & $20 \%$ & $60 \%$ \\
\hline
\end{tabular}




\subsubsection{Student intentions compared with reported parental attitudes to the relative importance of English}

There proved to be a significant association $(\mathrm{p}<.001)$ between student responses to survey item 8.1.25 and student intentions for year 9 FLL, shown in Table 5.19. It is not surprising that the majority, 27 , of the 50 students who reported that their parents thought they should spend more time on their English than learning a FL would decide against FLL, but it is interesting that 12 of them had decided that they would nevertheless study a FL, showing again that students felt free to follow their own interest in FLL, despite their parents' opinions. Parental experience of FLL or lack of it had some influence on the opinions reported by students. Of the 122 students who reported that at least one parent had learnt a FL at school, 50\% disagreed with the item, compared with $41 \%$ of the 125 students who thought that their parents had had no FLL experience. Thus, according to the students, parents who had no FLL experience were more likely to believe that concentrating on English language development was more important than FLL, although this did not necessarily dissuade students from taking up a FL at secondary school.

Table 5.19 Student intentions for FLL in year 9 compared with responses to item 8.1.25 "My parents think I should spend more time on my English than learning another language."

\begin{tabular}{|l|c|c|c|c|}
\hline \multirow{2}{*}{$\begin{array}{l}\text { Item } \\
\text { responses }\end{array}$} & N & \multicolumn{3}{|c|}{ Intending FLL in year 9 } \\
\cline { 3 - 5 } Agree & 50 & $24 \%$ & $22 \%$ & $54 \%$ \\
\hline Not sure & 100 & $48 \%$ & $25 \%$ & $27 \%$ \\
\hline Disagree & 142 & $58 \%$ & $15 \%$ & $27 \%$ \\
\hline
\end{tabular}

For association between item responses and intentions $p<.001$

\subsubsection{Student intentions compared with parental FLL experience}

Of the 122 students who reported that at least one parent had learnt a FL at school ${ }^{1}$, $43 \%$ stated that their parents wanted them to learn a FL and 56\% intended to opt into FLL. The interest in FLL among parents and students where parents had no FLL experience was much less. In the case of these 125 students, $32 \%$ reported that their parents wanted them to learn a FL and only $41 \%$ of them intended to do so, as shown in Table 5.20.

\footnotetext{
${ }^{1}$ Students were asked if their parents learnt any LOTEMs at school in New Zealand. Parents reported to have been at school overseas were not included.
} 
Table 5.20 Student intentions for FLL in year 9 compared with parents' FLL at school in NZ

\begin{tabular}{|l|c|c|c|c|}
\hline \multirow{2}{*}{$\begin{array}{l}\text { Parents' experience } \\
\text { according to students }\end{array}$} & & \multicolumn{3}{|c|}{ Intending FLL in year 9 } \\
\cline { 3 - 5 } & N & Yes & Maybe & No \\
\hline Parent(s) had FLL & 122 & $56 \%$ & $17 \%$ & $27 \%$ \\
\hline Parents had no FLL & 125 & $41 \%$ & $24 \%$ & $35 \%$ \\
\hline No information & 61 & $49 \%$ & $15 \%$ & $36 \%$ \\
\hline
\end{tabular}

The lack of parental FLL experience also created more doubt among students. It is impossible to know how many of the whole year 8 sample who reported that they might study a FL in year 9 actually did so, but Chapter 7, which examines the subsample of students who participated in both surveys, will show that the majority of 'doubters' decided against it.

\subsection{The influence of intermediate schools and teachers on intentions}

\subsubsection{School encouragement of FLL}

All students were asked to respond to item 8.1.18, "My school encourages everyone to try a language". Since most of them had no choice about it this item was not particularly relevant. $28 \%$ of the students were unsure, but $57 \%$ agreed $(\mathrm{N}=299)$, which perhaps shows that the majority of students recognised the value place on FLL by the two larger schools. Neither school used the word 'compulsory' but, in practice, it was accepted by the classes involved as a normal part of their intermediate programme.

\subsubsection{Teacher encouragement of FLL}

Students were also asked if their class teacher said they should do a language the following year (item 8.1.16). Only a few students, $16 \%$, agreed while $47 \%$ disagreed $(\mathrm{N}=278$, 'No opinion' $=30)$. It could not be assumed from this that teachers had advised against future FLL, since it was possible that the matter had not been discussed. In fact, class teachers who were interviewed indicated that they had not stated an opinion in most cases, either because they had not been asked by students or parents, or because they did not feel qualified to give advice about subjects which were unfamiliar to them.

\subsubsection{Intentions compared with primary/intermediate schools}

Table 5.21 shows significant differences $(\mathrm{p}<.05)$ in the intentions of students compared with their year 8 schools. The proportions of students intending to study a FL in year 9 were consistent with the overall pattern discussed in 5.6.1, but students at Rose were noticeably more unsure about further FLL. This could be explained by 
the fact that only $83 \%$ of Rose students had learnt a FL, compared with nearly $100 \%$ at Salvia, and much of that was of Spanish which was available at only one local secondary school. However, the pattern of intentions of students at Aster, where almost none had any FLL experience and the only language offered was Spanish, was similar to that at Salvia and even more positive towards future FLL. Another factor had to be involved, most probably socio-economic, owing to the difference in decile ratings, which supports previous findings that community perceptions of the value of FLL are crucial (Enderwick \& Gray 1992, Johnson 2001) and generally conveyed to students through their parents (Gardner 1985).

Table 5.21 Student intentions for FLL in year 9 compared with year 8 school

\begin{tabular}{|l|c|c|c|c|}
\hline \multirow{2}{*}{ Year 8 school } & N & \multicolumn{3}{|c|}{ Intending FLL in year 9 } \\
\cline { 3 - 5 } & 37 & Yes & Maybe & N o \\
\hline Aster & 152 & $50 \%$ & $17 \%$ & $33 \%$ \\
\hline Rose & 119 & $48 \%$ & $25 \%$ & $25 \%$ \\
\hline Salvia & & $13 \%$ & $39 \%$ \\
\hline
\end{tabular}

For association between school and intentions $\mathrm{p}<.05$

\subsection{The influence of secondary schools and teachers on intentions}

\subsubsection{Schools which students expected to attend}

The majority of students, 270 or about $88 \%$, were expecting to attend one of four of the five local secondary schools. Nearly half were expecting to go to Freesia, the mixed school next door to the intermediate school, Rose. Approximately one third were going to Salvia, mostly because they were already attending that school. The rest were divided between the two single sex schools in the district, $12 \%$ destined for the boys' school and 9\% for the girls' school. Only 6 students were destined for Arum which will therefore be omitted from this discussion. There was a significant association $(\mathrm{p}<.001)$ between chosen schools and student intentions for FLL, see Graph 5.10.

\subsubsection{School influence on student intentions}

The influence of the single sex schools will be discussed in Chapter 10 on gender differences. The difference in intentions among students going to the two mixed schools is interesting. Just under half the 131 students expecting to go to Freesia intended to study a FL and only $30 \%$ had decided not to study a FL whereas over half the 83 students expecting to attend Salvia were in the latter category. Differences in intentions could be attributed to several factors, including the languages offered (see next section) and the different option structures explained in the school profiles. Socio-economic factors seem an unlikely explanation since the two schools have 
similar decile ratings, and the majority of students going to both schools had previous FLL experience.

\section{Graph 5.10 Year 8 student intentions for FLL in year 9 compared with expected secondary school \\ Notes: $\mathrm{F}=$ Freesia, $\mathrm{N}=131 . \mathrm{S}=$ Salvia, $\mathrm{N}=83$. \\ Boys only $N=32$, Girls only $N=24$}

(For association between intentions and school $\mathrm{p}<.001$ )

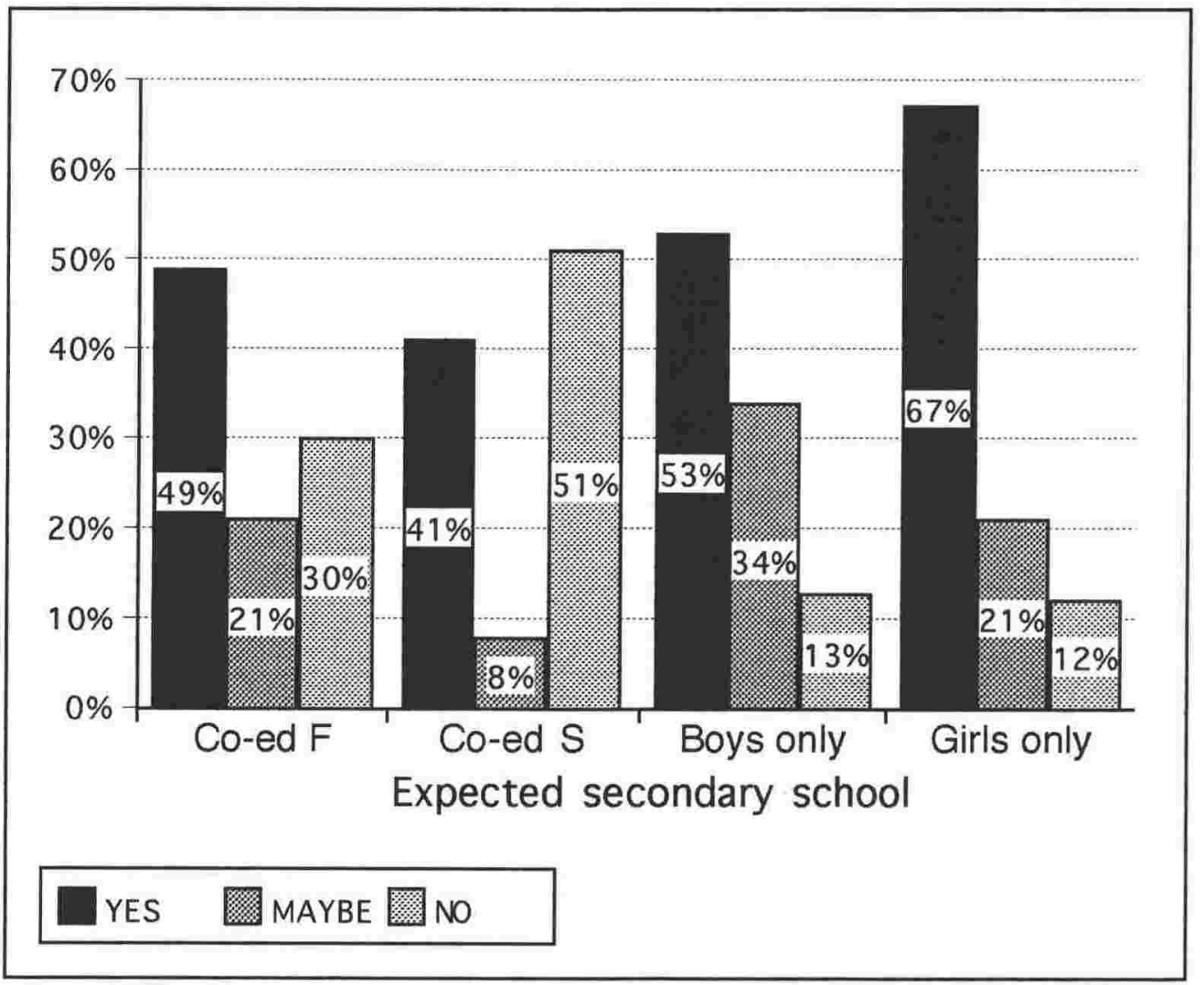

\subsubsection{The influence of available languages}

Table 5.22 shows the numbers of students who reported that they would or might study a particular language, according to intended secondary school. Freesia offers the greatest choice and the only possibility of studying Spanish. The majority of its intake comes from the neighbouring intermediate school which was, at the time of the survey, teaching Spanish or Japanese to most year 8 classes. The higher proportion of students opting to study a FL, compared with students expecting to attend Salvia, 
seems to reflect particular enjoyment of Spanish and satisfaction in being able to continue learning it. The lack of a permanent French teacher at Salvia may have influenced student intentions. Changing relief teachers can put students off and cause doubt about the future availability of a subject. Salvia students may have felt that their FL option was limited to Japanese. So few students were interested in German that the German class at Freesia did not eventuate the following year and the future of the language at Gerbera was in doubt.

Table $\mathbf{5 . 2 2}$ Year 8 student choices of language according to intended school

\begin{tabular}{|l|c|c|c|c|}
\hline & \multicolumn{4}{|c|}{ FLs students would or might study in year 9 } \\
\hline $\begin{array}{l}\text { Future } \\
\text { school }\end{array}$ & French & Spanish & German & Japanese \\
\hline Begonia & 10 & & 7 & 10 \\
\hline Freesia & 13 & 28 & 7 & 48 \\
\hline Gerbera & 9 & & 2 & 11 \\
\hline Salvia & 16 & & & 28 \\
\hline
\end{tabular}

In response to item 8.1.15, "There isn't a good range of languages for me to choose from for next year", 35\% of students agreed while $43 \%$ disagreed $(\mathrm{N}=304)$. The responses were also compared with the choice of secondary school, see Table 5.23.

Table 5.23 Responses to item 8.1.15: "There isn't a good range of languages for me to choose from for next year" $(\mathrm{N}=271$, 'No opinion' $=1)$

\begin{tabular}{|l|c|c|c|c|}
\hline & \multicolumn{3}{|c|}{ Student responses } & \\
\hline Future school & Agreed & Not sure & Disagreed & Totals \\
\hline Begonia & 5 & 8 & 20 & 33 \\
\hline Freesia & 32 & 34 & 65 & 131 \\
\hline Gerbera & 7 & 4 & 13 & 24 \\
\hline Salvia & 48 & 11 & 23 & 82 \\
\hline Totals & 92 & 57 & 121 & 270 \\
\hline
\end{tabular}


The least concerned about the range of languages available were the 33 boys destined for Begonia, 20 of whom disagreed with the item. About a half of the 155 students destined for Gerbera and Freesia disagreed. Not surprisingly the most disappointment was expressed by the 82 students staying at Salvia, where $58 \%$ of them were dissatisfied with the languages offered. Apart from the lack of interest in French, discussed above, students were probably aware that other secondary schools in the district offered more FL options, a choice of three or four, compared their two.

\subsubsection{The influence of different course lengths}

Item 8.1.7, "It's a pity that language options next year are only for half the year", proved to be directly relevant only to those students expecting to go to Freesia, although language options at Salvia also lasted less than one year. Student responses could, however, be considered a useful indication of their general attitudes to FLL and proved to have a significant association $(\mathrm{p}<.001)$ with their intentions for year 9 , see Graph 5.11. .

\section{Graph 5.11 Responses to item 8.1.7: “It's a pity that language options next year are only for half the year", compared with intentions for year 9.}

(For association between item responses and intentions $\mathrm{p}<.001$ )

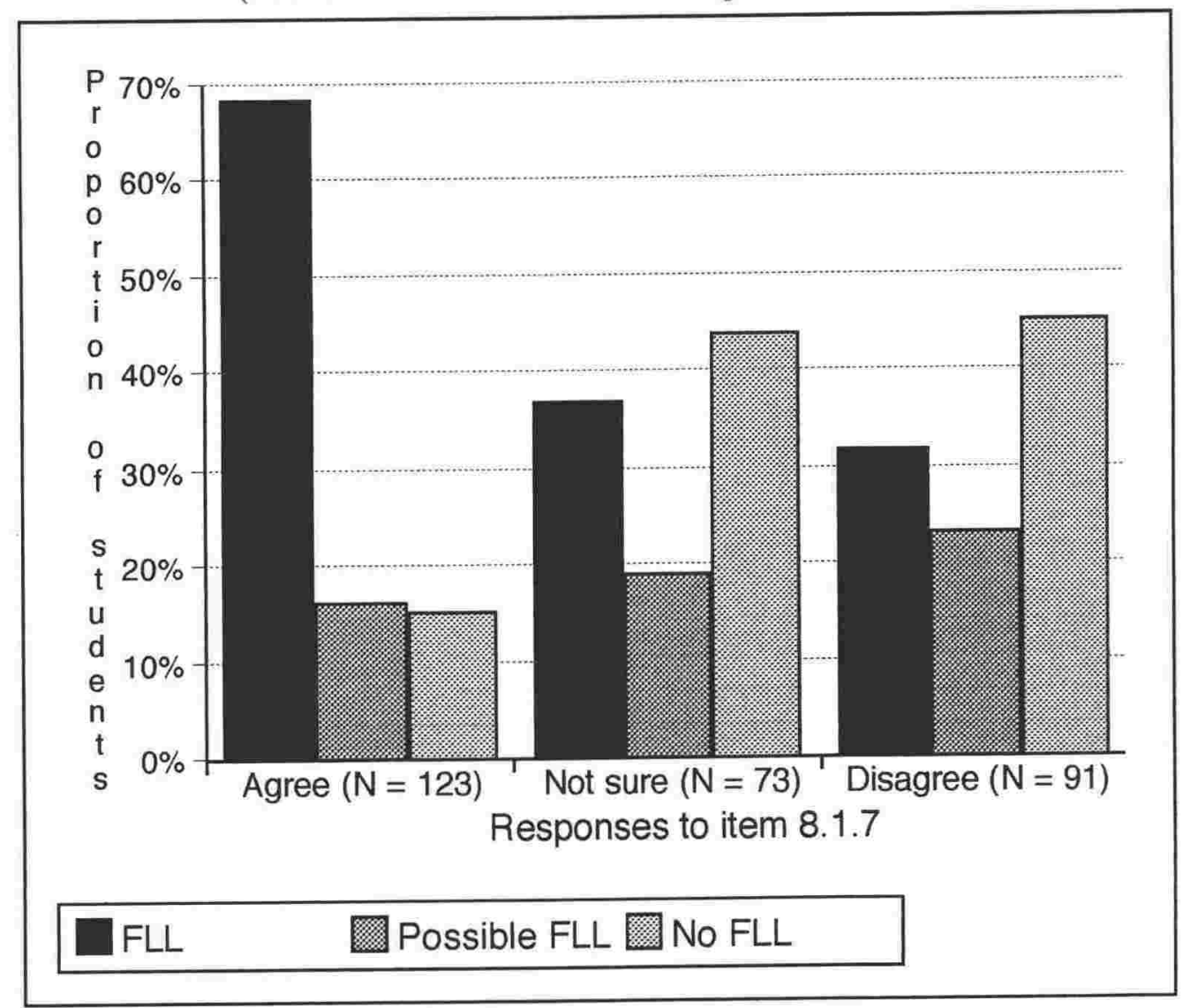


Despite this, it appears that the length of course was not a deciding factor in student choices. Rather the responses support the hypothesis that students who intended to study a FL had a clear personal interest in FLL, as they were clearly more disappointed at the prospect of short courses than those who were undecided or not intending FLL. The fact that 18 students indicated 'no opinion' suggests that students who were unsure or disagreed with the item may not have known how long option courses were or that they had not thought about the matter.

\subsubsection{The importance of the FL teacher}

One factor of ten cited as a barrier to continued FLL after year 9 is the fact that in most secondary schools there is usually only one teacher of a particular FL. Thus, if students take a dislike to a FL teacher they may decide against continuing that FL, knowing that they would always have the same teacher. By comparison, it is usual for students to meet a different teacher of, say, English or Mathematics each year. However, $61 \%$ of the students who reported that they would or might study a FL (N = 239) disagreed with item 8.1.9, "I'll try a language next year but I won't go on with it if I don't like the teacher". It should be noted that most of the students were giving a completely hypothetical opinion, not knowing anything about the teachers they might have. Nevertheless, some students would have heard of FL teachers through older siblings or friends, and students at Salvia would certainly know the Japanese teacher. These responses seem to lend more support to the hypothesis that students who intended FLL had a great interest in such learning and would therefore be keen to pursue their learning despite any perceived failings in their teacher.

\subsubsection{Summary of comparison of intentions with future secondary school}

Overall, intentions to study a FL or not in year 9 varied according to the secondary schools which students expected to attend, for a variety of reasons. It is, however, difficult to judge to what extent the secondary schools actually influenced student choices, apart from the differing range of FLs offered and the particular class structure at Begonia which will be discussed in the next chapter. Teachers did not seem to have much influence over student intentions, directly or indirectly. This is similar to ERO's (1994) findings, but contrasts clearly with research showing that teachers have a great influence over student progress in L2 acquisition (Tremblay \& Gardner 1990, Dörnyei 1996). Chapter 9 will show that teachers themselves believe that some of their colleagues do play a role in dissuading students from undertaking or continuing FLL.

\subsection{The influence of peers and older siblings on intentions \\ 5.12.1 The influence of peers}

In response to the suggestion that staying with their best friend was more important than which option subjects they chose, (item 8.1.3, N = 300), students were fairly evenly divided between agreement (37\%) and disagreement (42\%), and there was no significant association between responses to this item and student intentions. 
Another item, 8.1.5, was also designed to ascertain if students wanted to be learning the same things as their friends, although more indirectly. The suggestion that they would like to be able to practise another language with their friends provoked a particularly large majority $(80 \%, \mathrm{~N}=303)$ in agreement among students intending to study a FL. While the prospect of sharing learning with friends may not necessarily influence the choice of option subjects it may do so if students have experienced FLL which, these days, generally involves a considerable amount of interaction between students as well as between students and teachers.

\subsubsection{The influence of older siblings}

Item 8.1.20, similar to the previous one discussed above, was designed to see if the fact that a student's older brother or sister had learnt a FL might influence their own decision about FLL. 38\% of the students intending or considering FL study ( $\mathrm{N}=$ 178), who responded to the item, agreed that they would like to practise another language with an older sibling, but $48 \%$ disagreed. Disagreement could be interpreted in several ways, not necessarily indicating that their older siblings had no influence on students' subject choices.

\subsection{Intentions for Year 9 compared with attitudes}

\subsubsection{Intentions compared with general attitudes to FLL}

The general attitude scores discussed in 5.5 were aggregated on a scale of 1 to 3 , representing respectively negative, neutral or mixed, and positive attitudes. These scores were then compared with the students' intentions for year 9. The results are shown in Table 5.24. Not surprisingly, two thirds of students with positive attitudes towards FLL intended to study a FL, with a further $10 \%$ still undecided. What is more interesting is that under half of those with a negative score had decided against FLL with the rest evenly divided between intending and thinking about FLL. Of those with neutral or mixed attitudes towards FLL one half intended nevertheless to study a FL.

Table 5.24 Student intentions for FLL in year 9 compared with their general attitudes to FLL

\begin{tabular}{|l|c|c|c|c|}
\hline \multirow{2}{*}{$\begin{array}{l}\text { General } \\
\text { attitude to FLL }\end{array}$} & N & \multicolumn{3}{|c|}{ Intending FLL in year 9 } \\
\cline { 3 - 5 } & 90 & $67 \%$ & $10 \%$ & $22 \%$ \\
\hline Positive & 117 & $50 \%$ & $22 \%$ & $27 \%$ \\
\hline Neutral/mixed & 98 & $27 \%$ & $27 \%$ & $46 \%$ \\
\hline Negative & \multicolumn{4}{|c|}{} \\
\hline
\end{tabular}

Thus it seems that positive attitudes do encourage students to undertake FLL, but that less than positive or outright negative attitudes do not necessarily mean that students will not opt to study a FL. Whether this intention comes from the students 
themselves or is the result of external forces is open needs further investigation. What will be particularly disappointing for language teachers is that $22 \%$ of students with positive attitudes did not intend to study a FL. This, alongside other evidence of interest in languages among those not intending FLL, already discussed, shows clearly that there are other factors outweighing students' own attitudes in their decisions about option subjects, as shown by previous research (ERO 1994, Williams \& Burden 1997, Johnson 1998).

\subsubsection{Intentions compared with attitudes to FLL experience}

Student attitudes towards FLL experience were compared to their intentions in a similar way to the comparison made with their general attitudes towards FLL. As shown in Table 5.25, the 105 students with positive attitudes were divided in their intentions in very much the same way as discussed in the previous section. Differences were more apparent among the 120 students with mixed or neutral feelings towards their FLL experience. They were less likely to opt for future FLL, only $38 \%$, compared with the $50 \%$ of students with mixed or neutral attitudes towards FLL in general. Very few students had a completely negative attitude towards their FLL experience, and only just over a half of the latter had decided against further FLL.

Table 5.25 Student intentions for FLL in year 9 compared with

their attitudes towards their FLL experience

\begin{tabular}{|l|c|c|c|c|}
\hline \multirow{2}{*}{$\begin{array}{l}\text { Attitudes to } \\
\text { prior FLL }\end{array}$} & & \multicolumn{3}{|c|}{ Intending FLL in year 9 } \\
\cline { 3 - 5 } & N & Yes & Maybe & No \\
\hline Positive & 105 & $66 \%$ & $13 \%$ & $21 \%$ \\
\hline Neutral/mixed & 120 & $38 \%$ & $24 \%$ & $38 \%$ \\
\hline Negative & 17 & $12 \%$ & $35 \%$ & $53 \%$ \\
\hline
\end{tabular}

Comparing the results in Tables 5.24 and 5.25 seems to indicate that a positive experience of FLL at intermediate level did not increase the likelihood that students would intend to study a FL in the following year, nor reduce the proportion of those who did not intend to study a FL. On the other hand, less than positive experiences had a negative impact and considerably reduced the proportions of students intending to opt into FLL in year 9.

\subsection{Final analysis}

The analysis of the data discussed above reveals much about what the year 8 students knew, believed or thought about FLL, their intentions regarding FLL the following year and the reasons for those intentions. The above results above were compared with the literature discussed in chapters 2 and 3 which shows that certain notions, which some would call 'myths', are prevalent in other English-speaking countries, namely that FLs are difficult 
subjects and, therefore, suitable only for the brightest students, or that FLs are not as important as other subjects, largely because they are not 'useful', particularly as far as employment is concerned.

FLL is considered difficult in Australia, the United Kingdom and the United States as well as in New Zealand (for example: ALLC 1994, Gregory 2000, Trim 1999, ERO 1994). This belief is also supported by considerable anecdotal evidence in New Zealand. However, the belief was not found to be prevalent among the majority of the surveyed year 8 students, except in relation to learning to write in a FL, see section 5.5.1. This was only to be expected since, as most FL teachers would agree, it is usually the hardest skill for students to master, and the last to be taught in depth. The students involved in this research were usually complete beginners, undertaking only short courses with limited objectives, which focussed on oral skills. That FLL might be considered suitable only for the most intelligent students would probably not have occurred to most of these students because they were in classes of mixed ability where all were involved in all areas of learning, including FLL if they being taught. In the two larger schools involved the fact that some classes missed out on FLL was usually due to the lack of a suitable teacher, not to the nature of the students. Only in the case of the small primary school was FLL an optional extra. Thus it was not being indicated in any way to the majority of students that FLL might be considered as suitable for only an intelligent minority of them. In addition, most students were likely to have been unaware of the situation which still exists in some schools where FLL is encouraged, or even allowed, only in top stream classes.

Furthermore, when it came to stated reasons for not intending to undertake FLL in year 9, difficulty was not a factor mentioned by many students, see section 5.6.2.4. This could have resulted from the fact that they were only exposed to short FL courses, which might mean that they were still enjoying something new and challenging, see section 5.4.1. Perhaps the novelty had not worn off, which might have been the case if they had continued and found the learning more demanding, especially as they needed to do more reading and writing. Some students may well have realised that further study might be more difficult for them since confidence in their ability to cope with future FLL, see section 5.6.2.4, was a significant factor associated with their stated intentions for the following year.

The notion that FLs are not as important as other subjects was supported to some extent by the year 8 students, probably because they saw other subjects, such as English or mathematics, allocated much more class time and for the whole year. On the other hand, although the majority of the students knew that FLs were among optional subjects at their future secondary schools, and that they could choose to study one or not, it is unlikely that they were aware of how much FLs have to compete with other subjects for timetable space, how little FLL they might actually experience in year 9 even if they did choose a FL as one of their options and the extent to which senior classes might be combined or disappear completely. It is encouraging to note that the findings do not suggest that the students thought that FLL would detract from their development of English language skills at all, although this is another 'myth' for which there is considerable anecdotal evidence among 
adults in New Zealand.

It was also encouraging to discover that, despite the commonly accepted idea that English is becoming 'the' global language, most of the students manifested an awareness of the importance of LOTEs in the world, see section 5.4.4. Students might have been aware of the growing multicultural nature of the New Zealand population, a factor mentioned by many of the adults interviewed as a reason for favouring more FLL, at primary or intermediate level. On the other hand, the belief which many students expressed, that knowledge of a FL would be useful for future employment, was obviously misguided since research has shown that most job recruitment in New Zealand takes very little account of FL skills (Enderwick \& Gray 1992, Shackleford 1996a). This situation was reflected in the opinions of parents and teachers involved in this research, discussed in chapters 8 and 9, especially among teachers responsible for advising students about tertiary study and careers, and consequent subject choices. It is possible that intermediate and primary teachers were justifying the introduction of FLL into the curriculum by stating that FL skills would be 'useful' to students. Unfortunately, the findings suggest that most students were unaware of the usefulness of FLL in terms of the transference of skills, see section 5.5.2. It may be that neither the class teachers responsible for teaching English nor the language specialists (where these existed) were attempting to make the links, or simply that students were not absorbing such information. This will be disappointing for those who propose skills transference as one of the main arguments for FLL in schools (MoE 02).

As shown in sections 5.4.6 and 5.5.5 students had largely positive attitudes towards FLL in general and their own experience of FLL, but analysis shows that their knowledge of this area of education was, on the whole, limited by their own or their parents' experience, or by what they had read in secondary school prospectuses. There was some disparity between what students reported they were really interested in and what they thought they could or should study at school, in terms of their ability, career ideas or the often limited advice they were receiving. This may account for the fact that, when it came to analysing the conative aspect of student attitudes towards FLL, the positive cognitive and affective aspects of their attitudes revealed by the findings this research did not translate into such positive readiness for action, a possibility foreseen in section 2.2. As shown in section 5.6.1, the proportion of students intending to study a FL in year 9 reflected the typical national average of about one half.

Lastly the analysis of the year 8 survey data sought to ascertain what the main reasons were for the students' intentions concerning FLL in year 9. The fact that a particularly large proportion of students had prior FLL experience, which most had enjoyed, seemed to have had little effect on the overall numbers intending to study a FL the following year, see section 5.8.1. Nevertheless, about half of the reasons cited by students for their stated intentions to study a FL the following year, shown in section 5.6.2.1, were related to their prior experience of FLL, their enjoyment of it, their sense of achievement or their desire to continue something they had started. This raises the question as to whether a similar proportion of this particular sample would have intended to undertake FLL at secondary 
school if none of them had had any prior FLL experience.

There was little evidence that the schools or individual teachers had much influence on student intentions, as ERO (1994) found, nor that students were greatly influenced by their peers or older sibling. On the other hand, parents were clearly responsible for many of their children's attitudes and intentions, either directly or indirectly, see section 5.9. One of the criticisms made by the adults interviewed was that students often had to make uninformed decisions about FL options because of their own and their parents' lack of FLL experience. While most students felt that they were making independent decisions about studying a FL or not, it is clear from the data that they were influenced by their parents' views and experience, as many researchers have shown (Turner 1974, Oskamp 1977, ERO 1994, Gardner, Masgoret \& Tremblay 1997)

All of the above leads to the conclusion that year 8 student attitudes towards FLL were generally positive. Because of the small number of students in the sample who had had no experience of FLL it is impossible to judge to what extent these attitudes were the result of this experience or might have existed anyway. Judging from what the year 8 students reported about their intentions regarding FLL in year 9 it can be seen that the influences on their choices were numerous and interlinked. It is evident that positive attitudes alone did not ensure that students would decide to study a FL at secondary school, nor is it clear that any of the influences discussed above can be considered predominant.

The next chapter will discuss the attitudes and choices made by the whole year 9 student sample. That will be followed by a comparison of the year 8 and 9 results for the small sample of students who participated in both surveys. 


\section{RESULTS AND DISCUSSION - YEAR 9 STUDENT ATTITUDES TO FOREIGN LANGUAGE LEARNING AND CHOICES MADE}

Learn a new language and get a new soul. Old Czech proverb (Roskens 1979)

\subsection{Introduction}

This chapter addresses three research questions:

- Were the year 9 students studying a FL or not?

- What were the reasons for their decision to study a FL or not?

- Who or what were the main influences on their decisions?

The students surveyed were in year 9 at five secondary schools in the same area as the schools involved in the year 8 survey. Owing to greater cooperation from secondary schools than from intermediate schools I was able to survey a much larger student sample in 2000. Unfortunately, only 169 students participated in both surveys, fewer than I had anticipated. Chapter 7 will present a comparison of the results for this sub-sample in order to ascertain to what extent they carried out their reported intentions in regard to FLL, and the extent to which previous experience of FLL influenced their final choices. This chapter examines the responses of the whole year 9 sample in relation to the above questions.

Having established whether or not students were studying a FL in year 9, I compared their decisions with all the relevant Likert scale survey items to determine who or what had had the most influence on their choices regarding FLL. Students involved in FLL were asked if they intended to continue in year 10 and what reasons they had for their decisions. As with year 8 students, I analysed all the results for gender differences which will be discussed in Chapter 10.

Detailed profiles of the schools will be followed by some basic facts about the surveyed students and the choices they had made about FLL. The following sections will discuss the attitudes of students towards FLL as revealed by their responses in part 2 of the survey, completed by all the students, part 3 for those who had chosen to study a FL, and part 4 , for those who were not undertaking any FLL. The final section will examine possible influences on student choices.

\subsection{School Profiles}

\subsubsection{General information}

I obtained information from school prospectuses, ERO reports, information booklets for year 9 students and discussions with principals and other staff. Appendix 2 gives full details of the ethnic and gender balance of students and school decile ratings. Salvia, the only Catholic secondary school in the area, serves students from the whole area, as do the two single sex schools, Begonia (boys) and Gerbera (girls). The other two co-educational schools, Freesia and Arum, serve particular catchment areas. In 2000, no two schools offered the same opportunities for FLL. Table 6.1 
(see Appendix 12) gives full details of the place of FLs in the each school. FL class numbers in Tables 6.2 to 6.7 were supplied by the FL teachers.

\subsubsection{Arum}

ERO (1998a) reported that the school was "in a period of transition", with a new principal, board, management structures and style of leadership, all of which seemed to be improving the standing of the school in the community and increasing the school roll. One innovation has been the introduction, in 1999 after community consultation, of some SLL for all year 9 students. The principal reported that there there had been good feedback about the module system as a whole and no resistance from students or parents to the compulsory language element, probably because the initial commitment was for only one term. The principal was the most supportive of FLL among the secondary principals interviewed.

All year 9 students had to study three modules per term (i.e. 12 per year), for three 50 minute spells per week. Art, music, Maori studies and a language were compulsory and students had a wide range of subjects from which to choose five other modules. In term 4 they had to continue three of their previous modules. The principal acknowledged that the module system was very complicated, especially as far as languages were concerned. For example, a student could study Maori or ESOL in all four terms. French and Japanese could be started in terms 1 or 2, but had to be continued in terms 3 and 4 if a student wished to continue FLL in year 10. The more language modules students chose, the fewer other subjects they could sample. All the languages were offered up to Bursary level but the prospectus noted that if classes were not possible for any reason, students and parents would be consulted and alternatives discussed. The school acknowledged, however, that very limited advice and guidance about optional subjects was offered to students entering the school or moving up each year.

Table 6.2 Arum FL student numbers 2000

\begin{tabular}{|c|c|c|}
\hline Year group & French & Japanese \\
\hline 9 & 55 & 37 \\
\hline 10 & 20 & 22 \\
\hline 11 & 18 & 8 \\
\hline 12 & $7 *$ & 6 \\
\hline 13 & $2 *$ & 4 \\
\hline * Indicates students in combined classes \\
\hline
\end{tabular}


The school had full-time teachers of French and Japanese (a native speaker) but neither had the status of head of department. I interviewed both and they supplied details of the general trends in their subjects. Class numbers for the year 2000 , shown in Table 6.2, were reported to be similar to those of the previous year although there had been some reduction in Japanese, previously the most popular language. The FL teachers surmised that some students had chosen French or Maori because they had already learnt some Japanese. Both stated that they had little or no contact with feeder schools and little knowledge of students' any previous FLL experience, but the Japanese teacher was not impressed with the level of achievement of students who claimed to have learnt some of her language.

The drop-out rate from language classes in year 10 was usually about $50 \%$, but the principal considered that the year $10 \mathrm{FL}$ numbers were reasonable. The French teacher reported that year 11 numbers fluctuated between 10 and 24 students and that it was always necessary to combine years 12 and 13 . The Japanese teacher reported that the retention rate in Japanese had been good up until 2000 and she had always been able to have separate senior classes. She attributed the decline in numbers to changes in fashion, political and economic relations or the media portrayal of Japan. The main disincentive for continuing FLL was seen to be timetabling which put languages into competition with subjects that students saw as more interesting, more important or easier.

The French teacher reported that her students were of mixed ability and that not all those in year 11 were really "School Certificate material". She was not concerned about examination results provided students were enjoying the subject and learning something. She reported that she did not give individual students advice about continuing French unless they asked. She did not always know if they intended to continue and did not like to ask or put pressure on them in any way. Japanese seemed to attract and retain brighter students as the teacher reported that her examination results were excellent. She was more active in encouraging students to continue if they were interested, regardless of ability, but felt that some of her colleagues advised students against continuing without consulting her. She was pleased to report that the careers adviser had developed a more positive towards Japanese since her own son's experiences and was encouraging students to continue FLL. The Japanese teacher particularly valued the role of exchange trips with Japan for encouraging students to continue Japanese.

\subsubsection{Begonia}

In 1999, ERO reported that this large, traditional boys' school, with approximately 1500 students, predominantly Pakeha, had "a broad range of conventional, academic subjects' and a good record of student achievement. However, ERO asserted that the school needed to modify its conservative approach to curriculum content, assessment and reporting to meet the requirements of the National Education and Administration Guidelines and the needs of an increasingly diverse student body. 
Begonia had the most extensive streaming system of all the surveyed secondary schools. On entering the school boys were tested and sorted into classes according to academic ability. The streaming process had substantial implications for students as far as FLL was concerned. The school strongly supported the traditional view that SLL should be part of the education of academically brighter students and that it was not suitable for those of lesser ability. Thus students in the top four streams had to study performance music or a language as one of their two options. Students in the next three streams could opt to study a language and a few did so in 2000. These top seven streams chose two full-year options from a range of twelve subjects, including three FLs, Maori and ESOL. Option classes had 4 spells over a 7-day cycle. Of the remaining classes, five were "mixed ability" and one a small "development class" for special needs students. These lower streams had only one option subject and spent more time on core subjects. In theory, the students could choose a FL as their option but in practice this did not occur because it was not encouraged and because classes were filled by students in the upper streams. All students had to indicate at least two option choices on enrolment, including music or a language since they did not know at that stage which stream they would be in. The parents of a boy in one of the mixed ability classes reported that their son had listed French as one of his choices. When their son was placed in his class he was allocated his other choice without consultation. While the boy was apparently quite happy with this subject the parents did admit to some surprise at the school's action.

With regard to senior classes, the prospectus noted that "obviously the existence of subjects is dependent upon there being viable class numbers and staff availability". In fact there were rarely classes available for students in year 12 and 13 except in Japanese and that was likely to be a combined class. Senior students of French or German, if there were any, usually had to study by correspondence. Table 6.3 shows the fluctuating student numbers in FL classes over four years. The drop-out rates for students moving into year 10 ranged from $56 \%$ to $63 \%$ over those years, which the principal considered disappointing. He thought that the school could do better but that languages suffered from competition with other subjects which boys found more relevant for their future employment.

Once again, the major disincentive for continuing FLL was seen to be the clash of languages with other subjects that students saw as more interesting, more important or easier. In addition, most year 9 FL students were usually in the top three classes which were accelerated to sit their first examinations a year earlier than usual and move on to year 12 levels in year 11. This system, in the opinion of most FL teachers, did not permit adequate progress in FLL, given limited option time and tended to discourage students. In any case, according to some staff, students had little incentive to continue their FLL unless they were particularly keen, and in some cases influential colleagues actively discouraged students from continuing. This could be a reflection of the extremely monocultural outlook of the entire school, identified by the 1999 ERO report. 
Table 6.3 Begonia FL student numbers 1997 to 2000

\begin{tabular}{|c|c|c|c|c|c|c|c|c|c|c|c|c|}
\hline \multirow{2}{*}{$\begin{array}{l}\text { Year } \\
\text { group }\end{array}$} & \multicolumn{4}{|c|}{ French } & \multicolumn{4}{|c|}{ German } & \multicolumn{4}{|c|}{ Japanese } \\
\hline & 197 & 198 & 199 & 100 & 197 & 198 & 199 & 100 & 197 & 198 & 199 & 100 \\
\hline 9\# & 52 & 59 & 57 & 49 & 27 & 24 & 25 & 30 & 52 & 41 & 37 & 42 \\
\hline 10 & 25 & 21 & 19 & 21 & 11 & 8 & 10 & 8* & 18 & 25 & 17 & 23 \\
\hline 11 & 4 & 13 & 12 & 6* & 0 & 7 & 6 & 4* & 17 & 9 & 10 & 11 \\
\hline 12 & 0 & 0 & $3 \dagger$ & 3* & 0 & 0 & $1 \dagger$ & $1 \dagger$ & 3 & 6 & 0 & 4* \\
\hline 13 & 0 & 0 & $1 \dagger$ & 0 & 0 & $2+$ & $2 \dagger$ & 0 & 6 & 8 & 6 & 4* \\
\hline $\begin{array}{l}\text { \# Usual } \\
\text { * Stude } \\
\text { † Studen } \\
\text { Y } 12 \text { stuc }\end{array}$ & $\begin{array}{l}\text { ly } 2 \mathrm{c} \\
\text { nts in } \\
\text { ts stu }\end{array}$ & $\begin{array}{l}\text { asses } \\
\text { combi } \\
\text { dying } \\
\text { Gern }\end{array}$ & $\begin{array}{l}\text { of Fr } \\
\text { ned c } \\
\text { by co } \\
\text { han in }\end{array}$ & $\begin{array}{l}\text { nch a } \\
\text { lasses } \\
\text { rresp } \\
2000\end{array}$ & $\begin{array}{l}\text { nd Jap } \\
\text { ndenc } \\
\text { also a }\end{array}$ & anese & and & of $\mathrm{G}$ & & & & \\
\hline
\end{tabular}

\subsubsection{Freesia}

This large, co-educational school offered, according to ERO (1998b), "a stimulating academic, social and cultural environment", emphasised individual needs and saw education as more than academic achievement, offering a wide range of cultural, physical and social activities. It attracted students from a wide range of backgrounds and ability. There was a larger proportion of girls than boys, suggesting that parents of boys in the area tended to prefer a more traditional style of education for their sons.

Year 9 classes were broadly streamed, the majority of students being in mixed ability classes, with two "extension" and two "support" classes in 2000. The year 9 programme had a large degree of subject integration involving English, social studies, information technology and health, and a strong bicultural focus, all taught by one teacher per class. According to ERO (1998b), the programme seemed effective in terms of student achievement and enjoyment.

Year 9 students had to choose two "major" options, studied for half the year. These options included the largest range of languages of all the local secondary schools: French, German, Japanese, Spanish and Maori, although the numbers opting for German in 2000 proved too small for the class to run. Other major options were practical subjects, some of which were also available as one term, "minor" options, of which students studied four. Compared with the other schools, language subjects had less competition. In 2000, major options were taught for only two spells per 6 
day cycle, a reduction in class time from the previous system. The new system meant also that students who studied a FL in the first half of the year would be at a

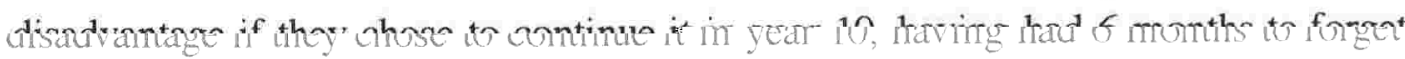
what they had learnt. In previous years all students proposing to continue FLL had been grouped into an extension class at the end of year 9 to bring them all up to the same level. The principal reported that pressure was being put on all curriculum areas, but especially the options, because of the need to incorporate new core curriculum, such as health and technology.

Freesia had the highest drop-out rate of language students of all the surveyed schools, approximately $75 \%$ of students moving into year 10 . Students were not warned that there might be no classes at senior level though that would probably occur, except for Japanese, if trends continued. I was surprised to discover that the ESOL department had instigated beginner Mandarin classes for fee-paying Asian students in years 12 and 13, and without any discussion with the languages department. There seemed to be no possibility that such classes would be available to the main student body at any level in the foreseeable future.

The 2000 prospectus stated "students who have studied a language previously are asked to consult the Language Department for their appropriate course". The HOD reported that it was rare for students to arrive with sufficient prior knowledge to go straight into year 10 courses. However, Freesia is next door to its main feeder school, Rose, and there was close co-operation between the two schools for the teaching of Spanish and Japanese which the secondary teachers were keen to continue. The Spanish teacher was able to run accelerated classes so that the year 10 students were going to enter School Certificate and the year 11 and 12 students were studying for Sixth Form Certificate and Bursary respectively. There was little communication with other feeder schools about FLL programmes. The HOD reported that there was no consistency in FLL teaching at intermediate level and that students tended to arrive with a wide variety of levels of achievement, but they usually recognised the considerable gaps in their knowledge and fitted well into classes.

Table 6.4 shows the FL student numbers in 2000. The HOD reported that the year 9 roll had increased but that the proportion of students in FL classes had dropped. There was a similar decline in numbers continuing in year 10 which could be attributed partly to the reduced number of options available in year 10. The HOD was particularly pessimistic about the future of German. Given the small numbers in year 10 and 11 European language classes it was surprising that those classes were allowed to run, and the HOD admitted that there was some resentment from colleagues because other option subject classes would have been particularly large. Some compulsory FLL had been attempted in the past but abandoned because too many students were unmotivated and resented the classes. Although the principal believed that FLL was very important and was concerned about dropout rates, he clearly had more urgent priorities to consider. He suggested that the school might 
have to reduce the number of FLs offered in order to maintain viable class numbers and seemed to favour Spanish and Japanese.

Table 6.4 Freesia FL student numbers in 2000

\begin{tabular}{|c|c|c|c|c|}
\hline Year group & French & German & Spanish $\diamond$ & Japanese \\
\hline $\mathbf{9}$ & 37 & 0 & 43 & 60 \\
\hline $\mathbf{1 0}$ & 9 & 4 & 9 & 17 \\
\hline $\mathbf{1 1}$ & 5 & $5 \#$ & $4 \dagger$ & 17 \\
\hline $\mathbf{1 2}$ & $2 \dagger$ & & $2 \dagger$ & 17 \\
\hline $\mathbf{1 3}$ & $4 \dagger$ & $1 \dagger$ & & 10 \\
\hline $\begin{array}{l}\text { † Students studying by correspondence } \\
\text { \# Includes one Y12 student repeating School Certificate }\end{array}$ \\
$\begin{array}{l}\text { Y13 student of German and 2 Y13s in French were overseas exchange } \\
\text { students }\end{array}$ \\
\hline
\end{tabular}

\subsubsection{Gerbera}

This large, decile 9 girls' school had students from the most prosperous socioeconomic backgrounds. In 1995, ERO reported that the school provided a balanced curriculum for academic, social, sporting and cultural learning needs and that "all students are counselled in their subject choices", although the principal reported that, owing to large numbers, this was no longer possible.

In 2000 year 9 students had to choose two whole-year options from a list of ten, including French, German, Japanese and Maori, and three eight week modules out of ten subjects including French, German and Japanese culture modules. These included some elementary language skills according to the prospectus. Student choices were restricted by the necessity of including art, music and food technology as either an option or a module, and they were not allowed to study the same or similar subjects as both. Option classes consisted of three spells a week.

Whole-year FL options, not culture modules, were a prerequisite, except in exceptional circumstances, for continuing FLL in year 10, when students again chose two whole-year options. The school had by far the highest retention rates in FL classes between year 9 and year 10. Combined classes for years 12 and 13 French and German had only existed since the previous year. It was reported that year 9 numbers in Japanese had dropped by about 30 (i.e. a whole class) from the previous year, with more students opting for French instead. At year 11, the Japanese 
numbers were half those of the previous year. German had had very stable numbers over the previous ten years but had suddenly declined in popularity to the extent that its future was uncertain. The FL teachers seemed uncertain as to the reasons for these changes. Table 6.5 shows the full 2000 FL student numbers.

Table 6.5 Gerbera FL student numbers in 2000

\begin{tabular}{|c|c|c|c|}
\hline Year group & French & German & Japanese \\
\hline $\mathbf{9}$ & 47 & 12 & 45 \\
\hline $\mathbf{1 0}$ & 32 & 14 & 31 \\
\hline $\mathbf{1 1}$ & 22 & 9 & 19 \\
\hline $\mathbf{1 2}$ & $13^{*}$ & $7^{*}$ & 14 \\
\hline $\mathbf{1 3}$ & $6^{*}$ & $6^{*}$ & 16 \\
\hline * Students in combined classes \\
\hline
\end{tabular}

The school had discussed changing the range of languages offered. The principal had suggested replacing German with Mandarin when the previous German teacher left and student numbers were declining. She considered Mandarin more relevant although there had not been any community demand for the language. The Board of Trustees favoured Spanish as an easier language to learn, but the school had not found a Spanish teacher and had therefore appointed a replacement German teacher. The teacher of Japanese believed that there was generally resistance to any increased emphasis on Asian languages in schools, and that New Zealand underestimated the importance of Asian countries, especially China and Korea.

As in other schools, students arrived in year 9 classes with a variety of prior FLL which the Japanese teacher considered a "nightmare" although the HOD, a native French speaker, did not consider it a problem because of gaps in the students' knowledge and the flexibility of their FL courses. The problem did not arise in German.

\subsubsection{Salvia}

Some basic information was given about this school in chapter 5. According to the MoE figures for 1 March 2000, the total school roll had dropped by about 100 students to just under 800 , but the gender balance remained the same with a slight majority of girls. As noted previously, most year 9 students had been at the school since year 7 and had learnt some French or Japanese, or both. Year 9 students had to choose 5 one-term modules from a list of 10 subjects, including French, Maori and Japanese. Students considering continuing French or Japanese in year 10 had to 
study the language for a further two terms, thereby reducing the number of other optional subjects they could sample. In 2000, the first French module was in term 1 and Japanese in term 2 so that it was possible for students to try both languages. Students could not continue both as the second and third modules were timetabled together, although the school had apparently intended that students should have the possibility of studying both languages. Module classes had 3 spells per 6 day cycle. I was surprised that students who had already had what might be termed 'taster' modules of FLL in years 7 and 8 should be faced with a similar system again, although some students, of course, entered Salvia from other primary or intermediate schools in year 9. The module system could also be considered somewhat at odds with the ERO (1997) report that the school was proposing an integrated programme for years $7-10$.

In year 10 , students chose two whole-year options. Students were warned in the prospectus that classes would not run if student numbers were too small, but were advised that they could continue by correspondence. Table 6.6 shows the FL student numbers in 2000. Those for year 9 were of the students opting for the first language module, as the survey was carried out during term 1 . The numbers were lower than in previous years, owing to the declining school 9 roll, and there had previously been two Japanese classes. This meant that the apparent drop-out rate for year 10 of about $70 \%$ was even higher, probably similar to that at Freesia.

The number of senior students studying French by correspondence was reported to be similar to previous years, and senior numbers in Japanese were relatively good. According to the HOD, the small senior numbers reflected the relatively low proportion of highly academic students, but those who persisted with FLL were successful in examinations, and she reported an $80 \%$ pass rate in School Certificate and $100 \%$ in Bursary. The small year 11 Japanese class was the result of a student intake of particularly poor academic ability. Such a small class at this level did not bode well for the survival of senior classes although that might have been only a temporary downturn.

The principal believed that FLL was very important and declared that he would like to see larger senior classes. On the whole, however, the principal was happy with the current situation for FLs. He reported that increased core curriculum was putting pressure on all optional subjects and students had to consider what subjects were more important to them. The HOD pointed out that, because of the nature of the school, compulsory religious studies was also putting pressure on the timetable. The principal had thought about replacing French with Spanish, which the HOD also saw as a possibility, partly because of the difficulty the school had in retaining a French teacher. The HOD was also considering the possibility of offering a starter Spanish course for year 12 . 
Table 6.6 Salvia FL student numbers 2000

\begin{tabular}{|c|c|c|}
\hline Year group & French & Japanese \\
\hline 9 & 19 & 26 \\
\hline 10 & 7 & 7 \\
\hline 11 & $1 \dagger$ & 14 \\
\hline 12 & $2 \uparrow$ & $9 *$ \\
\hline 13 & $1 \dagger$ & $3 *$ \\
\hline * Students in combined class \\
† Students studying by correspondence \\
\hline
\end{tabular}

\subsection{The year 9 sample}

\subsubsection{Numbers surveyed}

821 students took part in the survey which produced 664 useful sets of responses, representing about $81 \%$ of those surveyed and about $55 \%$ of the year group in the five schools in 2000, see Table 6.7. 157 survey forms were discarded, some because they were incomplete or extremely inconsistent, but most because English was not the $\mathrm{L} 1$ of the students or their parents. The sample was over twice the size of the year 8 sample, covering a higher proportion of the relevant schools, and thus should provide a more representative picture of student attitudes.

Table 6.7 Number of useful survey forms by school and gender

\begin{tabular}{|l|c|c|c|c|c|}
\hline School & Girls & Boys & Totals & $\begin{array}{c}\text { Total 2000 } \\
\text { cohort* }\end{array}$ & $\begin{array}{c}\text { \% of 2000 } \\
\text { cohort }\end{array}$ \\
\hline Arum & 74 & 65 & 139 & 199 & $70 \%$ \\
\hline Begonia & & 69 & 69 & 332 & $21 \%$ \\
\hline Freesia & 98 & 98 & 196 & 317 & $62 \%$ \\
\hline Gerbera & 175 & & 175 & 261 & $67 \%$ \\
\hline Salvia & 46 & 39 & 85 & 108 & $79 \%$ \\
\hline Totals & 393 & 271 & 664 & 1217 & $55 \%$ \\
\hline * 1 March 2000 figures from MoE \\
\hline
\end{tabular}

\subsubsection{Students' previous schools}

Table 6.8 shows which intermediate or primary school the year 9 students had attended in 1999. 104 of the 139 Arum students (73\%) had come from the two intermediate schools, Mimosa and Ixia, which did not participate in the year 8 
survey. Most students at the single sex schools came from small primary schools, or from Ixia. The largest group of the 196 Freesia students, 44\%, had come, as expected, from the neighbouring intermediate school, Rose, while most of the rest, $31 \%$, were from small primary schools. $87 \%$ of the 85 students at Salvia had been in year 8 at that school.

Table 6.8 Which schools year 9 students came from

Notes on schools:

1. * Schools with Year 8 students surveyed in 1999

2. Calla is a small Year 1-8 private girls' school.

3. Mimosa and Ixia are large Year 7-8 Intermediate Schools.

4. Other are mostly small rural primary schools.

\begin{tabular}{|l|c|c|c|c|c|c|}
\hline \multirow{2}{*}{$\begin{array}{l}\text { Year 7/8 } \\
\text { Schools }\end{array}$} & \multicolumn{7}{|c|}{ Number of student surveys from year 9 schools } \\
\cline { 2 - 7 } & Arum & Begonia & Freesia & Gerbera & Salvia & Totals \\
\hline Aster* & 0 & 0 & 16 & 1 & 0 & 17 \\
\hline Calla & 0 & 0 & 2 & 14 & 0 & 16 \\
\hline Mimosa & 52 & 7 & 2 & 20 & 0 & 81 \\
\hline Rose* & 3 & 4 & 87 & 12 & 2 & 108 \\
\hline Ixia & 50 & 17 & 21 & 68 & 1 & 157 \\
\hline Salvia* & 1 & 1 & 6 & 4 & 74 & 86 \\
\hline Others & 33 & 40 & 62 & 56 & 8 & 199 \\
\hline Totals & 139 & 69 & 196 & 175 & 85 & 664 \\
\hline
\end{tabular}

\subsubsection{Students' FLL decisions}

The proportion of students who had chosen to study a FL varied greatly from school to school, from $117(84 \%)$ at Arum to $38(45 \%)$ at Salvia. Details for each school are shown in Table 6.9. The average proportion for all the schools was $58 \%$ which is relatively high compared with the national trend. If a more representative sample of boys had been obtained the proportion of boys in FLL would have been much lower. Of the 69 Begonia boys surveyed, two thirds were in the top four streams and $83 \%$ of them had opted for FLL, whereas only $26 \%$ of the rest had chosen a FL, a significant difference $(\mathrm{p}<.001)$. The average proportion of students opting for FLL 
in the three schools where no pressure was put on students to study any languages was $50 \%$. The association between student choices regarding FLL and their secondary school proved highly significant $(\mathrm{p}<.001)$.

Table 6.9 Numbers of surveyed students studying one or more FLs in 2000

\begin{tabular}{|l|c|c|c|c|c|}
\hline School & N & In FLL & $\%$ & No FLL & $\%$ \\
\hline Arum & 139 & 117 & $84 \%$ & 23 & $17 \%$ \\
\hline Begonia & 69 & 44 & $64 \%$ & 25 & $36 \%$ \\
\hline Freesia & 196 & 92 & $47 \%$ & 103 & $53 \%$ \\
\hline Gerbera & 175 & 96 & $55 \%$ & 79 & $45 \%$ \\
\hline Salvia & 85 & 38 & $45 \%$ & 47 & $55 \%$ \\
\hline Totals & 664 & 387 & $58 \%$ & 277 & $42 \%$ \\
\hline S
\end{tabular}

Nos. include 2 students studying Spanish by correspondence and one boy learning German outside school

For association between school and FLL decisions $\mathrm{p}<.001$

Very few students, only 29 , reported that they would be studying two languages in year 9. Most were at Arum as it was possible for them to sample two FLs. Similar taster courses at Salvia encouraged only two girls to try two FLs, presumably because the majority had already learnt some of the two FLs available.

\subsection{General attitudes towards FLL}

\subsubsection{Introduction}

The attitudes towards FLL of the whole year 9 sample were investigated by the 20 items in Part 2 of the survey. These included statements suggesting that FLL is difficult, boring or not useful, or a subject more suitable for girls than boys. As with the year 8 analysis, student responses are shown with 'agree' and 'strongly agree' grouped together and similarly 'disagree' and strongly disagree', unless I felt it valuable to comment on the respective responses separately. The responses to all items were compared with the students' decision to study a FL or not to ascertain if there were significant associations. It must be noted that however significant a association it does not necessarily signify that the factor in question directly caused students to choose FLL or not. It is possible that students had formed their opinions after starting their FL classes. All responses were also investigated for gender differences which will be discussed in Chapter 10. 
Table 6.10 Student decisions about FLL compared with responses to item 9.2.12: "Learning a foreign language is too hard for most students."

\begin{tabular}{|l|c|c|}
\hline Item response & In FLL & Not in FLL \\
\hline Agree $(\mathrm{N}=112)$ & $44 \%$ & $56 \%$ \\
\hline Not sure $(\mathrm{N}=220)$ & $60 \%$ & $40 \%$ \\
\hline & & \\
\hline Disagree $(\mathrm{N}=311)$ & $65 \%$ & $35 \%$ \\
\hline $\begin{array}{l}\text { 'No opinion' }=21 \\
\begin{array}{l}\text { For association between item responses and FLL } \\
\text { decisions } \mathrm{p}<.001\end{array}\end{array}$ \\
\hline
\end{tabular}

\subsubsection{Beliefs about the difficulty of FLL}

Two items were designed to assess how difficult students considered FLL. $48 \%$ of students agreed with item 9.2.1 ( $\mathrm{N}=648)$, "New Zealanders think it's clever to speak more than one language", but $40 \%$ were unsure. The idea that FLL might be associated mostly with 'brighter' students had little effect on their FLL decisions, since a majority of students had opted for FLL, whatever their responses. Only a minority of students, $17 \%(\mathrm{~N}=643)$, agreed with item 9.2 .12 , "Learning a foreign language is too hard for most students". Whether or not students had experienced FLL at intermediate level made very little difference to their opinions about its difficulty. Not surprisingly, students' perceptions of the degree of difficulty of FLL were significantly associated $(\mathrm{p}<.001)$ with their choice to study a FL or not, see Table 6.10. Nearly two thirds of the 311 students who disagreed with the statement had chosen FLL while less than half of the 112 who agreed had done so.

\subsubsection{The relative importance of FLL}

Students were asked to consider the general importance of FLL, particularly in relation to English and Maori. A comparison between responses to the relevant items and student decisions to study a FL or not showed significant associations, see Table 6.11. For three of the statements the association was particularly significant ( $\mathrm{p}<.001$ ). Of the 150 who agreed that a FL should be part of the core curriculum at secondary school, $68 \%$ had chosen a FL option. Of the 247 who disagreed that there are many more important things to learn than FLs and the 343 who disagreed that learning Maori is more important than learning a FL in New Zealand, 75\% and 67\% of students respectively had chosen to study a FL. Responses to the statement, "Everybody speaks English these days" showed less significance $(\mathrm{p}<.02)$, but still 
$63 \%$ of the 408 students who disagreed with the item had chosen a FL option.

Table 6.11 Comparison between responses to items concerned with the relative importance of FLL and student decisions to study a FL or not

Key: $\quad 9.2 .11=$ "A foreign language should be part of the core curriculum at secondary school."

9.2.20 = "There are lots more important things to learn than foreign languages."

9.2.7 = "Everybody speaks English these days."

9.2.14 = "Learning Maori is more important than learning a foreign language in new Zealand."

\begin{tabular}{|c|c|c|c|c|c|c|c|c|}
\hline & Item & 9.2.11 & Item & 9.2 .20 & Item & 9.2 .7 & Item & 9.2.14 \\
\hline Item response & $\mathbf{N}$ & $\begin{array}{l}\text { \% in } \\
\text { FLL }\end{array}$ & $\mathbf{N}$ & $\begin{array}{l}\text { \% in } \\
\text { FLL }\end{array}$ & $\mathbf{N}$ & $\begin{array}{l}\% \text { in } \\
\text { FLL }\end{array}$ & $\mathbf{N}$ & $\begin{array}{l}\% \text { in } \\
\text { FLL }\end{array}$ \\
\hline Agree & 150 & $68 \%$ & 195 & $41 \%$ & 124 & $49 \%$ & 125 & $42 \%$ \\
\hline Not sure & 193 & $67 \%$ & 189 & $60 \%$ & 122 & $57 \%$ & 173 & $56 \%$ \\
\hline Disagree & 291 & $51 \%$ & 247 & $75 \%$ & 408 & $63 \%$ & 343 & $67 \%$ \\
\hline No opinion & 30 & $37 \%$ & 33 & $36 \%$ & 10 & $30 \%$ & 23 & $52 \%$ \\
\hline $\begin{array}{l}\text { Significance } \\
\text { of association }\end{array}$ & & $\mathrm{p}<.001$ & & $\mathrm{p}<.001$ & & $\mathrm{p}<.02$ & & $\mathrm{p}<.001$ \\
\hline
\end{tabular}

What is interesting is that, even among students who responded negatively to all these items, there was, nonetheless, never a proportion smaller than $41 \%$ who had chosen to study a FL. Large numbers of students were unsure about all the statements, ranging from $18 \%$ to $29 \%$ of the whole sample, but the majority of them, between $56 \%$ and $67 \%$, had chosen a FL option. There are clearly other influences on the students' subject choices besides the perceived importance of FLL compared with other subjects. These students might have chosen FLL out of personal interest, because of parental encouragement or because they felt pressured by their school to do so, not because they saw FLL as relatively valuable. It is also possible that reluctant students who were obliged to learn a LOTE might have opted for a FL only to avoid Maori. In the case of three of these items, fairly large numbers, up to 33 , expressed no opinion and these students were the least likely to have opted into FLL, perhaps because most had not even considered it a possibility. 


\subsubsection{The usefulness of FLL}

Students were presented with four items which sought to ascertain their beliefs about the value of FLL for future employment, for developing cultural understanding and for improving English language skills. A comparison between responses to all four items and student decisions about FLL showed significant associations for only two of the items, those suggesting that FL knowledge would help students obtain employment or help them understand English better, see Table 6.12. The proportions of students showing positive attitudes who had opted for FLL ranged from $62 \%$ to $69 \%$, but among those who were unsure the majority who had still opted for FLL, was not much less, ranging from $54 \%$ to $61 \%$.

\section{Table 6.12 Comparison between responses to items concerned with} the usefulness of FLL and student decisions to study a FL or not

Key: $9.2 .15=$ "Knowing a foreign language will help students get a good job."

9.2.8 = "Lots of jobs involve working with people who don't speak English."

9.2.6 = "Students who learn a foreign language are better at understanding another culture."

9.2.19 = "Learning another language helps students understand English better."

\begin{tabular}{|l|c|c|c|c|c|c|c|c|}
\hline & Item & $\mathbf{9 . 2 . 1 5}$ & \multicolumn{1}{|c|}{ Item } & $\mathbf{9 . 2 . 8}$ & Item & $\mathbf{9 . 2 . 6}$ & Item & $\mathbf{9 . 2 . 1 9}$ \\
\hline Item response & $\mathbf{N}$ & $\begin{array}{c}\text { \% in } \\
\text { FLL }\end{array}$ & $\mathbf{N}$ & $\begin{array}{c}\text { \% in } \\
\text { FLL }\end{array}$ & $\mathbf{N}$ & $\begin{array}{c}\text { \% in } \\
\text { FLL }\end{array}$ & $\mathbf{N}$ & $\begin{array}{c}\text { \% in } \\
\text { FLL }\end{array}$ \\
\hline Agree & 337 & $67 \%$ & 260 & $62 \%$ & 423 & $62 \%$ & 153 & $69 \%$ \\
\hline Not sure & 208 & $55 \%$ & 225 & $57 \%$ & 140 & $54 \%$ & 297 & $61 \%$ \\
\hline Disagree & 111 & $44 \%$ & 164 & $57 \%$ & 82 & $55 \%$ & 185 & $50 \%$ \\
\hline $\begin{array}{l}\text { No opinion } \\
\begin{array}{l}\text { Significance } \\
\text { of association }\end{array}\end{array}$ & 8 & $\mathrm{~N}=3$ & 15 & $\mathrm{~N}=6$ & 19 & $\mathrm{~N}=9$ & 29 & $\mathrm{~N}=11$ \\
\hline
\end{tabular}

Responses to the suggestion that knowing a FL would help students get a good job (item 9.2.15) were slightly less positive than those of year 8 students. $51 \%$ of year 9 students agreed with the statement $(\mathrm{N}=656)$ compared with $56 \%$ of year 8 students $(\mathrm{N}=300$ ), discussed in 5.4.4, suggesting that the older ones were more realistic about the usefulness of FLL. It should be noted that the wording of the item had been changed from 'another' to a 'foreign' language so that the year 9 students would be quite clear that the survey was not about learning English or Maori. 
Considerably fewer students, only $39 \%(\mathrm{~N}=649)$, believed that many jobs involve working with people who do not speak English (item 9.2.8), compared with the 51\% of year 8 students, as discussed in 5.4.4. The older students probably had a more realistic idea of the sort of language skills required in employment. Whatever students thought about this item it had little effect on their decisions regarding FLL. This could mean that those students who believed in the value of FLL as a job qualification did so in only vague terms and had not considered FL skills in relation to specific employment.

Of all the survey items, the notion that FLL could improve English language skills elicited the most uncertainty among students. $47 \%$ were unsure $(\mathrm{N}=635)$ and 29 expressed no opinion. It is clear that the great majority of students were not receiving the message that FLL involves the development of important skills which can be transferred to other subjects, especially their L1, although advocates of FLL would cite this as one of the major supporting reasons for such study.

Of the four items discussed above, the apparent lack of job usefulness had the greatest negative influence on students, as Table 6.12 shows. Only $44 \%$ of the 111 students who disagreed with item 9.2.15 had chosen a FL option, compared with half or more of the students who showed negative attitudes to FLL in their responses to the other items. This seems to be another indication that the choice to study a FL is to a large extent a matter of personal interest rather than a pursuit of something useful. The idea that FLL is useful for developing cultural understanding, item 9.2.6, produced the most positive response of all the items presented to all students. $48 \%$ of students agreed and a further $18 \%$ strongly agreed that students who learn a foreign language are better at understanding another culture. These positive attitudes did not, however, have much influence on student choices regarding FLL.

As there has been much discussion about the relative importance of the European languages traditionally taught in schools and Asian languages which might be needed for the country's trade development, students were asked to consider if Asian languages were the most useful ones for school students to learn, item 9.2.4 ( $\mathrm{N}=$ 635). Responses were evenly divided, whether or not students were studying a FL, with the largest group, $38 \%$, unsure and 19 students offering no opinion. This seems to indicate that students have only a vague idea of the value of FLL in terms of employment, and probably only limited knowledge of what sort of language skills the country requires. This will be considered further in the discussion of what the teachers reported, especially in relation to careers advice.

\subsection{The attitudes of students who were studying a FL \\ 6.5.1 Reasons for choosing to study a FL}

In Part 1 of the year 9 survey, I asked students who had chosen a FL option why they had done so. Table 6.13 shows their responses and the number and proportion of students citing each reason. 
Table 6.13 Reasons given by students for choosing FLL

\begin{tabular}{|l|c|c|}
\hline Comments & $\begin{array}{c}\text { Number of } \\
\text { Mentions }\end{array}$ & $\begin{array}{c}\text { Proportion of } \\
\text { students }\end{array}$ \\
\hline For travel & 90 & $23 \%$ \\
\hline General interest/just wanted to & 79 & $20 \%$ \\
\hline Compulsory & 68 & $18 \%$ \\
\hline General enjoyment/fun & 46 & $12 \%$ \\
\hline To build on previous learning/ enjoyment & 32 & $8 \%$ \\
\hline $\begin{array}{l}\text { For something new/ different/ } \\
\text { challenging }\end{array}$ & 27 & $7 \%$ \\
\hline Considered useful & 25 & $6 \%$ \\
\hline Job qualification & 22 & $6 \%$ \\
\hline $\begin{array}{l}\text { Influence or pressure from parents/other } \\
\text { family }\end{array}$ & 19 & $5 \%$ \\
\hline
\end{tabular}

Many students gave no reasons and some mentioned more than one. The four main reasons were for travel, general interest, enjoyment, or because they felt they had to learn a FL. In addition to the reasons listed, other reasons cited by less that $5 \%$ of students were:

- interest in other cultures;

- to improve contacts with speakers of other languages;

- lack of other desirable option;

- interest aroused by travel/residence overseas;

- to improve English.

Although, technically, FLL was not compulsory in any of the schools, many students clearly felt under pressure to study a FL. It is possible that, if learning Maori was the only alternative to learning a FL, the choice of the latter may be a sign of a reluctance to learn Maori rather than a real desire for FLL, but some students who stated that FLL was compulsory also gave other reasons for choosing a FL option. The only other alternative for top stream students at Begonia was studying performance music which might have been perceived as an impossible option for some boys.

The degree to which perceived compulsion was responsible for students choice of a FL was further tested by an item in Part 3 of the survey, completed only by students in FLL. The responses to this item, 9.3.13, from students at Arum, where all year 9 
students had to study a LOTE and Begonia, where some students were under pressure to do so, are shown in Graph 6.1. 45 students (29\%) disagreed, and 43 (27\%), a particularly high proportion, strongly disagreed, that they had chosen to study a FL because it was compulsory. This shows that most of these students were not reluctant FL learners despite the degree of perceived compulsion.

\section{Graph 6.1 Student responses to item 9.3.13: "I'm only doing a} foreign language because my school makes it compulsory." $\quad($ No opinion $=4)$

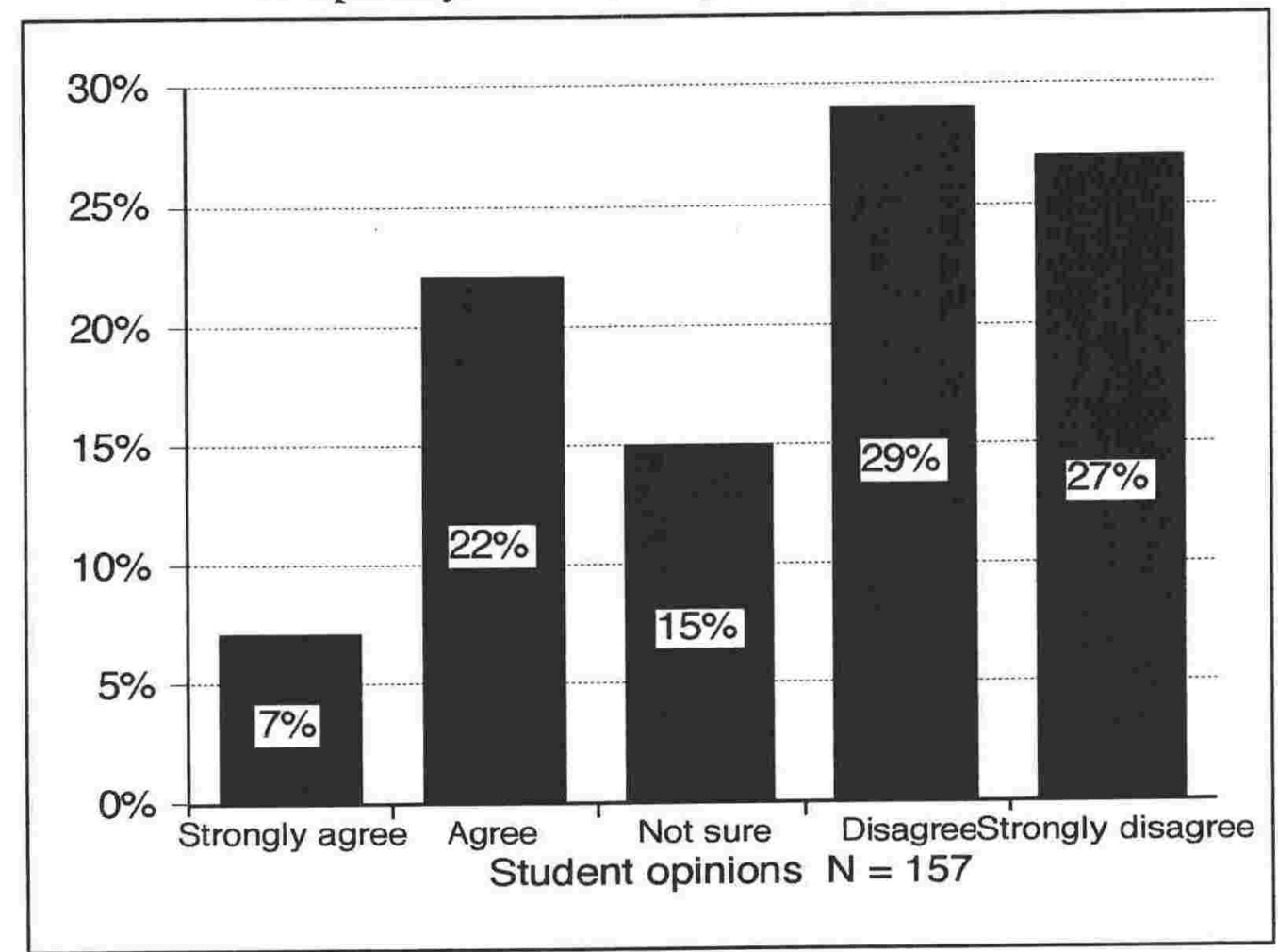

In fact, pure enjoyment seems to have been a powerful motive for FLL. Survey item 9.3.2, elicited especially positive responses from students, $47 \%$ agreed and a further $34 \%$ strongly agreed $(\mathrm{N}=385)$ with the statement, "It's fun to learn another language", see Graph 6.2. Given the timing of the survey it is not possible to assess whether their opinions were based on previous or current experience or both. Nor is it possible to gauge precisely what students meant when they used the word "fun" in their comments. 
Graph 6.2 Student responses to item 9.3.2: "It's fun to learn another language." (No opinion $=2$ )

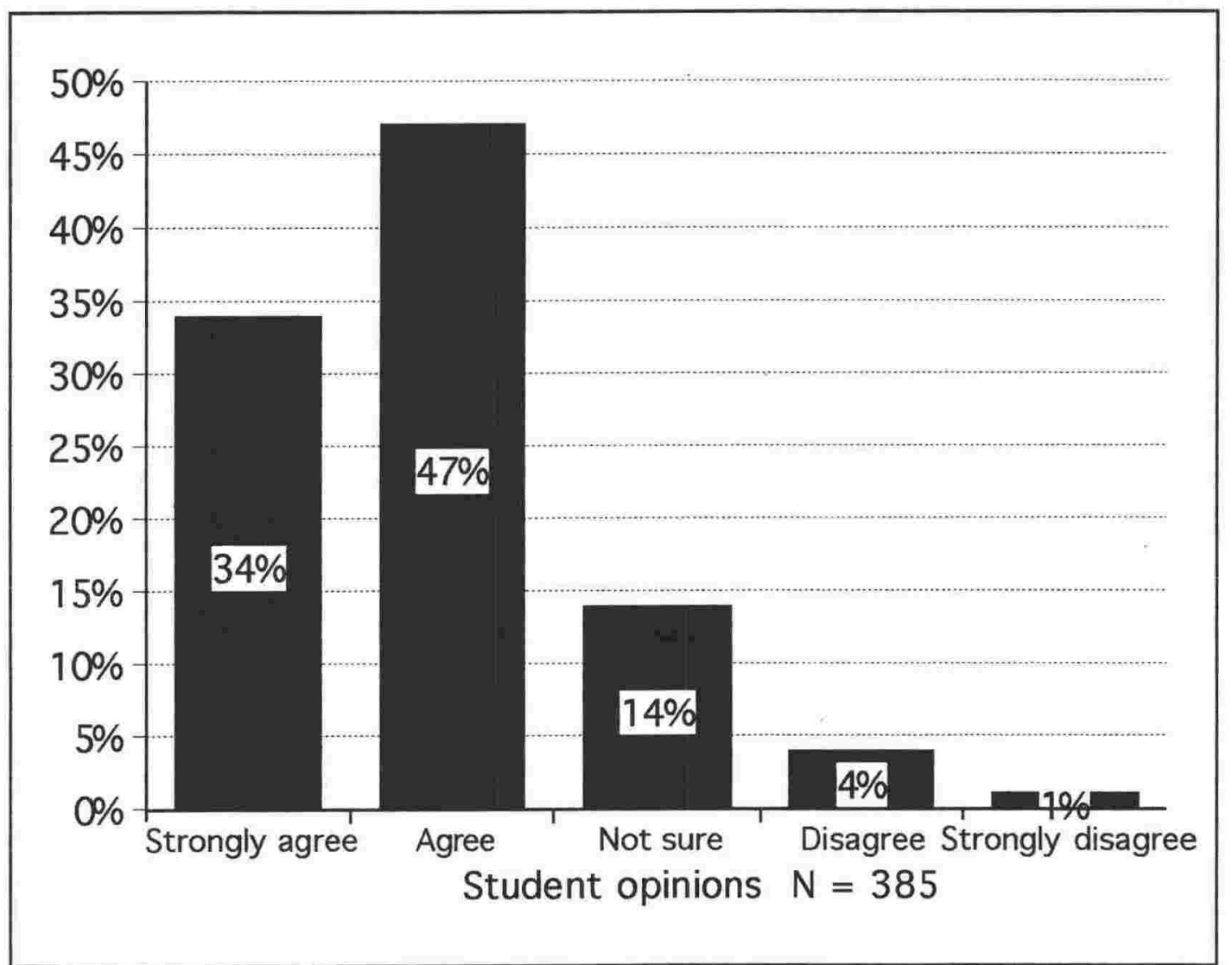

\subsubsection{The languages students had chosen to study}

The majority of FL students were studying French or Japanese, because they were the FLs offered by all the surveyed schools and the only FLs available in two schools. Even where German and Spanish were available only small numbers of students had chosen them, representing under $10 \%$ of FL students in any one school. Table 6.14 shows the complete figures. Only 29 students (7\% of all FL students) had chosen to study more than one language, mostly trying French and Japanese at Arum where it was possible to study each language for a term before deciding whether or not to continue one or the other (or neither). 
Table 6.14 Numbers of students studying each language

\begin{tabular}{|l|c|}
\hline Language & $\begin{array}{c}\text { Number of } \\
\text { students }\end{array}$ \\
\hline French & 201 \\
\hline German & $20 \dagger$ \\
\hline Japanese & 167 \\
\hline Spanish & $28^{*}$ \\
\hline $\begin{array}{l}\dagger \text { Includes 1 on correspondence } \\
\text { * Includes 2 on correspondence }\end{array}$ \\
\hline
\end{tabular}

Table 6.15 shows the relative popularity of French and Japanese in 2000, with some interesting differences. French was much more popular than Japanese at the single sex schools and at Arum, but the reverse was true at Freesia and Salvia. This may be the result of the previous experience of many of the students at the latter schools. Most of the Salvia students would have learnt Japanese and French, and possible reasons for the greater popularity of Japanese were discussed in the previous chapter. Many Freesia students would have learnt Japanese at the neighbouring intermediate school but few would have had the opportunity to learn any French.

Table 6.15 Numbers of year 9 students studying French and/or Japanese, by school

\begin{tabular}{|l|c|c|c|c|c|}
\hline \multirow{2}{*}{ School } & Student N* & \multicolumn{2}{|c|}{ French } & \multicolumn{2}{|l|}{ Japanese } \\
\hline Arum & 116 & 79 & $68 \%$ & 54 & $47 \%$ \\
\hline Freesia & 93 & 26 & $28 \%$ & 45 & $48 \%$ \\
\hline Salvia & 38 & 15 & $39 \%$ & 25 & $66 \%$ \\
\hline Single sex & 140 & 81 & $58 \%$ & 43 & $31 \%$ \\
\hline Totals & 387 & 201 & $52 \%$ & 167 & $43 \%$ \\
\hline
\end{tabular}

* $\mathrm{N}=$ number of students involved in FLL at each school.

Percentages are proportions of $\mathrm{N}$ and add up to more than $100 \%$ where students are studying both languages.

\subsubsection{Reasons for students' choice of language}

Students were asked why they had chosen their particular language options and the greatest influence, as I had surmised, seemed to be their previous experience of FLL. One third of the 389 students reported that they had chosen the FL because they had 
enjoyed the same one at intermediate level, found it interesting or felt they were good at it, and another $13 \%$ of students specifically stated that they wanted to continue a FL started previously. $19 \%$ were interested in visiting a particular country and $16 \%$ admitted to being influenced by family or friends. Some other reasons cited by small numbers (under 10\%) of students were:

- languages might be useful;

- a desire to study something new and challenging;

- FL was the easiest or the only acceptable option available;

- a desire to enhance contacts with family/friends in NZ or overseas.

Students were also asked if they would rather have learnt another language. 53 students indicated that this was the case and details of their alternative preferences are set out in Table 6.16. Some named a FL they could have studied, which suggests that either they found the question ambiguous or the option system had made their first choice difficult. In many cases students had not yet started their FL options and, if they had no prior FLL experience, could not judge their interest in or enjoyment of a particular FL. Others might not have been enjoying the FL they had chosen.

Table 6.16 Languages mentioned as preferred alternatives or additional FLs

\begin{tabular}{|l|c|}
\hline Language named & $\begin{array}{c}\text { Number of } \\
\text { students }\end{array}$ \\
\hline French & 9 \\
\hline Spanish• & 8 \\
\hline German $\diamond$ & 13 \\
\hline Japanese & 13 \\
\hline Italian & 4 \\
\hline Other & 6 \\
\hline Total & 53 \\
\hline $\begin{array}{l}\text { - } 3 \text { could have learnt Spanish } \\
\diamond 2 \text { could have learnt German, 5 were } \\
\text { at Freesia where class was cancelled }\end{array}$ \\
\hline
\end{tabular}

Satisfaction with the language options they had chosen was further tested by item 9.3.10, "I wish I'd chosen a different language". An exceptionally high proportion of students, $48 \%$ strongly disagreed and another $33 \%$ disagreed with this statement $(\mathrm{N}=378)$, showing that the vast majority of students were happy with their choice 
of language. A few students would like to have learnt a language not available in any schools, such as Italian, Irish or Tongan, usually because of their family background, but about half the students stated that they were interested in a particular language or had enjoyed learning it previously. It was interesting that no students wanted to learn Chinese but two expressed an interest in Latin.

\subsubsection{Beliefs about the difficulty of FLL}

As in year 8 , all students were asked to respond to the suggestion that knowing more than one language is confusing, item 9.3.8. Among the year 8 students, $48 \%$ of students disagreed with the statement. Of the older students who had chosen to study a FL, well over half, 58\%, disagreed and only $19 \%$ indicated that they were finding FLL confusing $(\mathrm{N}=376)$. The older students appeared to be more confident about their FLL, or were simply finding it easier than the younger ones, but it must be remembered that the majority of the year 9 student were doing something they had chosen whereas the year 8 students had mostly had no choice in the matter of FLL. Another item, 9.3.4, "I think I'll do well in my language learning this year", was designed specifically to test students' confidence in their FLL. Two thirds of the students $(\mathrm{N}=382)$ were confident that they would do well and only $6 \%$ were pessimistic about their progress.

\subsubsection{The usefulness of FLL}

As shown in 6.5.1, the reason for choosing a FL option cited by the largest proportion of the whole year 9 sample was an interest in future travel. Only a few students used the word "useful" on its own or connected FLL with jobs. Relevant items in Part 3 of the survey were concerned with the value of FLL for the development of useful skills, which could be applied to any language: listening, speaking, reading and writing.

Students who had opted for FLL did not, on the whole, believe that it would improve their listening and reading skills. Item 9.3.5, "Learning another language will help me listen more carefully", produced a particularly large proportion, $42 \%$, of "not sure' responses $(\mathrm{N}=385)$, although nearly one third of students agreed with the statement. Item 9.3.16, "Learning another language will help me read more carefully", produced an even higher proportion, $47 \%$, of 'not sure' responses $(\mathrm{N}=$ 382) and a third of students disagreed.

The majority of students, $57 \%$, agreed $(\mathrm{N}=383)$ with item 9.3 .9 , "I enjoy trying to say things correctly", which suggests a positive attitude towards improving speaking skills or perhaps just enjoyment at the challenge of producing strange sounds in a FL. In contrast, very few believed that FLL would help them improve their written work. Item 9.3.18, "Now that I'm learning another language I take more care with my written work", elicited disagreement from half the students $(\mathrm{N}=366)$, and uncertainty from another 37\%, with a particularly large number (23) of 'no opinion' responses. This may reflect the fact that many students had only just started their FL 
options and may not have done much writing at that stage. Nearly half the students $\mathrm{N}=384$ ) agreed that some FLL would help them in any future FLL they might undertake, item 9.3.1, although $35 \%$ were unsure. Overall, as with the year 8 students, it seems that the message about the value of FLL for the acquisition of transferable language skills is being received to only a limited extent. Once again, if this is to be used as a justification for FLL in schools the notion needs to be 'sold' more effectively to students, and perhaps their parents and teachers.

\subsubsection{Attitudes to speakers of the FLs studied}

Two items designed to investigate the attitudes of FL students towards speakers of the language(s) they were studying and their cultures, elicited very positive responses. Nearly two thirds of students $(\mathrm{N}=383)$ were interested in knowing more about the life of the people who speak the FL they were learning, item 9.3.12, see Graph 6.3. Asked if they were interested in meeting speakers of the FL they were learning, $42 \%$ agreed and a particularly high proportion, $30 \%$, strongly agreed $(\mathrm{N}=382)$ that this was the case showing even more positive attitudes (item 9.3.15), see Graph 6.4. These responses clearly show a clear interest not just in the language learning itself but also in the people and cultures represented by the FLs, an aspect of FLL that is emphasised in most FL classrooms these days.

Graph 6.3 Student responses to item 9.3.12: "I'd like to know more about the life of the people who speak the language I'm

learning." (No opinion $=4$ )

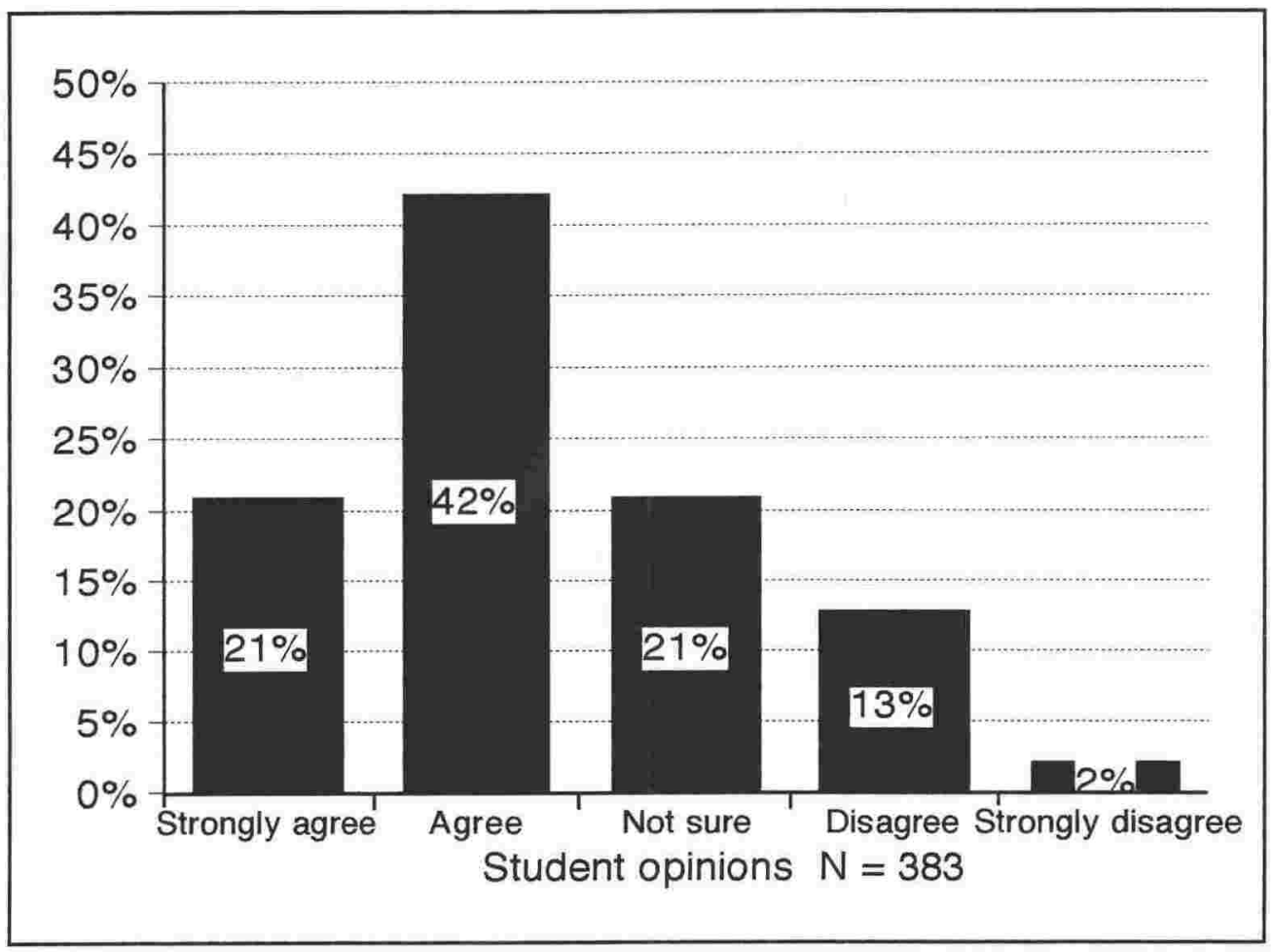


Graph 6.4 Student responses to item 9.3.15: “I'm interested in meeting people who speak the language I'm learning."

$($ No opinion $=5)$

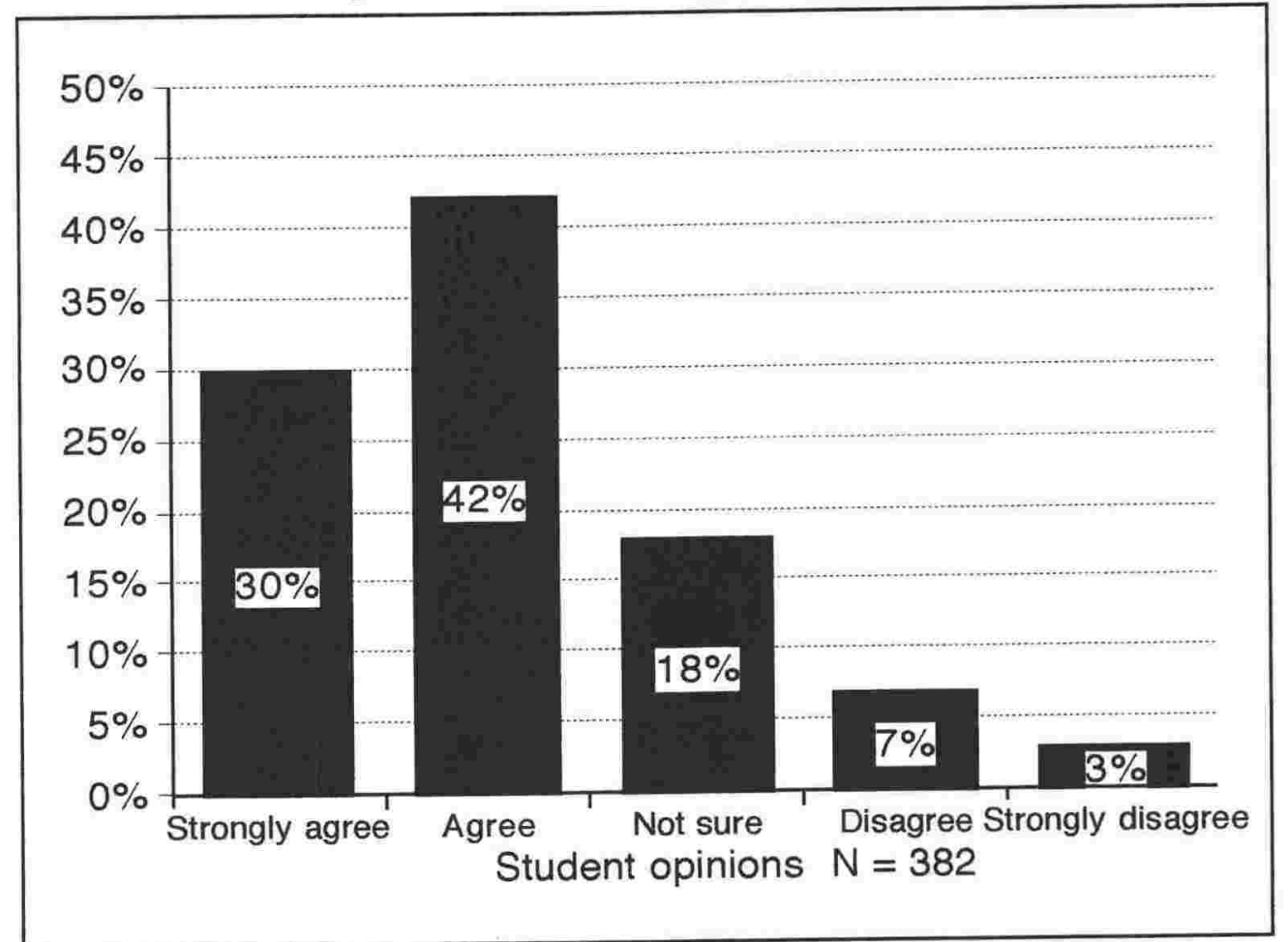

\subsubsection{Attitudes to length of option courses}

In the single sex schools year 9 option subjects were studied for the whole year, and so I analysed responses to the statement "I wish that foreign language options in year 9 were for the whole year" (item 9.3.14), from students in FLL only in the other three schools. $39 \%$ of the students who responded $(\mathrm{N}=238)$ agreed with the statement, but about one quarter did not want longer options. It is possible that, among students who had started their FLL, some might not have been enjoying their FL option, but it is also possible that students realised that increased time spent on any one option would reduce the number of subjects they could study during the year. It was naturally hard for students to comment if they had no previous experience and had not started their options.

\subsection{Intentions regarding FLL for year 10}

$39 \%$ of the 389 students involved in FLL reported that they would continue FLL in year 10, but over half, $57 \%$, were uncertain, mostly because the survey was carried out early in year 
9. Many wanted to see how well they progressed and others had not started their FLL. Only a tiny minority, 4\%, had definitely decided against further FLL and this was never more than $5 \%$ in any one school.

\subsubsection{Intentions compared with schools}

The high degree of uncertainty can be seen in Graph 6.5 where the results are shown by school. It is impossible to estimate why more students should be certain about continuing at Freesia than at the other two mixed schools since so many factors could be involved. The differing intentions of students at the single-sex schools will be discussed in chapter 10 on gender differences.

Graph 6.5 Proportions of students, by school, intending to continue FLL in year 10 or uncertain

Key: $\mathrm{A}=$ Arum, $\mathrm{B}=$ Begonia, $\mathrm{F}=$ Freesia, $\mathrm{G}=$ Gerbera, $\mathrm{S}=$ Salvia

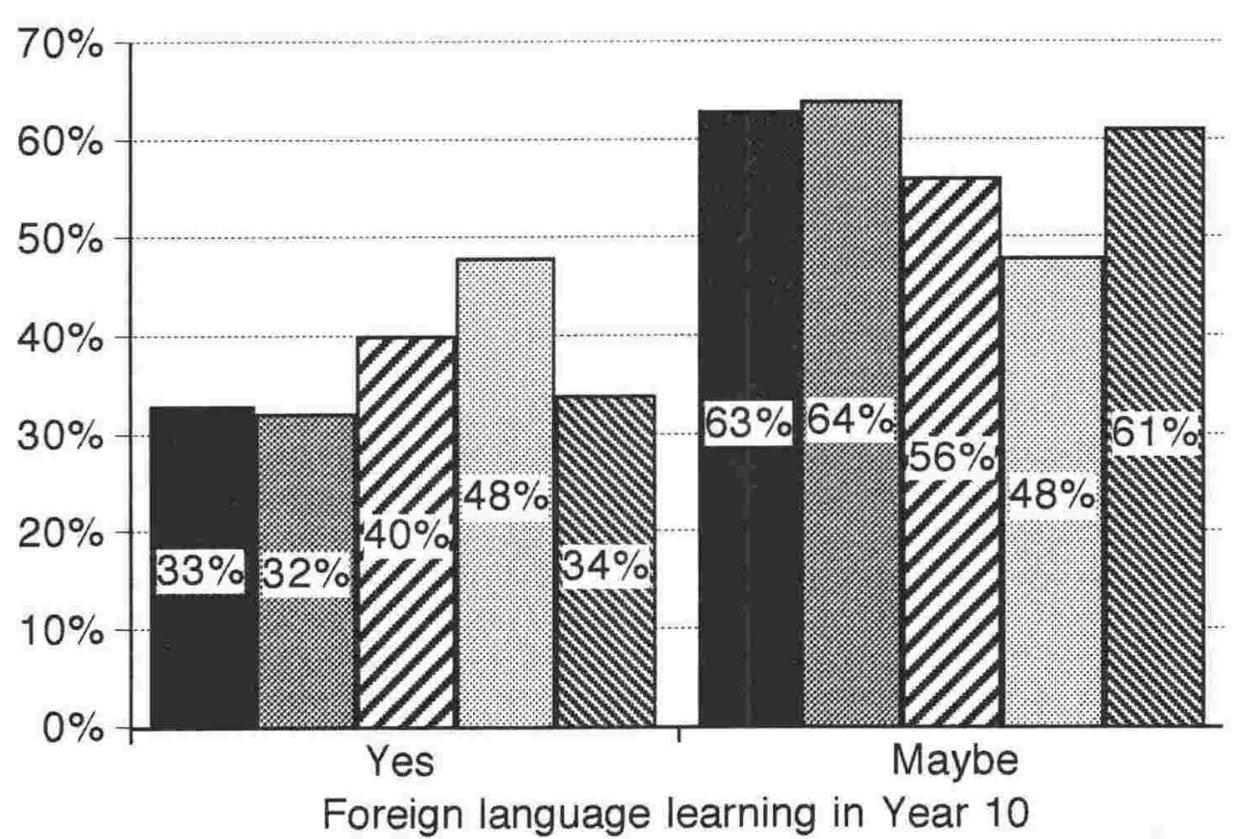

$A(N=116) \square B(N=44) \quad \square F(N=93) \quad \square G(N=96) \quad \mathbb{S}(N=38)$ 


\subsubsection{Intentions according to FL studied in year 9}

Graph 6.6 shows that students learning French or Japanese were the least likely to definitely intend continuing their FL studies, and had the highest proportions of uncertainty. The difference between the languages could, however, be due to the much larger numbers of students in French and Japanese, rather than less favourable attitudes to those languages.

\section{Graph 6.6 Student intentions for year 10 compared with FLs studied in year 9 \\ Key: $\mathrm{F}=$ French, $\mathrm{G}=$ German, $\mathrm{S}=$ Spanish, $\mathrm{J}=$ Japanese, 2 FLs $=$ students studying 2 FLs in year 9.}

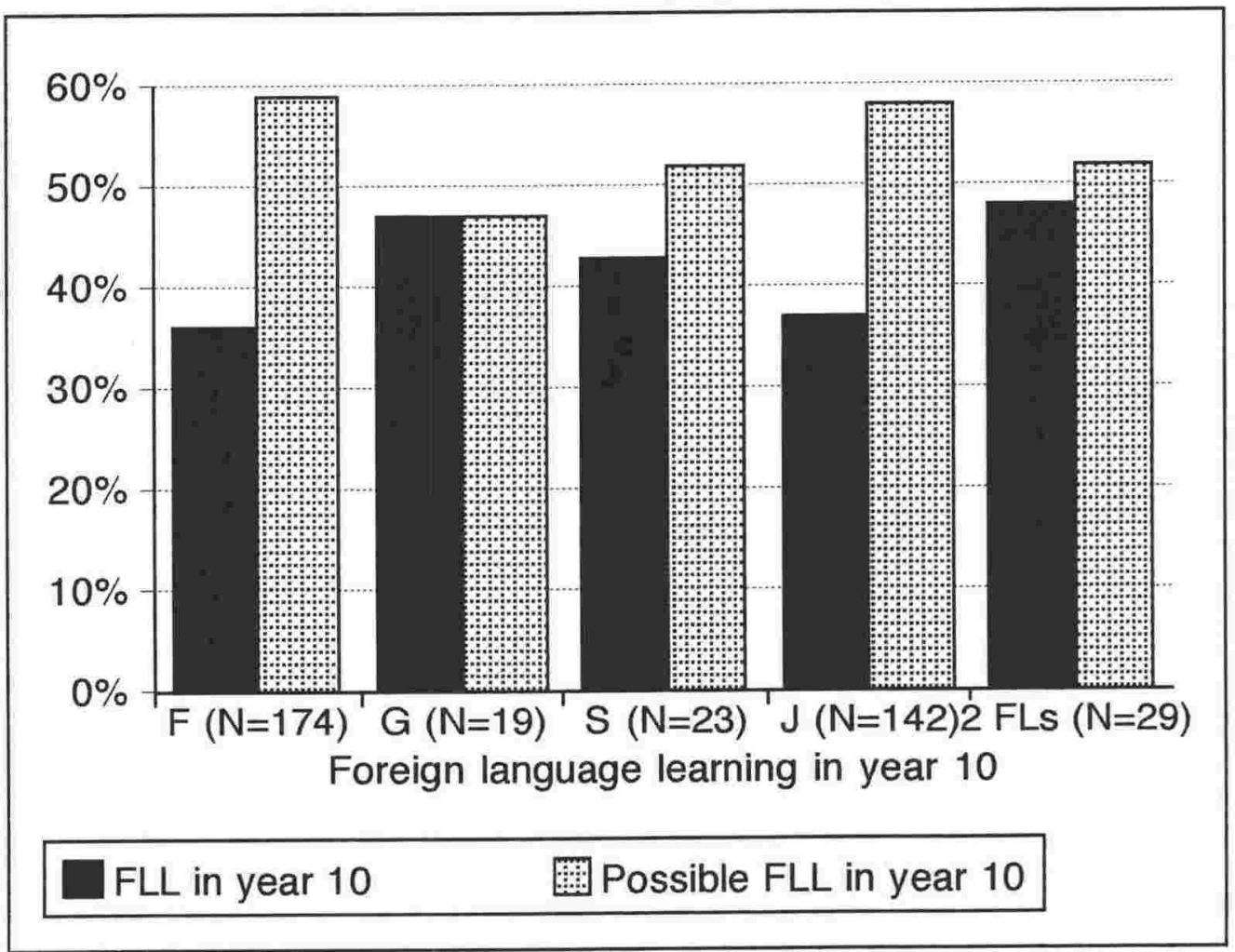

\subsubsection{Reasons for intentions to continue FLL}

Reasons given for definite intentions to continue FLL in year 10 are shown in Table 6.17. Some students gave more than one reason but very few mentioned a desire to obtain a particular qualification or that they were aiming for something useful for employment. Nearly half simply indicated that they were enjoying their learning. About one third were keen to improve their language skills, especially their oral 
fluency, or to learn more about the culture represented by the language they were studying. Once again, travel was a clear influence. Although a few reported that they might have to continue FLL it should be noted that it was not compulsory for any year 10 students. Their choices might, however, be limited according to what subjects were available in each option line of the timetable and what they had studied in year 9.

Table 6.17 Reasons given for continuing FLL in year 10

\begin{tabular}{|l|c|}
\hline Comments & $\begin{array}{c}\text { No. of } \\
\text { mentions }\end{array}$ \\
\hline Enjoying the subject & 72 \\
\hline Wants to improve, develop fluency, learn more & 46 \\
\hline For future travel & 23 \\
\hline May have no choice & 8 \\
\hline For examination goal (SC or UB) & 6 \\
\hline General usefulness & 5 \\
\hline Job qualification & 4 \\
\hline Might as well continue & 3 \\
\hline
\end{tabular}

Item 9.3.6, "I want to learn more of the same language next year", elicited agreement from half the students, but most of the rest were unsure, another indication of the high degree of uncertainty due to the timing of the survey as, discussed at the beginning of this section.

Table 6.18 Reasons given for not continuing FLL in year 10 or being uncertain

\begin{tabular}{|l|c|}
\hline Comments & $\begin{array}{c}\text { No. of } \\
\text { mentions }\end{array}$ \\
\hline Generally uncertain or have mixed feelings & 121 \\
\hline Depends on success and/or enjoyment in Year 9 & 69 \\
\hline Too hard/boring/no use/not enjoying & 15 \\
\hline Might want to try another option & 9 \\
\hline Wants to continue Maori only & 1 \\
\hline
\end{tabular}




\subsubsection{Reasons for not intending to continue FLL or uncertainty}

Table 6.18 presents the reasons cited by students who had decided against FLL in year 10 or who were unsure about it. Most students were keeping an open mind to see if they enjoyed FLL. Students who were able to try out a large number of modules during year 9 would not have to decide which ones to concentrate on until term 4. Those who were waiting to see how much they had achieved by the end of the year might decide on the basis of test or examination results. No students would be able to choose more than two optional subjects in year 10 and there might be new options available to them in year 10 .

Table 6.19 Significant item responses in relation to student intentions for FLL in year 10

\begin{tabular}{|c|c|c|c|}
\hline Survey item & $\mathbf{N} *$ & $\begin{array}{c}\text { Intending } \\
\text { FLL\# }\end{array}$ & Significance $\dagger$ \\
\hline 9.3.2. It's fun to learn another language. & 310 & $45 \%$ & $\mathrm{p}<.001$ \\
\hline $\begin{array}{l}\text { 9.3.17. I like practising another language } \\
\text { with my friends. }\end{array}$ & 183 & $55 \%$ & $\mathrm{p}<.001$ \\
\hline $\begin{array}{l}\text { 9.3.4. I think I'll do well in my language } \\
\text { learning this year. }\end{array}$ & 253 & $53 \%$ & $\mathrm{p}<.001$ \\
\hline $\begin{array}{l}\text { 9.3.6. I want to learn more of the same } \\
\text { language next year. }\end{array}$ & 192 & $70 \%$ & $\mathrm{p}<.001$ \\
\hline $\begin{array}{l}\text { 9.3.15. I'm interested in meeting people } \\
\text { who speak the language I'm learning. }\end{array}$ & 277 & $45 \%$ & $\mathrm{p}<.001$ \\
\hline $\begin{array}{l}\text { 9.3.12. I'd like to know more about the } \\
\text { life of the people who speak the language } \\
\text { I'm learning. }\end{array}$ & 241 & $49 \%$ & $\mathrm{p}<.001$ \\
\hline $\begin{array}{l}\text { 9.3.5. Learning another language will } \\
\text { help me listen more carefully. }\end{array}$ & 124 & $47 \%$ & $\mathrm{p}<.001$ \\
\hline $\begin{array}{l}\text { 9.3.9. I enjoy trying to say things } \\
\text { correctly. }\end{array}$ & 219 & $51 \%$ & $\mathrm{p}<.001$ \\
\hline $\begin{array}{l}\text { 9.3.16. Learning another language will } \\
\text { help me read more carefully. }\end{array}$ & 76 & $46 \%$ & $\mathrm{p}<.001$ \\
\hline $\begin{array}{l}\text { 9.3.18. Now that I'm learning another } \\
\text { language I take more care with my written } \\
\text { work. }\end{array}$ & 46 & $57 \%$ & $\mathrm{p}<.01$ \\
\hline $\begin{array}{l}\text { 9.3.8. Knowing more than one language } \\
\text { is confusing. }\end{array}$ & 72 & $24 \%$ & $\mathrm{p}<.005$ \\
\hline $\begin{array}{l}9.3 .11 \text { I'm only doing a foreign language } \\
\text { because my parents said I had to. }\end{array}$ & 14 & $\begin{array}{l}\text { Only } 2 \\
\text { students }\end{array}$ & $\mathrm{p}<.001$ \\
\hline $\begin{array}{l}\text { 9.3.10. I wish I'd chosen a different } \\
\text { language. }\end{array}$ & 20 & $\begin{array}{l}\text { Only } 1 \\
\text { student }\end{array}$ & $\mathrm{p}<.001$ \\
\hline
\end{tabular}




\subsubsection{Intentions compared with attitudes to FLL}

Student intentions for year 10 were compared with their responses to each item in Part 3 of the survey and, not surprisingly, there proved to be a high degree of association between attitudes and intentions. Table 6.19 summarises the proportions of students agreeing with each relevant item who intended to continue FLL and the degree of significance of the association. If students thought that FLL was fun, especially if they enjoyed practising with their friends, and if they were confident about their learning, up to $55 \%$ reported that they would continue.

If students were keen to learn more of the language they were studying $70 \%$ stated the firm intention of continuing. Students who showed appreciation or enjoyment of developing general language skills were also likely to continue. In particular, $57 \%$ of those who agreed that FLL helped them take more care with their written work intended to continue. It was not surprising that the minority of students who agreed that knowing more than one language is confusing, and the small numbers who wished they had chosen another language or who felt they were involved in FLL only because their parents had forced them into it, were highly unlikely to intend to continue FLL in year 10. However, it should be reiterated that, even among students revealing these negative attitudes, the majority reported that they were unsure if they would continue, ranging from $64 \%$ to $75 \%$.

\subsection{The attitudes of students who were not studying a FL}

\subsubsection{General attitudes to FLL}

I asked the 277 students who were not involved in FLL in year 9 why they were not studying a FL and if they would like to have studied one. If their response to the second question was positive I asked what language(s) they would like to have learnt and why. I also asked if they thought they might learn a FL one day. Students were presented with items to assess their ideas about the difficulty, the relative importance and the usefulness of FLL, their general attitudes to FLL and their attitudes to speakers of other languages.

Some broad indications of these students' attitudes are indicated by their responses to two particular items. $40 \%$ of students who offered an opinion $(\mathrm{N}=257)$ reported that they were glad they did not have to learn a FL in year 9 (item 9.4.9). The most negative attitudes were shown by the students at Salvia, perhaps because that school had the highest proportion of students with previous FLL experience. On the other hand, $48 \%$ agreed that FLL would be fun (item 9.4.15, N = 263). Students made comments such as:

"I think it would have been cool to be able to speak another language."

"The language (French) sounds pretty cool."

"I would have done French if I'd had more options."

"My friends said it (Japanese) was fun."

"I really enjoyed learning Spanish." (not available for student in year 9) 


\subsubsection{Reasons for choosing not to study a FL}

As shown in Table 6.20, the most common reason given by about one third of students for not opting into FLL was that they simply did not want to do so. They were not interested or they thought it would be boring. Almost another third reported that preferred another option, about half of whom mentioned Maori. In response to item 9.4.13, suggesting that they had chosen not to study a FL because they were more interested in practical subjects than those involving a lot of reading and writing, a particularly high number (17) gave no opinion and $46 \%$ of the rest $(\mathrm{N}=260)$ agreed that this was the case.

In addition to the reasons listed, other reasons cited by less that $5 \%$ of students were:

- Parental influence,

- Preference for short culture module at Gerbera,

- Interest only in unavailable FL,

- Thinking of starting FLL in Year 10 (not usually possible),

- Done enough FLL (only 4 girls) (contrary to NZCTE 1998),

- School decision/influence,

- Needed extra English.

It should be noted that nearly $60 \%$ of 83 students who reported a lack of interest in FLL had not had any experience of it, and were therefore not making an informed judgement. Only a few reported that they had been put off by their previous experience of FLL, which supports the findings of Fitzsimons (1999). Three quarters of the students were confident that they could always learn a FL later on if they needed it (item 9.4.5, $\mathrm{N}=269$ ).

\subsubsection{Beliefs about the difficulty of FLL}

As shown in Table 6.20, very few students reported that they thought FLL would be too difficult, but it has been found in recent research into children's playground language that "boring" can mean "difficult" these days (Bauer 2001). If this is the case, many of those who cited "boring" as a reason for not learning a FL, may have really meant that it would be too difficult. However, as discussed in 6.4 .2 above, only a minority of all the year 9 students agreed with the statement that learning a FL is too hard for most students.

Two further items tested students' perceptions of the difficulty of FLL. Less than a third agreed that "Knowing more than one language is confusing" (item 9.4.3), and the majority of students, $48 \%$, did not agree $(\mathrm{N}=268)$. Item 9.4.14, "Learning a foreign language would be too difficult for me", elicited even stronger disagreement. $29 \%$ of the students disagreed and another $22 \%$ strongly disagreed, while only $22 \%$ agreed. These responses suggest that most students were not avoiding FLL because they thought it would be too difficult for them, although they might have been reluctant to report their own perceived lack of ability or confidence. 
Table 6.20 Reasons for not studying a FL in Year $9(\mathrm{~N}=277)$

\begin{tabular}{|l|c|c|}
\hline Comments & $\begin{array}{c}\text { Number of } \\
\text { Mentions }\end{array}$ & $\begin{array}{c}\text { Proportion } \\
\text { of students }\end{array}$ \\
\hline Did not want to/not interested/would be boring & 83 & $30 \%$ \\
\hline Preferred another option/no time for FL & 46 & $17 \%$ \\
\hline Preferred Maori & 32 & $12 \%$ \\
\hline No need/no use for jobs & 28 & $10 \%$ \\
\hline Did not enjoy previous experience & 18 & $7 \%$ \\
\hline Did not know & 18 & $7 \%$ \\
\hline Too difficult & 17 & $6 \%$ \\
\hline
\end{tabular}

\subsubsection{The relative importance of FLL}

Students were asked to respond to the idea that they needed to concentrate on developing their English language skills (item 9.4.2), rather than learn a FL, and to the proposition that they could learn about the life and customs of other people without needing to understand any of their language (item 9.4.6). Neither suggestion produced any clear opinions from the students.

\subsubsection{The usefulness of FLL}

It was perhaps surprising that the majority of students, $49 \%$, did not believe that they would only ever need to be able to speak English or Maori (item 9.4.4, $\mathrm{N}=273$ ). Only about $10 \%$ of students appeared to think that they would never need knowledge of a FL. When asked to consider if they might need FL skills specifically for future employment, item 9.4.12, there was a great degree of uncertainty. Only 20\% of students $(\mathrm{N}=260)$ agreed with the statement "I won't need to know any foreign languages in any jobs I might do". The majority of these students seemed to be uncertain about the possible usefulness of FLs in future employment but this probably reflects a common idea that FLL is about learning a specific FL with a view to using that specific skill in a job. Most supporters of FLL would claim that it is the general experience of learning a language other than one's L1 and the broadening of one's cultural horizons that are its main values. 


\subsubsection{Attitudes to speakers of other languages}

Although they were not studying a FL, students showed largely positive attitudes in their responses to item 9.4.11, "I'm interested in meeting people who speak other languages", as shown in Graph 6.7. The decision not to choose a FL option in year 9 was clearly not made by the majority of students out of hostility towards speakers of other languages, which is a heartening finding.

\section{Graph 6.7 Student responses to item 9.4.11: "I'm interested in} meeting people who speak other languages."

(No opinion $=12$ )

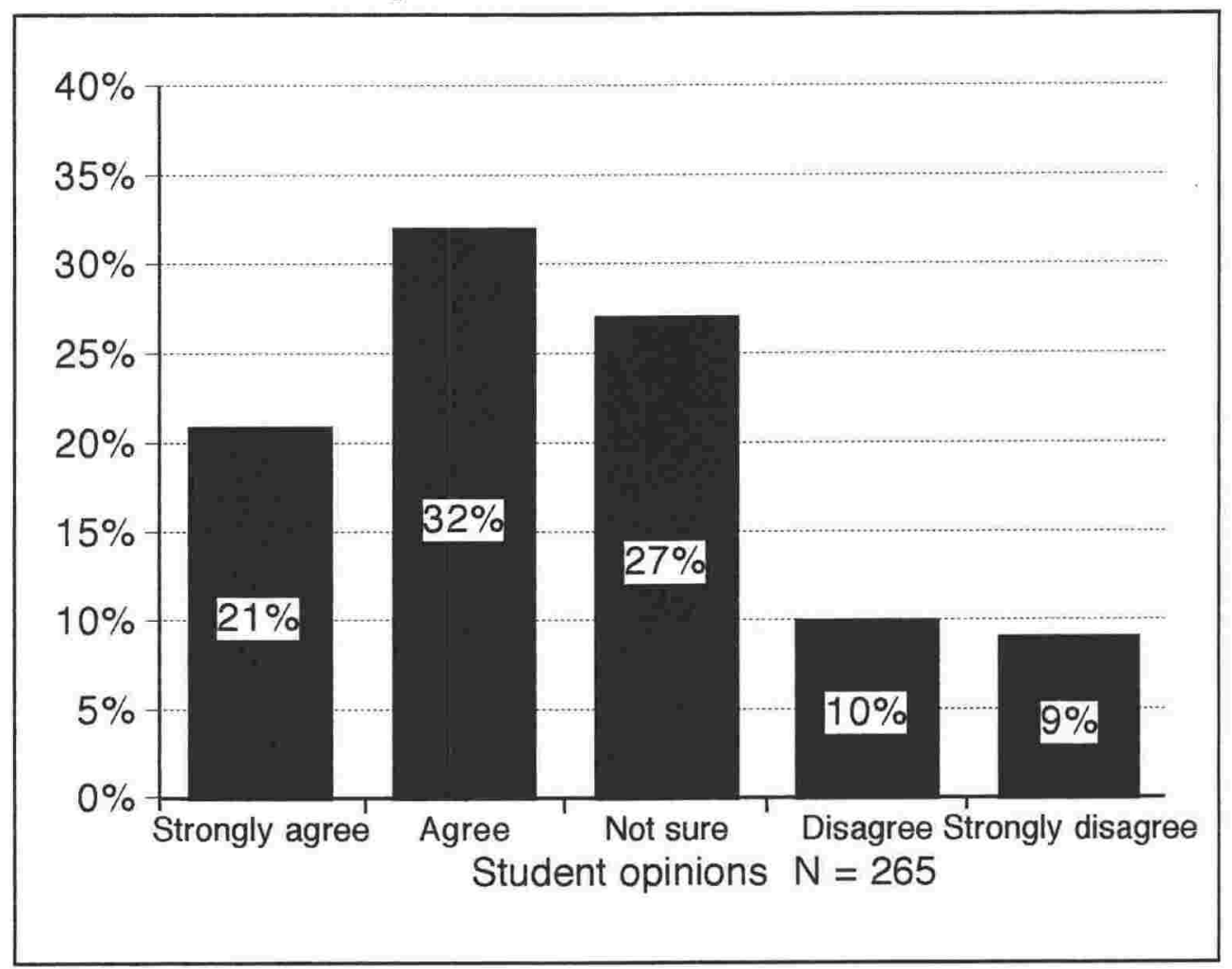

\subsubsection{Regrets about not studying a FL}

Students were asked if they would like to have studied a FL and the majority of the 275 students, $61 \%$, indicated that this was not the case. While a small number of students reported that they were not sure or did not answer the question, this still left 93 students who would like to have learnt a FL. In response to item 9.4.16, "I wanted to learn a foreign language but languages clashed with more important subjects", $27 \%$ of students agreed $(\mathrm{N}=249$, 'No opinion' $=28)$, which largely 
confirmed what students had reported in Part 1 of the survey. These students were, therefore, not avoiding FLL because of negative attitudes towards it but because as previously speculated, option subjects were competing for limited space in their timetables.

When asked which languages they would like to have learnt, most named languages which were available to them. Some were interested in German or Spanish, which were not always available, and a few students mentioned languages not available in any schools. Most of the latter reported having friends or relatives who spoke languages such as Greek, Italian, Fijian or Samoan. There was therefore a wide range of cultural and linguistic interests among this sample of students.

Students were asked why they would like to have learnt a FL and their reasons largely followed the same pattern as those of students who did opt for FLL, shown in Table 6.13. The majority cited an interest in a particular language or culture, or the desire to build on previous FLL experience which they had enjoyed. Travel was mentioned by only 12 students.

\subsubsection{Possibility of future FLL}

When students were asked if they thought they might learn a FL one day two thirds of them indicated 'maybe'. Later they were asked to respond to the statement, item 9.4.10, "I'd like to learn a foreign language one day", which revealed much more positive attitudes from all the students. Over half of the students $(\mathrm{N}=268), 53 \%$, revealed positive attitudes towards the idea of learning a FL in the future, including $19 \%$ who strongly agreed with the item, even if they were not sure that they would ever undertake such learning. It was not surprising that there were a highly significant associations $(p<.001)$ between student responses to two of the items discussed above, 9.4.15 and 9.4.11, and those to item 9.4.10 about possible future FLL. 69\% of the 141 students who were interested in meeting people who speak other languages and $81 \%$ of the 126 who thought that learning a FL would be fun indicated a desire to learn a FL at some time in the future. these responses offer more evidence of positive attitudes to FLL, even among students not involved in it at school.

\subsection{The influence of prior FLL on student option choices for year 9 \\ 6.8.1 FLL experience at intermediate level}

All year 9 students were asked if they had learnt any languages, apart from English or Maori at intermediate school, whether they had enjoyed the classes or not and whether they thought they had been good at FLL. Table 6.21 shows the proportions of students at each school with previous FLL experience. On average, just over half the students fell into this category but there was a particularly low proportion of boys, under one quarter, at Begonia because many of them had come from small primary schools where FLL was not available. The reverse was true for Salvia because of the FLL built into the intermediate level curriculum. 
Opportunities for FLL before year 9 in the area involved in this research varied greatly according to school. Information gleaned from various sources (teachers, advisers, parents) suggested that there was also considerable variation in the languages offered, the time allocated to FLL and the quality and methods of teaching.

Table 6.21 Students with FLL experience in Years 7 or 8,

1998-99, by year 9 school (Missing data $=6$ )

\begin{tabular}{|l|c|c|c|}
\hline Year 9 school & N & $\begin{array}{c}\text { No. with } \\
\text { previous FLL }\end{array}$ & $\begin{array}{c}\text { \% with } \\
\text { previous FLL }\end{array}$ \\
\hline Arum & 139 & 62 & $45 \%$ \\
\hline Begonia & 69 & 16 & $23 \%$ \\
\hline Freesia & 196 & 101 & $52 \%$ \\
\hline Gerbera & 175 & 89 & $51 \%$ \\
\hline Salvia & 85 & 72 & $85 \%$ \\
\hline Totals & 658 & 340 & $52 \%$ \\
\hline
\end{tabular}

\subsubsection{Reasons for previous experience}

Most students reported that they had had to learn a FL, but many cited other reasons for such learning. Some students for whom it was compulsory still gave other reasons, possibly reporting what their school or teacher had said. The main reason given by most students, apart from compulsion, was an interest in a particular language and culture, followed by a general interest in FLL and the wish to try something new, see Table 6.22 .

Table 6.22 Principal reasons cited by students for FLL in Years 7 or $8(\mathrm{~N}=340)$

\begin{tabular}{|l|c|c|}
\hline Comments & $\begin{array}{c}\text { Number of } \\
\text { mentions }\end{array}$ & $\begin{array}{c}\text { Proportion } \\
\text { of students }\end{array}$ \\
\hline $\begin{array}{l}\text { Interest in a particular language or } \\
\text { culture }\end{array}$ & 45 & $13 \%$ \\
\hline $\begin{array}{l}\text { General interest in FLL } \\
\begin{array}{l}\text { To try something new or different, } \\
\text { or for fun }\end{array}\end{array}$ & 21 & $6 \%$ \\
\hline
\end{tabular}

Very few students, no more than $2 \%$, mentioned other reasons, such as the influence of friends or relations, usefulness for jobs or travel, being selected for special, 
extension class, or not being interested in alternative options. In addition, students rarely had a choice of language as this depended on the availability of a suitable, willing teacher. There was no significant association between the existence of prior FLL and students' decisions to study a FL or not in year 9. In fact, the proportion of students choosing FLL in year 9 without previous FLL experience was higher than the proportion of those with such experience. This may have resulted more from what each school demanded of the students rather than their individual interests.

\subsubsection{Reactions to previous experience}

The extent to which students had enjoyed learning a FL at intermediate level was tested in two ways. In Part 1 of the survey, if students reported that they had studied a FL they were asked if they had enjoyed the classes and their reasons for responding 'yes' or 'no'. Of the 340 students who had learnt a FL at intermediate level 250 , over $73 \%$, reported that they had enjoyed it. Later, the same students were asked to respond to the statement: "I enjoyed learning a foreign language at intermediate school" (item 9.2.2). Their particularly positive responses are shown in Graph 6.8. Overall, $71 \%$ of students agreed, and only $20 \%$ disagreed $(\mathrm{N}=328)$. This result will be particularly encouraging to teachers of FLs at year 7 and 8 level.

Graph 6.8 Responses to item 9.2.2: "I enjoyed learning a foreign language at intermediate school." (No opinion $=12$ )

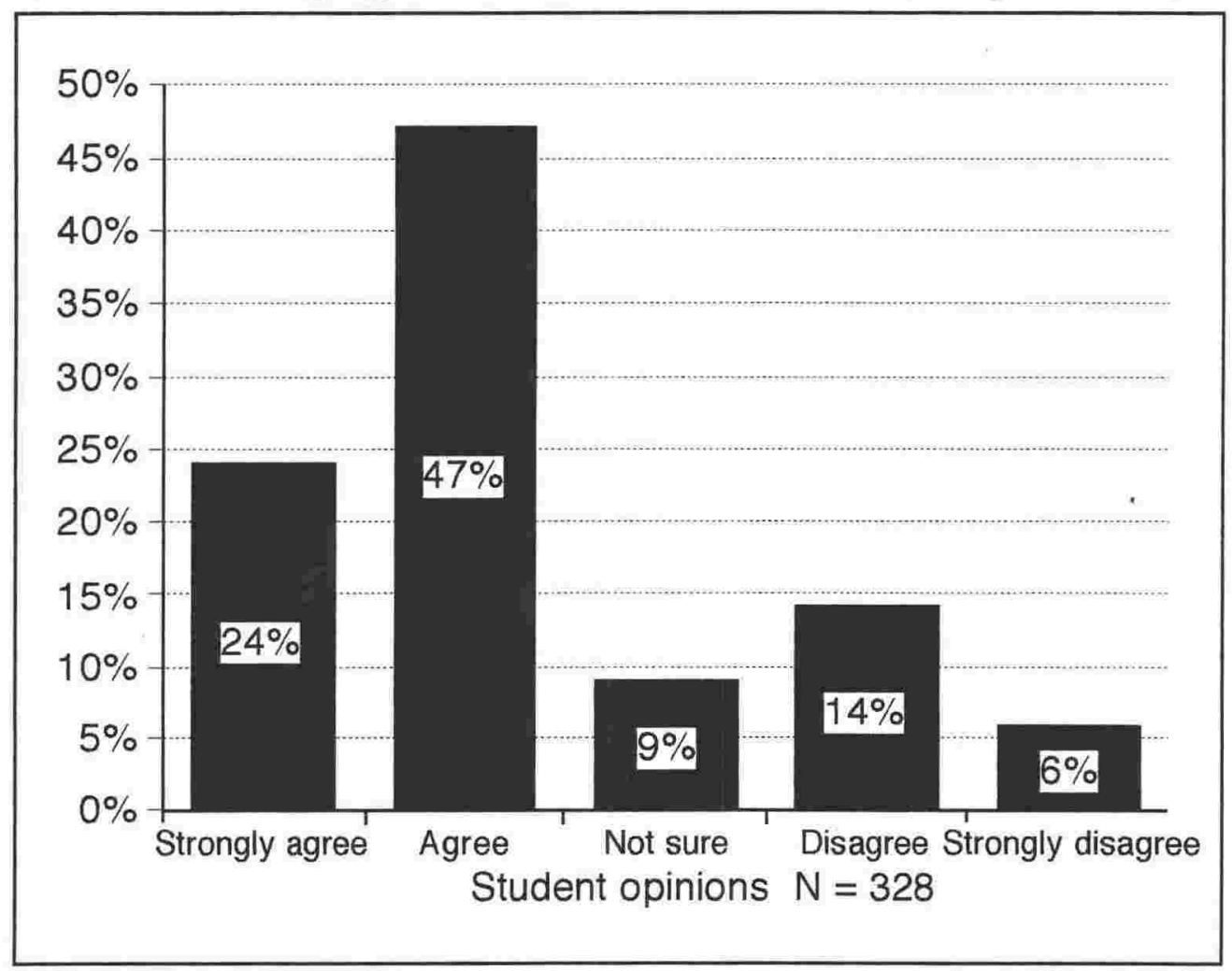




\subsubsection{Reasons for reactions to previous experience}

Reasons that students gave for enjoying or not enjoying their FLL at intermediate level are shown in Table 6.23. Students of ten gave more than one reason, but the number of positive comments (260), compared with negative ones (80), reflects the proportions of students who enjoyed or did not enjoy FLL discussed above. 24 students reported mixed feelings and some negative reasons were mentioned by only 5 or fewer students:

- they resented being forced to learn a FL

- they considered it a waste of time or that they had learnt nothing new

- they were simply pleased to get out of another subject

- they thought their class too big or disruptive

Table 6.23 Reasons for enjoying or not enjoying FLL at intermediate level $(\mathrm{N}=340)$

\begin{tabular}{|l|c|c|}
\hline Reasons for enjoying & $\begin{array}{c}\text { Number of } \\
\text { mentions }\end{array}$ & $\begin{array}{c}\text { Proportion } \\
\text { of students }\end{array}$ \\
\hline Fun, general enjoyment & 97 & $29 \%$ \\
\hline Interesting & 36 & $11 \%$ \\
\hline Something different/new/exciting & 33 & $10 \%$ \\
\hline Enjoyed learning about culture & 22 & $6 \%$ \\
\hline Enjoyed activities & 21 & $6 \%$ \\
\hline Liked teacher & 15 & $4 \%$ \\
\hline Enjoyed challenge/sense of achievement & 15 & $4 \%$ \\
\hline Reasons for not enjoying & & \\
\hline Boring & 38 & $11 \%$ \\
\hline Found it hard & 22 & $6 \%$ \\
\hline Disliked teacher & 9 & $3 \%$ \\
\hline
\end{tabular}

6.8.5 The effect on option choices for year 9 of enjoyment of, and confidence in, previous FLL experience

Although there was little link between the fact of having had some FLL experience and opting into it in year 9, there proved to be, not surprisingly, a significant association $(\mathrm{p}<.001)$ between previous enjoyment of FLL and the choice of further FLL. Approximately two thirds of students who had enjoyed their previous 
experience, according to either of the measures discussed in 6.8.3, had decided to learn a FL in year 9.

Asked if they thought they were good at language learning, 205 students, 60\%, reported that they did think so. A comparison of responses to this question with their decisions regarding further FLL also showed, not surprisingly, a significant association $(\mathrm{p}<.001)$. In this instance, $72 \%$ of those 205 students had decided to do more FLL. Thus, having confidence in their ability to succeed seemed to have had an even more positive effect than enjoyment of the subject on students' decision about FLL in year 9.

\subsection{The influence of schools}

The degree to which students felt compelled to study a FL varied according to each school's attitude towards the place of SLL in the year 9 curriculum, as discussed above. In order to obtain more evidence of school influence over subject choices, students were asked to respond to the statement, "My school encourages everyone to try a foreign language", item 9.2.16. There was a significant association between responses and schools $(\mathrm{p}<.001)$. Not surprisingly, at Arum, where all students had to do a LOTE module, over $80 \%$ of them reported that their school encouraged FLL. Nearly two thirds of students at Salvia (51 out of 80) were aware of encouragement to study a FL, probably because the majority of students had come through the intermediate classes where some FLL was compulsory and they were probably aware that the HOD was keen to see successful and interested students continue their FLL. At Freesia just under half the students (93 out of 193) supported the statement although there was no other evidence that the school encouraged FLL, apart from the exceptionally wide range of languages offered. Only 55 out of 169 girls at Gerbera perceived any encouragement to study a FL, but nearly half of the boys at Begonia ( 33 out of 68 ) did so, probably because most were under considerable pressure to study a language in one of the top stream classes.

Table 6.24 Student decisions about FLL compared with responses to item 9.2.5: “My school's prospectus made learning a foreign language look really boring." (No opinion $=37$ )

\begin{tabular}{|l|c|c|}
\hline Item response & In FLL in Y9 & Not in FLL in Y9 \\
\hline Agree $(\mathrm{N}=118)$ & $33 \%$ & $67 \%$ \\
\hline Not sure $(\mathrm{N}=187)$ & $60 \%$ & $40 \%$ \\
\hline Disagree $(\mathrm{N}=322)$ & $68 \%$ & $32 \%$ \\
\hline
\end{tabular}

For association between item responses and FLL p $<.001$

The possible influence of school prospectuses on students' subject choices was explored by comparing the responses to item 9.2.5, "My school's prospectus made learning a foreign 
language look really boring", with students' choices to study a FL or not. This showed a significant association $(\mathrm{p}<.001)$ between the two factors, shown in Table 6.24. Only a minority of students, $118(19 \%)$, agreed $(\mathrm{N}=627)$ with the statement, and over two thirds of those had not chosen a FL option. A similar proportion of the even larger numbers who were unsure, 187 (30\%) and of the particularly high number, 37, who gave no opinion had not opted for FLL. By contrast, over two thirds of those who disagreed with the statement had opted for FLL. It must be admitted, of course, that the attitudes, positive or negative, may have already existed before students saw the prospectuses and simply have been reinforced by whatever they read. Many students may simply have ignored the relevant parts of the prospectus if they had no prior interest in FLL. On the other hand, it may be considered desirable by those who wish to promote FLL to make the subjects appear more attractive in the school's information materials.

\subsection{The influence of siblings and peers}

The survey attempted to ascertain the degree to which student decisions about FLL had been influenced by older brothers and sisters or friends. Students who had opted for FLL were presented with item 9.3.7, "My older brother/sister encouraged me to try foreign language learning this year". Only 70 of the 272 students for whom the item was relevant agreed with it, but the fact that the majority disagreed could be interpreted to mean that older siblings had either not expressed an opinion on the subject, or had tried to discourage students from studying a FL but had been ignored. Overall, it would appear that older children did not greatly influence the decision of their younger siblings to study a FL.

When students not in FLL were presented with a similar item, 9.4.1, "My older brother/ sister says I should have tried a foreign language", only $15 \%$ of those who expressed an opinion $(\mathrm{N}=195)$ agreed and had obviously ignored the advice. Over two thirds disagreed but it is not possible to tell if that means that the subject was simply never discussed or that older siblings had actively discouraged younger ones from studying a FL. If the latter is the case then the influence of older siblings might have been quite considerable in dissuading younger ones from studying a FLL.

All the year 9 students were asked to indicate if they had chosen their options in order to stay with their best friends, item 9.2.10, but the overwhelming majority, $88 \%$ of those who responded $(\mathrm{N}=638)$, disagreed. Although over $80 \%$ of year 8 students intending FLL reported that they would like to be able to practise another language with their friends, just under half of the year 9 students who were studying a FL agreed that they liked practising another language with their friends, item 9.3.17 ( $\mathrm{N}=376)$. Such attitudes may, of course, have little influence on students' option choices.

The year 9 students not in FLL were presented with an item, 9.4.7, designed to gauge the general attitude to FLL among their friends. Only $22 \%$ of those who expressed an opinion $(\mathrm{N}=259)$ agreed that most of their friends thought that FLL was stupid, and nearly half disagreed, which suggests that general attitudes towards FLL are not negative. 


\section{4}

Parents who were interviewed were asked if they thought that their children's decision to study a language or not, or to choose a particular language, had been influenced by their friends or an older sibling. The parents offered some interesting examples which showed that peer or sibling influence could be powerful, positively or negatively. For example, two children had chosen a language option to be with their friends and another two because older siblings had studied the same language. On the other hand, one girl would not do a language, despite parental encouragement, because, as her mother put it, "learning a language is not a cool happening thing to do". Another parent believed that her son might have been put off studying a language because some of his friends had said it was boring, although others were studying a language.

\subsection{The influence of connections with, or travel to, non-English speaking places}

Students were asked if they had friends or relations living overseas and if these people spoke any LOTEs. The majority of students had no such connections, but 157 students reported that this was the case, and $71 \%$ of these students had decided to study a FL, significantly greater $(p<.02)$ than the overall proportion of students opting for FLL. This suggests that personal contacts with speakers of other languages encouraged interest in FLL.

Students were also asked if they had spent time anywhere where a LOTE is spoken, whether they had learnt any of the language and if they had enjoyed trying to speak it. Many students had travelled overseas, mostly to Australia or America, but 100 students reported that they had been somewhere a LOTE is spoken. As in the case of students with overseas connections, $71 \%$ of them had decided to study a FL. Thus another type of contact with speakers of other languages seems to have encouraged a greater interest in FLL than the average. Of these 100 students, 56 reported that they had learnt some of another language while overseas. They offered interesting comments about their language learning experiences on their travels. A few had made little effort or found it too difficult, but the majority, 47 out of 56 , reported that they had enjoyed that experience, especially the challenge of trying something completely new and different. One remarked: "some of the noises you had to make were hilarious". Several commented that it was fun to be able to communicate with the local people, even if only a little, and one student observed that "people appreciate you attempting their language". Others were as interested in the local culture as in learning the language.

On the whole, those students lucky enough to have travelled overseas had enjoyed their contact with other people and languages and it cannot be assumed that lack of any FLL learning while overseas was necessarily due to children's indifference or hostility to the idea. They may simply not have had the opportunity. Also, any positive attitudes they showed towards FLL may well have existed prior to their travels. In any case, students' experience of overseas FLL had no significant association with their decisions about FLL at school.

The parent interviews revealed some other types of contact with FL speakers which seemed to be having a positive attitude on their own and their children's attitudes to FLL, see Table 6.25. Many parents had travelled and had had opportunities to try out their school French, 
for example, and had usually appreciated the value of even their limited knowledge. There seemed to be growing numbers of overseas students being hosted by local families, often by friends or neighbours of the people interviewed, that children would meet at school. Some parents also reported that the local population was becoming more multicultural and that their children were becoming more used to mixing with NESB children.

Table 6.25 Family contacts with NESB people

\begin{tabular}{|l|c|}
\hline Type of contact & $\begin{array}{c}\text { Number of } \\
\text { mentions }\end{array}$ \\
\hline Parents' travel, especially using school French & 8 \\
\hline Family hosting overseas students & 8 \\
\hline Neighbours, family friends, work contacts & 7 \\
\hline $\begin{array}{l}\text { Children's contacts through school, scouts and } \\
\text { overseas students hosted by friends }\end{array}$ & 6 \\
\hline Family, eg immigrant grandparents & 5 \\
\hline Occasional visitors & 3 \\
\hline
\end{tabular}

\subsection{Final analysis}

In many ways the findings from the year 9 survey were similar to those from the previous year. As shown by the above discussion, the overwhelming majority of students in year 9 had positive attitudes towards FLL, although these were not always strong enough to persuade them to opt for a FL option. Like the majority year 8 students, most year 9 students did not believe that FLL was too difficult for most of them, although they did think that the ability to speak more than one language is considered clever by people in general and is likely to help them get a good job. Similarly, like the year 8 students, they were well aware that not everybody speaks English but were unsure about which languages would be most useful to learn. They did not consider learning Maori to be more important than learning a FL. They were uncertain about the relative importance of FLL in the curriculum and most did not think that it should be compulsory. The greatest value of FLL perceived by the students seems to lie in improving cultural understanding, while little value was attached to the development of general language skills. So the older students were no more aware than the younger ones of the transferability of language skills. The notion of 'usefulness' was not a major factor cited for or against FLL. Positive attitudes were based largely on interest in other languages and cultures, with future travel often in mind, and in language learning itself.

The attitudes of students who were learning a FL were generally positive, although many felt 
they had had little choice about undertaking such learning. Their motivation seemed largely integrative according to Gardner's (1985) broad definition. They were interested in other languages and cultures and in language learning itself, as other research has found (ERO 1994, Kern 1995). The only motivation which could be described as instrumental would be the students' interest in travel, see section 6.5. The overwhelming impression is that students were enjoying FLL and were confident about their learning, and those would be the main reasons why they would continue. Efforts to ascertain how many student would actually continue their FLL in year 10 were not particularly successful because the majority of students were uncertain, see section 6.6, largely as a result of the timing of the survey near the beginning of the year.

The largely positive attitudes among all the year 9 students is particularly well illustrated by the attitudes of those who had decided not to choose a FL option, see section 6.7. Less than half of these students had made that decision for negative reasons. This minority was simply not interested or thought it would be difficult, although most had no FLL experience on which to base their judgement. In fact, perceived difficulty was not a major factor, contrary to previous findings (Turner 1974, ERO 1994, Calderon et al. 2000). Over half the students thought FLL would be fun and about one quarter of them regretted not having been able to learn a FL. Very few ruled out the possibility of undertaking some FLL at some later time. Students with positive attitudes towards FLL had preferred other options but it is impossible to judge from the data whether this was the result of personal interest or a belief in the greater usefulness of other subjects.

As far as extrinsic factors are concerned, even before student attitudes were investigated it was clear that all optional areas of the year 9 curriculum were under timetable pressure in all the schools. Only in two of the schools could students study a FL for the whole year. In two other schools three terms of FLL were possible, but only at the expense of other subjects which students might like to have sampled, a "sacrifice" (as some teachers called it) which students choosing other subjects did not have to make.

Like the year 8 students, most of those in year 9 denied that they were influenced in their option subject choices by siblings or friends, but limited information from parents suggests that some of them at least were quite strongly influenced by their peers. Some degree of contact with speakers of LOTEs, through travel or other types of personal contact, was associated with a higher than average interest in FLL. The influence of parents will be examined in chapter 8 .

In answer to the research questions posed at the beginning of this chapter, it was found that the numbers of year 9 students involved in the five surveyed schools was, overall higher than the usual national average but that this figure was distorted by the different emphases placed on FLL in each school, see section 6.3.3. The reasons why there were or were not studying a FLL were similar to, and as complex as, the reasons given by the previous year's student sample. Also similar to the year 8 results were the variety and complexity of influences on the students' attitudes and option choices. 
One factor which will be of particular interest to all those who have been pushing for an earlier start to FLL in New Zealand schools is the fact that starting FLL in year 7 or 8 did not necessarily encourage students to choose a FL option in year 9 unless they had enjoyed that previous experience. If students thought they were good at such learning they were even more likely to choose further FLL. These factors will be explored in more depth in the following chapter, in the context of the results from the sub-sample who participated in both surveys. 


\section{COMPARISON OF YEAR 8 INTENTIONS AND YEAR 9 CHOICES}

A lack of language learning is one symptom of a lack of imagination. (Manhire 1999)

\subsection{Introduction}

As mentioned in the previous chapter, a comparison between year 8 students' intentions and their actual choices regarding FLL in year 9 could only be made with respect to the 169 students who were 'captured' in both student surveys. This chapter discusses the extent to which these students carried out the intentions, stated in the year 8 survey, to study or not study a FL when they started secondary school the following year, in answer to my fourth research question. One of the crucial questions asked by language teachers and advisers is whether or not the increased participation by schools in the Ministry of Education's SLLP is increasing the take-up of FL subjects at secondary level, research recommended by Peddie et al. (1999). So discussion will focus on the amount of FLL experience these students had had in years 7 and 8 , their feelings about the experience and the extent to which this influenced their choice to study a FL or not in year 9. As with the results for the whole year 8 and year 9 samples, any gender effects on results will be discussed in Chapter 10 .

\subsection{The sample, their intentions and final choices}

Of the 169 students in the sample, 77 intended to study a FL in year 9 (46\%), 38 were unsure $(22 \%)$ and $54(32 \%)$ did not intend such study. Graph 7.1 shows the relative proportions of the three groups. For comparison, in the whole year 8 sample $49 \%$ reported that they would be opting for FLL, 32\% were unsure and 19\% stated that they would not be learning a FL in year 9. For some reason, therefore, there was much less uncertainty among this sub-sample of students.

When they reached year 9 , small numbers of students had changed their minds about whether or not to opt into FLL. 9 students who had intended to study a FL had decided against it and 6 of those not intending FLL had opted for a FL, as had 12 of the undecided students. Thus, in the end, just over half the students were undertaking FLL in year 9, as shown in Table 7.1, compared with 58\% of the whole year 9 sample which, as discussed in the previous chapter, was a particularly high proportion. The pattern of choices among this sub-sample is much closer to the national trend.

Table 7.1 Student choices concerning FLL in year 9

\begin{tabular}{|l|c|c|}
\hline $\begin{array}{l}\text { Students in FLL in } \\
\text { year 9 }\end{array}$ & Number & Proportion \\
\hline Yes & 86 & $51 \%$ \\
\hline No & 83 & $49 \%$ \\
\hline
\end{tabular}


Graph 7.1 Student intentions for FLL in year 9

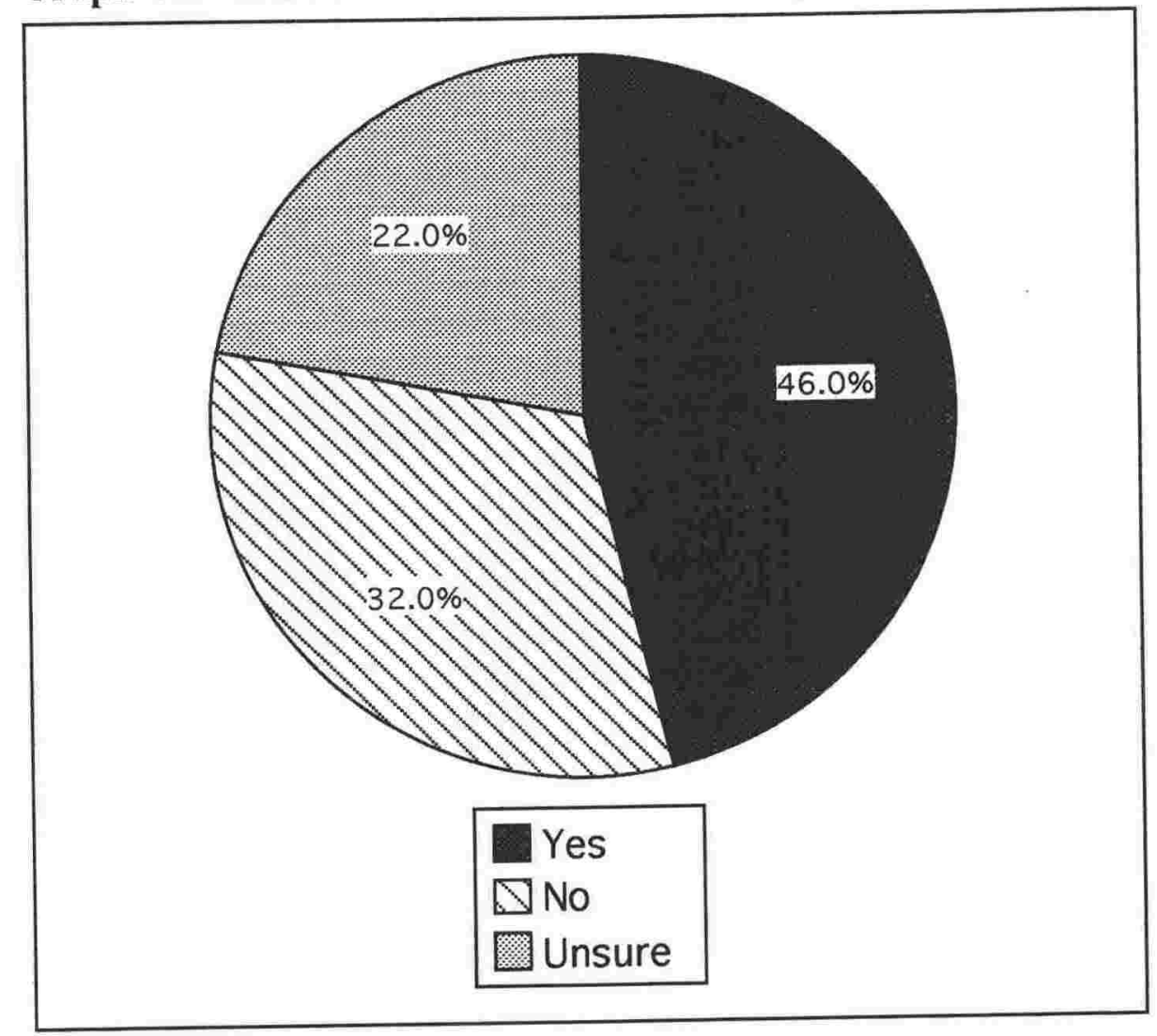

\subsection{The influence of previous FLL experience}

\subsubsection{Numbers of students with previous FLL}

The majority of the 142 students $(84 \%)$ had had some experience of FLL in years 7 and/or 8 , most of whom had had no choice in the matter. This proportion is slightly higher than that of the whole year 8 sample, $81 \%$ of whom had some experience of FLL, which might go some way to explaining the greater degree of certainty in the students' intentions for the following year.

\subsubsection{The effect of previous experience on intentions and final choices}

Graph 7.2 shows the comparison between students' intermediate FLL experience and their expressed intentions to study a FL or not in year 9. The largest proportion of students with FLL experience, $47 \%$, intended further FLL in year 9, while the largest proportion of students without FLL experience, $41 \%$, did not intend to study a FL. 
A similar small proportion of each group, under one quarter, was undecided. There was a much greater gap between the proportions of students opting for or against FLL among those with previous experience, than among those without. In fact, as only a very small proportion of the sample, 27 students, had not experienced any FLL in years 7 or 8 , the gap between the $37 \%$ and the $41 \%$ represents only one student. It appears that the fact of having some previous FLL experience was to some extent associated with intentions for future FLL. This contrasts with the results for the whole year 8 sample, discussed in 4.8.1, where previous FLL experience seemed to have had little effect on student intentions. In the smaller sample the lack of prior FLL seems to have had a more negative effect on students' intentions, but the apparent difference may simply be due to the small number of students concerned.

Graph 7.2 Proportions of students intending FLL in year 9 compared with previous FLL

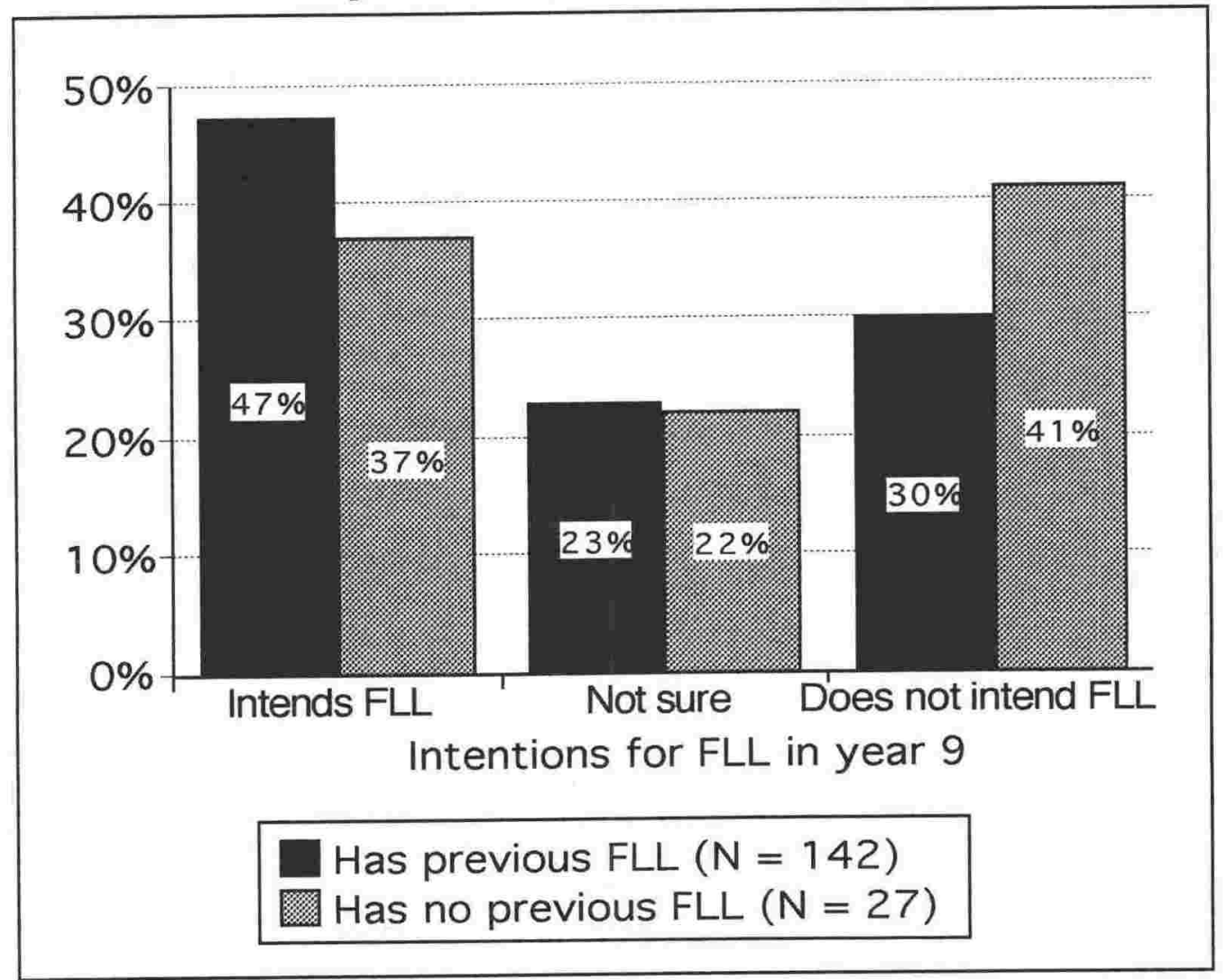


When these 142 students reached year 9, those with previous FLL were fairly evenly divided in their final decision about FLL, with just over half opting for it. Those without such experience were more likely to have decided against learning a FL, see Table 7.2. These results differed form those of the whole year 9 sample, discussed in 5.8.2, where the proportion of students choosing FLL without previous FLL experience was higher than the proportion of those with such experience. It is not possible, thus far, to draw any firm conclusions about the influence on previous FLL experience on year 9 choices in that regard.

Table 7.2 Choice of FLL in year 9 compared with previous FLL experience

\begin{tabular}{|l|c|c|}
\hline \multirow{2}{*}{$\begin{array}{c}\text { FLL in } \\
\text { years 7/8 }\end{array}$} & \multicolumn{2}{|c|}{ Students in FLL in year 9 } \\
\cline { 2 - 3 } Number & Proportion \\
\hline Yes $(\mathbf{N}=\mathbf{1 4 2})$ & 75 & $53 \%$ \\
\hline No $(\mathbf{N}=\mathbf{2 7})$ & 11 & $41 \%$ \\
\hline Total $(\mathbf{N}=169)$ & 86 & $51 \%$ \\
\hline
\end{tabular}

Graph 7.3 Students' reported enjoyment of FLL at intermediate level $($ Missing data $=11$ )

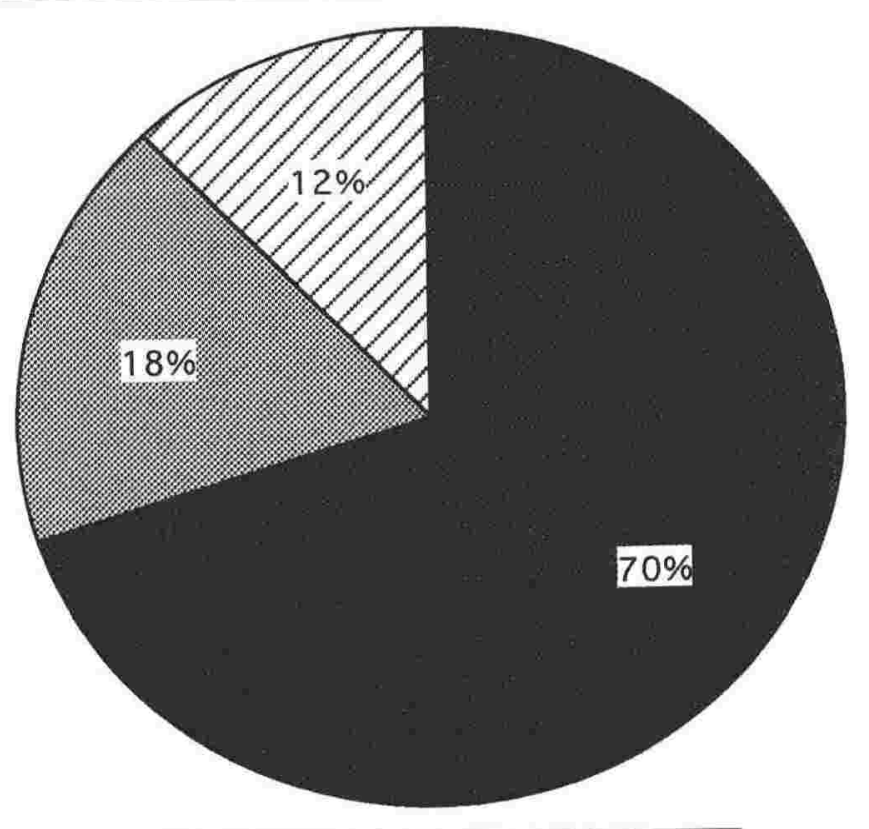

Enjoyed $(\mathrm{N}=92)$

Did not enjoy $(\mathrm{N}=23)$

$\square$ Mixed feelings $(N=16)$ 
7.3.3 The importance of enjoyment of previous FLL experience

Of the 142 students who had studied one or two FLs in years 7 and 8, $92(65 \%)$, reported that they had enjoyed the experience. A few expressed mixed feelings, including those who had enjoyed one FL and not the other, and only 23 , about $16 \%$, reported not having enjoyed their FLL. Graph 7.3 shows the relative proportions of positive, negative and mixed responses to the question "Did you enjoy the [FL] classes?"

\subsubsection{The effect of enjoyment on intentions and final choices}

A comparison between Graph 7.2 and Graph 7.4 shows that if students enjoyed their FLL at intermediate level they were more likely to express the intention of opting for into FLL in year 9. Of the 92 who enjoyed their experience, $55 \%$ were intending to study a FL compared with $47 \%$ of all students with previous experience of FLL. The proportion of students who did not enjoy their previous FLL and who were not intending future FLL, 39\%, was not much greater than the proportion of all students with previous experience of FLL, $37 \%$.

\section{Graph 7.4 Proportions of students intending FLL in year 9 compared} with enjoyment of previous FLL (Missing data = 11)

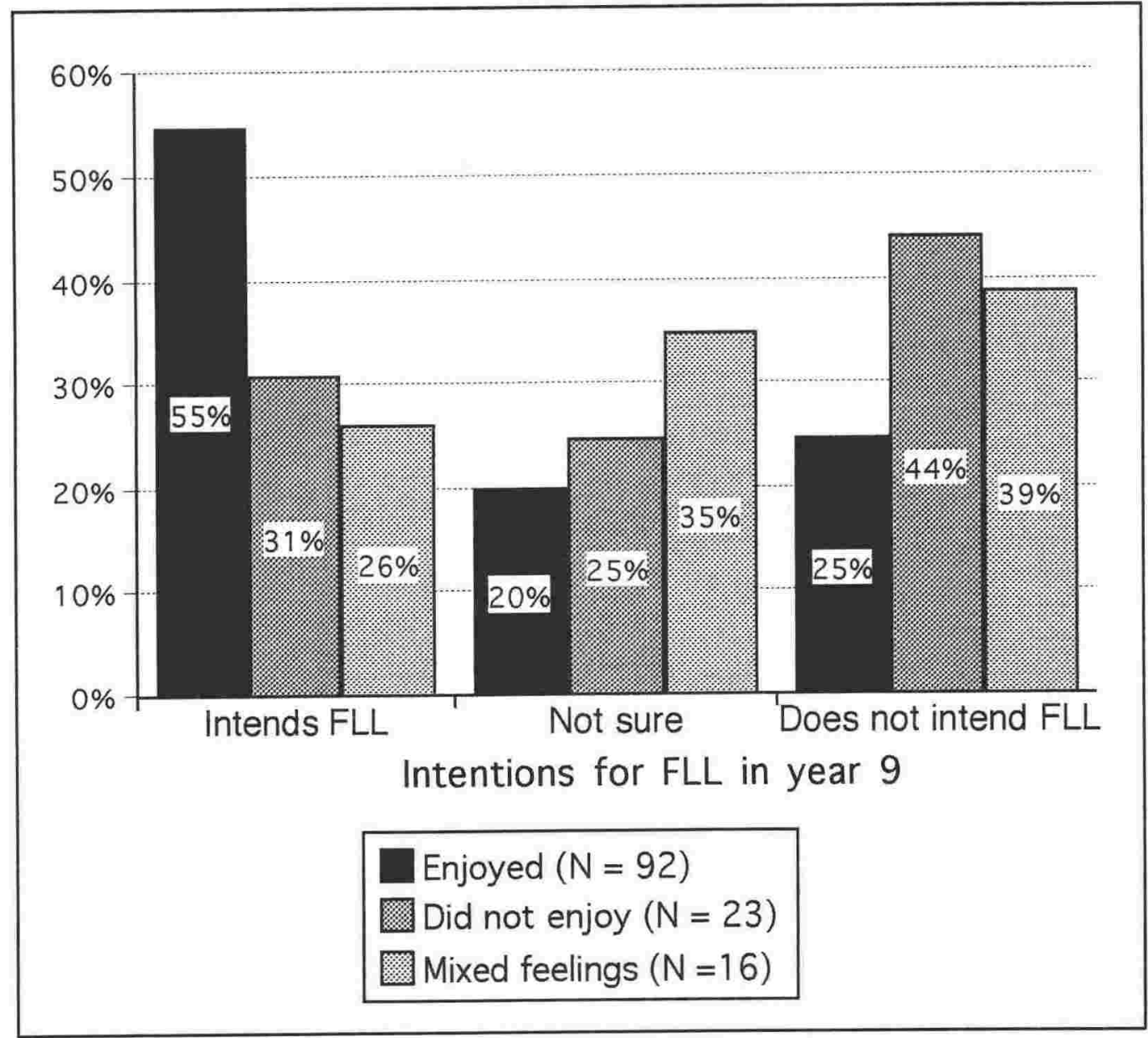


However, lack of enjoyment had considerably increased the proportion of students who were unsure if they would do any future FLL, from under one quarter to over one third. It is not surprising that students who did not enjoy a subject should intend to avoid it, if that was possible, in the future. However, over one quarter of students who reported not enjoying or who had mixed feelings about FLL at intermediate school still intended to choose a FL option in year 9.

Table 7.3 Choice of FLL in year 9 compared with enjoyment of previous FLL (Missing data $=11$ )

\begin{tabular}{|l|c|c|}
\hline \multirow{2}{*}{$\begin{array}{c}\text { Enjoyment of FLL } \\
\text { in years 7/8 }\end{array}$} & \multicolumn{2}{|c|}{ Students in FLL in year 9 } \\
\cline { 2 - 3 } & Number & Proportion \\
\hline Yes $\mathbf{N}=\mathbf{9 2})$ & 58 & $63 \%$ \\
\hline Mixed feelings (N = 16) & 6 & $37 \%$ \\
\hline No (N = 23) & 6 & $26 \%$ \\
\hline Total N = 131 & 70 & $53 \%$ \\
\hline
\end{tabular}

A comparison between Tables 7.2 and 7.3 shows that students' enjoyment of their previous FLL not only increased the likelihood that they would intend further FLL at secondary school but also increased greatly the proportion who finally opted for FLL. $63 \%$ of the 92 students who had enjoyed their intermediate FLL opted for a FL in year 9, compared with $67 \%$ of the whole year 9 sample who had reported enjoyment of prior FLL. This proportion of students opting into FLL is considerably higher than the overall proportion of $53 \%$, and the association between enjoyment of previous FLL and the decision to study a FL in year 9 proved to be statistically significant $(\mathrm{p}<.005)$. Students were clearly encouraged by their personal enjoyment of FLL, be it of language learning in general or of a specific language.

\subsubsection{The importance of confidence in previous FLL experience}

Although $65 \%$ of the 142 students with intermediate FLL experience had enjoyed it, only 77 of them, about $54 \%$, responded positively to the question "Do you think you were good at language learning?". Only three reported that they did not know if they were good at it. Graph 7.5 shows the relative proportions of students who responded to the question. 
Graph 7.5 Students' reported confidence in FLL at intermediate level (Missing data $=13$ )

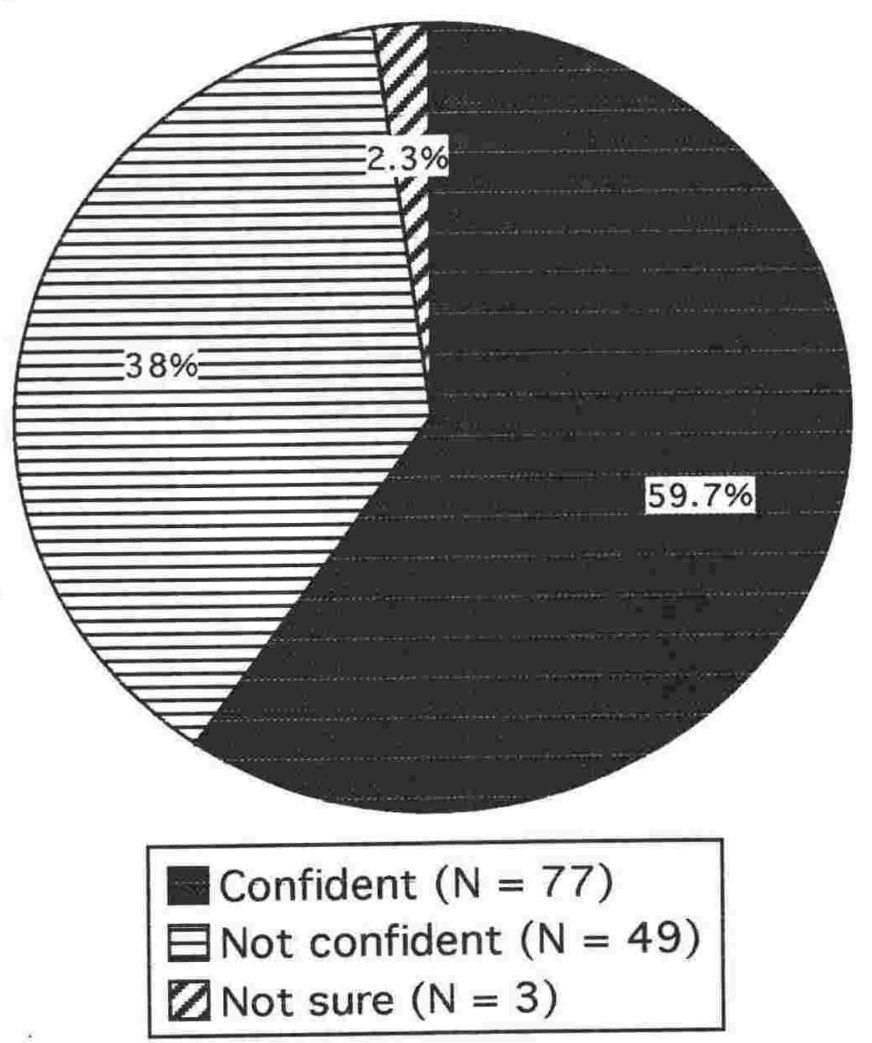

\subsubsection{The effect of confidence on intentions and final choices}

Graph 7.6 shows the effect of students' confidence in their previous FLL on their intentions for year 9. A comparison with Graph 7.4 shows that if students felt that they were good at their FLL they were even more likely to intend to study a FL in year 9 than if they had enjoyed their previous experience. $60 \%$ of the 77 who were confident in their previous FLL intended future FLL compared with 55\% of the 92 who had enjoyed their FLL. Among the students who were unsure or not intending future FLL there were greater differences between the proportions of students who were confident or not confident about their past FLL than between those who enjoyed or did not enjoy their previous FLL. Statistical analysis showed a significant association $(\mathrm{p}<.002)$ between students' confidence in their ability to learn a FL and their intentions for the following year. 
Graph 7.6 Proportions of students intending FLL in year 9 compared with confidence in their previous FLL (Missing data=16)

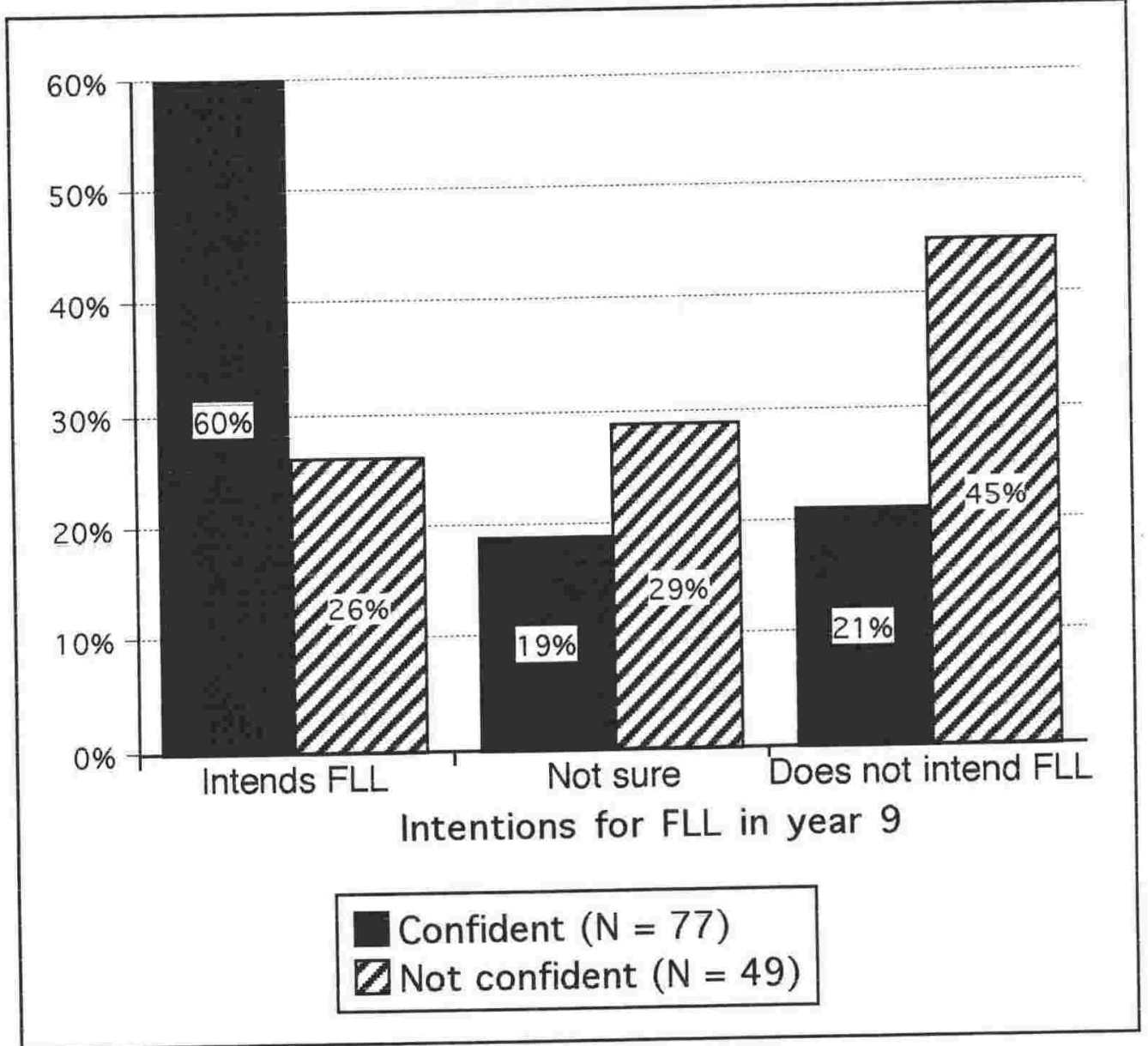

Confidence in earlier FLL also proved to be a more important factor in students' final decisions about FLL than the other factors already discussed, as shown in Table 7.4. $69 \%$ of the 77 who thought they were good at language learning decided to chose a FL option in year 9 compared with the $63 \%$ of the 92 who had enjoyed their previous FLL. The association between students' confidence and their final choices was statistically highly significant $(\mathrm{p}<.001)$. It must be pointed out that over one quarter of the students who did not think they were good at FLL at intermediate school were still prepared to try further FLL at secondary school, although it must also be reiterated that some were under pressure to study a language and may have chosen a FL in preference to Maori or, in some cases, music. 
Table 7.4 Choice of FLL in year 9 compared with confidence in previous FLL (Missing data $=16$ )

\begin{tabular}{|l|c|c|}
\hline \multirow{2}{*}{$\begin{array}{c}\text { Confidence in FLL } \\
\text { in years } \mathbf{7 / 8}\end{array}$} & \multicolumn{2}{|c|}{ Students in FLL in year 9 } \\
\cline { 2 - 3 } & Number & Proportion \\
\hline Yes $(\mathbf{N}=\mathbf{7 7})$ & 53 & $69 \%$ \\
\hline No $(\mathbf{N}=\mathbf{4 9})$ & 15 & $31 \%$ \\
\hline Total $\mathbf{N}=\mathbf{1 2 6}$ & 68 & $54 \%$ \\
\hline
\end{tabular}

\subsubsection{The influence of specific FLs on intentions}

Table 7.5 shows the FLs studied by this sub-sample. Among the three main groups of students, totalling 58, who had learnt Japanese, Spanish or a combination of French and Japanese, 45\% intended to study a FL in year 9. The least likely to intend further FLL and the most likely to be unsure were those who had learnt only Spanish, which is not surprising, given the limited availability of the subject at the local secondary schools.

Table 7.5 Intentions for FLL in year 9 compared with FLs studied at intermediate level

\begin{tabular}{|l|c|c|c|}
\hline \multirow{2}{*}{$\begin{array}{l}\text { FLs studied at } \\
\text { intermediate }\end{array}$} & \multicolumn{3}{|c|}{ Students intended to study a FL in Y9 } \\
\cline { 2 - 4 } & Yes & Not sure & No \\
\hline $\begin{array}{l}\text { French + Japanese } \\
(\mathbf{N}=\mathbf{6 4})\end{array}$ & 30 & 8 & 26 \\
\hline Spanish (N= 34) & 13 & 15 & 6 \\
\hline Japanese (N = 31) & 15 & 8 & 8 \\
\hline $\begin{array}{l}\text { Spanish + Japanese } \\
(\mathbf{N}=\mathbf{8})\end{array}$ & 7 & 1 & 0 \\
\hline Chinese & 0 & 0 & 2 \\
\hline French & 1 & 0 & 0 \\
\hline French + Spanish & 0 & 0 & 1 \\
\hline German & 1 & 0 & 0 \\
\hline
\end{tabular}


Students were asked which FL they would or might study in year 9. A few students intended to study two languages and a few were not sure which of two named languages they would study. Some students who reported that they might choose a FL option did not specify which FL they might learn. Table 7.6 shows the numbers of students from the three main groups of intermediate FL learners who reported that they would or might study a FL in year 9 and which languages they intended to study. Those who had learnt only Japanese were very unlikely to consider studying another language. Those who had learnt both French and Japanese were more likely to choose Japanese than French in year 9. Those who had learnt Spanish were, predictably, the most divided in their future choices of language. It is noticeable that no students intended to study Spanish if they had not already been introduced to it. Similarly, there was very little reported interest in German, which could be attributed to the fact that only one student had learnt it at intermediate.

Table 7.6 Intended FLs for study in year 9 compared with FLs studied at intermediate level

\begin{tabular}{|l|c|c|c|c|}
\hline \multirow{2}{*}{$\begin{array}{l}\text { FLs studied at } \\
\text { intermediate }\end{array}$} & \multicolumn{4}{|c|}{ Languages intended for study in Y9 } \\
\cline { 2 - 5 } & French & German & Japanese & Spanish \\
\hline $\begin{array}{l}\text { French + Japanese } \\
(\mathbf{N}=\mathbf{6 4})\end{array}$ & 13 & 1 & 20 & 0 \\
\hline Spanish (N = 34) & 2 & 2 & 5 & 9 \\
\hline Japanese (N = 31) & 1 & 2 & 16 & 0 \\
\hline
\end{tabular}

\subsubsection{Did students carry out their intentions?}

Table 7.7 shows which languages students finally chose in Year 9 compared with their intentions reported the previous year, where these were clearly stated. The majority of students who intended to study Spanish in year 9 did so. The two who did not could have done so at their secondary school and did not choose another FL instead. In contrast, five of the six students who intended to study German were unable to do so because the language was not available, and only two of them were prepared to study another FL instead.

Although French and Japanese were available at all the secondary schools a small number of students changed their minds about which language to study. Three who had intended to study French and but only one who had intended to study Japanese chose another FL. Over three quarters of students who had stated their intention to study Japanese did opt for that language, compared with only just over half of those who had intended to study French. It seems that, when it came to choosing among competing options, Japanese had a greater appeal than French. Most students did not 
change their minds about which FL they wanted to study, but other factors persuaded about one quarter of them not to study a FL at all.

Table 7.7 FLs chosen for study in year 9 compared with reported intentions in year 8

\begin{tabular}{|l|c|c|c|c|}
\hline \multicolumn{2}{|l|}{} & \multicolumn{4}{|l|}{ No. of students studying in Y9 } \\
\hline $\begin{array}{l}\text { Would or } \\
\text { might study }\end{array}$ & $\begin{array}{l}\text { No. of } \\
\text { students }\end{array}$ & $\begin{array}{c}\text { Intended } \\
\text { FL }\end{array}$ & $\begin{array}{c}\text { Another } \\
\text { FL }\end{array}$ & No FL \\
\hline French & 22 & 12 & 3 & 7 \\
\hline German & 6 & 0 & $2 *$ & 4 \\
\hline & 50 & 38 & 1 & 11 \\
\hline Japanese & 10 & 8 & 0 & $2 \dagger$ \\
\hline
\end{tabular}

7.3.9 Summary of the influence of prior FLL experience on year 9 decisions

Table 7.8 summarises the information discussed above and shows clearly that simply having tried FLL in years 7 or 8 did not necessarily encourage students to continue it in year 9 .

Table 7.8 Proportions of students who intended FLL in year 9 compared with their final choices, according to their prior FLL experience

\begin{tabular}{|l|c|c|c|}
\hline \multicolumn{2}{|l|}{} & \multicolumn{2}{c|}{ Proportions of students } \\
\hline Relevant sample & N & $\begin{array}{c}\text { Intended } \\
\text { FLL in y9 }\end{array}$ & $\begin{array}{c}\text { Opted for } \\
\text { FLL in y9 }\end{array}$ \\
\hline All students & 169 & $46 \%$ & $51 \%$ \\
\hline Those with prior FLL & 142 & $47 \%$ & $53 \%$ \\
\hline $\begin{array}{l}\text { Those who enjoyed prior FLL } \\
\text { Those who thought they were } \\
\text { good at FLL }\end{array}$ & 92 & $55 \%$ & $63 \%$ \\
\hline
\end{tabular}


If students had enjoyed their prior experience they were, not surprisingly, more likely to continue, but believing that they were good at FLL had a much more positive influence on both their intentions and final choices for FLL in year 9. This supports the findings of Gardner (1985), Dörnyei (1996) and Holt et al. (2001) that achievement improved attitudes towards FLL.

\subsection{Student profiles}

I thought it valuable to examine closely some individual student choices about FLL in year 9 compared with the intentions they stated in year 8 , where these were different or unclear. Of the 77 students who reported that they would study a FL in year 9, only 9 changed their minds, and only 6 who had not intended to study a FL opted into FLL. Of the 38 students who were not sure if they would study a FL in year 9, including two who did not give any indication, the majority, 26, decided against it. Some examples of students in each of these categories, with their beliefs and attitudes, will be examined in detail in the following sections. All the students have been given pseudonyms.

\subsubsection{Students who intended to study a FL in year 9 but chose not to}

Jane had no experience of FLL in years 7 and 8 but intended to study French the following year because "it sounds like a good language". She also stated that "it should be fun learning different languages at high school". She strongly agreed that it was a pity that language options the following year were only for half the year and that a FL should be part of the core curriculum at secondary school. She strongly disagreed that there were lots more important things to learn than languages or that knowing more than one language was confusing. She gave clear indications that her option choices would be made independently from her friends and parents. Altogether Jane seemed to have positive attitudes towards learning French but in year 9 she had chosen the short ( 8 week) culture model instead of the whole year option, which almost certainly precluded her from any further FLL at that school. She still stated that she would like to have studied French but gave no indication as to why she had not chosen the full language option. One can only conclude that other options seemed more interesting or important to her.

Carol enjoyed Japanese classes at intermediate school "because they were fun" and thought she was good at learning the language. She stated that she would be continuing to study Japanese the following year although she did not expect it to be easy. She indicated that she was interested in the chance to go overseas on a school trip and agreed that it was a pity that language options the following year were only for half the year. She also agreed that a FL should be part of the core curriculum at secondary school. Carol's survey responses suggested that her parents and friends might influence her subject choices to some extent. In year 9 she had not chosen a FL option "because I may be doing it next year and I had already chosen what I wanted to do". This reveals that other options had taken priority, although she stated that she would like to have studied Japanese and/or French. She strongly agreed that learning a FL would be fun and that she'd like to learn a FL one day. She strongly 
ruled out the possibility that the difficulty of the subject had deterred her. Carol was under the misapprehension that it was possible to start FLL in year 10 which was extremely unlikely.

Mark had just started learning Japanese in year 8 and had already enjoyed a term of French, agreeing that their language teacher made learning really fun. He was also interested in Latin which his father had learnt, and thought it would be "easy to pick up". He strongly suggested that he could choose his subjects quite independently from his parents but other responses indicated that he might be influenced by his friends. He did not think that knowing more than one language was confusing and, like Carol, was interested in the chance to go overseas on a school trip. Mark strongly agreed that knowing another language would help him get a good job. However, some of his responses seemed to contradict these apparently positive attitudes towards FLL. He strongly agreed that it was better for him to spend time doing more English than learning another language and that there were lots more important things to learn than languages. It is, therefore, not surprising that, when he reached secondary school, he had decided not to study a FL. In the end he had not enjoyed Japanese and thought he was not very good at it. Furthermore, he agreed that learning a FL would be too difficult for him and strongly disagreed that it should be compulsory. Mark also agreed that he was more interested in practical subjects than those with lots of reading and writing. Thus Mark was dissuaded from learning a FL by preferences for other options and by his intermediate experience.

\subsubsection{Students who considered studying a FL in year 9 but decided against it}

Kim learnt some French and Japanese at intermediate school and especially enjoyed the Japanese. She agreed that their language teacher made learning really fun. She also expressed an interest in learning German because she wanted to travel there one day, but her secondary school did not offer German so she thought she would continue Japanese. In year 8 she agreed that everyone should learn a European language, but by year 9 she was agreeing that learning a FL is too hard for most students. Kim agreed that knowing more than one language was confusing and did not think she would find FLL easy the following year. In year 9 she reported that she did not think she was good at language learning and had not chosen a FL option because she had found it quite hard, although she would like to have studied a language. She wanted to be a teacher and thought it would help her get a job, which reinforced the belief she expressed in year 8 , that knowing another language would help her get a good job. In year 8 she disagreed that there were lots more important things to learn than languages, but in year 9 strongly agreed that languages clashed with more important subjects. She reported that she would like to learn another language one day and thought it would be fun. Kim, despite more positive attitudes than Mark's, similarly had other priorities for year 9 options and was discouraged by the apparent difficulty of FLL. 
Sally also studied French and Japanese at intermediate school and thought she might continue Japanese "because I liked learning it this year" and "because it is fun and the people are very nice". She enjoyed learning about the Japanese lifestyle as well as the language. She strongly agreed that learning another language was easier than she had expected and the next year she reported that she had been good at language learning. She strongly disagreed that knowing more than one language was confusing or that learning a FL would be too difficult for her. In year 8 she agreed that it was a pity that language options the following year were only for half the year and that knowing another language would help her get a good job. However, in year 8 Sally also strongly agreed that there were lots more important things to learn than languages, a statement she still agreed with in year 9. In fact, she gave as the reason for not studying a FL in year 9: "There are other options I think are more important." She added, however, that would like to have studied Japanese or Spanish and thought she would learn a language one day. Sally strongly agreed that learning a FL should be part of the core curriculum at secondary school and did not think FLL was too hard for most students. She also strongly agreed that she wanted to learn a FL but languages clashed with more important subjects, and that learning a FL would be fun. In Sally's case, very positive attitudes towards FLL did not outweigh apparently more important options.

Guy learnt Japanese at intermediate school and thought he might continue because "it was fun". He agreed that what he had seen of languages he could learn the following year made them look really interesting. In year 9 he reported that he had enjoyed the Japanese classes because of the different activities and food, agreeing that their language teacher made learning really fun, but he did not think he was good at language learning. Despite his enjoyment he strongly agreed that knowing more than one language was confusing, and by year 9 he agreed that learning a FL would be too difficult for him. In year 8 Guy strongly agreed that it was better for him to spend time doing more English than learning another language, an idea that seemed to be supported by his parents, and that there were lots more important things to learn than languages. When asked why he was not studying a FL in year 9 Guy said: "I like the majors where you build things and use different tools" and "languages aren't my subject". Not surprisingly, he agreed that he was glad he did not have to learn a FL that year and that he was more interested in practical subjects than those with lots of reading and writing. Guy may have been influenced by his peers since he agreed that most of his friends thought that learning FLs was stupid.

Sam learnt some German and Spanish at intermediate school "because I wanted to do something different". Although German was available at the secondary he intended going to he thought he might learn French because he wanted to go to France when he was older. He strongly agreed that everyone in New Zealand should learn a European language and that knowing another language would help him get a good job. Although he strongly agreed that knowing more than one language was confusing he thought he would find learning a language easy the next year. 
However, Sam also strongly agreed that there were lots more important things to learn than languages and that it was better for him to spend time doing more English than learning another language, an idea which his parents seemed to support. Sam's responses to statements about his prior FLL experience showed mixed feelings and he clearly did not want to do more of the same languages. He strongly agreed, nevertheless, that he would like to try another language. In year 9, Sam reported that he had not enjoyed his previous FLL because "my teacher was very slow", although he thought he was good at language learning. He strongly agreed that FLs should be part of the core curriculum at secondary school and that knowing a FL would help students get a good job, but also that languages clashed with more important subjects. Like Guy he agreed that most of his friends thought that learning FLs was stupid but he indicated that he would like to learn a FL one day, indicating a desire to learn more Spanish. He did not think it would be too difficult for him but that it would be fun.

\subsubsection{Students who did not intend to study a FL in year 9 but did choose a FL option}

Ellie learnt French and Japanese at intermediate school and enjoyed them because she like learning about different cultures. She stated: "I think that it's a good thing to know a language but I want to learn Gaelic and we don't have the option". She indicated later that she was proud of her part-Celtic heritage and wanted to learn Gaelic partly because it was a dying language. Ellie agreed that what she had seen about languages she could learn the following year made them look really interesting, but also that there were lots more important things to learn than languages and that her parents thought she should spend more time on her English than learning another language, although she indicated that they did not mind what subjects she chose. By year 9 she had decided to learn French because "I think it would be fun and it may help me in jobs later". She indicated that her parents had wanted her to study a FL. She did not think that it should be part of the core curriculum but nor did she think that FLL was too hard for most students.

Mel learnt French and Japanese at intermediate school and, although he did not intend to study a FL in year 9, he did express an interest in learning more Japanese, with the idea of going to work there. With regard to his prior FLL he agreed that he would like to know more about the life and customs of the people who speak the language. He was unsure about most of the year 8 survey items but did agree that there were many more important things to learn than languages. In year 9 he reported that he had not enjoyed his earlier experience of French, because of the teacher, but that Japanese was fun. He thought he was good at language learning and that he would do well in the subject that year. He indicated clearly that he was studying Japanese out of personal interest, not through any pressure from his parents or the school. Mel clearly appreciated the value of FLL for a variety of reasons. 


\subsubsection{Students who thought they might study a FL in year 9 and did}

so

Don had no experience of learning a FL before starting secondary school but thought he might learn French or German, most probably the former as his family knew some French. He reported that his parents, who had lived in France for 3 years, wanted him to learn a language the following year but that they let him choose whatever subjects he wanted to do. Don agreed in both year 8 and 9 that there were lots more important things to learn than another language. In the end, however, he did opt to learn French, saying "it would be cool to be able to speak another language". He strongly disagreed that he was studying a language because his parents insisted, although he agreed that they were pleased that he was doing so. He also firmly denied that he had chosen his options to stay with his best friend. Don seemed to have mixed feelings about whether FLL was fun but indicated that he wanted to continue the following year. However, it was interesting to note that he did not want to know more about the life of French-speaking people, nor was he interested in meeting any of them. There were indications that he was finding French a bit difficult. In year 8 he strongly disagreed that knowing another language was confusing but in year 9 he agreed that learning a FL was too hard for most students. $\mathrm{He}$ also did not think he would do well in his language learning, noting that it was a "little bit boring".

Lisa thought she might learn Spanish at secondary school because she had already learnt some, and might go to Spain one day. She strongly agreed that she would like to know more about the life and customs of Spanish-speaking people. She disagreed that knowing more than one language was confusing or that FLL was too hard for most students, adding the comment: "It can be exciting if you really want to learn the language." Lisa also reported that she was attracted to FLL because of the chance to go overseas on a school trip. She agreed that it was a pity that language options for the following year were only for half the year. In year 9 she reported that she had not always enjoyed Spanish classes at her previous school, but she was clearly still keen on future travel. She was also confident that she would do well in her language learning. Lisa disagreed strongly that she was only studying a FL because her parents had forced her, but they were obviously pleased that she was doing so. As regards the possible usefulness of FLL for jobs Lisa made the perceptive comment: "Just because you know another language doesn't mean you can get a job easier. It just depends on what sort of job you want when you are older."

\subsubsection{Summary of profiles}

To sum up these profiles, it seems that most of the students had positive attitudes towards FLL, especially at intermediate school, which supports previous findings in New Zealand (Mahon 1989, Vine 1993, Fitzsimons 1999, Peddie et al. 1999). All but one of the above students who had stated in year 8 the intention or the possibility of studying a FL the following year had very positive attitudes but had decided against FLL citing mostly their preference for other optional subjects. Only the boys 
thought that it was better to spend more time on their English and two out of three seem to have been influenced by their parents in this respect. Only boys agreed that their peers thought FLL was "stupid" and only boys stated a preference for practical subjects over those involving reading and writing. The boys more than the girls seem to have been put off by the difficulty of the FLL they had encountered at intermediate level. In other words, the boys seem largely to have fallen into the stereotypical pattern of rejecting FLL. All four girls but only one of the boys thought that all students should have some FLL experience, which suggests that they would have been happier if FLL had been built into their year 9 timetable rather than competing with other attractive subjects. On the other hand, if the boys who stated a preference for practical subject were able to do these as well as some language learning, and if this was considered acceptable by the majority of their peers, there are clear indications that they might actually appreciate the opportunity.

Those who had decided in favour of FLL, despite expressing doubts in year 8 or a firm intention not to study a FL, all showed a personal interest in language learning, usually for both enjoyment and future use, and they all seemed to reflect positive parental encouragement. One of the boys was particularly confident about learning Japanese, building on his previous experience, was interested in the culture and intended to travel to Japan. The other boy, on the other hand, had no prior experience of FLL and seemed to have little interest in French culture or people. His responses suggest some parental pressure to learn French. As he was finding the subject difficult and boring it might be predicted that he would not continue the following year. It would be interesting to know whether, if he had made an earlier start and had enjoyed the experience, he would have had a more positive attitude towards his year 9 FLL experience.

While it is impossible to generalise from such a small sample of students, they do tend to reflect the main trends revealed by the data as a whole, in particular the gender differences which will be discussed in detail in chapter 10. On the whole the mostly positive student attitudes do not seem to have changed much from one year to the next, but external factors appear to have had a strong negative influence on final choices. For the girls this was chiefly the fact that FLs compete against other optional subjects, but for the boys peer and parental attitudes and negative FLL experience seem to be crucial factors.

\subsection{Final analysis}

Typically, about half the students in this sub-sample decided to study a FL in year 9. The majority, $89 \%$, of those who stated a clear intention in year 8, to study a FL or not, carried out their intentions. Only 15 students changed their minds. Students who had been unsure about FLL in year 9 were twice as likely to decide against such study as for it. Thus it seems that, by the end of year 8 , over two thirds of the students in this sample had developed a fixed attitude about the relative value of studying a FL the following year. Their attitudes were, on the whole, positive towards both their experience of FLL and the idea of under- 
taking it in the future, but the necessity of making choices between FLs and many other options which seemed more 'useful' seemed to be the main reason that discouraged them from FLL at secondary school. Those that opted for FLL mostly did so out of strong personal interest rather than any instrumental motivation. These results support the findings of ERO (1994) and Johnson (2001) that timetable pressures in the secondary school system tend to diminish the chances of students opting into FLL, however keen they might be.

Enjoyment of, and confidence in, prior FLL experience proved important in encouraging these students to continue but the small size of the sample, especially the limited number of students without prior FLL, probably make it impossible to generalise about these results. As Peddie et al. (1999) recommended, further research needs to be undertaken in relation to the SLLP to ascertain if an earlier start to FLL increases the involvement of students in FLL at secondary level, especially beyond year 9 . In the meantime, while the competitive climate among option subjects persists in secondary schools, it seems that the onus remains on FL teachers to create positive attitudes towards FLL and increase the numbers of FL students, if this is considered desirable (ERO 1994).

The next chapter presents the FLL experiences and attitudes of the small sample of parents who were interviewed, and investigates the extent of their influence on their children's option choices in year 9 . 
information. Most of the parents would have been at school at the time when many, if not most, had no choice about FLL. Students' academic ability was assessed and they were placed in classes and subjects considered appropriate to their ability. For example, students in a 'professional' class would find that they were expected to learn a FL and perhaps Latin as part of their core curriculum for at least one or two years, while students in another class might not have the opportunity to study a FL. In general, students did not have a choice of FL since French was often the only one available.

\subsubsection{The influence of parental FLL experience on student choices}

Clearly, many parents would have been unable to give their children advice about the nature or value of FLL based on first-hand experience and might have consider FLL suitable only for the most intelligent students. Whatever parents' views about their school experience of FLL, they might have been unaware of the enormous changes in teaching methodology and materials in the last 20-30 years. In particular, parents might have found FLL boring or difficult, and hence have discouraged their children from trying a FL.

Table 8.1 compares what students reported about their parents' FLL at school and their own decisions to study a FL or not in year 9. It is clear that if parents had studied a FL their children were significantly more likely $(p<.001)$ to have opted into FLL. It could be assumed that the majority of such parents had enjoyed their FLL or appreciated its value and had therefore directly or indirectly encouraged their children to study a FL.

Table 8.1 Student decisions regarding FLL in year 9 compared with their parents' experience of FLL at school

\begin{tabular}{|l|c|c|}
\hline $\begin{array}{l}\text { One or both parents studied a } \\
\text { FL at school }\end{array}$ & $\begin{array}{c}\text { In FLL in } \\
\text { Y9 }\end{array}$ & $\begin{array}{c}\text { Not in FLL } \\
\text { in Y9 }\end{array}$ \\
\hline Yes $(\mathbf{N}=\mathbf{2 4 3})$ & $72 \%$ & $28 \%$ \\
\hline No $(\mathbf{N}=\mathbf{2 7 9})$ & $45 \%$ & $55 \%$ \\
\hline $\begin{array}{l}\text { For association between parents' experience and students' decisions } \\
\text { p<.001 }\end{array}$ \\
\hline
\end{tabular}

\subsubsection{The general extent of parental influence on students' subject choices}

All students were asked to respond to the statement, "My parents let me choose whatever option subjects I wanted to do this year", item 9.2.18. 87\% of students (N $=657$ ) asserted that they had chosen exactly what they wanted to study without interference from their parents. In fact, over half strongly agreed with the statement. A similar picture was obtained from students involved in FLL when they responded 
to item 9.3.11, "I'm only doing a foreign language because my parents said I had to". The overwhelming majority of students, $92 \%$, disagreed that this was the case. I will show below that the parent interviews tended to support what the students reported about parental influence over the choice of option subjects. It seems that if parents did influence their children's choices it was mostly indirectly, without either parents or students realising it.

\subsubsection{Parental attitudes towards FLL in New Zealand schools}

From what the students reported, it appears that very few parents expressed negative attitudes towards FLL to their children. $80 \%$ of the 635 students who responded to item 9.2.3 disagreed with the statement, "My parents think that studying a foreign language is a waste of time for New Zealand students". The relatively high number who gave no opinion, 29, had presumably never discussed the topic with their parents. Cross tabulating responses to this item and what students had decided about FLL added support to the notion that parents' attitudes had affected student choices even if the students were not aware of this. $67 \%$ of the 507 students who disagreed with the item, but only $31 \%$ of the 39 who agreed, had decided to study a FL, a significant association $(\mathrm{p}<.001)$, see Table 8.2 .

\section{Table 8.2 Student decisions regarding FLL compared with responses} to item 9.2.3, "My parents think that studying a foreign language is a waste of time for New Zealand students" (No opinion $=29$ )

\begin{tabular}{|c|c|c|}
\hline Item response & In FLL in $Y 9$ & $\begin{array}{c}\text { Not in FLL in } \\
\text { Y9 }\end{array}$ \\
\hline Agree $(\mathrm{N}=39)$ & $31 \%$ & $69 \%$ \\
\hline Not sure $(\mathrm{N}=89)$ & $33 \%$ & $67 \%$ \\
\hline Disagree $(\mathrm{N}=507)$ & $67 \%$ & $33 \%$ \\
\hline
\end{tabular}

The proportion of students who were unsure about their parents' views about FLL and who did not opt into FLL was similar to that of those whose parents held negative attitudes. This seems to be an example of what Gardner (1985) termed passive discouragement. Apparent indifference on the part of parents can be as powerful an influence over children as active discouragement.

\subsubsection{Parental attitudes towards FLL for their own children}

Item 9.2.13, "My parents wanted me to do a foreign language this year", produced very divided responses and considerable doubt amongst students, see Table 8.3. Only $42 \%$ of students $(\mathrm{N}=633)$ believed that their parents had wanted them to study 
a FL and a particularly large number, 31, gave no opinion. Once again there was a significant association $(\mathrm{p}<.001)$ between what students reported about their parents' attitudes and their decisions about FLL. In this case $81 \%$ of the 263 students who agreed with the item had chosen a FL. The fact that students disagreed with the statement does not, of course, mean that parents were actually hostile to the idea of FLL for their children. It could simply reflect indifference or the fact that the option had not been discussed. $37 \%$ of the 212 who disagreed that their parents had wanted them to study a FL had anyway decided to study a FL.

Table 8.3 Student decisions regarding FLL compared with responses to item 9.2.13, "My parents wanted me to do a foreign language this year" (No opinion $=31$ )

\begin{tabular}{|l|c|c|}
\hline Item response & In FLL in Y9 & Not in FLL in \\
\hline Agree $(\mathrm{N}=263)$ & $81 \%$ & $19 \%$ \\
\hline Not sure $(\mathrm{N}=158)$ & $55 \%$ & $45 \%$ \\
\hline Disagree $(\mathrm{N}=212)$ & $37 \%$ & $63 \%$ \\
\hline
\end{tabular}

For association between item responses and students' decisions $\mathrm{p}<.001$

These responses seem to provide further evidence of the influence of parental attitudes over student choices even though it was not perceived by most students and parents. Responses to item 9.3 .3 by students involved in FLL provided further support. $78 \%$ of those who responded $(\mathrm{N}=382)$ agreed with the statement, "My parents are pleased that I'm doing a foreign language this year". Although the parents might not have put pressure on their children to undertake FLL they had clearly provided positive reinforcement for the decision. It seems fair to assume that there would have been some subtle encouragement towards FLL during family discussions about option choices.

Students not in FLL were presented with the statement, "My parents think I should spend more time on my English than learning a foreign language", item 9.4.8, in order to investigate further the possibility that students were put off FLL by their parents. When year 8 students' responses to the same statement were compared with their intentions regarding FLL in year 9 it was found that only $28 \%$ of those not intending to study a FL agreed and a similar proportion were unsure. In the case of the year 9 students not in FLL, only $25 \%$ agreed and $28 \%$ were unsure $(\mathrm{N}=253)$. In both cases the results suggest that only a minority of parents had made known to their children their belief that learning a FL might detract from their children's development of their English skills. Students may have disagreed with the item, been unsure or have expressed no opinion because the possibility of studying a FL 
had not even been discussed with their parents.

\subsection{The parent sample and their education}

I aimed to interview the parents of 30 Year 9 students who had been surveyed, and having selected a suitable group, as discussed in 4.7 , I made arrangements to meet them, either couples or individuals. In the end, the parents of three students were unavailable, but one mother gave some opinions by telephone and then filled in a written version of the interview questions. The final interviewees consisted of three couples, one man (a solo parent) and twenty four mothers, the parents of 11 girls and 17 boys. In a few cases male partners were hovering in the background or arrived late during the course of the interview and added a few comments.

All the interviewees were born in New Zealand except one mother from Australia and one father from the USA. English was the L1 of all the interviewees and their parents, although a few reported that they had NESB grandparents or more distant forebears. All of them provided general background information about their ages, school leaving ages and educational qualifications. The age range, shown in Table 8.4, was very similar to that of the majority of teachers interviewed.

Table 8.4 Age range of parents

\begin{tabular}{|l|c|c|c|}
\hline Age group & Mothers & Fathers & Total \\
\hline $35-39$ & 6 & 1 & 7 \\
\hline $40-44$ & 16 & 2 & 18 \\
\hline $45-49$ & 5 & 0 & 5 \\
\hline $50+$ & 0 & 1 & 1 \\
\hline Totals & 27 & 4 & 31 \\
\hline
\end{tabular}

The majority of the parents born in New Zealand would have started secondary school between 1973 and 1977 which means that any experience they had of FLL would have been at a time when:

- many schools were streamed and FLs were usually taught only to top streams;

- FLL was often compulsory for the top streams;

- the only FL offered in most schools would have been French;

- language teaching methods were substantially different from present ones, being

largely based on the 'grammar-translation' method.

The ages at which the parents had left school and their educational qualifications are shown in Tables 8.5 and 8.6 . 
Table 8.5 Ages at which parents left school

\begin{tabular}{|l|c|c|c|}
\hline $\begin{array}{l}\text { School } \\
\text { leaving age }\end{array}$ & Mothers & Fathers & Totals \\
\hline 14 & 1 & 0 & 1 \\
\hline 15 & 2 & 0 & 2 \\
\hline 16 & 3 & 3 & 6 \\
\hline 17 & 14 & 0 & 14 \\
\hline 18 & 7 & 1 & 8 \\
\hline Totals & 27 & 4 & 31 \\
\hline
\end{tabular}

Table 8.6 Educational qualifications of parents

\begin{tabular}{|l|r|r|r|}
\hline $\begin{array}{l}\text { Highest qualification } \\
\text { achieved }\end{array}$ & Mothers & Fathers & Totals \\
\hline Not given & 1 & 0 & 1 \\
\hline None & 3 & 1 & 4 \\
\hline School Certificate & 2 & 1 & 3 \\
\hline $\begin{array}{l}\text { University Entrance/ Sixth } \\
\text { Form Certificate }\end{array}$ & 6 & 0 & 6 \\
\hline University Bursary & 1 & 0 & 1 \\
\hline $\begin{array}{l}\text { Trade or professional diploma } \\
\text { or certificate }\end{array}$ & 8 & 1 & 9 \\
\hline University degree & 6 & 1 & 7 \\
\hline Totals & 27 & 4 & 31 \\
\hline
\end{tabular}

As far as the three couples were concerned, two of the wives had left school a year later than their husbands. The one man who left school at 18 was an American university academic who also had the highest qualification, $\mathrm{PhD}$. All the women with university qualifications had Bachelors' degrees. Eight of the women had teaching qualifications although not all were involved in teaching. Those who were included kindergarten, primary, secondary and polytechnic teachers, but none with any experience of teaching languages other than English. Five of the women described themselves as "homemaker" or as "wife and mother", but two of the "home-makers" were studying part-time. Two other women were studying, one fulltime at university. 


\subsection{Parental FLL experience}

\subsubsection{FLs studied}

Table 8.7 shows the experience of school FLL reported by the parents. In just over half of the families one or both parents had studied at least one FL. The mothers were much more likely to have studied a FL than the fathers and, as expected, they had mostly learnt French. The figures given under 'Fathers' represent information given by women about the experiences of their husbands who were not interviewed. Three reported that their husbands had not studied any FLs. None of the four men interviewed had ever learnt a FL at school.

Table 8.7 Parents' FLL experience at school

\begin{tabular}{|l|c|c|c|}
\hline Language(s) & Mothers & Fathers & Totals \\
\hline French & 11 & 6 & 17 \\
\hline Latin & 0 & 1 & 1 \\
\hline French and Latin & 3 & 0 & 3 \\
\hline French and German & 4 & 0 & 4 \\
\hline Totals & 18 & 7 & 25 \\
\hline
\end{tabular}

\subsubsection{Reasons for FLL choices}

Three women reported that FLL had been a compulsory part of their schooling but none expressed any resentment about this. Another stated that if she had learnt French she could not have done secretarial work, suggesting that she had a choice between programmes. Two stated that they could have chosen German instead of French and another indicated that German was an option at her school. One woman had chosen French as being more "feminine" while her brother had chosen German. Their language studies ranged from 1 to 4 years. One woman reported that her husband had been in a top stream class but had been able to opt for woodwork instead of French.

\subsubsection{Attitudes to their FLL experiences}

Table 8.8 summarises the parents' reported feelings about the FLL experience at school or the lack of it. All the comments were made by women except one of the last. Several reported their husbands' experiences, which were mostly negative:

- He hated French but she did not know why;

- He got 5\% in French (in School Certificate?) so is very hostile towards it;

- Thinks her husband might have failed French;

- Husband was a practical man and hopeless at French, could see no relevance in it and shows no interest in his daughter's French work. 
One woman reported that her husband appreciated the opportunity to study Latin because it had helped him to understand English. The American academic considered his inability to speak anything but English to be the result of a deficient education and a handicap in his many travels and professional contacts.

Table 8.8 Parents' feelings about their FLL or lack of it

\begin{tabular}{|l|c|}
\hline Comments & $\begin{array}{c}\text { Number of } \\
\text { mentions }\end{array}$ \\
\hline Generally enjoyed & 13 \\
\hline Not used (much) since, of doubtful value & 6 \\
\hline $\begin{array}{l}\text { Generally worthwhile } \\
\text { Learning involved little speaking (only one thought } \\
\text { this boring) }\end{array}$ & 5 \\
\hline Difficult & 4 \\
\hline Enjoyed Latin but not French & 2 \\
\hline Wished they had done some/more & 1 \\
\hline None learnt, no regrets, no interest & 3 \\
\hline
\end{tabular}

Table 8.9 Parents' thoughts about the value of FLL for presentday New Zealand school students

\begin{tabular}{|l|c|}
\hline Comments & $\begin{array}{c}\text { Number of } \\
\text { mentions }\end{array}$ \\
\hline $\begin{array}{l}\text { Knowledge/understanding/tolerance of other cultures, improved } \\
\text { relations - not everyone uses English }\end{array}$ & 13 \\
\hline $\begin{array}{l}\text { General educational value : broadening of horizons, experience, } \\
\text { knowledge. Most of world is not monolingual }\end{array}$ & 11 \\
\hline Usefulness in jobs, contacts with non-English speakers & 11 \\
\hline Useful for travel & 6 \\
\hline Challenge for brightest students & 4 \\
\hline Transferable skills & 4 \\
\hline Better understanding/appreciation of English & 3 \\
\hline Fun & 2 \\
\hline Not much use & 4 \\
\hline
\end{tabular}


8.5 Parental beliefs about the value of FLL in New Zealand schools

The majority of parents saw considerable merit in FLL in school for reasons shown in Table 8.9. Most did not seem concerned about which FLs were offered, speaking of the general value of breaking out of the monolingual, monocultural tradition of most New Zealanders. Of those who favoured specific languages, with employment in mind, 8 mentioned Japanese, 4 Chinese and one simply nominated Asian languages. Two parents remarked that New Zealanders are great travellers but that they have always coped with English only, implying, it seemed, that this situation would probably continue. One individual commented that travellers with only English missed a lot. Another noted the impossibility of working in a non-English speaking country without learning the language.

\subsection{Parental attitudes towards the starting age for FLL}

Table 8.10 shows a clear majority of parents in favour of an earlier start to FLL. There also seems to be a majority in favour of all students having at least a taste of such learning. It is probably fair to say that, given an earlier start to learning and with all students having some experience of language learning before they reach secondary school, the great majority of parents would see language learning as entirely optional at secondary level.

\subsection{Parental attitudes towards the place of foreign language learning in their children's schools.}

The comments listed below were summarised according to the school that their children were attending. The number of families involved at each school and the number of times similar comments were made are given in brackets. If no number is given a comment was made by only one parent.

Table 8.10 Parents' opinions about the best starting age for FLL

\begin{tabular}{|l|c|}
\hline Approximate age & $\begin{array}{c}\text { Number of } \\
\text { mentions }\end{array}$ \\
\hline Younger than at present & 7 \\
\hline Pre-school & 3 \\
\hline At primary school - compulsory & 2 \\
\hline At primary school - optional & 3 \\
\hline At intermediate level - compulsory & 10 \\
\hline At intermediate level - optional & 2 \\
\hline At secondary level - compulsory & 1 \\
\hline At secondary level - optional & 1 \\
\hline Not sure but all need a taste & 4 \\
\hline $\begin{array}{l}\text { Secondary schools need to provide chance of } \\
\text { later start }\end{array}$ & 1 \\
\hline
\end{tabular}


At Arum, where one term of language learning was compulsory (5): 
- It was a pity that a later start at FLL was not possible as this more or less ruled out the possibility of studying two languages. (One parent erroneously believed that a later start was possible.)

- The school did not seem to promote languages.

At Salvia, where language learning was entirely optional, for one or three terms, depending on whether students wished to continue in Year 10 (4):

- Parents liked the Year 7 and 8 modules which gave students a taste of French and Japanese and the means of making an informed choice in Year 9 (4).

- There was a satisfactory choice of languages (3).

- There should be more choice of languages, e.g. Spanish for seniors.

- It was unsatisfactory that it was impossible to do both French and Japanese in Year 9.

- Languages tended to be overlooked, especially in favour of sport (new module subject available in 2000) (2).

- The school had trips to Japan (2).

\subsection{Parental views on choosing year 9 options}

\subsubsection{Lack of information}

A major problem experienced by parents trying to help their children choose optional subjects was their own or their children's lack of experience of FLL. Nearly half of them stated that, had the children had at least a taste at intermediate level, they would know what FLL involved, whether they enjoyed it and whether they had any particular ability for it. Even if parents had had some experience it was not necessarily a good one, most likely of French and so long ago that at least a third of them believed (correctly) that teaching methods had changed. Many found it very hard to judge, simply from reading a prospectus, what FLL might be like, and school visits were of limited help given time constraints and the impossibility of observing real. One mother spoke of feeling "totally blind" when it came to advising her children. Another thought that choosing option was a "bit of a nightmare", and a "hit and miss affair". Yet another suggested that students might rely on hearsay from their peers and could easily be discouraged if they heard that a FL was difficult or boring.

\subsubsection{Curriculum pressures}

Another major problem for parents was the limited number of subjects students could choose in some schools, combined with the pressure to focus on those considered directly useful for a future job. In addition there were usually a number of apparently interesting new subjects on offer. Students who had to choose two whole year options in Year 9 had the least choice. Because of timetable arrangements, students could, in some cases, find themselves obliged to study something they resented, which created an unacceptable situation for all concerned, student, subject teacher and parents. Fortunately this proved to be the case for only one family among those interviewed. 
Two parents indicated that they might have accepted FLL for their children if shorter courses had been available. On the other hand, where schools offer more, shorter options, students have less time to study subjects although the traditional year $11 \mathrm{FL}$ examinations have been based on a three year course. One parent commented on the discouraging factor, often cited by FL teachers, that students learning a FL for only the first half of year 9 may forget much of what they have learnt, however keen they are to continue in year 10 .

\subsubsection{How option subjects were chosen}

Table 8.11 shows what the parents reported about how they and their children approached the matter of choosing option subjects. Eight families reported that they were already familiar with the schools that their children were to attend, through older children or through teaching there. One woman had done some research into the pros and cons of single sex schools for girls. Only a minority (9) of the families found the written information received from the school "helpful" or "useful". Five considered it unclear or insufficient and one mother was particularly critical, calling it "scant".

Table 8.11 How Year 9 option subjects were chosen

\begin{tabular}{|l|c|}
\hline Aspects of decision-making & $\begin{array}{c}\text { Number of } \\
\text { mentions }\end{array}$ \\
\hline Visited school on open day/evening & 21 \\
\hline Studied school prospectus/other information & 19 \\
\hline Family discussions & 17 \\
\hline Discussions with other parents & 1 \\
\hline
\end{tabular}

Table 8.12 How the eventual decisions were made regarding FLs

\begin{tabular}{|l|c|}
\hline Extent of parental influence & $\begin{array}{c}\text { Number of } \\
\text { mentions }\end{array}$ \\
\hline Very little - student made ultimate choice & 14 \\
\hline Some influence & 5 \\
\hline Parental insistence & 3 \\
\hline Last minute decision by child & 2 \\
\hline
\end{tabular}

\subsubsection{The extent of parental influence in the decision-making}

Table 8.12 shows what the parents reported about their involvement in the decisionmaking process. Half of them claimed to have had very little influence over their 
children's choice of option subjects, which supports what the students themselves reported, see 9.3 below. They were happy to leave it up to the students even if this led to a substantial degree of hesitation and last minute decisions. Only three sets of parents reported that they had insisted that their child study French (1) or not (2). It seems that in only one of the 28 families were both parents hostile to language learning of any sort.

\subsubsection{Individual reasons for student choices as reported by their parents}

At Arum three students had chosen French for diverse reasons. One had been put off Japanese by his intermediate experience, especially by the difficult writing system, and the other two both had some French ancestry. One of the latter had some previous experience, an older sister doing French, and his father had always assumed his son would study the language. (He probably would have insisted had his son been reluctant.) Two girls chose Japanese, one did French as well, both without previous experience. One was fascinated by what she saw of Japanese at the open day and her mother encouraged her to do French as well. The other's choice was supported by her parents who considered it a useful language.

Six of the boys at Begonia were in the top four streams. Parents of two of them had not realised (or had forgotten) that music was an option as opposed to a FL. Three boys were already interested in languages, and two of them were not musical anyway. One of the three had chosen German chiefly to stay with his friends, according to his parents. A fourth boy had chosen music but would have been prepared to do German. The other two boys were adamantly against learning a language although neither had had any previous experience on which to base their opposition. Their parents, however, took different views of FLL. The mother of one would have liked him to study a language but, as the family is musical, let him do music. The parents reported being told that if they did not like the streaming and option system there were plenty of other schools the boy could attend.

The parents of the other boy were angry about the lack of choice and considered a FL a "lesser evil than music". (None of the family seemed to have considered Maori.) The older brother had been in a similar position and had opted for Japanese, hated it because it was difficult and advised his brother to try German instead. The school had also advised the family that it was the easier language, but the boy was not doing any work and expecting to fail, which the parents considered inevitable. They stated that learning any LOTE was a complete waste of time for their sons and had vigorously challenged the school's policy. The only solution they were offered was to move the boy to a lower stream which they were not prepared to accept as it would disrupt the boy's academic and social development. They also reported having been told that top stream students were needed to fill the language classes to keep the language teachers fully employed. 
Three boys were in streams where they had a free choice of any two whole-year options but none chose a language. One mother, whose son wanted to be a chef, would have recommended more firmly that he do French if there had been more, shorter options to study. One boy was in a stream where he could choose only one option. Although he put down French as one of his possible options, the school decided that he would do the other subject without further consultation with parents. This clearly reinforced the school's position that FLL was only suitable for boys of higher academic ability. The parents were not upset about the decision as their son was perfectly happy but were surprised at the lack of consultation.

At the other three schools, where language learning was entirely optional, 7 students had chosen a FL. Four girls were studying French. Two of them had enjoyed their previous experience of the language. One had been in a school with long-term contacts with a French school, from which pupils had come to stay and the other had an uncle living in Paris. One seemed to have chosen French because her mother had insisted she study a FL and that was the one her older sister was studying. Another had chosen German after enjoying previous experience and being impressed by what she had seen on a visit to the school, particularly the booklet about the advantages of learning German. Two students had chosen Japanese because they had enjoyed their previous experience, and one of them had a Japanese grandmother.

Of the girls who had not opted into FLL, one had initially chosen French but both her parents were against it. Her mother believed that she had made the choice only to stay with friends and reported that the girl's father was of the opinion that, if she were going to do a language, she should do Maori. (I assumed that the father was Maori, but the girl had not chosen to study that language either.) Two girls had enjoyed FLL at intermediate school but had no inclination to continue. One of them was learning sign language as she had a deaf friend. The mother of the other thought French would be useful for her daughter but reported that the girl's father was adamantly against the idea.

Of the boys who had not chosen a FL, two had enjoyed their previous experience of Japanese but could see no point in continuing. Their parents could see the value of FLL but the boys considered other subjects more important and one of them did not think he was good enough at it anyway. The third boy in this group had no previous experience of language learning, except a little Maori and was adamantly against FLL as being of no use and no interest, although his mother was disappointed about this.

According to the parents no students had regretted their choice of language, except the one extremely reluctant learner. Three parents stated that, if it had been available, they might have preferred their children to study Spanish.

\subsubsection{Other possible influences on student decisions about FLL}

I asked the parents if their children had any experience of overseas travel or family 
contacts with people from non-English speaking backgrounds. I also asked to what extent they thought that their children's option choices had been influenced by their friends and older siblings, and whether their children had any clear ideas about possible future FLL or future career choices.

\subsubsection{Past or future overseas travel}

It was clear that, although many of the children had been out of New Zealand, very few had ever been anywhere where languages other than English are commonly spoken. Parents of the boys who had been to Club Med in Noumea and the Philippines reported that being in what was at least partly a non-English speaking environment had not aroused any interest in their sons in language learning. Table 8.13 shows the places mentioned by parents that their children had been.

Table 8.13 Students' travel experience overseas

\begin{tabular}{|l|c|}
\hline $\begin{array}{l}\text { Overseas travel } \\
\text { experience }\end{array}$ & $\begin{array}{c}\text { Number of } \\
\text { mentions }\end{array}$ \\
\hline None & 11 \\
\hline Australia & 10 \\
\hline USA & 2 \\
\hline Pacific Islands & 2 \\
\hline UK/Ireland & 1 \\
\hline Singapore/Philippines & 1 \\
\hline
\end{tabular}

Table 8.14 Possible future overseas travel of students

\begin{tabular}{|l|c|c|}
\hline $\begin{array}{l}\text { Parents' comments about } \\
\text { children's attitude to travel }\end{array}$ & Girls & Boys \\
\hline Interested in travel & 4 & 4 \\
\hline Will probably travel & 2 & 5 \\
\hline Might travel & 1 & 1 \\
\hline Total & 7 & 10 \\
\hline
\end{tabular}

According to parents' reports, previous overseas travel did not seem to be an influence in student decisions regarding FLL. Parents were therefore also asked if they thought their children would travel in the future. Responses to the question are shown in Table 8.14. Approximately two thirds of parents 
thought that their children were interested in overseas travel, but most gave no indication of where their children might go unless they already had some overseas connections. One boy was reported by his parent to be interested in travelling only with the All Blacks. (Whether as a player or a spectator was not specified!)

\subsubsection{Family contacts with people from non-English speaking backgrounds}

Table 8.15 shows that the families had a variety of contacts with people who spoke LOTEs, both in New Zealand and overseas.

Table 8.15 Family contacts with people from non-English speaking backgrounds

\begin{tabular}{|l|c|}
\hline Type of contact & $\begin{array}{c}\text { Number of } \\
\text { mentions }\end{array}$ \\
\hline Parents' travel, especially using school French & 8 \\
\hline Family hosting overseas students & 8 \\
\hline Neighbours, family friends, work contacts & 7 \\
\hline $\begin{array}{l}\text { Children's contacts through school, scouts and } \\
\text { Overseas students hosted by friends }\end{array}$ & 6 \\
\hline Family, eg immigrant grandparents & 5 \\
\hline Occasional visitors & 3 \\
\hline
\end{tabular}

It was evident from other parts of the research that the hosting of overseas students, particularly from Latin America and Japan, was a familiar concept to students in the area, if not in their own families, then through school friends or neighbours, for example. If parents had travelled, their use of other languages was largely limited to attempts at reviving their school French. Many parents commented also on the extent to which New Zealand, and their community in particular, were becoming more multicultural.

\subsubsection{Peer/sibling influence}

Some indications of this type of influence have already been discussed in 8.8.5 above. One woman reported that her daughter would not study a language, although her mother was in favour of it, because, as her mother put it, "learning a language is not a cool happening thing to do". Another woman believed that her son might have been put off studying a language because his friends had said it was boring, although some of them were studying a language. 


\subsubsection{Possible future FLL}

None of the parents of students who had chosen to study a FL were certain that their children would continue with it in year 10. On the other hand, only one third of them believed that it would definitely or probably not happen. Parental responses to the question about future FLL are shown in Table 8.16. One father who thought his son would probably continue also stated that he might insist on it, and one mother who thought that her son might continue certainly hoped so.

\subsubsection{Possible future career choices}

According to their parents, few children had any clear ambitions for future employment. While many had expressed ideas about what they wanted to do, these tended to change from time to time, and some of their ambitions were probably pure fantasy. It was interesting to note that the mother who mentioned that her daughter's latest idea was to become an air hostess only realised while talking to me that this had implications for language learning.

Table 8.16 Students' future language learning

\begin{tabular}{|l|c|c|}
\hline \multirow{2}{*}{$\begin{array}{l}\text { Student will continue } \\
\text { FL in Year 10 }\end{array}$} & Number of & mentions \\
\cline { 2 - 3 } & Girls & Boys \\
\hline Probably & 1 & 2 \\
\hline Possibly & 4 & 2 \\
\hline Don't know & 2 & 0 \\
\hline Probably not & 1 & 3 \\
\hline Definitely not & 0 & 1 \\
\hline
\end{tabular}

\subsection{Teachers' views of parent attitudes towards FLL}

Teachers at the schools with year 8 classes believed that parents had generally favourable attitudes towards the introduction of FL modules in years 7 and 8 , as found also be Mahon (1989) and Peddie et al. (1999). About half of the teachers who commented on parental attitudes reported that the modules had been positively or keenly welcomed while the rest claimed that the changes had been accepted without resistance and that their schools had received no negative reactions from parents.

By contrast, the largest group of teachers who commented on the attitudes of parents of secondary students believed they were generally hostile or indifferent towards FLL. Parents were thought to disapprove of FLL mostly because they did not see any need for languages other than English, which seemed universal to them, and they were most concerned with 
what they deemed the "basics". That is, they saw education as a preparation for employment and making money ("looking after no. 1"), and were not concerned with anything aesthetic or learning for pleasure. Some teachers felt that parents may have had bad memories of their own FLL at school, or have thought that their children had "done" languages at intermediate school. Others believed that parents might accept their children choosing to study a language option initially, especially if it were a short module, with a comment such as: "very nice if you want to", but would be likely to discourage continued study beyond year 9 as they saw the need to focus obtaining job qualifications.

There were teachers who believed that parents had positive attitudes towards FLL, especially at Arum where compulsory language learning modules had been introduced in Year 9 without negative reactions from families. Teachers attributed a growing interest in and acceptance of FLL to a variety of causes. They felt that parents saw the world shrinking, with more New Zealanders travelling overseas for pleasure or developing business contacts with non-English speaking people. More and more parents were coming into contact with foreign students. Some regretted not having done any FLL themselves. These views seem to be a more accurate reflection of the parents' attitudes as discovered by this research.

There were teachers who believed that some parents had chosen their child's school for its academic record and, believing that languages are for brighter students, liked to see their child a FLL class as a sign of their academic ability. (Two teachers suggested that this revealed a certain element of snobbery.) On the other hand, one teacher believed the absolute opposite, that an increasing number of parents were wondering why students need to learn any other language apart from English. The attitudes of parents of secondary students, as perceived by teachers, are summarised in Table 8.17 .

Table 8.17 Attitudes of secondary students' parents to compulsory and optional FLL, according to teachers

\begin{tabular}{|l|c|}
\hline Parents' attitudes & $\begin{array}{c}\text { Number of } \\
\text { mentions }\end{array}$ \\
\hline Negative attitudes or indifference & 16 \\
\hline Positive attitudes & 9 \\
\hline Growing acceptance & 6 \\
\hline $\begin{array}{l}\text { Changeable feelings towards Japanese } \\
\text { they value it according to their ambitions for }\end{array}$ & 4 \\
\hline
\end{tabular}

In general, parents' attitudes to FLL were thought to vary according to their ambitions for their children. Parents tended to accept the traditionally taught languages, French and German, more than others, although Spanish was seen to have a lot of parental support. 
Attitudes towards Japanese in particular were seen to be changeable according the the economic situation, perhaps reflecting the view, especially of boys' parents, that it was worth learning largely for its usefulness for future employment. Students who chose Japanese were seen by teachers to be more influenced by parents and older siblings than other language learners. According to one teacher, parents might even put pressure on a "hopeless" student to study Japanese for apparent economic advantage.

One or two teachers noted that positive parental attitudes did not always influence students to start or continue FLL. This had to be the case as far as French and German were concerned in the surveyed secondary schools since, despite the positive parental attitudes towards these languages perceived by teachers, there were substantial drop-out rates at every level. These teachers seem to reflect more accurately what was happening in these schools, according to my findings. Although numerous researchers have claimed that parents are a major influence on student attitudes towards FLL (Turner 1974, Oskamp 1977, Gardner 1985, ERO 1994, Gardner et al. 1997) it was perhaps the general climate of some of the institutions which gave students the subtle message that FLs were not as important as other subjects. This was the situation identified by Lai (1999), for example, in Hong Kong.

\subsection{A summary of general comments made by the parents interviewed 8.10.1 The need for languages other than English for the country's future}

- New Zealand depends on overseas trade and increasing tourism, so the number of students studying FLs should be increasing;

- New Zealand is at a disadvantage compared with European or Asian countries where FLL is more thorough and taken for granted;

- New Zealand should not rely on everyone else speaking English;

- The most successful businesses will understand the culture of their customers and understand what people are saying to each other in negotiations;

- There is nothing worse than dealing with foreigners and knowing nothing of their language - it is disrespectful;

- Students keen enough to pursue FLL will find jobs to use their skills.

\subsubsection{The value of FLL for personal development}

- Education is for personal development as well as work preparation;

- The language learning process is different from that needed to learn other subjects, as Gardner (1985) stressed, requiring much more active involvement from students something that all students can benefit from;

- Students need to be encouraged to broaden their horizons, "think outside the square", not be put off by people who do not appreciate the value of FLL because they have closed minds or have had bad experiences;

- Students at intermediate and secondary schools are not stretched enough. 


\subsubsection{The value of FLL for cultural understanding}

- New Zealand society is becoming more diverse;

- Much more general awareness is needed of other languages and cultures, especially of Maori and immigrants.

\subsubsection{What parents need to know}

- Parents need more education about FLL. They can only judge according to their own experience but things have changed.

- Parents need to know more about what students get out of continued FLL and what they can do with their language skills.

\subsubsection{The need for an earlier start to FLL}

- All children should start L2 learning at an early age. For NESB students this may, of course, mean English.

- Younger children are more eager to learn and find language learning easier.

Secondary school is too late.

- No-one can know what language they might need in the future and thus language learning skills are valuable for all.

- Students need to try things before they can know if they are interested in them or good at them.

\subsection{Final analysis}

In order to test the validity of what the parents and their children reported I compared the relevant data obtained from each parent interview with what their children had reported. In all respects there were very few, usually minor discrepancies which could mostly be explained by parents and children not knowing or having forgotten things. The results encouraged me to believe that, on the whole, all the surveyed students had accurately reported information about their parents, to the best of their ability. The impression given by all the above evidence was that most parents had positive attitudes towards FLL and could see its value for all children, for a variety of reasons, if it were introduced at a younger age. This supports what I have encountered in my experience as a FL teacher. Positive attitudes among parents often emerged at parent-teacher interviews when they offered comments about how glad they were that they had studied French, for example, or how they wished they had had an opportunity to learn another language.

Whatever influence parents had over their children's decisions with regard to FLL remained mostly indirect or unspoken and students most felt free to make their own decisions about option subjects. Teachers, on the whole, were pessimistic about parental attitudes towards FLL and their views did not always seem to be an accurate reflection of those attitudes. They were, perhaps, blaming parents for what they saw as poor student numbers and retention rates in FL classes, although parents themselves felt they had little influence over their children's choices. What teachers themselves thought about FLL and the extent of their influence on student choices is discussed in the next chapter. 

OVER STUDENT CHOICES

...the more milk you have the more cream will rise to the top (Calloway 1999)

\subsection{Introduction}

This chapter discusses the results of the teacher interviews carried out in the schools where I surveyed students. I asked teachers about their own experiences of FLL at school and their opinions about FLL for present-day students. In addition, I attempted to ascertain what they thought their colleagues' attitudes were. I interviewed 56 teachers, with a wide range subject specialities and positions in the schools. Most were extremely co-operative and pleased to give of their scarce free time to help the research. Not surprisingly, FL teachers were particularly keen to be involved and offered a large amount of information about the place of FLs in their schools and attitudes towards it. .

\subsection{Teachers' experience of and attitudes towards FLL}

\subsubsection{School experiences}

All but nine of the interviewed teachers had been educated in New Zealand, and mostly at a time when FLL was available almost exclusively at secondary school and French was often the only language available. Most teachers had been in top academic or 'professional' streams where FLL language learning (and/or Latin) had been a compulsory subject for at least one year, but two teachers reported that they had not been able to study a language because of the classes in which they had been placed. Thus 34 out of the 47 New Zealand educated teachers had learnt French at school. For 28 of them that was the only modern FL they studied although six had also studied Latin. Over half of those who had studied French had done so for between 3 and 5 years. Only six teachers had studied German, all but one in addition to French, but one woman had only studied the language because she had the opportunity to do so while living overseas for 6 months. Eleven of the teachers educated in New Zealand had not learnt a modern FL. (One teacher gave no information on the subject.) Two teachers came from Dutch-speaking homes and one was a native Spanish speaker.

By comparison with the experience of those educated in New Zealand, the 9 teachers educated overseas, in South Africa, the UK, Ireland, France, Germany, China and Japan, all female, had all studied a language other than their L1, usually more than one, although French was still the language most commonly studied. Not surprisingly, it was the FL teachers who had studied the widest range of languages. What was surprising was that, of the 13 FL teachers employed at the 5 secondary schools involved in the research, 6 had been educated overseas. This might suggest that, no matter what emphasis schools may put on FLL, there may not be enough adequately educated New Zealand teachers to staff FL classes. Table 9.1 shows teachers' school language learning experience, according to their place of education 
and involvement in language teaching.

\subsubsection{Teachers' attitudes to their school FLL experience}

I asked teachers what they had liked or disliked about school FLL. The following factors were commented on favourably (numbers in brackets indicate the number of teachers making similar comments):

- the teacher (7)

- the sound or flow of the language (5)

- the links to English, including the challenge of "decoding" and learning new vocabulary (5)

- learning about the culture of native speakers (4)

- communication with native speakers (4)

- oral work (3)

- reading magazines and comics (2)

Aspects of FLL which teachers reported that they disliked included:

- grammar (7)

- the lack of real communication, because of the emphasis on reading and writing (7)

- the difficulty of language learning (6)

- the teacher (4)

- the rote learning (4)

- the exam preparation (3)

- the poor textbooks (1)

- the apparent lack of relevance of FLL

Table 9.1 FLs studied at school by teachers

\begin{tabular}{|l|c|c|c|c|}
\hline & \multicolumn{3}{|c|}{ Teaching a FL } & \multicolumn{2}{c|}{ Not teaching a FL } \\
\hline $\begin{array}{l}\text { FLs studied } \\
\text { at school }\end{array}$ & $\begin{array}{c}\text { Educated in } \\
\mathbf{N Z}(\mathbf{N + 1 1})\end{array}$ & $\begin{array}{c}\text { Educated } \\
\text { elsewhere } \\
(\mathbf{N = 6})\end{array}$ & $\begin{array}{c}\text { Educated in } \\
\mathbf{N Z}(\mathbf{N = 3 6})\end{array}$ & $\begin{array}{c}\text { Educated } \\
\text { elsewhere } \\
(\mathbf{N = 3})\end{array}$ \\
\hline French & 10 & 4 & 24 & 2 \\
\hline German & 4 & 1 & 2 & 1 \\
\hline EFL & & 1 & & 1 \\
\hline Japanese & 1 & & & 1 \\
\hline Russian & 1 & 1 & & \\
\hline Mandarin & & & & \\
\hline Afrikaans & & 2 & & \\
\hline Irish & & & & \\
\hline Italian & & 1 & & \\
\hline
\end{tabular}


Most of the dislikes could be attributed to the nature of much FL teaching in the past. Current FL teachers would claim that most of the negative aspects listed above have been overcome by considerable changes in teaching methods and the materials used.

\subsubsection{Further FLL by teachers not specialising in languages}

Apart from those who became FL teachers, very few teachers did any further language study once they had left school. Four English teachers did one or two years of French or German at university. Another English teacher sought some knowledge of Italian and Swedish before she went overseas. One special needs teacher of Dutch heritage studied German and Indonesian at university. Four teachers were studying Spanish at evening classes, one a computer/maths specialist, and three intermediate teachers helping their students learn, but all out of personal interest. One woman had studied ancient Greek at evening school in connection with religious studies. One man mentioned that reading knowledge of a foreign language (he chose German) was a prerequisite for his degree.

\subsubsection{Teachers' reactions to FLL}

The positive comments far outweighed the negative ones, although most teachers had been obliged to do some FLL. Even teachers who did little or no FLL at school had some positive attitudes. Table 9.2 presents a summary of the positive comments. Globalisation and New Zealand's traditional isolation (geographical and mental) were concepts frequently mentioned. The few negative opinions came from teachers who had never used the language learnt and consequently had doubts about the value of FLL, or thought that they might have done something more useful. One expressed frustration at the lack of jobs making use of FL skills.

Table 9.2 The value of their own FLL to teachers

\begin{tabular}{|l|c|}
\hline Comments & $\begin{array}{c}\text { Number of } \\
\text { mentions }\end{array}$ \\
\hline $\begin{array}{l}\text { Useful for travel } \\
\text { Generally useful/worthwhile, sense of achievement, } \\
\text { personal fulfilment }\end{array}$ & 17 \\
\hline $\begin{array}{l}\text { Knowledge/understanding/tolerance of other cultures, } \\
\text { improved relations }\end{array}$ & 13 \\
\hline $\begin{array}{l}\text { Transferable skills e.g. memorisation, training of the } \\
\text { mind }\end{array}$ & 10 \\
\hline $\begin{array}{l}\text { General understanding of language - structures, } \\
\text { differences }\end{array}$ & 9 \\
\hline $\begin{array}{l}\text { Better understanding/appreciation of English, } \\
\text { empathy with EFL learners }\end{array}$ & 7 \\
\hline Broadening of horizons, recognition of wider world & 6 \\
\hline
\end{tabular}


FL teachers were not necessarily teaching a FL they had learnt at school. Only one New Zealand educated teacher of Japanese had studied it at school. The other secondary teachers of Japanese had studied the language as adults, one of whom had had no FLL at school. The intermediate teacher of Japanese had learnt French and was using the MoE course, Hai!. The intermediate teachers of Spanish were using the MoE course, Sí!, and were also studying the language in their own time at evening class. Four secondary FL teachers were teaching their L1.

Seven teachers expressed regret that they had had little or no FLL at school. Some had travelled overseas, particularly in Europe, and been frustrated trying to communicate with local people. Others intended to travel and foresaw the value of knowing a LOTE. Some were embarrassed at meeting multilingual overseas people. Some declared that they would like to have a better appreciation of French films. Two teachers admitted not valuing FLL at school but they do see its value now that the younger generation, of ten their own children, are enjoying FLL or travelling overseas.

\subsection{Teachers' attitudes towards FLL for present-day students}

\subsubsection{How important is FLL?}

Teachers were asked to rate FLL as 'very important', fairly important' or 'not important' to New Zealand students these days. Table 9.3 shows the teachers' responses according to their positions. All but one teacher were of the opinion that FLL is very or fairly important. One year 8 class teacher was not sure although she valued her own experience of learning French throughout secondary school and had encouraged her daughters to learn it. All the language HODs and teachers in charge of FL subjects considered FLL very important - one used the word "essential".

I found it surprising that four of the other FL teachers thought FLL only fairly important. Discussions with the teachers showed that, mostly, they were reflecting the general attitude they saw in schools and the wider society, rather than their personal feelings. This may have been the result of ambiguity in my questionnaire, or they may have modified their stance in conversation with me, knowing my background. One HOD originally circled the option 'not important', but it soon became obvious that this was not his personal opinion, merely a reflection of what he perceived to be general opinion in New Zealand. He considered FLL very important. In another example, a principal chose the response, 'fairly important' but stated later that all students should study a language up to year 11 (i.e. at least three years). It was particularly interesting that the two teachers of Maori who were interviewed both firmly supported FLL. There were no noticeable differences of opinion between the different types of school. 
Table 9.3 Value placed on FLL for New Zealand students

\begin{tabular}{|l|c|c|}
\hline Type of teacher & $\begin{array}{c}\text { Very } \\
\text { important }\end{array}$ & $\begin{array}{c}\text { Fairly } \\
\text { important }\end{array}$ \\
\hline Principal & 3 & 3 \\
\hline Other senior management & $2 *$ & $3 *$ \\
\hline Deans/ guidance/ careers adviser & 5 & 8 \\
\hline Foreign language HOD & 4 & 0 \\
\hline Other FL specialists & 6 & 3 \\
\hline Maori language teachers & $2 \bullet$ & 0 \\
\hline Others involved in FL teaching & $2 \diamond$ & 0 \\
\hline $\begin{array}{l}\text { Others } \\
\text { Totals }\end{array}$ & $4 \#$ & 9 \\
\hline * Includes an intermediate DP teaching a FL \\
- One intermediate teacher and a secondary HOD Maori \\
$\begin{array}{l}\text { - Both at intermediate school } \\
\text { \# Includes one intermediate teacher }\end{array}$ \\
\hline
\end{tabular}

\subsubsection{Why is FLL important?}

Table 9.4 sets out a summary of the values teachers saw in FLL. Most teachers mentioned several of the factors listed, but by far the majority believed that students need to learn that New Zealand is part of a wider world, where not everybody speaks English. Several considered arrogant the notion that everyone here or overseas will speak English and noted that students have to understand that people from other cultures think and do things differently, and express their ideas differently. Some pointed out that New Zealand society is becoming more multicultural and hence greater understanding and tolerance is required at home as well as in overseas relations. Teachers realised that New Zealand's trade and tourism links with nonEnglish-speaking countries are expanding and the concept of "globalisation" was frequently referred to, often in relation to New Zealand's geographical isolation and traditionally self-centred attitudes. It was generally thought that language skills are important combined with other qualifications. 
Table 9.4 Why FLL is important for New Zealand school students

\begin{tabular}{|l|c|}
\hline Comments & $\begin{array}{c}\text { Number of } \\
\text { mentions }\end{array}$ \\
\hline $\begin{array}{l}\text { Knowledge/understanding/tolerance of other cultures, } \\
\text { improved relations - not everyone uses English }\end{array}$ & 38 \\
\hline $\begin{array}{l}\text { General educational value: broadening of horizons, } \\
\text { experience, knowledge. Most of world is not } \\
\text { monolingual }\end{array}$ & 21 \\
\hline $\begin{array}{l}\text { Transferable skills, mental discipline e.g. memorisation, } \\
\text { attention to detail }\end{array}$ & 19 \\
\hline $\begin{array}{l}\text { NZ's economic need of communication skills for trade, } \\
\text { commerce, tourism }\end{array}$ & 16 \\
\hline \begin{tabular}{l} 
Better understanding/appreciation of English \\
\hline $\begin{array}{l}\text { Useful for travel (increasing) } \\
\text { differences, links }\end{array}$
\end{tabular} & 12 \\
\hline
\end{tabular}

Other comments, apart from those relating to the country's economic needs, were broadly divided between concern for students' personal development and the importance of skills acquisition. One year 8 teacher firmly believed that her students were never going to travel beyond the nearest city, but most teachers appreciated the need for students to be as flexible as possible in rapidly changing world. At the age of 12 or 13 they cannot know where they might travel in the future, for pleasure or for work, nor what skills they might need for the several or many jobs they might have. A few teachers appreciated the value of FLL but added that it is important only for the most able students, although it should be offered to all students. Three teachers thought that FLL was fine if that is what students wanted, but that the choice to study a language would depend on personal needs. Three teachers saw FLL as important but thought that students (presumably those whose first language is English) need to learn Maori first.

\subsubsection{Which languages should New Zealand students learn?}

This question provoked a wide range of responses, see Table 9.5. Some mentioned one or two languages only, some listed three, four or five, with the implication that students should have a good variety of options. Some chose 'any European' or 'any Asian' language. About half of the teachers thought that students' choices should include a mix of Asian and European languages. The rest were evenly divided between favouring European or Asian languages only. 
Eight teachers stated that it does not matter which second language a child learns, for the basic educational advantages discussed above, especially the transferability of skills and the breaking out of the monolingual mode. "All languages are enlightening." Some felt uncertain or not confident to judge. Six teachers noted that the future linguistic needs of either the country or of individuals would vary and were difficult to predict. Two teachers mentioned Pacific Island languages, commenting that they might be more relevant in the Auckland area, or that Pacific Island students, like NESB students, need different courses from English-speaking students (i.e. language maintenance programmes). Three teachers noted the need to focus on the Asia/Pacific region, in connection with Spanish and/or Asian languages. The only negative comments against a specific language concerned Japanese which was, ironically, the most popular one. One teacher suggested that the best choice for any student would be one community and one international language, another that "a language that might teach them about their roots".

Although I made it clear to everyone I interviewed that this research was about LOTEMs, a number of secondary teachers mentioned the importance for New Zealand students of Maori or the value of Latin. Three teachers believed that both Latin and Maori should be taught to all students before they were offered the choice to study other languages. There was little support for the introduction of Korean but some interest in Chinese, seen also among the students surveyed, and a growing recognition of the value of Spanish. The teachers' views seem to reflect what was happening in their schools, including what was feasible in terms of teacher availability and student choices. It is doubtful that many, if any, of the teachers below the level of senior management had much influence over what FLs were offered in schools, or whether classes continued in the face of low student numbers.

Table 9.5 FLs considered the most appropriate for students

\begin{tabular}{|l|c|c|c|}
\hline & \multicolumn{3}{|c|}{ Number of mentions } \\
\hline $\begin{array}{l}\text { Language } \\
\text { mentioned }\end{array}$ & $\begin{array}{l}\text { As only FL } \\
\text { to be taught }\end{array}$ & $\begin{array}{c}\text { To be offered } \\
\text { with another FL }\end{array}$ & $\begin{array}{c}\text { Total number } \\
\text { of mentions }\end{array}$ \\
\hline Japanese & 5 & 20 & 25 \\
\hline French & 0 & 17 & 17 \\
\hline Spanish & 4 & 12 & 16 \\
\hline German & 0 & 11 & 11 \\
\hline Chinese & 0 & 10 & 10 \\
\hline Korean & 0 & 2 & 2 \\
\hline $\begin{array}{l}\text { Any European (or } \\
\text { Romance) lang. }\end{array}$ & 0 & 4 & 4 \\
\hline Any Asian lang. & 3 & 4 & 7 \\
\hline Maori & 0 & 7 & 7 \\
\hline
\end{tabular}




\subsubsection{Opinions about specific languages}

Asian languages were considered important for economic reasons. Teachers noted that New Zealand has increasing numbers of visitors and immigrants from Asian countries. It was deemed important to provide opportunities to explore their languages even though such languages have had much less influence on our English language and largely British culture than European languages. In particular, teachers perceived a growing number of contacts with Japanese people through trade and tourism, for example. Two teachers considered Japanese too hard for students to reach a reasonable level of achievement, but another asserted that its structure was close to Maori which eased the learning task. Chinese was also supported largely for economic reasons, but was also seen to be an important community language. Two teachers commented that there seemed to be little demand from parents for the teaching of Chinese. One school principal was interested in offering Mandarin and the school had a native Chinese speaker eager to teach it, but the Board of Trustees favoured Spanish (for which there was no student demand nor teacher available). According to this principal, Chinese needed to become "fashionable" in order to create student demand. Another teacher considered Chinese important for tourism. A few teachers mentioned Korean, generally for the same reasons as Chinese. One teacher was against teaching any language purely for commercial reasons.

Some teachers remarked that most New Zealanders still travel to Europe, and those supporting the study of European languages stressed the cultural heritage of most New Zealanders. Few reasons were given in support of specific languages. Typically French was associated with dance and cookery, though it was also seen to be useful for travel and attractive for its romantic associations. Teachers reported that French was still popular with students destined for law or medicine, and considered easier than German. Two teachers remarked that it would be good for students to learn the FL which parents were most likely to be able to help with, but one teacher felt that it should not continue to have such a dominant place in the FL curriculum. German was perceived as important for economic cooperation with Europe, a useful lead into other European languages and easier than Asian languages. It was recognised that Spanish is widely spoken and that New Zealand has increasing links with Spanish-speaking countries. Spanish was also considered easy and fun to learn and it represents a large range of cultures.

Two teachers commented that, for most students, Maori is a second, if not a FL and so the study of it has most of the advantages of any FLL. Another stressed the need to understand and respect our own New Zealand culture and its official language first, before learning other languages. In particular it was felt that Maori need their own language for self-esteem. For these reasons it was suggested, as mentioned above in section 9.3.2, that Maori should be the first language taught after English to all students, after which students should be offered the choice of a FL. It was claimed that Maori has been successfully introduced into primary schools as a natural part of everyday learning, and that children learn easily and enjoy it. One bilingual 
intermediate teacher reported some initial hostility from students and parents towards her use of Maori in class, but as she did not force students to use the language it had gradually been accepted. At one secondary school it was reported that only a few parents had resisted the introduction of a compulsory Maori Studies module in year 9. Only one intermediate teacher was hostile to the teaching of Maori, considering it a "dead" language, and taking the attitude that the community can maintain it if they wish but it should not be pushed elsewhere.

\subsubsection{The best starting age for FLL}

The opinions of teachers are summarised in Table 9.6, divided according to whether teachers were in secondary or other schools. The great majority of teachers (40) favoured FLL beginning before intermediate level, that is before the age of 11 years, although six of them would accept a Year 7 start at the latest.

Table 9.6 The best starting age

\begin{tabular}{|l|c|c|c|}
\hline \multirow{2}{*}{$\begin{array}{c}\text { Preferred } \\
\text { starting age }\end{array}$} & \multicolumn{3}{|c|}{ Number of mentions } \\
\cline { 2 - 4 } & $\begin{array}{c}\text { By secondary } \\
\text { teachers }\end{array}$ & $\begin{array}{c}\text { By other } \\
\text { teachers }\end{array}$ & Totals \\
\hline The younger the better & 16 & 11 & 27 \\
\hline Pre-school & 1 & 6 & 7 \\
\hline Sometime at primary level & 1 & 4 & 5 \\
\hline 4 to 6 years & 3 & 1 & 4 \\
\hline 7 to 10 years & 8 & 1 & 9 \\
\hline Year 7 or 8 & 7 & 4 & 11 \\
\hline Year 9 & 1 & 0 & 1 \\
\hline Not sure & 1 & 1 & 2 \\
\hline No opinion & 2 & 0 & 2 \\
\hline
\end{tabular}

\subsubsection{Reasons for preferred starting ages}

General comments made in favour of starting FLL before secondary level are shown in Table 9.7. Teachers who believed that FLL should begin at intermediate level gave a variety of reasons. One stated that, at that age, "students absorb knowledge 
like a sponge". Others believed that younger students can have more fun in FLL. Many observed that most overseas students start FLL at an earlier age. Teachers frequently stressed the need for students to be able to make an informed choice when deciding whether or not to choose a FL option at secondary school. Some believed that L2 learning started too early slows down L1 development, and that, by year 7 or 8 , students have mastered sufficient basic English to be ready for L2 learning. Others asserted that intermediate students are mature enough to start investigating the wider world, and will therefore enjoy learning more new concepts.

Table 9.7 Reasons for starting FLL earlier than year 7

\begin{tabular}{|l|c|}
\hline Comments & $\begin{array}{c}\text { No. of } \\
\text { mentions }\end{array}$ \\
\hline $\begin{array}{l}\text { Younger students learn much more easily and } \\
\text { quickly }\end{array}$ & 12 \\
\hline $\begin{array}{l}\text { Younger children have fewer inhibitions, less } \\
\text { 'baggage' to overcome }\end{array}$ & 4 \\
\hline Secondary school is too late & 3 \\
\hline $\begin{array}{l}\text { Students can achieve more; secondary courses } \\
\text { are inadequate to cover exam syllabuses }\end{array}$ & 2 \\
\hline
\end{tabular}

About half of those favouring a start at primary school used phrases such as "as young as possible". Four teachers specified that year 9 was too late. One was unsure about the best starting age but emphasised that all students should have the opportunity to learn an L2 at some stage. Another was unwilling to give an opinion but did specify that, if language learning were compulsory, it should start at Year 7. Some teachers suggested that intermediate students needed more serious study, i.e. that current programmes did not stretch students enough, especially in English, geography and history, and that more needed to be done to develop their thinking and communication skills. In addition, there was too much repetition in year 9 of work already covered at intermediate level. According to some, the obvious way to overcome these shortcomings was to begin secondary schooling at year 7 and include at least 2 years FLL.

\subsubsection{Should all students have some experience of SLL?}

While primary and intermediate teachers were evenly divided over the question of making FLL compulsory, secondary teachers were twice as likely to favour compulsion as not, see Table 9.8, although three quarters of the secondary teachers favoured compulsory FLL at primary or intermediate level, not in their own schools. Most responses to the question of compulsion were qualified in some way and teachers tended to reflect the current situation in their school. Many teachers in favour of FLL for all stressed the cultural value of FLL, but were unable to overlook 
obvious problems of staffing, curriculum and resource development, choosing which FLs to offer and adding another subject to an already overcrowded curriculum. Those against compulsory FLL tended to doubt the ability of all students to cope with L2 learning, but most were in favour of offering all students the opportunity for FLL at primary or, more often, at intermediate level. Some pointed out that for NESB students, English is an L2 so perhaps they would not need or want to learn another 'FL'. There was considerable variation in opinions about how much FLL students should have, what the best starting age was and what sort of course younger students should have.

Table 9.8 Teacher opinions about the status of FLL in schools

\begin{tabular}{|l|c|c|c|}
\hline Status of FLL & $\begin{array}{c}\text { Secondary } \\
\text { teachers }\end{array}$ & Other teachers & Totals \\
\hline Compulsory & 24 & 9 & 33 \\
\hline Optional & 12 & 8 & 20 \\
\hline Not sure & 1 & 0 & 1 \\
\hline No comment & 2 & 0 & 2 \\
\hline
\end{tabular}

Teachers in favour of compulsory FLL emphasised the value of continuous courses, as against short "taster" modules, which secondary FL teachers generally considered "too little, too late", and it was secondary teachers who were most aware of the issue of 'articulation' which, as pointed out by Peddie et al. (1999) needs much more discussion. It was suggested that the only way to overcome all the inconsistencies in current FL courses was to have, at intermediate level at least, some sort of common curriculum to enable all students to arrive at secondary school at similar levels and be able to make reasonable choices about further FLL. One of the major points, made particularly by FL teachers, was that FLL would not be taken seriously at primary/intermediate level unless it were a separate 'essential learning area' in the Curriculum Framework.

Whatever their views on making FLL compulsory or not, the majority of teachers wanted to see much more FLL, especially in primary and intermediate schools. Ideally such learning should be integrated into the curriculum by having some content-based language teaching, making the most of whatever language skills teachers might have. Teachers believed that more positive attitudes to FLL should be encouraged by, for example, inviting foreign visitors into schools or offering more overseas travel opportunities. One of the main reasons for wanting to see more, earlier FLL was to give students a more informed choice about languages when they reached secondary school. Some teachers felt that, if students had had a good grounding in FLL, it would not matter if they did not then continue it at secondary level. Most teachers emphasised the need for younger students to have as wide a 
range of learning experiences as possible. Supporters of compulsory FLL pointed out that it is "no big deal" to learn another language at school overseas, and that FLL is not necessarily too difficult, even for the weakest students. In fact, it can and should be fun for all.

The issue of 'articulation', identified by Peddie et al. (1999) was mentioned by a number of teachers who saw the need for agreement between secondary schools and their local primary and intermediate feeder schools about the languages to be taught and starting ages, so that students could benefit from continuous courses. Teachers realised that this would not address the problems of students who moved to schools in different areas with different language learning schemes. Thus it was suggested that there should be SLL courses at intermediate level, of consistent length and content, so that students would arrive at secondary school with similar levels of experience and achievement.

Only a small number of teachers believed that FLL was suitable only for the most able students. Similarly, only a tiny minority wanted to see FLL compulsory for secondary students. There was more support for the notion that students need to be well grounded in their L1 before tackling an L2, so that FLL for all stating in year 7 might be acceptable. None of these teachers seemed to appreciate the irony that NESB students were generally expected to become proficient in English as soon as possible, regardless of the state of development of their L1.

Two other teachers claimed that students cannot be forced to learn another language as it is "too personal a matter". This seems to reflect another issue which Gardner (1985) identified - the fact that language learning is not like learning any other subject - although Gardner argued that this was a reason for every student to have SLL experience, not that this made it unsuitable for some students. The attitude of these teachers seems to be the traditional one of restricting learning opportunities for students according to their academic ability. One teacher speculated that involving greater numbers of students in FLL would increase general confidence in such learning among those who doubt the suitability or value of FLs for many students, in contrast to the present, unfortunately common, view that FLL is a "luxury not a necessity". This seems to be another example of societal attitudes being a selffulfilling prophecy, as Gardner (1985) identified in relation to the perception of SLL as a difficult subject, discussed in section 2.7.

Two teachers seemed to suggest that FLL should not be part of the secondary curriculum at all. One believed that children responded best to exposure to a FL when very young and in the home, not seeming to distinguish between first language acquisition and school language learning. Another thought that FLL should be part of travel experience, that is, practical communication and the absorption of culture on the spot, not taught in school. 
The one teacher who admitted to being unsure about whether or not all students should be exposed to FLL was a secondary principal who asserted that some primary and intermediate schools were introducing FLL for the wrong reason, that is, to attract high-calibre students, not necessarily because they believed in its educational value. This type of pursuit of a 'competitive edge' was noted by Williams (1996). Some recent media reports suggest that there is a growing demand for an earlier start to FLL or the maintenance of community languages (O'Flaherty 2001, Schumacher, 2001, Walsh 2001) in schools.

\subsection{Teachers' views of the current situation}

\subsubsection{Which students study FLs?}

When asked what of type of students tended to opt into FLL at secondary level, half the teachers reported that it was those above average academically, see Table 9.9. The teachers' opinions suggest that, however positive the attitudes towards FLL of teachers, parents or students, in the end it was only the more intelligent students who chose FLL, although a few teachers reported signs that a wider range of students was opting to learn FLs.

Table 9.9 Which students choose to study FLs

\begin{tabular}{|l|c|}
\hline Comments & $\begin{array}{c}\text { Number of } \\
\text { mentions }\end{array}$ \\
\hline The most academic, most able & 28 \\
\hline Mostly girls & 8 \\
\hline $\begin{array}{l}\text { Those with some family involvement in using } \\
\text { LOTEs }\end{array}$ & 9 \\
\hline $\begin{array}{l}\text { Those particularly interested, keen enough to } \\
\text { sacrifice other subjects }\end{array}$ & 6 \\
\hline No pattern, a wider range than before & 4 \\
\hline $\begin{array}{l}\text { Those with previous, good experience of language } \\
\text { learning }\end{array}$ & 4 \\
\hline Those from middle class, professional homes & 3 \\
\hline $\begin{array}{l}\text { Those influenced by the good reputation of the } \\
\text { subject or the teacher }\end{array}$ & 1 \\
\hline Those who want to stay with their friends & 1 \\
\hline Don't know/no comment & 4 \\
\hline
\end{tabular}


According to most teachers, the more able students were:

- the more studious and prepared to do the necessary (hard) work;

- those who saw the need for languages other than English, often influenced by overseas (especially Japanese) visitors;

- the "risk takers", wanting challenges;

- the most curious with the widest interests;

- good with, and interested in, language and languages;

- usually more mature than average, good all rounders and aiming at tertiary education.

Several teachers used the word "sacrifice" when talking about FL students, implying that the students had to be particularly keen in order to forego other optional subjects which might interest them.

Parents were thought to be another influence on student choices. According to teachers, parents of FL students placed more value on education in general than many others and were themselves interested in languages. FL students usually had parents who travelled or had business connections overseas. The students themselves travelled or had other opportunities to use a language other than English outside school. One Japanese teacher had surveyed the Year 9 intake and found that most of the students were in the class because their parents had made the decision or because older siblings had done the subject. Other teachers also reported that students were influenced by older siblings who had enjoyed FLL or been on an overseas exchange, for example. The reports of the influence of foreign travel and association with speakers of other languages tend to support the findings of my student surveys. On the other hand, students themselves did not think that they had been particularly influenced by their parents or siblings, but one FL teacher reported that interest in FLL still reflected a student's socio-economic background, as has been reported overseas (Burstall 1979, Loulidi 1990, Peddie 1993). On the whole, the teachers' perceptions supported my hypotheses that parents were an important influence over children's attitudes towards FLL.

\subsubsection{What governs student choices of FL?}

Teachers indicated that gender and previous experience were the main influences on student choices. To some extent, the choice of language seemed to depend on the "flavour of the moment" which perhaps explained the fluctuation in popularity of Japanese, although three teachers claimed that boys tended to choose Japanese anyway. Several teachers commented that students tended to opt into FLL in year 9 if they had had a good previous experience of FLL, which also supports my findings. However, most of such comments referred to students continuing Spanish. In any case, according to teachers, a bad experience does not necessarily put students off. They may choose a different language when they start secondary school (whether or not language learning is compulsory) because they feel they have "done", say, French. If they did not enjoy their previous experience it was most likely to be because they found it difficult, especially if they had been learning 
Japanese. These last two comments support what Vine (1993) found in her study.

\subsubsection{Why students reject FLL in year 9 or beyond}

When asked to say why they thought most students dropped out of FLL as soon as it was no longer compulsory, or after trying a Year 9 option, teachers gave the opinions listed in Table 9.10. The first two groups of comments, made by over half the teachers, are largely two sides of the same coin, that is, they are positive and negative reasons for the students' choices, depending on the perceived future value of FLL. According to several teachers, this lack of value was often associated with the idea that everyone can or will use English, although this was not supported by the students themselves in my findings. The general attitude towards FLs perceived by the teachers was vividly illustrated by some of the phrases they used. They saw FLs treated as:

- a "luxury"

- the "poor relations" in schools

- a "fringe element"

- part of the "old world" of education

Table 9.10 Reasons for not undertaking or continuing FLL

\begin{tabular}{|l|c|}
\hline Comments & $\begin{array}{c}\text { Number of } \\
\text { mentions }\end{array}$ \\
\hline $\begin{array}{l}\text { Career choices, need more 'useful', 'safer' } \\
\text { subjects' }\end{array}$ & 21 \\
\hline Not important, not needed, no long-term value & 15 \\
\hline Too difficult, too much work, want easier subjects & 14 \\
\hline Put off by teacher & 5 \\
\hline $\begin{array}{l}\text { Just not interested } \\
\text { Senior students put off by having to do } \\
\text { correspondence study }\end{array}$ & 1 \\
\hline
\end{tabular}

Teachers attributed the marginalisation of FLs to the trend to reduce the amount of time available to option classes in Year 9, i.e. shorter courses or fewer classes all year (e.g. twice every 6 days). FL teachers, more than others, saw these conditions as particularly disadvantageous to successful FLL and reinforcing the notion that languages are difficult subjects. According to the teachers, students were often aware that it is considered hard enough to get good marks in year 13 FL examinations compared with maths and sciences, without the amount of study time being reduced. 
Several teachers made the point that, even when students were interested in languages, enjoyed studying them and wanted to continue, schools sometimes made it too hard for them. Their choices were limited by timetable clashes and by pressure to choose options which were seen as priorities for their goals of occupation or further study. Another factor thought to dissuade students from continuing FLL was the need for many senior students to study by correspondence. It should be stated, however, that the teacher in charge of correspondence students on at one school assured me that the language students coped extremely well because they aware highly motivated and well supervised. My personal experience tells me that this is not the case in all schools. Teachers observed particularly unfavourable conditions for FLs where there were accelerated classes in a school and one felt that, in his school, FLL was being "snuffed out" or "strangulated".

\subsubsection{The extent to which student choices were guided by schools}

Teachers were asked what information and advice was given to students, specifically in relation to FLL, before they started year 9 and as they moved up the school.

\subsubsection{For students moving from Year 8 to 9}

All the secondary schools distributed prospectuses with varying amounts of detail about the school, courses and other activities offered, and so on. Some produced booklets with additional information for specific year groups with details about optional subjects. All the schools held some sort of open day or evening when any interested students and their families could visit and talk to teachers. The Year 7 - 13 school had the advantage that most year 9 students came through from its own intermediate classes so they were able to visit secondary classes in action and talk to the teachers on site. Most secondary schools sent out one or two staff, usually including a year 9 dean, to visit year 8 classes in the main contributing schools.

The smaller schools were able to interview each new student on enrolment with their parents, in order to discuss such matters as option choices. The larger ones did not find this feasible and generally invited families to an enrolment evening where a wide range of senior staff handled the enrolments and gave information and advice. Most teachers felt that, in general, students arrived having made their option choices, usually in consultation with their parents. Year 9 deans reported that they had very little influence on subject choices made by incoming students. Some FL teachers, however, expressed concern that, whatever the method of enrolment, the students might not always receive dispassionate advice about option choices and might even be dissuaded from studying a FL if the enrolling teacher considered them "unsuitable".

\subsubsection{For students moving from Year 9 to $\mathbf{1 0}$}

Teachers gave the impression that student choices were based largely on their 
year 9 experiences and on the information supplied about the options available in year 10 and beyond. General career advice, delivered to small groups or whole classes, was not usually organised until year 10. Individual junior students (i.e. in years 9 and 10) would normally receive advice only if they requested it. Thus, any messages students might receive about, for example, maintaining as broad a range of subjects as possible in order to keep their options open for future study or jobs, would come after many had already given up some of their optional subjects, including FLs.

\subsubsection{For senior students (years 11-13)}

At each school I tried to interview teachers responsible for advising students about subject choices and their comments are summarised in Table 9.11. The main impression I obtained was that, in class or individual discussions about employment or tertiary education, very little mention was ever made of FLs by students or staff. This was mostly because, by the time careers advisers and senior deans had contact with students, most of those who had undertaken some FLL had given it up. Teachers reported, too, that employers and tertiary institutions made very little mention of FLs as desirable qualifications or components of further study.

Table 9.11 Advice about FLL given to senior students in relation to employment or tertiary courses

\begin{tabular}{|l|c|}
\hline $\begin{array}{l}\text { Comments from senior deans and careers } \\
\text { advisers } \quad(\mathbf{N}=\mathbf{8})\end{array}$ & $\begin{array}{c}\text { Number of } \\
\text { mentions }\end{array}$ \\
\hline $\begin{array}{l}\text { Little or no mention, little or no demand for jobs or } \\
\text { in tertiary studies }\end{array}$ & 8 \\
\hline $\begin{array}{l}\text { Some mention of Japanese + business, economics, } \\
\text { computing }\end{array}$ & 4 \\
\hline Some mention of Japanese + tourism & 4 \\
\hline $\begin{array}{l}\text { Only see senior students, rarely any studying a } \\
\text { language }\end{array}$ & 3 \\
\hline $\begin{array}{l}\text { Some possibility of language electives in tertiary } \\
\text { courses, e.g. business }\end{array}$ & 2 \\
\hline $\begin{array}{l}\text { No demand from local employers for language } \\
\text { skills }\end{array}$ & 2 \\
\hline
\end{tabular}

Comments made by individual teachers about the place of FLL in further education included the following:

- Universities welcomed FL students but there were hardly any prerequisites for tertiary courses in any subjects any more; 
- Not even the Ministry of Foreign Affairs and Trade required FL skills in its new employees, preferring to organise its own intensive courses;

- FL students tended to be more focussed in their goals and not consult careers advisers;

- "What can I do with my languages?" was a worrying question for an adviser as tertiary institutions hardly mentioned FLs in their reports on what careers their graduates went into;

- Although tourism courses at schools and tertiary institutions had "mushroomed" it was not always obvious that FLL had any place in these;

- If there was a FL component students learnt no more than basic phrases;

- One local institution had a tourism course which included Japanese but its high cost deterred many students;

- The only career which specifically mentioned the desirability of FL skills was that of flight attendant;

- In order to major in business at one local institution it was necessary to spend two years on compulsory courses, with no room for FLL, however keen a student might be.

Even if careers advisers did consider FLL important in terms of students keeping their options open, in view of the uncertainty about future employment, they did not see students early enough to give such advice. One adviser, spoken to only briefly, wanted to stress that, given the chance, she always advised students to continue their FLL. Furthermore, if advisers saw the benefit of FLL in relation to specific career paths, they could not honestly tell students that such value was perceived by tertiary institutions or employers. For example, one teacher believed that there were big opportunities for students with a good knowledge of Japanese language and culture, which might explain why, of all the FLs offered at local secondary schools, Japanese had the best retention rates, but local tertiary institutions did not seem to be acknowledging these opportunities or offering appropriate courses to help students make the most of them.

\subsection{Teachers' views of their colleagues' attitudes towards FLL}

Teachers were largely pessimistic about what they perceived to be the attitudes of most of their colleagues, see Table 9.12. This seems paradoxical compared with the largely positive attitudes reported above and may result, to some extent, from the limited sample of teachers interviewed. On the other hand, it may reflect a gulf between what teachers personally believed and what they said in public, to students and parents, or in staff discussions, which tended to support the status quo of FLL in schools. In other words, whatever value teachers put on FLL, the majority, apart from the FL teachers themselves, felt unable or unwilling to take action to support their ideas. The most disturbing aspect of negative attitudes perceived 


\section{4}

by some teachers among their colleagues was a tendency to deliberately dissuade students from continuing FLL. While some teachers admitted that this might be for laudable motives, one felt passionately that it was undermining the whole place of FLL in the school and used the word "sabotage".

Some teachers were aware of the resentment caused by the existence of small FL classes, especially at senior level, and believed that some of their colleagues would have such classes abolished and let "market forces" prevail. With growing demands on curriculum time and the continued compartmentalisation of subjects there was increasing competition for "a piece of the cake". Teachers who reported such attitudes were clearly distressed that the central issue of students' education was being lost in such "political battles". Others noted that teaching is an "aging profession" (McCutcheon 2002 gives the average age as 43), and it was only to be expected that the majority of teachers would have conservative views about the nature of school FLL and its place in the curriculum. Some saw their colleagues' attitudes tending to improve as their children started to work and travel, which, as shown in section 9.2.4, some teachers themselves admitted.

Table 9.12 Teachers' attitudes towards FLL, according to their colleagues

\begin{tabular}{|l|c|}
\hline Comments & $\begin{array}{c}\text { Number of } \\
\text { mentions }\end{array}$ \\
\hline $\begin{array}{l}\text { Influential teachers dissuade some students from } \\
\text { language study }\end{array}$ & 8 \\
\hline Language learning is not important & 5 \\
\hline Some resent small language classes & 5 \\
\hline $\begin{array}{l}\text { A little is OK for all but it is too hard for some to } \\
\text { continue }\end{array}$ & 2 \\
\hline $\begin{array}{l}\text { Some are put off by bad experiences, do not know } \\
\text { FLL has changed }\end{array}$ & 3 \\
\hline Generally supportive & 3 \\
\hline
\end{tabular}

\subsection{Teachers' influence according to students}

In section 4.5.3 I discussed the responses of year 8 students to survey items specifically referring to their FL teachers and their methodology. These response were largely positive and I showed that positive experience of prior FLL tended to have positive effects on student decisions about FLL in the following year. I also endeavoured to find out if year 8 class teachers had played any role in influencing student choices about FLL for year 9 by asking all the students to respond to item 8.1.16, "My class teacher says I should do a language next year". Only a small number of students, $16 \%$, agreed with this while $47 \%$ disagreed 
$(\mathrm{N}=278)$. It could not be assumed from this that teachers had advised against future FLL, since it was possible that the matter had not been discussed. In fact, from what class teachers reported in their interviews, they had not stated an opinion in most cases, either because they had not been asked by students or parents or because they did not feel qualified to give advice about subjects about which some of them knew very little. From what the year 8 students reported, it appears that their teachers had little direct influence over students' decisions about future FLL, although FL teachers who succeeded in making FLL an enjoyable experience indirectly encouraged further learning. This absence of teacher influence was reinforced by the response of the year 9 students when asked why they had or had not enjoyed their previous FLL, discussed in section 6.8.4. Very few students mentioned teachers for positive or negative reasons.

One barrier that FL teachers sometimes perceive to continued FLL after year 9 is the fact that in most secondary schools there is unlikely to be more than one teacher of any particular FL. Thus, if students take a dislike to the teacher of the FL they have chosen to study in year 9 they may decide against further study of that FL, knowing that they would always have the same teacher. By comparison, it is usual for students to meet a different teacher of, say, English or Mathematics each year. However, 58\% of 206 year 8 students who reported that they would or might study a FL in year 9 disagreed with the statement, "I'll try a language next year but I won't go on with it if I don't like the teacher"1. Of course, for most of the students, not knowing anything about the teachers they might have, this would be a completely hypothetical opinion. Nevertheless, some students would have heard of FL teachers through the experiences of older siblings or friends, and students at the Salvia would certainly know the Japanese teacher. It does suggest a determination among students to study what they were interested in, and not be put off by a teacher.

\subsection{Staffing issues}

\subsubsection{The general situation}

All the teachers interviewed were asked if they were aware of any problem in obtaining suitable FL teachers and if this might be responsible for the apparent declining interest in FLL among students. Most teachers felt unable to comment but the school principals and FL teachers all had something to say on the subject. The two largest secondary schools, Begonia which offered 3 FLs, with some pressure on top stream Y9 students to study a language, and Freesia (mixed), which offered 4 entirely optional FLs, could each sustain only one full-time FL teacher (of Japanese). The other FL teachers in these two schools also needed to teach other subjects, whether they wanted to or not. The other two mixed schools had some compulsory language learning and offered only two FLs. One was able to employ full time French and Japanese teachers, but the other had only one full time teacher teaching both languages and a part time French teacher. Of necessity, this constrained timetabling and limited students' options.

\footnotetext{
'It is recognised that this questionnaire item was of dubious value, consisting of two statements in one, in addition to containing a negative statement. Such statements were largely eliminated in the piloting and consultation processes as being hard for the students to respond to clearly.
} 
In addition, it was interesting to discover that seven of the 13 FL teachers in the secondary schools were born overseas, and all except one of the seven had been educated overseas where FLL was a compulsory part of their secondary education. One teacher reported that the local teachers' college only catered for Japanese teachers, which was perhaps responsible for improving that particular teacher supply, if not helping the supply of teachers of other FLs. The New Zealand education system may not be providing sufficient numbers of FL teachers for what schools would like to offer, as noted by Williams (1996). Any attempt to improve teacher supply would need a great increase in numbers of tertiary FL students and for this to happen far more school students would need to start and continue FLL.

The question of part-time FL teachers is problematic. Personal experience shows that part-time teachers often play only a minor part in the general life of the school and lack of visibility may lead to lack of interest in their subjects, both among their colleagues as well as the students, if they are the sole teachers of that subject. Furthermore, they may not be guaranteed employment from one year to the next, depending on student numbers. In some cases, interviewed teachers taught FLs part time because of lack of classes, especially at senior levels, and had to teach other subjects, not necessarily of their own volition. Such arrangements can put constraints on timetabling and limit students' options. It is often a "chicken and egg" situation, of course. Lack of students opting for a particular subject usually means a reduction in classes, which in turn may drive a teacher away. If a school has trouble replacing a teacher it may not be able to continue offering a subject. Two of the schools involved in this research were having problems of this nature. In every secondary school surveyed some senior language students had to work in combined classes or study by correspondence. This was not a result of teachers not being available in the schools but of the inability of the staffing budgets to cope with more than one or two very small senior classes. All the teachers concerned claimed that correspondence courses are very difficult and require a high degree of student motivation and independence.

\subsubsection{The principals' perspective}

One school had sought a Spanish teacher but being unable to find one had employed a new German one instead, despite declining interest in the subject. The same principal was worried about replacing the HOD languages who was about to leave, as the school needed someone able to teach French and Japanese in order to maintain its current curriculum. At another school the future of French was uncertain as there was no permanent teacher, owing substantially to the fact that the school could never offer a full-time French teaching position. The school could not guarantee that French classes would go ahead, a situation which naturally tended to put students off opting for the subject, but the lack of students reduced the chances of offering classes in the first place - a vicious circle. There had been some thought that it might be worth trying a different language to attract more students and hence a teacher. 
At another school the supply of FL teachers had not been an issue for the principal, but he was aware of anecdotal evidence that Japanese teachers could be hard to find. One school had had difficulty when first establishing Japanese but was very pleased with the current situation. Even when their teacher had taken leave the school had had a number of applicants for the position of long term reliever and found a good replacement. The only problem that arose each year was the small numbers of students interested in French and German and hence the need to decide if classes would run or not. Another principal declared that small senior numbers created an ongoing staffing problem although he was determined to support the classes for as long as possible. In his experience, once classes were lost they were never restored, a point also made by one of the language teachers. In schools which were generous in staffing very small senior language classes, the senior administration staff were well aware of the resentment this could cause, discussed above in section 9.5, and usually made efforts to compensate staff in some other way. One principal considered correspondence courses totally unsatisfactory. He was not criticising the courses themselves but felt that putting students on correspondence was unfair for the students who did not have the same interaction with fellow students and teachers as their peers did in other subjects (cf Spence 1998).

Whatever the staffing problems or the attitudes of principals towards FLL, it was clear that they would be loath to lose all senior FL classes for fear that some parents would send their children elsewhere. These would tend to be the more academic students who get the best examination results and assist in maintaining the good reputation of the school, a necessity in a system which forces many schools to compete for students.

\subsubsection{The language teachers' perspective}

The FL teachers tended to have a slightly different perspective from their principals. In their experience, obtaining relief teachers of Japanese had often been a problem in the past, whereas there was usually no shortage of French or German teachers. However, the HOD languages in the school without a permanent French teacher agreed with her principal that this was a major problem for student motivation. In general, there seemed to be a low turnover of FL teachers, provided there were sufficient students to keep classes going and provide them with satisfying jobs. FL teachers tended to feel that student retention rates depended to a great extent on their energy, commitment, enthusiasm of individual teachers and their ability to make classes sufficiently fun and interesting to 'capture' the students. In particular, teachers of European languages needed to make up for geographical distance with good resources and enthusiasm. While this responsibility falls on teachers of all optional subjects, FL teachers felt that it was unfair of principals to claim that they valued FLL and suggest that teachers might be to blame for high drop-out rates, when many other factors were contributing to students' decisions to discontinue FLL. One such factor was the problem of students' lack of basic English skills, which some FL teachers perceived as a barrier to successful FLL. Other comments 
about teacher availability were made by immigrant teachers, particularly speakers of Asian languages, who felt that there should be more support for native speakers of LOTEs to retrain as teachers of their languages.

Commenting on combined classes, one teacher claimed that these can have advantages although they may put some students off. The lower level students can be stretched while the others get more revision. All the students need to develop the habit of independent learning to a greater extent than students in single level classes.

On the 'problem' of students arriving in year 9 with different amounts of FLL experience, teacher opinions varied. Some found it very difficult to cope with such students. They saw FLL in primary and intermediate schools as very "hit-and-miss" and were critical of the FL knowledge and teaching ability of their primary colleagues. Such negative attitudes have been observed by some of the language advisers who have reported (personal communications) that the attitudes of secondary language teachers towards FLL in primary and intermediate schools are one of the barriers to the successful development of FL programmes in years 7-10, and that secondary FL teachers tend to underestimate the expertise and enthusiasm of their primary colleagues.

However, the majority of secondary FL teachers involved in this research did not view the different levels of expertise among their year 9 students as a serious problem because most of the intermediate courses were short "taster" courses and students had considerable gaps in their knowledge. Students with some prior knowledge and confidence could help and encourage beginners. A greater problem from the point of view of the advisers is that students may arrive at secondary school having enjoyed "taster" FL classes, ready for more in-depth learning, only to be faced with similar short beginners' courses which offer little that is new and which may discourage them from continuing FLL. As already discussed, this seemed to be happening at one of the surveyed schools.

\subsubsection{The views of other teachers}

Some teachers could not understand why New Zealand does not value FLs as other cultures do. There seems to be no pattern or consistency in the FLs offered by schools, and the popularity of individual languages among students seems to vary from place to place. Offering different FLs was not seen to attract more students but simply reduce the numbers learning traditionally taught languages. Teachers wanted the profile of FLL raised in schools so as to increase public awareness of its value. Limited exposure in schools limits interest and understanding of what is possible and desirable. Some schools had thought about introducing senior FL beginners' courses, for example, business Japanese or German for tourism, but teachers foresaw problems arising from pressure on the timetable from other subjects. Sceptical teachers believed that class numbers would be too small to warrant the staffing, or that year 12 FL classes might be considered "soft options", like drama 
and media studies, which attract students not prepared to do the necessary hard work. Schools would need to develop their own courses and resources. This would involve time and money they are reluctant to spend without support or encouragement at national level.

Teachers in general, like the interviewed parents, appreciated the value of FLL, and had ideas about improving student numbers. However, they perceived extensive barriers to increasing FLL in secondary schools (cf Spence 1997, 1998, Johnson 2001).

\subsection{General comments about education in New Zealand}

The following sections present a range of views from individual teachers. Not all the interviewed teachers commented on these topics, nor would they all agree with the comments listed, but these comments do build up a coherent picture of what teachers perceive to be happening in schools.

\subsubsection{General attitudes towards schooling}

- Poor attitudes towards FLL reflect generally poor attitudes towards education.

- New Zealanders are very relaxed about education which is not as standardised nor as demanding as in other countries, where even some kindergartens have FLL.

- Life is still too easy for most people and improved education is not seen as the answer to any socio-economic problems which do exist.

- New Zealanders are "too laid back", "lazy", "isolated".

- A section of the community is not "in tune" with education, with low aspirations and expectations. The challenge is to get all to value it so that the gap does not widen between this section of the community and the better educated "elite".

Ironically, the very organisation and ethos of one school was identified by some of its teachers as encouraging such a division because of the rigid streaming of classes. Accelerated classes which pushed students through examinations early caused them to miss out on social development, and cramming tended to push some subjects, such as FLs, out of the curriculum. The students in the stop streams were given the sense of being an elite while those below felt inferior, though it was admitted that some other students were glad not to be subjected to the the same academic pressures.

\subsubsection{The limited nature of secondary education}

- There are signs of growing awareness among teachers and students of the contrast between schooling in New Zealand and overseas because of increasing contacts with foreign exchange students.

- There are not enough hours in the school day to improve the range of subjects taught compared with overseas.

- General knowledge is "appalling".

- Secondary schooling should begin two years earlier, as is common overseas. 
- The answer to the problem of the "crowded curriculum" might be more integration of subjects, instead of the creation of more, separate core subjects. (A degree of subject integration did exist at Freesia which, as discussed in 6.2.4, was considered effective by ERO (1998b).

Several teachers were particularly bitter about the creation of new timetable space for technology which was reducing time for other subjects, especially the options. They believed that technology is an essential tool which should be integrated into all subjects, not taught separately. Teachers also objected to the trend they perceived for education to become more like job preparation with too little "brain training". In their opinion, job training should be carried out on the job or during tertiary studies. Schooling a child is not about producing an "economic unit".

\subsubsection{More pressing demands on scarce resources}

Principals in particular reported that their schools were facing major problems which were straining their limited resources. For example, a kura kaupapa Maori (a Maori language primary school) had decided to designate a particular secondary school as the "preferred destination" for its students, although the school in question had no resources to provide bilingual education. Most schools were coping with an increase in NESB students with what they considered inadequate support from the government, (one principal called it "peanuts"). Schools often had to rely on the chance availability of native speakers, especially of Asian languages, to help immigrant students with some subjects, like maths and science.

\subsection{Final analysis}

The principal ideas which emerged from the teacher interviews and which were supported by a majority of the teachers can be summarised as follows:

\subsubsection{Children need to start SLL an an earlier age}

Although special needs and NESB students may be better served by concentrating on developing basic English and other skills, they should not be excluded from other language learning if they want to be involved. Maori was considered a priority for primary children by many teachers, but no teachers suggested that immigrant children might be offered the option of maintaining their L1 alongside learning English.

\subsubsection{All students should have some FLL at intermediate level}

There was clearly a considerable amount of disagreement among teachers regarding the desirability and possibility of teaching FLs to all students, and the best starting age for compulsory or optional FLL. On the whole, teachers were reluctant to use the word 'compulsory', but most saw advantages in FLL for all or most students in FLL. They understood that an earlier start might enable students to attain greater proficiency in a FL, and believed that increasing the number of students starting FLL would improve the retention rates in later FL classes. From their responses to the question of where they would ideally like to see FLL in schools, it was evident that 
the majority of teachers felt that it should begin before students reach secondary school and that, preferably, it should in some way be integrated into primary education, but it was not at all clear how that might be done if it were to remain optional. Considerable disagreement remained between teachers favouring short "taster" courses and those wanting longer, on-going courses leading to more advanced studies at secondary level.

The general impression was that specialist language teachers are not needed at intermediate level because adequate resources now exist for enthusiastic teachers to teach French, German, Spanish and Japanese in years 7 and 8. However, on-going teacher training is still needed and more language specialists are still desirable. Courses of consistent length and content are needed so that students arrive at secondary school with similar levels of achievement. Students and their parents need to be able to make more informed choices about optional FLL at secondary school. An earlier start will facilitate achievement of higher standards at senior levels. This is turn should help reduce the perception of FLs as "difficult" subjects. Students need to realise that FLL is an on-going process and cannot be "done" in one short module.

\subsubsection{Other changes needed}

There needs to be continued improvement of FL curriculums, teaching methods and materials in order to increase students' enjoyment, and enhance their perception of the "relevance" of FLL. More students need to be encouraged to continue FLL at secondary school and beyond. This, in turn, would help to produce more teachers with language skills. Students, their parents (and some teachers) and the wider community need to be persuaded about the importance of SLL for personal improvement and the benefit of the country. To this end, 'Languages' needs to be made an 'essential learning area', separate from 'Language' (i.e. English) in the New Zealand curriculum framework.

\subsubsection{The importance of gender}

A major factor identified by teachers as an influence on student decisions regarding FLL, discussed in section 9.4.2, was gender. This factor will be examined in detail in the following chapter. 

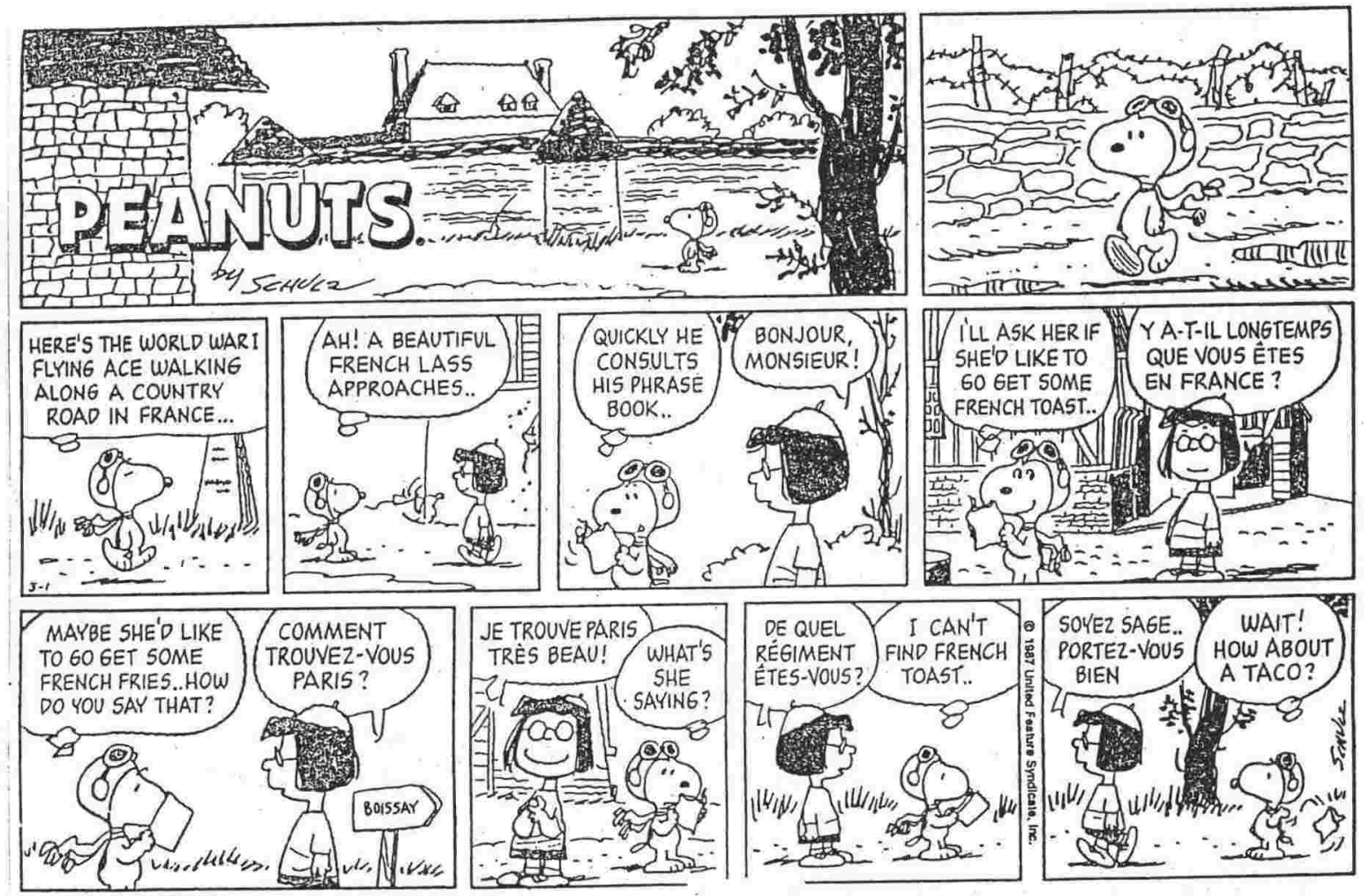

\subsection{Introduction}

The literature on gender differences in attitudes to FLL in schools, mostly in Englishspeaking countries, asserts that it is seen by students, parents and of ten by schools as more relevant for girls than for boys. This has been shown, for example, in the UK (Burstall 1979), the USA (Bacon \& Finnemann 1992), Europe (Coleman 1996), Australia (Carr 1997) and New Zealand (Waite 1992, ERO 1994). Where FLL is compulsory, boys on the whole are less successful than girls (Clark \& Trafford 1995) and opt out of it as soon as possible in greater numbers than girls (Aplin 1991, Ashenden \& Milligan 2001). Where it is optional, fewer boys choose to study a FL and fewer continue each year (Fullerton \& Ainley 2000, Holt et al. 2001), a situation which is clearly illustrated by MoE statistics in New Zealand.

An examination of enrolments numbers in the two FLs studied most in New Zealand secondary schools, French and Japanese, shows a clear discrepancy in the numbers of girls and boys starting and continuing their FLL, see Table 10.1. Drop out rates were high in all years and always higher for boys than girls except between years 12 and 13 .

In this research I analysed all the data obtained to ascertain if any gender differences existed in the attitudes of the students surveyed, their parents, their teachers or their schools. In most cases there were no significant gender differences, but some interesting differences emerged which are discussed in this chapter. 
Table 10.1 Numbers and drop out rates of students, by gender, in one cohort studying French and Japanese 1994-1998

(MoE Statistics)

\begin{tabular}{|c|c|c|c|c|c|c|}
\hline & French & Japanese & \multicolumn{2}{|c|}{ Gender balance } & \multicolumn{2}{|c|}{ Drop out rates } \\
\hline Year/Level & Girls & Boys & Girls & Boys & Girls & Boys \\
\hline $\mathbf{1 9 9 4 / Y 9}$ & 15164 & 11178 & $58 \%$ & $42 \%$ & & \\
\hline $\mathbf{1 9 9 5 / Y 1 0}$ & 8066 & 4868 & $62 \%$ & $38 \%$ & $47 \%$ & $56 \%$ \\
\hline $\mathbf{1 9 9 6 / Y 1 1}$ & 4833 & 2331 & $67 \%$ & $33 \%$ & $40 \%$ & $52 \%$ \\
\hline $\mathbf{1 9 9 7 / Y 1 2}$ & 2735 & 1141 & $71 \%$ & $29 \%$ & $43 \%$ & $51 \%$ \\
\hline $\mathbf{1 9 9 8 / Y 1 3}$ & 1752 & 760 & $70 \%$ & $30 \%$ & $36 \%$ & $33 \%$ \\
\hline
\end{tabular}

10.2 Perceptions of gender differences in interest and ability in FLL 10.2.1 Student perceptions

Asked to respond to the statement: "Girls are more interested in learning a language than boys", students in both years 8 and 9 revealed a high degree of uncertainty, with no significant gender differences in the responses. The suggestion that boys are better at FLL than girls, put to Year 8 students who had had some FLL experience, elicited almost no agreement and a high degree of uncertainty, but significant $(p<.001)$ gender differences in the responses, shown in Table 10.2. The girls' responses are not surprising and suggest that most of them considered themselves to be as competent as the boys at FLL, though not necessarily any better. The boys were more divided in their views.

Table 10.2 Responses by gender to survey item 8.2.16: "Boys are better at learning a language than girls."

\begin{tabular}{|c|c|c|}
\hline Item response & $\begin{array}{c}\text { Proportion of } \\
\text { girls }\end{array}$ & $\begin{array}{c}\text { Proportion of } \\
\text { boys }\end{array}$ \\
\hline Agree & Only 2 students & $15 \%$ \\
\hline Disagree & $66 \%$ & $34 \%$ \\
\hline Unsure & $32 \%$ & $51 \%$ \\
\hline 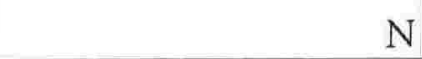 & 114 & 108 \\
\hline
\end{tabular}


Given the large number of 'not sure' and 'no opinion' responses, it would seem that the students did not feel able to judge the abilities of their peers, or that they were reluctant to do so. Several students added comments indicating any difference in ability depends on individuals, not their gender. For example, two girls stated:

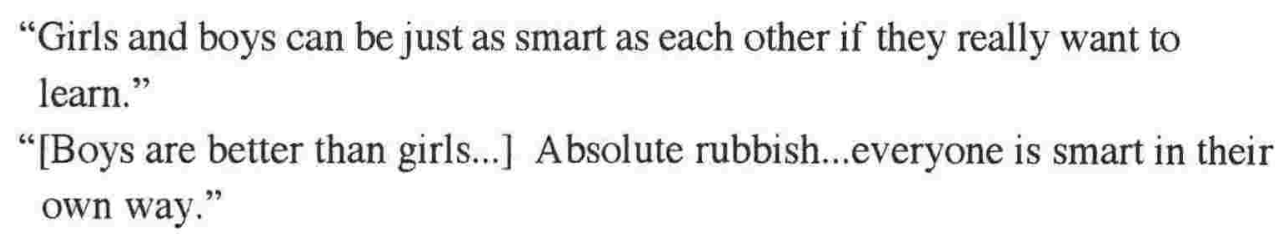

The older students were also very uncertain, but with no significant gender differences. The year 9 students seemed equally reluctant or unable to judge the ability of their peers.

Overall, there was little evidence that the students perceived any gender differences in attitudes towards FLL nor in aptitude for FLL. In the literature I found little reference to students' own perceptions of such differences. However, the findings of this research support those of Powell and Batters (1985) who found that both genders vigorously rejected the idea that FLL is more important to one group or that one group is more capable of FLL.

\subsubsection{Parents' perceptions}

Judging from their personal experience, the parents I interviewed could have been forgiven for perceiving FLs as mostly 'girls' subjects' since, as discussed in 7.4.1, most of the mothers, but very few of the fathers, had learnt a FL at school. I did not seek their views about FLL in relation to gender and none expressed any such views. It was interesting that it was mostly mothers who made themselves available for interviews, which could indicate that they were more prepared to talk about their children's education than the fathers, or perhaps that they were more interested in FLL. No parents seemed aware of any gender bias in their children's FL classes or in the schools in general. The possible differences in parental influence on girls and boys will be discussed in 10.5.1 below.

\subsubsection{Teachers' perceptions}

The New Zealand educated teachers interviewed for this research might similarly have perceived FLs as mainly for girls. 22 of the 27 women but only 12 of the 20 men had studied French at school, although similar small proportions of both had studied Latin or German. Of the 11 teachers who had not learnt a FL at school seven were male, though two had studied Latin. By comparison, all the 9 teachers educated overseas, all female, from Europe, Africa and Asia, had experience of SLL or FLL and had usually learnt more than one language.

As shown in other countries (Wright 1993, Carr 1997) the FL teaching profession tends to be dominated by women, partly because most tertiary FL students are female 
(Coleman 1996). I found a similar situation in the schools I surveyed. Of the 13 secondary FL teachers, 9 were female and it appears, that schools might have trouble recruiting FL teachers were it not for qualified immigrants. Seven of the FL teachers were born overseas, and all but one had been educated overseas where FLL was compulsory at secondary school. Whatever the students reported about FLs in relation to gender it seems evident that, subconsciously, they might, at least in the long run, come to associate FLL with females since most of their FL teachers were female.

As discussed in Chapter 9, teachers reported that FL students tended to be girls, especially beyond year 9. Two HODs of languages reported that this was particularly the case in European language classes. One called girls the "lifeblood" of such classes as most boys dropped out after year 11. Both reported that boys were more interested in Japanese which led to a better gender balance in classes. A third school reported that Japanese was the most popular language and that it usually attracted more boys than girls. Gender was reported to be one of the main influences on students' choice of FL, alongside previous FLL experience. Once again, whatever the students reported about FLL and gender, the boys were clearly receiving subtle messages that FLL was not for most of them.

Regarding gender stereotypes, one female teacher commented that the old notion that FLs were "poofie" or "wussie" subjects, and only for girls, was changing. On the other hand, a male teacher at Begonia still saw peer pressure against FLL. He also used the word "poofie" to describe the attitudes of some boys to his own subject, English, as well as languages or any subjects seen as "arty". Another FL teacher reported that girls were usually willing and confident about FLL, which only applied to the more academic boys. Where such male prejudice against FLL, and language study in general, comes from is open to speculation and would be an interesting topic for further research

\subsection{Year 8 intentions}

\subsubsection{To study a FL or not - the gender gap appears}

Among the year 8 students, there were clear indications that girls were likely to dominate FL classes the following year. More than twice as many girls had decided to study a FL as had decided against it. Boys were more evenly divided, while more boys than girls were not sure about FLL for the following year. These differences proved statistically significant $(\mathrm{p}<.05)$ and are illustrated by Graph 10.1 . When the intentions for year 9 were compared with the schools which the students expected to attend there were marked gender differences, shown in Table 10.3.

The smallest gender difference in intentions was at Salvia, where most students had studied both the FLs offered. Among students expecting to attend the single sex schools, girls were more likely than boys to be intending FLL, but the greatest discrepancy was for the large mixed school, Freesia. Although these students were 
offered the greatest range of languages, their general socio-economic background was poorer than that of students destined for the single sex schools. This seems to have had a greater negative effect on the attitudes of boys than girls. Overall, whichever school they expected to attend, more girls than boys intended to study a FLL in year 9.

\section{Graph 10.1 Student intentions for FLL in year 9, by gender}

(For gender association $\mathrm{p}<.05$ )

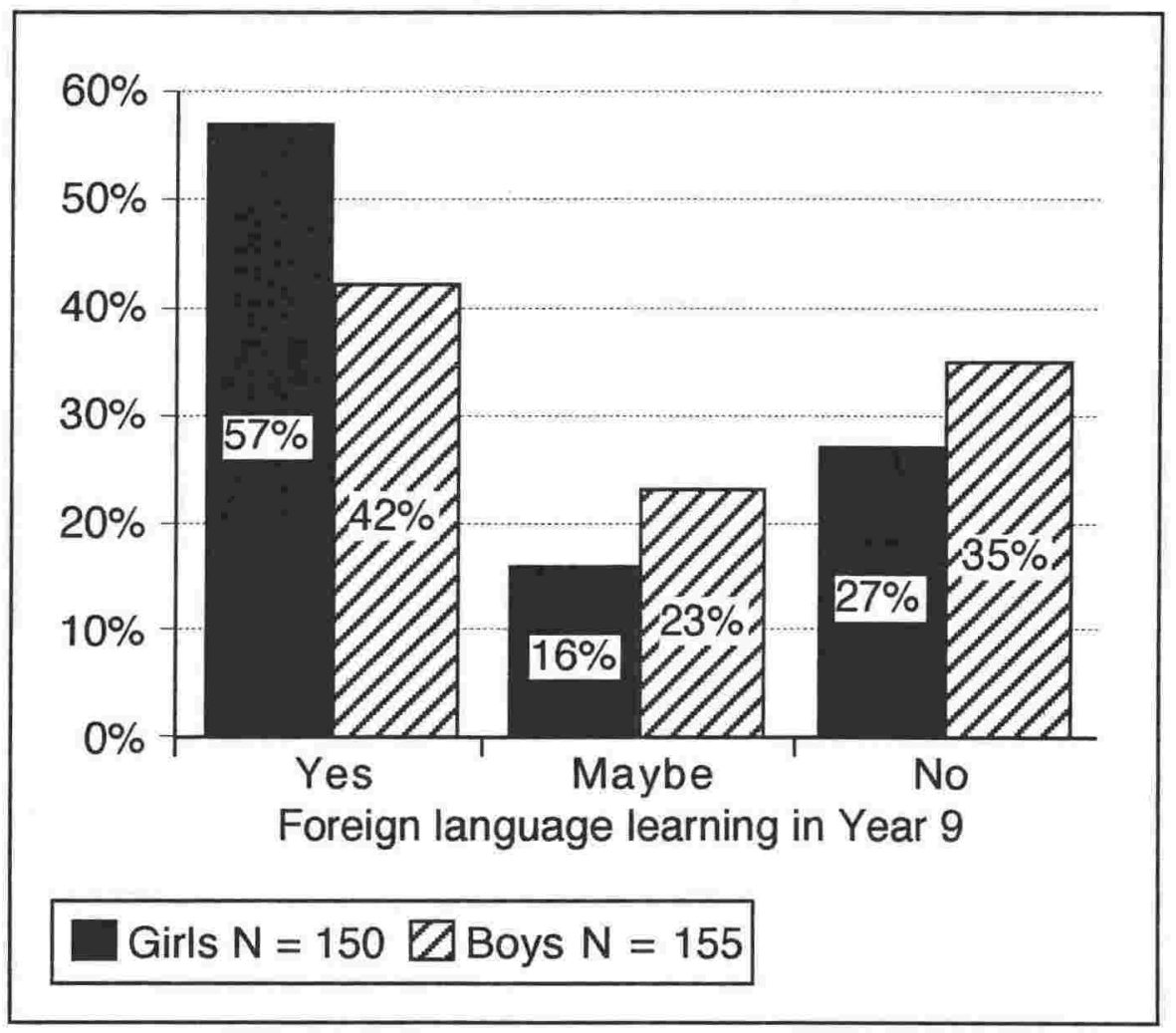

\subsubsection{Reasons for intending FLL}

Some gender differences are apparent in students' motivation for opting into FLL. Table 10.4 shows that girls were much readier to give reasons for their choices than boys. Nearly half the girls, but only a quarter of the boys, wanted to continue what they had started at intermediate school. About a quarter of the girls were interested in a particular FL, a reason cited by proportionately only half as many boys. Other reasons listed were given by similar proportions of both genders. A few students, mostly girls, mentioned the influence of parents, siblings, friends or overseas connections, or the desire for something new and challenging. Given the necessity 
of interpreting students' wording, it is possible that the reasons reported by each gender do not necessarily differ greatly but may simply reflect different ways of expressing their interest.

Table 10.3 Year 8 student intentions for FLL according to expected secondary school

\begin{tabular}{|l|c|c|c|c|c|c|}
\hline & \multicolumn{7}{|c|}{ Will study a FL in year 9 } \\
\hline $\begin{array}{l}\text { Future } \\
\text { school* }\end{array}$ & $\begin{array}{c}\text { Girls } \\
\text { N }\end{array}$ & $\begin{array}{c}\text { Girls } \\
\text { yes }\end{array}$ & $\begin{array}{c}\text { Girls } \\
\text { maybe }\end{array}$ & $\begin{array}{c}\text { Boys } \\
\text { N }\end{array}$ & $\begin{array}{c}\text { Boys } \\
\text { ye s }\end{array}$ & $\begin{array}{l}\text { Boys } \\
\text { maybe }\end{array}$ \\
\hline $\begin{array}{l}\text { Single sex } \\
\text { colleges }\end{array}$ & 24 & $67 \%$ & $21 \%$ & 34 & $50 \%$ & $32 \%$ \\
\hline & 65 & $60 \%$ & $19 \%$ & 66 & $38 \%$ & $24 \%$ \\
\hline Freesia & 41 & $44 \%$ & $7 \%$ & 41 & $39 \%$ & $10 \%$ \\
\hline
\end{tabular}

Table 10.4 Reasons for intending to study a FL in year 9

\begin{tabular}{|l|c|c|}
\hline & Number of mentions \\
\hline Comments & $\begin{array}{l}\text { Girls } \\
(\mathbf{N}=\mathbf{8 5})\end{array}$ & $\begin{array}{l}\text { Boys } \\
(\mathbf{N}=\mathbf{6 5})\end{array}$ \\
\hline $\begin{array}{l}\text { To build on prior experience, enjoyment } \\
\text { Or success in FLL }\end{array}$ & $38^{*}$ & $17 \diamond$ \\
\hline Interest in a specific FL, culture & $23 \#$ & $8 \diamond$ \\
\hline Useful for jobs & $11^{*}$ & $8 \dagger$ \\
\hline For travel & $10 \#$ & $7 \diamond$ \\
\hline FLL or a specific FL sounds 'cool', 'fun' & 7 & $7 \dagger$ \\
\hline General interest in FLs and/or cultures & 7 & $2 \dagger$ \\
\hline $\begin{array}{l}\text { * Mostly Japanese } \\
\text { \# Mostly French } \\
\dagger \\
\begin{array}{l}\text { Only Japanese mentioned } \\
\diamond \text { Japanese cited most often }\end{array}\end{array}$ \\
\hline
\end{tabular}


10.3.3 Reasons for being unsure about or not intending FLL in year 9 There was little difference between genders in the reasons given for being uncertain about FLL in year 9. Some factors, including overseas connections, influence of older sibling and usefulness for jobs were mentioned by 1 or 2 boys but none of the girls. Among the few students who gave a reason for definitely not intending to study a FL the following year there were no real gender differences.

\subsubsection{FLs which year 8 students intended to study}

Graph 10.2 shows the numbers of students intending to study a FL in year 9, according to gender and the FL chosen. The girls were more evenly divided in their choices than the boys who manifested a clear preference for Japanese. $43 \%$ of the 85 girls, but only $20 \%$ of the 65 boys, opted for a European language, French being a clear favourite among the girls, followed by Spanish, while boys were slightly more interested in German. Two thirds of the students who intended FLL but had not decided on a language were girls, most of whom were tossing up between a European language or Japanese. Among the students who reported that they might study a FL in year 9 the boys tended to report a preference for Japanese, but there were no other discernible differences.

\section{Graph 10.2 Choice of language for year 9 made by students} intending to study a FL, by gender

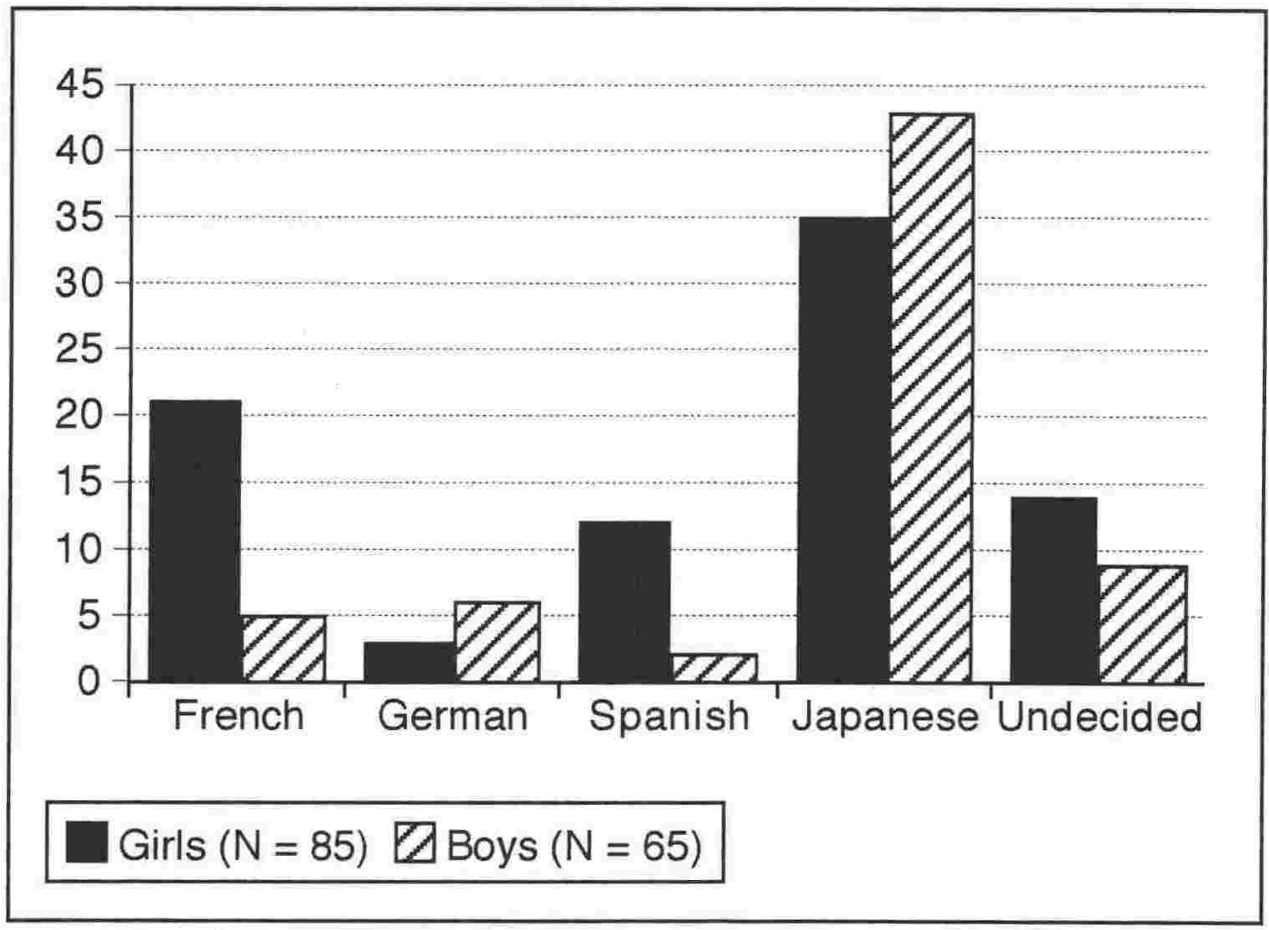


10.3.5 Intended choice of FL compared with expected year 9 school

There were few differences in the choices of FL among students expecting to attend Begonia or Gerbera. It did not appear, as has been suggested from time to time, and shown to be untrue (Phillips 1982, Powers and Batters 1985), that German is seen as a 'boys' subject' compared with French as a 'girls' subject' (Pritchard and Loulidi 1994). Japanese was the most popular FL for all students expecting to attend Freesia, but more popular for the boys. European languages were almost equally popular with the girls, but very few boys intended to study one. These differences were even more pronounced at Salvia where most students had learnt some French and Japanese. Only two of the 18 boys intending to study a FL in year 9 had chosen French, while the girls were evenly divided between the two languages. The boys seem to have reacted differently to their previous FLL experience. Either they had found Japanese particularly interesting or had taken a particular dislike to French for some reason.

\subsubsection{Intended choice of FL compared with preferred FL}

As discussed in section 5.7, students were asked what FL they would most like to learn and responses were compared with actual FLL intentions. Table 10.5 shows that a much larger proportion of girls (45\%) than boys (only 29\%) had decided to study the FL they most wanted to learn. A larger proportion of boys (34\%) than girls (29\%) had decided against FLL, even if their preferred FLL was available to them, and boys were twice as likely as girls to be unsure whether they would do any FLL in year 9.

Table 10.5 Student intentions for year 9 if preferred FL was possible at intended secondary school

\begin{tabular}{|c|c|c|c|c|}
\hline Intentions & Girls & $\%$ of $\mathbf{N}$ & Boys & $\%$ of $\mathrm{N}$ \\
\hline $\begin{array}{l}\text { Intending to study } \\
\text { preferred FL }\end{array}$ & 35 & $45 \%$ & 21 & $29 \%$ \\
\hline $\begin{array}{l}\text { Not sure which FL to } \\
\text { study } \dagger\end{array}$ & 5 & $6 \%$ & 4 & $5 \%$ \\
\hline Will study another FL* & 6 & $8 \%$ & 4 & $5 \%$ \\
\hline Not sure about FLL & 9 & $12 \%$ & 19 & $26 \%$ \\
\hline Will not study a FL\# & 23 & $29 \%$ & 25 & $34 \%$ \\
\hline $\mathrm{N}=$ & 78 & & 73 & \\
\hline \multicolumn{5}{|c|}{$\begin{array}{l}\dagger \text { Most mentioned Japanese as a possibility } \\
\text { * All had opted for Japanese } \\
\text { \# Boys were nearly twice as likely to have decided against FLL if their } \\
\text { preferred FL was a European one }\end{array}$} \\
\hline
\end{tabular}


When student intentions were compared with their interest in learning French or Japanese, the FLs available at all the local secondary schools, substantial gender differences were evident, as shown in Table 10.6. The boys interested in learning French were much more uncertain about future FLL and more likely to have decided against it than the girls. The boys' preference for Japanese is revealed once again.

Table 10.6 Intentions to study French or Japanese compared with students' ultimate interest in FLL

\begin{tabular}{|c|c|c|c|c|}
\hline GIRLS & French & $\%$ & Japanese & $\%$ \\
\hline Would most like to study & 36 & & 38 & \\
\hline Intend to study & 10 & $28 \%$ & 17 & $45 \%$ \\
\hline May study* & 8 & $22 \%$ & 8 & $21 \%$ \\
\hline Will/may study another FL $\dagger$ & 7 & $19 \%$ & 0 & $0 \%$ \\
\hline Will not study a FL & 11 & $31 \%$ & 13 & $34 \%$ \\
\hline BOYS & French & $\%$ & Japanese & $\%$ \\
\hline Would most like to study & 19 & & 37 & \\
\hline Intend to study & 2 & $11 \%$ & 18 & $49 \%$ \\
\hline May study* & 10 & $53 \%$ & 9 & $24 \%$ \\
\hline Will/may study another FL $\dagger$ & 0 & $0 \%$ & 1 & $3 \%$ \\
\hline Will not study a FL & 7 & $37 \%$ & 9 & $24 \%$ \\
\hline \multicolumn{5}{|c|}{$\begin{array}{l}\text { * Students reported that they would or might study a FL, but not } \\
\text { necessarily their preferred language } \\
\dagger \text { Students reported that they would or might study a FL but not } \\
\text { their preferred FL, or no choice was specified. }\end{array}$} \\
\hline
\end{tabular}

\subsubsection{Reasons given by year 8 students for their choice of FL}

Very few students stated why they had chosen specific FLs. All comments but one about Japanese were made by boys and focussed on its usefulness for jobs. There were obviously some misconceptions as one boy stated "it is the most spoken language in the world" and one girl declared it the most useful language available. Two boys chose Spanish because it is widely spoken. Each of the four boys who commented on their choice of German had a different reason for choosing that 
language:

"I've always wanted to go there"

"the main car manufacturers are there"

"it's a pretty rich country"

"easy to learn".

These comments suggest that few students had clear ideas of why any FLL might be interesting or valuable. In any case, their choices were limited by what was available in schools and, if they had no prior FLL, dependent on limited information gained from parents or secondary school prospectuses. Nevertheless, boys were evidently getting the message that Japanese was somehow more acceptable and desirable a subject than any other FL.

\subsection{Year 9 decisions}

\subsubsection{To study a FL or not - the gender gap narrows}

The majority of both genders had decided to study a FL but the proportion of girls was higher than that of the boys. Since the year 9 sample was not exactly the same as the year 8 one a direct comparison between intentions and final decisions cannot be made. It must also be remembered that the sample of year 9 boys was less representative than that of the girls and would have distorted all the data concerning the boys. However, as these distortions are most likely to be in favour of FLL, it might be expected that, given a more representative sample of the year 9 boys, any identified gender differences might have been greater.

This supposition was supported by the data obtained from the sub-sample of students who participated in both surveys. In year 9 the majority of this sample were attending Freesia and Salvia, where FLL was entirely optional. The sample included only four boys at Begonia and 13 girls at Gerbera. The overall pattern of their FLL intentions in year 8 were similar to those of the whole year 8 sample, although fewer students of both genders reported that they would be studying a FL in year 9 . However, the pattern of their final choices was quite different from that of the whole year 9 sample as the majority of girls had opted for FLL, whereas the majority of boys had chosen not to study a FL. These proportions are much closer to the typical pattern illustrated in Table 10.1. The two sets of data are illustrated in Graph 10.3.

The actual choices made by the year 9 students in each school, see Table 10.7, proved to be very similar to the overall patterns of FLL intentions among year 8 students shown in Table 10.3. It is interesting that only a small majority of girls at Gerbera had chosen a FL compared with the intentions of over two thirds of the Year 8 girls expecting to attend that school. This suggests that the year 8 girls may have been affected by my presence as a female teacher and have been, possibly unconsciously, trying to please me or fit what they perceived to be my expectations. A comparison of the year 8 and year 9 figures for Freesia suggest that most of the year 8 students intending to go to Freesia and who stated that they might study a FL probably did not. At Salvia it seems that, among the indecisive year 8 students, the 
girls were more likely to have finally opted for FLL than the boys. Since about $90 \%$ of the 85 year 9 Salvia students were involved in both surveys, this is probably an accurate reflection of the situation.

\section{Graph 10.3 Student decisions regarding FLL in year 9, by gender,} for whole sample and sub-sample

Notes: SS (Sub-sample) $N=169,93$ girls, 76 boys

All $\mathrm{Y} 9 \mathrm{~N}=664,393$ girls, 271 boys

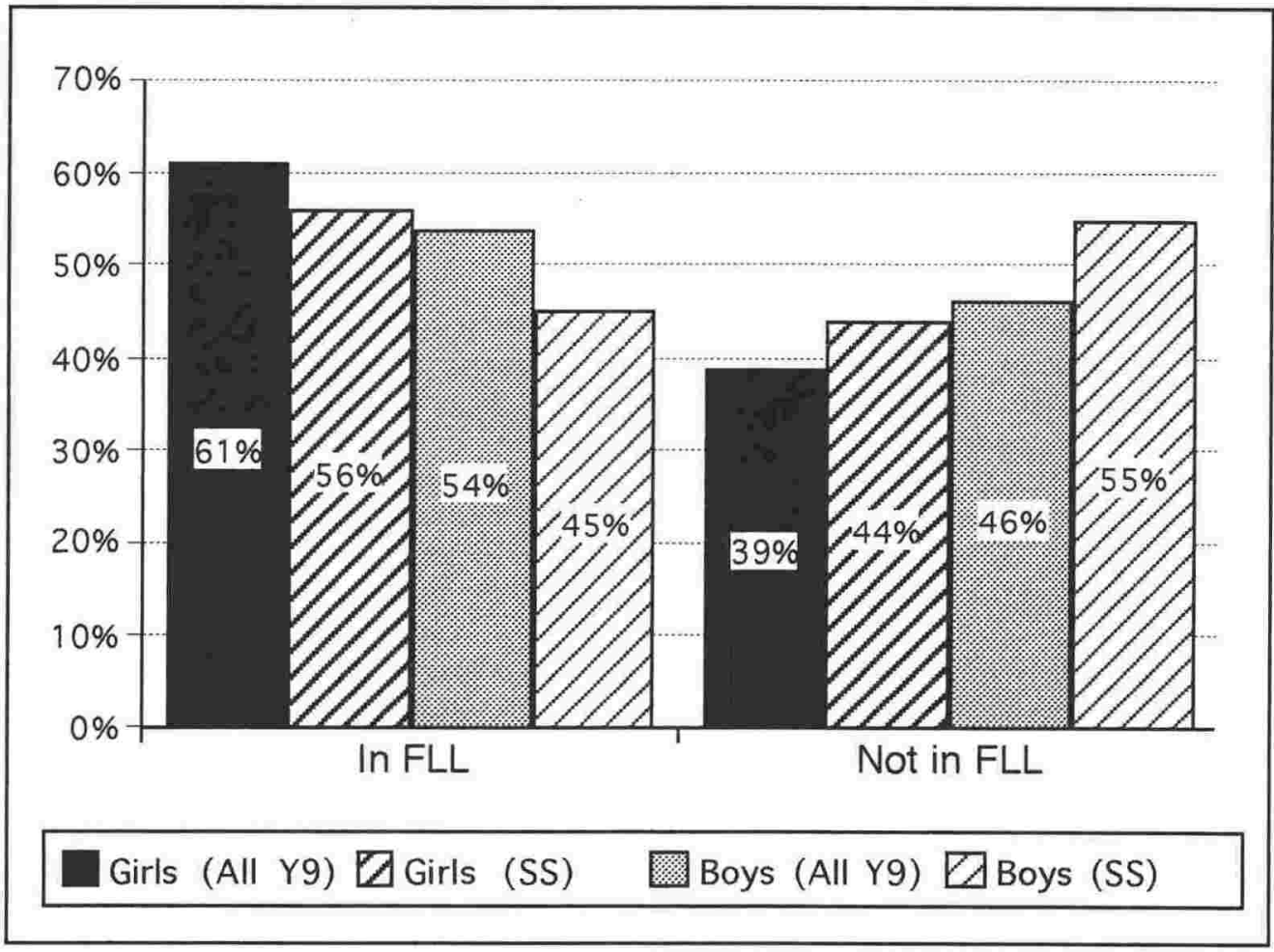

For both genders there was a significant association $(\mathrm{p}<.001)$ between the decision to study a FL or not in year 9 and the school. At the two mixed schools where FLL was entirely optional girls clearly outnumbered boys in language classes, particularly at Freesia. At Arum, where at least one term of language learning was compulsory, the proportions of girls and boys studying a FL (i.e. not Maori) were almost identical. The major surprise was that at the single sex schools a considerably higher proportion of boys than girls was involved in FLL. The proportion of girls matched approximately the usual national figure for FLL while the proportion at the boys' school was exceptionally high, which was probably not an accurate reflection of the 
school as a whole. As mentioned before, most of the boys were in the top four streams and thus under pressure to study a language. It is highly likely that, if the whole year 9 group had been surveyed, the proportion of boys involved in FLL would have been much lower, but it is impossible to speculate what that proportion might have been.

Table 10.7 Student decisions regarding FLL in year 9, by gender and school

\begin{tabular}{|c|c|c|c|c|c|}
\hline \multirow[b]{2}{*}{ School } & \multirow[b]{2}{*}{$\begin{array}{l}\text { Decile } \\
\text { rating }\end{array}$} & \multicolumn{4}{|c|}{ Students studying a FL in 2000} \\
\hline & & Girls & \begin{tabular}{|c|}
$\%$ of all \\
girls
\end{tabular} & Boys & $\begin{array}{c}\% \text { of all } \\
\text { boys }\end{array}$ \\
\hline Arum & 7 & 62 & $84 \%$ & 55 & $85 \%$ \\
\hline Freesia & 6 & 59 & $60 \%$ & 33 & $34 \%$ \\
\hline Salvia & 7 & 23 & $50 \%$ & 15 & $38 \%$ \\
\hline Single sex & G 9, B 8 & 96 & $55 \%$ & 44 & $64 \%$ \\
\hline Totals & & 240 & $61 \%$ & 147 & $54 \%$ \\
\hline
\end{tabular}

Particularly striking was the fact that, of the 28 students who reported that they were going to study two languages, 25 were girls. It appears that boys were rarely sufficiently interested in FLs to "sacrifice" other options in order to study two languages, even if they were interested in FLL.

\subsubsection{Reasons for the decisions of year 9 students 10.4.2.1 Reasons for choosing to study a FL}

Some interesting differences emerged between girls' and boys' motivation for FLL, shown in Table 10.8. For girls the most important reason, cited by nearly one third of them, and nearly three times as many as boys, was an interest in overseas travel. The boys' main reason for studying a FL was the perception that it was compulsory (although this was not so at any of the surveyed secondary schools). Thus, the majority of girls had opted into FLL mostly out of personal interest, whereas a considerable proportion of the boys felt forced into it. In addition, girls were twice as likely as boys to say that they wanted to continue a subject previously experienced. Among other reasons cited less frequently, boys were more interested in learning something new or challenging, while girls tended to mention job qualifications, other people's influence or an interest in other cultures, which might relate to their interest in travel. Gender differences with regard to the influence of prior FLL experience, and notions of the 'usefulness' and 
difficulty of FLL will be discussed below.

Table 10.8 Reasons for studying a FL in Year 9

\begin{tabular}{|l|c|c|c|c|}
\hline \multirow{2}{*}{ Comments } & \multicolumn{4}{|c|}{ Number of Mentions } \\
\cline { 2 - 5 } & Girls & $\begin{array}{c}\text { \% of } \\
\mathbf{2 4 1}\end{array}$ & B o y s & $\begin{array}{c}\text { \% of } \\
\mathbf{1 4 9}\end{array}$ \\
\hline For travel & 74 & $31 \%$ & 16 & $11 \%$ \\
\hline $\begin{array}{l}\text { General interest/just } \\
\text { wanted to }\end{array}$ & 50 & $21 \%$ & 29 & $19 \%$ \\
\hline Compulsory & 23 & $10 \%$ & 45 & $30 \%$ \\
\hline $\begin{array}{l}\text { General enjoyment/fun } \\
\text { To build on previous }\end{array}$ & 30 & $12 \%$ & 16 & $11 \%$ \\
\hline learning/enjoyment & 24 & $10 \%$ & 8 & $5 \%$ \\
\hline
\end{tabular}

\subsubsection{Gender perceptions of compulsion.}

As shown in Table 10.8, the perception that FL was compulsory affected year 9 boys three times as much as girls. This difference could be explained, at least partly, by the particular student sample at Begonia, already discussed. It is also possible that at Arum, where all students were obliged to study a LOTE for at least one term, the boys resented this more than the girls. They might not have distinguished between learning Maori and a FL, or they might have been particularly hostile to the idea of learning Maori and viewed FLL as a lesser evil.

The influence of perceived compulsion was further explored by survey item 9.3.13, "I'm only doing a foreign language because my school makes it compulsory". Responses to this were analysed by school since it was only relevant to students at Arum and Begonia. Graph 10.4 shows that the girls were less influenced by school pressure to study a FL than the boys. However, there was an even greater difference between the responses of boys at the two schools. The most likely explanation for this would seem to be the fact that the Arum boys were from mixed ability classes in a decile 7 school whereas the Begonia boys were in top streams at a decile 8 school, and therefore more likely to come from educated families with an appreciation of the value of FLL. However, of the 46 boys surveyed at Begonia who were in the top four streams, and therefore obliged to study music or a language, one third agreed that they were only in FLL because it was 
compulsory. This shows a considerable reluctance on the part of boys to study a FL, either because it was at the expense of preferred options or because they disliked FLL or the idea of it. It is also possible that the Begonia boys resented the degree of compulsion when they could see boys in lower streams having a freer choice of subjects.

\section{Graph 10.4 Responses to item 9.3.13: "I'm only doing a foreign language because my school makes it compulsory", by school and gender}

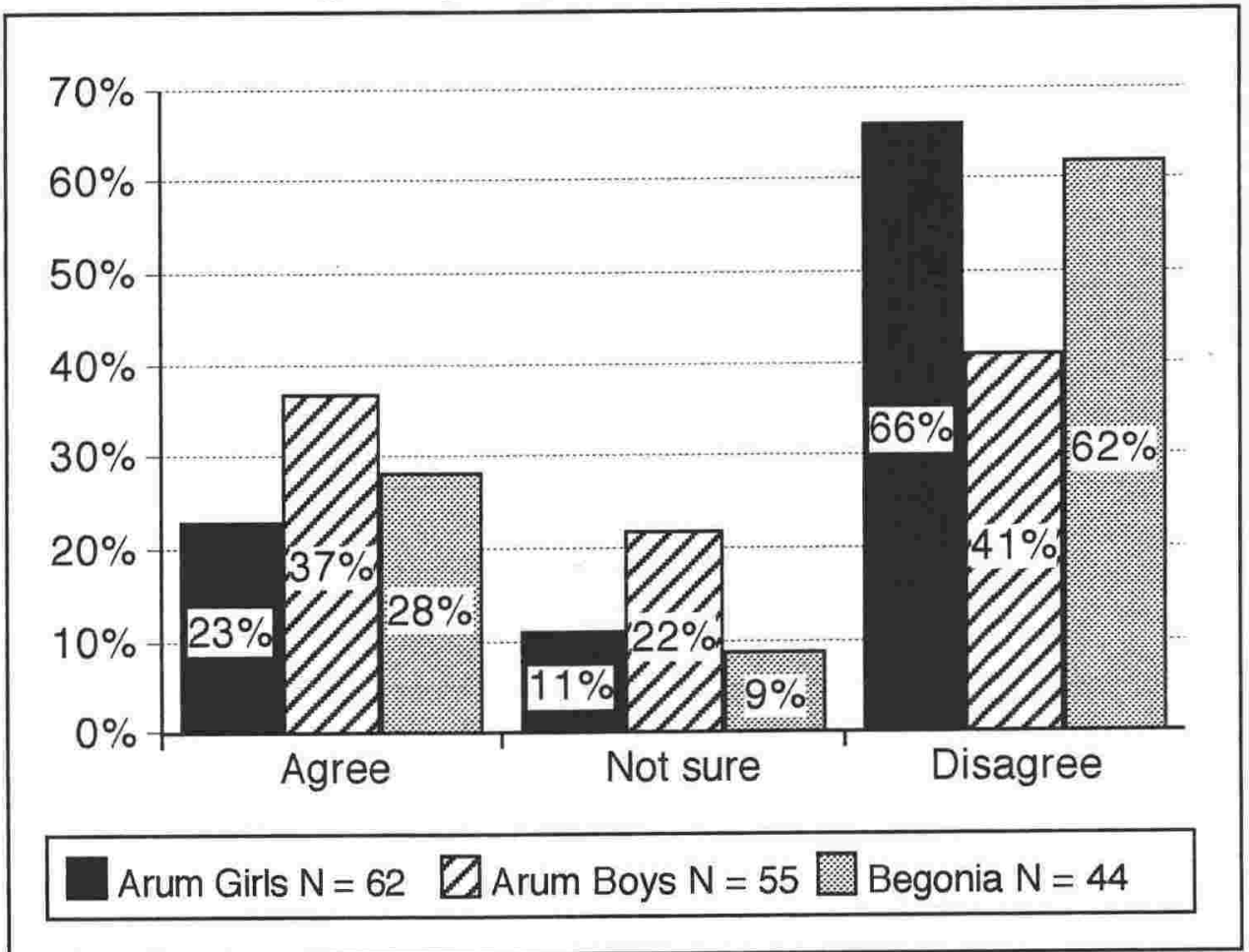

\subsubsection{Reasons for choosing not to study a FL}

Boys were more than twice as likely as the girls to cite lack of interest as a reason for not opting into FLL, 43\% $(\mathrm{N}=122)$, see Table 10.9. On the other hand, $37 \%$ of the girls $(\mathrm{N}=152)$, compared with only $17 \%$ of the boys, reported that they had preferred another option, often stating or implying that they would like to have studied a FL but it could not be fitted into their timetable. Nearly half of these girls but only about one third of the boys specified that Maori was their preferred option. Thus, even among students who did not opt into FLL, it appears that girls generally had a greater 
interest in LOTEs and other cultures. A handful of girls reported that they had already done enough FLL or needed extra English, neither of which reasons were mentioned by any boys. The only other apparent difference, though still involving small numbers of students, was that the girls seem to have been more influenced by their parents than the boys.

Table 10.9 Reasons for not studying a FL in Year 9

\begin{tabular}{|l|c|c|c|c|}
\hline \multirow{2}{*}{ Comments } & \multicolumn{4}{|c|}{ Number of Mentions } \\
\cline { 2 - 5 } & Girls & $\begin{array}{r}\text { \% of } \\
\mathbf{1 5 2}\end{array}$ & Boy s & $\begin{array}{r}\text { \% of } \\
\mathbf{1 2 2}\end{array}$ \\
\hline $\begin{array}{l}\text { Did not want to/not } \\
\text { interested/would be boring }\end{array}$ & 31 & $20 \%$ & 52 & $43 \%$ \\
\hline $\begin{array}{l}\text { Preferred another option } \\
\text { or no time for FL }\end{array}$ & 31 & $20 \%$ & 15 & $12 \%$ \\
\hline Preferred Maori & 26 & $17 \%$ & 6 & $5 \%$ \\
\hline No need/no use for jobs & 17 & $11 \%$ & 11 & $9 \%$ \\
\hline $\begin{array}{l}\text { Did not enjoy previous } \\
\text { experience }\end{array}$ & 10 & $7 \%$ & 8 & $7 \%$ \\
\hline Did not know & 8 & $5 \%$ & 10 & $8 \%$ \\
\hline Too difficult & 8 & $5 \%$ & 9 & $7 \%$ \\
\hline Parental influence & 8 & $5 \%$ & 1 & $1 \%$ \\
\hline
\end{tabular}

The apparent relief at not having to learn a FL was significantly greater ( $\mathrm{p}<.005$ ) among boys, see Table 10.10 , providing more evidence that hostility to FL subjects was a greater factor in boys' choices than in the decisions made by girls. Some students might have chosen to study a FL if they had not been faced with more attractive, competing options. Although there was no significant association between gender and responses to the statement, "I wanted to learn a foreign language but languages clashed with more important subjects" item 9.4.16, slightly more girls than boys agreed. When these responses were analysed by school some striking differences emerged at Salvia and between the single sex schools, see Table 10.11. 
Although only small numbers of students were involved, it is apparent that most of the Salvia girls regretted not being able to study a FL, while the reverse was true for the Salvia boys. By contrast, at the single sex schools, a higher proportion of boys than girls felt excluded. It would be interesting to know what proportion of boys in the lower streams not covered by the survey, who had little opportunity to learn a FL, might have felt disappointed.

Table 10.10 Responses by gender to survey item 9.4.9: "I'm glad I don't have to learn a foreign language this year."

\begin{tabular}{|c|c|c|}
\hline Item response & $\begin{array}{c}\text { Proportion } \\
\text { of girls }\end{array}$ & $\begin{array}{c}\text { Proportion } \\
\text { of boys }\end{array}$ \\
\hline Agree & $32 \%$ & $50 \%$ \\
\hline Disagree & $44 \%$ & $24 \%$ \\
\hline $\mathrm{N}$ & 144 & 113 \\
\hline
\end{tabular}

Table 10.11 Responses by gender to survey item 9.4.13: "I wanted to learn a foreign language but languages clashed with more important subjects", at Salvia and single sex schools.

\begin{tabular}{|l|c|c|c|c|}
\hline $\begin{array}{l}\text { Item } \\
\text { response }\end{array}$ & $\begin{array}{l}\text { Girls at } \\
\text { Salvia }\end{array}$ & $\begin{array}{l}\text { Boys at } \\
\text { Salvia }\end{array}$ & $\begin{array}{l}\text { Boys at } \\
\text { Begonia }\end{array}$ & $\begin{array}{l}\text { Girls at } \\
\text { Gerbera }\end{array}$ \\
\hline Agreed & 12 & 3 & 8 & 17 \\
\hline Not sure & 1 & 6 & 3 & 22 \\
\hline Disagree & 7 & 10 & 12 & 33 \\
\hline Totals & 20 & 19 & 23 & 72 \\
\hline
\end{tabular}

\subsubsection{FL choices made by year 9 students}

When the year 9 students' final choices of FL were analysed the apparent preference of boys for Japanese all but disappeared. Table 10.12 shows that the proportions of girls and boys in Japanese were identical but that French was more popular among the girls. German proved slightly more popular with the boys and Spanish with the girls. A major gender difference lay in the numbers opting to try two languages, the great majority ( 25 out of 28 ) being girls. 14 girls but only 2 boys were trying both 
French and Japanese at Arum. One other boy was studying the same two languages at Begonia. Another 11 girls were studying various combinations of languages at the other schools, including one girl at Gerbera who was being permitted to study Spanish by correspondence as well as attend French classes. This does not necessarily indicate that boys were not interested in learning more than one language. It could mean that the really keen linguists among the boys met greater barriers than the girls. On the other hand, other findings suggest that the boys usually lacked sufficient interest in FLs to "sacrifice" other options in order to study two of them.

Table 10.12 Proportions of students in each language by gender ( $\mathrm{N}=$ number of students in FLL)

\begin{tabular}{|l|c|c|c|c|c|}
\hline Gender & N & French & German & Spanish* & Japanese \\
\hline Girls & 240 & $54 \%$ & $4 \%$ & $9 \%$ & $43 \%$ \\
\hline Boys & 147 & $48 \%$ & $7 \% \dagger$ & $4 \%$ & $43 \%$ \\
\hline
\end{tabular}

$\uparrow$ Includes one boy on correspondence

* Two students were studying by correspondence

Totals $=$ more than $100 \%$ as some students were studying more than one FL

As French and Japanese were taught at all the schools I thought it useful to examine the gender balance in these two languages by school. Table 10.13 shows that the pattern of choices varied from school to school which is hard to interpret, but may be explained by socioeconomic status, ethnicity or by students' previous experience of FLL.

Table 10.13 Numbers of students studying French and/or Japanese by school and gender

Notes: 1. Percentages are proportions of students in FLL.

2. Percentages add up to more than $100 \%$ where students are studying both FLs.

\begin{tabular}{|l|c|c|c|c|c|c|c|c|}
\hline \multirow{2}{*}{} & \multicolumn{4}{|c|}{ French } & \multicolumn{4}{c|}{ Japanese } \\
\cline { 2 - 10 } & Girls & \multicolumn{3}{|c|}{ Boys } & \multicolumn{3}{l|}{ Girls } & \multicolumn{2}{c|}{ Boys } \\
\hline School & N & $\%$ & N & $\%$ & N & $\%$ & N & $\%$ \\
\hline Arum & 40 & $65 \%$ & 39 & $72 \%$ & 36 & $58 \%$ & 18 & $33 \%$ \\
\hline Freesia & 19 & $32 \%$ & 7 & $21 \%$ & 24 & $41 \%$ & 21 & $62 \%$ \\
\hline Salvia & 13 & $57 \%$ & 2 & $13 \%$ & 12 & $52 \%$ & 13 & $87 \%$ \\
\hline Single sex & 58 & $60 \%$ & 23 & $52 \%$ & 32 & $33 \%$ & 11 & $25 \%$ \\
\hline Totals & 130 & $54 \%$ & 71 & $48 \%$ & 104 & $43 \%$ & 63 & $43 \%$ \\
\hline
\end{tabular}


Among those at the higher decile single-sex schools, with the highest proportions of Pakeha students, both girls (decile 9, 175 students, 51\% with previous FLL) and boys (decile 8,69 students, $23 \%$ with previous FLL) seemed to prefer the traditional FL, French, by a ratio of about 2:1. At both Arum (decile 7, 139 students, 70\% Pakeha, $45 \%$ with previous FLL), and at Salvia (decile 7, 85 students, $76 \%$ Pakeha, $85 \%$ with previous FLL), girls were fairly evenly divided between French and Japanese but Japanese was the clear favourite with the boys at Salvia while French was much more popular among the boys at Arum. On the other hand, at Freesia (decile 6, 196 students, 81\% Pakeha, 52\% with previous FLL), Japanese was the first choice of most girls (41\%) and boys (62\%). One evident factor is that the majority of students at Salvia had prior experience of FLL, mostly of both French and Japanese, and so it could be assumed that this experience had influenced their choices.

A major gender imbalance showed up when the proportions of girls and boys studying each language across all the schools was analysed, see Table 10.14. In all the languages except German, where the gender balance was even, girls substantially outnumbered the boys. In Spanish at Freesia the ratio of girls to boys, $4: 1$, was the highest.

When the students were asked if they would rather have learnt another language, one third of the girls reported that they would like to have learnt Japanese and a similar proportion of the boys would like to have studied German. As previously discussed, the data provides some evidence of boys' particular interest in German, but it might not have been available to all the interested students. One could speculate that the girls' reported interest in Japanese might indicate that even more girls would have studied two languages had they been able to accommodate them in their timetable.

Table 10.14 Proportions of girls and boys studying each FL

\begin{tabular}{|l|c|c|c|}
\hline Language & $\mathbf{N}$ & $\%$ of girls & $\%$ of boys \\
\hline French & 201 & $65 \%$ & $35 \%$ \\
\hline German & 20 & $50 \%$ & $50 \%$ \\
\hline Spanish & 28 & $79 \%$ & $21 \%$ \\
\hline Japanese & 167 & $62 \%$ & $38 \%$ \\
\hline Totals & 390 & $62 \%$ & $38 \%$ \\
\hline
\end{tabular}




\subsubsection{Reasons given by year 9 students for their choice of FL}

Similar reasons were given by most girls and boys for choosing a FL option, see Table 10.15, but with different emphases. The reason cited most of ten, wanting to continue a language started previously, was provided by a larger proportion, 53\%, of the girls $(\mathrm{N}=241)$ than the boys, $38 \%(\mathrm{~N}=149)$. Nearly one quarter of the girls, but only $11 \%$ of the boys, expressed an interest in visiting a particular country, which ties in with the girls' clear association of FLL with travel, as discussed above. More boys, $12 \%$, attributed their decisions to adult influence, compared with only $7 \%$ of the girls, possibly because of the strong perceptions of many of them that FLL was compulsory. Small numbers reported that FLL would be vaguely 'useful' but only 4 girls and no boys mentioned the idea that the FL they had chosen might be useful for a future job.

Table 10.15 Positive reasons for choice of language(s)

\begin{tabular}{|l|c|c|c|c||}
\hline & \multicolumn{4}{|c||}{ Number of Mentions } \\
\hline Comments & Girls & $\begin{array}{r}\text { \% of } \\
\mathbf{2 4 1}\end{array}$ & B o y s & $\begin{array}{c}\text { \% of } \\
\mathbf{1 4 9}\end{array}$ \\
\hline $\begin{array}{l}\text { Desire to build on previous } \\
\text { FLL }\end{array}$ & 127 & $53 \%$ & 56 & $38 \%$ \\
\hline $\begin{array}{l}\text { Desire to visit/interest in } \\
\text { particular country }\end{array}$ & 56 & $23 \%$ & 17 & $11 \%$ \\
\hline $\begin{array}{l}\text { Influence/pressure from } \\
\text { parents or other adults }\end{array}$ & 17 & $7 \%$ & 18 & $12 \%$ \\
\hline General usefulness & 22 & $9 \%$ & 10 & $7 \%$ \\
\hline
\end{tabular}

The boys (12\%) were noticeably more likely than the girls (2\%) to report negative reasons for choosing a FL option, such as a preference for what seemed the easiest or the only acceptable option available. It appears that girls generally felt more independent than boys in choosing a FL option and made their choice overwhelmingly out of personal interest.

\subsection{General attitudes to FLL experience}

\subsubsection{Girls enjoy FLL more than boys}

In accordance with much of the literature (for example, Burstall 1979, Powell \& Batters 1985, Aplin 1991) and my personal experience I hypothesised that girls enjoy FLL more than boys. Table 10.16 shows the differing reactions of year 8 girls and boys to three survey items indicating their general attitudes to the FLL they had experienced, and where the gender differences were significant. Equal numbers of girls and boys, 125 of each, had learnt a FL in year 7 or 8 , but overall, the girls showed more positive attitudes than the boys. 
Nearly twice as many boys as girls indicated that they did not want to learn more of the same language, a significant difference $(\mathrm{p}<.03)$. When asked to respond to the prospect of trying a different language the following year, the largest proportion of girls ( $40 \%, \mathrm{~N}=118$ ) was in favour whereas the largest proportion of boys $(37 \%, \mathrm{~N}$ $=123$ ) was against, revealing again a more positive attitude among the girls than the boys.

\section{Table 10.16 Proportions of year 8 students reporting agreement or disagreement with survey items indicating reactions to}

FLL, by gender

\begin{tabular}{|c|c|c|c|c|c|c|c|}
\hline \multirow[b]{2}{*}{ Item } & \multirow[b]{2}{*}{ Statement } & \multirow[b]{2}{*}{$\mathbf{N}$} & \multicolumn{2}{|c|}{ Agree } & \multicolumn{2}{|c|}{ Disagree } & \multirow{2}{*}{$\begin{array}{c}\text { Gender } \\
\text { sig.* }\end{array}$} \\
\hline & & & Girls & Boys & Girls & Boys & \\
\hline 8.2 .9 & $\begin{array}{l}\text { Our language } \\
\text { teacher made } \\
\text { learning really fun. }\end{array}$ & $\begin{array}{c}\text { Girls } \\
117 \\
\text { Boys } \\
119 \\
\end{array}$ & $50 \%$ & $49 \%$ & $20 \%$ & $33 \%$ & $\mathrm{p}<.04$ \\
\hline 8.2 .3 & $\begin{array}{l}\text { I don't want to } \\
\text { learn more of the } \\
\text { same language. }\end{array}$ & $\begin{array}{c}\text { Girls } \\
118 \\
\text { Boys } \\
118 \\
\end{array}$ & $16 \%$ & $31 \%$ & $49 \%$ & $41 \%$ & $\mathrm{p}<.03$ \\
\hline 8.2 .8 & $\begin{array}{l}\text { I enjoyed language } \\
\text { learning so much } \\
\text { I'd like to try } \\
\text { another language. }\end{array}$ & $\begin{array}{c}\text { Girls } \\
118 \\
\text { Boys } \\
123 \\
\end{array}$ & $40 \%$ & $32 \%$ & $25 \%$ & $37 \%$ & None \\
\hline
\end{tabular}

In year $974 \%$ of 215 girls but only $66 \%$ of 113 boys reported that they had enjoyed learning a FL at intermediate school (item 9.2.2). When asked for reasons for these response, considerably larger proportions of girls reported that they had found FLL interesting and enjoyed learning about other cultures, see Table 10.17. It is impossible to infer whether this is attributable to an inherent lack of interest among this sample of boys or to a failure of teachers to make the FL classes sufficiently stimulating for the boys.

Among students who had decided to opt into FLL in year 9, there was a significant difference $(p<.005)$ between genders in reactions to item 9.3.2, "It's fun to learn another language". $86 \%$ of 239 girls, compared with $71 \%$ of 146 boys, agreed or strongly agreed with the item, see Table 10.18. The boys were four times as likely to disagree, possibly because they, more than the girls, felt forced to study a FL. The boys were also almost twice as likely to be unsure which may be the result of 
lack of experience, since the majority of the $146,58 \%$, had had no prior FLL, whereas the majority of the 239 girls, $58 \%$, had had FLL experience.

Table 10.17 Reasons for enjoying or not enjoying FLL at intermediate level, by gender

\begin{tabular}{|l|c|c|c|c|}
\hline \multirow{2}{*}{ Reasons } & \multicolumn{4}{|c|}{ Number of mentions } \\
\hline For enjoying & Girls & $\%$ of $\mathbf{2 2 7}$ & Boys & \% of 113 \\
\hline Interesting & 31 & $14 \%$ & 5 & $4 \%$ \\
\hline Enjoyed learning about culture & 20 & $9 \%$ & 2 & $2 \%$ \\
\hline For not enjoying & & & & \\
\hline Boring, difficult & 38 & $17 \%$ & 21 & $19 \%$ \\
\hline
\end{tabular}

Table 10.18 Responses by gender to survey item 9.3.2: "It's fun to learn another language."

\begin{tabular}{|c|c|c|}
\hline Item response & $\begin{array}{c}\text { Proportion } \\
\text { of girls }\end{array}$ & $\begin{array}{c}\text { Proportion } \\
\text { of boys }\end{array}$ \\
\hline Agree & $86 \%$ & $71 \%$ \\
\hline Not sure & $11 \%$ & $20 \%$ \\
\hline Disagree & $2 \%$ & $9 \%$ \\
\hline $\mathrm{N}$ & 239 & 146 \\
\hline
\end{tabular}

Even among students who had not opted into FLL (153 girls and 124 boys), girls showed more positive attitudes than boys. Asked if they would like to have studied a FL the proportion of girls who responded positively, $41 \%$, was significantly higher $(\mathrm{p}<.02)$ than that of boys, $24 \%$, as shown in Table 10.19. 
Table 10.19 Responses to the question: "Would you like to have studied a foreign language?"

\begin{tabular}{|c|c|c|}
\hline Item response & $\begin{array}{c}\text { Proportion } \\
\text { of girls }\end{array}$ & $\begin{array}{c}\text { Proportion } \\
\text { of boys }\end{array}$ \\
\hline Yes & $41 \%$ & $24 \%$ \\
\hline No & $54 \%$ & $69 \%$ \\
\hline $\mathrm{N}$ & 153 & 124 \\
\hline
\end{tabular}

When asked why they would like to have studied a FL, the majority of both genders expressed interest in a particular language or culture, but, surprisingly, the proportion of boys was considerably higher than that of the girls, see Table 10.20. This might result from the fact that fewer of the boys had had the chance to try a FL, only $43 \%(\mathrm{~N}=124)$, compared with $60 \%$ of the girls $(\mathrm{N}=153)$. It suggests that they would have liked the opportunity but that they, more than the girls, had been put off opting for FLL, perhaps for lack of suitable opportunities or encouragement. Once again, the girls mentioned travel more often than the boys.

Table 10.20 Reasons why Year 9 students not in FLL would like to have learnt a FL

\begin{tabular}{|l|c|c|}
\hline Comments & $\begin{array}{c}\text { \% of Girls } \\
(\mathbf{N}=\mathbf{6 3})\end{array}$ & $\begin{array}{c}\% \text { of Boys } \\
(\mathbf{N}=\mathbf{3 0})\end{array}$ \\
\hline $\begin{array}{l}\text { Interest in a particular language } \\
\text { and/or culture }\end{array}$ & $41 \%$ & $60 \%$ \\
\hline $\begin{array}{l}\text { To build on previous } \\
\text { experience/enjoyment }\end{array}$ & $21 \%$ & $23 \%$ \\
\hline For travel & $14 \%$ & $10 \%$ \\
\hline
\end{tabular}

The same students were asked to respond to items suggesting that they would enjoy FLL, with significant gender differences $(p<.001$ or $p<.002)$ in both sets of responses, see Table 10.21. Girls were nearly twice as likely as boys to think that FLL would be fun. When it was suggested that they might like to learn a FL one day, three times more boys than girls disagreed. However, the substantial proportions of boys responding positively to the two items reinforce the notion that more boys would have opted for FLL given more opportunity or encouragement. Asked if they would learn a FL one day, the majority of students were uncertain, but nearly three times as many girls as boys indicated that they would, a significant gender difference $(p<.01)$. 
Table 10.21 Proportions of year 9 students not in FLL reporting agreement or disagreement with survey items indicating attitudes to FLL, by gender

\begin{tabular}{|c|c|c|c|c|c|c|c|}
\hline \multirow[b]{2}{*}{ Item } & \multirow[b]{2}{*}{ Statement } & \multirow[b]{2}{*}{$\mathbf{N}$} & \multicolumn{2}{|c|}{ Agree } & \multicolumn{2}{|c|}{ Disagree } & \multirow{2}{*}{$\begin{array}{c}\text { Gender } \\
\text { sig.* }\end{array}$} \\
\hline & & & Girls & Boys & Girls & Boys & \\
\hline 9.4 .15 & $\begin{array}{l}\text { Learning a } \\
\text { foreign language } \\
\text { would be fun. }\end{array}$ & $\begin{array}{c}\text { Girls } \\
145 \\
\text { Boys } \\
138 \\
\end{array}$ & $60 \%$ & $33 \%$ & $14 \%$ & $34 \%$ & $\mathrm{p}<.001$ \\
\hline 9.4 .10 & $\begin{array}{l}\text { I'd like to learn a } \\
\text { foreign language } \\
\text { one day. }\end{array}$ & $\begin{array}{c}\text { Girls } \\
147 \\
\text { Boys } \\
121 \\
\end{array}$ & $56 \%$ & $49 \%$ & $9 \%$ & $26 \%$ & $\mathrm{p}<.002$ \\
\hline
\end{tabular}

Gender differences also showed up in response to item 9.3.14, "I wish that foreign language options in year 9 were for the whole year" at the secondary schools where FL courses were for less than a whole year. The girls at Salvia (13 out of 23, 56\%) were much keener than the boys ( 5 out of $15,33 \%$ ) to have whole year options As most of the students had already had "taster" FL modules, this adds weight to the surmise that boys had enjoyed the experience less than the girls. At Arum, 27 out of 60 girls $(45 \%)$, compared with only 16 out of 53 boys (30\%), wished that FL options were for the whole year, which may reflect the fact over half the girls, but only $35 \%$ of the boys had had some previous FLL, as well as different reactions to such experience. On the other hand, the gender differences in the attitudes at these two schools may be more evidence of a greater interest in FLL among the girls.

The above arguments are contradicted by the responses at Freesia where a larger proportion of boys, (16 out of $31,52 \%$, than girls, (17 out of $56,30 \%$ ), wanted longer options, although only $44 \%$ of the boys had any previous experience compared with $59 \%$ of the girls. However, the Freesia boys in FLL who were also keen on whole year options, represented only $18 \%$ of the 98 year 9 boys at that school, and may have been simply the dedicated few. There was probably a complex combination of factors involved in all the student responses, but the overall picture remains of greater enthusiasm for FLL among the girls than the boys.

\subsubsection{Girls are less monocultural in outlook than boys}

The literature suggests that girls tend to be more interested in overseas travel, meeting speakers of other languages and exploring other cultures (Powell \& Batters 1985, Aplin 1991). Among year 8 students there were no significant gender differences in attitudes to FL speakers and their cultures, but there were indications that the girls 
were more open to learning more about the culture of people whose languages they were learning, see Table 10.22. Their positive responses may, of course, simply reflect greater enjoyment of FLL among the girls rather than inherently less monocultural attitudes.

Table 10.22 Responses by gender to survey item 8.2.7: "I'd like to know more about the life and customs of the people who speak the language."

\begin{tabular}{|c|c|c|}
\hline Item response & $\begin{array}{c}\text { Proportion of } \\
\text { girls }\end{array}$ & $\begin{array}{c}\text { Proportion of } \\
\text { boys }\end{array}$ \\
\hline Agree & $57 \%$ & $43 \%$ \\
\hline Disagree & $20 \%$ & $24 \%$ \\
\hline $\mathrm{N}$ & 118 & 120 \\
\hline
\end{tabular}

Among Year 9 students in FL classes (240 girls and 147 boys) there were striking gender differences in the responses to the two items designed to investigate attitudes towards speakers of the languages they were studying and their cultures, see Table 10.23. The girls were significantly more positive $(p<.05)$ in responses to the idea of knowing more about the life of speakers of the languages they were learning and the

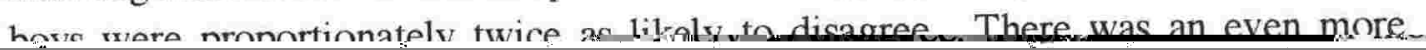


More girls than boys responded positively, which again suggests the association made by girls between FLL and travel, and over twice as many boys as girls disagreed. Among students not studying a FL in year 9 (153 girls and 124 boys) the attitude of the girls to the possibility of meeting speakers of other languages was significantly more positive $(p<.001)$ than that of the boys, also shown in Table 10.23. Overall, these items support the idea that girls are more interested than boys in people of other cultures.

\subsubsection{Boys find FLL more difficult than girls}

As has been found in Australia (Zammit 1992, Carr 1997) FLL was perceived as difficult by more boys than girls. Of the whole year 8 sample, significantly more boys than girls $(\mathrm{p}<.03)$ agreed that knowing more than one language was confusing and significantly more boys than girls $(\mathrm{p}<.02)$ disagreed that they would find FLL easy the following year, see Table 10.24 .

Table 10.24 Proportions of year 8 students reporting agreement or disagreement with survey items indicating perceptions of difficulty in FLL, by gender

\begin{tabular}{|c|c|c|c|c|c|c|c|}
\hline \multirow[b]{2}{*}{ Item } & \multirow[b]{2}{*}{ Statement } & \multirow[b]{2}{*}{$\mathbf{N}$} & \multicolumn{2}{|c|}{ Agree } & \multicolumn{2}{|c|}{ Disagree } & \multirow{2}{*}{$\begin{array}{c}\text { Gender } \\
\text { sig.* }\end{array}$} \\
\hline & & & Girls & Boys & Girls & Boys & \\
\hline 8.2 .1 & $\begin{array}{l}\text { Learning another } \\
\text { language was } \\
\text { easier than I } \\
\text { expected. }\end{array}$ & $\begin{array}{c}\text { Girls } \\
120 \\
\text { Boys } \\
117\end{array}$ & $57 \%$ & $37 \%$ & $24 \%$ & $38 \%$ & $\mathrm{p}<.01$ \\
\hline 8.2 .2 & $\begin{array}{l}\text { Trying to write it } \\
\text { was really } \\
\text { difficult. }\end{array}$ & $\begin{array}{c}\text { Girls } \\
119 \\
\text { Boys } \\
119\end{array}$ & $42 \%$ & $55 \%$ & $44 \%$ & $29 \%$ & None \\
\hline 8.2 .11 & $\begin{array}{l}\text { Reading the } \\
\text { language was too } \\
\text { hard. }\end{array}$ & $\begin{array}{c}\text { Girls } \\
119 \\
\text { Boys } \\
118 \\
\end{array}$ & $32 \%$ & $35 \%$ & $46 \%$ & $39 \%$ & None \\
\hline 8.2 .10 & $\begin{array}{l}\text { Now I've learnt } \\
\text { some of one } \\
\text { language it'll be } \\
\text { easier for me to } \\
\text { learn another. }\end{array}$ & $\begin{array}{c}\text { Girls } \\
118 \\
\text { Boys } \\
120\end{array}$ & $34 \%$ & $24 \%$ & $33 \%$ & $37 \%$ & None \\
\hline 8.1 .8 & $\begin{array}{l}\text { Knowing more } \\
\text { than one language } \\
\text { is confusing. }\end{array}$ & $\begin{array}{c}\text { Girls } \\
150 \\
\text { Boys } \\
154\end{array}$ & $27 \%$ & $42 \%$ & $56 \%$ & $42 \%$ & $\mathrm{p}<.03$ \\
\hline 8.1 .6 & $\begin{array}{l}\text { I think I'd find } \\
\text { learning a } \\
\text { language easy } \\
\text { next year. }\end{array}$ & $\begin{array}{c}\text { Girls } \\
151 \\
\text { Boys } \\
155\end{array}$ & $20 \%$ & $21 \%$ & $28 \%$ & $43 \%$ & $\mathrm{p}<.02$ \\
\hline
\end{tabular}


The particularly high degree of uncertainty among the girls might signify a reluctance to boast about their confidence or ability or to admit that FLL might be difficult for them, as well as a genuine uncertainty about their aptitude for FLL. The boys, on the other hand, seemed more convinced that they would find FLL difficult.

Table 10.24 also shows that the year 8 girls had notably more positive reactions than the boys to their experience of FLL. Significantly more girls than boys $(p<.01)$ found it easier than they expected. It is possible that the girls had worked harder and therefore experienced more success than the boys. On the other hand, the boys may have underestimated the amount of effort required to succeed or have been overconfident to begin with. Alternatively, the teaching styles and resources may have suited the girls better than the boys. In terms of particular aspects of FLL, more boys than girls agreed that reading the FL was too hard and that trying to write the FL was "really difficult". Noticeably more girls than boys believed that prior learning would make future FLL easier.

Items designed to see if students thought that FLL was only for the brightest students drew no significant gender responses from year 8 students but among the total year 9 sample boys were twice as likely as girls to agree that FLL is too hard for most students, see Table 10.25. It is not possible, however, to distinguish whether boys see FLL as particularly difficult because of their individual lack of confidence or because it is a belief widely held by their community, a belief upheld by the boys' school.

Table 10.25 Responses by gender to survey item 9.2.12: "Learning a foreign language is too hard for most students."

\begin{tabular}{|c|c|c|}
\hline Item response & $\begin{array}{c}\text { Proportion of } \\
\text { girls }\end{array}$ & $\begin{array}{c}\text { Proportion of } \\
\text { boys }\end{array}$ \\
\hline Agree & $12 \%$ & $25 \%$ \\
\hline Disagree & $54 \%$ & $41 \%$ \\
\hline $\mathrm{N}$ & 381 & 262 \\
\hline
\end{tabular}

Among year 9 students not in FLL, significantly more boys than girls $(p<.001)$ agreed that "Knowing more than one language is confusing", and that "Learning a foreign language would be too difficult for me", see Table 10.26. 
Table 10.26 Proportions of year 9 students not in FLL reporting agreement or disagreement with survey items indicating perceptions of difficulty in FLL, by gender

\begin{tabular}{|c|c|c|c|c|c|c|c|}
\hline \multirow[b]{2}{*}{ Item } & \multirow[b]{2}{*}{ Statement } & \multirow[b]{2}{*}{$\mathbf{N}$} & \multicolumn{2}{|c|}{ Agree } & \multicolumn{2}{|c|}{ Disagree } & \multirow{2}{*}{$\begin{array}{c}\text { Gender } \\
\text { sig.* }\end{array}$} \\
\hline & & & Girls & Boys & Girls & Boys & \\
\hline 9.4 .3 & $\begin{array}{l}\text { Knowing more } \\
\text { than one } \\
\text { language is } \\
\text { confusing. }\end{array}$ & $\begin{array}{c}\text { Girls } \\
149 \\
\text { Boys } \\
119\end{array}$ & $24 \%$ & $39 \%$ & $60 \%$ & $32 \%$ & $\mathrm{p}<.001$ \\
\hline 9.4 .14 & $\begin{array}{l}\text { Learning a } \\
\text { foreign language } \\
\text { would be too } \\
\text { difficult for me. }\end{array}$ & $\begin{array}{c}\text { Girls } \\
145 \\
\text { Boys } \\
116\end{array}$ & $12 \%$ & $35 \%$ & $62 \%$ & $36 \%$ & $\mathrm{p}<.001$ \\
\hline
\end{tabular}

All these items appear to support the idea that girls in both these year groups experienced more success and felt more confident in FLL. It is possible that more of the boys than the girls judged FLL to be difficult because of actual differences in cognitive development in the two genders at the age of the students surveyed which has been found in the NEMP $(1998,2001)$. On the other hand, as has been suggested by some teachers, it may arise from a difference in attitudes to the amount and type of work required to make satisfactory progress in FLL (Powell and Batters 1985, Clark and Trafford 1995). The tendency of some the boys to avoid subjects involving a lot of reading and writing is discussed in section 10.5 .5 below.

Table 10.27 Responses by gender to survey item 9.3.4: "I think I'll do well in my language learning this year."

\begin{tabular}{|c|c|c|}
\hline Item response & $\begin{array}{c}\text { Proportion } \\
\text { of girls }\end{array}$ & $\begin{array}{c}\text { Proportion } \\
\text { of boys }\end{array}$ \\
\hline Agree & $68 \%$ & $63 \%$ \\
\hline Disagree & $2.5 \%$ & $11 \%$ \\
\hline $\mathrm{N}$ & 237 & 145 \\
\hline
\end{tabular}

When year 9 students with prior FLL experience were asked if they thought they were good at it, a notably higher proportions of girls, $65 \%(\mathrm{~N}=227)$, than boys, $50 \%(\mathrm{~N}=113)$, responded positively. Of those who had chosen a FL option, 
similar proportions, about two thirds, of both boys and girls agreed that they would do well in their FLL, but significantly more boys than girls disagreed $(\mathrm{p}<.005)$, see Table 10.27.

\section{0.5.4 Boys consider FLs less useful than girls \\ 10.5.4.1 The relative importance of FLL}

All the surveyed students were asked to consider the place of FLs in relation to other curriculum areas. Table 10.28 shows that boys had significantly less favourable attitudes than girls to the relative importance of FLL. Over half of year 8 boys compared with less than a third of girls agreed that there were "lots more important things to learn than languages". Fewer year 9 students agreed with this notion and the boys were more evenly divided than the younger group, but the girls were still much more likely to disagree that there were many more important subjects than languages. This suggests that girls valued FLL more highly than boys, but also that boys' attitudes improved somewhat with age.

As previously discussed, there was also a highly significant association $(p<.001)$ between the responses to item 9.2.20 and the degree to which students had opted for FLL. The proportions of each gender who agreed that there are many more important subjects to learn than languages but who had nevertheless opted for FLL, presumably out of personal interest, was similar, approximately $40 \%$ ( 37 out of 94 girls and 42 out of 101 boys). Among the students who did not agree with the item, there was a noticeable discrepancy between the genders. $78 \%$ of the 162 girls but only $69 \%$ of the 85 boys had opted for FLL. Thus, even if boys thought that FLL was as important as other options they were less likely than girls to choose it, presumably because of lack of personal interest or because parents or schools were pushing them into other subjects.

Table 10.28 Responses by gender to survey items 8.1.17 and 9.2.20: "There are lots more important things to learn than languages."

\begin{tabular}{|c|c|c|c|c|}
\hline \multirow[b]{2}{*}{ Item response } & \multicolumn{2}{|c|}{ Year 8} & \multicolumn{2}{|c|}{ Year 9} \\
\hline & Girls & Boys & Girls & Boys \\
\hline Agree & $32 \%$ & $54 \%$ & $25 \%$ & $39 \%$ \\
\hline Disagree & $40 \%$ & $24 \%$ & $43 \%$ & $33 \%$ \\
\hline $\mathrm{N}$ & 146 & 152 & 373 & 258 \\
\hline
\end{tabular}


When year 9 students were asked to consider whether FLL should be part of the core curriculum, slightly more girls than boys agreed and over half the boys disagreed compared with $42 \%$ of the girls, as shown in Table 10.29 . This could mean that the boys were less interested in FLL than the girls but it could also be interpreted as a sign that the boys objected more vigorously to being forced into such learning.

Table 10.29 Responses by gender to survey item 9.2.11:

"A foreign language should be part of the core curriculum at secondary school."

\begin{tabular}{|c|c|c|}
\hline Item response & $\begin{array}{c}\text { Proportion of } \\
\text { girls }\end{array}$ & $\begin{array}{c}\text { Proportion of } \\
\text { boys }\end{array}$ \\
\hline Agree & $25 \%$ & $21 \%$ \\
\hline Disagree & $42 \%$ & $51 \%$ \\
\hline $\mathrm{N}$ & 380 & 254 \\
\hline
\end{tabular}

Among students who agreed that FLL should be compulsory, a substantially larger proportion of girls, 68 out of $96(71 \%)$, than boys, 34 out of 54 (63\%), had "put their money where their mouth was" and opted into FLL. This could partly be attributed to the current necessity of making choices where boys are perhaps more likely than girls to prefer other available subjects even if they think that learning a FL would be a good thing. There was a significant association between the attitudes of girls and their subject choices $(p<.005)$ but none for the boys.

A more general item presented to the year 9 students who had not opted for FLL, designed to gauge the general attitude to FLL among their friends, showed a significant difference $(\mathrm{p}<.001)$ between the attitudes of boys and girls, see Graph 10.5. A small majority of boys, $37 \%(\mathrm{~N}=113)$, agreed that most of their friends thought that FLL was stupid, but a large majority of girls, $67 \%(\mathrm{~N}=146)$, disagreed. This seems a useful indication that, even among girls not involved in FLL, general attitudes are still quite positive. 
Graph 10.5 Responses by gender to survey item 9.4.7: "Most of my friends think that learning foreign languages is stupid." (For gender association, p<.001)

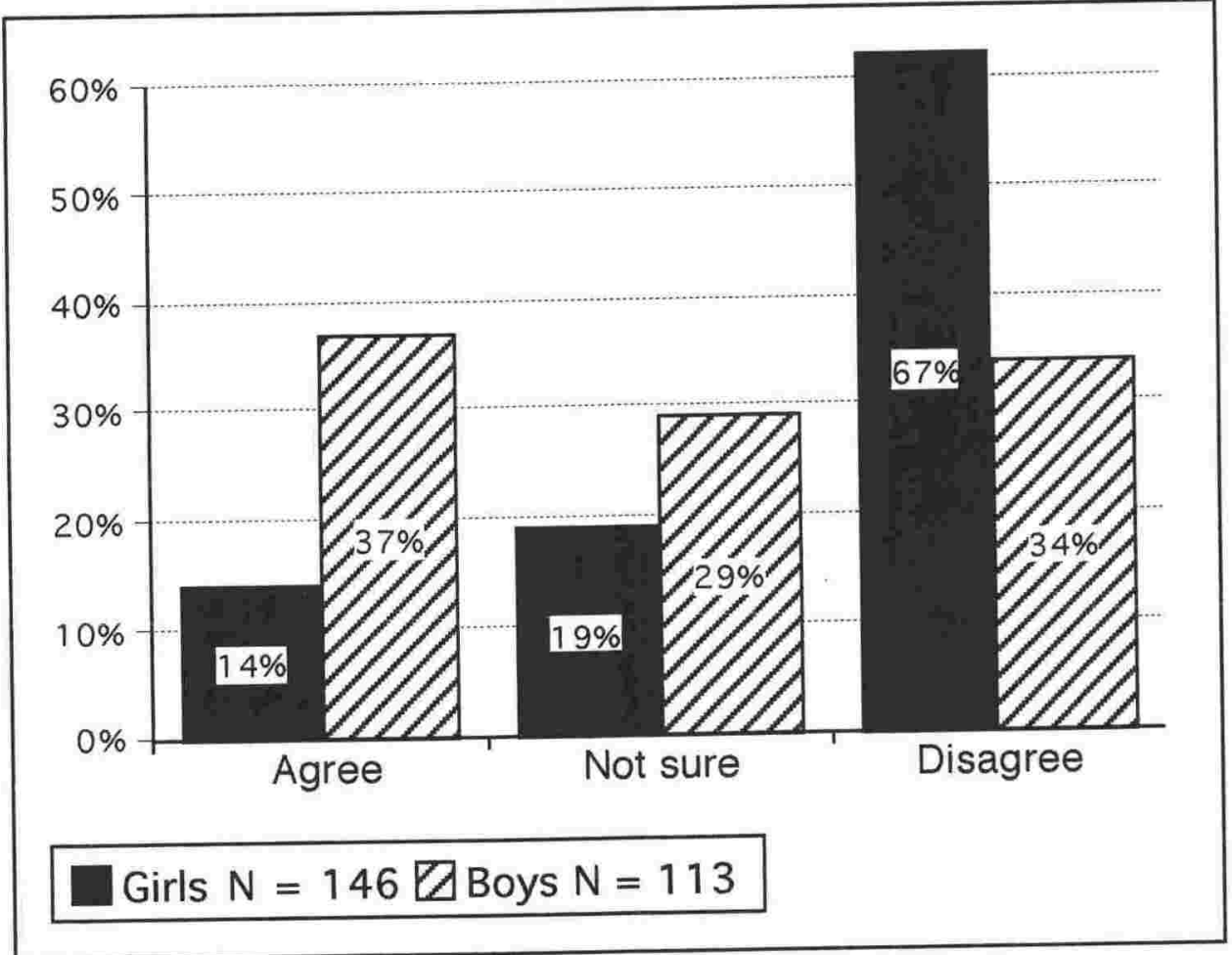

When the year 8 students were asked if they thought that learning Maori was more important than a FL in New Zealand, item $8.1 .14,31 \%$ of the boys (N $=151)$ but only $21 \%$ of the girls $(N=149)$ agreed. When the same item was put to all the year 9 students (item 9.2.14) a similar proportion of girls agreed $(\mathrm{N}=382)$ but the boys appeared much less inclined to believe in the value of learning Maori - only $17 \%$ agreed $(\mathrm{N}=259)$. Views about the relative importance of Maori seemed to have little effect on the girls' decisions regarding FLL. The 82 girls who agreed about the greater importance of Maori were fairly evenly divided in their choice to study a FL or not, but the 43 boys who agreed with the item were nearly twice as likely to have decided not to study a FL. This does not necessarily mean, of course, that these boys had chosen to study Maori instead. Even if they had, they may have been uninterested in any language learning, but have considered Maori of greater relevance for New Zealand students if they had to learn an L2. 
The views of the year 9 students not involved in FLL seem to contradict the above as nearly half disagreed with the statement, item 9.4.4 "I will only ever need to be able to speak English, or perhaps a bit of Maori". There was a marked gender difference in these responses as $55 \%$ of the girls $(\mathrm{N}=152)$, but only $40 \%$ of the boys $(\mathrm{N}=121)$ disagreed. This suggests a greater degree of openness among the girls to the possibility of learning a FL in the future, which supports evidence already cited in 10.4.1.

\subsubsection{The relative importance of English}

In year 8 , boys were more likely than girls to believe that concentrating on English would be more valuable than FLL. Girls disagreed with item 8.1.12 significantly more strongly than boys $(p<.005)$, see Table 10.30. Significant differences were also found among the year 9 students not involved in FLL who were asked about their feelings towards concentrating on English, see Table 10.31 . Only about $28 \%$ of girls $(N=148)$, compared with $45 \%$ of boys $(\mathrm{N}=118)$, felt the need to work harder at their English, and the girls disagreed with the statement much more strongly than the boys.

Table 10.30 Responses by gender to survey item 8.1.12: "It's better for me to spend time doing more English than learning another language."

\begin{tabular}{|c|c|c|}
\hline Item response & $\begin{array}{c}\text { Proportion of } \\
\text { girls }\end{array}$ & $\begin{array}{c}\text { Proportion of } \\
\text { boys }\end{array}$ \\
\hline Agree & $21 \%$ & $40 \%$ \\
\hline Disagree & $48 \%$ & $36 \%$ \\
\hline $\mathrm{N}$ & 149 & 154 \\
\hline
\end{tabular}

Table 10.31 Responses by gender to survey item 9.4.2: "I need to concentrate on developing my English language skills."

\begin{tabular}{|c|c|c|}
\hline Item response & $\begin{array}{c}\text { Proportion of } \\
\text { girls }\end{array}$ & $\begin{array}{c}\text { Proportion of } \\
\text { boys }\end{array}$ \\
\hline Agree & $28 \%$ & $45 \%$ \\
\hline Disagree & $51 \%$ & $30 \%$ \\
\hline $\mathrm{N}$ & 148 & 118 \\
\hline
\end{tabular}


These results could indicate that the girls felt more confident about their general language ability than the boys, or that the boys thought they were less able than the girls. On the other hand, it might indicate that the boys, much more than the girls, did not see the need for knowing any language other than English, or that they were more aware of the emphasis put on English skills by schools, and possibly parents, because of a common perception in society that boys are falling behind girls.

Table 10.32 Proportions of year 9 students in FLL reporting agreement or disagreement with survey items indicating perceptions of the transferability of language skills, by gender

\begin{tabular}{|c|c|c|c|c|c|c|}
\hline \multirow[b]{2}{*}{ Item } & \multirow[b]{2}{*}{ Statement } & \multirow[b]{2}{*}{$\mathbf{N}$} & \multicolumn{2}{|c|}{ Agree } & \multicolumn{2}{|c|}{ Disagree } \\
\hline & & & Girls & Boys & Girls & Boys \\
\hline 9.3 .16 & $\begin{array}{l}\text { Learning another } \\
\text { language will help } \\
\text { me read more } \\
\text { carefully. }\end{array}$ & $\begin{array}{l}\text { Girls } \\
235 \\
\text { Boys } \\
147\end{array}$ & $16 \%$ & $26 \%$ & $34 \%$ & $33 \%$ \\
\hline 9.3 .9 & $\begin{array}{l}\text { I enjoy trying to say } \\
\text { things correctly. }\end{array}$ & $\begin{array}{l}\text { Girls } \\
236 \\
\text { Boys } \\
147\end{array}$ & $59 \%$ & $54 \%$ & $17 \%$ & $22 \%$ \\
\hline 9.3 .18 & $\begin{array}{l}\text { Now that I'm } \\
\text { learning another } \\
\text { language I take more } \\
\text { care with my written } \\
\text { work. }\end{array}$ & $\begin{array}{l}\text { Girls } \\
226 \\
\text { Boys } \\
140\end{array}$ & $12 \%$ & $14 \%$ & $47 \%$ & $54 \%$ \\
\hline 9.3 .1 & $\begin{array}{l}\text { Knowing some of } \\
\text { one foreign language } \\
\text { will make it easier for } \\
\text { me to learn another } \\
\text { later on. }\end{array}$ & $\begin{array}{c}\text { Girls } \\
236 \\
\text { Boys } \\
148\end{array}$ & $45 \%$ & $54 \%$ & $17 \%$ & $15 \%$ \\
\hline
\end{tabular}

\subsubsection{Awareness of transferable language skills}

Only about one quarter of all the year 9 students agreed that learning another language helps students understand English better, item 9.2.19. In a rare instance, among this minority there was a higher proportion of boys ( $29 \%, \mathrm{~N}$ = 261) than girls $(21 \%,(\mathrm{~N}=374)$, another reflection, possibly, of the skewed sample at the boys' school. However, belief in the value of FLL had 
little association with the boys' choices of options although it proved significant for the girls $(\mathrm{p}<.02)$. Only $64 \%$ of the 75 boys who agreed with the item had chosen a FL compared with $74 \%$ of the 78 girls. This may simply reflect, yet again, the greater interest among girls in FLL, rather than the influence of this particular factor on their choices.

Items designed to ascertain if students who were learning a FL perceived any value in it for developing specific, transferable skills produced no significant gender differences, see Table 10.32. Boys responded much more positively than girls to the idea that earning another language would help them read more carefully. In relation to the "active" skills of speaking and writing, responses suggest that boys cared less than girls about accuracy and, therefore, probably perceived less value in FLL for developing these skills. Another instance of the boys having more positive attitudes than the girls related to the idea that FLL would help them in any possible future FLL. The girls tended to be more unsure. On the whole, evidence from my surveys tends to indicate that messages about the value of FLL for the acquisition of transferable skills were being received to only a limited extent by students.

\subsubsection{Awareness of the value of FLL for cultural understanding}

Year 9 boys were, perhaps surprisingly, significantly more likely than girls to appreciate the value of FLL for understanding other cultures, see Table 10.33. Nearly three quarters of them $(n=264)$ agreed with the relevant item 9.2.6, compared with only $60 \%$ of the girls $(\mathrm{N}=381)$.

Table 10.33 Responses by gender to survey item 9.2.6:

"Students who learn a foreign language are better at understanding another culture."

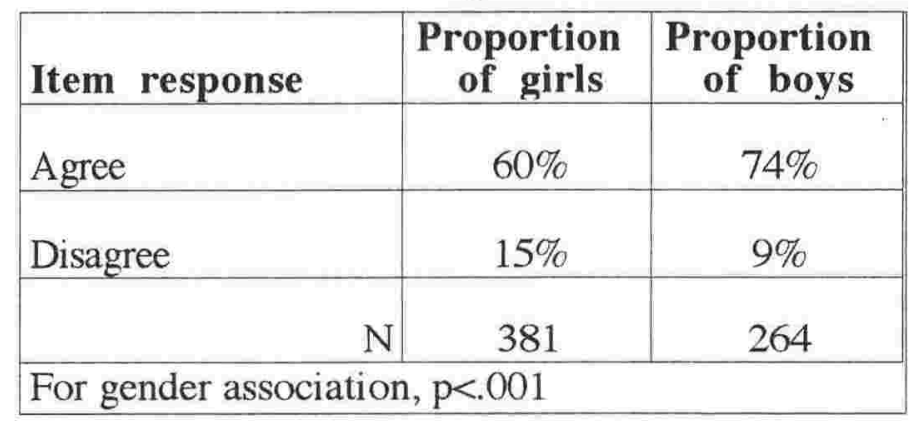

It is hard to estimate why, in this rare instance, the attitudes of boys towards FLL seemed more positive than those of the girls. It may be another consequence of the unbalanced sample at the boys' school, where the majority, being in top streams, were most likely to come from well-educated homes 
with an appreciation of other languages and cultures or experience of foreign travel. Among students who were not studying a FL, more boys, (42\%, N = $114)$, than girls, $(35 \%, N=145)$ agreed that they could learn about the life and customs of other people without needing to understand any of the their language, item 9.4.6. These results suggest that girls more than boys are aware of the links between language learning and the acquisition of cultural knowledge and understanding, although why this should be is open to debate.

\subsubsection{Usefulness of FLs for employment}

As shown above in 10.3.2 and 10.4.2.1, neither girls nor boys made much mention of the usefulness of FLL for future employment as a reason for opting into FLL. It was therefore somewhat surprising that just over half of the year 9 students $(\mathrm{N}=656)$ responded positively to the suggestion that knowing a FL would help them get a good job. There was no significant gender difference in the association between this belief and the choice to study a FL, but the girls seemed slightly more influenced by the possible benefits of FLL for future employment than the boys as 69.5\% of the 197 girls who agreed with item 9.2.15, compared with only $62 \%$ of the 140 boys, had opted for FLL. Among the $17 \%$ of students who did not believe in the value of FLL as a job qualification, and the $32 \%$ who were unsure, there were greater proportions of girls than boys who had nevertheless opted for FLL, possibly yet another indication that FLL simply appealed more to girls than boys.

Table 10.34 Responses by gender to survey item 9.2.8: "Lots of jobs involve working with people who don't speak English."

\begin{tabular}{|c|c|c|}
\hline Item response & $\begin{array}{c}\text { Proportion } \\
\text { of girls }\end{array}$ & $\begin{array}{c}\text { Proportion } \\
\text { of boys }\end{array}$ \\
\hline Agree & $39 \%$ & $41 \%$ \\
\hline Not sure & $39 \%$ & $29 \%$ \\
\hline Disagree & $22 \%$ & $30 \%$ \\
\hline $\mathrm{N}$ & 385 & 264 \\
\hline
\end{tabular}

Similar proportions of both genders in both year groups agreed that lots of jobs involve working with people who do not speak English but there was a 
significant difference $(\mathrm{p}<.02)$ among the rest of the year 9 students, see Table 10.34. The boys disagreed more strongly than the girls who were more unsure. Despite the similar proportions agreeing with the item, $67 \%$ of girls $(\mathrm{N}=151)$ but only $55 \%$ of boys $(\mathrm{N}=109)$ had opted for FLL. Yet again, whatever the perceived value of FLL in relation to employment, boys were still less likely than girls to choose to study a FL.

Students not in FLL in year 9 were asked to consider the possibility that they might need some FL knowledge in future employment. The great majority of the girls were evenly divided between uncertainty and disagreeing with item 9.4.12, "I won't need to know any foreign languages in any jobs I might do". The boys were even more uncertain but were significantly more likely to agree $(\mathrm{p}<.05)$ than the girls, see Graph 10.6.

\section{Graph 10.6 Responses by gender to survey item 9.4.12: "I won't need to know any foreign languages in any jobs I might do." (For gender association, $\mathrm{p}<.05$ )}

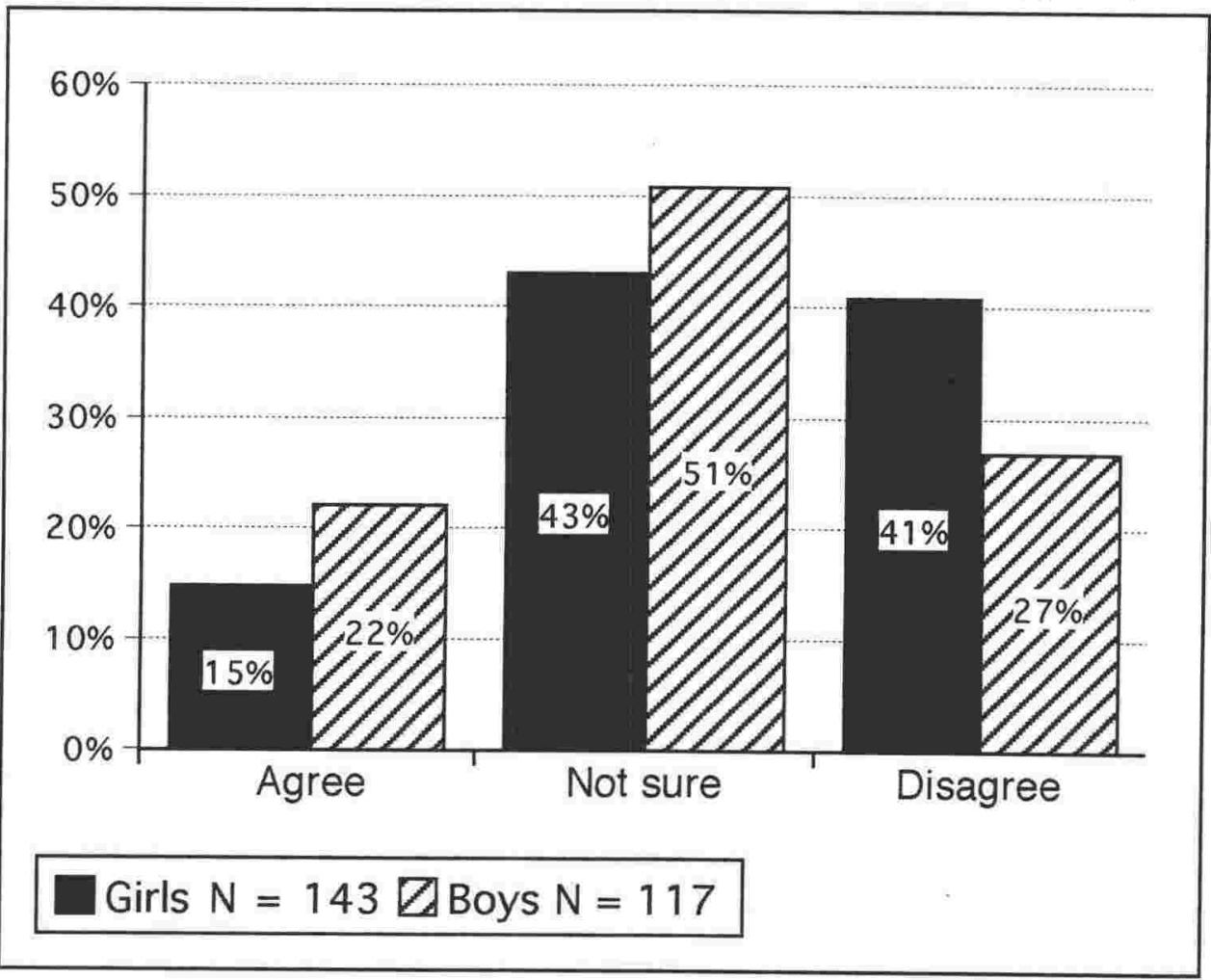




\subsubsection{Boys prefer other subjects}

Although, only $12 \%$ of boys who had not opted for FLL reported that they preferred other subjects other than Maori, see 10.3.4.3, anecdotal evidence suggests that boys tend to prefer practical options, such as subjects that used to be called 'woodwork' or 'metalwork'. This perception is supported by the responses to item 9.4.13, suggesting that students preferred subjects with less reading and writing than FLL. Responses showed a significant gender difference ( $\mathrm{p}<.001$ ), shown in Graph 10.7. $60 \%$ of the boys $(\mathrm{N}=111)$, but only $35 \%$ of the girls $(\mathrm{N}=146)$ agreed that they were more interested in practical subjects.

Graph 10.7 Responses by gender to survey item 9.4.13: 'I'm more interested in practical subjects than subjects with lots of reading and writing." (For gender association, $\mathrm{p}<.001$ )

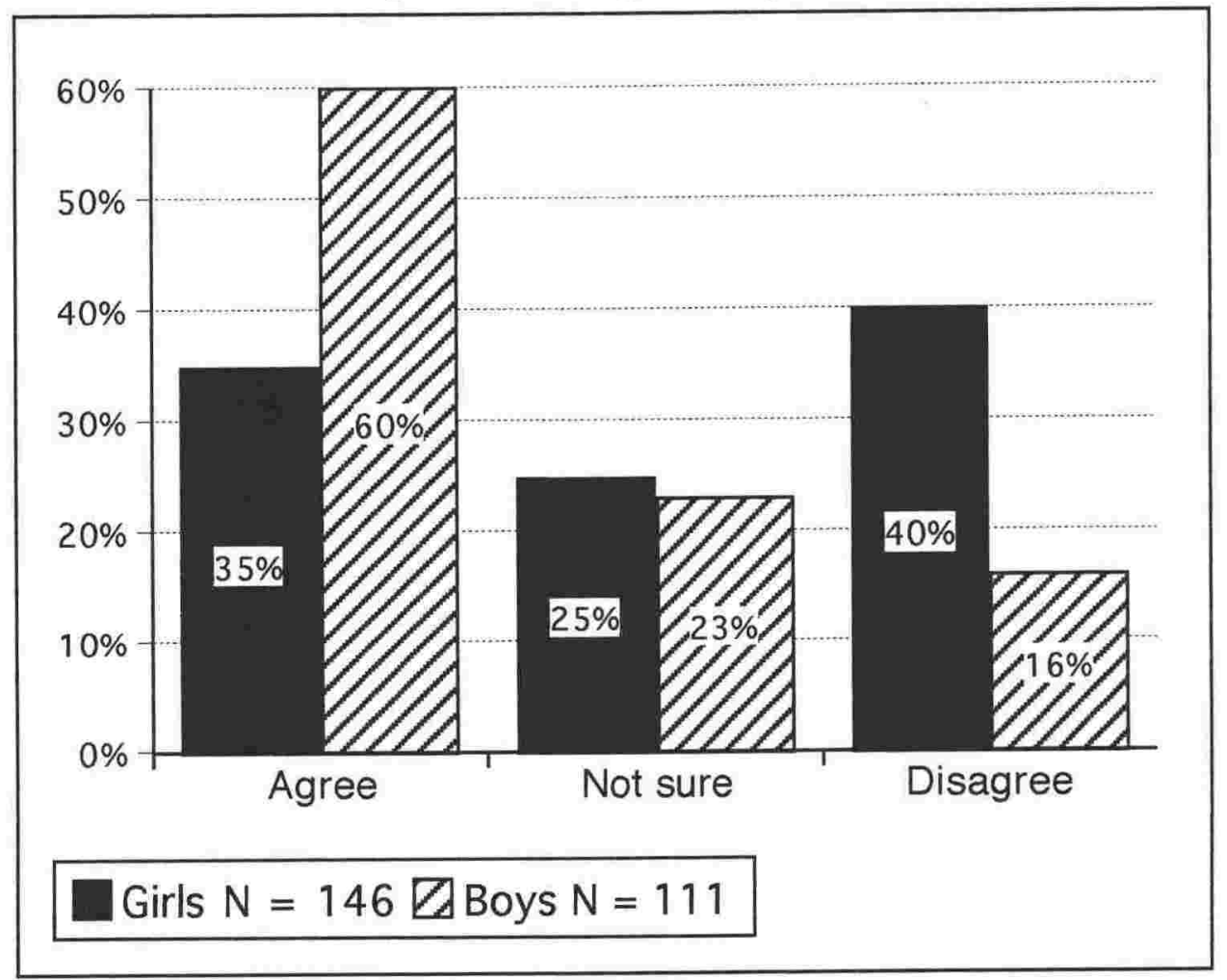

\subsection{External influences}

\subsubsection{The influence of parents}

It has been suggested that English-speaking societies, like New Zealand, transmit to students, generally through their parents, the belief that FLs are not important and are 
mainly for girls (Burstall 1979, Waite 1992). Among all the students involved in this research girls and boys felt equally independent from their parents in their choice of option subjects. As discussed in chapter 8 , the majority of parents similarly reported that their children had made the final decision about optional subjects independently. In talking to me, the parents had not given any hint that they considered FLL more suitable for girls than boys, see 10.2.2 above, but it must be noted that my being female and a FL teacher may have influenced their comments, and most of my interviewees were female too. It was clear that, in the parents' experience, FLL at school was very much the province of girls.

A lack of FLL experience on the part of parents seemed to have a more powerful influence on sons than on daughters. For example, if neither parent had learnt a FL at school, as far as students were aware, year 8 boys were much less likely than girls to opt into FLL, see Table 10.35 .

\section{Table 10.35 Year 8 student intentions and year 9 student choices regarding FLL compared with parents' FLL at school in $\mathbf{N Z}$}

Notes: * = proportion of $\mathrm{N}$ intending or having chosen FLL in year 9 NA = not applicable (parents not educated in NZ)

\begin{tabular}{|l|r|r|r|r|r|r|r|r|}
\hline \multirow{2}{*}{$\begin{array}{c}\text { Parents' experience } \\
\text { according to } \\
\text { students }\end{array}$} & \multicolumn{4}{|c|}{ Year 8 students } & \multicolumn{4}{|c|}{ Year 9 students } \\
\cline { 2 - 11 } & $\begin{array}{l}\text { Girls } \\
\text { N }\end{array}$ & $*$ & $\begin{array}{c}\text { Boys } \\
\text { N }\end{array}$ & $*$ & $\begin{array}{c}\text { Girls } \\
\text { N }\end{array}$ & $*$ & $\begin{array}{c}\text { Boys } \\
\text { N }\end{array}$ & $*$ \\
\hline $\begin{array}{l}\text { One or both parent } \\
\text { had learnt FL }\end{array}$ & 62 & $60 \%$ & 60 & $52 \%$ & 146 & $71 \%$ & 97 & $73 \%$ \\
\hline $\begin{array}{l}\text { Neither parent had } \\
\text { learnt FL }\end{array}$ & 55 & $55 \%$ & 70 & $30 \%$ & 144 & $53 \%$ & 135 & $37 \%$ \\
\hline No info/NA & 34 & $53 \%$ & 27 & $44 \%$ & 103 & $58 \%$ & 39 & $67 \%$ \\
\hline
\end{tabular}

Among year 9 students who had decided to study a FL, boys perceived much more parental pressure than the girls. Only a small minority of these students (29) agreed that they were only learning a FL because their parents said they had to, but the boys outnumbered the girls, 3:1, a significant gender difference $(\mathrm{p}<.05)$.

As shown in 8.2.4, year 9 students did not perceive a great deal of hostility from their parents towards FLL in general. Graph 10.8 shows that both girls and boys disagreed with the statement, "My parents think that studying a foreign language is a waste of time for New Zealand students" but that there was a significant gender difference $(p<.005)$ in the responses suggests that parents of the boys were more likely to have had doubts about the value of FLL. 
Almost identical proportions of girls and boys agreed, disagreed or were unsure about item 9.2.13, "My parents wanted me to do a foreign language this year", and equal proportions of those who agreed had opted into FLL, over $80 \%$. Thus the great majority of both genders had done what their parents wished despite being confident that they could choose whatever options they wanted. It seems that students either wanted to please their parents (cf Hofman 1974), without admitting it, or did not realise how much they had been influenced by them. As already discussed, disagreement could mean that parents were hostile or simply indifferent to the idea of FLL for their children, or even that the option had not been discussed. What is interesting is that $42 \%$ of the 124 girls in this category, but only $31 \%$ of the 88 boys, had nevertheless chosen a FL. Similarly, 59\% of the 92 girls who were unsure of their parents' opinions, as against $50 \%$ of the 66 boys, had opted into FLL. Both cases apparently offer more evidence of a greater interest in FLL among the girls, and possibly a greater determination to learn a FL whatever their parents' opinions might be.

\section{Graph 10.8 Responses by gender to survey item 9.2.3: "My parents} think that studying a foreign language is a waste of time." (For gender association, $\mathrm{p}<.005$ )

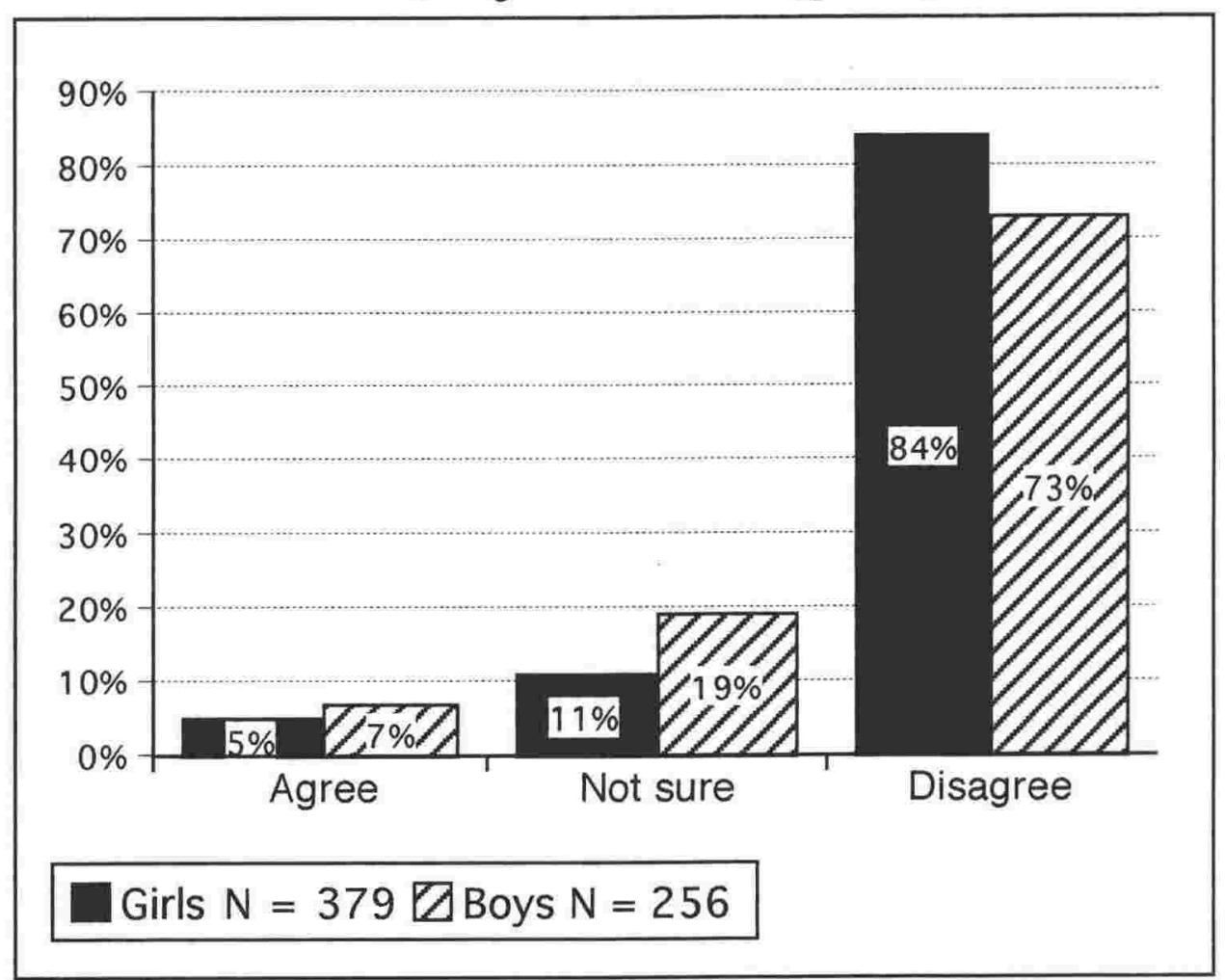


Boys were less likely than girls to agree that their parents were pleased that they were involved in FLL, and much more likely to report that they were unsure of their parents' feelings, see Table 10.36. It is impossible to determine from the available data whether the student reports were an accurate reflection of their parents' opinions. Even if they were, it is equally impossible to know for certain why the boys' parents should be less enthusiastic about their sons learning a FL, although the views of the parents with sons at Begonia, discussed in 8.8.5, offer some hints.

Table 10.36 Responses by gender to survey item 9.3.3: "My parents are pleased that I'm doing a foreign language this year.'

\begin{tabular}{|c|c|c|}
\hline Item response & $\begin{array}{c}\text { Proportion } \\
\text { of girls }\end{array}$ & $\begin{array}{c}\text { Proportion } \\
\text { of boys }\end{array}$ \\
\hline Agree & $83 \%$ & $71 \%$ \\
\hline Not sure & $16 \%$ & $26 \%$ \\
\hline Disagree & $2 \%$ & $3 \%$ \\
\hline $\mathrm{N}$ & 235 & 147 \\
\hline
\end{tabular}

All students were asked to respond to the statement, "My parents think I should spend more time on my English than learning another language". Only a minority of year 8 students agreed but proportionately twice as many boys as girls, see Table 10.37, a significant difference $(\mathrm{p}<.02)$. There was an even more significant gender difference $(p<.005)$ among the year 9 students. The boys were fairly evenly divided in their responses but over half the girls again firmly disagreed with the idea that their parents might prefer their daughters to concentrate on English than learn a FL.

Table 10.37 Responses by gender to survey items 8.1.25, 9.4.8: "My parents think I should spend more time on my English than learning another language."

(For year 8: 'No opinion' = 13. For gender association, $\mathrm{p}<, 02$.

For year 9: 'No opinion' $=22$. For gender association, $p<.005$ )

\begin{tabular}{|l|c|c|c|c|}
\hline & \multicolumn{3}{|c|}{ Proportions of } & \multicolumn{2}{c|}{ students } \\
\hline $\begin{array}{l}\text { Item } \\
\text { response }\end{array}$ & $\begin{array}{c}\text { Year 8 } \\
\text { girls }\end{array}$ & $\begin{array}{c}\text { Year 8 } \\
\text { boy }\end{array}$ & $\begin{array}{c}\text { Year 9 } \\
\text { girls }\end{array}$ & $\begin{array}{c}\text { Year 9 } \\
\text { boys }\end{array}$ \\
\hline Agree & $11 \%$ & $22 \%$ & $19 \%$ & $32 \%$ \\
\hline Disagree & $55 \%$ & $42 \%$ & $56 \%$ & $35 \%$ \\
\hline & 143 & 152 & 144 & 109 \\
\hline
\end{tabular}


In summary, it seems that any parental influence on the attitudes of students towards FLL and the choices they made in year 9 remained largely unperceived by the students themselves. As interviews showed, there was almost no suggestion made by any of the parents that FLs were considered by themselves or their children as 'girls' subjects', but negative attitudes on the part of parents seem to have discouraged more boys than girls from learning a FL. Such attitudes might have resulted from parents' lack of FLL experience as this seems to have had a greater negative influence on sons than on daughters. Of course, boys' parents may have simply encouraged interest in other subjects, without necessarily expressing disapproval of FLL, and some boys and their parents may simply not have considered the possibility of FLL. On the other hand, girls' parents seem, consciously or unconsciously, to have signalled that it was more acceptable for girls to try FLL, even where there was no tradition of such learning in the family.

\subsubsection{The influence of connections with or travel to non-English speaking places}

As discussed in 6.11, the minority of students who reported having overseas friends or relations who spoke any LOTEs were significantly $(p<.02)$ more likely to have decided to study a FL. When examined by gender the association proved even more significant for $(p<.005)$, with a particularly positive effect on boys' interest in FLL. When asked about FLL experiences while travelling overseas, nearly two thirds of the girls reported that they had learnt something of another language, compared with just under half of the boys, but it is impossible to judge whether the difference was due to different attitudes among the children or different opportunities they had. Graph 10.9 shows that having some overseas FLL experience did not make much difference to girls' FLL decisions, but that boys who had had such experience were much more likely to opt into FLL (13 out of 18 ) than boys without (12 out of 21).

\subsubsection{The influence of peers and older siblings}

Among the year 8 students $44 \%$ of boys $(N=151)$ but only $30 \%$ of girls $(N=149)$ agreed that they wanted with to stay with their best friend the following year, whatever subjects they took, item 8.1.3, a significant difference $(p<.05)$. The great majority of year 9 students showed much more independence from their friends as $92 \%$ of the girls $(\mathrm{N}=377)$ and $83 \%$ of the boys $(\mathrm{N}=271)$ disagreed with the suggestion that they had chosen their option subjects so that they could stay with their best friend, item 9.2.10. Nevertheless this was still significant difference $(p<.005)$. Generally, the boys seem to have been influenced by friends in their option choices to a greater extent than the girls, or else the girls were not admitting to such influence.

\subsubsection{The influence of prior FLL}

Experience of FLL, or lack of it, at intermediate school seems to have had little effect on the intentions of year 8 girls to study a FL in year 9. Graph 10.10 (see Appendix 13) shows that higher proportions of girls than boys were intending to study a FL in 
year 9, whether or not they had already learnt a FL. On the whole, boys were much less likely to consider FLL for year 9 if they had not had any prior experience. Only one quarter of such boys $(\mathrm{N}=31$ ) reported that they would study a FL, in comparison with nearly half of those with prior experience $(\mathrm{N}=124)$. Nearly half the boys without prior experience reported that they definitely would not be studying a language, compared with under one quarter of the girls (only 6) in that category. The girls were apparently not deterred from opting for FLL option by lack of previous experience. If anything, this seems to have encouraged them to try it, although it must be pointed out that the figure of $69 \%$ represents only 18 students.

\section{Graph 10.9 Student choices for year 9 FLL compared with experience of FLL during overseas travel}

Note: Numbers in brackets are totals for each group of students

$\mathrm{Y}=$ had experience of FLL overseas

$\mathrm{N}=$ did not have experience of FLL overseas

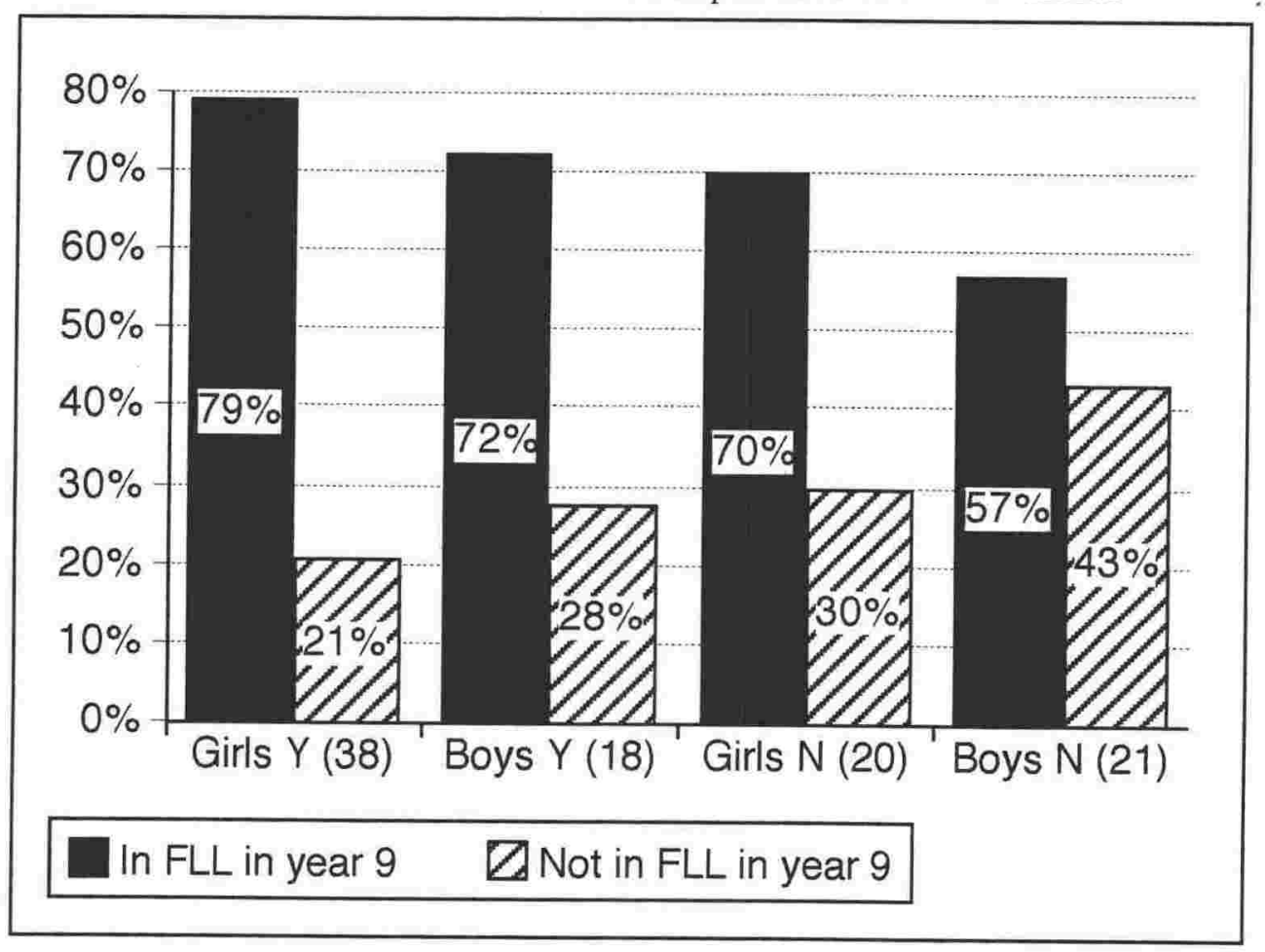

The prior learning of specific languages seems to have influenced the intentions of girls and boys differently, see Graph 10.11 (Appendix 13). Whatever their previous FLL experience, girls were more likely than boys to choose to study a FL in year 9, and what FLs they had learnt made little difference to their intentions. Boys were 
least likely to intend further FLL if they had only learnt a European language. Learning some Japanese seemed to have a particularly positive effect on boys' intentions, and learning two languages, including Japanese, considerably reduced their uncertainty about future FLL. Although such experience produced the largest proportions of boys with definite intentions for or against further FLL, the proportion of boys not intending further study, $37 \%(\mathrm{~N}=40)$, was still considerably smaller than the $48 \%$ of boys without any FLL experience $(\mathrm{N}=31)$ who did not intend to study a FL in year 9. Prior experience of FLL, provided it included Japanese, seems to have had a particularly positive influence on the attitudes of the boys towards FLL in year 9.

Student intentions were also analysed to ascertain whether those who intended further FLL were going to continue studying the FL they had already learnt, see Table 10.38. Japanese was the preferred FL with all students, but the boys' showed little interest in continuing other languages and no interest at all in starting French or Spanish if they had not learnt any previously. The fact of having some FLL experience at intermediate level and the actual FLs learnt appear to have been more crucial for boys than for girls in terms of their attitudes towards further learning.

Table 10.38 Year 8 student choices of FL for Year 9 compared with FL previously studied

\begin{tabular}{|c|c|c|c|c|c|c|c|c|}
\hline \multirow{2}{*}{$\begin{array}{l}\text { Year } 9 \\
\text { choice of } \\
\text { language }\end{array}$} & \multicolumn{2}{|c|}{$\begin{array}{l}\text { Totals } \\
\text { to study }\end{array}$} & \multicolumn{2}{|c|}{$\begin{array}{l}\text { Continuing } \\
\text { same FL }\end{array}$} & \multicolumn{2}{|c|}{$\begin{array}{l}\text { Intend to } \\
\text { start new }\end{array}$} & \multicolumn{2}{|c|}{$\begin{array}{l}\text { No prior } \\
\text { FLL }\end{array}$} \\
\hline & G & B & $\mathbf{G}$ & B & G & B & G & B \\
\hline French & 21 & 5 & 12 & 3 & 4 & 2 & 5 & 0 \\
\hline Spanish & 13 & 2 & 9 & 2 & 2 & 0 & 2 & 0 \\
\hline Japanese & 35 & 43 & 22 & 25 & 7 & 13 & 6 & 5 \\
\hline Totals & 69 & 50 & 43 & 30 & 13 & 15 & 13 & 5 \\
\hline
\end{tabular}

Reactions to their previous FLL experience also affected boys' choices for year 9 more than girls'. Similar proportions of both year 9 girls $(69 \%, N=158)$ and boys $(65 \%, N=75)$ who had enjoyed their previous FLL had decided to learn a FL in year 9 , but there was a striking difference between those who reported that they had not enjoyed it. The girls in this group were, proportionately, nearly three times as likely (18 out of 39) as boys (4 out of 25 ) to have chosen a FL option, see Table 10.39. Similar high proportions, over $70 \%$, of the 148 girls and 57 boys who reported that they had been good at FLL decided to continue it in year 9. Among those who did not have such confidence about their previous FLL there was a much smaller gender difference in choices than among those who had not enjoyed their 
FLL, although the girls ( 24 out of 64 ) were still more likely than the boys ( 15 out of 47) to undertake further FLL.

It seems that the girls, whatever their feelings about their previous experience, were generally more interested in FLL and prepared to try some more, perhaps hoping that a different teacher in a different school would make it more enjoyable. On the whole, the boys in the year 9 sample had not enjoyed their intermediate school FL classes as much as the girls, nor did they think they were good at FLL to the same degree as the girls, and these factors had a greater negative influence on the boys than on the girls. It is possible that the few boys who had not enjoyed their prior experience, or felt they were not good at FLL, but who nevertheless opted for FLL in year 9, were the ones who felt under pressure to study a FL in their particular school.

Table 10.39 Proportions of students in year 9 FLL by gender, according to responses to item 9.2.2, "I enjoyed learning a foreign language at intermediate school" ('No opinion' = 12)

\begin{tabular}{|l|c|c|c|c|}
\hline $\begin{array}{l}\text { Item } \\
\text { response }\end{array}$ & $\begin{array}{l}\text { \% of girls in } \\
\text { Y9 FLL }\end{array}$ & N & $\begin{array}{l}\text { \% of boys } \\
\text { in Y9 FLL }\end{array}$ & N \\
\hline Agree & $69 \%$ & 158 & $65 \%$ & 75 \\
\hline Disagree & $46 \%$ & 39 & $16 \%$ & 25 \\
\hline $\begin{array}{l}\text { Significance of association between responses: } \\
\text { for girls p<.005, for boys p<.001 }\end{array}$ \\
\hline
\end{tabular}

\subsubsection{The influence of schools}

In the area where this research was carried out boys and girls attending the mixed secondary schools had equal opportunities to study a FL, but opportunities differed at the single sex schools. The latter both offered whole year options for the same FLs, but the girls had the additional choice of studying short cultural modules related to each language. The availability of these modules suggests that the school believed that as many students as possible should have some experience of learning at least a little of another language and culture. As previously explained, whether Begonia boys could study a FL depended on the stream to which they were allocated. All boys had to indicate two option choices on enrolment, including music or a language, but only the top 7 of 13 classes could study 2 optional subjects, and, ultimately, the language classes consisted almost entirely of boys from the top four streams, because the school upheld the traditional view that FLL should be part of the education of brighter students and that it was not suitable for those of lesser ability. To some extent the boys with an interest in FLL were doubly disadvantaged at Begonia since a particularly large proportion of them had come from small rural schools where they had no previous opportunity to learn a FL. 
Whichever school they expected to attend, the year 8 girls were more likely than the boys to intend to study a FL, see Graph 10.12, but the proportions of each gender and the gap between them varied from school to school. The girls were most likely to be contemplating FLL if they were going to Gerbera (16 out of 24) but this might have included students opting for the short module. The 17 out of 34 boys who reported that they would study a FL at Begonia and the particularly high proportion, 11 , who indicated uncertainty, probably reflect the fact that they could not know for certain to which stream they would be allotted and that they knew there was a degree of compulsion with regard to FLL at that school.

\section{Graph 10.12 Year 8 student intentions for FLL in year 9 compared} with expected secondary school and gender

$$
\text { Key: } \begin{aligned}
G & =\text { girls, } B=\text { boys, } F=\text { Freesia }(65 \mathrm{G}, 66 \mathrm{~B}) \\
\mathrm{S} & =\text { Salvia }(41 \mathrm{G}, 41 \mathrm{~B}) \mathrm{SS}=\operatorname{single} \operatorname{sex}(24 \mathrm{G}, 34 \mathrm{~B})
\end{aligned}
$$

Note: For significance of association between school and FLL intentions, $p<.001$

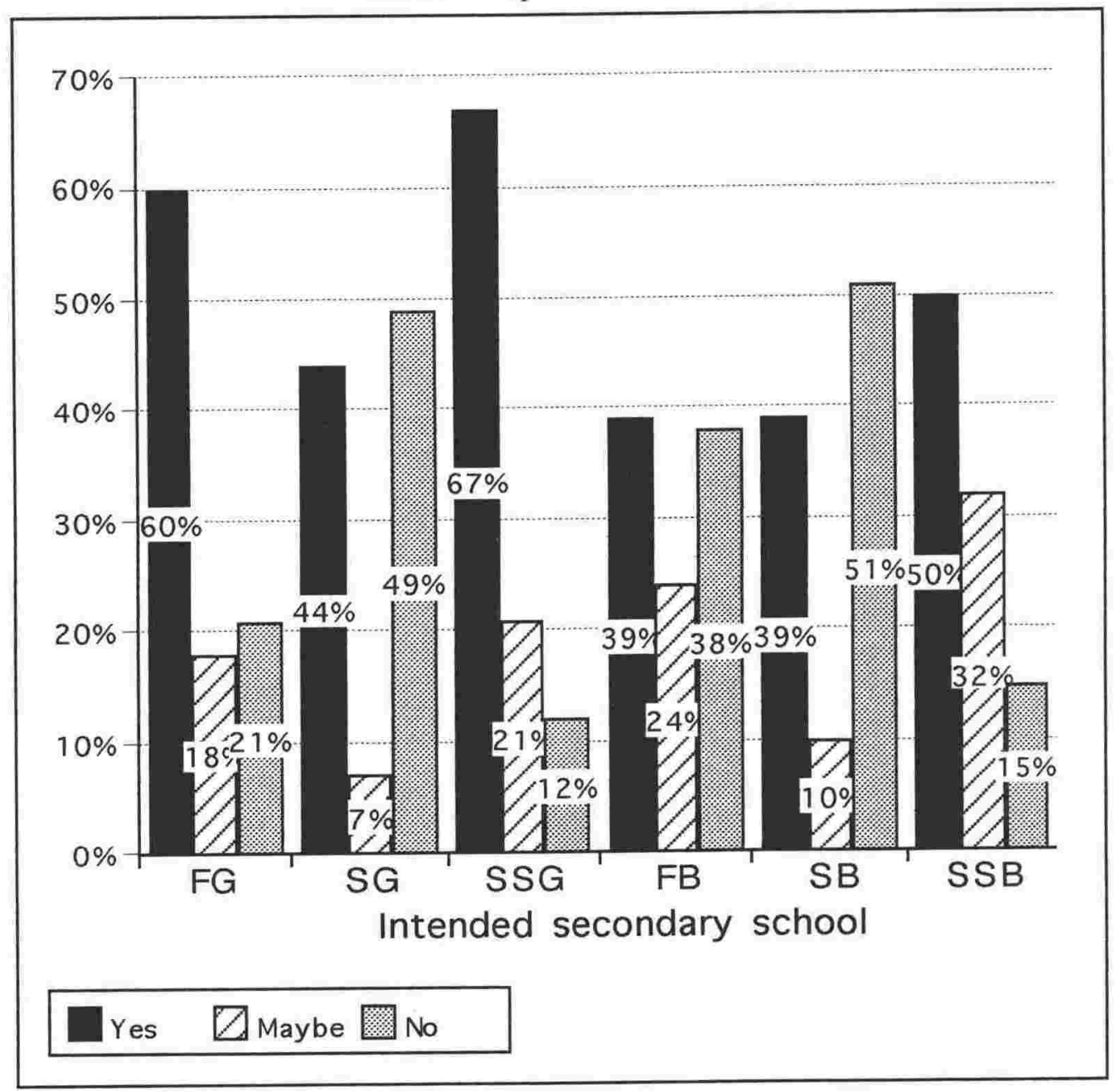


The widest gap between girls and boys intending FLL was among students expecting to attend Freesia, $60 \%$ of girls $(\mathrm{N}=65)$ and $39 \%$ of boys $(\mathrm{N}=66)$. One can only speculate on the possible explanations for this. The school had the second lowest decile rating, next to Salvia, of all the surveyed secondary schools, and the boys might therefore have come from homes traditionally uninterested in FLL. All the alternatives to the language options were practical subjects which, as has already been demonstrated, the boys tended to find more attractive.

What is more notable is the narrowest gap between the genders among students intending to remain at Salvia. These findings seem to contradict the overall picture of the influence of prior experience of FLL on intentions discussed in the previous section. Not only did prior experience not encourage a larger proportion of boys than average to opt for FLL in year 9, but it also seems to have put off a particularly large proportion of girls. Perhaps many students felt that they had had enough FLL, as suggested by NZCTE (1998). This could be the result of several factors, including the socioeconomic background of the Salvia students, the particular problems that the school had in retaining a French teacher, the number of more attractive options offered to the students, or simply that they had not enjoyed their previous FLL.

I attempted to assess the influence on students' option choices of the information available to them, through prospectuses or open evenings, for example. Among year 8 students who responded to item 8.1.24, "What I've seen about languages I could learn next year makes them look really interesting", $53 \%$ of girls $(N=143)$ agreed but only $40 \%$ of boys $(\mathrm{N}=151)$. While the gender difference was not significant it is another indication of more favourable attitudes towards FLL among girls. This is reinforced by the responses to a similar item put to all year 9 students which did produce statistically significant gender differences $(\mathrm{p}<.001)$, shown in Table 10.40.

Table 10.40 Responses by gender to survey item 9.2.5: "My school's prospectus made learning a foreign language look really boring."

\begin{tabular}{|c|c|c|}
\hline Item response & $\begin{array}{c}\text { Proportion of } \\
\text { girls }\end{array}$ & $\begin{array}{c}\text { Proportion of } \\
\text { boys }\end{array}$ \\
\hline Agree & $15 \%$ & $25 \%$ \\
\hline Disagree & $58 \%$ & $42 \%$ \\
\hline $\mathrm{N}$ & 376 & 251 \\
\hline \multicolumn{3}{|c|}{ For gender association, $\mathrm{p}<.001$} \\
\hline
\end{tabular}


It will be of concern to FL teachers that one quarter of boys might have been put off FLL by what they read about it in their school's prospectus, compared with only $15 \%$ of girls. When the association was analysed in more detail by school, it was found that the reactions of the boys to prospectus information about FLL varied significantly according to school $(\mathrm{p}<.005)$. The most negative responses were from boys at Salvia where 18 out of 37 agreed with the statement, and Begonia, where 20 out of 67 agreed that the prospectus had made FLL appear boring. While FLL remains largely optional in the secondary curriculum, these reactions must be a cause of concern to schools or teachers wanting to encourage more students to begin and continue it, especially those trying to attract more boys into FLL. On the other hand, it must be conceded that these negative attitudes may have already existed among the boys and have coloured their reaction to the information about FLL provided by their school prospectuses.

\subsubsection{The influence of teachers}

Year 8 boys tended to be more critical than girls about their FL teachers, as shown in Table 10.41. It is impossible to judge whether boys perceived real inadequacies that the girls tolerated more, or if they were reflecting a dislike of their teacher or their teaching methods. It was a boy who commented:

"They should teach us more instead of just making us write in books."

Table 10.41 Proportions of year 8 students reporting agreement or disagreement with survey items indicating reactions to FL teachers, by gender

\begin{tabular}{|c|c|c|c|c|c|c|c|}
\hline \multirow[b]{2}{*}{ Item } & \multirow[b]{2}{*}{ Statement } & \multirow[b]{2}{*}{$\mathbf{N}$} & \multicolumn{2}{|l|}{ Agree } & \multicolumn{2}{|c|}{ Disagree } & \multirow{2}{*}{$\begin{array}{c}\text { Gender } \\
\text { sig.* }\end{array}$} \\
\hline & & & Girls & Boys & Girls & Boys & \\
\hline 8.2 .12 & $\begin{array}{l}\text { Our language } \\
\text { lessons could } \\
\text { have been better } \\
\text { organised." }\end{array}$ & $\begin{array}{l}\text { Girls } \\
120 \\
\text { boys } \\
119\end{array}$ & $26 \%$ & $37 \%$ & $44 \%$ & $32 \%$ & None \\
\hline 8.2 .15 & $\begin{array}{l}\text { The things ... we } \\
\text { used in class } \\
\text { didn't really help } \\
\text { me learn. }\end{array}$ & $\begin{array}{l}\text { Girls } \\
117 \\
\text { Boys } \\
114\end{array}$ & $20 \%$ & $32 \%$ & $59 \%$ & $51 \%$ & None \\
\hline 8.2 .9 & $\begin{array}{l}\text { Our language } \\
\text { teacher made } \\
\text { learning really } \\
\text { fun }\end{array}$ & $\begin{array}{c}\text { Girls } \\
117 \\
\text { Boys } \\
119\end{array}$ & $50 \%$ & $49 \%$ & $20 \%$ & $33 \%$ & $\mathrm{p}<.04$ \\
\hline 8.1 .9 & $\begin{array}{l}\text { I'll try a language } \\
\text { next year but I } \\
\text { won't go on with } \\
\text { it if I don't like } \\
\text { the teacher. }\end{array}$ & $\begin{array}{l}\text { Girls } \\
146 \\
\text { boys } \\
153\end{array}$ & $16 \%$ & $28 \%$ & $64 \%$ & $54 \%$ & None \\
\hline
\end{tabular}


More boys than girls agreed that their language lessons could have been better organised (item 8.2.12), and more boys than girls agreed that the materials used in class were not very helpful for their learning (item 8.2.15). The boys were significantly more likely than the girls to disagree $(p<.04)$ that their teacher had made their FL lessons fun. This may reflect a greater dislike of FLL among the boys or a greater reluctance among the girls to criticise their teacher. According to students' comments, fun is certainly an important factor, and both girls and boys commented about how they enjoyed learning through games. However, boys were much less likely to offer comments, and tended to qualify their otherwise positive comments, for example:

\section{"Can be fun sometimes" \\ "Sometimes it's cool" ('cool' seems to be a synonym for 'fun')}

When looking ahead, the year 8 girls seemed to be appreciably more determined than the boys to carry on with FLL whatever they might think of their future teacher (item 8.1.9).

All these items seem to imply that the boys enjoyed FLL less than the girls, but also that the girls were more reluctant to criticise their teachers. Perhaps what was fun for the girls was not enjoyed as much by boys. Perhaps the general organisation and content of FL lessons suited the girls better, something that teachers need to consider carefully. There is clearly need for research into both teaching methods and materials in relation to the gender of students, especially at pre-secondary level, to ascertain if changes might encourage more boys to study FLs at secondary school.

Student attitudes to FLL could possibly be subtly influenced by the FL teachers they encountered in secondary schools. As discussed in section 8.7.1, the secondary schools involved in this research usually had only one person teaching any particular FL, and often only part of their time was devoted to FL teaching. The two largest schools, Begonia and Freesia, could each sustain only one full-time FL teacher, both male and both of Japanese. This might to some extent reinforce the perception that it was acceptable for boys to study Japanese, if not other FLs. In contrast, Gerbera had 3 full-time FL teachers, all female. Arum was able to employ full time French and Japanese teachers, but Salvia had only one full time teacher covering both languages and a part time French teacher. All four of these were female. In addition, most of the FL teachers which year 8 students had met were also female. Boys may be deterred from learning subjects which seem to be taught almost exclusively by females, especially if they find themselves in a minority in classes.

\subsection{Beyond year 9}

\subsubsection{Student intentions for year 10}

There was a significant gender difference $(p<.05)$ in the reported intentions for FLL in year 1.0, shown in Graph 10.13. The girls tended to be more decided for further 
FLL whereas the boys were more unsure. This could be partly the result of different proportions of each gender having already started their FLL or not, but, given the information from the schools about student numbers in FL classes beyond year 9, it could fairly safely be predicted that girls would continue to dominate in most cases and that the gender imbalance would increase in each succeeding year.

There was a marked difference in the intentions of students at Begonia and Gerbera. The girls were evenly divided between being certain that they would continue in year 10 or being unsure, $48 \%(\mathrm{~N}=96)$ in each group. Only 4 girls reported that they would definitely not be studying a FL in year 10. By contrast, only about a third of boys $(\mathrm{N}=44)$ reported that that they would be studying a FL in year 10 , and twice that number were uncertain, showing that FLs had a lower priority for the boys compared with other subjects. The usually high drop out rate from FL classes was also, according to the FL teachers at Begonia, a result of the school's policy of accelerating the top three streams, see 6.2.3, not necessarily a reflection of the boys' personal interests.

It is particularly interesting that among students of Japanese there was little difference in intentions between girls and boys. 30 out of 82 girls and 23 out of 59 boys intended to continue their FLL in year 10. In contrast, among those learning French $42 \%$ of girls $(\mathrm{N}=107)$, but only $27 \%$ of boys $(\mathrm{N}=67)$, reported that they would continue in year 10 .

\section{Graph 10.13 Student intentions for year 10 by gender}

(For gender association, $\mathrm{p}<.05$ )

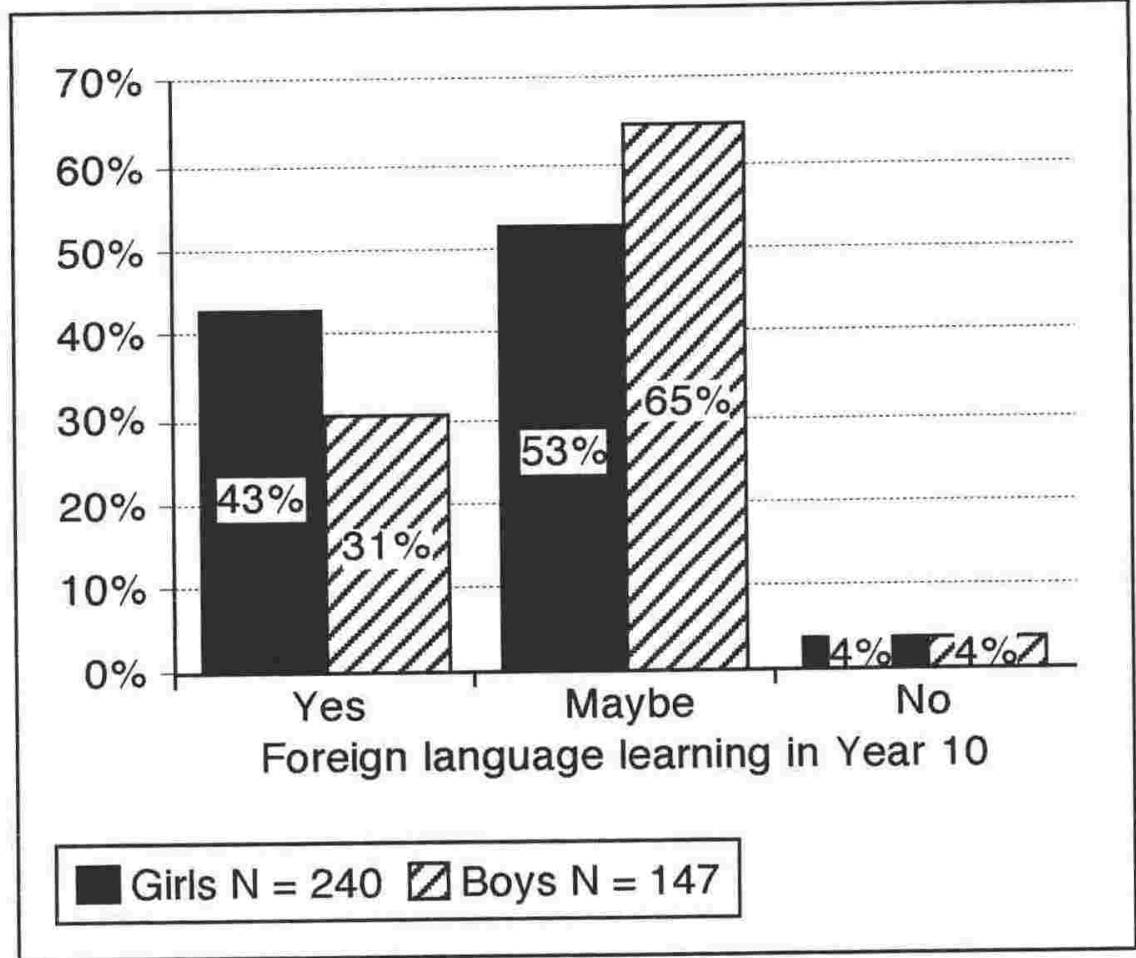


Among the small numbers studying German and Spanish the girls were more likely to intend further FL study than the boys. Thus, not only were the girls more likely to continue FLL than the boys but, except for a few keen individuals, the boys were only likely to continue if they were learning Japanese.

Students cited diverse reasons for intending to continue FLL year 10, see Table 10.42 , and interesting gender differences emerged. The boys were much more likely to attribute their intention to enjoyment of the subject. The girls tended to have specific aims of improving their knowledge and language skills or of travelling in the future.

Table 10.42 Reasons given for continuing FLL in year 10

\begin{tabular}{|l|c|c|}
\hline \multirow{2}{*}{ Comments } & \multicolumn{2}{|c|}{ No. of mentions } \\
\cline { 2 - 3 } & $\begin{array}{c}\text { Girls } \\
(\mathbf{N}=\mathbf{1 0 4})\end{array}$ & $\begin{array}{c}\text { Boys } \\
(\mathbf{N}=\mathbf{4 6})\end{array}$ \\
\hline Enjoying the subject & $43 \%$ & $59 \%$ \\
\hline $\begin{array}{l}\text { Wants to improve, develop } \\
\text { fluency, learn more }\end{array}$ & $35 \%$ & $22 \%$ \\
\hline For future travel & $17 \%$ & $11 \%$ \\
\hline
\end{tabular}

The girls showed significantly more positive attitudes $(p<.05)$ than the boys to the idea of learning more of the same FL the following year, see Table 10.43. The majority of the girls, 55\%, agreed with item 9.3.6, but a small majority of the boys, $46 \%$, were undecided. Boys were, proportionately, twice as likely as girls to indicate that they did not want to continue learning the same FL.

Table 10.43 Responses by gender to survey item 9.3.6: "I want to learn more of the same language next year."

\begin{tabular}{|c|c|c|}
\hline Item response & $\begin{array}{c}\text { Proportion } \\
\text { of girls }\end{array}$ & \begin{tabular}{|c|} 
Proportion \\
of boys
\end{tabular} \\
\hline Agree & $55 \%$ & $43 \%$ \\
\hline Not sure & $39 \%$ & $46 \%$ \\
\hline Disagree & $6 \%$ & $11 \%$ \\
\hline $\mathrm{N}$ & 234 & 149 \\
\hline
\end{tabular}




\subsubsection{Gender differences in general retention rates}

In terms of retention rates in FL classes beyond year 9, no detailed comparison could be made between the genders in the mixed schools as the teachers supplied only total class numbers. As discussed in 10.2.3 above, FL teachers reported that girls tended to predominate in European language classes, especially in years 12 and 13. Better gender balances usually continued in Japanese classes. One school reported that boys outnumbered girls in year 13 Japanese in 2000, a fact which was obviously sufficiently unusual to be worth mentioning.

What is apparent is the large discrepancy between senior student numbers at the single sex schools. Gerbera had by far the highest retention rates in FL classes of all the surveyed schools and combined classes for years 12 and 13 French and German had only existed since the previous year. At Begonia there were rarely classes available for students in year 12 and 13 except in Japanese, and that was likely to be a combined class. Senior students of French or German, if there were any, usually had to study by correspondence.

Table 10.44 shows the numbers in years 12 and 13 in 2000, as reported by the language HODs of the two schools. Except where indicated they were in combined classes. It is clear that the general organisation of Begonia and its attitude towards FLL were proving unfavourable towards the existence of senior language classes. As the HOD supplied figures for the previous three years it was possible to see that the pattern was typical. The general impression I gained from visits to the school was that FLs were not considered relevant for most boys and not really encouraged, although the principal claimed to be disappointed that more boys did not continue FLL.

Table 10.44 Students numbers in years 12 and 13 at single sex schools in 2000

\begin{tabular}{|l|c|c|c|}
\hline & \multicolumn{3}{|c|}{ Languages } \\
\hline School & French & German & Japanese \\
\hline Gerbera & 19 & 13 & $30 \diamond$ \\
\hline Begonia & $3 *$ & $1 \dagger$ & 8 \\
\hline $\begin{array}{l}*=\text { combined with year 11 class } \\
\dagger=\text { on correspondence } \\
\diamond=\text { in separate classes }\end{array}$
\end{tabular}

\subsection{Final analysis}

Some clear patterns of gender differences appear in the above results, showing that, as I hypothesised, girls in the year 8 and 9 samples surveyed had generally more favourable 
attitudes towards FLL. These attitudes seem to derive both from differing personal interests and from external influences, although, for the most part, students seemed unaware of such influences. Most of them did not perceive any gender differences in interest and ability in FLL, and the small number of parents I interviewed made no comments on the subject, but the teachers perceived discrepancies which my findings support.

Given completely free choices about optional subjects in year 9, more girls than boys chose FL options and intended to continue FLL. Senior FL classes were dominated by girls, except in Japanese. Girls reported that they found FLL more fun and less difficult than boys. Girls also showed more interest in other cultures and overseas travel. Although there were signs that many boys had an inherent interest in FLs, when it came to making choices, they tended to prefer more practical subjects. If they did opt into FLL, they preferred Japanese, mostly because they considered it useful for the future. Although girls who had not chosen a FL option also had other priorities they were much more likely than boys to regret not being able to learn a FL as well.

Patterns of FLL choices varied according to each school's organisation of the curriculum and their attitude to the value of FLL. In particular there were substantial differences between the single sex schools. Girls tended to be more favourably disposed to FLL than the boys, but the boys had more limited opportunities for FLL, as Holt et al. (2001) also found. Boys were generally more inclined to perceive, and at times resent, the pressure to learn a FL.

The gender differences revealed by my findings must be influenced by the whole range of complex factors discussed above and it is impossible to pinpoint which factors had the most influence over students' decisions about FLL. However, there is clear evidence that a lack of FLL experience prior to starting secondary school and parental hostility or, possibly, indifference to FLL had a particularly negative influence on the boys. The idea that FLL is a 'girls' subject', as shown by the range of literature discussed, is supported by most of the above evidence. More research would be valuable to discover why this continues to be the case. 


\section{CHAPTER 11 CONCLUSION}

The Japanese eat very little fat and suffer fewer heart attacks than the British, Americans or Australians.

The French, on the other hand, eat a lot of fat and suffer fewer heart attacks than the British, Americans or Australians.

The Japanese drink very little red wine and suffer fewer heart attacks than the British, Americans or Australians.

The Italians, on the other hand, drink a lot of little red wine and suffer fewer heart attacks than the British, Americans or Australians.

The conclusion to be drawn from this is that you can eat and drink what you like.

It's speaking English that kills you.

(Anonymous)

\subsection{Discussion of hypotheses}

\subsubsection{Reasons for choosing FLL}

Hypothesis 1. Students have a particular interest in language and languages.

Hypothesis 2. Students who choose to study a particular FL are more interested in learning about the target community or communities and their culture(s) than in how useful the language will be.

My findings largely support previous research in New Zealand (e.g. ERO 1994, Holt et al. 2001) and show that the major reason for studying a FL at secondary level was an interest in languages and other cultures, be it general in nature or interest in a specific FL. Another important reason was an interest in future travel, which is probably closely related to the former. Students tended to choose particular FLs mostly on the basis of their past experience, although Japanese was the most popular FL, especially for boys whose motivation appeared to be largely instrumental. Some students reported their interest in visiting a particular country or admitted to being influenced by family or friends. Many students wanted to continue something they had enjoyed at intermediate school, or to build on what they had already achieved. Students were much more likely to continue FLL beyond year 9 if they were enjoying their classes, were confident about making progress, were interested in learning more of the language and more about the people who speak it, and appreciated the value of the language skills they were developing.

Only a few students used the word 'useful' to explain why they had chosen a FL option, although it could be assumed that they had that in mind when they reported their wish to travel overseas. As far as future employment was concerned, year 9 students were much more likely to opt for FLL if they thought that knowing a FL would help them obtain a good job. Students appreciated that FLL could give them a better under-standing of other cultures and of English. In general, students' interest in FLL was sufficiently strong for them to pursue it, whatever their friends or family thought about it, or whatever they thought about the teaching. 
Overall it appears that my first hypothesis was correct but that hypothesis 2 was only partly true, and only for some students. The preference for Japanese seems to have been dictated to a large extent by instrumental motives or extrinsic factors, not necessarily by students' interest in the Japanese language or culture.

\subsubsection{Reasons for not choosing FLL}

Hypothesis 3. Most students do not see the need to learn a FL for the purposes of future employment.

Hypothesis 4. Students think that languages are 'difficult' subjects and therefore only suitable for brighter ones.

The idea that FLL might be a waste of time was barely mentioned by year 8 students, but they were much less likely to opt for FLL if they thought there were more important subjects to study, if they thought they should concentrate on improving their English and if they believed their parents thought the same. A general lack of interest in FLL seemed to be a major factor in student decisions. If neither students nor parents had any experience of FLL the possibility of the students, especially boys, undertaking it in year 9 without some degree of pressure from schools was small. In fact, only a minority of year 9 students believed that there were many more important things to learn than FLs, that learning Maori is more important than learning a FL, that everybody speaks English these days or that knowing a FL would not help them get a good job. Although these beliefs correlated significantly with the decision not to choose a FL option, the proportions of these students who nevertheless opted into FLL was never less than $40 \%$. This lends more support to my first hypothesis, although it might also be explained by the demands of their particular secondary schools.

The fact that FLL remains optional signals to students, especially those in whom an interest in other languages and culture has not been aroused, that it is not important. Teachers reported considerable pressure on schools these days to prepare students for future employment, which can detract from their role of providing a broad general education. There is, in addition, limited understanding of the skills and knowledge to be gained from FLL among students, non-language teaching staff and employers. Although very few students indicated specifically that FLL would not help them obtain employment, hypothesis 3 is largely supported by my findings.

The idea that FLL might be too difficult was barely mentioned by year 8 students as a reason against future FLL, but they were significantly more likely to intend to study a FL in year 9 if they thought they would do well in it, whether or not they had any FLL experience. Only a minority of the whole year 9 sample, with or without FLL experience, agreed that FLL was too hard for most students, but those who did so were significantly more likely not to opt for FLL in year 9. Many more students claimed that FLL would be boring, which, as discussed in chapter 6 , might have been another way of saying that it would be difficult. However, nearly half of them 
did not agree that knowing more than one language is confusing, and less than one quarter agreed that learning a FL would be too difficult for them personally. It is possible that more students than those willing to report it may have been dissuaded from studying FL by a belief that it would be difficult, but, in any case, nearly half of them were making relatively uninformed judgments.

The notion that FLL is only for the most intelligent students was strongly reinforced by the boys' school which put pressure on top stream students to learn a LOTE and made FLL virtually unavailable for other boys. Among year 9 students in all the surveyed schools who were learning a FL only a small minority indicated that they were finding it confusing or were pessimistic about their likely progress. It is not possible to tell if this was because the majority of FL learners were above average academically, as the teachers reported, or if it was the case that, given opportunity and encouragement, and presumably good teaching, most students did not find FLL any harder than other subjects. Hypothesis 4 is only partly supported by findings since the attitude that FLL is only for brighter students seems to come from the adults more than the students.

The current situation in New Zealand seems to resemble closely that of the USA nearly thirty years ago, identified by Turner (1974) who attributed the poor take-up of FLL and high drop-out rates can be to lack of motivation, lack of parental interest, and lack of institutional support. In the context of this research, however, lack of institutional support appears to outweigh other factors, as will be discussed in section 11.1.6 below.

\subsubsection{Gender differences}

Hypothesis 5. Girls have more favourable attitudes towards FLL than boys.

As discussed at the end of the previous chapter, this hypothesis is largely supported by my findings. The differing attitudes among girls and boys were not openly stated, nor even recognised by most of the students themselves, but revealed indirectly by many of the research results. The most disturbing finding is that, despite positive attitudes, year 9 students were often dissuaded or prevented from choosing a FL option because of the limited opportunities available to them, and this particularly applied to boys in the single sex school. Differing attitudes may be partly the result of a 'chicken and egg' situation. If boys find themselves in the minority in language classes they are likely to see FLL as a 'girls' subject'. While the majority of students who continue FLL in senior classes and at tertiary level are female, the majority of FL teachers will continue to be female. As long as there are schools which perpetuate the belief that FLL is suitable only for the most intelligent students, FLL will remain undervalued by the majority of the population. If, in addition, the schools which take this attitude are mostly boys' schools, then FLL will remain a 'girls' subject'. My findings offer some cause for optimism in terms of restoring the gender balance. Having an enjoyable and successful experience of FLL in year 7 or 
8 had a particu-larly positive effect on the decision of boys to pursue FLL in year 9, especially the study of Japanese.

\subsubsection{Parental influence}

Hypothesis 6. Parents' attitudes towards FLL in general, based on previous experience or lack of it, are an important influence on student attitudes.

Hypothesis 7. Parents' attitudes towards specific languages they have studied have an influence on student attitudes.

The influence of parents on the intentions of year 8 students and the decisions of year 9 students were largely unperceived by the students. However, analysis showed that, indirectly, parents had a strong influence over students' attitudes towards FLL, as shown by previous research, (Oskamp 1977, Gardner 1985, Williams \& Burden 1997), and probably reflected the generally indifferent attitudes of New Zealand society towards the value of FL skills (Enderwick \& Gray 1992, Johnson 1998). Students were more likely to opt for FLL if they thought their parents were in favour of it. They were much less likely to do so if their parents had had no FLL experience themselves, if they had shown no interest in FLL or had expressed disapproval of FLL for their children. Thus my findings seem to support hypothesis 6.

If parents had studied a FL at school it was most likely that they had had no choice in the matter and that they had studied French. Their attitudes to FLL for today's students may have been coloured by the extent to which they had enjoyed learning French or found it of use in later life. However, lack of parental FLL, rather than a negative experience, seemed to have had a greater influence on student choices, although not all parents who lacked FLL experience were indifferent or hostile towards it. Some of those interviewed felt that a major problem was their inability to give their children informed advice about FLL, especially if their children also lacked experience of it. They recognised that opportunities for FLL had widened and that the nature of FLL had changed, but they felt unsure about its value for their children. Hypothesis 7 remains largely unproved.

\subsubsection{The influence of prior experience}

Hypothesis 8. Prior experience of FLL may improve a student's attitudes towards FLL but positive attitudes may not necessarily encourage continued study.

Prior FLL experience per se did not seem to have a strong influence on year 8 students' intentions since, typically, about half the students intended to study a FL in year 9, whether or not they had such experience. They were not deterred from further FLL by the idea that they had learnt enough and did not need to continue, contrary to what advisers have surmised (NZCTE 1998). However, the lack of FLL experience produced a larger proportion of students definitely not intending to study a FL in year 9. Thus prior experience may have had a slightly positive effect on student intentions. Closer analysis showed that students were significantly more 
likely to intend further FLL if they:

- had enjoyed their language learning in year 7 or 8;

- considered their language classes had been well organised;

- wanted to learn more of the same FL; or

- wanted to know more about the people who speak the FL studied, or actually meet them.

Just over half the year 9 sample had learnt a FL in years 7 and/or 8 and over $70 \%$ of them had enjoyed that experience. Prior experience did not increase the uptake of FLL in year 9, but students were much more likely to choose a FL option if they had enjoyed their experience, and even more likely to do so if they thought they would do well in the subject. These findings were confirmed by the analysis of the sub-sample surveyed in both years, where it was possible to compare the year 8 intentions with their actual choices in year 9. Prior FLL experience had a positive effect not only on students' attitudes but also on decisions about continuing FLL in year 9, providing that their experience was positive. To some extent hypothesis 8 is disproved.

\subsubsection{The role of attitudes in students' choices}

Hypothesis 9. Ultimately, attitudes towards FLL play only a minor role in final subject choices for Year 9.

The majority of all students revealed positive attitudes towards the idea of studying a FL and towards any FLL experience they had had. Positive attitudes were also generated by contact with LOTE speakers, either through friends and family overseas or through travel, and the minority of students with such contacts were more likely to opt for FLL than others. In response to the hypothetical question of which language they would like to learn if they had a free choice, nearly three quarters of year 8 students expressed an interest in FLL. Nevertheless, nearly one quarter of those with positive attitudes towards FLL in general did not intend future FLL.

Analysis of the data relating to year 9 students who had not opted for FLL shows clearly that, for many students, however positive their attitudes or whatever their previous experience of FLL, other external factors outweighed these in their choice of option subjects. Many students who had decided against FLL, may not have considered the possibility, for lack of experience or parental encouragement, but one third of them reported that they would like to have studied a FL, and most of those indicated that they would have done so if their timetables had allowed it, as language teachers and advisers have found (NZCTE 1998). One third reported that they had preferred other options, nearly half of whom had chosen Maori. Nearly half of these students thought that it would be fun to learn a LOTEM and over half of them wished to learn one in the future for reasons of interest in a particular language or culture, or a desire to build on previous FLL which they had enjoyed. Thus a substantial number of students with positive attitudes felt excluded from FLL by timetable constraints. 
Such constraints varied from school to school and the intentions of year 8 students with regard to FLL in year 9 were closely related to the school they expected to attend. About one quarter of year 9 students reported that they were only studying a FL because it was compulsory, although it did not necessarily mean that they were unhappy about this. The varied uptake of FLL in different schools might also have been due to differing socio-economic backgrounds. Parents of higher socioeconomic status seemed to prefer the single sex schools, but this does not explain the great disparity in the status and uptake of FLL between the two single sex schools, unless the parents generally support the view that FLL is more suitable for the most intelligent students and/or girls. This would be a particularly interesting area for future research.

External factors were also clearly influencing students' choice of FL. Many year 8 students expressed a desire to learn a FL which would have been possible, but only a minority availed themselves of the opportunity. Students who had learnt Japanese were most likely to continue FLL and most, with or without previous FLL experience, especially boys, intended to study Japanese, even if their ultimate preference was to learn another FL, and even if that was possible. Year 8 students undecided about FLL or their choice of FL might have been waiting to see what combinations of options were possible in year 9. The majority of year 9 students were happy with the FL they had chosen to study. Those who were not might simply have been disappointed in their classes for some reason, but it might also mean that an external factor, such as a timetable clash or parental influence, had prevented them from opting for their first choice.

All the evidence from this research shows that there were numerous, interlinked influences on student attitudes towards and choices made regarding FLL. Figure 2 (see Appendix 14) attempts to show the interrelationship between the main factors. The degree of boldness of elements in the diagram indicates the strength of the influence of these elements, as suggested by my research findings which support what many social psychologists have found - that attitudes may not be directly related to behaviour (Fishbein \& Ajzen 1975), and that measuring attitudes will not necessarily lead to an accurate prediction of actions (Cooper \& Fishman 1974). In particular, students need the opportunity to act on their positive attitudes because:

People who believe that they have neither the resources or the opportunities to perform a certain behavior are unlikely to form strong behavioral intentions to engage in it even if they hold favorable attitudes toward the behavior and believe that important others would approve of their performing the behavior. (Ajzen 1988:133-134)

This research shows that New Zealand students do not always have the opportunities they would like to pursue FLL. 
It is not clear that any of the factors discussed could be considered the predominant influence on students' decisions, but schools seemed to be discouraging FLL by leaving FLs to compete with other subjects and by not advising students about the value of FLL, no matter how much individual teachers, including principals, reported that they valued it. From secondary principals' accounts it was clear that schools had other priorities in terms of curriculum content, timetabling and resources. For New Zealand school students, on the whole, contextual factors seem to outweigh all but the most positive attitudes towards FLL. Positive attitudes alone, of students, parents or teachers, may not ensure that students decide to study a FL at secondary school. The hypothesis that attitudes play only a minor role in final subject choices is supported by my research, as it has been by others (ERO 1994, Williams \& Burden 1997, Johnson 1998).

\subsection{The outlook for the future - official encouragement for FLL in schools}

\subsubsection{The latest initiative}

The publication of Learning Languages: A Guide for New Zealand Schools (MoE 2002b), developed with the assistance of NZALT and launched at their 2002 national conference, provides a wealth of resources for schools which want to introduce FLL or consolidate their existing programmes. The guide incorporates advice based mostly on Australian experiences of developing LOTE programmes and offers assistance to teachers and Boards of Trustees for 'selling' FLL to their communities, as well as practical advice for curriculum and class planning.

\subsubsection{Support for Asian languages}

Indirectly the government continues to support the learning of Asian languages through Asia 2000, an organisation also supported by large private companies, such as Fonterra, Air NZ and the Wool Board, which shows that some enterprises do value FL skills and cultural understanding, at least for relations with Asian countries. From time to time Asia 2000 offers teachers financial assistance for professional development, resources or student activities. The latest announcement (Asia 2000 2002) specified that equal consideration would be given for grants towards the teaching of Bahasa Malay, Indonesian, Japanese, Korean and Mandarin Chinese.

\subsubsection{The Second Language Learning Project}

Schools continue to become involved in the SLLP and the MoE has invited applications for funding for 2003 (MoE 2002a). Funds remain contestable with proposals undergoing a rigorous selection process. In 2001 only half the schools which applied received funding, and not always as much as they requested. Nor was money guaranteed for future years, so it remains uncertain whether schools whose schemes are accepted for the SLLP will continue L2 teaching funds disappear.

According to Spence (2001), the development of resources for teaching Spanish, Japanese, French and German for years 7 and 8 have been "very successful in introducing more widespread second language learning than has previously been the 
custom at this age". Unfortunately, she adds, retention rates and achievement levels have not improved because it has proved impossible to plan coherent and progressive programmes for reasons including the following:

- second language learning is not compulsory;

- time-tabling is extremely varied, flexible (often giving way to other events) and of ten makes choosing a language difficult;

- teaching is not continuous.

Nevertheless, materials development continues and Chinese should be available in 2004 (Spence 2002). The MoE is developing case studies to be available on-line for schools to see what can be achieved through the SLLP. Lack of coordination between the primary and secondary sectors remains problematic, despite ongoing efforts of the language advisers, and there is continued hostility from some secondary FL teachers. Despite the recommendations of Peddie et al. (1999) there seems to be no ongoing evaluation of the SLLP, especially to ascertain if an earlier start to FLL is affecting student numbers in year 9 and beyond.

\subsubsection{The new national qualifications}

The National Certificate of Educational Achievement (NCEA) which replaced School Certificate in 2002 is available in a wide range of languages: French, German, Spanish, Chinese, Japanese, Korean, Maori, Samoan, Cook Islands Maori and Indonesian. Thus qualifications are in place, at least at level 1 (aimed at year 11 students), for students who want to learn a language and have official recognition of their achievement. Unfortunately, the existence of the achievement standards in no way guarantees that students will have access to learning any of these languages at any stage of their schooling. In addition, development of consistent achievement standards across all languages has proved extremely difficult because of wide variations in the curriculum documents. Spence (2001) sees the need to "seek the development of a languages curriculum framework for all languages".

\subsubsection{The curriculum stocktake}

A complete revision of the NZ curriculum began in 1991. At the time, Capper (1991) claimed that debate about LOTE learning resembled debate about the purposes of education as a whole, in the absence of any clear community view on the subject. He identified three broad arguments for FLL:

- commercial reasons;

- educational reasons - FLL helps to improve overall cognitive development;

- cultural reasons - for maintenance of community or indigenous languages. Capper asserted that the government would be most supportive of FLL for commercial interests, but that their support would be pointless if the business community were not persuaded to value language skills when employing people. According to Capper, "Industry demands pre-packaged linguists, or at least it says it does" (1991: 3), and FL skills are rarely taken into account in recruitment, even combined with other skills and qualifications. The lack of employment opportunities 
might result in a decline in FLL in schools and at tertiary level. Capper could see "no concerted effort by anybody to clearly state what the issues in language learning are in a way which helps the lay public to sort out the contradictions" (1991: 6).

Supporters of FLL hope that the curriculum stocktake will address such contradictions and clarify the role FLL as well as improve its status in schools. Many teachers and some of the parents interviewed for my research identified FLL as a means of addressing one of the principal weaknesses they saw in the current education system - that students, especially at intermediate level, are not being stretched enough.

The curriculum has undergone extensive revision during the last decade. Since 2002, schools have had what the MoE considers to be the complete set of Englishmedium curriculum statements for the essential learning areas. This, however, includes only 'gazetted' statements (documents which have to be adhered to by schools for years 1-10). It does not include curriculum statements for LOTEs.

The MoE decided to carry out a complete stocktake of the curriculum over two years, starting in 2000, with the aims of investigating how it has been implemented, how it is working and how it compares with overseas practice (MoE 2001b). The stocktake was to "focus on how to ensure the curriculum is effective in schools and how to maintain and update it so it remains effective at national and local levels" (Velde 2000). In particular, the stocktake was to examine the issue of the "crowded curriculum', evidence for which was already identified by Marshall (cited in Velde 2000). One cause of this problem was seen to be the introduction of the curriculum statement for technology which had to be implemented by all schools. Some schools have integrated technology into other curriculum areas, but many have created a separate compulsory element in timetables with the result that:

Increasingly, Year 9 and 10 students have less time for options in their courses - a major headache for schools that offer foreign languages. (Marshall cited in Velde 2000)

The MoE (2000) report on the progress of the stocktake produced a review of literature concerning curriculum developments since 1990. The section entitled Manageability begins:

Most of the anecdotal evidence from teachers, and the correspondence to the Minister on curriculum matters, has been centred around the manageability or crowdedness of the curriculum. The ongoing implementation of the curriculum has been perceived as difficult, particularly in the timetabling...

The review reports comments made about six essential learning areas of the curriculum but mention of any LOTEs is conspicuous by its absence. It seems to be assumed that the English curriculum statement represents the whole essential learning 
area of Language and Languages. Although several ERO reports are cited, their 1994 report on language learning is not mentioned. ERO itself (2001) produced two reports relating to the curriculum stocktake. The first deals with the curriculum as a whole and how it translates into classroom programmes and student achievement, and the extent to which it meets society's needs at national or local level. The second examines the seven National Curriculum Statements, that is only the statements gazetted for years 1-10. Yet again, Language and Languages is represented solely by the English curriculum statement.

Eventually, in 2001, the MoE acknowledged that there is concern about the place of LOTE learning in the curriculum. A statement, released to give background details about curriculum revision since 1991 and the aims of the stocktake, declares that it will "investigate a number of problems/issues associated with the NZ curriculum and its development which have been raised in and outside the [education] sector". There follows a list of nine issues, the last of which is "the status of second language learning" (MoE 2001c).

As part of the stocktake a series of meetings was held in 2001 to discuss the effectiveness and appropriateness of each essential learning area and the relevant curriculum statements. I was fortunate to be invited to participate in the day of discussions about the Language and Languages area, among a wide range of subject experts from all parts of the education sector and some representatives of the broader community, such as employers.

Participants were divided into three groups - English, Maori and 'the others', representing a wide range of languages, including Indonesian and Latin. Some discussions were held in these groups and some in mixed sessions. The 'others' were informed that the issue of the status of SLL related to:

- the value of SLL in the curriculum;

- teaching methods according to starting age;

- teacher training; and

- possibilities of curriculum integration, e.g. content-based instruction.

All participants were invited to submit a written list of key issues which, they were assured, would be included in the final analysis.

Very strong feelings were expressed by participants representing LOTEMs that there was a need for "policy and coherent government strategy to support and fund two separate essential areas: (i) languages on instruction, English and te reo Maori, and (ii) other languages" (MoE 2001a: 2) because all LOTEs felt marginalised and New Zealand has a growing need of at least bilingual, if not multilingual, people. Spence (2001) reported that the MoE accepts that current policy concerning LOTEs is an "opportunity-to-learn-policy" and that consequently teaching varies widely from school to school. The Ministry view was reiterated at the 2002 NZALT Annual Conference (Spence 2002), acknowledging that FLL opportunities are not equal for 
all students. Some schools believe in the importance of L2 learning and give it some prominence; others tend to say that it is impossible to do everything, so they will do only what is mandated. A monolingual school community may not see the advantages of SLL.

As part of the curriculum stocktake two overseas educational research bodies, in the UK and Australia, were invited to review the New Zealand curriculum. These independent reviews were largely favourable but both noted the low priority given to FLL. In particular, the UK body recommended that "consideration be given to building on New Zealand's bilingual culture to motivate and support students to learn further languages at school and beyond." (Evans 2002).

\subsection{The remaining major barriers to FLL}

\subsubsection{The lack of continuity between primary and secondary education}

This issue was to be investigated by the curriculum stocktake with regard to all areas of schooling (e.g. Aikin 2001). Continuity has been a major concern of the MoE since it offered a vision of a 'seamless education system' (MoE 1993a, Hawk \& Hill 2000), and the transition from year 8 to year 9 was an important focus of the ERO (2001) review of the curriculum. Lack of continuity has been identified as a major barrier to the development of effective SLL programmes, despite positive moves by schools, advisers and the MoE which has stressed the:

need to arrange for all students to be able to continue their learning as they move between schools. In this way, students will achieve positive learning outcomes and so be motivated to continue. (MoE 2002b: 34)

\subsubsection{The lack of compulsory LOTE learning}

One obvious way to overcome the problems of the current situation, as suggested by Spence (2001) and supported by most FL teachers and many of the adults interviewed for my research, is to make SLL a compulsory part of the years 7-10 curriculum. This would be greatly assisted by adopting an eighth essential learning area for LOTEs, as exists in Australia. Optional subjects are, by definition, nonessential and tend to be marginalised where timetables are under pressure and the core curriculum seems to be expanding.

Since, in social psychological terms, SLL offers experiences that cannot be gained by studying any other subjects, it might be inferred that, when students choose optional subjects, they are not comparing like with like when considering SLL against other subjects. Furthermore, without expert guidance, students may not have a realistic notion about what SLL entails. Without prior experience students faced with a bewildering range of option subjects are not making informed choices, a point supported by many of my interviewees. Many people, within and outside education circles, still perceive SLL to be too hard for the majority of students, or that it is 
simply of no use to them. Such preconceptions, could effectively be countered by giving all students at least some sort of 'taster' courses in FLL before they start secondary school, so that both they and their parents have a good idea of what SLL involves and can make more informed choices so long as it remains optional.

If a certain amount of SLL were to be mandated, schools would need long-term support to accommodate it. An initial reaction would inevitably be that it was yet another element to squeeze into an already overcrowded core curriculum. This overcrowding may be the result of attempts to extend the curriculum to match the breadth of education existing in other highly educated countries with which NZ likes to compare itself, while not requiring that children spend the equivalent amount of time in school. On the other hand, there are schools with innovative programmes which manage to incorporate all the compulsory elements through a degree of integration, rather than trying to timetable all core elements separately.

The positive effects on student attitudes towards FLL of enjoyable and successful intermediate FLL, shown by my research, have implications for FL teaching at intermediate level. There needs to be ongoing research into the suitability and effectiveness of methodology and resources for these students with regard to both achievement levels and attitudes.

My findings also support what many FL teachers feel: that the uptake and continuation of FLL at secondary level depends almost entirely on their efforts to attract and retain students, without a great deal of institutional support. In fact, with the increase of FLL at intermediate level while secondary FLL remains optional, this responsibility seems to be shifting onto intermediate FL teachers. The latter are receiving a great deal of support from the advisory service but are not always equally supported by their secondary counterparts, largely owing to a lack of communication, which is a feature of the gap between intermediate and secondary education discussed above.

\subsubsection{The lack of public support}

If the country seriously wants to improve available language skills and cultural understanding it must develop clear and coherent policy about the place of SLL in schools, combined with long-term planning for the development of teachers and resources. In addition, the government would need to undertake a campaign to educate the wider public, especially employers, about the value of SLL. Efforts to interest the wider public in FLL still depend largely on FL teachers and organisations such as the Alliance Française and the Goethe Institute. They were responsible for the International Languages Week held from 14-20 July 2002, one of the main aims of which was:

To shift the thinking of all the New Zealanders who think they can get by just with English, and open their minds to other languages (NZALT 2002: 15). 
FL teachers could be seen to be pushing their own barrow, that is, trying to save their jobs, but they would argue that they believe that FLL is an essential part of a good, general education for all students, as implied in the Curriculum Framework (MoE 1993b: 10).

Even the introduction of a compulsory element of FLL at, say, intermediate level, will not guarantee that any more than the usual dedicated few students will continue FLL throughout their schooling, or beyond. For this to happen, there is probably a need to develop more demand for language skills in employment. My research has shown very positive attitudes to FLL in general, but students and their parents need to see the education and employment sectors demanding FL skills to give them the necessary incentive to pursue FLL. Some valuable proposals to promote FLL were presented by Watts (1991) as a result of his research:

- FL students should be encouraged to do business studies;

- businesses should support FL programmes with business applications;

- tertiary institutions need to extend opportunities for combining relevant courses;

- business needs to understand cost-effectiveness of training language graduates rather than improving language skills of business graduates;

- businesses should offer incentives for increasing language skills.

Judging from what careers guidance counsellors in schools reported during this research not much progress has been made towards a more general recognition of the value of FLL.

\subsection{Suggestions for further research}

If FLL remains optional in New Zealand schools, and if it is considered desirable to increase the number of students developing FL skills, further long-term research needs to be undertaken into the effects of the SLLP, as Peddie et al (1999) recommended. Specifically, it needs to be ascertained if an earlier start to FLL increases the number of students choosing to study a FL at secondary level, and, perhaps more importantly, whether more students continue FLL beyond year 9. It is not clear why FLL continues to be a 'girls' subject' in New Zealand and elsewhere. The domination of FLL by female students would therefore be a valuable subject for further research. Similarly, the continuing belief in some sectors of the community that FLL is only possible or desirable for the most able students would be an interesting area of investigation.

\subsection{Final thoughts}

It is forty years since UNESCO issued the following statement:

a second language must become part of the total education experience process, not something reserved for the gifted, but a normal educational experience for the ordinary child (cited by Watts 1991)

It remains to be seen what the final outcomes of the curriculum stocktake will be, and if there 


\section{6}

is any change to the place of FLL in schools. Supporters hope that the government will address the wider issue of what constitutes a good broad education for the increasingly globalised world, and that the role of SLL will seen to be an essential part of that education. It is encouraging that the Prime Minister, Helen Clark, has expressed concern that most New Zealanders speak only one language and believes the country needs to look at a national language strategy that would emphasise learning languages at school and in the curriculum:

It's going to be good for us commercially and good for us in terms of building our ties with the rest of the world. In a sense it doesn't matter whether that language is Spanish, Chinese, Japanese, Korean, French of Italian. The key thing is that make an effort to communicate in other people's languages. (New Zealand Newspapers Association 2001)

As Clark's government was re-elected in 2001 there seems room for some optimism that a more positive language-in-education policy may be developed in the not too distant future.

My research has uncovered a wealth of positive attitudes towards the value of FLL among students, parents and teachers, despite the pessimistic views of many of the latter and the institutional obstacles faced by students. Although the research was limited to only one small part of the country and the findings cannot be said to represent attitudes in any other areas, it seems to me that any move by the government to improve the standing of SLL, and more particularly FLL, in schools would receive a great deal of support.

I began this research from a strong personal interest in SLL and a belief in its importance for the education of all students. My feelings are best summed up by the words of one of the regional international languages advisers:

I believe passionately in the value of learning other languages, whether they be indigenous, community or international. A person growing up in a monolingual, monocultural environment is deprived of the wonder of new worlds. Language teachers provide links to a global society; they are often the catalyst needed to engender a love of things different, a love of things new and exciting. The contribution of International Languages teachers to a school community is therefore much more than teaching French or German or Spanish or Japanese. They teach a life style, enlightened attitudes and values of tolerance and acceptance of difference. They teach students to think and experience internationally. (Gilbert, cited in NZCTE 1998: 64)

\section{He taonga te reo katoa}

The learning of all languages is to be treasured

(Whakatauki - Maori proverb) 


\section{REFERENCES}

Ager, Dennis, George Muskens and Sue Wright (eds.) 1993. Language Education for Intercultural Communication. Clevedon: Multilingual Matters.

Agnihotri, R. K., A. L. Khanna and I. Sachdev 1998. Social Psychological Perspectives on Second Language Learning. New Delhi: Sage.

Aikin, Sandra 2001. Initial comments on transition from primary to secondary school. Retrieved 6/9/01 fromhttp://www.tki.org.nz/r/stocktake/ postings/initial_e.php.

Ajzen, Icek 1988. Attitudes, Personality and Behavior. Milton Keynes: Open University Press.

Allen, Edward D. 1975. The teacher as catalyst: motivation in the classroom. In Frank M. Grittner (ed.), Student Motivation and the Foreign LanguageTeacher. A Guide for Building the Modern Curriculum. Skokie, Illinois: National Textbook Co, 1-10.

Altman, Howard B. 1974. The making of a precedent: foreign language education. In Frank M. Grittner (ed.), Careers, Communication \& Culture in Foreign Language Teaching. Skokie, Illinois: National Textbook Co., 12-25.

Aplin, Richard 1991. Why do pupils opt out of foreign language courses? a pilot study, Educational Studies 17 (1), 3-13.

Ashenden, Dean and Sandra Milligan 2001. Mind your languages, Education Age April 4 2001, 8-9.

Asia 2000 2002. Asia 2000 funds awards call for proposals, Polyglot 25,43.

Australian Bureau of Statistics 2001. 2001 Yearbook. Canberra: ABS.

Australian Education Council 1989. The Hobart Declaration on Schooling. Canberra: AEC.

Australian Language and Literacy Council (ALLC) 1996. Language Teachers: The Pivot of Policy. The Supply and Quality of Teachers of Languages Other Than English. Canberra: AGPS.

Bacon, Susan M. and Michael D. Finnemann 1992. Sex differences in self-reported beliefs about foreign-language learning and authentic oral and written input, Language Learning 42, 471-95.

Bailey, Kenneth D. 1987 (3 ed.). Methods of Social Research. New York: Free Press.

Baker, Colin 1988. Key Issues in Bilingualism and Bilingual Education. Clevedon: Multilingual Matters.

Baker, Colin 1992. Attitudes and Language. Clevedon: Multilingual Matters.

Ball, Peter, Howard Giles and Miles Hewstone 1984. Second language acquisition: the intergroup theory with catastrophic dimensions. In Henri Tajfel (ed.), The Secret Dimension Vol. 2. Cambridge: Cambridge University Press, 668-94.

Baranick, William A. and Paul L. Markham 1986. Attitudes of elementary school principals toward foreign language instruction, Foreign Language Annals 19 (6), 481-9.

Barber, Faith 1990a. Sharing the secrets of success, Export News April 1990, 23-27. 
Barber, Faith 1990b. The right word works wonders in the target market, Export News May 1990, 4-7.

Barber, Faith 1990c. Marketing strategy: bridging the cultural gap, Export News August, 9-13.

Barrett, M. and J. Short 1992. Images of European people in a group of 5-10 year old English schoolchildren, British Journal of Developmental Psychology 10, 339-363.

Bauer, Winifred 2001. Personal communication.

Beard, Joe L. 1984. Attitudes of secondary school counselors and superintendents toward foreign language teaching: a descriptive study, Foreign Language Annals 17 (1), 2932 .

Bell, Terrel 1981. Secretary Bell encourages study of non-English Languages in challenge to the States, Foreign Language Annals 15 (3), 219.

Benton, Richard A. 1996. Language policy in New Zealand: defining the ineffable. In M. Herriman and B. Burnaby (eds.), Language Policies in English-Dominant Countries. Clevedon: Multilingual Matters, 62-98.

Birch, Cyril 1971. Why a foreign language breadth requirement? In James W. Dodge (ed.),The Case for Foreign-Language Study. New York: Northeast Conference on the Teaching of Foreign Languages, 26-27.

Board of Studies (Victoria) 2000. Languages Other Than English. Curriculum and Standards Framework II. Carlton, Victoria: Board of Studies.

Boekman, Barry 1998. The serious state of language learning in New Zealand, New Zealand Association of Language Teachers Members' Newsletter 14, 17-19.

Boston, Carol 1998. Federal support for foreign language education, ERIC Review. K-12 Foreign Language Education 6 (1). Retrieved 15/4/99 from http://www.accesseric. org/resources/ericreview/vol6nol.

Bouffard, Edward N. 1980. Foreign language study in the U.S., Foreign Language Annals 13(4), 309-314.

Bourhis, Richard Y. and Howard Giles 1977. The language of intergroup distinctiveness. In Howard Giles (ed.), Language, Ethnicity and Intergroup Relations. London: Academic Press, 119-135.

Bowen, Eugene 1990. Export News April 1990, 18.

Bradac, James J. and Howard Giles 1991. Social and educational consequences of language attitudes, Moderna Sprak 85 (1), 1-11.

Brannon, R. Marshall and David E. Cox 1976. Coping with the real problems in the secondary schools. In Gilbert A. Jarvis (ed.), An Integrative Approach to Foreign Language Teaching: Choosing Among the Options. Skokie, Illinois: National Textbook Company, 151-199.

Brickell, Henry M. and Regina H. Paul 1982. Ready for the '80s? A look at foreign language teachers and teaching at the start of the decade, Foreign Language Annals 15 (3), 169-187.

Broadcasting Publications, Inc. 1994. Broadcasting and Cable Yearbook. Washington, D.C.: Broadcasting Publications. 
Brown, Kim 2000. Conclusion: Creative thinking about a new modern languages pedagogy. In Simon Green (ed.), New Perspectives on Teaching and Learning Modern Languages. Clevedon: Multilingual Matters, 183-194.

Brumfit, Christopher 2000. Modern languages within a policy for language in education. In Simon Green (ed.), New Perspectives on Teaching and Learning Modern Languages. Clevedon: Multilingual Matters, 94-104.

Bryant, William H. 1978. Publicity: key to higher enrollment, Foreign Language Annals 11 (1), 9-14.

Bugos, Thomas J. 1980. Defending the FL requirement in the liberal arts curriculum, Foreign Language Annals 13 (4), 301-306.

Burch, Sylvia 1999. Personal communication.

Burns, Robert, B. 1997 (3 ed.) Introduction to Research Methods. Melbourne: Longman.

Burstall, Clare 1979. Primary French in the balance. In J. B. Pride (ed.), Sociolinguistic Aspects of Language Learning and Teaching. Oxford: Oxford University Press, 136-143.

Busch, Michael 1993. Using Likert scales in L2 research: a researcher comments ..., TESOL Quarterly 27 (4), 733-736.

Calderon, Angel J., Ian R. Dobson and Neil Wentworth 2000. Recipe for success: Year 12 subject choice and the transition from school to university, Journal of Institutional Research 9 (1), 111-123.

Callister, P. 1993. Tomorrow's Skills. 1993 Update. Wellington: The Careers Service.

Calloway, Sir Frank 1999. Interview with Charles Southwood, ABC recording broadcast 9/8/00 by Concert FM.

Campana, Phillip J. 1983. Assessing support for high school foreign language programs: the Tennessee model, Foreign Language Annals 16 (1), 27-33.

Capper, Phillip 1991. Address to Wellington Association of Language Teachers, Voices February 1992, 3-8.

Carr, Jo 1997. Where do the boys go? - The problematic LOTE gender agenda, Australian Language Matters 5 (4), 12-13.

Carter, President Jimmy 1978. Announcement of the setting up of President's Commission on Foreign Language and International Studies, Foreign Language Annals 11 (6), 619-620.

Catholic Education Commission of Victoria 2000. Unpublished notes prepared for the 2000 CECV Annual Report.

Catholic Education Office (Melbourne). CECV Policy 1.19. Languages other than English: LOTE. Retrieved 2/5/01 fromhttp://www.ceo.melb.catholic.edu.au/.

Cavalier, R. 1996. Preface. In Australian Language and Literacy Council, Language Teachers: The Pivot of Policy. The Supply and Quality of Teachers of Languages Other Than English. Canberra: AGPS, xi. 
Center for Applied Linguistics 1997. National K-12 Foreign Language Survey Results. Retrieved 11/5/99 from http://www.cal.org/public/results.htm.

Chamberlain, Mary 1998. Towards the future. Address to the NZALT Conference, Dunedin, July 1998, New Zealand Association of Language Teachers Members' Newsletter 15, 9-14.

Chambers, Gary 2000. Motivation and the learners of modern languages. In Simon Green (ed.), New Perspectives on Teaching and Learning Languages. Clevedon: Multilingual Matters, 46-76.

Clark, Ann and John Trafford 1995. Boys into modern languages: an investigation of the discrepancy in attitudes and performance between boys and girls in modern languages, Gender and Education 7 (3), 315-325.

Clark, Helen 2001. Cited by New Zealand Newspapers Association 2001.

Clark, Margaret L. and Marjorie H. Rudolph 1981. Why or why not foreign languages, Foreign Language Annals 14(4 \& 5), 317-324.

Clément, Richard, Zoltán Dörnyei and Kim A. Noels 1994. Motivation, self-confidence, and group cohesion in the foreign language classroom, Language Learning 44, 417448.

Clyne, Michael 1997. Language policy in Australia - achievements, disappointments, prospects, Journal of Intercultural Studies 18 (1), 63-71.

Clyne, Michael, Catherine Jenkins, Imogen Y. Chen, Roula Tsokalidou and Theresa Wallner 1995. Developing Second Language From Primary School: Models and Outcomes. Canberra: NLLIA.

Coleman, J.A. 1996. Studying Languages: a Survey of British and European Students. London: CILT.

Commission on Foreign Language and International Studies 1979. Strength through wisdom: a critique of U.S. capability. A report to the President, Modern Language Journal 64, 9-57.

Cook, Vivian 1991. Second Language Learning and Language Teaching. London: Edward Arnold.

Cooper, Robert L. and Joshua A. Fishman 1974. The study of language attitudes, International Journal of the Sociology of Language 3, 5-19.

Cooper, Thomas C. 1985. A survey of teacher concerns, Foreign Language Annals 18(1), $21-4$.

Council of Australian Governments (COAG) 1994. Asian Languages and Australia's Economic Future. (The Rudd Report) Canberra: AGPS.

Council of Chief State School Officers 1985. International dimensions of education: position paper and recommendations for action, Foreign Language Annals 19 (3), 243-7.

Crawford, James 1997. Language Policy. Retrieved 14/4/01 from: http://ourworld. compuserve.com/homepages/JWCRAWFORD/langpol.htm.

Crawford, Jane 1995. Where are we really going?, Babel 30 (1), 20-23 \& 30. 
Crawford, Jane 1999. Should we be talking languages up or talking them down?, Australian Language Matters 7 (4),9-10.

Crawford-Lange, Linda M. 1985. Foreign language enrollments: Why are they declining? What can be done?, NASSP Bulletin 69 (February 1985), 14-21.

Crocombe, Graham T., Michael J. Enright and Michael E. Porter 1991. Upgrading New Zealand's Competitive Advantage. Oxford: Oxford University Press. (The Porter Report)

Crookall, David and Rebecca L. Oxford 1988. Review of Robert C. Gardner, Social Psychology and Second Language Learning: The Role of Attitudes and Motivation, Language Learning 38 (1), 127-140.

Crookes, Graham and Richard Schmidt 1991. Motivation: Reopening the research agenda, Language Learning 41, 469-512.

Curnow, Simon 1998. Newsletter from the President, New Zealand Association of Language Teachers Members' Newsletter 15, 3.

Curriculum Corporation 1994. A Statement on Languages Other Than English for Australian Schools. Carlton, Victoria: Curriculum Corporation.

Dandonoli, Patricia 1986. Recent trends in support by private foundations for foreign language education, Foreign Language Annals 19 (1), 49-56.

De Felippis, Dominick 1979. Views of secondary school superintendents on foreign language study: a support-constraint analysis, Foreign Language Annals 12 (2), 139-144.

Department for Education and Employment (DfEE UK) 2001. Modern Foreign Languages: a Response by the Department for Education and Employment to the Final Report and Recommendations of the Nuffield Languages Inquiry. Retrieved 19/2/01 from http://www.dfee.gov.uk/nuffield/.

Department for Education and Employment and Qualifications and Curriculum Authority (DfEE/QCA UK) 1999. The National Curriculum for England: Modern Foreign Languages. Retrieved 7/2/01 from http://www.nc.uk.net/home.html.

Department of Education, Training and Youth Affairs (DETYA Australia) 1997. Annual Report 1996-97. Retrieved 1/5/01 from http://www.detya.gov.au.

Department of Education, Training and Youth Affairs (DETYA Australia) 2000. Commonwealth Programmes for Schools: Quadrennial Administrative Guidelines 2001 to 2004 . Retrieved 1/5/01 fromhttp://www.detya.gov.au/schools/guidelines/ quadrennial/2001-04/2001-04.pdf.

Department of Employment, Education and Training (DEET Australia) 1991. The Australian Language and Literacy Policy. Canberra: AGPS.

De Vaus, David A. 1995 (4 ed.). Surveys in Social Research. London: Allen and Unwin.

Djité, Paulin G. 1994. From Language Policy to Language Planning: An Overview of Languages Other Than English in Australian Education. Deakin, ACT: NLLIA.

Dodick, David J. 1996. A study of the attitudes and motivation of high school foreign language students, Canadian Modern Language Review 52, 577-595. 
Dörnyei, Zoltán 1990a. Analysis of motivation components in foreign language learning. Paper presented at the 9th World Congress of Applied Linguistics, ThessalonikiHalkidiki, Greece.

Dörnyei, Zoltán 1990b. Conceptualizing motivation in foreign-language learning, Language Learning 40, 45-78.

Dörnyei, Zoltán 1996. Moving language learning motivation to a larger platform for theory and practice. In Rebecca Oxford (ed.), Language Learning Motivation: Pathways to the New Century. Honolulu: University of Hawai'i, Second Language Teaching and Curriculum Center,71-80.

Dörnyei, Zoltán 1998. Motivation in second and foreign language learning, Language Teaching 31, 117-135.

Dörnyei, Zoltán, E. Nyilasi and Richard Clément 1996. Hungarian school children's motivation to learn foreign languages: a comparison of five target languages, Novelty 3: 6-16.

Downes, Peter 2001. Why are the British such poor language learners? Institutional issues. Paper presented to LAGB Autumn Meeting 2001, Reading University. Retrieved 12/9/01 from http://serlinux3.essex.ac.uk/ ewa/ECmflDowAbs.htm.

Dutcher, Nadine 1995. Overview of Foreign Language Education in the United States. Washington, D.C.: Center for Applied Linguistics. Retrieved 2/10/01 from http://www.ncbe.gwu.edu/ncbepubs/resource/foreign.htm.

Eckstein, Anne 2000. LOTE strategies and directions for the new millennium. Unpublished paper presented to the National LOTE Conference 2000.

Eddy, Peter A. 1979. Foreign languages in the USA: a national survey of American attitudes and experience, Modern Language Journal 64, 58-63.

Edgerton, Mills R. Jr. 1971. A philosophy for the teacher of foreign languages. In James W. Dodge (ed.), The Case for Foreign-Language Study. New York: Northeast Conference on the Teaching of Foreign Languages, 44-52.

Education Department of Western Australia 2001. LOTE Beyond 2000. East Perth, WA: Education Department of Western Australia.

Education Review Office 1994. Second Language Learning. Wellington: ERO.

Education Review Office 1995. Assurance Audit Report: "Gerbera" High School. Wellington: ERO.

Education Review Office 1997. Accountability Report: "Salvia" College. Wellington: ERO.

Education Review Office 1998a. Accountability Review Report: "Arum" College. Wellington: ERO.

Education Review Office 1998b. Accountability Review Report: "Freesia” High School. Wellington: ERO.

Education Review Office 1999. Assurance Audit Report: “Begonia” High School. Wellington: ERO. 
Education Review Office 2001. The New Zealand Curriculum: An ERO Perspective. Retrieved 1/5/02 from http://www.ero.govt.nz/ Publications/pubs2001/Curriculum /CurricContents.htm.

Eiser, J. Richard 1984. Attitudinal Judgement. New York: Springer-Verlag.

Eiser, J. Richard 1987. The Expression of Attitude. New York: Springer-Verlag.

Eiser, J. Richard and Joop van der Pligt 1984. Attitudes in social context. In Henri Tajfel (ed.), The Secret Dimension Vol. 2. Cambridge: Cambridge University Press, $363-$ 378.

Enderwick, Peter and Michele Akoorie 1996. Fast Forward: New Zealand Business in World Markets. Auckland: Longman Paul.

Enderwick, Peter and Denise Gray 1992. Foreign languages in international business: the case of New Zealand, Journal of Teaching International Business 4 (1), 49-68.

Evans, Sarah 2002. Curriculum needs more attention to diversity and languages, $N Z$ Education Review August 28-September 3 2002, 5.

Farr, Sam 1997. In opposition to making the English the official language of the U.S. government, Modern Language Journal 81, 438.

Fishbein, Martin and Icek Ajzen 1975. Belief, Attitude, Intention and Behavior: An Introduction to Theory and Research. Reading, Mass.: Addison-Wesley.

Fishman, Joshua A. and Barbara R. Markman 1979. The Ethnic Mother-Tongue School in America: Assumptions, Findings, Directory. Unpublished final report to the National Institute of Education (NIE Grant G-78-0133).

Fitzsimons, Barbara 1999. Personal communication.

Foster, Lois and Kay Harman 1992 (3 ed.). Australian Education: A sociological perspective. Sydney: Prentice Hall.

Fowler, Floyd J. 1995. Improving Survey Questions. Thousand Oaks, Calif.: Sage.

Fullerton, Sue and John Ainley 2000. Subject Choice by Students in Year 12 in Australian Secondary Schools. Longitudinal Surveys of Australian Youth, Research Report Number 15. Camberwell, Victoria: ACER.

Gall, Meredith D., Walter R. Borg and Joyce P. Gall 1996 (6 ed.). Educational Research: An Introduction. White Plains, N.Y.: Longman.

Gao, Mobo 1996. Learning Asian languages in Australia: a reply to Andy Kirkpatrick, Babel 31 (1), 14-17.

Gardner, Robert C. 1985. Social Psychology and Second Language Learning: The Role of Attitudes and Motivation. London: Edward Arnold.

Gardner, Robert C. and Richard Clément 1990. Social psychological perspectives on second language acquisition. In Howard Giles, and W. Peter Robinson (eds.), Handbook of Language and Social Psychology. Chichester: John Wiley \& Sons, 495-517.

Gardner, Robert C., R. Lalonde and R. Pierson 1983. The socio-educational model of second language acquisition: an investigation using LISREL causal modeling, Journal of Language and Social Psychology 2, 51-65. 
Gardner, Robert C. and Wallace E. Lambert 1972. Attitudes and Motivation in SecondLanguage Learning. Rowley, Mass.: Newbury House.

Gardner, Robert C. and Peter D. MacIntyre 1993. A student's contributions to secondlanguage learning. Part II: affective variables, Language Teaching 26, 1-11.

Gardner, Robert C., A-M. Masgoret and P.F. Tremblay 1997. Home background characteristics and second language learning. Manuscript submitted for publication. Cited in Dörnyei 1998.

Gardner, Robert C. and P. C. Smythe 1975. Second language acquisition: a social psychological approach, Research Bulletin No. 332. London, Ontario: Department of Psychology, University of Western Ontario.

Gearon, Margaret 2001 (Education Department, Monash University). Personal communication.

Giles, Howard, Richard Y. Bourhis and Donald, M. Taylor 1977. Towards a theory of language in ethnic group relations. In Howard Giles (ed.), Language, Ethnicity and Intergroup Relations. London: Academic Press, 307-348.

Giles, Howard and P. Johnson 1987. Ethnolinguistic identity theory: a social psychological approach to language maintenance, International Journal of the Sociology of Language 68, 66-99.

Giles, Howard and Robert St. Clair 1979. Language and Social Psychology. Oxford: Basil Blackwell.

Gregory, Ann 2000. The primary sector. In Simon Green (ed.), New Perspectives on Teaching and Learning Modern Languages. Clevedon: Multilingual Matters, 77-93.

Grittner, Frank M. (ed.) 1975. Student Motivation and the Foreign Language Teacher. A Guide for Building the Modern Curriculum. Skokie, Illinois: National Textbook Co.

Groennings, Sven 1980. Developments and prospects following the President's Commission on foreign language and international studies, Foreign Language Annals 13 (6), 447-450.

Guthrie, Jo 1998. French or word-processing? How do year 9 students choose? Unpublished MA research paper for Griffith University, Brisbane.

Haddon, Rosemary 1998. The bittersweet experience of teaching Chinese in New Zealand, New Zealand Association of Language Teachers Members' Newsletter 14, 26-27.

Hanimann, Joseph. 2001. You don't say! Speaking out in favor of foreign languages, Frankfurter Allgemeine Zeitung 25/1/01.

Harwood, Jake, Howard Giles and Richard Y. Bourhis 1994. The genesis of vitality theory: historical patterns and discoursal dimensions, International Journal of the Sociology of Language 108, 167-206.

Hatch, Evelyn and Anne Lazaraton 1991. The Research Manual: Design and Statistics for Applied Linguistics. Boston, Mass.: Heinle and Heinle.

Hawk, Kay and Jan Hill 2000. Bridging Educational Islands: Making the Aotearoa/New Zealand system a 'seamless experience for students'. Paper presented to the NZARE conference, Waikato University, November 2000. 
Henerson, Marlene E., Lynn Lyons Morris and Carol Taylor Fitz-Gibbon 1987 (2 ed.). How to Measure Attitudes. Newbury Park, Calif.: Sage Publications.

Hernick, M. and D. Kennedy 1968. Multi-level grouping of students in the modern foreign language program, Foreign Language Annals 2, 200-4.

Herriman, Michael 1996. Language policy in Australia. In Michael Herriman and Barbara Burnaby (eds.), Language Policies in English-dominant Countries. Clevedon: Multilingual Matters, 35-61.

Hoegl, Juergen K. 1986. Education in the world system: the demand for language and international proficiencies in economic development and national security, Foreign Language Annals 19 (4), 281-7.

Hofman, John E. 1974. A review of Gardner and Lambert 1972, International Journal of the Sociology of Language 3, 117-120.

Hollis, Ray (NZQA) 1993. Languages in the NZ Curriculum. An address to Langsem 1993, New Zealand Association of Language Teachers Members' Newsletter 1, 2635.

Holmes, Janet 1976. A review of some methods on investigating attitudes to languages, dialects and accents. In W. Viereck (ed.), Sprachliches Handeln- Soziales Verhalten. Munich: Wilhelm Fink, 301-330.

Holt, Ron, Hiromi Maeda, Yoshitaka Sasai, Carolyn Shaw, Yuka Waller and Stuart Young 2001. Year 10 Japanese: A Study of Retention Factors in New Zealand Secondary Schools. Christchurch: School of Languages, Christchurch Polytechnic Institute of Technology.

Hotho, S. and N. Reimann 1998. Learner motivation: from dilemma to dialogue, Forum for Modern Language Studies 34 (2), 130-143.

Huber, Bettina J. 1992. Characteristics of foreign language requirements at US colleges and universities: findings from the MLA's 1987-89 survey of foreign language programs, ADFL Bulletin 24 (1), (cited by Dutcher 1995, no page nos. supplied).

Ingram, David 2001. Interpreters - can they be trusted? Retrieved 28/3/01 from http://www.nuffield.org.

Jackson, Ken and Roger Peddie 1998. Learning Smarter: The Economics of Learning International Languages in Secondary Schools. Auckland: University of Auckland.

Jenkins, Madeleine 2001 (Key Learning Area Manager LOTE, Victorian Curriculum and Assessment Authority). Personal communication.

Johnson, Diane 1994. Keynote address presented at the NZALT Conference 11 May 1994, New Zealand Language Teacher 20, 29-42.

Johnson, Diane 1998. Teaching and Learning Modern Languages: A New Zealand Perspective, New Zealand Language Teacher 24, 72-84.

Johnson, Diane 2001. The New Zealand teacher at work: a survey of attitudes, opinions and professional practices, New Zealand Language Teacher 27, 71-80.

Johnson, Dora 1998. Less commonly taught languages, ERIC Review. K-12 Foreign Language Education 6 (1), Fall 1998. Retrieved 15/4/99 from http://www. accesseric.org/resources/ericreview/vol6nol. 
Jones, W.R. 1949. Attitude towards Welsh as a second language. A preliminary investigation, British Journal of Educational Psychology 19 (1), 44-52.

Jones, W.R. 1950. Attitude towards Welsh as a second language. A further investigation, British Journal of Educational Psychology 20 (2), 117-32.

Jones, W.R. 1966. Bilingualism in Welsh Education. Cardiff: University of Wales Press.

Kaplan, Alice 1993. French Lessons. Chicago \& London: University of Chicago Press.

Kaplan, Robert B. 1993. Language policy and planning in New Zealand, Annual Review of Applied Linguistics 14, 156-176.

Kern, Richard G. 1995. Students' and teachers' beliefs about language learning, Foreign Language Annals 28 (1), 71-92.

King, Lid 1999. Challenges to multilingualism. In Arturo Tosi and Constant Leung (eds.), Rethinking Language Education: from a monolingual to a multilingual perspective. London: Royal Holloway University of London/CILT.

Kipp, Sandra, Michael Clyne and Anne Pauwels 1995. Immigration and Australia's Language Resources. Canberra: AGPS.

Kirkpatrick, Andy 1995. Learning Asian languages in Australia - which languages and when?, Babel 30 (1), 4-11 \& 26-29.

Kirkpatrick, Andy 1997. A reply to the replies, Babel 32 (1), 22-25.

Klayman, Norma Enea 1975. Views of secondary school educators on the foreign language requirement in higher education, Modern Language Journal 59, 168-173.

Knop, Constance K.1975. Gaining better student support for the foreign language program. In Frank M. Grittner (ed.), Student Motivation and the Foreign Language Teacher. A Guide for Building the Modern Curriculum. Skokie, Illinois: National Textbook Co., 95-106.

Knüfermann, Volker 1993. Study programmes and career opportunities for language students, New Zealand Language Teacher 19 (2), 69-73.

Koppel, Irene E. 1982. The perceived contribution of foreign languages to high priority educational goals, Foreign Language Annals 15 (6), 435-439.

Labrie, Normand and Richard Clément 1986 Ethnolinguistic vitality, self-confidence and second language proficiency: an investigation, Journal of Multilingual and Multicultural Development 7 (4), 269-282.

Lai, Eva Fung-kuen 1999. Motivation to learn English, Language, Culture and Curriculum $12(3), 280-285$.

Lambert, Richard D. 1985. Foreign language instruction: a national agenda, Foreign Language Annals 18 (5), 379-383.

Lambert, Wallace E. 1974. Culture and language as factors in learning and education. In F. E. Aboud and R. D. Meade (eds.), Cultural factors in learning and education. Bellingham, Washington: Fifth Western Washington Symposium on Learning.

Larsen-Freeman, Diane and Michael H. Long 1991. An Introduction to Second Language Acquisition Research. London and New York: Longman. 
Lawson, John H. 1971. No title. In James W. Dodge (ed.) 1971, The Case for ForeignLanguage Study. New York: Northeast Conference on the Teaching of Foreign Languages, 3-7.

Leal, R. B., C. Bettoni and I. Malcolm 1991. Widening Our Horizons: Report of the Review of the Teaching of Modern Languages in Higher Education. Canberra: AGPS.

Lett, John A., Jr. 1977. Assessing attitudinal outcomes. In June K. Phillips (ed.), The Language Connection: From the Classroom to the World. Skokie, Illinois: National Textbook Company, 267-302.

Levett, Allan and Anna Adams 1987. Catching up with Our Future: The Demand for Japan Skills in New Zealand. Wellington: New Zealand Japan Foundation.

Lewelling, Vickie and Jeanne Rennie 1998a. National standards: preparing for the future, ERIC Review. K-12 Foreign Language Education 6 (1), Fall 1998. Retrieved 15/4/99 from http://www.accesseric.org/resources/ericreview/vol6nol.

Lewelling, Vickie and Jeanne Rennie 1998b. State initiatives for foreign language instruction, ERIC Review. K-12 Foreign Language Education 6 (1). Retrieved 15/4/99 fromhttp://www.accesseric.org/resources/ericreview/vol6nol.

Liddicoat, Anthony 1996. Australia's changing language policy, Babel 31 (1), 4-7 \& 33.

Lippmann, Jane N. 1974. Rationale for language study. In Gilbert A. Jarvis (ed.) The Challenge of Communication. Skokie, Illinois: National Textbook Co., 37-69.

Liston, David and Nigel Reeves 1985. Business Studies, Languages and Overseas Trade. London: Pitman/Institute of Export.

Lo Bianco, Joseph 1987. National Policy on Languages. Canberra: AGPS.

Lo Bianco, Joseph 1997. English and pluralistic policies: the case of Australia. In William Eggington and Helen Wren (eds.), Language Policy: dominant English, pluralist challenges. Amsterdam and Philadelphia: John Benjamins, 107-119.

Lo Bianco, Joseph 2000. Making Britons bilingual: joined-up or switched-on thinking, Australian Language Matters 8 (4): 1, 6-8.

Lo Bianco, Joseph, Anthony J. Liddicoat and Chantal Crozet (eds.)1999. Striving for the Third Place. Intercultural Competence through Language Education. Melbourne: Language Australia.

Loulidi, Rafik 1990. Is language learning really a female business? Language Learning Journal 1, 40-43.

Mackiewicz, Wolfgang 2001. Universities need a language lesson, Guardian Weekly 21-27 June 2001.

Mahon, Justine 1989. Foreign language teaching in intermediate schools, New Zealand Language Teacher 15 (1), 20-25.

Manhire, Bill 1999. Interview with Kim Hill on National Radio 22/1/99.

Mathews, Jay 2000. Young linguists flourish in US immersion experiment, Guardian Weekly. TEFL Supplement January 2000. 
McCollow, John and John Graham 1997. Not quite the national curriculum.

Accommodation and resistance to curriculum change. In Bob Lingard and Paige

Porter (eds.), Essays on the Development of National Policies in Schools Education.

Canberra: Australian College of Education, 60-75.

McCutcheon, Jen 2002. Striking for a better system, Education Review March 20-26, $2002,6$.

McGroarty, Mary 1996. Language attitudes, motivation and standards. In Sandra Lee McKay and Nancy H. Hornberger (eds.), Sociolinguistics and Language Teaching. Cambridge: Cambridge University Press, 3-46.

McKinnon, Don 1995. Learning languages: enhancing the skills of New Zealanders. (Address to NZALT Annual Conference), New Zealand Language Teacher 21, 1922.

Met, Myriam 1985. Decisions! Decisions! Decisions! Foreign language in the elementary school, Foreign Language Annals 18 (6), 469-473.

Meyer, Gertrud 1975. Making the foreign language program visible to the public: the language festival. In Frank M. Grittner (ed.), Student Motivation and the Foreign Language Teacher. A Guide for Building the Modern Curriculum. Skokie, Illinois: National Textbook Co., 45-65.

Ministerial Council on Education, Employment, Training and Youth Affairs (MCEETYA) National Report on Schooling in Australia: 1999. Retrieved 22/8/01 from http://www.curriculum.edu.au/mceetya/public/pub325.htm\#download.

Ministry of Education 1993a. Education for the 21st Century. Wellington: Learning Media.

Ministry of Education 1993b. The New Zealand Curriculum Framework. Wellington: Learning Media.

Ministry of Education 1997. Curriculum Development Updates 24, 38. Wellington: Ministry of Education.

Ministry of Education 2000. Literature Review - Published Critiques and Commentary on the New Zealand Curriculum. Retrieved 13/7/01 from http://www.tki.org.nz/r/ stocktake/postings/litreview_e.php.

Ministry of Education 2001a. Curriculum Stocktake - Discussion Day Notes. Language and Languages learning area of the New Zealand Curriculum. Unpublished document. Also available from http://www.tki.org.nz.

Ministry of Education 2001b. Notice about Curriculum Stocktake in Education Gazette 80 (1). Retrieved 12/2/01 from http://www.edgazette.govt.nz/articles/show_articles. cgi?id=5886.

Ministry of Education 2001c. What is the Curriculum Stocktake Project? Retrieved 13/7/01 from http://www.tki.org.nz/r/stocktake/to_date/what_e.php.

Ministry of Education 2002a. Curriculum Support. Second Language Learning Funding Pool. Retrieved 22/5/02 fromhttp://www.edgazette.govt.nz/notices/ show_notices. cgi?id=58305.

Ministry of Education 2002b. Learning Languages: A Guide for New Zealand Schools . Wellington: Learning Media. 
Ministry of Foreign Affairs and Trade 1995. Latin America and the Caribbean: Trade and Economic Prospects. Wellington: MFAT.

Moore, Helen 1996. Language policies as virtual reality; two Australian examples, TESOL Quarterly 30 (3), 473-497.

Morizumi, Fumi 1999. Why do more girls than boys want to study languages in Japan? Unpublished paper presented to 12 th World Congress of Applied Linguistics, Tokyo, August 1999.

Muchnick, Arlene G. and David E. Wolfe 1982. Attitudes and motivations of American students of Spanish, Canadian Modern Language Review 38 (2), 262-281.

National Asian Languages and Studies in Australian Schools (NALSAS). Introduction to the NALSAS strategy. Retrieved 8/8/01 from http://www.curriculum.edu.au/ nalsas/newproj.htm.

National Council for Languages and International Studies (NCLIS USA) 1999. About the Association. Retrieved 13/4/99 from http://www.languagepolicy.org/about.html.

National Education Monitoring Project (NEMP) 1998. Listening and viewing report. Retrieved 1/3/02 from http://nemp.otago.ac.nz/PDFs/list_view_98/chapter5.pdf.

National Education Monitoring Project (NEMP) 2000. Reading and speaking report. Retrieved 1/3/02 from http://nemp.otago.ac.nz/PDFs/read_speak_00/chapter9.pdf.

National Standards in Foreign Language Education Project 1996. Standards for Foreign Language Learning: Preparing for the 21st Century. Yonkers, NY: National Standards in Foreign Language Education Project.

New Zealand Association of Language Teachers (NZALT) 1995. Policy on Language Teaching and Learning in Secondary Schools. (No place of publication): NZALT.

New Zealand Association of Language Teachers (NZALT) 2002. Languages can take you places, in every sense of the word, Polyglot 25, 15-16.

New Zealand Council for Teacher Education 1998. Second Language Learning in International Languages. Wellington: NZCTE.

New Zealand Educational Institute (NZEI) 2000. Drive for compulsory Maori in schools. Press release 26 September 2000. Retrieved 2/10/00 from http://www.nzei.org.nz.

New Zealand Newspapers Association 2000. Kiwis Need More Languages - PM. Press release retrieved 29/11/01 from http://www.stuff.co.nz.

New Zealand Tourism Board (NZTB) 1991. Tourism in New Zealand: A Strategy for Growth. Wellington: NZTB.

New Zealand Tourism Board (NZTB) 1996. Tourism in NZ: Strategy and Progress. Wellington: NZTB.

Nicholas, Howard, Helen Moore, Michael Clyne and Anne Pauwels 1993. Languages at the Crossroads: the Report of the National Enquiry into the Employment and Supply of Teachers of Languages Other Than English. Canberra: NLLIA. 
Noels, Kimberly A. 2001. New orientations in language learning motivation: towards a model of intrinsic, extrinsic, and integrative orientations and motivation. In Zoltán Dörnyei and Richard Schmidt (eds.), Motivation and Second Language Acquisition. Honolulu: University of Hawai'i, Second Language Teaching and Curriculum Center, 43-68.

Nuffield Foundation 2001a. Languages at Work in 21st Century Britain: time to change attitudes. Press release retrieved 12/9/01 from http://www.nuffieldfoundation. org/languages/news/nw0000000346.asp.

Nuffield Foundation 2001b. Ambassadors plan to "give young children a flying start". Press release retrieved 12/9/01 from http://www.nuffieldfoundation.org/languages/ news/nw0000000364.asp.

Nuffield Foundation 2001c. £500,000 from Nuffield to support the development of languages in the UK. Press release retrieved 12/9/01 from http://www.nuffield foundation.org/languages/news/nw0000000305.asp.

Nuffield Languages Inquiry 2000. Languages - the next generation. London: The Nuffield Foundation.

Nunan, David 1992. Research Methods in Language Learning. Cambridge: Cambridge University Press.

Nussenbaum, Gladys 1983. Sociolinguistic dimensions of foreign and second language learning, Foreign Language Annals 16 (2), 121-124.

Oakes, Judy 2001 (at Association of Independent Schools, Victoria). Personal communication.

O'Flaherty, Brendan 2001. 'Ow you say, Listener August 18 2001, 28-29.

Oppenheim, A. N. 1992. Questionnaire Design, Interviewing and Attitude Measurement. London: Pinter.

Oskamp, S. 1977. Attitudes and Opinions. Englewood Cliffs, N.J.: Prentice-Hall.

Oxford, Rebecca L. 1996. New pathways of language learning motivation. In Rebecca Oxford (ed.), Language Learning Motivation: Pathways to the New Century. Honolulu: University of Hawai'i, Second Language Teaching and Curriculum Center, 1-8.

Oxford, Rebecca L. and Jill Shearin 1996. Language learning motivation in a new key. In Rebecca Oxford (ed.), Language Learning Motivation: Pathways to the New Century. Honolulu: University of Hawai'i, Second Language Teaching and Curriculum Center,121-144.

Ozolins, Uldis 1993. The Politics of Language in Australia. Cambridge: Cambridge University Press.

Papalia, Anthony 1978. Students' beliefs on the importance of foreign languages in the school curriculum, Foreign Language Annals 11 (1), 21-23.

Paquette, R. André 1971. Why study foreign languages? In James W. Dodge (ed.), The Case for Foreign-Language Study. New York: Northeast Conference on the Teaching of Foreign Languages, 28-31.

Parkinson, Wendy 1999. Language wisewhys: a re-evaluation of the rationale for languages education in today's world, Babel 34 (3), 12-15 \& 37-38. 
Peddie, Roger A. 1992. Language and languages policy in NZ, English in Aotearoa 18, 4050 .

Peddie, Roger A. 1993. From Policy to Practice: The Implementation of Languages Policies in Victoria, Australia and New Zealand. Auckland: Centre for Continuing Education, Auckland University.

Peddie, Roger, Cathy Gunn and Marilyn Lewis 1999. Starting Younger: The Second Language Learning Project Evaluation. Wellington: Ministry of Education.

Perkins, James A. 1978. No title, Foreign Language Annals 11 (5), 621.

Perkins, James A. 1979. Chairman's remarks introducing Strength through Wisdom: A Critique of U.S. Capability. A report to the President from the President's Commission on foreign language and international studies, November 1979, Modern Language Journal 64, 9-57.

Pfister, Guenter G. 1989. High school foreign language programs: a renewed challenge, The Clearing House 62 (7), 309-313.

Phillips, D. 1982. A thoroughly respectable language: Pupils views on German and other 'second' foreign languages, British Journal of LanguageTeaching 20 (1), 23-29.

Phillipson, Robert and Tove Skutnabb-Kangas 1994. Lessons for Europe from language policy in Australia. In M. Puitz (ed.), Language Choices: Conditions, Constraints and Consequences. Amsterdam/Philadelphia: John Benjamins, 115-159

Pound, Ezra 1934. ABC of Reading. London: George Routledge and Sons.

Powell, Robert C. and Julia D. Batters 1985. Pupils' perceptions of foreign language learning at 12+: some gender differences, Educational Studies 11 (1), 11-23.

Preller, Arno G. 1974. Building better bridges in a kaleidoscopic society. In Frank M. Grittner (ed.), Careers, Communication and Culture in Foreign Language Teaching. Skokie, Illinois: National Textbook Co., 72-83.

Pritchard, Rosalind M.O. 1987. Boys' and girls' attitudes towards French and German, Educational Research, 29, 65-72.

Pritchard, Rosalind M.O. and Rafik Loulidi 1994. Some attitudinal aspects of foreign language learning in Northern Ireland: focus on gender and religious affiliation, British Journal of Educational Studies, 42, 388-401.

Quinn, Terry 1998. "What a long way we've come!”, Australian Language Matters 6 (1), 8$8 \& 13$.

Quirk, Randolph 1990. British must get their tongues around 1992, The European, Weekend June 1-3, 1990, reprinted in New Zealand LanguageTeacher 17 (1), 50.

Ramage, Katherine 1990. Motivational factors and persistence in foreign language study, Language Learning 40 (June 1990), 189-219.

Raso, Elina 2001 (at Catholic Education Office, Victoria). Personal communication.

Reeves, Dona B. 1974. Public awareness: what can individual teachers do? In Gilbert A. Jarvis (ed.), The Challenge of Communication. Skokie, Illinois: National Textbook Co., 91-112. 
Rizvi, Fazal 1997. Asian languages and Australian schools. In Bob Lingard and Paige Porter (eds.), Essays on the Development of National Policies in Schools Education. Canberra: Australian College of Education,111-121.

Rogers, Betty S. and Joe Arn 1998. Language requirements for baccalaureate degrees in AACSB-accredited schools: deans; opinions, Journal of Education for Business 73 (6), 347-50.

Rolland, Barbara 1974. Careers for our foreign language graduates. In Frank M. Grittner (ed.), Careers, Communication and Culture in Foreign Language Teaching. Skokie, Illinois: National Textbook Co., 41-50.

Romano, Tubichi (ed.) 1995. Why not Australia: a Cross-National Study of Language Education Systems. Adelaide: South Australia Institute of Languages.

Roskens, Ronald W. 1979. Legacies of the Tower of Babel, Modern Language Journal 64 , 173-8.

Ryan, Ellen B., Howard Giles and Richard J. Sebastian 1982. An integrative perspective. In Ellen B. Ryan \& Howard Giles (eds.), Attitudes towards Language Variation. London: Edward Arnold, 1-19.

Ryan, George 1984. A 1980 survey referred to in a speech to International Business Council MidAmerica, reported in Foreign Language Annals 18 (1), 73-77.

Saunders, Keith 1998. Modern languages in crisis? Language Learning Journal 18, 63-67.

Scarino, Angela 1996. Editor's spot, Babel 31(1), 3.

Scarino, Angela 1998. Languages at a national level, Australian Language Matters 6 (1), 12 13.

Schmidt, Richard, Deena Boraie and Omneya Dassabgy 1996. Foreign language motivation: internal structure and external connections. In Rebecca Oxford (ed.), Language Learning Motivation: Pathways to the New Century. Honolulu: University of Hawai'i, Second Language Teaching and Curriculum Center, 9-70.

Scholfield, Phil 1995. Quantifying Language: A Researcher's and Teacher's Guide to Gathering Data and Reducing it to Figures. Clevedon: Multilingual Matters.

Schulz, Renate A. 1998. Foreign language education in the United States: trends and challenges, ERIC Review. K-12 Foreign Language Education 6 (1). Retrieved 15/4/99 from http://www.accesseric.org/resources/ericreview/vol6nol.

Schumacher, Shiree 2001. Talking point, Next July 2001, 56-62.

Schuman, Howard and Stanley Presser 1981. Questions and Answers in Attitude Surveys. New York: Academic Press.

Shackleford, Nick 1996. Languages policy and international languages of trade and tourism: rhetoric and reality. Thesis submitted as part of Master of Education (Educational Administration). Auckland: University of Auckland.)

Shannon, Jean 1991. "Language is good business" conference report, Vox 5, 16-17.

Skehan, Peter 1989. Individual Differences in Second-Language Learning. London: Edward Arnold. 
Smith, Lockwood 1994. Address to NZALT annual conference. Cited in ERO 1994.

Smith, Steve 1995. Languages in Britain. Interview in New Zealand Language Teacher 21, 51-53.

Spence, Gail 1997. Newsletter from the President, New Zealand Association of Language Teachers Members' Newsletter 11, 1-2.

Spence, Gail 1998. The art of communication, New Zealand Association of Language Teachers Members' Newsletter 14, 13-15.

Spence, Gail 2001.Languages and the NCEA. Paper distributed by the Ministry of Education as appendix to the curriculum stocktake - discussion day notes for the language and languages learning area of the New Zealand curriculum.

Spence, Gail 2002, cited in Education Gazette 18,4. Retrieved 5/8/02 from: http://www. edgazette.govt.nz/articles/show_articles.cgi?id=6236.

Spolsky, Bernard 1978. Educational Linguistics: An Introduction. Rowley, Mass.: Newbury House.

Stark, Anne 1990. Something special to offer Hungary, Export News March 1990, 26-27.

Starks, Donna 1998. Monolingual speakers of New Zealand's languages, New Zealand Studies in Applied Linguistics 4, 71-75.

Statistics NZ 2002. Country of Residence of Overseas Visitors. Retrieved 2/9/02 from http://www.stats.govt.nz/domino/external/pasfull/pasfull.nsf/7cf46ae26dcb6800cc 256a 62000a2248/4c2567ef00247c6acc256bde 0077b92f?OpenDocument\#2D.

Stefanik, Heinrich 1990. Australia: a languages graveyard no more?, Vox 4, 33-38.

Stirling, Pamela 1999. Polyglots paramount, Listener February 13 1999, 28.

Strasheim, Lorraine A. 1979. The issue: multi-level classes, Foreign Language Annals 12 (5), 423-425.

Strasheim, Lorraine A. 1980. Interdisciplinary cooperation: will teachers in "average" schools be left out?, Foreign Language Annals 13 (1), 59-61.

Supit, Trina 1996. Language policy: quo vadit?, Australian Language Matters 4 (3), 12.

Tajfel, Henri 1974. Social identity and intergroup behaviour, Social Science Information 13, 65-93.

Tajfel, Henri 1981. Human Groups and Social Categories: Studies in Social Psychology. Cambridge: Cambridge University Press.

Tajfel, Henri 1982. Social Identity and Intergroup Relations. Cambridge: Cambridge University Press.

Taifell. Henri_C Flament_M.Billig and R. Bundy 1971. Social categorization and 
Tremblay, P. F. and R. C. Gardner 1995. Expanding the motivation construct in language learning, Modern Language Journal 79: 505-520.

Trim, John 1999. Language education policies for the twenty-first century. In Arturo Tosi and Constant Leung (eds.), Rethinking Language Education: from a Monolingual to a Multilingual Perspective. London: Royal Holloway University of London/CILT, $6-18$.

Turner, Paul R. 1974. Why Johnny doesn't want to learn a foreign language, Modern Language Journal 58, 191-200.

Vale, David 2000. Editor's spot, Babel 35 (3), 3.

Velde, Matt 2000. Meeting the challenge, Education Gazette 79 (16). Retrieved 11/9/00 from: http://www.edgazette.govt.nz/articles/show_articles.cgi?id=5809.

Victorian Department of Education, Employment and Training 2000. Languages Other Than English in Government Schools, 1999. Melbourne: DEET, Victoria.

Victorian Department of Education and Training 2002. Languages for Victoria's future. An Analysis of Languages in Government Schools. Melbourne: Department of Education and Training.

Vine, Bernadette 1993. Attitudes, orientation, gender and the decision (not) to continue Japanese. Unpublished LING 402 research paper, Victoria University of Wellington.

Voght, Geoffrey M. and Ray Schaub 1992. Foreign languages and international business, ERIC Digest. ED347851.

Waite, Jeffrey 1992. Aoteareo: Speaking for Ourselves. Wellington: Learning Media.

Walker, John L. 1976. The student view: attitudes toward foreign language learning. In Gilbert A. Jarvis (ed.), An Integrative Approach to Foreign Language Teaching: Choosing Among the Options. Skokie, Illinois: National Textbook Company,129179.

Walsh, Rebecca 2001. French lessons de rigueur, New Zealand Herald 9/10 June 2001.

Watts, Noel 1987. The use of foreign languages by New Zealand exporters in the 1980's: a Massey University investigation, New Zealand LanguageTeacher 13 (2/3), 39-42.

Watts, Noel 1991. Foreign languages in the international marketplace. Keynote address to NZALT Conference, New Zealand Language Teacher 17 (2/3), 11-18.

Watts, Noel 1992. The Use of French in Exporting and Tourism. Palmerston North: Department of Modern Languages, Massey University.

Waugh, Ingrid 1990. Adjusting across cultures, Export News April 1990, 3-5.

Weatherford, H. Jarold 1982. Foreign language attitudes survey: Georgia superintendents and high school principals, Foreign Language Annals 15 (1), 31-34.

White, Carol and Susan Gray 1997. Creating a culture of respect: a state secondary school's journey, Proceedings of the NZARE Conference. No place of publication: NZARE.

Whitehead, Kenneth D. 1985. Foreign language study: one of the basics, American Education 19 (2), 5-7 \& 14. 
Wiley, Terence G. 1996. Language planning and policy. In Sandra Lee McKay and Nancy $\mathrm{H}$. Hornberger (eds.), Sociolinguistics and LanguageTeaching. Cambridge: Cambridge University Press, 103-147.

Wilkes, Helen 1983. Title pages: a simple device for culture consciousness-raising in the teenaged student of French, Foreign Language Annals 16 (2), 107-112.

Williams, Lynn 1994. Language learning in New Zealand: its present and its future, New Zealand LanguageTeacher 20, 51-55.

Williams, Lynn 1996. Letter from the President, New Zealand Association of Language Teachers Members' Newsletter 8, 3-12.

Williams, M. and R. Burden 1997. Psychology for Language Teachers. Cambridge: Cambridge University Press.

Williss, Mike 1995. An illusion of difficulty - a reply to Andy Kirkpatrick, Babel 30 (2), 28-32.

Wittgenstein, Ludwig 1922. Tractatus Logico-Philsophicus. Cited in Elizabeth Knowles 1998 (ed.), The Oxford Essential Quotations Dictionary. New York: Berkley Books, 237.

Wray, Alison, Kate Trott and Aileen Bloomer 1998. Projects in Linguistics: A Practical Guide to Researching Language. London: Edward Arnold.

Wright, Sue 1993. Language learning in England and Wales: policies and attitudes in flux. In Dennis Ager, George Muskens and Sue Wright (eds.), Language Education for Intercultural Communication. Clevedon: Multilingual Matters, 39-55.

Zammit, Susan A. 1992. The Challenge: Choosing to Study a Language Other Than English Through High School. Canberra: Report to the Department of Employment, Education and Training.

Zammit, Susan A. 1993. Attitude to Language, Test Results, Gender Differences and Foreign Language: How do They Connect? Hawthorn, Victoria: Australian Council for Educational Research.

Ziegler, Carl 1975. The language teacher and amateur language student. In Frank M. Grittner (ed.), Student Motivation and the Foreign Language Teacher. A Guide for Building the Modern Curriculum. Skokie, Illinois: National Textbook Co., 107118. 


\section{APPENDIX 1}

\section{Interpreters - can they be trusted?}

Some years ago I was on an Australian aid mission to China. Since neither my two colleagues nor I spoke Chinese, we used interpreters supplied by the Chinese authorities. One of them was meticulous in interpreting, even apologising if he believed something said by the other party was erroneous and explaining later why he thought so. The other interpreter was a young recent graduate whose English was excellent.

At an official banquet for the Australian party, while the younger fellow was interpreting, there was also present an official from the relevant Australian government department who had a little Chinese language. He warned us at the conclusion of the banquet that, though he didn't have much Chinese, he believed that the Chinese interpreter was not translating accurately.

Eventually we challenged the interpreter who gave a salutary but, in retrospect, entirely reasonable response: "It's not my job to translate accurately for you", he said. "It's my job to convince you that the project should go ahead."

A multi-million dollar aid project was in question: our advice would determine whether it proceeded or not at the Australian taxpayer's expense and our judgement had to rely on the information we obtained in the course of the negotiations! Eventually the project did go ahead, operated for several years, and was highly successful but it is a lesson that any English-only trader or any negotiator who relies on the other party's interpreter would do well to heed.

Ingram (2001) 


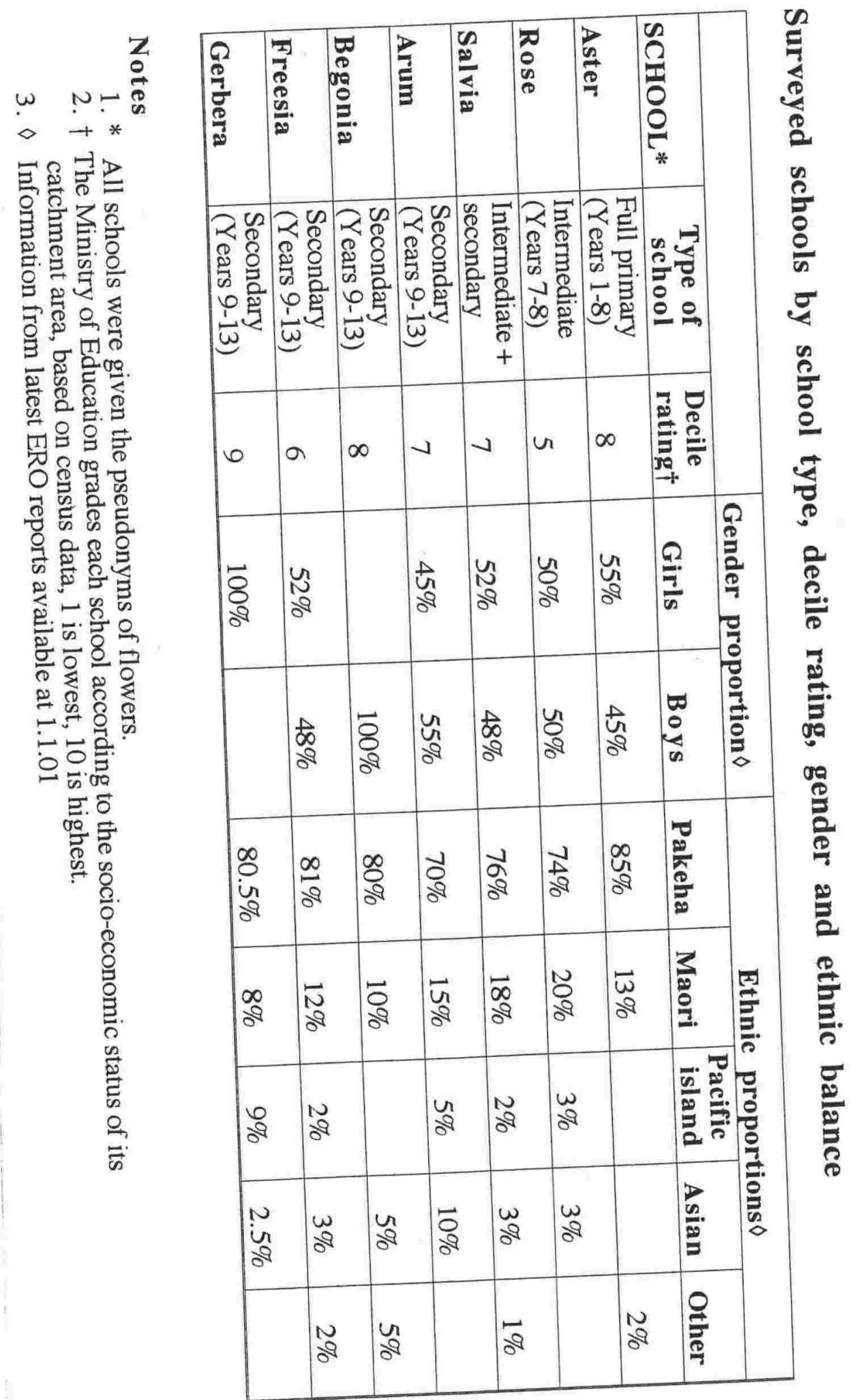




\section{APPENDIX 3}

\section{Sample information sheet and consent form for students and parents}

Victoria University of Wellington

School of Linguistics and Applied Language Studies

\section{Research into attitudes towards foreign language learning}

\section{INFORMATION SHEET}

Research in connection with a $\mathrm{PhD}$ in Linguistics

Researcher: Sandra Shearn

To all year 8 students in (name of class) and their parents/caregivers

I am an experienced language teacher, mostly of French and Spanish, and most recently at the Correspondence School. I am at present studying for a $\mathrm{PhD}$ in Linguistics at Victoria University. I am investigating what Year 8 students know and feel about learning a foreign language, that is a language other than English or Maori, either this year or at secondary school next year. I propose to ask as many of the same students as possible to fill in a similar questionnaire next year, if they are attending a secondary school in the same area, in order to find out what decisions they have made regarding language learning, if languages are among the optional subjects for Year 9 students.

I will ask all the students in this class, who have their parents' permission, to fill in a questionnaire for me, which should take about a quarter of an hour. All the information will be strictly confidential. All questionnaires will be kept secure and available only to myself and my supervisor, Professor Janet Holmes, and they will be destroyed at the end of my research. Neither the school, its location, nor any individual student or teacher will be identified in my report.

When I have completed my thesis I will supply a readable summary of my findings to the school, and I will be happy to discuss the results of my research with students and their families, if requested.

I should be grateful if parents would sign and return to your child's class teacher the attached form, indicating if you consent to your child's participation in this research and that $s /$ he is willing to participate. Please contact me if you have any questions about the project.

Yours sincerely

Contact write: c/o School of Linguistics and Applied Language Studies Victoria University of Wellington

P.O. Box 600

Wellington

e-mail: sandra.shearn@vuw.ac.nz

Supervisor Professor Janet Holmes

work phone: 04 472-1000 ext. 8796

(consent form attached) 
Victoria University of Wellington

School of Linguistics and Applied Language Studies

Research into attitudes towards foreign language learning

\section{CONSENT FORM}

My child and I have received and understood an explanation of this research project. We have had the opportunity to seek further information. My child is willing to participate and I give my consent.

Signed (parent/caregiver)

(child)

Name of child (please print clearly) 


\section{APPENDIX 4}

\section{Sample information sheet and consent form for teachers}

\section{Victoria University of Wellington}

School of Linguistics and Applied Language Studies

\section{Attitudes to foreign language learning in New Zealand schools \\ INFORMATION SHEET \\ Research in connection with a PhD in Linguistics \\ Researcher: Sandra Shearn}

I am investigating what a sample of Year 9 students know and feel about learning languages other than English or Maori. I aim to discover the extent to which their attitudes influenced their decisions to study or not study one or more foreign languages, where such study is optional, when they started secondary school.

I wish to survey students by means of a written questionnaire to be administered by myself to whole core classes. I undertake to supply in advance suitable letters for form teachers to distribute in order to inform students and their parents, and to seek their consent to participate. The timing of the administration of the questionnaire will be arranged in consultation with the Principal and class teachers willing to assist. I intend to follow up the survey by interviewing a sample of students and their parents.

I would also like to interview Year 9 form teachers and a sample of other staff members, including the Principal, Year 9 Dean(s), careers adviser and language specialists, in order to assess the place of foreign language learning in the school. Interviews will take about half an hour and be arranged at a time and place to suit each teacher. With the permission of each staff member I would like to tape the interviews to ensure an accurate record of facts and opinions. Teachers interviewed may, at any time before data collection is completed and without having to give reasons, withdraw from the project themselves and any information they have provided.

No school, teacher or student will be identified in the report of my findings, nor will the location of any schools be revealed, although statements of fact or opinion may be attributed to 'a Principal', a 'form teacher' or a 'language teacher', for example. All notes, tapes and questionnaires will be kept securely and accessed only by myself and my supervisor, Professor Janet Holmes. At the end of my research all notes and questionnaires will be destroyed and tapes electronically wiped to ensure confidentiality for all involved.

When the project is complete I will supply a readable summary of my findings to each school involved. I would be happy to discuss the results of my research with teachers, or students and their families. The findings will be published in my thesis and submitted to relevant national and international journals.

Signed:

Contact: e-mail: sandra.shearn@vuw.ac.nz

fax: 044732243

write: c/o School of Linguistics and Applied Language Studies

Victoria University of Wellington

P.O. Box 600

Wellington

Supervisor: Professor Janet Holmes work phone: 044635614 
Victoria University of Wellington

School of Linguistics and Applied Language Studies

\section{Research into attitudes towards foreign language learning}

\section{CONSENT FORM}

\section{Researcher: Sandra Shearn}

I have been given and have understood an explanation of this research project. I have had an opportunity to ask questions and have them answered to my satisfaction. I understand that I may withdraw from this project myself or any information I have provided, before data collection is completed, without having to give reasons.

I agree to take part in the research and consent to the taking of notes and the recording on cassette tape of my interview about the place of foreign languages in my school and student attitudes towards them. I understand that, at the end of the research, the tape will be electronically wiped and all notes destroyed.

Signed:

Name of participant: (please print clearly)

Date: 


\section{APPENDIX 5}

Year 8 student survey questionnaire

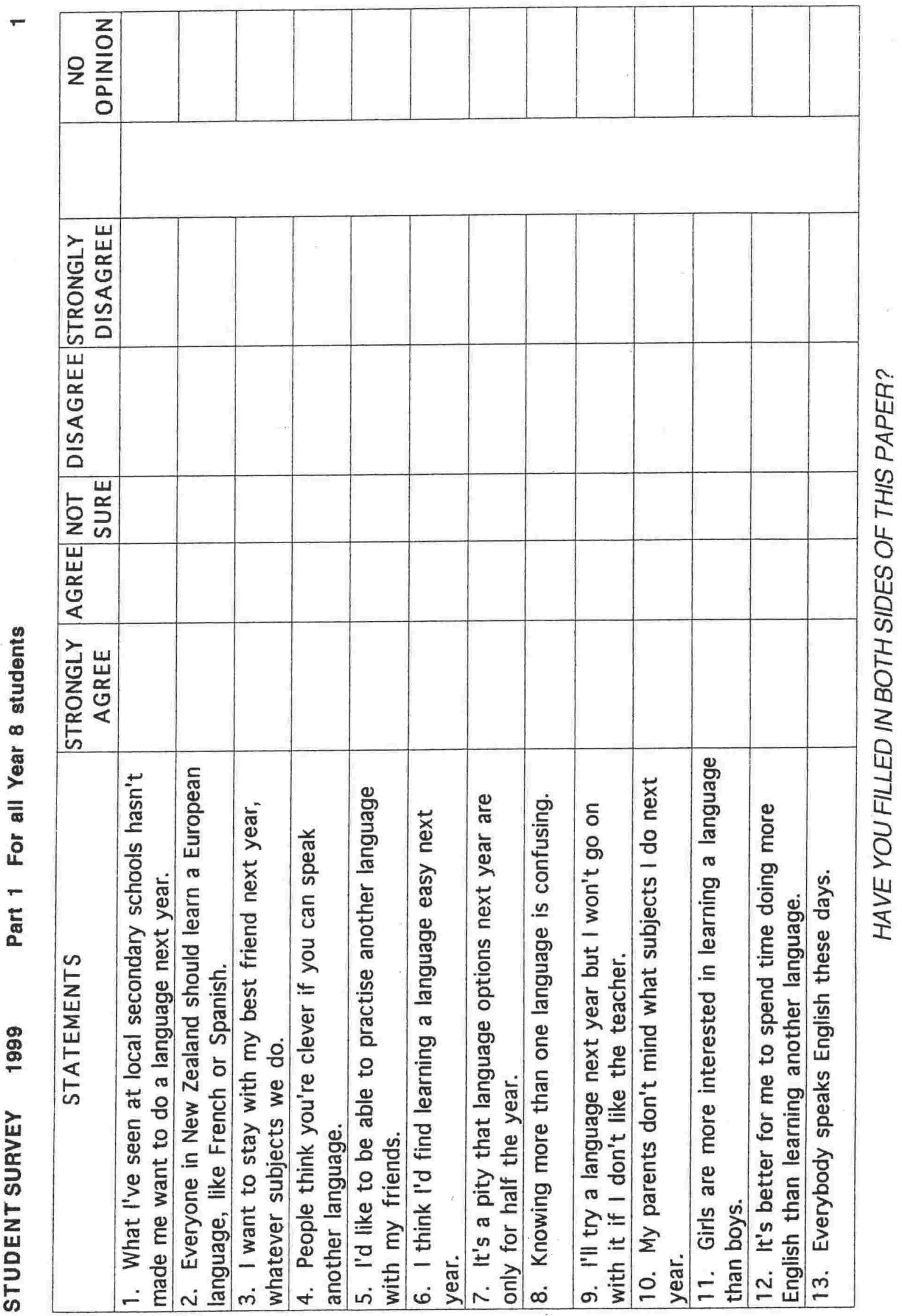




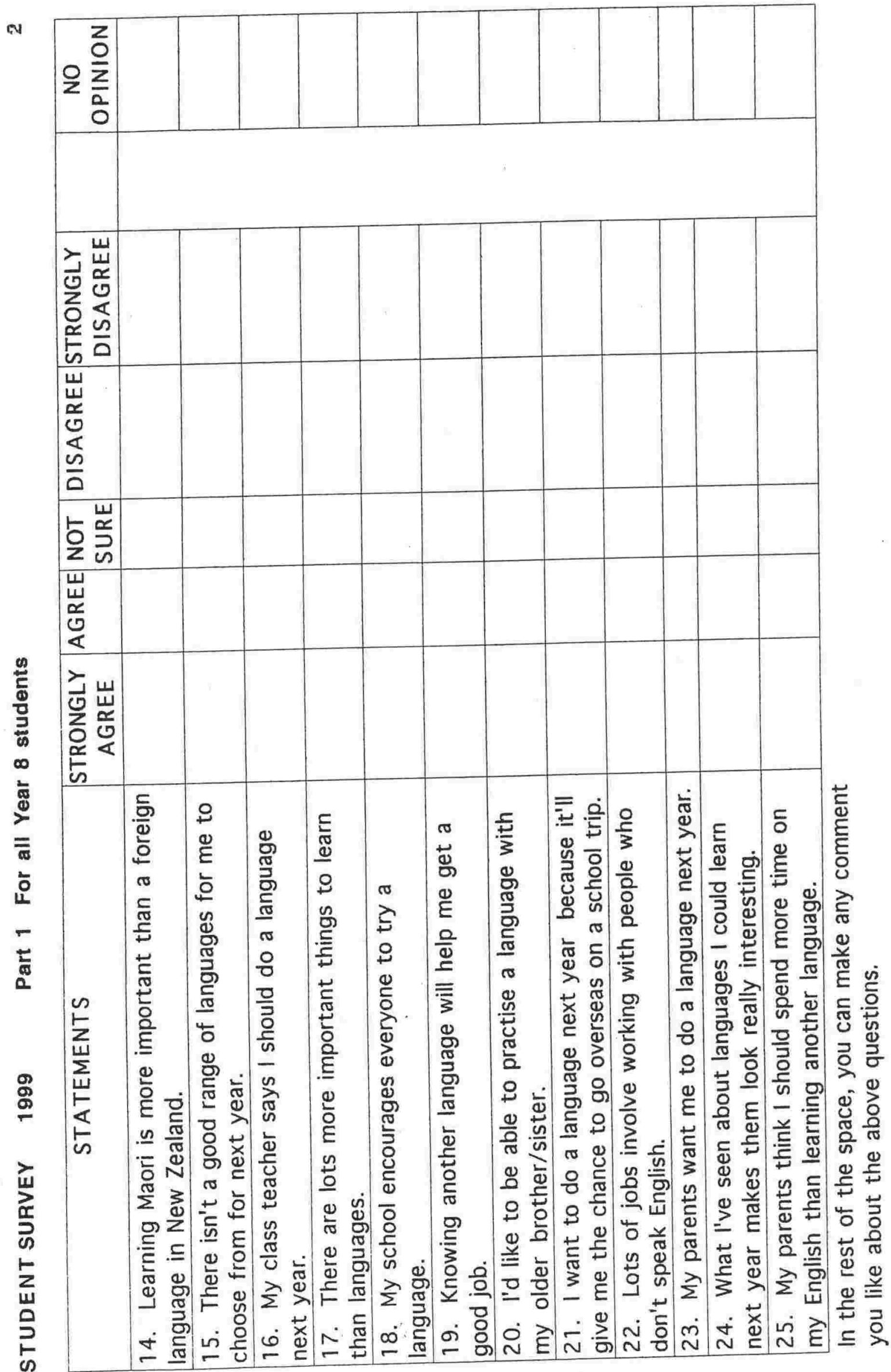




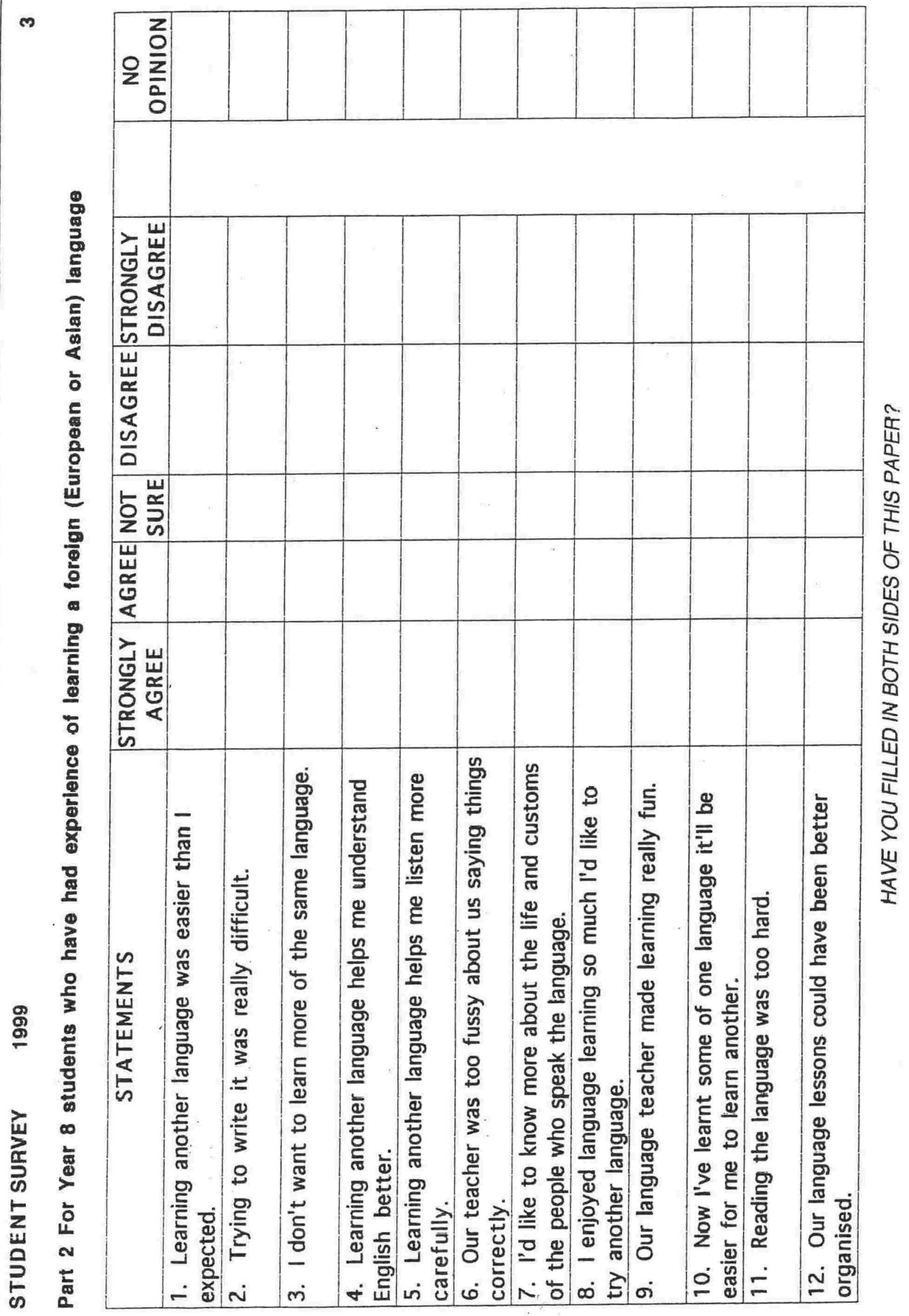




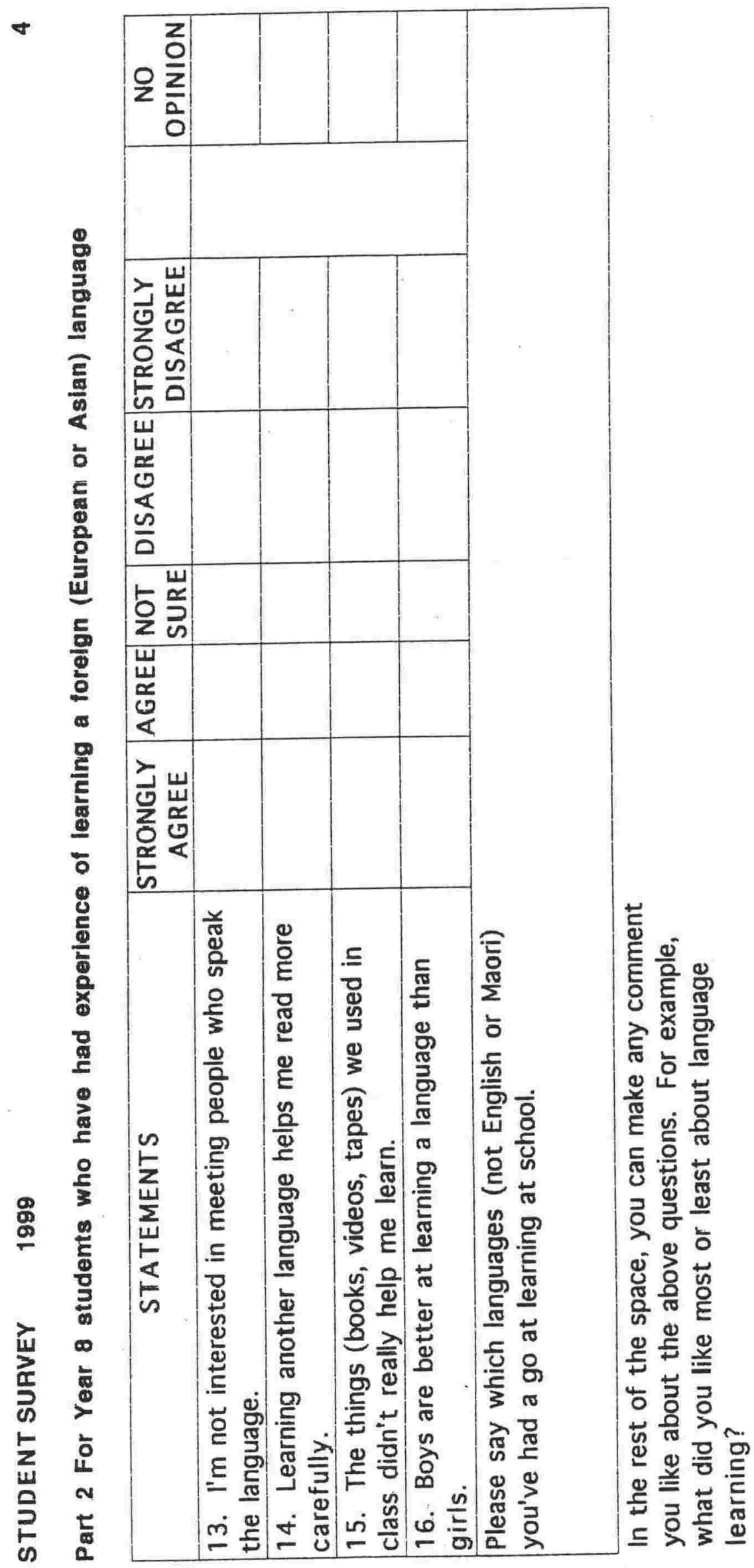

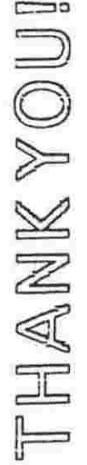


STUDENT SURVEY 1999

\section{Part 3 For all Year 8 students}

NAME

(please print clearly)

\section{CLASS/SCHOOL}

GIRL or BOY?

1. Have you ever learnt a language at school, apart from English or Maori?

YES [ ] (now go to Q2) NO [ ] (now go to Q8)

2. Did you have to learn a language? YES [ ] (now go to Q4) NO [ ] (now go to Q3)

3. Why did you choose to do a language?

4. Did you have a choice of language? YES [ ] (now go to Q5) NO [ ] (now go to Q6)

5. Which language(s) did you choose?

Why? (now go to Q7)

6. Which language(s) did you have to do?

7. Give a rough idea of how long you spent on each language you've learnt at school.

8. Which secondary school do you think you'll be attending next year?

9. Will you study a language next year? YES [ ] or MAYBE [ ] (now go to Q10)

NO [ ] (now go to Q11)

10. Which language(s) will or might you do?

Why?

11. If you could learn any language at all, what would you most like to learn?

Why?

12. Have you spent time in a country where they speak another language?

YES [ ] (now go to Q13) NO [ ] (now go to Q14)

HAVE YOU FILLED IN BOTH SIDES OF THIS PAPER? 
13. Which country did you go to?

For how long?

Did you learn any of the language?

Did you enjoy being there/speaking the language?

14. Did your parents grow up speaking any languages apart from English or Maori at home? YES [ ] NO [ ]

If YES, which language(s)?

15. Did your parents learn any languages at school apart from English or Maori?

YES [ ] NO [ ]

If YES, what language(s)?

How much did they learn?

16. Which language(s) do you and your family usually speak at home?

17. Do you have friends or relations overseas? YES [ ]NO [ ]

If YES, where are they?

Do they speak any languages apart from English? YES [ ] NO [ ]

If YES, what language(s)?

Is there anything else you'd like to say about language learning?

Do you have any comments about this questionnaire?

TIEANI YOU! 
APPENDIX 6

Year 9 student survey questionnaire

STUDENT SURVEY 2000

Part $1 \quad$ For all Year 9 students

FULL NAME

GIRL [ ] or BOY [ ]

(please print clearly)

CLASS

SCHOOL

\section{Section A}

1. Did your parents grow up speaking any languages at home, apart from English or Maori?

YES [ ] NO [ ]

If YES, which language(s)?

2. Did your parents learn any languages at school in New Zealand, apart from English or Maori?

$$
\text { YES [ ] NO [ ] PARENTS NOT EDUCATED IN N.Z. [ ] }
$$

If YES, can you say what language(s)?

3. Which language(s) do you and your family usually speak at home?

4. Do you have friends or relations living overseas at present?

YES [ ] NO [ ] (If NO, go to Question 6)

If YES, where are they?

5. Do they speak any languages apart from English?

YES [ ] NO [ ]

If YES, what language(s)?

6. Have you spent time anywhere where they speak another language?

YES [ ] (If YES, go to Question 7) NO [ ] (If NO, go to Question 11)

7. Where did you go?

8. How long did you spend there?

9. Did you learn any of the language?

YES [ ] NO [ ]

If YES, what language?

10. Did you enjoy trying to speak the language?

YES [ ] NO [ ]

Can you explain why? 
STUDENT SURVEY 2000

11. Did you study any languages, apart from English or Maori, at intermediate school?

YES [ ] NO [ ] (If NO, go to Question 14)

If YES, which language(s)?

Why?

12. Did you enjoy the classes?

YES [ ] NO [ ]

Why or why not?

13. Do you think you were good at language learning? YES [ ] NO [ ]

14. Are you learning a language, apart from English or Maori, at school now or later this year?

YES [ ] (If YES, go to Section B below)

NO [ ] (If NO, go to Section C on page 3)

\section{Section B}

1. Why are you studying a foreign language?

2. Which language(s) are you studying?

3. Did you have a choice of language? YES [ ] (If YES, go to Question 4) NO [ ] (If NO, go to Question 5)

4. Why did you make your particular choice?

5. Would you rather have done another language?

YES [ ] NO [ ]

If YES, which one?

Why?

6. Do you think you'll continue studying a foreign language next year?

Why or why not?

$$
\text { YES [ ] MAYBE [ ] NO [ ] }
$$

(Now please go to section D on page 3) 
STUDENT SURVEY 2000

\section{Section C}

1. Why are you not studying a foreign language this year?

2. Would you like to have studied a foreign language?

$$
\text { YES [ ] NO [ ] }
$$

If YES, which language(s)?

Why?

3. Do you think that you might learn a foreign language one day?

$$
\text { YES [ ] MAYBE [ ] NO [ ] }
$$

4. In your opinion, what are the main reasons why students choose to study languages?

\section{Section D}

Which school did you go to last year?

Did you take part in a similar survey last year?

$$
\text { YES [ ] NO [ ] }
$$

Are you willing to be interviewed with other students to discuss your ideas about language learning in more detail?

$$
\text { YES [ ] NO [ ] }
$$

In the space below please feel free to comment about language learning, for yourself or other students, at intermediate or secondary school?

Do you have any comments about this questionnaire? 


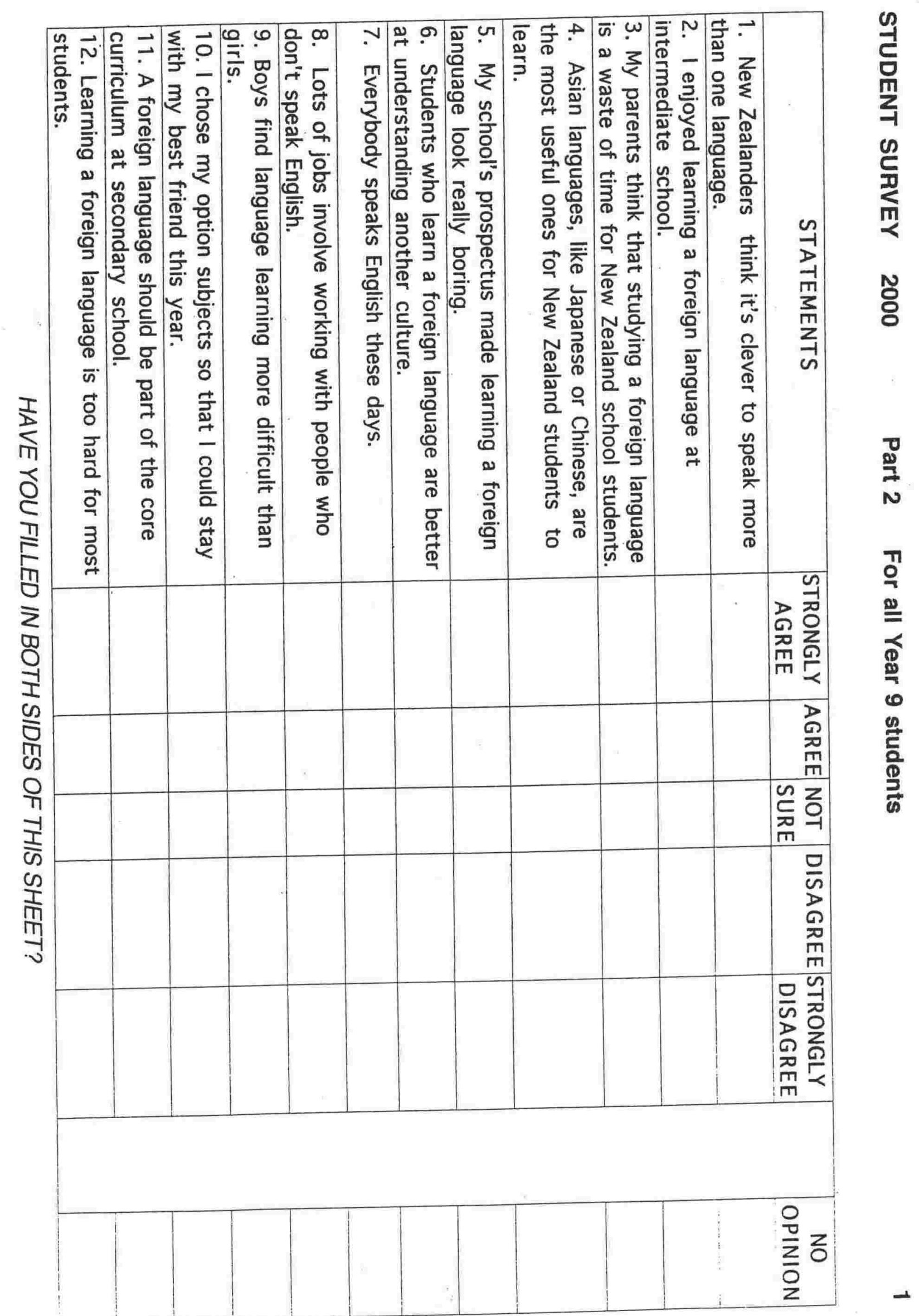




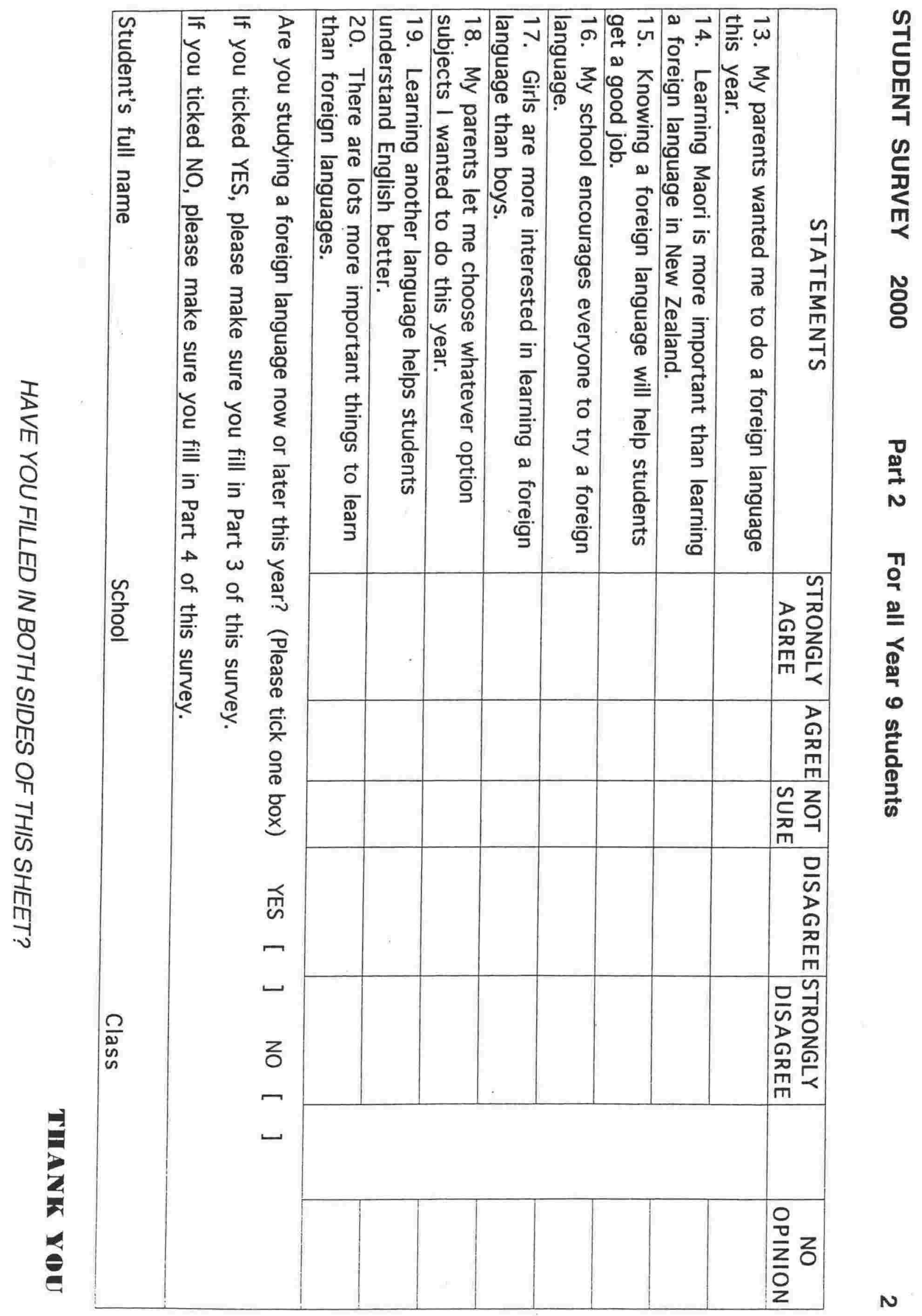




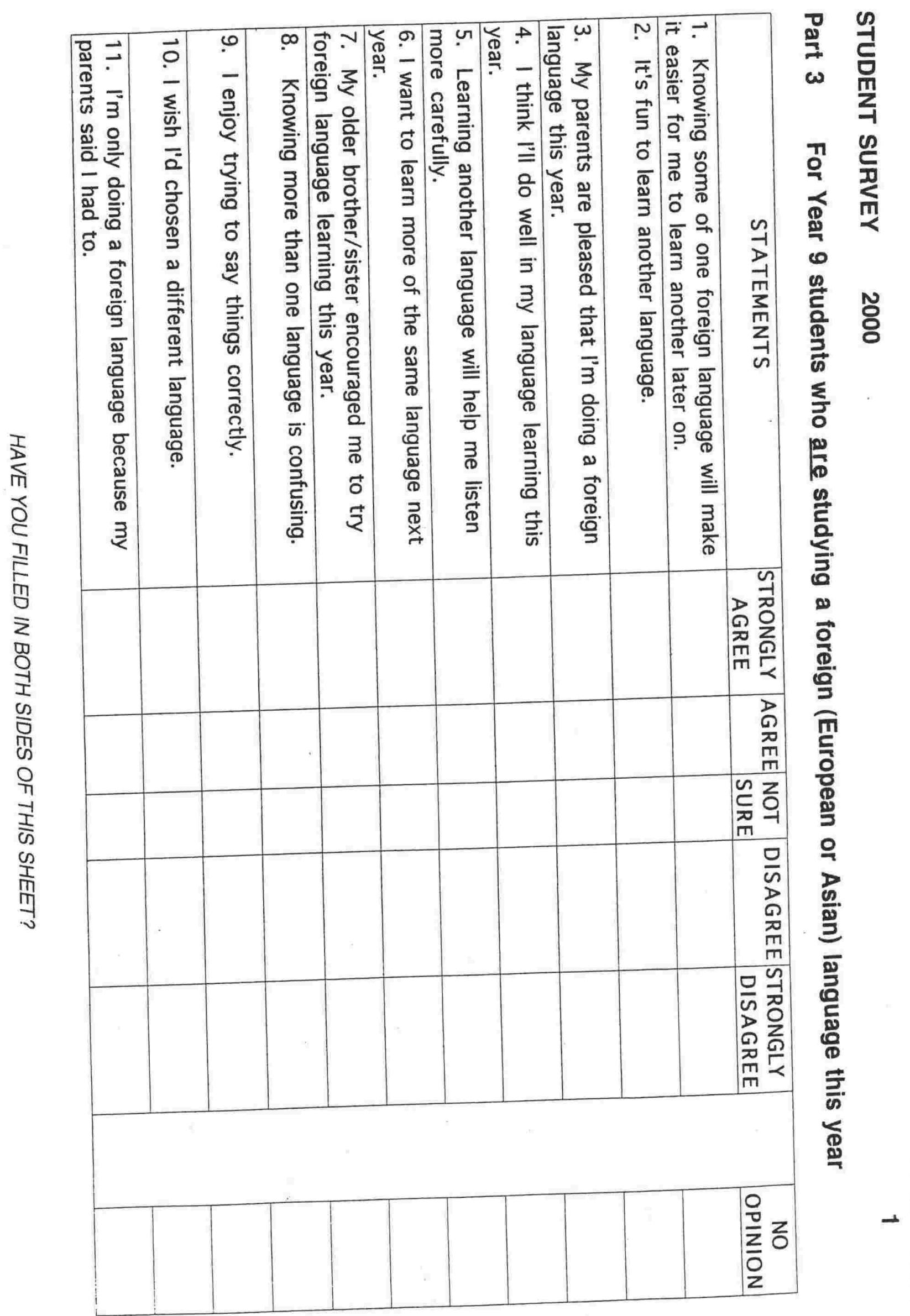




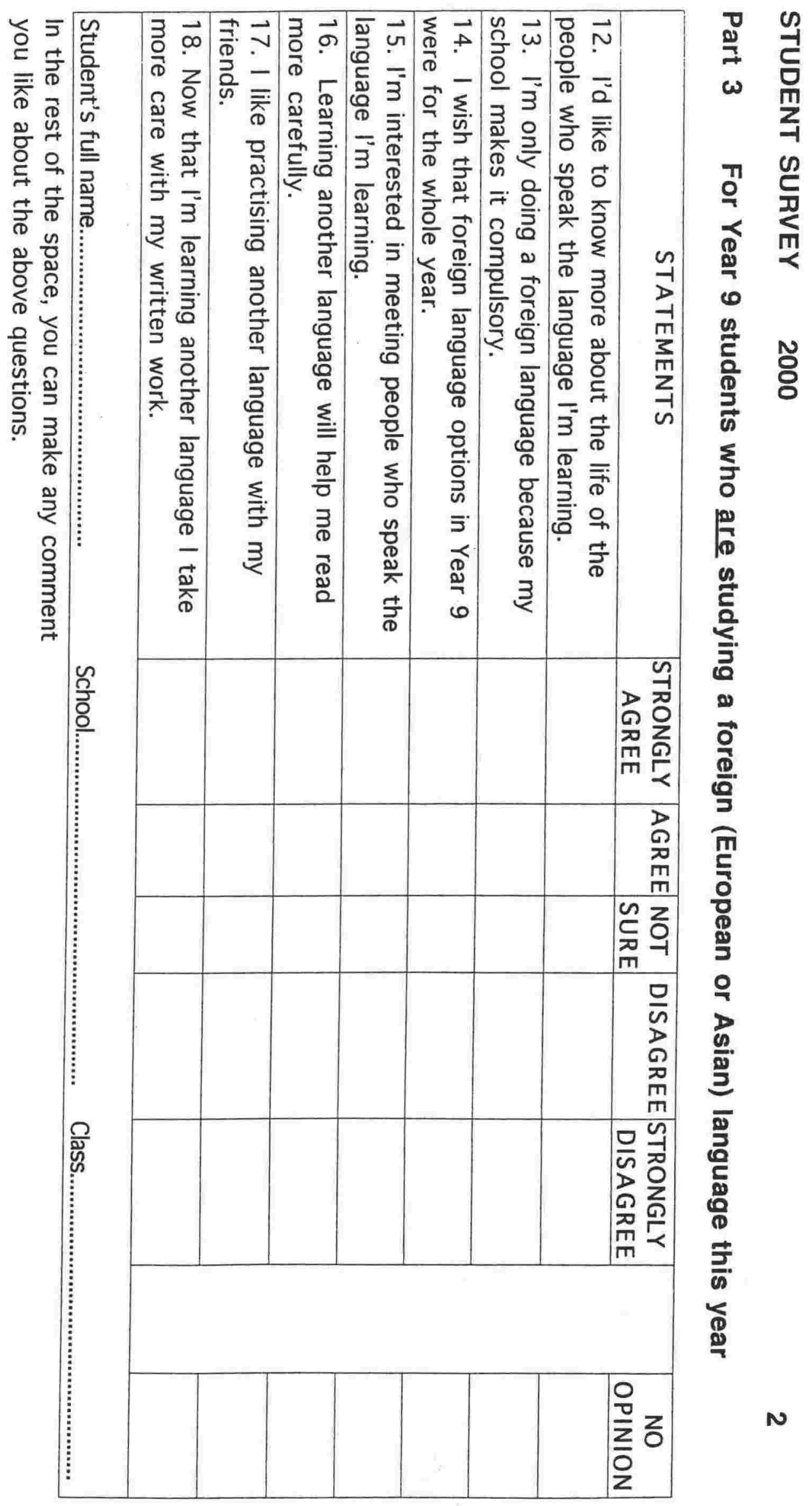




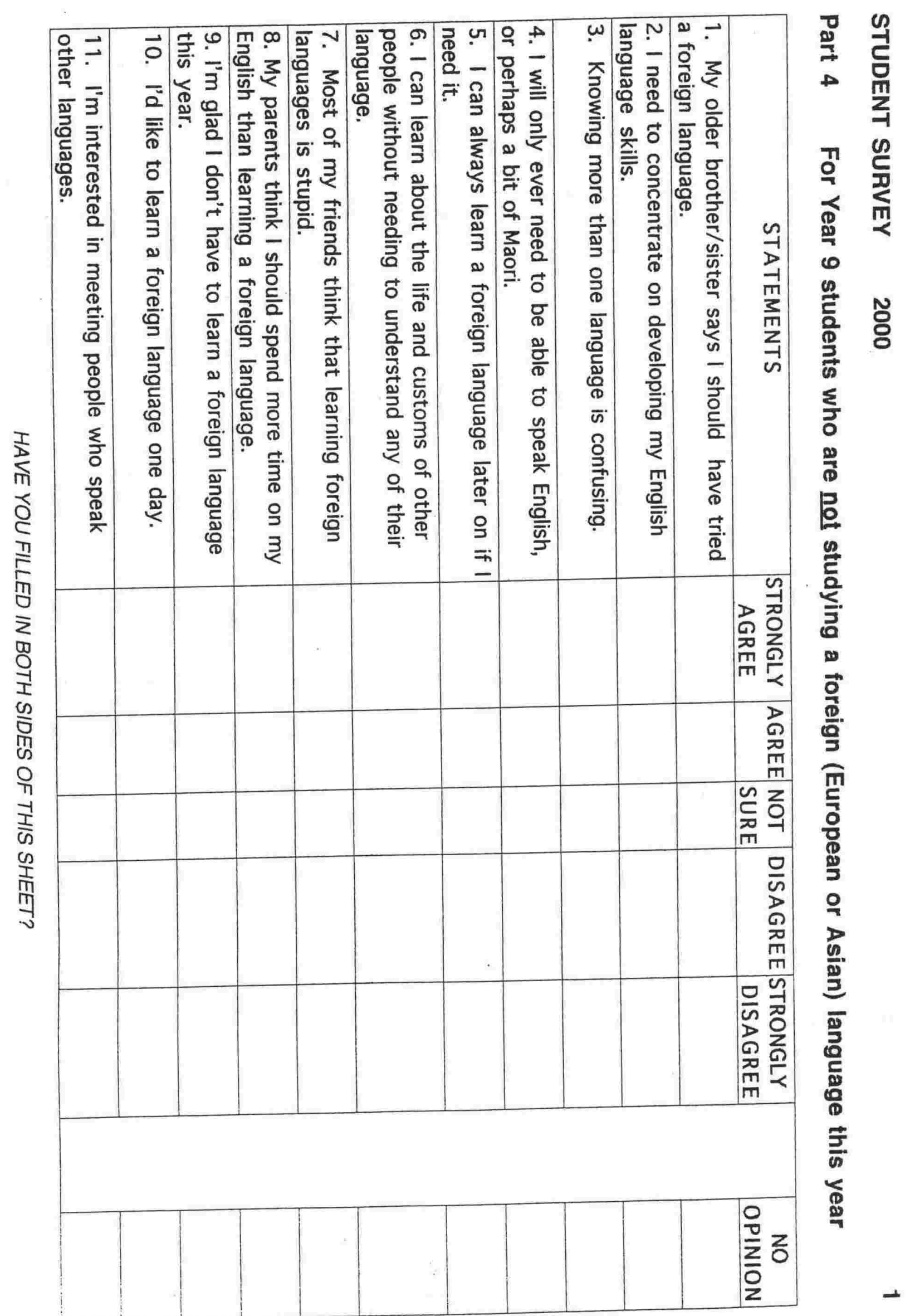




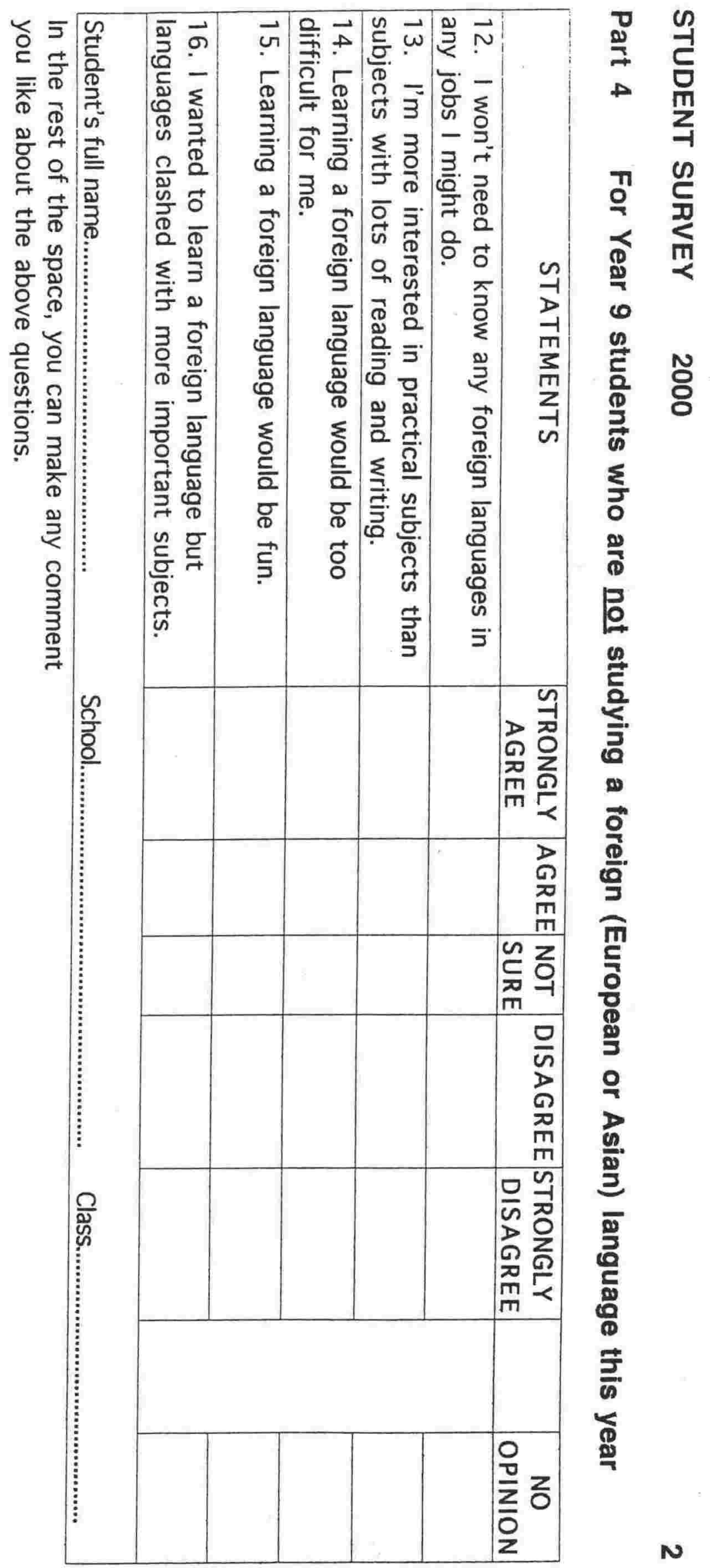




\section{APPENDIX 7}

Questions for primary and intermediate teacher interviews

A. PRELIMINARY QUESTIONNAIRE (To be filled before interview if possible)

1. What is your position in the school?

2. How long have you been here?

3. What class level do you teach?

4. What is your mother tongue?

5. Have you ever learnt a language other than English? If so, please give brief details of what, when and where

6. Have you ever taught a language apart from English? If so, please give brief details of your experience.

7. In your view, is learning a language other than English important for New Zealand students? Please indicate by circling one option:

Very importantFairly important Not important Not sure

8. What are the best languages for New Zealand students to learn?

9. What is the best age for students to start learning another language? 


\section{B. INTERVIEW QUESTIONS}

\section{Follow up answers to Preliminary Questionnaire}

1. Why did you learn

2. What did you enjoy or not enjoy about the experience?

3. What did you gain most at the time? (the learning process or the end result?)

4. What have you felt about it since? (Have you used the language? How?)

5. What are your general feelings about the time and effort you spent on language learning?

2. Expand views on language learning in general in NZ schools:

1. Ask for reasons for view of importance of language learning (Preliminary Q8)

2. Ask for reasons for choice language(s) (Preliminary Q9)

3. If fairly or very important, should all students have some experience?

4. If compulsory, how much should they do? Per week? For how many years?

5. If optional, how much should they do?

6. Where language learning is optional, what sort of students usually choose to do languages? What do you think about that? (gender, socio-economic status, parental education?)

\section{Language learning in this school (if there is any):}

1. What is the general picture of FLL at your school? -the good and the bad points? How long has it been going? Who instigated it? Why?

2. How important is it in the eyes of other teachers, students, the wider school community (families, local employers, tertiary institutions, etc.)?

3. Does your school do anything to encourage or discourage language learning? (e.g. make it compulsory, fee-paying)

4. If FLL is optional, what sort of reasons do you think students have for choosing to study a language or for dropping out of such classes (if this is possible?

5. Are there any problems finding suitable language teachers? Why?

6. What would be the ideal situation, in your opinion, as far as FLL is concerned in NZ? (class sizes, streaming, frequency/length of lessons, length of courses, choice of languages, compulsion, starting age, etc)

7. Have you any other comments to make about language learning in your school?

8. Would you like to make any comments about this interview or the research? 


\section{APPENDIX 8}

\section{Questions for secondary teacher interviews}

A. PRELIMINARY QUESTIONNAIRE (To be filled before interview if possible)

1. What is your position in the school?

2. How long have you been at this school?

3. What subjects do you teach?

4. What other area(s) of responsibility do you have?

5. What is your mother tongue?

6. Have you ever learnt a language other than English or Maori? If so, please give brief details of what, when and where

7. Have you ever taught a language apart from English or Maori? If so, please give brief details of your experience.

8. In your view, is learning a language other than English or Maori important for New Zealand students? Please indicate by circling one option:

Very important Fairly important Not important Not sure

9. What are the best languages for New Zealand students to learn?

10. What is the best age for students to start learning another language? 


\section{B. INTERVIEW QUESTIONS}

1. Follow up questions to Preliminary Questionnaire

1. Why did you learn ?

2. What did you enjoy or not enjoy about the experience?

3. What did you gain most at the time? (the learning process or the content?)

4. What have you felt about it since? (Have you used the language? How?)

5. What are your general feelings about the time and effort you spent on language learning?

\section{Expand views on language learning in general in $\mathrm{NZ}$ schools:}

1. Ask for reasons for view of importance of language learning (Preliminary Q8)

2. Ask for reasons for choice of language(s) and starting age (Preliminary Q9, 10)

3. If fairly or very important, should all students have some experience?

4. If compulsory, how much should they do? Per week? For how many years?

5. If optional, how much should they do?

6. Where language learning is optional, what sort of students usually choose to do languages? (gender, socio-economic status, parental education?)

\section{Language learning in this school:}

1. What is the general picture of FLL at your school? -the good and the bad points? Appropriate range of languages? Why/not?

2. How important is it in the eyes of other teachers, students, the wider school community (families, local employers, tertiary institutions, etc.)?

3. Does your school do anything to encourage or discourage language learning? (e.g. make it compulsory - for all/some)

4. If FLL is optional, what sort of reasons do you think students have for choosing to study a language or for dropping out of such classes (if this is possible?)

5. Are there any problems finding suitable language teachers? Why?

6. What would be the ideal situation, in your opinion, as far as FLL is concerned in NZ? (class sizes, streaming, frequency/length of lessons, length of courses, choice of languages, compulsion, starting age, etc)

7. Have you any other comments to make about language learning in your school?

8. Would you like to comment on this interview or the research? 


\section{For HOD or teachers in charge of foreign languages}

1. What are current class numbers in each language at each level?

2. How do these compare with previous years?

3. What are general trends in foreign language learning in your school:

- students' choices of language,

- drop-out rates,

- running of Y12 and 13 classes (any combined, on correspondence),

- teacher supply,

- disincentives,

- exam results?

4. Is there any gender bias noticeable in choices of language or drop-out rates?

5. Please give details of the place of languages in core curriculum or option system at Years 9 and 10, length of courses, frequency of classes. Can students start a foreign language after Year 9?

6. Does the school have a curriculum committee? Who are the members? Have they had any noticeable influence over the place of foreign languages in the school?

7. What contact do you have with feeder schools? Is there any cooperation over foreign language learning? Does the school have problems with students who have previous experience of foreign language learning? How are they catered for?

8. What guidance do students receive about their option choices on entering the school and at later stages? Is such advice only given on request from students or parents? Who gives the advice? Apart from the prospectus is there anything in writing about option choices? 9. Please add any other information particular to the place of foreign languages (not Maori) at your school which you think might be useful. 


\section{APPENDIX 9}

\section{A. Summary of parent interview schedule for parents to consider in advance}

Interviews will be based on the following topics and you might like to think about or discuss them in advance.

\section{How Year 9 option subject decisions were made}

- Who was involved in discussions

- What information was available - sources, amount, value

- Who made final decisions

2. How decision to do a foreign language or not (if optional) was made and/or how choice of language was made

- Who or what influenced choice, including child's previous experience of language learning, contact with speakers of languages other than English or Maori, travel, etc.

- Main reason(s) for final decision

3. Parents' experience of learning/using languages other than English or Maori education, travel etc.

- What experience of formal foreign language learning at school or beyond

- Feelings about this experience

- Personal or business connections with speakers of other languages here or overseas

- Influence of any of these on child's choices

\section{Present and future}

- Feelings about current foreign language learning

- Likelihood of continuing foreign language learning or starting it at a later stage

- Future possibilities for education, work, travel

\section{General feelings about current situation at child's school}

- availability of languages and their place in the curriculum

- school's general attitude to foreign language learning

6. General feelings about the place of foreign language learning in NZ schools

- $\quad$ starting age, choice of languages, place in the curriculum

- $\quad$ general value for individuals, for New Zealand 


\section{APPENDIX 9}

\section{B. Parent interview schedule}

Name of child and school

Studying a foreign language or not Yes No Compulsory (Start at B)

Language

\section{A IF FOREIGN LANGUAGES ARE PART OF OPTION SYSTEM}

\section{How option decisions were made}

Can you tell me how it was decided which option subjects was going to take in

Year 9 (third form)

What sort of discussions took place?

Who was involved - parents, child, teachers, siblings, other students/ parents?

What info was available/consulted - prospectus, option booklet, school visit?

What did you think of info, advice you received?

Did schools encourage students to try a FL? How?

Were options a factor in choice of school?

Who made ultimate decisions?

2. How decision to do a foreign language or not was arrived at

- Can you remember any discussion specifically about languages?

- Did ....... have any previous experience of foreign language learning at school?

- if yes, what language(s)?

- part of normal schoolwork or optional extra?

- for how long?

- what did parents/child think of that - useful?

- interesting?

- enjoyable?

- reasons???

- Has..........had any experience of overseas travels or residence?

- Did either experience influence their choice - to do a foreign language or not ?

Was choice influenced by - languages (not) offered by secondary school?

- length of options?

- child's friends, siblings etc?

- possibility of overseas school trip?

- any contact with people speaking languages other than

English

- any preconceptions about cultures 


\section{B IF LANGUAGE LEARNING (INC. M) IS COMPULSORY}

\section{How choice of language was made}

Can you tell me how it was decided which language was going to take in Year 9 (third form)

What sort of discussions took place

Who was involved - parents, child, teachers, siblings, other students/ parents What info was available/consulted - prospectus, option booklet, school visit, Opinions of info, advice received

Was the nature of the option system or the place of languages a factor in choice of school?

Who made ultimate decisions

\section{Child's previous experience}

Can you remember any discussion specifically about languages?

Did ........ have any previous experience of foreign language learning at school?

- if yes, what language(s)?

- part of normal schoolwork or optional extra?

- for how long?

- what did parents/child think of that - useful?

- interesting?

- enjoyable?

- reasons???

Has...........had any experience of overseas travels or residence?

Did either experience influence their choice - to do a foreign language or not?

- to choose a particular language?

Was choice of language influenced by

- languages (not) offered by secondary school?

- child's friends, siblings etc?

- possibility of overseas school trip?

- any contact with people speaking languages other than English?

- any preconceptions about cultures?

\section{FOR ALL}

3. Parents' experience of foreign languages - education, travel etc.

Can you tell me what experience you've had of formal foreign language learning at school or beyond

- What language(s)

- for how long

- what you thought of

- the learning process/cultural info at the time - the outcome - subsequent use/value 
- has this influenced what you've said to your child and the extent to which you've encouraged/discouraged him/her?

- What personal or business connections have you (had) with speakers of other languages here or overseas?

- has this influenced their decisions re foreign languages in your opinion?

\section{Present and future}

A If child is studying a foreign language

$$
\text { So . }
$$
is studying

What are main reasons for this? If compulsory - would your child have preferred not to have

$\rightarrow$ Would you have preferred that s/study Challenging? Are you/child happy with How is it going so far - fun? Inter of hours per week, teacher, course content, general choices made, length of course,

- Do you think is likely to continue next year? Why/why not?

- What influence do you expect to have about his/he

- On what do you think the choices will be based? (what sort?)

- possible future carest/enjoyment

- possible future travel - with school or family, eg exchange,

- teacher(s) or contact with foreign language assistants

- what child's friends are going to do

B If child is not studying a foreign language

- Would your child like to have studied a/nother FL?

- Would you like him/her to have studied a/nother FL?

- Would you like to see your child learn a foreign language at some stage?

- Why/not?

- Do you think s/he would like to, be interested in doing so, find it useful in further education, work or travel?

\section{Foreign language learning at your child's school}

- What do you think about the place of languages? Are you happy with the nature of choices available (or not) for foreign language learning:languages offered/length of courses/hours per week, availability of senior classes/correspondence, possibility of doing more than one foreign language possibility of starting beyond Year 9 (e.g. multi-level learning)

whether optional or compulsory

availability of native speakers (eg assistants)

Does school organise any overseas trips?

Are foreign exchanges encouraged? 
Are students given any other incentives to do Fls - opportunities for use, reasons for learning?

\section{The place of foreign language learning in $\mathrm{NZ}$ schools}

- Do you have any general feelings about the place of foreign language learning in NZ schools -primary or secondary?

- too much/too little/just right

- choice of languages

- optional/compulsory

- starting age

- supply or competency of teachers

- Do you think NZ needs foreign language speakers? Why (not)?

- If, yes, what is the best way to produce them?

- Reasons???

- What do you think NZers in general think FLL in NZ schools? Do they see any value in FLL for:- cross-cultural understanding?

Awareness of their own language and others?

Economic reasons - jobs in tourism and trade?

general education - cultural and linguistic

enrichment, access to wider range of ideas?

Practical use in overseas travel 


\section{APPENDIX 10}

\section{BACKGROUND INFORMATION SHEET}

1. Were you born in New Zealand?

Yes $\square \quad$ No $\square$

2. If no, (a) where were you born?

(b) at what age did you come to New Zealand?

3. (a) Which language did you speak first in your home?

(b) Which language did your mother speak first at home as a child?

(c) Which language did your father speak first at home as a child?

4. Are you Female $\left[\begin{array}{l}\square \text { Male } \\ \text { ? }\end{array}\right.$

$\begin{array}{llcl}\text { 5. Please circle your age group: } & \text { Under } 30 & 30-34 & 35-39 \\ 40-44 & 45-49 & 50+\end{array}$

6. How old were you when you left school?

7. What is your highest educational qualification?

8. What is your current job?

I give my permission for any information $I$ have supplied to be used for research purposes and $I$ understand that my identity will not be disclosed without my permission in any circumstances.

Signed:

Date: / /

Print full name: 
APPENDIX 11

Chapter 5 graphs

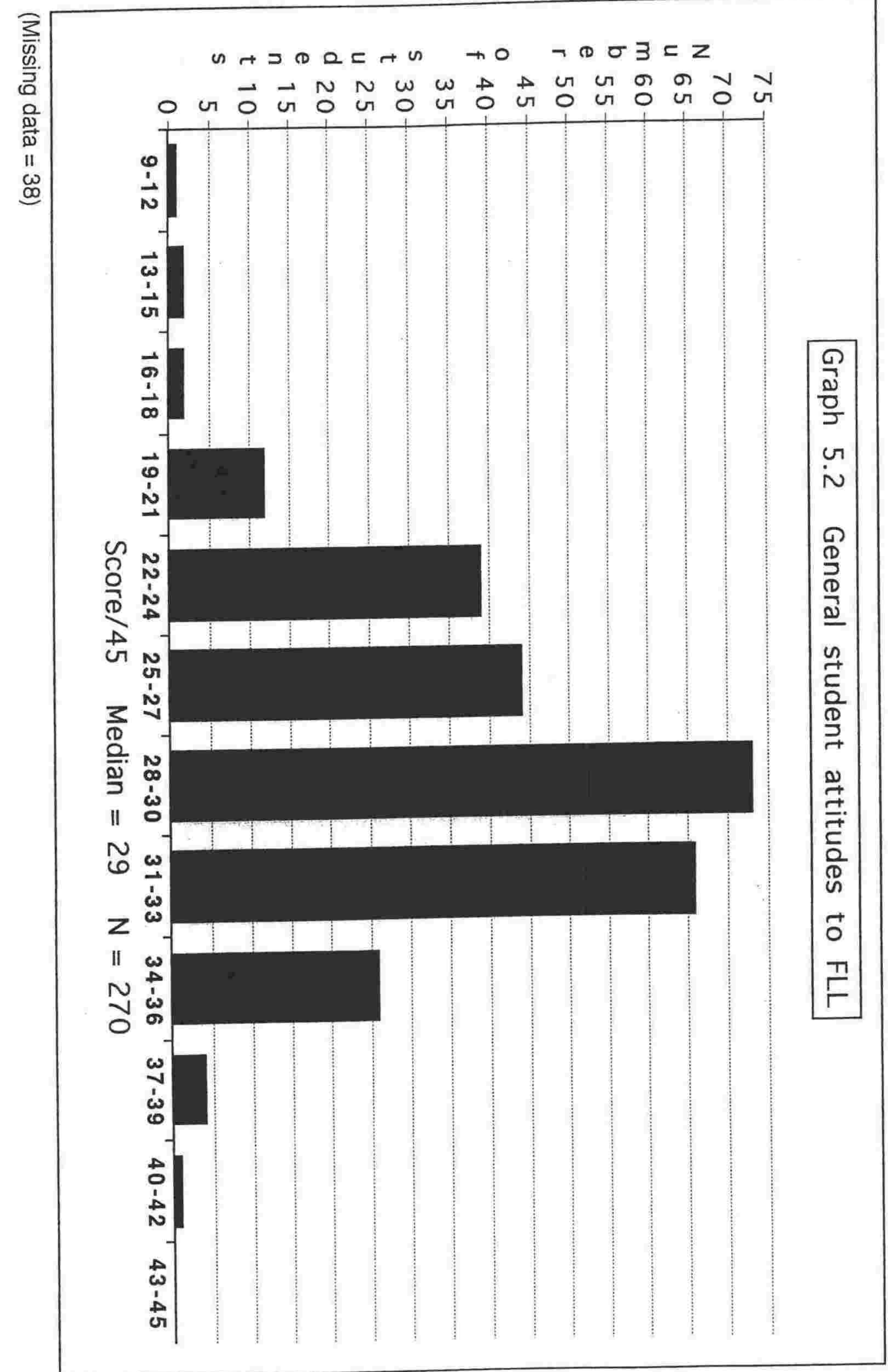




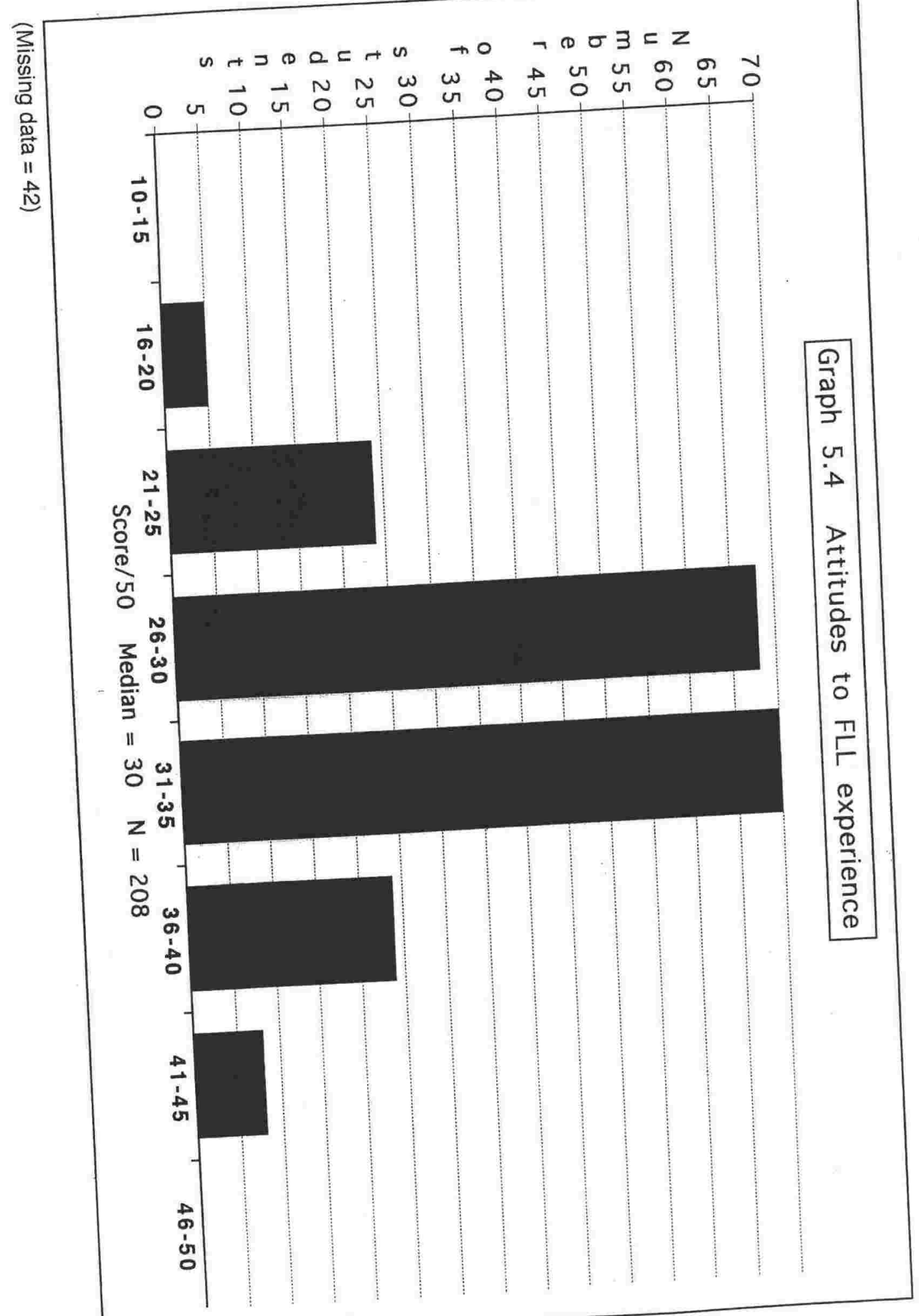




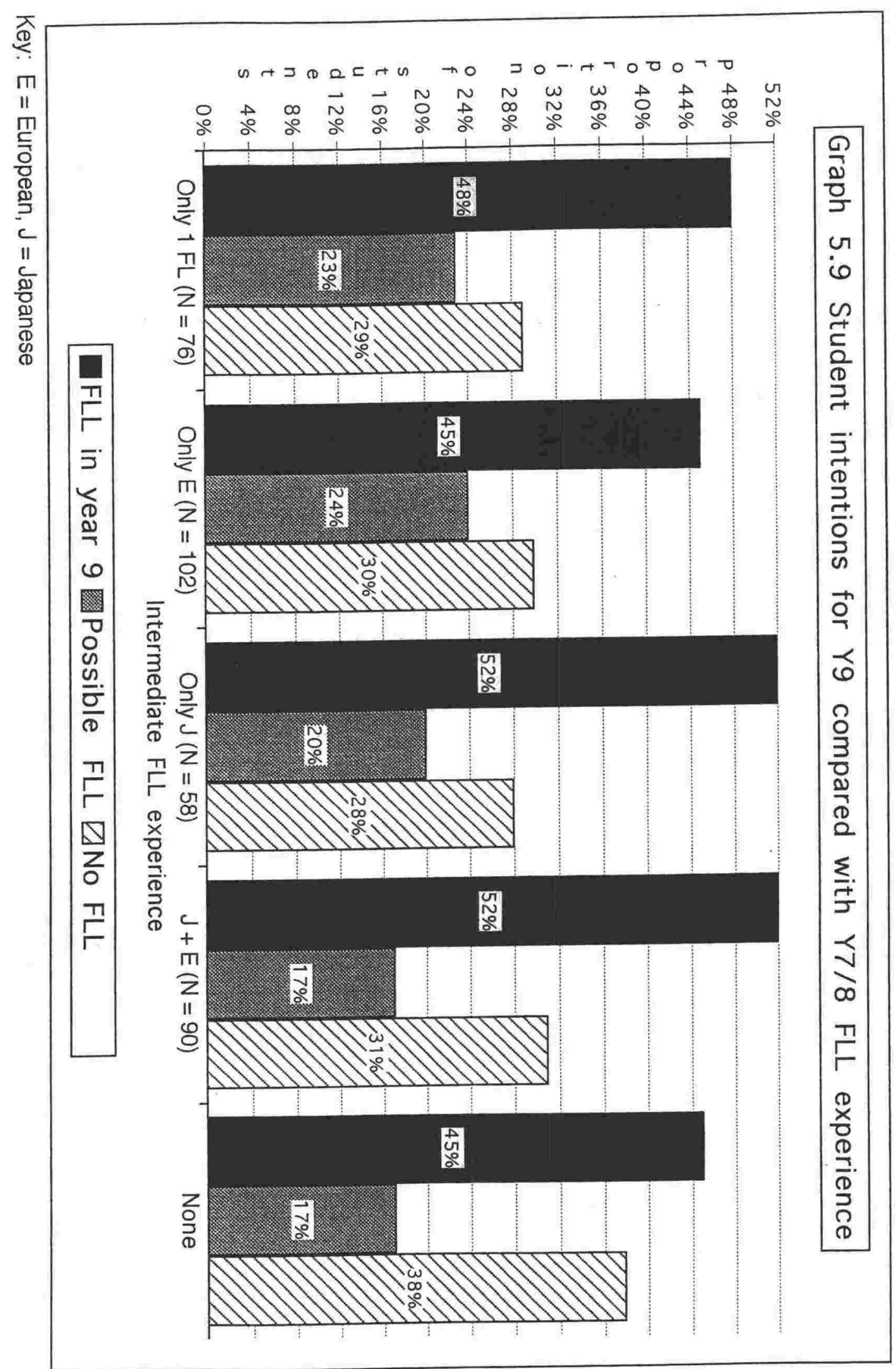


APPENDIX 12

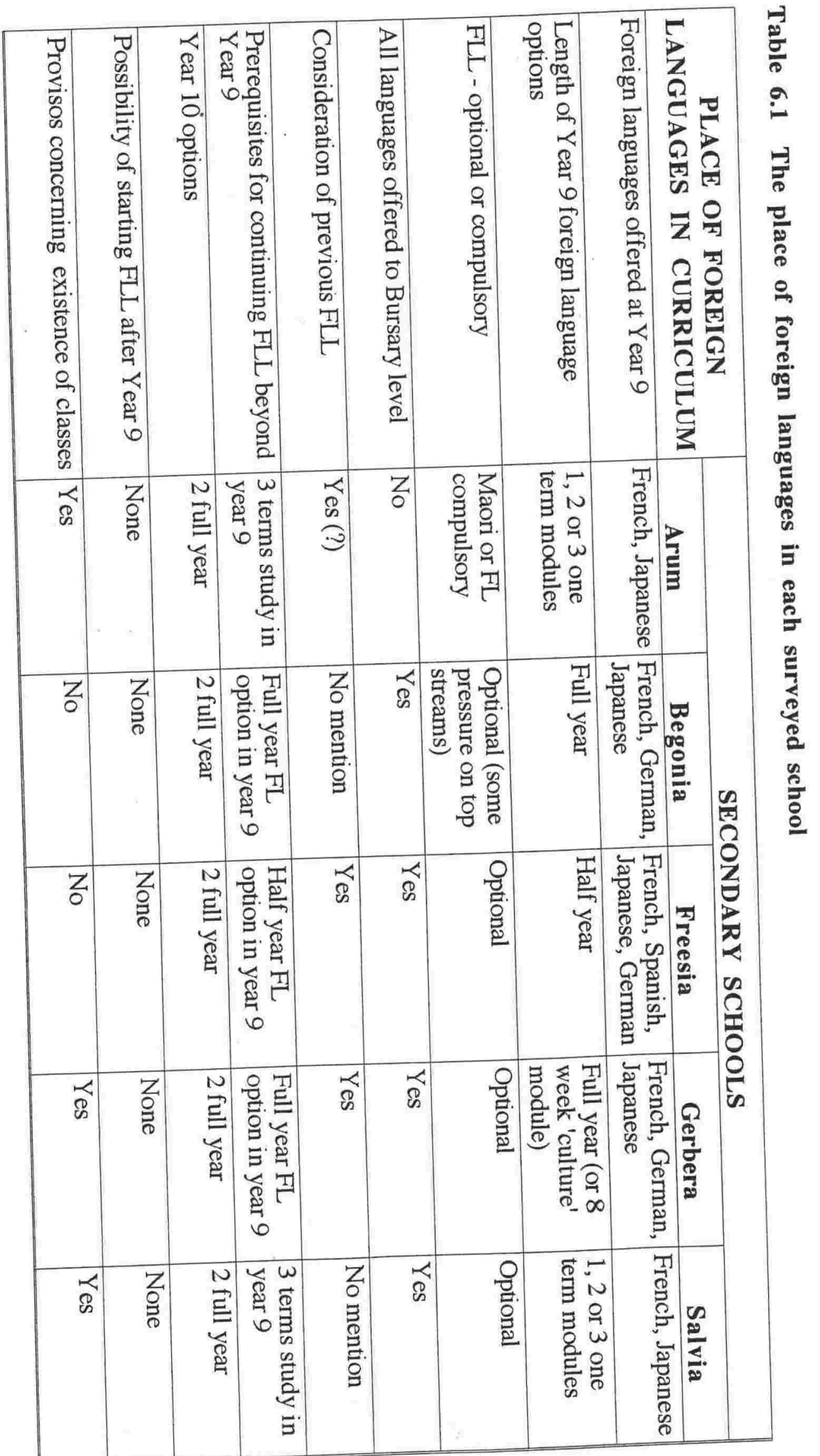


APPENDIX 13

Chapter 10 graphs

Graph 10.10 Year 8 student intentions for FLL in year 9 compared with previous experience, by gender

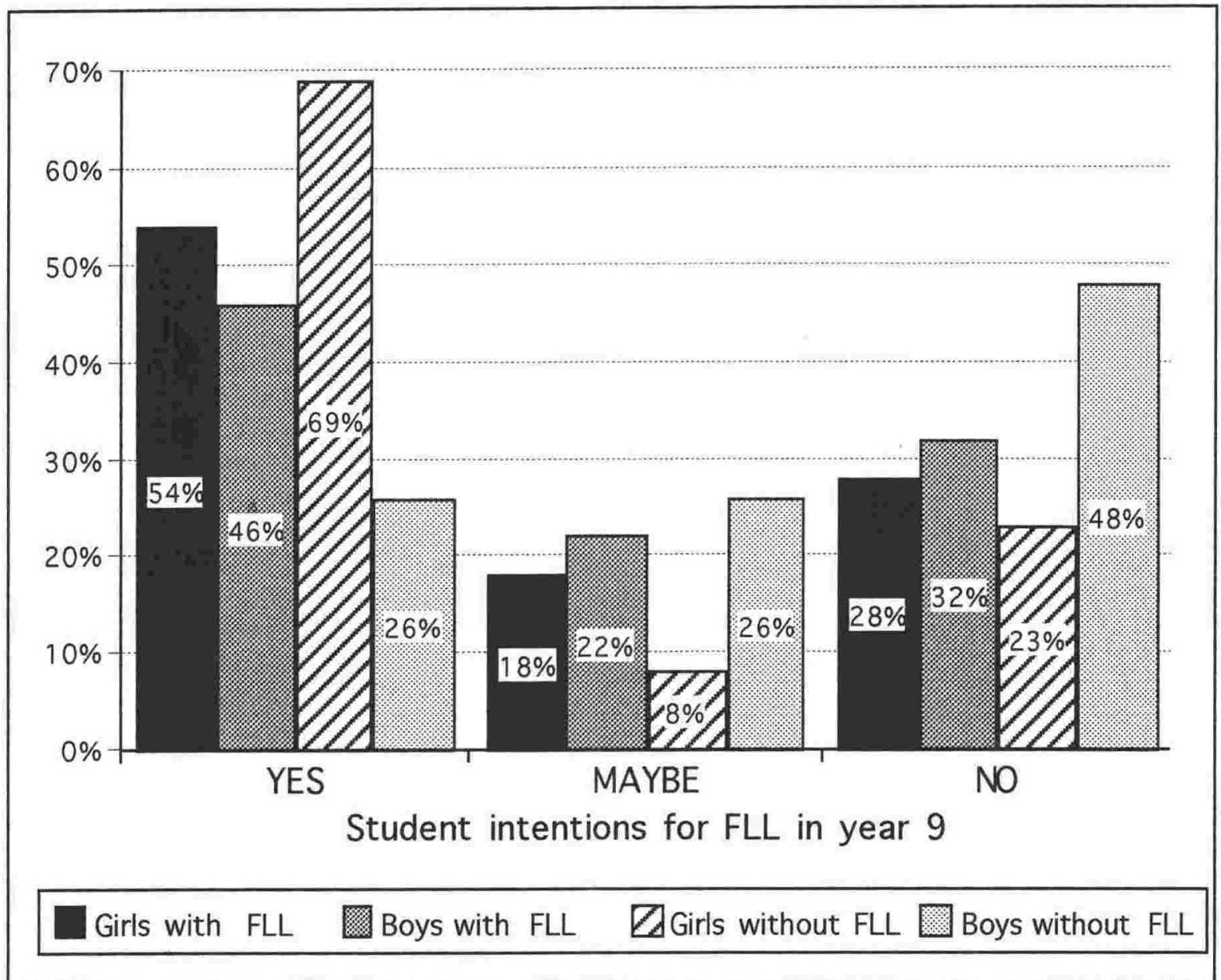

Notes: Students with FLL experience girls $N=124$, boys $N=124$

Students without FLL experience girls $\mathrm{N}=26$, boys $\mathrm{N}=31$

Excludes 3 students who gave no information 


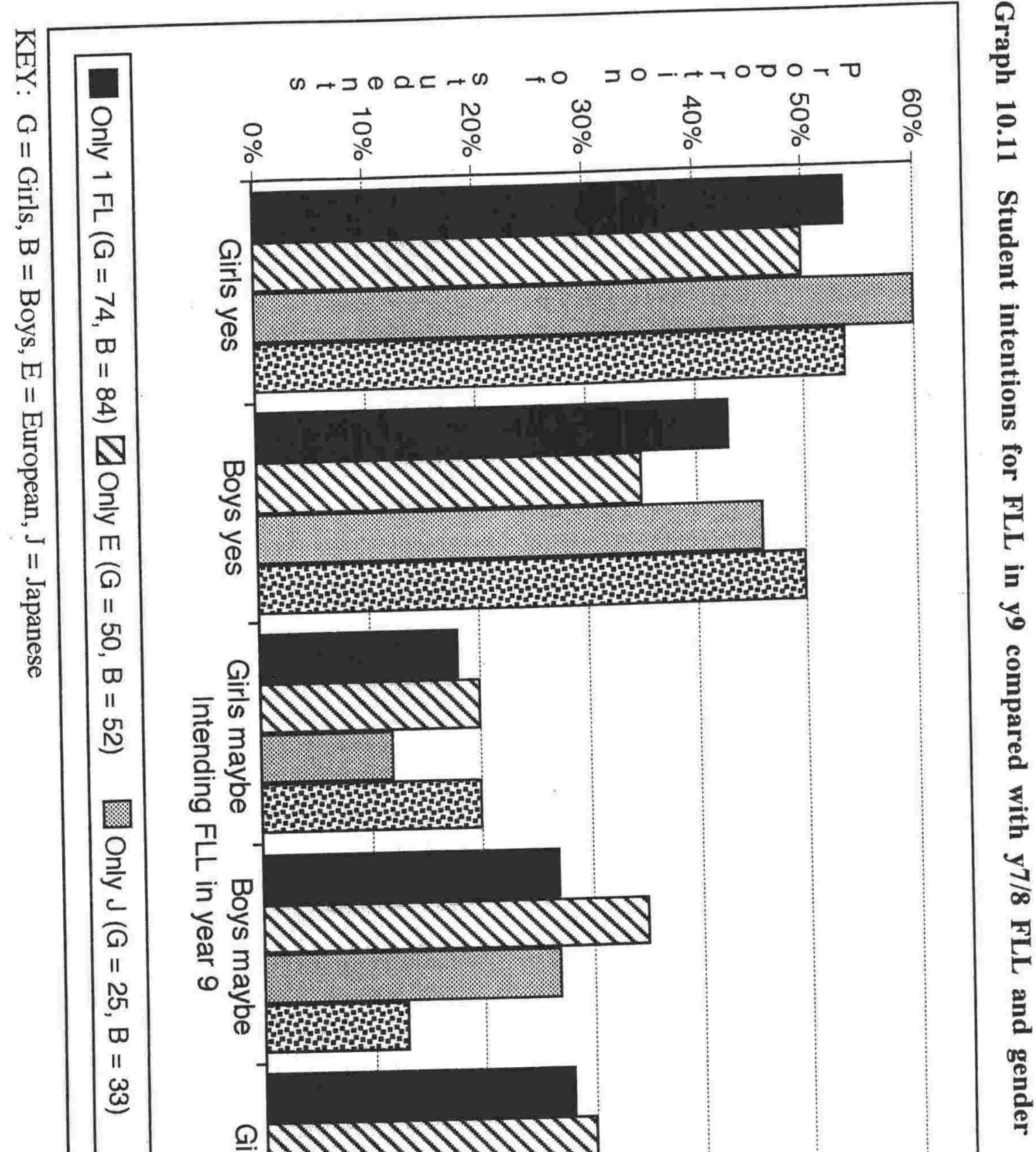




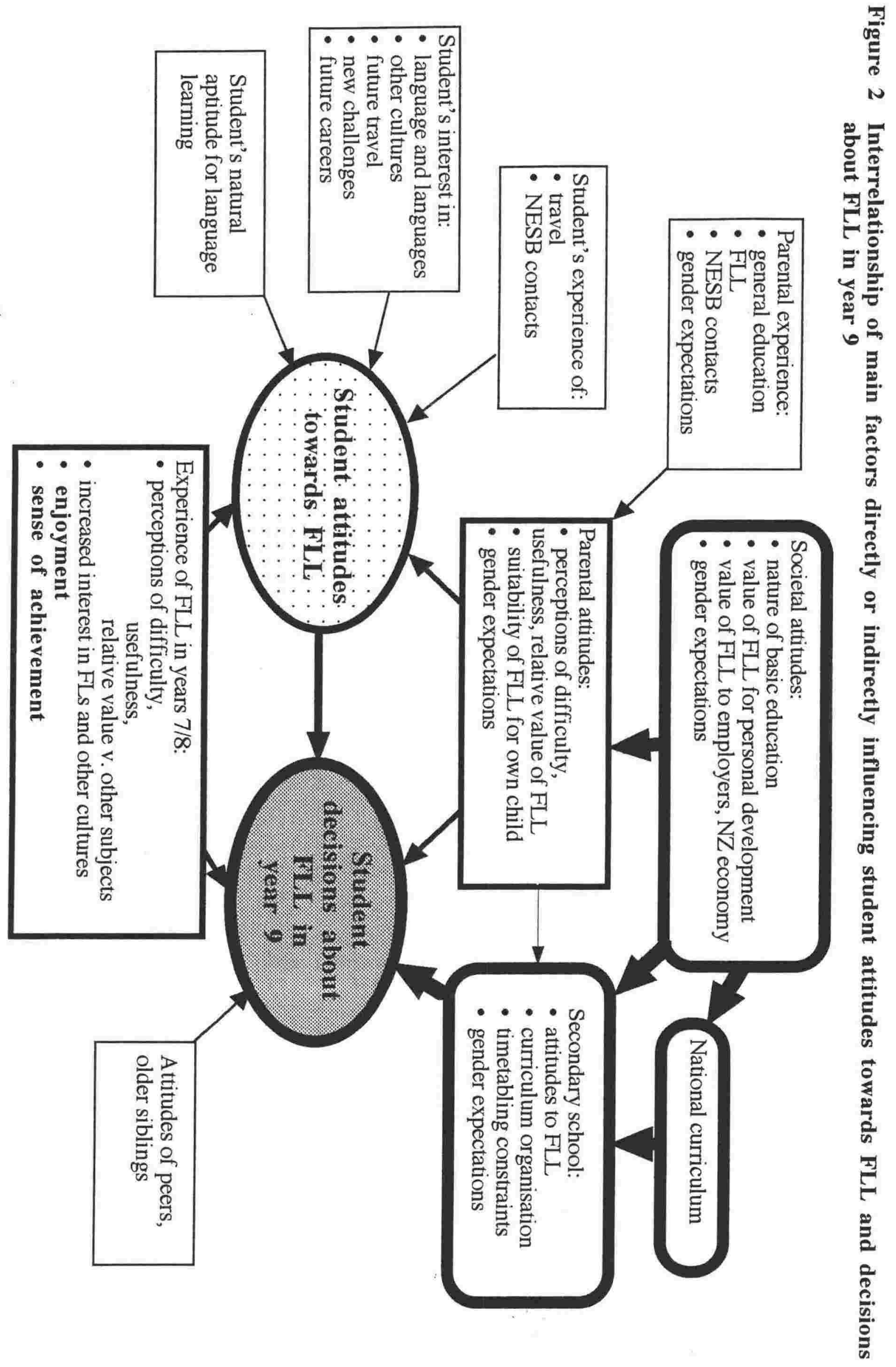




\section{APPENDIX 15}

Year 8 questionnaire Parts 1 and 2 - response frequencies

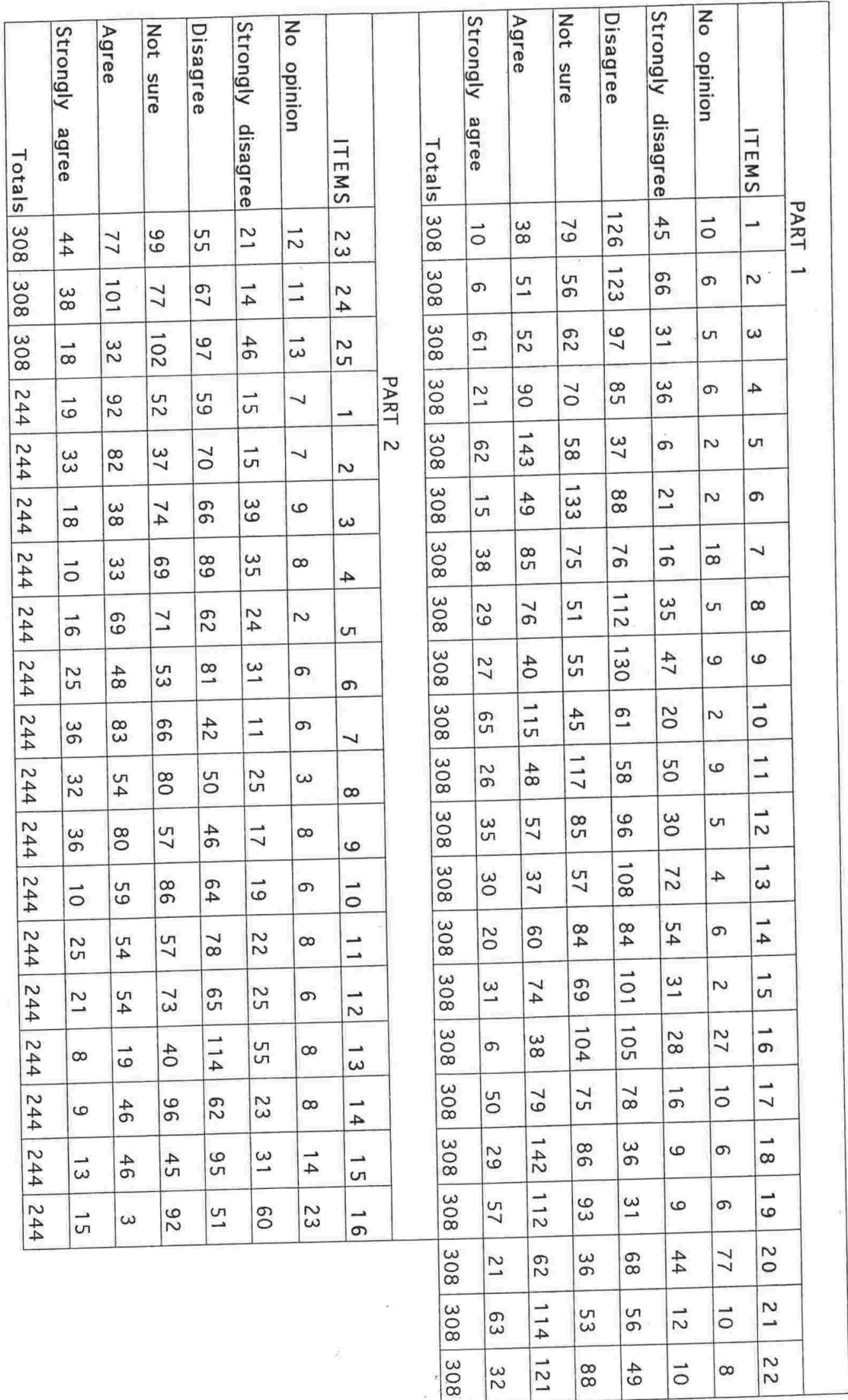


Year 9 questionnaire Parts 2,3 and 4 - response frequencies

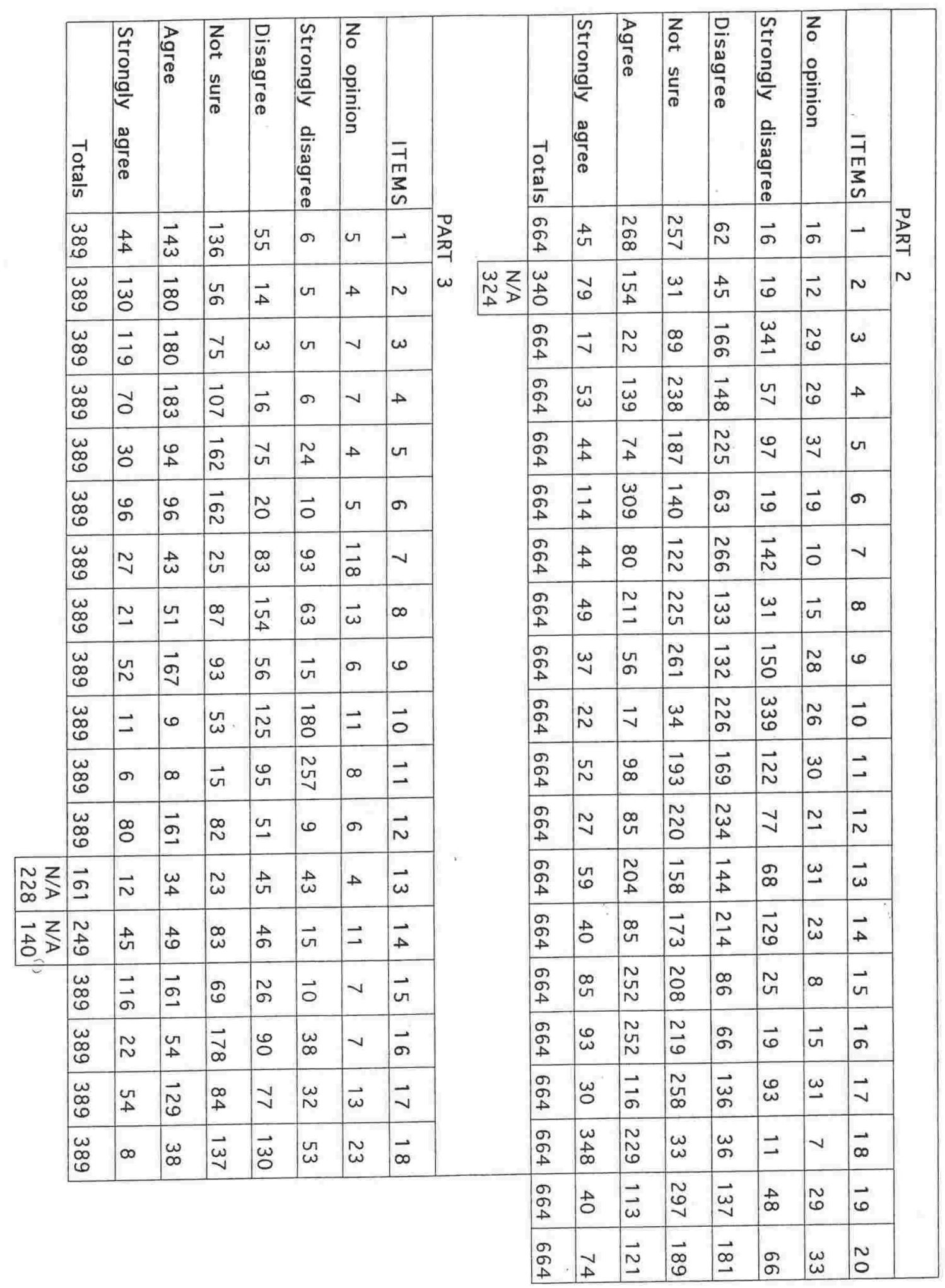




\begin{tabular}{|c|c|c|c|c|c|c|c|c|}
\hline $\begin{array}{l} \\
-1 \\
\frac{-1}{0} \\
\frac{+}{\omega} \\
\frac{\omega}{n}\end{array}$ & 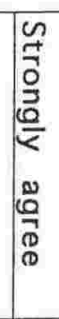 & $\begin{array}{l}\vec{D} \\
\stackrel{0}{7} \\
\mathbb{D} \\
\mathbb{D}\end{array}$ & 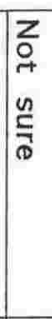 & 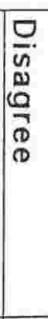 & 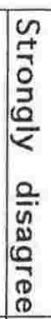 & $\begin{array}{l}z z \\
0 \\
0 \\
\frac{0}{3} \\
3\end{array}$ & $\begin{array}{l}\overline{7} \\
\text { m } \\
\text { n }\end{array}$ & \\
\hline $\begin{array}{l}\tilde{N} \\
\text { v } \\
\text { v }\end{array}$ & 6 & $\tilde{0}$ & $\underset{N}{\omega}$ & ร) & $\infty$ & 必 & - & 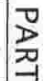 \\
\hline $\begin{array}{l}\tilde{N} \\
\text { U }\end{array}$ & $\vec{\omega}$ & v & I & $N$ & $\underset{\infty}{\omega}$ & 6 & N & $\rightarrow$ \\
\hline $\begin{array}{l}\tilde{N} \\
\text { un }\end{array}$ & $\tilde{\infty}_{\infty}^{\sim}$ & U & I & $\stackrel{\omega}{-}$ & $\underset{v}{\omega}$ & $v$ & $\omega$ & \\
\hline $\begin{array}{l}\tilde{N} \\
\text { U } \\
\text { T }\end{array}$ & $\vec{A}$ & $\tilde{\omega}$ & 2 & $\begin{array}{c}\infty \\
\omega\end{array}$ & J & $N$ & $\rightarrow$ & \\
\hline $\begin{array}{l}\tilde{N} \\
\text { ur } \\
\end{array}$ & $\underset{A}{G}$ & $\vec{\sim}$ & \pm & $\vec{v}$ & 6 & 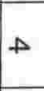 & ur & \\
\hline $\begin{array}{l}N \\
v \\
v\end{array}$ & $\tilde{N}$ & $\forall$ & $\overrightarrow{8}$ & $\underset{\omega}{\omega}$ & $\stackrel{\sim}{\sim}$ & $\vec{\sigma}$ & $\sigma$ & \\
\hline $\begin{array}{l}\tilde{U} \\
\text { uv }\end{array}$ & 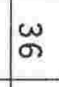 & न & 9 & 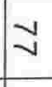 & ज़ & $\vec{\sigma}$ & $v$ & \\
\hline $\begin{array}{l}N \\
v \\
v\end{array}$ & $\underset{+}{\sim}$ & $\omega$ & $N$ & () & Gr & 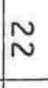 & $\infty$ & \\
\hline $\begin{array}{l}N \\
\text { u }\end{array}$ & $\stackrel{\vec{v}}{ }$ & $\begin{array}{l}\sim \\
\infty\end{array}$ & శ్ & I & $\stackrel{\omega}{v}$ & $\vec{\infty}$ & 0 & \\
\hline$\underset{N}{\tilde{U}}$ & 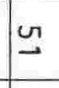 & 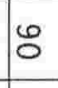 & $\stackrel{\infty}{\rightarrow}$ & $\tilde{\tilde{N}}$ & $\stackrel{\sim}{\sim}$ & $v$ & $\vec{\circ}$ & \\
\hline $\begin{array}{l}N \\
\text { v } \\
\text { ur }\end{array}$ & สั & ळ & $\tilde{\omega}$ & $\stackrel{N}{v}$ & $\tilde{\omega}$ & $\vec{\circ}$ & 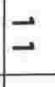 & \\
\hline$\underset{\sim}{N}$ & $\vec{v}$ & $\stackrel{\omega}{\underline{\omega}}$ & $\vec{\sim}$ & ज़ & $\stackrel{\omega}{\underline{\omega}}$ & $\vec{v}$ & $\vec{N}$ & \\
\hline$\underset{\text { viv }}{\sim}$ & 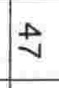 & N & I & ज] & $\vec{\infty}$ & $\vec{\infty}$ & $\vec{\omega}$ & \\
\hline $\begin{array}{l}N \\
\text { uv }\end{array}$ & $\tilde{O}$ & $\underset{\infty}{\omega}$ & $\stackrel{v}{ }$ & vै & $\mathrm{G}$ & $\vec{A}$ & $\vec{A}$ & \\
\hline $\begin{array}{l}\tilde{v} \\
\text { v }\end{array}$ & $\frac{\vec{\infty}}{\infty}$ & ఎ & $\checkmark$ & $\mid \begin{array}{l}\omega \\
\omega\end{array}$ & $\tilde{V}$ & $\vec{\sim}$ & $\vec{v}$ & \\
\hline $\begin{array}{l}\tilde{U} \\
\text { U }\end{array}$ & $\stackrel{\sim}{v}$ & 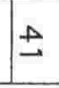 & హె & న & $\vec{\omega}$ & $\tilde{\sigma}$ & $\vec{\sigma}$ & \\
\hline
\end{tabular}

